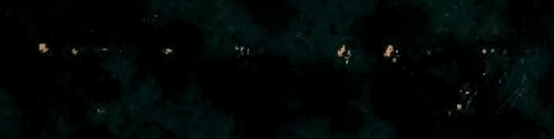

… … 


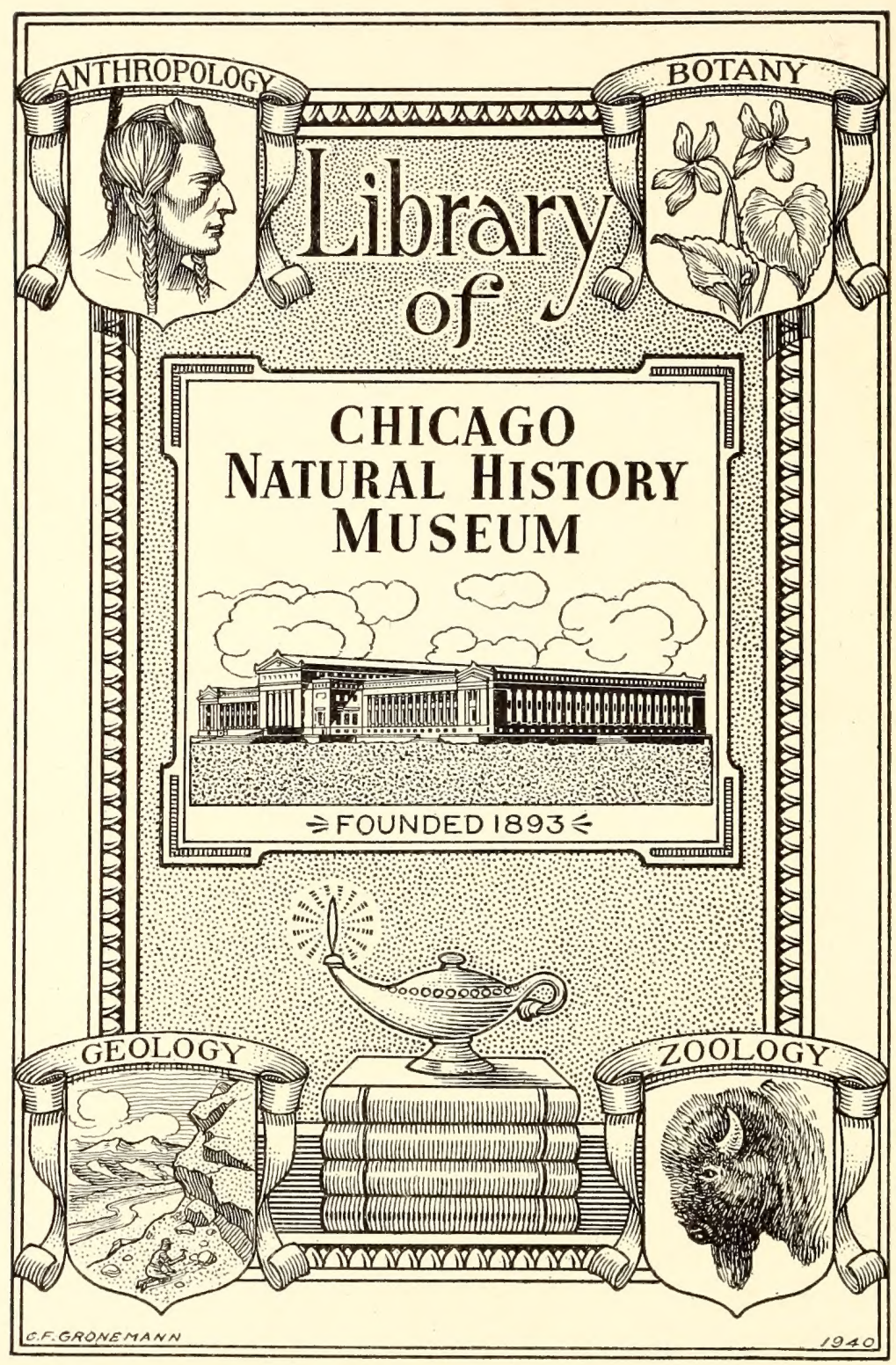





\section{CATALOGUE SYNONYMIQUE}

DES

COLÉOPTÈRES D’EUROPE ET D’ ALGÉRIE. 
Digitized by the Internet Archive in 2011 with funding from

The Field Museum's Africa Council 


\section{LCATALOGUE SYNONYIIOUE}

DES

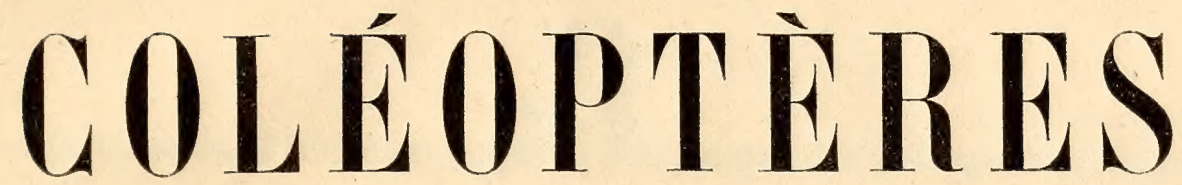

\section{D'EUROPE ET D'ALGÉRIE,}

\section{PAR J. GAUBIL,}

Capitaine de carabiniers au $17^{\mathrm{c}}$ régiment d'infanterie légere, chevalier de la Légion d'Honneur', membre des Sociétés entomologiques de France et de Steltin, membre correspondant de la Société d'histoire naturelle du déparfement de la Moselle, etc.

\section{PARIS,}

MAISON, LIBRAIRE, RUE CHRISTINE, 3.

1849. 


\section{QL \\ $5 \% 1$ \\ 628}

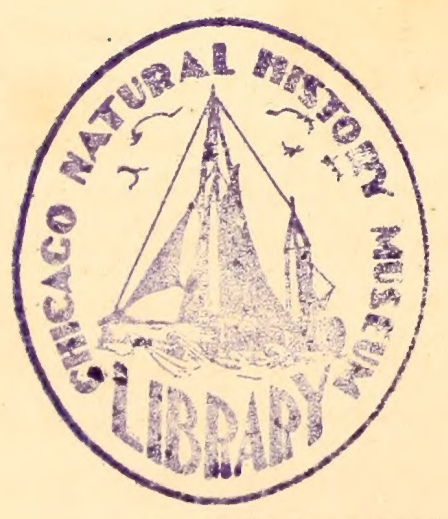

16003 Strasbovt, imprimerie DE G. SLLBERMaNN.

$2.4-63$ Q gunk. Sra?. 


\section{PREFACE}

En publiant aujourd'hui le catalogue synonymique des Coléoptères d'Europe et d'Algérie, je crois devoir exposer les motifs qui m'ont déterminé à cette publication, faire connaitre les principaux ouvrages que j'ai consultés pour mettre mon travail à la hauteur de la science, répondre à quelques observations qui m'ont été adressées et remercier les amis désintéressés qui, par leurs conseils, et la part active qu'ils y ont prise, en m'envoyant les notes que je leur demandais, ont rendu mon travail plus facile, et ont soutenu mon courage souvent prêt à reculer devant les nombreuses difficultés que j'ai rencontrées.

Pendant mon séjour à Paris, de 1841 à 1844 , je m'occupai du classement de ma collection. Aidé par MM. Chevrolat, Guérin et Reiche et gràce à leurs bontés toutes particulières pour moi, je pus, en me servant des monographies publiées jusqu'alors, non-seulement parvenir au but que je m'étais proposé, mais encore recueillir, dans les bons ouvrages qu'ils voulurent bien me confier, un grand nombre de notes qui devaient me servir à continuer le classement de ma colleclion, dans quelque localité que je me trouve. De là à la première idée d'un catalogue, il n'y avait qu'un pas.

Entré en relations d'échanges avec un grand nombre d'entomologistes, je ne tardai pas à ressentir combien il me serait utile d'avoir la synonymie de tous les coléoptères que j'envoyais ou que je recevais. Bien souvent, de différents points de la France ou de l'Allemagne, on m'adressait le même insecte sous quatre ou cinq noms différents; et, cet insecte que j'avais demandé, le plus ordinairement je le possédais moi-même sous un autre nom.

J'entassai donc notes sur notes, je consulıai tons les ouvrages qu'il me fut possible de me procurer, et, à force de travail, j'obtins pour moi un catalogue, qu'à la demande de plusieurs de mes amis et de mes correspondants, j'allais livrer à l'impression lorsque parut le premier cahier de la Fauna austriaca de M. Ludwig Redtenbacher, ouvrage très-remarquable, que plusieurs entomologues distingués qui s'intéressent à la publication de mon livre m'engagèrent à adopter comme guide. 
Si mon catalogue offre quelques défectuosités sous le rapport du classement, on doit l'attribuer à la nécessité où j'ai été d'intercaler des genres et des espèces qui m'étaient inconnus et qui ne se trouvent pas dans la Faune d'Autriche.

Puissé-je avoir réussi à satisfaire les souscripteurs qui m'ont mis à même de couvrir les frais de cette publication.

Plusieurs de ces derniers m'ont demandé pourquoi, à un catalogue de coléoptères d'Europe, je joignais ceux de l'Algérie. Voici ma réponse : C'est que, outre la grande facilité que l'on a de se les procurer, la plus grande partie des espèces algériennes se retrouvent sur le littoral de l'Europe méridionale.

Un mot maintenant à tous les amis de la science qui ont bien voulu me seconder. Que MM. Putzeys, de Bruxelles; Riehl, de Cassel; Schaum, de Stettin; Truqui, de Turin; Chevrolat, Doné, Dupont, Guérin-Méneville, Lucas, Mellié et Reiche de Paris ; Allibert, de Puimoisson ; Billot, de Haguenau; C. Boulard, Colombain et F. Schultz, de Bitche ; Ecoffet et Hochstetter, de Colmar ; Gehin, de Metz; le colonel et le général Levaillant; Mulsant, de Lyon; Ott et Silbermann, de Strasbourg, reçoivent ici le témoignage public de ma sincère reconnaissance. Tous ont contribué à rendre mon travail plus facile, l'un en déterminant les insectes de ma collection, l'autre en me confiant ses travaux inédits; celui-ci en transcrivant pour moi des extraits d'ouvrages que je ne pouvais me procurer ; celui-là en me communiquant les insectes que je désirais examiner. S'il y a quelque chose de bien dans mon travail, il leur appartient tout entier. Mon livre n'est qu'une pierre apportée pour l'édifice que d'autres pourront entreprendre un jour. Puissent-ils y trouver quelques-uns des renseignements que j'ai eu tant de peine à me procurer, et je me trouverai récompensé de mon travail et de mes veilles.

Bitche, 15 juillet 1848. 


\section{AUTEURS CITÉS DANS CE VOLUME.}

Annales de la société entomologique de France. Paris 1832 et suivants. in-8 ${ }^{\circ}$. fig.

Annales des sciences physiques et naturelles d'agriculture et d'industrie, publiées par la société d'agriculture, etc., de Lyon. Lyon 1838 et suiv., gr. in-8 $\mathbf{8}^{\circ}$ fig.

Allibert (Alph.) . . . . Monographie du genre Trichopterix (Revue zoologique, par la société Cuviérienne, publiée sous la direction de M. F. G. Guérin-Méneville. Février 18\$4, p. 51). Aragona (A.) . . . . . De quibusdam coleopteris Italiæ novis aut rarioribus. Tentamen inaugurale Aloysius Aragona Submittit. Ticini Regii 1830. in-8.

Auné (CH.) . . . . . . . . Iconographie et histoire naturelle des coléoptères d'Europe, Hydrocanthares. Paris 1836. 1 vol. in-8o. fig.

Idem. Essai sur le genre Monotoma (Annales de la société entomologique de France. 1836, p. 453).

Idem......... Révision de la famille des Pselaphiens (Annales de la société entomologique de France. 1844, p. 73).

Idem. . . . . . . . Descriptions d'un nouveau genre et de quelques espèces nouvelles, par M. Ch. Aubé (Annales de la socièté entomol. de France, années 1844, p. 227; 1842 , p. 231 et suiv.).

Audinet-Serville . . Nouvelle classification de la famille des Longicornes (Annales de la société entomologique de France, 1833-1835).

Bassi (C.) . . . . . . Description de quelques nouvelles espèces de coléoptères de l'Italie, par M. C. Bassi (Annales de la société entomologique de France, 1834, p. 463 et suiv.).

Idem. . . . . . . Notice sur le genre Cardiomera, nouveau genre de coléoptères de la fämille des carabiques, par M. C. Bassi, de Milan (Annales de la société entomologique de France, 1834, p. 320).

Billberg (E. J.). . . . . Monographia Mylabridum. Holmiæ 1813. in-8o.

Borsduval et Lacondare. Faune des environs de Paris. Paris 1835.1 vol. in-12. Brandt (J. F.), et Erich- Monographia generis Meloe, cum tabula picta, 1831, son (W. F.). .... in-4. (Nova acta curiosorum natura, t. XVİ. Breslau et Bonn 1832, p. 103 à 142).

Baulcé (A.). . . . . Expédition scientifique de Morée, t. III. Zoologie (Arachnides et insectes). Paris 1822, in-4. fig.

Idem. . . . . . . . . Observations critiques sur la synonymie des carabiques (Revue entomologique, par M. G. Silbermann, 18341835, $\mathrm{II}^{\mathrm{e}}$ et $\mathrm{III}^{\mathrm{e}}$ yol.).

Bulletin de la société impériale des naturalistes de Moscou. Moscon 1829 et suiv., in-8. 
BuRveister

Castelnau (de).

Chaudolr (le baron DE) . . Description de quelques espèces de carabiques (Bulletin de la société impériale des naturalistes de Moscou. 1843).

Cinevrolat (A.) . . . . . . Centurie de Buprestides, par M. Aug. Chevrolat (Revue entomologique, par M. G. Silbermann, t. V, p. 41).

Idem.

Description des Mylabrides de Barbarie, par M. A. Chevrolat (Revue entomologique, par M. G. Silbermann, t. V, p. 267).

Idem.

Mémoire sur un coléoptère tétramère de la famille des Xylophages, et observations sur plusieurs espèces de cet ordre rencontrées dans diverses fourmilières, par A. Chevrolat (Revue entomologique, par M. G. Silbermann, t. III, p. 263).

Idem. . . . . . . . Description de quelques coléoptères de la Galicie et du Portugal (Revue zoologique, 1840, p. 8).

ComoltI. . . . . . . De coleopteris novis ac rarioribus minusve cognitis provinciæ Novocomi. Eicini Regii 1837, in-80.

DahL (G.) . . . . . . . (Col. u. Lepida) Coleoptera, ein systematisches Verzeichniss, von G. Dahl. in-8. Wien 1823.

Dejean (le comte) . . . Catalogue des coléoptères de sa collection, troisième édition, revue, corrigée et augmentée. Paris 1837. 1 vol. in-8o.

Dejean (le comte) el Bois- Iconographie et histoire naturelle des coléoptères d'Euduval. . . . . . . . rope. Paris 1829.4 vol. in-8. fig.

Dufour (LÉon) . . . . Description de dix espèces nouvelles ou peu connues d'insectes recueillis en Espagne, par M. L. Dufour (Ann. génér. des sc.phys., VI, p. 307).

Idem. . . . . . . Excursion entomologique dans les montagnes de la vallée d'Ossan. Pau 1843. in-80.

Duftschmidt (K.) . . . . Fauna austriæ, oder Beschreibung der österreichischen Insekten für angehende Freunde der Entomologie. Leipzig 1812-1825. in-8.

Entomologische Zeitung, herausgegeben von dem entomologischen Vereine zu Stettin. Leipzig 1840-1847. 8 vol. in $-8^{\circ}$. fig.

Erichson (W. F.). . . . Naturgeschichte der Insekten Deutschlands. Berlin 1847. 1848.

Idem. . . . . . . . Genera Dyticeorum, etc. Berolini 1842. in-80.

Idem. . . . . . . . Entomographien. Berlin 1840. in-8\%. fig.

Idem. . . . . . . . Die Käfer der Mark. Brandenburg. Berlin 1837-1839. 1 vol. in-8o.

Idem . . . . . . Genera et species Staphylinorum, insectum Coleopterorum familiæ. Berolini 1840. 1 vol. in-8 ${ }^{\circ}$ fig.

Idem

Apercu des Histeroides de la collection du muséum de Berlin, extrait traduit du mémoire de M. W. F. Erichson, inséré dans les Annales de M. Klug (Revue entomologique de M. G. Silbermann. 1835. $3^{\mathrm{e}}$ vol. p. 181). 
Erichson (W. F.). . . . Rapport sur les travaux entomologiques en 1836, par M. W. F. Erichson. Traduit des Archives d'histoire naturelle de M. Wiegmann, par M. G. Silbermann (Revue entomolog., 1837, t. V).

Ferté-Senecterre (de LA). Notice sur le genre Anthicus, par M. de Laferté-Senecterre (Annales de la société entom. de France. 1842, p. 247).

Fischer (G.). . . . . . Entomographia imperii Russici 4. Mosquæ 1820, 1823, 1825. 3 vol.

Fischer (de Waldheim, G.). Entomographia Rossicæ (Bulletin de la société impériale des naturalistes de Moscou, 1844).

GaUbiL (JEAN). . . . . Description de quelques carabiques nouveaux pour la Faune de la France et de nos possessions d'Algérie, par M. Gaubil, officier au $17^{\mathrm{e}}$ léger (Revue zoologique. Septembre 1844 et mars 1846).

Germar (Ern. Fréd.) . . . Zeitschrift für die Entomologie herausgegeben. Leipzig 1839-1844, in-8\%.

GLLmeister . . . . . Trichopterygia, Beschreibung und Abbildung der haarflügeligen Käfer. Nürnberg 1845, bei Jacob Sturm (Sturm, Deutschlands Fauna. Bd. 171).

Gonx et Percheron . . . Monographie des Cetoines. Paris 1833 . in-8\% . fig.

Gory et Laporte . . . . . Monographie des Buprestides. Paris. in-8o. fig.

Idem . . . . . . . Supplément à la Monographie des Buprestides. Paris. in-80. fig.

Gory (M. H.) . . . . . Histoire naturelle et Iconographie des insectes coléoptères ( Monographie des Clytus). Paris 1841 . in-8. fig.

Grells (le docteur) . . . Description de cinq espèces nouvelles de coléoptères d'Espagne (Annales de la soc. ent. de France. 1846, p. 305 et 309 ).

Grimmer (K. H. B.). . . . Steiermark's Coleoptern, mit ein hundert sechs neu beschriebenen Species. Gratz 1841. in-8 ${ }^{\circ}$.

Guérin-Meneville (F. E.). Revue zoologique, par la société Cuviérienne, publiée sous la direction de M. F. E. Guérin-Meneville. in- ${ }^{\circ}$.

Idem . . . . . . . Species et Iconographie des animaux articulés, etc. Paris 1844. 2 livr. fig.

Heer (Oswaldo) . . . . Fauna coleopterorum Helvetica. Turici. 1 vol. in-12.

Hummel. . . . . . . Essais entomologiques. St-Pétersbourg 1821 et sv. in-8².

Isis, oder encyclopädische Zeitung (Recueil dirigé par M. Oken). Leipzig 1817 et suiv. in-8º.

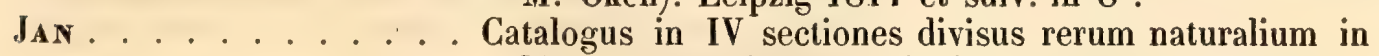
Museo extransium Josephi de cristofori et Georgii Jan. Milano 1832. in-80.

Kцv G (F.) . . . . . . . Jahrbücher der Insektenkunde, mit besonderer Rücksicht auf die Sammlung im köngl. Museum zu Berlin. Berlin 1834. in- $8^{\circ}$.

Küster . . . . . . . . . Die Käfer Europa's, etc. Nürnberg 1844 et suiv.

Kiesenwetter . . . . Beiträge zur Monographie der Gattung Heterocerus, von Kiesenwetter (Zeitschrift für die Entomologie, etc., von Germar. 4 Bände).

Lacordaire (Tн.) . . . . Monographie des coléoptères subpentamères de la famille des Phytophages, par M. Th. Lacordaire (Mémoires de la société royale des sciences de Liége, $\mathrm{t}$. III et V). Liége, Bruxelles, Paris, 2 vol. in-8 ${ }^{\circ}$. 
Lacordatre (Th.) . . . Monographie de la famille des Erotyliens. Paris 1842. 1 vol. in-8.

LAPorte (M. L. F. DE) . . Note monographique sur le genre Zuphium (Revue entomologique, par M. G. Silbermann. Paris, Strasbourg 1833 , t. Ier).

Idem ........ Études entomologiques ou descriptions d'insectes nouveaux et observations sur la synonymie (Revue ent., par M. G. Silbermann, 1837, t. IV, p. 5).

Latreille (P. A.). . . . Histoire naturelle générale et particulière des crustacés et des insectes. Paris 1802-1805. 14 vol. in-8 . fig.

Linnæa entomologica, Zeitschrift herausgegeben von dem entomologischen Vereine in Stettin, Berlin, Posen. 2 vol. $1846,1847$.

Lucas (H.) . . . . Exploration scientifique de l'Algérie pendant les années 1840,1841 et 1842 , publiée par ordre du gouvernement avec le concours d'une commission académique, etc. (Histoire naturelle des animaux articulés, par M. H. Lucas, membre de la commission scientifique de l'Algérie). Paris 1847-1848. 2 vol. in-4º fig.

Mannerheim (le comte) . . Ecunemis, Insectorum genus monographice tractatum iconibusque illustratum. Petropoli 1823. in-8\%

Idem ........ Revue critique de quelques ouvrages entomologiques (Extrait du Bulletin de la société impériale des naturalistes de Moscou. 1838). Broch. in-8º.

Idem . . . . . . . Énumération des Buprestides et description de quelques espèces nouvelles de cette tribu de la famille des Stirnoxes, de la collection de M. le comte Mannerheim. Broch. in-8o (Extrait du Bulletin de la soc. imp. des natural. de Moscou. 1838).

Idem . . . . . . . . Versuch einer monographischen Darstellung der Gattungen Corticaria und Lathridius, von Graf Mannerheim (Zeitschrift für die Entomologie, herausgegeben von Germar. Aus dem $5^{\text {ten }}$ Bande).

Mellí́ . . . . . . Monographie du genre Cis, par M. Mellié (Revue zoologique. Mars 1847).

Ménétriés (E.) . . . . Catalogue raisonné des objets de zoologie, recueillis dans un voyage au Caucase, par E. Ménétriés. in-4º Saint-Pétersbourg 1832.

Idem ......... Catalogue d'insectes recueillis entre Constantinople et le Balcan, par M. Ménétriés (Mémoire de l'académie de Saint-Pétersbourg).

Motschulsky (Victor De). Monographie du genre Georisus (Extrait du Bull. de la soc. imp. des natural. de Moscou. 1843. $4^{\mathrm{e}}$ cah.).

Idem ......... Note sur le genre Trychopteris, Kirby, et Ptilium, Schüppel (Revue zoologique, etc. Mars 1847).

Müllen et Kunze. . . . Müller, P. W. J., u. D. Gust. Kunze, Monographie der Ameisenkäfer (Scydmanus Latr.). 4 M. 1 Kpfrt.

Mulsant (ET.) . . . . Histoire naturelle des coléoptères de France (Longicornes). Paris et Lyon 1839. 1 vol. in-8º fig.

Idem . . . . . . . Hirtoire nat. des coléopt. de France (Lamellicornes). Paris et Lyon 1843. 1 vol. in-8 . fig. - Palpicornes. 1844. 1 vol. in-8 ${ }^{\circ}$. fig. - Sulcicolles et Securipalpes. 1846. 1 vol. in $-8^{\circ}$. fig. 
Paykule (G.) . . . . . Monographia Histeroidum. - Upsaliæ, Paris. 1 vol. in-8 ${ }^{\circ}$. fig.

Pecchroli. . . . . . . Description de deux coléoptères nouveaux d'Italie, par M. Pecchioli (Annales de lasoc. entomol. de France, 1836, p. 445).

Putreys (M. J.) . . . . Prémices entomologiques. Liége 1845. Broch. in-8o.

Idem ......... Monographie des Clivina et genres voisins (Extrait des Mémoires de la société royale des sciences de Liége, t. II). Liége 1846. in-80.

Idem . . . . . . . Broscosoma. Carabidum genus novum, descr. atque. fig. illust. Bruxellis, M. octobre 1846.

Idem ....... Trechorum europæorum conspectus (Entomologische Zeitung). Octobre 1847.

Ratzeburg (Тн. Сн.) . . . Die Forstinsekten (1 partie, les coléoptères). Berlin 1837. in $-4^{\circ}$. fig.

Redtenbacher (Ludwig) . Fauna austriaca, die Käfer nach der analytischen Methode. Bearbeitet von Ludwig Redtenbacher. Wien 1847-1848. 1 vol. in-8 .

Idem . . . . . . . Teutamen dispositionis generum et specierum coleopterorum Pseudotrimemorum archiducatus Austriæ. Vindobonæ 1843. Broch. in-8 ${ }^{\circ}$.

Revue entomologique, publiée par M. Gustave Silbermann. Paris 1833-1837. 5 vol. in-8*. fig.

Schaum (H. R.). . . . Analecta entomologica, Dissertatio inauguralis. Halis, saxonum 1841. in-8 ${ }^{\circ}$.

Idem . . . . . . . Observations critiques sur quelques Lamellicornes melitophiles, par M. le docteur H. R. Schaum (Ann. de la soc. ent. de France, année 1845).

Schiödte (IG. Сн.) . . . . Genera og species af Danmark's Eleutherata. Kjobenhaven 1840-1841. in-8 .

Sснмidt (H. M.) . . . . Dissertatio inauguralis zoologica de Pselaphis Faunæ Pragensis cum anatomia Clavigeri. Pragæ 1836. in-8.

Sснмidт (W.) . . . . . Revision der europäischen Edemeriden, von $\mathrm{D}^{\mathrm{r}}$ Wilh. Schmidt (in Linnoea entomologica, 1845).

Schmidt (Dr J.) . . . Monographie du genre Hoplia (Entomologische Zeitung, etc. Stettin 1840).

Idem .......... Monographie du genre Anthicus (Entomologische Zeitung, etc. Stettin 1842).

Schönherr (C. S.) . . . Genera et species Curculionidum. Paris 1833-1845. $8 \mathrm{vol}$. in- $8^{\circ}$.

Solier . . . . . . Essai d'une division des coléoptères Hétéromères et d'une monographie de la famille des Collapterides, par M. Solier (Annales de la soc. entomol. de France. 1844 et suiv.).

Spinola (le marquis Max.). Essai monographique sur les Clerites. Paris 1846. 2 vol. in- $8^{\circ}$.

Steffahny . . . . Teutamen monographiæ generis Byrrhi. Berolini 1842 (aus Germar's Zeitschrift. in-4\%).

Sturm (J.). . . . . . . . . Deutschlands Fauna Kæfer. Nürnberg 1805-1846. 18 vol. Idem . . . . . . Catalog der Käfersammlung. Nürnberg 1843.1 vol. in-8 .

Suffrian $\left(D^{r}\right)$. . . . . Revision der europäischen Arten der Gattung Cryptocephalus, von Direktor Suffrian in Siegen (Linnoxa en tomologica, etc. Stettin 1817). 
Suffrian $\left(\mathrm{D}^{\mathrm{r}}\right) \ldots \ldots$ Monographie du genre Cassida (Entomologische Zeitung, etc. Stettin 1844).

Truqur . . . . . . . Monographie du genre Amphicoma, par M. Truqui, de Turin, inédit.

Villa (Ant. et J. B.) . . Coleoptera Europæ Dupleta. Mediolani 1833-1838. Broch. in- $8^{\circ}$.

Walth . . . . . . Reise nach dem südlichen Spanien, 2te Ausg. Passau 1839.

Idem . . . . . . . Reise durch Tyrol, Oberitalien u. Piemont nach dem südlichen Spanien. Passau 1835. in- 8 .

Zimmermann . . . . . Mémoire sur le genre Amara, par Christophe Zimmermann. Traduit de l'allemand par M. G. Silbermann (Revue entomologique, etc. $1834.2^{\mathrm{e}}$ volume). 


\section{LISTE DE MM. LES SOUSCRIPTEURS.}

MM.

1. Catala. . . . . Capitaine en retraite, chevalier de la Légion d'Honneur, à Limoux (Aude).

2. Boisgiraud . . . . Doyen de la faculté des sciences de Toulouse.

3. Rient ...... Caissier général, membre de la société éntomologique de Stettin, à Cassel (électorat de Hesse).

4. Mulsant (E.) . . . . . Sous-bibliothécaire de la ville de Lyon, professeur d'histoire naturelle au collége national, membre de l'Académie nationale des sciences, belles-lettres et arts, président de la société Linnéenne de la même ville, etc.

5. Sommer. . . . . . Négociant, memhre de plusieurs sociètés savantes, à Altona, près de Hambourg.

6. Genıs . . . . . Pharmacien, membre de plusieurs sociétés savantes, à Metz (Moselle).

7. Colombain . . . . . Négociant à Bitche (Moselle).

8. Boulard (Gamilee). . Membre de la société entomologique de France, à Bitche (Moselle).

9. Maud'huit ..... Vérificateur des poids et mesures, à Sarreguemines (Moselle).

10. Sig NorET . . . . . Docteur en médecine et pharmacien, membre de la société

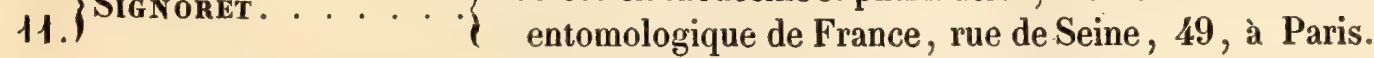

12. Feisthamel (le baron). Maréchal-de-camp, membre fondateur de la société entomologique de France, etc.

13. Paris . . . . . Notaire, membre de la société entomologiqne de France, à Epernay (Marne).

14. Godart . . . . . . Capitaine d'habillement au 67e de ligne, à Lyon (Rhône). 15. Antorne ..... Propriétaire, rue des Farges, 65, à Saint-Just, près de Lyon (Rhône).

16. Busson . . . . Sous-bibliothécaire de la ville du Mans, rue Tascher, 21, au Mans.

17. Mellié . . . . . Vérificateur de l'enregistrement et des domaines, membre de la société entomologique de France, rue MontThabor, 9, à Paris.

18. Thibesard ..... Fondé de pouvoir du receveur général du département de l'Aisne, membre de la société entomologique de France, à Laon (Aisne).

19. Gueneau D'Aumont. . Major au $18^{\circ}$ de ligne, membre de la société entomologique de France, à Paris. 
MII.

20. Jevin . . . . . . . Sous-lieutenant au 9e de ligne, à Alger (Afrique).

21. Va,chonrue (Alex.) . . Grand chemin d'Aix, 42, à Marseille (Bouches-duRhône).

22. De Marseul ...... Chef d'institution, membre de la société entomologique de France, à Laval (Mayenne).

23. Вомравт . . . . . . Naturaliste, à̀ Villefranche (Rhône).

24. Brumet (Johnny ... A Nice (Piémont).

25. Heretieu. . . . . . Inspecteur des contributions directes du département de Tarn-et-Garonne, membre du conseil du département du Lot et de plusieurs sociétés savantes, secrétaire perpétuel de la société agricole et industrielle du département du Lot, à Montauban (Tarn-et-Garonne).

26. Mocquerys . . . . . Chirurgien-dentiste, rue Grand-Pont à Rouen (SeineInférieure).

27. Buquet (LuCien) . . . Naturaliste, attaché au ministère de la marine, trésorier de la société entomologique de France, rue Dauphine, 35, à Paris.

28. Horeau. . . . . . Pharmacien en chef de l'armée d'Afrique, membre de la société entemologique de France, à Alger.

29. Millot ....... Docteur en médecine et pharmacien-major à Alger (Afrique).

30. LaUras . . . . . . Docteur en médecine et pharmacien-aide-major à l'hôpital du Dey à Alger (Afrique).

31. Capiomont ...... Docteur en médecine et pharmacien-aide-major à l'hôpital du Dey, à Alger (Afrique).

32. Movgodin . . . . . Docteur en médecine et pharmacien-aide-major à l'hôpital du Dey à Alger (Afrique).

33. Levaillant. . . . . Général de brigade, à Alger (Afrique).

34. Costa (Achilre) . . Bibliothécaire de l'académie des aspirants des naturalistes de Naples.

35. Truqui (Eugene) . . . Docteur en médecine, à Turin (Piémont).

36. Gadthier. . . . . A Nice (Piémont).

37. Ghevrolat ..... Rédacteur à l'administration de l'octroi de Paris, membre fondateur de la société entomologique de France, etc., à Paris.

38. Levaimlant (C.) . . Colonel du 17e léger, à Phalsbourg (Meurthe).

39. Von Heyden . . . . Sénateur, directeur de la société d'histoire naturelle de Seckenberg, à Francfort-sur-le-Mein.

40. Bertrand . . . . . Archiviste du département des Landes, à Mont-de-Marsan.

41. Naux . . . . . . . Officier au 22e léger, à Lyon (Rhône).

42. Blutel . . . . . . Direeteur des douanes, vice-président de la société des sciences naturelles de La Rochelle, membre de la société entomologique de France, à La Rochelle (Charente-Inférieure). 
MIM.

43. Lucas. . . . . . . Membre de la commission scientifique de l'Algérie, employé au laboratoire d'entomologie du muséum d'histoire naturelle, etc., à Paris.

44. Savove . . . . . . Colonel du 3 e régiment d'artillerie, à Metz (Moselle).

45. Germar. . . . . . . Professeur d'histoire naturelle, à Halle (Saxe).

46. Jour . . . . . . Professeur de zoologie à la faculté des sciences de Toulouse, membre de l'académie des sciences, à Toulouse (Haute-Garonne).

47. Prophète. . . . . Chirurgien-dentiste, à Nîmes (Gard).

48. Puton ....... Propriétaire, à Remiremont (Vosges).

49. Billor . . . . . Professeur des sciences physiques au collége de Haguenau (Bas-Rhin).

50. Dakbas . . . . . Juge de paix à Wintzenheim, à Colmar (Haut-Rhin).

51. Rue de Metz, 5, à Nancy (Meurthe), et chez le général 52. Whare (Adolphe) $\ldots\left\{\begin{array}{l}\text { Lewaschoff, près le jardin d'été à Saint-Petersbourg } \\ \text { (Russie). }\end{array}\right.$

53. Grúé (Marius) . . . C Cours de Villiers, 24, à Marseille (Bouches-du-Rhône).

54. Küren bU Rg (le comte). . Assesseur de la direction des mines et salines de Hall, en Tyrol.

ร̌̆. Levoiturier . . . . Membre de la société entcmologique de France, à Orival, près d'Elbeuf (Seine-Inférieure).

56. Nandon . . . . . Chez M. Buquet, à Paris (Seine).

57. Marson . . . . C Chez M. Buquet, à Paris (Seine).

58. Bottée de Toulmon. Chez M. Buquet, à Paris (Seine).

59. Lacordatre .... . Professeur de zoologie et d'anatomie comparée à l'université de Liége, etc., à Liége (Belgique).

60. Pfliiger . . . . . Registrateur, à Hildesheim (Hanovre).

61. Silbermany (G.) . . Imprimeur, l'un des administrateurs du muséum d'histoire naturelle de Strasbourg, chevalier de la Légion d'Honneur, etc., à Strasbourg (Bas-Rhin).

62. Chassun (DE) . . . . Capitaine adjudant de place, à l'ìle d'Oléron (CharenteInférieure).

63. Grandin . . . . Officier au $\mathbf{7 e}^{\mathrm{e}}$ chasseurs, à Huningue (Haut-Rhin).

64. Racine . . . . . . Horticulteur, à Dieppe (Seine-Inférieure).

65. Domergue de Saint-

Florent . . . . . Propriétaire, à Vandœuvre près de Nancy (Meurthe)

66. Renard. . . . . . A Saint-Quentin (Aisne).

67. Meckenueim (le bn DE). Faubourg Chartrain, 44, à Vendôme (Loir-et-Cher).

68. Pages (François) . A Nimes (Gard).

69. Doü̇́ . . . . . . Ancien chef de bureau au ministère de la guerre, officier de la Légion d'Honneur, etc., rue de l'Ancienne-Comédie, 13, à Paris.

71. Pierret (Alexandre). Rue Corneille, 3, à Paris. 
MM.

72. Blanchard ..... Major au $2^{\mathrm{e}}$ régiment de spahis, à Misserghin, province d'Oran (Algérie).

73. Écoffet . . . . . Directeur des contributions indirectes du département du Haut-Rhin, à Colmar.

74. OTr . Orfèvre, à Strasbourg (Bas-Rhin).

75. Goubert (Léon). . . Employé à la direction des tabacs, à Strasbourg (BasRhin).

76. Motschoulski (V. De). Capitaine, à Moscou.

77. Moye (G. E.) . . . E Étudiant en médecine, à Dieuze.

78. Arias Teijérro . . Ancien magistrat espagnol, place de la Gendarmerie, à Beaune (Côte-d'Or).

79. Rouson . . . . . Capitaine au 5́7 $7^{\mathrm{e}}$ de ligne, au Panthéon, à Paris (Seine).

80. Bottée de Toulmont. Chez M. Buquet, à Paris (Seine).

81. Degland . . . . . . Chez M. Buquet, à Paris (Seine).

82. Millet . . . . . . Chez M. Buquet, à Paris (Seine).

83. Roton (DE) . . . Capitaine de carabiniers au 17 e léger.

81. Perragallo . . . Employé chez le directeur des contributions indirectes, à Beaune (Côte-d'Or).

85. Fairmaire (Léon) ... Rue Ménars, 14, à Paris.

87. Grä́cls . . . . . Chef-directeur du muséum royal des sciences naturelles de Madrid.

88. Bonand. . . . . . Chirurgien en chef de l'hôpital de Calais, chevalier de la Légion d'Honneur, à Calais (Pas-de-Calais).

89. Foudras . . . . . Rue Sainte-Catherine, 9, à Lyon (Rhônej.

90. Perroud ..... . Rue Saint-Pierre, 23, à Lyon (Rhòne).

91. Rouget . . . . . . A Dijon (Côte-d'Or).

92. Garder. . . . . . Conservateur du muséum de la ville de Saint-Étienne, rue de la Bourse, 10, à Saint-Étienne (Loire).

93. Paparel . . . . Percepteur des contributions à Chasseredes (Lozère). 


\section{CATALOGUE SYNONYMIQUE}

DES

\section{COLÉOPTÈRES D'EUROPE ET D'ALGÉRIE.}

(Les espèces suivips d'une étoile font partie de ma collection.)

\section{FAM. CICINDELA.}

\section{TETRACHA. Guérin.}

Mggacephala. Latr. Gicindela. Fabr.

1 Euphratica. Olivier. Hisp. merid. Algiriana. Blanchard.Algiria.

GICINDELA. Linné.

1 (Concolor. Dej. Rouxii. Barthelemy. id.

Erea. Chevrolat. id.

2 Ismenia. Buquet.

3 Luctuosa. $D e j$.

4 Maura. Fabr.

Var. Arenaria. Fab. Hispania.

Sicula.Redtenbacher. Sicilia.

5 Saphirina. Géné. Sardinia.

6 Campestris. de Geer.

Var. Afinis. Baber. Sibiria.

- Marocana. Fab. Gall. merid.*

- Connata. Heer. Helvetia.

- Nigrescens. Heer. id.

- Farellensis.

- Guadaramensis.

$$
\text { Graells. Hispania. }
$$

Gralls. id.

- Nigrita. Dej. Corsica.

- Funebris. Sturm. Germania.

7 Decempunctata. Brullé. Gracia. ${ }^{*}$

8 Desertorum. Baber. Russ. merid.

9 f Peletieri. (Dej.) Lucas. Algiria.*

Ritschii. Lucas. id.

10 Audouinii. Barthélemy. (Laphyra Dup.) Algiria.*
11 Hybrida. Linné. Fabr. Gallia.* Commixta. Schönherr. Suecia. Integra. Sturm. Germania.

Monticola. Ménétriés. Russ.merid. Var.Riparia. (Megerle) Dej. Gallia. Aprica. Stephens. Anglia. Var. Maritima. Dej. Gall.bor. Altaïca. Gebler. Sibiria. Variet. Transversalis. Zieg. Dej. Austria.

12 (Sylvicola. (Meg.).Dej. Gall, orient. Hybrida. Duflschm. Austria.

13 Gallica. Brullé. - Gall. merid.* Alpestris.Heer. Helvetia. Chloris. Dej. Gall.merid. Var. Bilunata.Heer. Helvetia. Burmeisteri. Gistl. Germania.

14 Soluta. (Megerle). Dej. Hungaria.* Savranica. Besser. Volhynia. Insignis. Mannerh. Russ, merid. Desertorum. Ménétriés.id.

15 Sylvatica. Fabr. $\boldsymbol{P}^{*}$

16 Sturmii. Ménétriés. Russ. merid.

17 (Viennensis. Schrank. Austria.*

Sinuata. Panzer. Dej.id.

Litterata. Fuessly. Gistl. id.

18 Trisignata. Illiger. Gall, merid.* Infracta. Megerle. Illyria. Pavefacta. Schönherr. Barbar a.

19 Litterata. Sulzer. Gall. orient. * Lugdunensis. Dej. id. Sinuata. Clairville. Helvetia. Var. Padana. Cristof. Italia.

20 Strigata. Dej. Russ, merid. 
21 Cohærensis. Sturm. Cat. id.

22 Chiloleuca. Fischer. Podolia.

23 (Besseri. Dej.

Tibialis. Besser.

Russ, merid. id.

24 Intricata. Schönherr. Barbaria.

25 Volgensis. Besser. Dej. Russ . merid.

Elegans. Fischer.

id.

Stigmaphora. Fischer.id.

Var.Imperialis. Dahl.Italia.

26 Littorea. Forskal. Algiria.*

Goudotii. Dej.

Cruciata. Dahl.

27 Circumdata. $D e j$.

Sardinia.

id.

28 Dilacerata.(Parr.) Dej.Grocia.

Angulosa. Olivier. id.

29 Contorta. Steven. Russ. merid.

Tortuosa. Falderm. Turcomania.

Figurata. Chaudoir. Russ. merid.

30 (Melancholica. Fabr. Sicilia.*

$\{$ Egyptiaca. Klug.Dej. Egypt.

Punctum. Drapiez. Arabia.

31 Fasciatopunctata.Ger-

mar. Russ. merid.

32 Fischeri,Adams, Germ, id.

33 Infracta. Meg. St. Cat. Istria.

34 (Littoralis. Fabr. Algiria.*

Barbara. var, de Castelnau. id.

35 (Nemoralis. Olivier. Gall. merid. Littoralis. Dej. id.

$\{$ Lulunata. Fischer. Russ. merid.

Var.Discors.Meg. Dej. Dalmatia.

Barthelemyi. Dupont. Algiria.

36 Agnata. Sturm. Cat. Barbaria.

37 (Flexuosa. Fabr. Gall. merid. ${ }^{*}$

Var. Sardoa. Dej. Sardinia. ${ }^{*}$

- Circumflexu.Dej.Sicilia.

38 Lyonii. Vigors. Barbaria.

Latreillei. Dej. id.

39 Nitidula. Dej. Algiria.

40 (Distans. Fischer. Dej. Russ. merid.

Infuscata? Pallas. id.

41 Zwickii. Fischer. Dej. id.

42 Steveni. Dej. id.

43 (Paludosa. Dufour. Hispania.

Scalaris. Dej. : Gall. merid.

Equestris. Bonelli. Col. d'oliv. id:

44
45 Gracilis. Pallas.

$\{$ Var. Tenuis. Steven. Angustata. Fischer. Dahurica. Mannerh. id.

46
Sibiria.

Russ, merid.

Sibiria.
2. FAII, CARABI.

ELAPHRUS. Fabr.

1 Uliginosus. Fabr. Gallia.*

2 (Cupreus. Duftschmid. id.*

Uliginosus. Gyllenhal. Suecia.

Riparius? Olivier. Gallia.

Borealis. Andersch. Finlandia.

3 Lapponicus. Gyllenh. Lapponia.

Var. Elongatus. Esch. Kamtschatka.

4 Arcticus. Schönherr. Lapponia.

5 Splendidus.(Esch.) Dej.Pyrenais. *

6 Ulrichii. Schmidt. Redt.Carniolia. ${ }^{*}$

7 Riparius. Fabr. Suecia.*

$\{$ Paludosus. Olivier. Gallia.

8 Aureus. Muller. Germania.*

$\{$ Littoralis.(Meg.) Dej. Hungaria.

BLETHISA. Bonelli.

Nebria. Gyll. Garabus. Fabr.

1 Multipunctata. Linné. Gallia.*

Var. Aurata. Esch. Kamtschatka.

2 Eschscholtzii. Zubkoff.Russ. merid.

3 Arctica. Gyllenhal. Lapponia.

PELOPHILA. Dejean.

Blethisa. Bonelli. Nebria. Gyll. Carabes. Fabr.

1 (Borealis. Fabr. Suecia. Var. Aretica. Schönh. Lapponia. Dejeanii. Gebler. Sibiria.

\section{NOTIOPHILUS, Duméril.}

Elaphrus. Fabr. Gicindela. Livné.

1 Aquaticus. Iinné. Fab.
Dej.P.*
(Var. Astuans. Stéven. Russ. merid.
2 Palustris. Duftsch.Er. Austria.*
3 (Semipunctatus. Fabr. Germania.*
Var. Piguttatus, Fabr.
Dej. $P$. 
4 Quadripunctatus. Fab. . $^{*}$

5 Pallipes. Careno. Hispania.

6 Geminatus. Dej. … id.*

7 Obsoletus. Careno. id.

OMOPHRON. Latreille.

Scolitus. Fabr.

1 Limbatum. Fabr. P. ${ }^{*}$

2 Variegatum. Olivier. Hispania.*

NEBRIA. Latreille. Bonelli.

Carabus. Fabr. Alpezus. Bonelli.

1 Complanata. Linné. Gall. merid.* Arenaria. Fabr.

2 Livida. Linné. \{ Sabulosa. Fabr.

3 Lateralis. Fabr. Sabulosa. var. $\beta$. Algiria. Austria.* id.

\section{Schönherr.}

Livida.var. b. Gyll. id

4 Psammodes. Rossi. Gall. orient.*

5 Schreibersii. (Dahl.)

Dej. Sicilia. ${ }^{*}$

6 Rubicunda. Schönh. Algiria.*

7 Picicornis. Fabr. Gall. orient. ${ }^{*}$

Erythrocephala.Panz.Germania.

8 Orsini. Villa. Lombardia.

9 f Genei. Dej. $\quad$ id.

Pectoralis. Gené. id.

10 Brevicollis. Fabr. P.*

11 Lugdunensis. Chaud. Gall. orient.

12 Andalusia. Rambur. Hisp.merid. ${ }^{*}$ Yariabilis. Lucas. Algiria.

13 ? Barbara. Chaudoir. id.

14 Hyperboreus. Gyllenh. Lapponia.* A Aretica. Dej.

15 Nivalis. Paykull Gapponia*

\section{Balbi. Bonelli. Helvetia.}

16 Pareyssii. Chaudoir. Pedemont.

17 (Gyllenhalii. Schönherr. Gall.orient. ${ }^{*}$

Var. Jockischii. Duft. Styria.

- Balbi. Bonelli. Pedemont.

18 Höphneri. Dahl. Heer. Helvetia.

19 Heegeri. Dahl.Dej. Hungaria.

20 Jockischii. Sturm.Dej. Pyr. orient. * $\{$ Gyllenhalii. Duftsch. Germania.

21 Heydenii. Parreyss. Corfou.

22 Kratteri. Kollar. Ital. merid.

23 Faldermanni. Ménétr. Russ. merid.

24 Exarata. Fischer. id.

25 (Dahlii. Duftschmid. Corinthia.

I Var. Littoralis. Bonel. Italia.
26 Turcica. Chaudoir. Turcia.

27 Tibialis, Bonelli. Italia.

28 Rubripes. Beaud.Laf. Gall.orient.

29 Olivieri. Dej. Pyr orient.

30 Leistoides. Lasserre, Helvetia.

31 Reichii. Dahl. Dej. Hungaria. ${ }^{*}$

32 Laticollis. Bonelli. Pedemont.

33 Cordicollis. Chaudoir. Alp. helvet.

34 Escheri. Heer. Helvetia.

35 Germari. Heer. ... Tirolis. *

36 Fossulata. Lasserre. Helvetia. * Crenatopunctata.Bon.id.

37 Lafrenayei. Dej. Pyrenceis. ${ }^{*}$

Gagates. Bonelli. Pedemont.

38 Foudrasii. Dej. G Gall. orient. ${ }^{*}$

39 Hellwigii. Panz. Duft. Germania.*

40 Stigmula. Dej. Styria. ${ }^{*}$

41 Fasciatopunct.Schiod. Carniolia. ${ }^{*}$

42 Dejeanii. Ziegler. Styria.

43 Transylvanica.Kol.Dej.Transylvan.

44 Femoralis. Chaudoir. Gallizia.

45 (Castanea. Bonelli. Dej. Helvetia.*

Var. Umbrina. Germ. id.

-- Picea. Dej. id. ${ }^{*}$

- Concolor. Bonel. id.

Ferruginea. Bonelli. id.

46 Depressa. Lasserre. id.

Planiuscula? Chaud. id.

47 Brunnea. Duftschmid. Styria.* $^{*}$

Ferruginea. Sturm. id.

(Var. Fotida. Gysselen. Austria.

48 Atrata. Dej. Styria.

49 Jillæ. Dej. Pedemont.

Lafrenayei. Villa. id.

50 Angustata. Dej. Helvetia.

๖1 Angusticollis. Bonelli. id.*

52 Intricata. Stéven. Russ. merid.

53 Marschallii. Stéven. id.

54 Bremei. Heer. Helvetia.

55 Chevrierii. Heer. id.

56 Fulviventris. Bassi. Italia.

57 Junkii. Sturm. Germania.

\section{PTEROLOMA. Schönherr.}

1 Forstromii. Gyllenhal. Suecia.

LEISTUS. Frœhlich.

Pogonophorus. Latr. Carabus. Fabr. Manticona. Jurine. Panzer.

1 (Spinibarbis. Fabr. Gallia.*

Cœruleus. Latreille. $\boldsymbol{P}$.

Pallipes. Panzer. Germania. 
2 Rufipes. Chaudoir. Turcia.

3 Fulvibarbis. Hoffmsg.

Dej. Gall. merid.*

4 Rufomarginatus. Duft. Austria.

5 Nitidus. Duftschmid. Pyrenais.

6 Rhæticus. Heer. Helvetia.

7 (Spinilabris. Fabr. Gallia bor.*

Rufescens. Clairville. Duft. Schh. Germania.

8 (Præustus. Fabr. Gall. bor.* Rufescens. Fabr. Latr.

Terminatus. Panz.Dej. id.

9 Analis $^{1}$. Fabr. Dej. Holsatia.* Frœhlichii. Duft. Dej. Styria.

10 Angusticollis. Dej. Hispania.

CYCHRUS. Fabricius. Dej.

Carabus. Olivier. Tenebrio. Linné.

1 Angustatus. Hoppe. Carinthia.

2 Italicus. Bonelli. Dej. Alp. helvet. ${ }^{*}$ \{ Rostratus. Petagna. Italia.

3 Bovelinii. Heer. Alp. helvet.

Rotundicollis. Villa. Italia.

4 Schmidtii. Chaudoir. Laybach.

5 Rostratus. Lin. Fabr. Gallia bar.*

Caraboides. Linné. Suecia.

Var. Angustatus. Dahl. Hungaria.

- Convexus. Meger. Austria.

Coadunatus, de Geer. Suecia.

Var. Elongatus. Dej. Gallia bor.*

6 Semigranosus. Dahl.

Cat. Dej. Hungaria. ${ }^{*}$

7 Pygmæus. Chaudoir. Austria.

8 Attenuatus. Fabr. Gallia bor.* Proboscideus. Olivier.id.

9 Var. Intermedius. Heer. Helvetia. Cordicollis. Chaudoir. Tirolis. Glacialis. Cristofori. id.

10 Eneus. Fischer. Russ, merid.

\section{PROCERUS. Megerle. Dej.}

Carabus. Fabr.

1 Gigas, Creutzer. \{ Scabrosus. Dej.

Carniolia. ${ }^{*}$

id.

2 Duponcheli. Dej.

3 ( Scabrosus. Olivier. Olivieri. Dej.

Gracia.

Constantin. id.

1 Cette espece a élé trouvée par moi anx environs de Phalsbourg, où elle est très-rare.
4 Tauricus. Pallas. Dej. Russ. merid.

5 Vicinus. Friwaaldsky. Constantin.

6 Caucasicus. Adams.

Dej. Russ. merid.

\section{PROCRUSTES. Bonelli.}

Carabus. Fabr.

1 Coriaceus. Linné. $P^{*}$

$\{$ Var. Spretus. Dej. Dalmatia.

- Rugosus. Dej. id.

2 Foudrasii. Solier. Grocia.*

3 Cerisii. Dej. id.

4 Banonii. Dupont. Dej. id.

5 Græcus. Dej. id.*

6 Turkei. Erichson. Constantin.*

CARABUS. Linné. Fischer.

Tachypus. Weber. Plectes. Techenus. Fischer.

1 Colatus. Fabr. "Carniolia.

2 Dalmatinus. Duftsch. Dalmatia.*

3 Croaticus. Dej. Croatia.*

4 Lucasii. Gaubil. inlitt. Algiria.

Rugosus. Lucas. id.

$5 \quad$ Illigeri. Dej. Croatia.

6 Kollari. Dahl: Dej. Hungaria.*

7 Geneii. Dej. Sardinia.

\& (Scheidleri. Fabr. Austria. ${ }^{*}$

Stenzii. Villa. Hungaria.

Virens. Sturm. id.

Var, Purpurat. Sturm, id.

- Coruleus. Panz. Austria.

9 Preyssleri. Duftschm. id.*

10 Rothii. Kollar. Dej. Transylvan.*

11 Excellens. Fabr. Podolia.*

\{oldeggii. Duftschm. Russ, merid.

12 Erythromerus. Stéven.

Dej. id.

13 Hoffmanni. Falderm. Podolia.

14 Estreicheri. Bes. Dej. id.*

15 Zawadskii. Dej. Gallizia.

16 Adoxus. Stéven. Russia.

17 Krynickii. Fischer. id.

18 (Scabriusculus. Olivier, Hungaria.*

Agrestis. Creutzer. id.

Var. Erythropus. Zieg, Volhynia.

19 Lippii. Dahl.Dej. Hungaria.

20 Maillei. Solier. Algiria.*

Peletieri. Laporte. id.

Microcephalus, Dup.-id. 
21 Faminii. Dej. Sicilia.*

22 Alyssidotus. Illig. Dej.Gall. merid.

23 Ramburii. Dej.

Corsica.

24 Widmanni. Ménétriés. Constantin.

Geoffroyi. Dej. id.

25 Acuminatus. Ménétr. id.

26 Bonplandii. Ménétriess. id.

27 Rossii. Bonelli. Italia.*

28 Concolor. Fabr. Carinthia.

Mollii. Dej. id

Carinthiacus. Sturm. var. id.

Catenulatus. Fabr. Gallia.*

Intricatus. Olivier. $P$.

Var. Harcynia. Sturm. Germania.

- Cyanescens. Stm. id.

- Ausonius.Ziegler. Styria.

- Duponcheli. Dej. Gall. merid.

- Austriacus. Sturm. Austria.

Herbstii. Dej.

Croatia.*

Ahenus. Sturm.

Dalmatia.

Errans. Gory.

Croatia.*

Catenatus.Meg. Panz. Carniolia. ${ }^{*}$

Dufourii. Dej.

Hisp. merid.*

Pareyssii. Kollar.

Croatia. $^{*}$

Hohlbergi. Mannerh. Russ, merid.

Bohemannii. Mannerh. id.

38 Monilis. Fabr. P. ${ }^{*}$

Catenulatus. Olivier. id.

Var. Affinis. Panzer.

Schmidt. Germania.

- Consitus. Panzer. P.*

- Oblongus.Sturm. Germania.

- Regularis. Knoch. id.

- Kronii. Hoppe. id.

- Granulatus. Oliv. P.

- Morbillosus. Latr. id.

Arvensis. Fabr. Gallia. ${ }^{*}$

Rupicola. Jurine. Helvetia.

Var.Pomeranus Oliv. Germania.

- Seileri. Heer. Helvetia.

Ereus. Ziegler. Styria.

Schrikellii. Dej. . id.

40 Varians. Fischer.

41

42

43

44

45

46

47

48

49

Russ. merid

Deyrolii. Rambur. Lusitania. ${ }^{*}$

Cristoforii. Spence. Pyrenceus. ${ }^{*}$

Cordatus. Sturm. Hispania.

Sacheri. Zawadski. Galizia.*

Euchromus. Palliardi. Hungaria. ${ }^{*}$

Montivagus. Pall. id. ${ }^{*}$

Gall. merid.*

Pedemont. *
50 Castillianus. Dej.

52 Guadaramanus (de

Brême). de Laferté.

53 Lusitanicus. Fabr.

Antiquus. Dej.

53. ( Schaumii. Gaubil. Lusitania. *

Lusitanicus. Dej. Icon. id.

54 Latus. Dej.

55 Complanatus. Dej. id.

56 Brevis. Dej. id.

57 Helluo. Bonelli. Dej. id.

58 Mor:illosus. Fabr. Corsica.*

Alternans.Pallas. Dej.id.

Var. Servillei. Solier. Sicilia.

(- Mittrai. Solier. Algiria. ${ }^{\star}$

59 Celtibericus. Illiger. Lusitania.*

Taganus. Schneider. id.

60 Rugosus. Fabr. *Hisp. merid.*

\{ Barbarus. Dej. id.

61 Numida. Le Laporte. Alyiria.*

Varvasi. Solier. id.

Gerardii. Buq. inlitt. id.

62 Cancellatus. Illiger. Gallia.*

Granulatus. Fabr. Germania.

Var. Merlachii. $\left(D a h^{\prime}\right.$.) Hungar $a$.

- Tuberculatus. (Me-

$$
\text { gerle.) Germania. }
$$

- Excisus. (Meg.) Austria.

- Nigricornis.(Zieg.) Gallia.*

- Verrucosus.(Ziegl.) Helvetia.

- Saproniensis. Oes-

kay. Hungaria.

- Fuscus.Palliardi. Helvetia.

- Sciticus. Kollar. id.

- Dahlii. (Megerle.) Lombardia. ${ }^{\star}$

63 Emarginatus. Duft. Carniolia.*

64 Graniger. (Dahl.) Palliardi. Dej. Hungaria.*

65 Intermedius. Dejean. Dalmatia.

66 Ulrichii. Germar. Germania.*

Morbillosus.Panz.Dej.id.

Var. Fastuosus. Pall. Hungaria.

Cumanus. Stév. Fisch. Russ. merid.

Vagans. Olivier.
Granulatus. Linné. Gallia.*

Cancellatus. Fabr. id.

Var. Interstitialis.

$\{$ Duftschmid. Carinthia.

- Assimilis. Duft. Austria.

- Palustris. (Dahl.) Italia.

- Leander. Ménétr. Russ. bor.

68 Schartowii. Heer. Helvetia.

69 Ménétriesii. Falderm.

Fisch. Russ, bor. 
Sodulosusi. Fabr. Dej.Holsatia * $^{\star}$

Variolosus. Fabr. Germania.

Weigelii. Panzer. Gallia orient.

73 Auratus. Fabr. $P$. *

Var.Honoratii.Banon.

75

Lotharingus. Dej.

Dej. Alp. gaill.*

76

77

78

79

80

Punctatoauratus. Dej. Pyr. orient. *

Farinesii. Dej.

Festivus $D e j$.

id.*

Escheri. Dahl. Dej.

f Auronitens. Fabr.

$\{$ Var. Atratus. Heer.

Gall. merid. *

Helvetia. *

Gall. bor.*

Helvetia.

Hispania.

81 Solieri. Dej.

82 Nitens. Linné. Fabr. Gall. merid. .

83 Melancholicus. Fabr. Gall. merid.*

84 Gallæcianus. Gory. Hispania.

85 Exaratus. Stephens.

Adams. Russ. merid. .

86 Dejeanii. Stév. Fisch. id.*

87 Stæhlini. Adams. id.

88 Purpurascens. Fabr. $\int_{\text {Dej. } P . *}$

Yar. Crenatus. Ziegler. Sturm. Austria.

89

90

91

92

Helveticus. Heer. Helveiia.

Cantabricus. Rambur. Hispania.

Lateralis. Chevrolat. id.?

Violaceus. Lin. Fabr. Germ. bor.*

Var. Glabrellus. Megerle. Dahl. Austria.*

Andrejuscii. Fischer. Volhynia.

Wolfi. Dahl. Hungaria.

Var. Candisatus.Duft.

St. Styria.
- Azurescens. Zieg-
ler. Dej. Croatia.
- Germarii. Sturm.
Dej. Carinthia.*
- Exasperatus. Dft.
Dej. Gall. orient.

93 Sphodrinus. Fischer. Russ. merid.

94 Aurolimbatus. Mann. id.

Var. Castaneipennis.

Ménétriés. id.

1 Ce carabus que j'ai pris abondamment aux environs de Phalsbourg, avait été déjà rencontré près de Neuwied sur les hords du Rhin, dans la vallée de Sainte-Marie-aux-Mines et sur les bords de I'Isere.
95 Neesii. Hoppe. Dej. Carinthia.* Yar. Kunzei. Heer. Helvetia. - Lavigatus.Dej. Styria.

96 Parallelus. Fischer. Russ, merid.

97 Marginalis. Fabr. Russia.

$\{$ Violaceus, var, b.

Schh. Berolinensis.

Chrysochlorus. Fisch. Russ. merid.

98 Glabratus. Fabr. Holsatia.*

$\{$ Violaceus. Müller. Germania.

99 Cribratus. Adams.

Fischer. Russ, merid.

100 Perforatus. Fischer. id.*

101 Calleyi. Fischer. id.

Y Var.Prasinus, Ménét. id.

102 Mingens. Stév. Dej. id. *

Mcotis. Stéven. id.

Hungaricus. Dahl. Hungaria.

103 Vomax. Schh. Dej. Russ. merid.

Gastridulus. Fischer, id.

104 Hungaricus. Fabr. Hungaria.

105 Græcus. Dej. Gracia.*

106 Carceli. Gory. id.

107 Tamsii. Ménétriés. Russ. merid.

108 Morio. Mannerheim. id.

\{ Smyrnensis, Dupont, Oriente.

109 Trojanus. Dej. Gracia.

110 (Bessarabicus. Stéven.

Dej. Russ. merid.

111

. id.

(Bosphoranus. Stëven.

Dej. id.*

Var. Campestris. Bes. Podolia.*

112

Sibiricus. Bæeber. Russ. merid.

113 Besseri. (Zieg.) Dej. Podol. mer.*

114 Concretus. Fischer. Russ. merid.

115 (Campestris.Stév.Dej.id.

Var.Pallasii. Stéven. id.

Perrini. Falderm. id.

116 Ledeburii. Fischer. Russia.

117 Agnatus. Sturm. Cat. id.

118 Karelini. Fischer. Russ. merid.

119 Nemoralis. Müller. Gallia.*

$\{$ Hortensis. Fabr. Dej. id.

120

121

122

Monticola. Dej. Gall. merid

Ghiliani. de Brême. Lusitania.

(Dilatatus. (Zieg.) Dej. Carinthia.*

Illyricus. Sturm. Illyria.

Funkii. Hoppe. id.

123 Convexus. Fabr. P.

124 Egesippii. de Laferté. Lusitania.

125 Hornschuchii. Hoppe Carinthia.

$\{$ Baldensis, de Cristof, Lombardia. 
126 Preslii. (Parr.) Dej. Gracia.

127 Hortensis. Linné. Suecia.*

$\{$ Gemmatus.Fabr.Dej.Germania.

128 Hoppei. Sturm. Dej. Carinthia.* ) Var.Alpestris. Ziegl. Styria.

- Alpinus. Villa. Lomb. alp. -Castanopterus.Vl.id. Sylvestris. Fabr. Gallia. ${ }^{\star}$ Arvensis. Olivier. Germania. Nivosus. Lasserre. Helvetia Nivalis. Godet. Heer. id. Var. Alpestris. (Ziegler.) St. Germania. - Angustatus. Heer. Helvetia.

130 Alpinus. Bonel. Dej. Alp. Gall.* Conspicuus. Sturm. Italia.

131 Latreillei.Bonel. Dej. Helvetia. * 132 Riedlii. Ménétriés. Russ. merid. 133 Implicatus.Stm.Cat. Italia. 134 Opacus. Sturm. Cat. Carinthia. 135 Linnei.Megerle.Panzer. Dej. Hungaria.* Var. Scopoli.Ziegler.Carniolia. - Macaieri. Dahl. Hungaria. - Angustatus. Pnz.Podolia.

136 Splendens. Fabr. Pyrenceis. * Carolinus. Fabr. id.

137 Rutilans. Fabr. Pyr. orient.* 138 Hispanus. Fabr. Gall. merid.* 139 Intricatus. Linné. Gallia bor. ${ }^{*}$ $\{$ Cyaneus. Fabr. Dej. Germania. Var. Gigas. Heer. Helvetia.

140 Lefebvrei. Dej. Sicilia. ${ }^{*}$ 141 Creutzeri. Fabr. Carniolia. ${ }^{*}$ 142 Depressus. Bonelli. Helvetia.* Var. Peyrolerii.Jan. Lomb. alp. - Intermedius.

Heer. Helvetia.

- Bonellii.Sturm. Carinthia.

143 Stevenii. Ménétriés. Kuss. merid.

144 Biebersteinii. Ménét. id.

145 Deplanatus. Fischer. id.

146 Fabricii. Meg. Panz. Helvetia. ${ }^{*}$

Var. Heerii. Germar. id.

- Bugnionii.Chaud. Alp. Helvet.

147 Boberi. Adams. Fisch. Russ. merid.

148 f Kircheri. Germar. Tirolis. $^{*}$ Parvicollis. Sturm. id.

149 Irregularis. Fab. Dej. Holsatia.* Var. Sculptis. Andr. Heer. Helvetia.
CALOSOMA. Weber.

Carabus. Linné. Calistuenes. Fischer.

1 Sycophanta. Linne. Gallia.*

2 (Inquisitor. Linné.Fabr.id.*

Y Var. Cupreum. Dej. Russ, merid.

Reticulatum. Stéven. id.

3 Indagator. Fabr. Oliv. Gall merid. ${ }^{*}$ Auropunstatum. Dej. P.

4 Auropunctatum. Payk. Suecia.* Indagator. Gyll. Dej. Gall. merid.

5 Sericeum. Fabr. Dej. Russia.* Investigator. Illiger. German. bor. Var. Caspium. Fischer. Russ. merid.

6 Reticulatum. Fabr. Oliv. Germ. bor. ${ }^{*}$

CALISTHENES. Fischer.

1 Panderi. Fischer. Russ. merid.

2 Orbiculatus. Motschuls. Caucasus.

3 Araraticus. Ericison. id.

DRYPTA. Fabricius.

CrCindela. Olivier.

1 (Emarginata. Fabr. Gallia. ${ }^{*}$

$\{$ Var. Italica. de Crist. Lombardia.

2 Distincta. Rossi. Sicilia.

Cylindricollis. Fabr.

Dej. Gall. merid.

3 Intermedia. Rambur. Hispania.

ODACANTHA. Fabricius. Payk.

Attelabus. Linné. Carabus. Olivier. Cicindela. Panzer.

1 Melanura. Linné. P.* \{ Angustata. Olivier. Gallia.

\section{ZUPHIUM. Latreille.}

Galerita. Fabr.

1 - Olens. Fabr. Gall. merid.

2 Chevrolatii. de Laporte. Gall. merid. INumidicum. Lucas. Algiria.

3 Unicolor. Germar. Dalmatia.

POLISTICHUS. Bonelli.

Galertita. Fabr. Zuphium. Latr. Carabus. Olivier.

1 Vittatus. Brullé. Gall. merid.* Fasciolatus. Oliv. Dej. id. 
2 Fasciolatus. Rossi. Discoideus. Dej.

\section{GYMINDIS. Latreille.}

TARUS. Claïr. Anomeus. Fisch. Lebia. Duftsch. Carabus. Fabr.

1 Picta. Pallas. \{ Cruciata.Fischer. Dej,id.

2 Discoidea. Dej. Russ. merid*

Humeralis. Fab

Humerosus. Schönh. Guecia. (Var. Diance. (Dahl.) Helvetia.

5 Hybrida. Lasserre. id.

6 Elongata Lasserre id.

7 Patruelis. Sturm. Cat. Russia.

8 Dorsalis Fischer. Russ, orient. ${ }^{*}$

9 Lineata. Schönherr. Gall. merid. ${ }^{*}$

Lineola. Dufour. Hispania.

(Vittata. (Dahl.) Sicilia.

10 Lævistriata. Lucas.

11 Setifensis. Lucas.

12 Leucophthalma. Luc. id.

13 Marginata. Lucas. id.*

14 Palliata. Stéven. Russ. merid.

15 (Homagrica. Duftsch. Gall. merid.

Y Var. Meridionalis. Dej.id.

(- Lunaris. Duftsch. Austria.

16 Cingulata. Dej. Styria. ${ }^{*}$

17 Coadunata. Dej. Gall. merid. ${ }^{*}$

18 Melanocephala. Dej. Pyr. orient. ${ }^{*}$

19 Axillaris. Duftschmid. Gall. merid. ${ }^{*}$

20 Angularis. Gyllenhal. Suecia.

Humeralis. var. $\beta$.

Payk. id.

21 Macularis. Mannerh. $\{$ Dej. id.*

Humeralis. Paykull. id.

Binotata. Sturm. Germania.

22 Binotata. Fischer. Dej. Russ, merid.

23 Apicalis. Mannerh. id.

24 Ornata. Stéven. (Glycina.) Chaud. id.

25 Gaubilii. Lucas.

Algiria.

26 Alternans. Rambur. Hisp. merid.

27 Signata. Sturm. Cat. Dalmatia.

28 (Basalis. Gyllenhal. Styria.*

Humeralis. Paykull. Suecia.

Punctata. Bonel. Dej. Pyr, orient.

29

Miliaris. Fabr. Dej. $\quad \boldsymbol{P}^{*}$

30 Picipes. Sturm. Cat. Sicilia.

31 Affinis. Rambur.

\section{2}

\section{3}

34 Baetica. Rambur. id.

Marmoræa. Géné. Bassi. Italia.

35 Etrusca. Bassi. id.

36 Sulcata. Rambur. Hisp. merid.

I Gracilis. Dej.

37 Onychina. Dej.

38 Dilaticollis. Lucas.

39 bufo. Fabr.

Faminii. Dej.

Mauritanica. Dej.

Hispania.

id.

Algiria.*

Sicilia. $^{*}$

Gall. merid.

Algiria. *

\section{DEMETRIUS. Bonelli.}

Demetrias. Dej. Cat. Lebia. Duftschm. Carabus. Fabr.

1 Unipunctatus. Germ. $\boldsymbol{P}^{*}$

2 Atricapillus. Linné. id.* $\{$ Var. Elongatus. Zenk. Duftsch. Dej. id.* - Confusus? Heer. Helvetia.

3 Fulviceps. Villa. Ital. bor.

\section{AËTOPHORUS. Schmidt. Gœbel.}

\section{Demetrias. Dej. Sturm.}

1 Imperialis. Megerle. Germar. Dej. Gall. orient.*

\section{DROMIUS. Bonelli.}

Lebia: Latr: Duftsch. Canabus. Fabr.

1 Longiceps. Dej. Volhynia.

2 Linearis. Bonel. Oliv. P.

Punctatostriatus. Dft. Austria.

Prosta (Odacantha.)

Stéven. Russ. merid.

3 Insignis. Lucas. Algiria.

4 Melanocephalus. Dej. P.*

5 Sigma. Rossi. id.*

Fasciatus. Fabr. Duft. Austria.

Sturmii. Babington. Anglia.

Bipennifer. Babingt. id.

6 Quadrisignatus. Dej. Gallia.*

7 Bifasciatus. Perroud. $P$.*

8 Fasciatus. Fabr. Dej. Gallia.*

9 Cruciferus, Lucas. Algiria.

10 Quadrinotatus. Duft. P.*

11 Quadrimaculatus.Pnz. id.*

12 Testaceus. Erichson. Germania.* 
13 Agilis. Fabr. P. ${ }^{*}$

Truncatus. Fabr. Dania.

Var. Fenestratus. Dej. P.

- Quadrimaculatus

Schh. Suecia.

- Atricapillus.Pnz. Germania.

- Biguttatus. Clair. Helvetia.

14 Fenestratus. Fabr. Germ. bor. ${ }^{*}$ Agilis, var. c.Gyllerh.Suecia.* Arcticus. Olivier. id.

15 Meridionalis. Dej. Gall.merid.*

16 Angustus. Brulle. Gallia.

17 Marginellus. Fabr. Germania.* \{ Agilis.var, d. Gyllenh. Suecia.

18 Scapularis. Dej. Hispania.

19 Glabratus. Duftschm. Gallia.* Var. Pygmaus. Che-

vrier. Helvetia.

20 Mauritanicus. Lucas. Algiria.*

21 Plagiatus. Duftschmid. Gall. merid. ${ }^{*}$ Corticalis. Duf. Dej. Hispania.

22 Pallipes. (Zieg.) Dej. Austria.*

23 Montenegrinus. Küst. Montenegro.

24 Andalusiacus. Rambur. Hisp. merid.

25 Obscuroguttatus. Duft. Germania * Spilotus. Dej. . Gallia.

26 Foveola. Gyllenhal. Suecia.* Punctatellus, Duftsch. Dej. $\boldsymbol{P}$. Truncalellus. Latr. id.

27 Striatipennis. Lucas. Algiria ${ }^{*}$.

28 Truncatellus. Linné.

Fabr. Pyr. orient.

29 Levipennis. Lucas. Algiria.*

30 Maurus. (Meg.) Sturm. Germania.*

31 Quadripustulatus. Fab. Gallia.* Quadrillum. Duftsch. Austria.

Var. Bipunctatus. Heer. Helvetia.

- Striatulum.Heer. id.

32 Albonotatus. Dej. Pyr. orient.

33 Albomaculatus. Lucas. Algiria.

34 Sturmii. Géné. Sardinia.

SINGILIS. Rambur. Lucas.

1 Bicolor. Rambur.

Hisp. merid.*

Soror. Rambur.

id.

3 Mauritanica. Lucas. Algiria.

LEBIA. Latreille. Bonelli.

Lamprias, Bonelli, Carabus. Fabr.

1 Pubipennis. Dufour.

Hispania. $^{*}$

2 Fulvicollis. Dej.

- 3 f Fulvicollis. Fabr.

Africana. Solier.

Algiria. ${ }^{*}$

id.

Gerardii. Buquet. id.

4 Cyanocephala. Fabr. Gall. merid.*

Var. Formosa. Villa. Lombardia.

5 Annulata. Brullé. Gall. bor.*

Femoralis. Chaudoir. id.

6 Numidica. Lucas. Algiria.

7 (Chlorocephala. Ent. Hefte. Gall. bor.*

Cyanocephala.de Geer.Germania.

8 Rufipes. Dej. Gall. merid.

9 Cyathigera. Rossi. id.* Anthophora. Dufour. Hispania.

10 Grux-minor. Lin. Fab. Gallia.*

11 (Nigripes. Dej. Gall. merid. Communimacula.

12 jTurcica. Fabr. Gall. merid.* (Dahl.) Sicilia.

Var. Quadrimaculata.

Dej. id.*

13 Humeralis. Sturm. Austria.

14 Hæmorrhoidalis. Fab. P.*

\section{APTINUS. Bonelli.}

Brachinus. Fabr.

1 Displosor. Dufour. Hispania.*

\{ Ballista. Illiger. Dej. Pyr. orient.

2 Mutilatus. Fabr. Austria.*

3 Atratus.(Ziegler.)Dej.id.

4 Alpinus. Dej.... Alp. Gall.*

5 Pyrenæus. Dej. Pyr. orient.

6 Italicus. Dej. Italia. ${ }^{*}$

7 Bellicosus. Dufour. Hispania.*

$\{$ Jaculans. Illiger. Dej. Lusitania.

8 Baeticus. Rambur. Hisp. merid.

9 Testaceus. Rambur. id.*

10 Andalusiacus, Rambur. id.*

\section{BRACHINUS. Weber.}

\section{Carabus. Olivier.}

1

2 Humalis.

\{ Causticus. Dej.

3 Nigricornis. Dej.

4 Crepitans. Linné.

5 Obscuricornis. Brullé. Gracia.

6 Etslans. Dej.

7 Græcus. Dej.

8 Immaculicornis. Dej.
Hisp. merid. * Gall. merid. ${ }^{*}$ id.

id.*

P. $^{*}$

Lusitania.

Gracia.*

Gall. merid 
9 Obscurus. Heer. Helvetia.

10 Patruelis. Sturm. Cat. Sicilia.

11 Dalmatinus. Stm. Cat. Dalmatia.

12 Atricornis. Stẻven. St.

$$
\text { Cat. Russ. merid. }
$$

13 Barbarus. Lucas. Algiria.

14 Siculus. Sturm. Cat. Sicilia.

15 (Explodens. Duftsch. P.*

Obscuricornis. Godet. Podolia.

16 Strepitans. Duftsch. Gall. merid.* Glabratus. Dej. id.

17 Psophia. Dej. id.*

18 Ejaculans. Fischer. Tauria.

19 Bombarda. Dej. Gall. merid.*

20 Sclopeta. Fabr. P.*

$\{$ Var.Suturalis. Dej. Gall.merid.

21 Fimbriolatus. Lucas. Algiria.

22 Bayardii. Solier. Gracia..

23 Bipustulatus. Stéven. Russ. merid.*

24 Quadripustulatus. Dej.id. $\{$ Quadrimaculatus.

Eschh. id.

25 Quadrinotatus. Ménét. id.

26 Exhalans. Rossi. Gall. merid. Var. Trimaculatus.

(Gaubil.) id.

27 Hammatus. Fischer. Russ, merid.

28 Cruciatus.Stéven. Dej.id.

29 Thermarum. Stéven.

(Mystax.) id.

Caspicus. Godet. id.

MASOREUS. (Ziegler.) Dejean.

Badister. Creutzer. Trechus. St.

1 (Wetterhalii. Gyllenhal.

$\begin{cases}\multicolumn{1}{c}{\text { (Harp.) }} & \text { Suecia.* } \\ \text { Luxatus. Dej. } & P . \\ \text { Laticollis. Sturm, } & \text { Germania. } \\ \text { Testaceus. Lucas. } & \text { Algiria. }\end{cases}$

CORSYRA. Stéven. Dej.

Cymindis. Fischer.

1 Fusula. Fischer. Russ. merid.

GRAPHIPTERUS. Latreille. Dej.

Anthia. Fabr. Garabus. Olivier.

1 Exclamationis. Fabr. Algiria.*

2 Multiguttatus. Latr. id.*
3 (Luctuosus. Dej.

Peleteri.de Castelnau.id.

-Multiguttatus. Oliv. id.

4 Barthelemyi. Solier. Barbaria.*

ANTHIA. Weber. Fabr.

Carabus. Olivier.

1 Sexmaculata. ${ }^{1}$ Fabr. Algiria. ${ }^{*}$

SIAGONA. Latreille. Dej.

Cucusus. Fabr.

1 Rufipes. Fabr.

2 Jennissonii. Dej.

3 Europæa. Dej.

4 Oberleitneri. Dej.

5 Gerardii. Buquet.

6. ? Rufa. Chaudoir.

7 Dejeanii. Rambur.

Algiria.*

id. ${ }^{*}$

Sicilia.*

Gracia.

Algiria.*

id.

Hisp. merid.*

\section{SCARITES. Fabr. Dej.}

Cucojus, Galerita. Fabr.

1 (Gigas. Fabr. Gall. merid.*

Var.Algiricus. Sturm. Algiria.

Pyracmon. Bonelli. Sicilia.

2 Bucida. Pallas. Russ. merid.*

3 Polyphemus. Bon.Dej.Hisp.merid. ${ }^{*}$

4 Euritus. Fischer. Russ. merid.

5 Salinus. Pallas Dej. id.*

6 Hespericus. Dej. Hisp.merid.

7 Collinus, Rambur. id.*

$\{$ Var. Levaillantii. Luc. Algiria.

8 Planus, Bonelli. Gall. merid.*

9 Arenarius. Bonelli. id.*

10 Terricola. Bonelli. id. ${ }^{*}$

11 Lævigatus. Fabr. id.*

$\{$ Var. Telonensis. Bonel. id.

12 Tauricus. Chaudoir. Tauria.

13 ? Sexpunctatus. Chaud.Algiria.

\section{MISCODERA. Eschscholtz.}

\section{Leiochiton. Curtis.}

\section{Arctica. Paykull. Lapponia.}

1 Cette espèce qui n'avait été trouvée que dans les environs de Tunis, a été rencontrée assez abondamment dans les vallées du Djebel-Amour par M. le colonel Levaillant du $36^{\circ}$ de ligne. 
CLIVINA. Latreille, Panz, Dej, Putzeys.

Tenebrio. Linné. Scarites. Fabr.

1 Fossor. Linné. ‥ P. Arenaria. Fabr. Dej. Germania. Var. Collaris. Herbst. Gallia. - Gibbicollis. Meg id. Ypsilon. Dej. Russ. merid.

3 Scripta. Putzeys. Luc. Algiria.

4 Blanchardii. Lucas?' id.*

DYSCHIRIUS. Bonelli. Putzeys.

Scarites. Fabr. Carabus. Herbst.

1 Thoracicus. Rossi. Germania.* Y Nigra. Sehiodte. Dannia.

Var. Riparius. Mannh. Finlandia.

-Aurichalceus.Stm.Pyr. orient.

2 Numidicus. Putzeys. Sicil. Algir. ${ }^{*}$

3 Africanus. Putzeys. Algiria.*

4 Obsoletus. Putzeys. id.*

5 Obscurus. Gyllenhal. Belgia.*

6 Fulvipes. Dej. Hispania.

7 Digittatus. Dej. Styria.

8 Globosus. Herbst. Gallia.*

Gibbus. Fabr. Panz. Germania.

Minimus. Ahrens. id

Remotus. Marsham. Anglia.

Var. Laevicollis? Ahr. Germania.

9 Rotundipennis. Chaud. Carniolia. *

Ferrugineus. Kok. in lit. Styria.

10 Gracilis. Heer. Helvetia.*

11 Rufipes. Dej. Austria.

12 Emulus. Schüppel. Sicilia.

\{ Rufo-cneus? Chaud. Russia.

13 Algiricus. Lucas. Algiria. ${ }^{*}$

14 Punctatus. Dej. P

15 Minutus. Dej. Gall.merid.

16 Semistriatus.Dej.Heer.Gallia.*

17 Læviusculus. Putzeys. id.

18 (Eneus. Dej. id.

Var. Paludosus. Ahr. Germania.

- Ahena. Ahrens. id.

- Aerea. Ahrens. id.

19 Intermedius. Putzeys. Belgia.

20 Lafertei. Putzeys. Pedemont.

21 Impressus. Putzeys. Lusitania.

22 Chalybeus. Strm. Cat.

Putzeys. Gall. merid.

23 Punctipennis. Putzeys. id.

24 Ruficornis. Ziegler.

Putzeys. Austria.
25 Uliginosus. Putzeys. Germania. Cylindricus. Sturm. id.

26 Bonellii. Putzeys. Italia.

Dyschiria. Bonelli. id.

27 Apicalis. Putzeys. Dalmatia. ${ }^{*}$

28 Striatopunctatus. Kollar. Putz. id.

29 Salinus. Erichson. Germania.* Eneus. Ahrens. Gall.merid. Metallicus. Germar. Hungaria.

30 Pusillus. Dej. Russ. merid.

31 (Angustatus. Dej. Ahr. Belgia.

Sabulicola. Boisd. et Lacordaire. $\boldsymbol{P}$.

Pusillus. Stephens. Anglia.

32 I Substriatus. Duftsch. Pedemont.

(Bipunctatus. Grim. Styria.

33 Bimaculatus. Bonelli. Gall, orient.

34 Cylindricus, Dej. Gall. merid. Extensus. Schaum.

Putzeys. Berolini.

36 Politus. Dej. … Pyrenais. ${ }^{*}$

Ereus? Herbst. Germania.

Elongatus. Ahrens. id.

Metallicus. Ahrens. id.

37. Strumosus. Hoffmg.

Erich. Russ. merid.

38 Arenosus. Steph. Putz.Anglia.

39 Nitidus. Dej. $\quad \boldsymbol{P}^{*}$

Thoracicus. Olivier. id.

Chalceus. Erichson. Gallia.*

41 Oblongus. St. Cat.

Putzeys. Russ. merid

42 Inermis. Curtis. Anglia.

CARTERUS. $D e j$.

1 Interceptus. Dej. Lusitania.*

2 Affinis. Rambur. Hisp. merid.

3 Gracilis. Rambur. id.

4 Rufipes. Lucas. Algiria.

DITOMUS. Bonelli. Latr.

Aristus. Ziegler. Charabus, Calosoma, Scaurus. Fabr. Scarites. Olivier. Rossi.

1 Tricuspidatus, Fabr. Gall, merid. * Calydonius. Fabr.Dej.Sardinia. Var. Cornutus. Dej. Hispania.

2 ? Spinicollis. Chaud. Algiria.

3 Cordatus. Dej. Hispania.*

4 Rotundicollis. Ramb. id. 
5 Dama. Rossi.

6 Barbarus,(Odogenius.)

$$
\text { Solier. Algiria.* }
$$

7 Fulvipes. Latr. Dej. P.*

8 Pilosus. Dej.

9 Dilaticollis. Lucas.

10 Tomentosus. Dej.

11 Distinctus. $D e j$.

12 Hirtus. Sturm. Cat.

13 Robustus. Parreyss.

14 Tenebricosus. Klug.

St. Cat. Constantin.

15 Cyaneus. Olivier. Gracia.*

Atrocæruleus. Waltl. Hispania.

16 Ruficornis. Lucas. Algiria.*

Parvulus. Chevrolat. in litt. id.

17 Interruptus. Schaum. id.

18 Capito. Dej.

19 Opacus. Erich.

20 Clypeatus. Rossi. Sulcatus. Fabr. Dej. id

21 Affinis. Sturm. Cat.

22 Eremita. Dej.

23 Nitidula. Stéven. Dej. id.

24 Sardeus. Sturm. Cat. Sardinia.

25 Obscurus. Stéven. Dej. Russ. merid.

26 Sphœrocephalus. Olivier. Dej. Gall. merid.

27 Difficilis. Chevr. in litt. Algiria. *

28 Rufipes. Chaudoir. Turcia.

APOTOMUS. Dejean.

Scanites, Rossi. Olivier.

1 Rufus. Rossi.

Gall. merid.*

2 Testaceus. Rossi. Russ.merid.

3 Rufithorax. Pechioli. Italia.

\section{PANAGEUS. Latreille.}

Garabus. Linné. Fabr.

\section{Crux-major. Linné. Gallia *}

Crux. Gyllenhal. Suecia.

Bipustulatus. Olivier. P.

Var. Quadripustulatus. Sturm. id.

- Trimaculatus.Dej. Gallia.
LORICERA. Latreille.

Carabus. Fabr.

1 Pilicornis. Fabr. Gallia. ${ }^{*}$

Seticornis. Müller. Germania.

Enea. Latreille. $\quad \boldsymbol{P}$.

Var. Alpina. Heer. Helvetia.

LICINUS, Latreille. Dej.

Carabus. Fabr.

1 Agricola. Olivier. Dej. Gall. merid.*

Silphoides. Rossi. Italia.

Croaticus. Dahl. Croatia.

2 Silphoides. Fabr. Gallia.*

3 Granulatus. Dej. Hispania.*

4 Siculus. Dej. Sicilia. ${ }^{*}$

5 Dalmatinus. Chaudoir. Dalmatia.

6 Brevicollis. Dej. Algiria.*

7 Peltoides. Illiger. Lusitania."

8 Equatus. Dej. Gall. merid.*

9 Cassideus. Fabr. P.*

Emarginatus,Olivier. Gallia.

Depressus. Sturm. Germania.

Var. Latus. Solier. Italia.

10 Depressus. Paykull. Gallia.*

Cossyphoides.Duftsch.Austria.

Cassideus. Illig. Schh. Germania.

11 Hoffmanseggii. Panz. Gallia.*

Var. Separatus, Dahl. Hungaria.*

Minutus. Ziegler. Styria.

Nebrioides. Stm. var. Carniolia.

12 Oblongus. Dej. Alp. Gall.

\section{CALLISTUS. Bonelli.}

Carabus. Fabricius.

1 Lunatus. Fabr. Plateosus. Fourcroy. P.

Germania.*

CHLENIUS. Bonelli.

Harpalus. Gyllenh. Carabus. Fabr.

1 Velutinus. Duftschm. Gall. merid.* Cinctus. Olivier. id. Marginatus, Rossi. Italia.

Zonatus. Panzer. Germania.

Var. Gracilipes. Ullr. Illyria.

2 Festivus. Fabr. Gall. merid.* $\{$ Var. Fischeri. Falder. Russ. merid. 
3 Borgiæ. Lefebvre. Dej. Sicilia.*

Fimbriolatus. (Dahl.) Sardinia.

4 Auricollis. Géné. Sardin. Alg. \{Eneicollis. Sturm. Sicilia.

5 Spoliatus. Rossi. Dej. Gall. merid.*

6 Variegatus. Fourcroy. P.*

IAgrorum. Oliv. Dej. Gall. merid.

7 Terminatus. $D e j$. Apicalis. Stéven. id.

8 Vestitus. Fabr. P.*

9 (Eratus. Quensel. in Schh. Dej. Algiria.*

Algerinus. Gory. id.

Yar. Varvasii. de Lap. id."

10 Flavipes. Ménétriẻs. Russ. merid.

11 (Schrankii. Duftschm. Gallia.*

Bombycinus. Bonelli. Italia.

IEschscholtzii. Fischer. Russ. merid.

12 Gratiosus. Chaudoir. Volhynia.

13 Chrysothorax. Stéven. Russ. merid.

14 /Nigricornis. Fabr. Gallia bor.* Holosericeus Schönh.

var. Suecia.

Var. Melanocornis.

(Ziegl.) Dej. Germania.

- Eneus. Dej.

15 Nigripes. Dej.

$P$.

Pyr.orient.

16 Dives. (Hoffmg.) Dej. Hispania.*

17 Melanopus. Sturm.

Cat. Russ. merid.

18 Tauricus. Sturm. Cat. id

19 Tibialis. Dej.

\{ Nigricornis. Dej. Cat. Gall. merid.

20 Holosericeus. Fabr. Gallia.*

Sulcicollis. Paykull. Gall. bor.*

(Cælatus. Weber. Dej. Germ.bor.*

Anaglypticus. Knoch.

Dahl. id.

Quadrisulcatus. Payk. Suesia.

\$ Sulcicollis. Gyllenh.

Germar. id.

Quadrisulcatus. Illig. German.bor. Chrysocephalus. Rossi, Gall. merid.* ? Distinguendus. Chaudoir. Algiria.

Eneocephalus. Dej. Russ. merid.

Dalmatinus. Erichson. Dalmatia.

Gracilis. Solier. Dej. Russ. merid.

Azureus. Dej. Hisp.merid.Alg. *

Cœruleus. Stéven.Dej. Russ. merid. *

Virens. Rambur. Hisp. merid.

Steveni. Schönherr. Russ, merid.
Infuscatus. Eschsch. id.

EPOMIS. Bonelli. Dej.

Chlenius. Latr. Garabus. Duftsch. Rossi.

1 Circunscriptus. Duft. Gall. merid.* Cinctus.Panzer. id.

2 Dejeanii. Solier. Dej. Gracia.

DINODES. Bonelli. Dej.

Chemenus. Latr. Carabus. Duftsch.

1 Azureus. Duftschmid. Gall. merid.* Rufipes. Dej. Sturm. id.

2 Maillei. Solier. Dej. Gracia.*

3 Viridis. Ménétriés. Russ. merid.

4 Boeticus. Rambur. Hisp. merid.

5 Laticollis. Chaudoir. Turcia.

OODES. Bonelli. Dej.

Harpalus. Gyll. Carabus. Fabr.

1 Helopioides. Fabr. P.* $\{$ Var. Notatus. Megerle. Austria. Obtusus. S urm. Germania.

2 Hispanicus. Dej. Hispania.

3 Mauritanicus. Lucas. Algiria.*

4 Abaxoides. Lucas. id.*

BADISTER. Clairville. Dej.

Amblychus. Gyll. Carabus. Fabr.

1 Unipustulatus. Bonelli. $\boldsymbol{P}$.*

\{Cephalotes. Dej. Gallia.

2 Bipustulatus. Fabr. id.*

Crux-minor. Olivier. $P$.

Anchora. Ménétriés. Russ. merid.

Var. Lacertosus. Knoch.

- Humeralis. Will.

Redtenbacher. Austria.

3 Binotatus. Fischer. Russ. merid.*

4 (Peltatus. Panzer. Gallia.*

Corruscus. Stéven. Russ. merid.

Chalybeum. Sturm.

(Agonum.) Germania.
5 Dilatatus. Chaudoir. id.

6 Humeralis. Duftsch. Austria. ${ }^{*}$

Sodalis. Duftsch. St. Germania.

Dorsiger. Duftschm. Austria.

Var. Xanthomus. Chau-

doir. Russia. 
OMPHREUS. Parreyss. Dej.

1 Morio. Parreyss. Dej. Montenegro.

\section{POGONUS. Ziegler. Dej.}

Rapton.Megerle. Platysma. Sturm. CaRABUS. Duftsch. Harpalus. Ahrens.

1 Pallidipennis. Dej. Gall. merid.*

2 Flavipennis. Dej. Hispania. Latipennis. Ullrich. Illyria.

3 Luridipennis. Germar. Gallia bor.* Burellii. Curtis. Germania.

4 Fulvipennis. Sturm. Italia.

5 Iridipennis. Nicolä̈. Germania.* Brevicollis. Mannerh. Sibiria.

6 (Littoralis. (Megerle.)

St.Dej. Gall. merid. * Pilipes.Germar. Dalmatia.

7 Chalceus. Marsham. Germania.* Parallelipipedus.

Marsh. Anglia.

Halophilus. Nic. Dej. Germania. Var. Oceanicus. Dej.

$$
\text { Cat. Gall. bor. }
$$

- Hispanicus. Dej. Cat. Hispania.

8 Flavipes. Sturm. Dej. Sicilia.

9 Viridanus. Dej. Hispania.*

10 Gilvipes. Dej. Gall. merid.* Flavipes. Sturm. Cat. id.

11 Riparius. Dej. id.*

12 Orientalis. Dej. Russ. merid.

13 Meridionalis. Dej. Gall. merid.*

14 Punctulatus. Dej. Russ. merid.

15 Gracilis. Dej. Gall. merid.* Pygmaus. Sturm. Cat. id.

16 Testaceus. Dej. id.*

17 Eruginosus. Stephens. Anglia.

18 Filiformis. Ziegl. (Sirdenus.) Dej. Sardinia.*

PATROBUS. Megerle. Dej.

Platysma. Sturm. Harpalus. Gyllenh. Carabus. Fabr. Velox. Fabr. Germania. Rufipes. Gyll. Dej. Suecia.

2 Septentrionis.Dej.Schh.Lapponia.* I Rufipes. Gyll.var.d. id.
3 Lapponicus. Chaudoir. Lapponia.

4 Rufipennis. Hoffmsg.

Dej. Gall. merid.*

DOLICHUS. Bonelli. Dej.

Harpalus. Gyll. Garabus. Fabr.

1 Flavicornis. Fabr. Italia.*

SPHODRUS. Clairville. Dej.

Harpalus. Gyll. Carabus. Fabr.

1 (Leucophthalmus. Lin. Gallia.*

Niger. Paykull. Suecia.

Planus. Fabr. Dej. Gallia.

2 Gigas. Fischer. Russ. merid.

3 Cellari. Baber. id.

\section{PRISTONYCHUS. Dejean.}

Sphodrus. Bonelli. St. Harpalus. Gyll.

Lemostenus. Bonelli. Carabus. Fabr.

1 f Subcyaneus. Illig.Gyll.Gallia.* Terricola. Illig. Dej. P.

2 Sardeus. Dahl. Erich. Gall. merid."

3 Algirinus. Gory. Algiria.*

4 Punctatus. (Meg.) Dej. Lombardia.*

5 Cimmerius. Stév. Dej. Gracia.*

6 Grandicollis, Stm. Cat. Sicilia.

7 (Tauricus. Dej. Russ. merid.*

Inoqualis. Stéven. id.

(Janthinus. Ménétriẻs. id.

8 Conspicuus. Klug. St.

Cat. Constantin.

9 Turcicus. Sturm. Cat. id.

10 Mauritanicus. Dej. Algiria.

11 Oblongus. Dej. Gall. merid.*

12 Angustatus. Dej. Alp. Gall.*

13 Elongatus. Dej. Croatia.*

Ovatus, Ziegler. Dalmatia.

14 Barbarus. Lucas. Algiria.

15 Dalmatinus. Dej. Dalmatia.

16 Oblongicollis. Sturm.

Cat. Russ, merid.

17 Cœruleus. Bonelli. Italia:*

18 Amethystinus. Dej. id.*

19 Violaceipennis. Sturm.

Cat. Alp. Ital.

20 (Janthinus. Duftschmid. Austria. *

Episcopus. Drapiez. id.

(Var.Purpuratus. Meg. id. 
21 Alpinus. Dej.

Chalybeus. Dej.

Cyanipennis. Eschsch. Russ. merid.

Complanatus. Dej. 'Gall. merid.*

Caspicus. Ménétriés. Russ. merid.

(Elegans. Dej.

Longicollis. (Megerle.) Carniolia.

27 Var. Schreibersii.

Schmidt. id.

28 Venustus. Clairville. Gall. merid.*

Subcyaneus. Stéven. Russ, merid.

Caruleus. Bonelli.

(Læmostenus'. Italia.

Amœnus, Fischer. Russ. merid.

30 Suturalis. Brulle. Grocia.

CALATHUS. Bonelli. Dej.

Harpalus. Gyllenh. Carabus. Fab. Oliv. 28
17 Deplanatus. Chaudoir. Turcia.

18 Obtusus. Sturm. Cat. Sardinia.

Ovalis. (Dahl.) id.

19 Rotundicollis. Dej. P.*

20 Gilvipes. Sturm. Cat. Dalmatia.

21 Elongatus. Dej. Germania.

22 Micropterus. Duftsch. id.

Melanocephalus. var.

b. Gyll. Suecia.

Glabripennis. Sturm. Germania.

Microcephalus. Dej. Gallia.

23 Ochropterus. Duft. St. id.*

24 Melanocephalus.Linné.

$$
\left\{\begin{array}{r}
\text { Fabr. P.* } \\
\text { Fej. }
\end{array}\right.
$$

Var. Alpinus. Dej. Styria.*

25 Obscurus. Lucas. Algiria.

26 Obscuricollis. Chaud.

27 Solieri: Bassi. Sicilia."

Opacus. Lucas. Algiria.

\section{SYNUCHUS. Gyllenhal.}

Taphria. Bonelli. Latr. Dej. Agonum. Sturm.

1 Vivalis. Illig. St. Gallia bor.*

Nivalis. Panzer. Dej. Germania.

$\{$ Rotundatus. var. $\beta$.

Schh. Suecia.

Var. Alpinus. Heer. Helvetia.

GARDIOMERA. Bassi.

1 Genei. Bassi.

Sicilia.

ANCHOMENUS. Erichson.

Platynus, Anchomenus, Agonum. Bonelli. Carabus. Fabr. Harpalus. Gyll.

(Platruns. Bonelli.)

1 Elongatus. Stéven. Russ. merid.

2 Complanatus. Bonelli. Pedemont.*

3 Depressus. Lasserre. Helvetia.*

4 Scrobiculatus. Fabr. Austria.*

5 Erythrocephalus. Peyroleri. Pedemont.

6 Peyrolerii. Bassi. id.*

(Anchomenus. Bonelli.)

7 Longiventris. Eschsch. Germ. hor.*

\{igilans. (Dolichus.)

Sturm. id. 
8 Uliginosus. Erichson. $N$,

9 Mannerheimii. Sahlberg. Dej. Finlandia.

10 Angusticollis. Fabr. Gyll. Dej. P.* Assimilis. Paykull. Suecia. Var.Rufpennis. OEsk. Hungaria.

11 Krynickii. Speck. Volhynia.

12 Algirinus. Lucas. Algiria.

13 Cyaneus. Dej. Pyr.Helvet.*

14 Livens. Gyll. Erich. Suecia.*

Memnonius. Nic. Dej. Gallia.

Bipunctatus. Sturm. Germania.

15 S Prasinus. Thunb. Fab. P.*

Y Viridanus. Olivier. id.

16 Melanocephalus. Dej. Hispania.

17 Albipes. Fabr. Erich. Dania.*

$\{$ Pallipes. Fabr.Dej. $\quad P$.

Pavidus. Panzer. Germania.

18 Oblongus. Fabr. Dej. Gallia.*

I Taniatus. Panzer. Germania.

\section{(Agonum. Bonelli.)}

19 Marginatus. Linné. $P_{\text {.* }}^{*}$

20 Impressus. Illiger. Germania.*

21 Austriacus. Fabr. Austria.*

Var. Dalmatinus. Dej. Dalmatia.

7-Punctatus. Eschsch. Russ. merid.

Karelinii. Falderm. id.

22 Modestus. Sturm. Dej. Gallia.*

Nigricornis.(Carabus.)

Panzer. Germania.

Metallicus. (Carabus.) Melsh. id.

23 Fulgidicollis. Erichs. Algiria.*

24 Ericeti. Knoch. Panz. Finlandia.

25 (Sexpunctatus. Linné. Gallia.*

Duodecimpunctatus.

Müller. Germania.

26 Bifoveolatus, Sahlberg.Lapponia.

Sexpunctatus. var. c.

Gyllenh. id.

27 Parumpunctatus. Fabr.P.*

Sexpunctatus, Müller. Germania.

Plicicollis. Nicolaï. id.

Clandestinus. Sturm. id.

Var. Tibialis. Ziegl.

Sturm. id.

- Melletii. Heer. Helvetia.

28 Elongatus. Dej. Styria.

Micans. Germar. Sibiria.

29 Alpestris. Heer. Helvetia.
30 Numidicus. Lucas. Algiria.

31 (Tristis, Dej.Erichs. Suecia.*

Versutus, var, c. Gyll. id.

Latipenne.Dej. German. bor.

Tarsatus, Zettersted. Suecia.

Viduus, var. c. Gyll. id.

32 Viduus. Kugellann.

Panzer. Germania.*

33 (Versutus, Gyll, Erich. Suecia.

Levis, Gyllenhal. id.

(Lave. (Agonum.) Dej. Austria.

34. Micans, Nicolä̈, Erich. Germania.* $\{$ Pelidnus. Duftsch. St. id.

35 Lugens. (Ziegl.) Duftschmid. Gallia.*

36 (Emarginatus. Gyllenh. Suecia.* Afer. Duftsch. Sturm. German. bor. Mostus. Duftsch. St. id.

37 Lugubris. Dej. Gallia.*

$\{$ Hungaricus, Friwalds, Austria.

38 Sordidus. Kollar. Dej. Corfou.

39 Atratus. Duftschmid. Germania.* Niger. Dej.

40 Angustatus. Dej. Hungaria. \{ Elongatus. Dahl. id.

41 Ménétriésii. Dej. Russ, merid Niger. Ménétriés. id.

42 (Subæneus. (Ziegl.) Dej. Helvetia. $\{$ Subcyaneus.Stm. Cat. Hungaria.

Crenatus, Latreille. id.

43 Chalconotus. Ménétr. Russ, merid.

44 (Pelidnus. Payk. Gyll.
$\left\{\begin{array}{l}\text { Er. Gallia.* } \\ \text { Puellus. Dej. }\end{array}\right.$
Longicollis. Lacord. P.*

45 Scitulus, Dej. German, bor.

46 Lehmanni. Chaudoir. Livonia.

47 Convexiusculus, Chau-

doir. Gracia.

48 Gracilis. Stm. Dej. Er. P.*

Picipes var, b. Gyll. Suecia.

49 Fuscicornis. Schaum. Germania.

50 (Picipes. Fabr. Dej. Gallia.*

Fuscipennis. Nicolaï. Germania.

Testaceus. Panzer. id.

Lutescens. Panzer. id.

Canellipes. Eschsch. Sibiria.

51 Thoreyi. von Vinthem.

Dej. Germ. bor.*

52 Quadripunctatus. de Geer. Suecia.*

Y Var. Cupratus. Sturm. id.

53 Bogemanni. Gyllenh. Suecia.

54 Ruficornis. Sturm. Cat,Italia. 
OLISTHOPUS. Dejean.

Agonum. Bonelli. Harpalus. Gyllenh.

1 (Rotundatus. Paykull.

$\left\{\begin{aligned} \text { Dej. } & \text { Gallia. }{ }^{\star} \\ \text { Vafer. Duftschmid. } & \text { Dalmatia }\end{aligned}\right.$

2 Puncticollis. Lucas. Algiria.*

3 Hispanicus. Dej. Gall. merid.*

Fuscatus, $D e j$

5 Glabricollis. Germar. id.

Punctulatus. Dej. id.*

6 Helferi. Sturm. Cat. Sicilia.

7 Sturmii. Duftschmid. Germania.* Flavipes. Panzer. Gallia.

\section{POECILUS, Bonelli.}

Feronia, Latr. Dej. Harpalus. Sturm. Carabus, Oliv, Fabr.

1 Punctulatus. Fabr. (So-

$$
\text { gines. Steph.) } P \text {.* }
$$

2 Barbarus, Lucas. Algiria.*

3 Cupreus. Linné. Fabr. Gallia.*

Var. Corulescens. Lin.

Versicolor. Sturm. id.

Medius. (Megerle.) Austria.

Var. Cupreoides, And. Lombardia.*

- Affinis. Sturm. Germania.*

Nemorensis. (Megerle.) Austria.

Erythropus. Stéven. Russ. merid.

4 Cursorius. Dej.

5 Quadricollis. Dej.

Var.Cyanescens.Gory. id.*

$\rightarrow$ Viridis. Gaub. Col.id.*

Dimidiatus. Olivier. Gallia.*

Tricolor. Fabr.

Galliabor.

Kugellani. Illig. (Ca-

rabus.) Helvetia.

Var. Eneus. Dej.

Gall merid.

7 Crenulatus, Dej.

8 Lepidus. Leske, Fabr.

Var. Cœrulescens.

$$
\text { Dej. } P \text {.* }
$$
Hispania. $^{*}$

Herbst. Austria.*

- Koyi. Germar. Hungaria.

- Viaticus. Bon. Dej. Italia.*

- Marginalis. (Meg.) Dalmatia.

Cyanescens. Besser. Volhynia.

Var. Transalpinus.

9 Ullrichii, Sturm. Cat. Hurgaria.
10 Böhmii. Sturm. Cat. Austria.

11 Gressorius. Dej. Alp. Gall.*

12 Mauritanicus. Dej. Algiria.*

13 Subcœruleus. Quensel

$\left\{\begin{array}{c}\text { in Schh. Gall. orient.* } \\ \text { Striatopunctatus (Me- } \\ \text { gerle.) Duft. Dej. Austria. }\end{array}\right.$

Cceruleovirens.Sturm. id.

14 Coarctatus, Lucas, Algiria.*

15 Cantabricus. Rambur, Hispania.

16 Splendens Géné. Sardinia.

17 Purpurescens, Dej. Algiria.*

18 Numidicus. Lucas. id.*

19 Boeticus. Rambur. Hispania.

20 Infuscatus. Hoffmsg.

Dej. Gall. merid.

Crenatus. Dej. $\quad$ Lusitania.*

22 Lugubris, Stéven. Dej. Russ, merid.

Nitidus. Dej. Hispania.*

24 Puncticollis. Dej. Gall.merid.*

\section{ARGUTOR. Megerle.}

Poecilus. Bonelli. Feronia. Dej. Carabus. Oliv, Fabr. Harpalus. Gyll.

1 Vernalis. Fabr. Gyll. P.*

Crenatus. Duftsch. Austria.

Var. Rotundicollis.

Duftsch. St. Germania.

_ Sedulus. Dej. $\quad \boldsymbol{P}$.

2 Maritimus, Gaubil. Gall. merid.*

3 Affinis. Sturm. Cat, Hungaria.

4 Salzmanni. Germar. Gall. merid.* Rubripes. Dej. id.

5 (Longicollis. Duftsch. Austria.*

Negligens. (Meg.) Dej. Gallia.

Nigerrimus. Sturm. Germania.

6 Inquinatus. Sturm. Hungaria.

Inquietus. (Meg.) Dej. id.

7 (Sturmii. Dej. Germania.*

Negligens. (Platysma.) Sturm. id.

8 Niceænsis, Villa. Pedemont.

9 Eruditus. Dej. P. ${ }^{*}$

Strenuus, Duftsch. Germania.

Interstinctus. Sturm, id.

Ovoideus. Sturm. id.

10 Strenuus. Illig. Panz. Gallia.*

Diligens. Sturm. Germania.

Var, Pullus, Gyll. Dej. id.*

Heyeri. Sturm. id.

Gagates. (Meg.) Duft. id.

Erythropus, Leach. Anglia. 
Pygmæus. St. Erich. Germania. Strenuus. Gyll, Dej. Suecia.

Soler. Sturm. Germania.

Nigriceps. Sturm. id.

Var. Testaceus. Sturm. id. Badius. Sturm, Cat. id.

13 Ochraceus.Sturm. Cat. Austria.

14 Piceus. Sturm. Cat. Styria.*

15 Pusillus. Dej.

16 Amoenus. Dej.

17 Pumilio. Dej.

18 Lusitanicus. Dej.

19 Nigerrimus. (Dahl.)

20

21

22

23

24

25

26

27

28

29

30

31

32

33

34

\section{Depressus. $D e j$.}

Nanus, Heer.

Calathoides. Dej.

Rufus. Duftschm.

Hispanicus. Dej.

Barbarus. Dej.

Spadiceus. Dej.

Subsinuatus. Dej.

Unctulatus. Duftsch.

Canaliculatus. (Lissotarsus.) Chaudoir.

Alpestris. Heer.

Appenninus. Dej.

Amaroides. Dej.

Abaxoides. Dej.

\{ Striatocollis. Dej.

\{ Picipes. Sturm.
Pyrenceis.

Pyrenais.*

id. *

Lusitania.

Sicilia.

Gallia.*

Helvetia.

Algiria.*

Austria.*

Hispania.*

Gall. merid. *

Gall. bor.*

Styria.

id. *

\section{Sicilia.}

Helvetia.

Alp. Lomb.

Pyrenais. *

id. $^{*}$

Croatia.

Hungaria.

\section{OMASEUS. Ziegler.}

Melanius. Bonelli, Feronia. Latr, Dej. Carabus. Fabr. Oliv. Harpalus. Sahlb. Gyll.

1 Cophosioides, Dej Hungaria. Cyclops. Sturm. Cat. id. Bannaticus. Sturm. id.

2 Melanarius. Illiger. $P_{0}^{*}$ Leucophthalmus, Fab. Dania. Var. Pennatus. Dej. Gallia. Nemoralis. Lat.inlitt. id. Nigerrimus. Sturm. Hungaria.

3 Melas. Creutzer. Dej. Dalmatia.* Maurus (Molops.) Stm. Hungaria. Var.Depressus. (Ziegl.)Dalmatia,* - Italicus. Bonelli. Gall. merid.*

4 Hungaricus. Dej. Hungaria.* Italicus. (Cophosus.) Sturm. Cat. Lombardia.

5 (Brevis. Sturm. Cat. Lombardia. Transversalis. (Abax.) Villa. id.

6 Wiedmanni. Stm. Cat. Gall. merid.

7 Nigritus. Fabr. Gallia. * Confluens. Panzer. Germania.

8 Anthracinus. Illiger. Gallia.* Maurus. Fabr. Schiod. Dania.

9 Distinctus. Lucas. Algiria.*

10 Rhæticus, Heer. Helvetia.

11 (Minor. Dej. Sahlb. P.* Anthracinus. Gyllenh. Suecia. Vernalis. Sturm. Germania. Var. Gracilis. Sturm.

Dej. $P{ }^{*}$

12 Tingitanus. Lucas. Algiria. ${ }^{\star}$

13 Elongatus. Duft. Dej. Gall. merid.*

14. Meridionalis. $D e j$. id,*

15. Aterrimus. Fabr. Gyll.P.*

16 Nigerrimus. Dej. Hispania.

17 Foveolatus. Stm. Cat. Germania.

18 Morio. (Ziegl.) St. Cat. Austria.

19 Affinis. Sturm. Cat. id.

\section{STEROPUS. Megerle.}

Feronia, Dej. Molops. Germar. St.

1 Madidus. Fabr. Dej. Gallia.* Var. Concinnus. Stm.

Humidus, St. Dej. Germania. * in Sch. Lusitania.

Var. Gagatinus. Germar. Dej. Hispania., Arrogans, Duftsch. id.

Var. Globosus. Fabr. Dej. Lusitania.*

3 Mannerheimii. Dej. Russia.

4. Ethiops 1.Illiger. Dej. Holsatia.* Marusiacus. Hummel. Russia.

5 Rufitarsis. Par. Dej. Hungaria.

6 Illigeri. (Megerle.) Dej. Austria.*

7 Placidus. (Erich.) Rosenhauer. Tirolis.

8 Cognatus. Dej. (Pla$\left\{\begin{array}{r}\text { tysma.) Hungaria.* } \\ \text { * }\end{array}\right.$ Schmidtii. Kunze. Carinthia.*

9 Cordatus. Sturm. Cat. Styria. 10 Brevis, Sturm. Cat. Constantin.

1 Cette espèce a été recueillie par moi aux environs de Phalsbourg, où elle est assez rare. 
PLATYSMA. Bonelli.

Feronia. Dej. Molops. Germar. CaraBUS. Fabr.

1 Variabilis. Ménétriés. Russ, merid.

2 Picimana. Duftsch. Gallia.*

3 Graja. Bonelli. Dej. Pedemont.

4 Extensa. (Parr.) Dej. Corfou.

5 Caucasica. Ménétriés. Russ. merid.

6 Marginepunctata. Dej. Italia.*

7 Senilis. Findel. Dej.

Cat. Pedemont.

9 Truncata. Villa. id.*

Nebrioides. Dej. Cat. id.

10 Gracilis. Sturm. Cat. Italia.

11 Maura. Duftsch.

Morio. Duftsch. Austria. *

Conformis. Sturm. id.

Bilineatopunctata.

12 Escheri. Heer.

(Dahl.) Italia.

13 Tamsii. Dej.

14 Findelii. Dej. $\quad H u$

Hafniersis. Gmel. Germania.

16 Angustata. (Meg.) Duft.Germania.

17 Arenosa. Leach. Anglia.

18 Borealis. (Harp.) Zett. Lapponia.

19 Ovata. Sturm. Cat. Constantin.

\section{COPHOSUS. Ziegler.}

Feronia. Dej.

1 Magnus. Dej.

Magnus. Dej. Hungaria.
Var. Cylindricus, Hbst.

$\left\{\begin{array}{c}\text { Dej. } \\ \text { - Filiformis. (Meg.) }\end{array}\right.$

- Dej. id.*

- Grandis. Gysselen.id.

2 Duponcheli. Dej. Gracia.

\section{PTEROSTICHUS. Bonelli.}

Feronia. Latr. Dej. Garabus, Fabr, Oliv. Harpalus. Gyll. Platysma. Germar.

Striatus. Payk. Suecia. *

Subcordatus. Chaud, Russ. merid.* Niger, Fabr, Dej. Gallia.

Var, Distinguendus.

Heer. Helvetia. ${ }^{*}$

2 Bacrii. Motschulsky. Russia.
3 Pestiensii. Schmidt. Carniolia.* Milleri. Kokeil. id.

4 Fasciatopunctatus. Fab. Austria. Striatopunctatus.

(Ullrich.) id.

5 Parumpunctatus. Dej. Gallia.* Cristatus. Dufour. Pyrencis.

Var. Lasserrei.(Dahl.) Gall. merid:*

כ믈 Impressicollis. Peirol. Pedemont. Intermedius. Mannerh. Pyrenais, *

6 Italicus. Chaudoir. Italia.

7 Micans. Lasserre.Heer. Helvetia. $\{$ Larien sis. Sol. St. Cat. id.

8 Deplanatus. Stm, Cat. Helvetia.

9 Hagenbachii. Sturm. Helvetia.* Honoratii. Dej. ․ Alp, Gall.

10 Modestus. Sturm. Cat. Volhynia.

11 Rufipes. Dejean. Gall. merid.*

12 Femoratus. Dejean. Gall. orient.*

13 Ambiguus. Dejean. Corsica.

14 Dufourii. Dejean. Pyrenais.*

15 (Truncatus. Dejean, Alp, Gall.*
Dilatatus. Villa.
Lombardia.

16 Obscurus, Steven. Russ. merid. Regularis? Fischer. id.

17 Imhoffii. Sturm. Cat. Alp, Helvet.

18 Aterrimus. Strm. Cat. Küster. Gall. merid.

19 Angustatus. Stm. Cat. Austria.)

20 Panzeri.(Megerle.) Dej.id.*

21 Biseriatus. Heer. Alp. Helvet.

22 Unicolor. Sturm, Cat, Italia.

23 Planatus. Sturm. Cat. id.

24 Instusii. Lw. Redtenb. Austria.

25 Ziegleri. Duftsch. Dej. Carinthia, *

26 Flavofemoratus. Bonell.

Dejean. Pedemont.

27 Pinguis. Bonelli. Dej. id.*

28 Depressus. Stm. Cat. Italia.

29 Cribratus. Bonell. Dej. Pedemont.

30 Drescheri. (Plectes.)

Fischer, Russia.*

31 Vagepunctatus. Bonll. Pedemont. ${ }^{*}$

32 Rutilans. Bonelli. Dej. Alp. Pedem.*

33 Fossulatus. Ahrens. Carniolia.*

$\{$ Welensii. Dahl. Dej. id.

34 Variolatus. Dejean. Styria.

35 (Interpunctatus. (Meg.)
Duftschmid.
Fossulatus. Schönher.
Dejean. Silesia.
Var.Minkwitzii.(Dhl.) id.

36 Klugii. (Dahl.) Dejean. Hungaria. 
37 Impressus. Peyroleri. Pedemont.

38 Selmanni. Duftschm. Austria.*

39 (Prevostii. Dejean. Helvetia.

Yar. Duvalii. Dejean. Helvetia * Selmanni. Sturm. id.

40 Stroblii. Knærlein. Austria.

41 Duratii. Villa. Pedemont.*

42 Xatartii. Dejean. Pyrencis.*

43 Jurinei. Panzer. Dufts. Austria.*

Var. Zahlbruckneri.

Gysselen, Alp. Helvet.

- Heydenii. Findel.

Heer. id.

- Clairvillei.St.Cat.Austria.

44 Bicolor. Peiroleri. . Pyrencis.

45 Dubius. Heer. Alp. Helvet.

46 Externepunctatus. Dej. Alp. Gall.*

Var. Sinuatopunctatus. Bonelli. Alp. Pedem.

(Multipunctatus. Dej. Helvetia.*

Var. Erythropus. Vill. Lombardia.

Cristoforii. Jan. Alp. Lomb.

Purpuratus, Lasserre, id.

48 Viridinitens. Stm. Cat. Italia.

49 Infuscatus, Stm. Cat. id.*

50 Spinolæ. Dejean. id.

51 Yvanii. Dejean. Alp. Gall.*

Bilineipunctatus. St.

Cat. id.

52 Muhlfeldii. Dufts. Dej. Carinthia. ${ }^{*}$

53 Affinis. Sturm. Cat. Hungaria.

54 (Baldensis.Jan.St.Cat. Italia.

\{ Var. Brunii. Cristofor. id.

55 Concolor. Sturm. Cat. id.

56 f Transversalis. Duftsch. Helvetia. ${ }^{*}$

$\{$ Var. Ellipticus. Lass. id.

57 Dissimilis. (Abax.) Vill.

Dej. Cat, Alp. Lomb.

\section{ABAX Bonelli.}

Fleronia. Latr. Dej. Ciarabus. Fab. Oliv. Harpalus. Gyllenh.

1 Striola. Fabr.

Var. Depressus. Oliv. P.

Subpunctatus. Ziegl. Croatia.

2 Pyrenæus. Dejean. Pyr. orient. ${ }^{*}$

3 Exaratus. Bonelli. Dej. Pyren.*

4 jOblongus. Dej. Italia.

Yar. Italicus. Cristof. id.
5 Parallelipipedus. (Meg.)

$\left\{\begin{array}{c}\text { Dej. Italia. } \\ \text { Elongatus. Lasserre. } \\ \text { Heer. Helvetia. } \\ \text { Porcatus, Villa. }\end{array}\right.$

6 Latus. (Megerle.) Dej. Hungaria.

7 (Carinatus. Duftsch. Austria.*

Var. Porcatus. Dufts. id.

Crenatus. (Dahl.) Hungaria.

8 Frigidus, Fabr. Gallia.*

Ovalis. Dufts. Dej. St. Germania.

9 Parallelus. Duftsch. Austria.*

Saxatilis, Panz.Germ. Gallia.

10 Contractus. Lass. Heer. Helvetia.

11 Assimilis. Sturm. Cat. Italia.

12 Beckenhauptii. Duftsch.

Sturm. Dej. Carinthia.*

13 Schüppelii. Dahl. Dej. Hungaria.*

14. Mellyi, Parreyss Stm.

Cat. Transylvan.

15 Rendschmidtii. Hart.

16

Zawadskii. Dej. Germar. Silesia. Gallizia.

\section{PERCUS, Bonelli.}

Abax. Sturm. Feronia, Latr. Dej.

1 Corsicus. Latreille. Corsica.*

\{ Lavigatus. Stm. Cat. id.

2 Genei. Dej.

3 Passerini. Dej. id.

4 Villæ. Durazo. Dej.

Sturm. Cat, Pedemont.

5 Bilineatus. Dej. Sicilia.*

6 Lineatus. Solier. Dej.

Cat. Algiria.*

7 Plicatus. Dupont. Dej. Ins. Balear.

8 Strictus. Dej. Gracia.

9 Ramburii. Dej. Cat. Corsica.*

10 Loricatus. Dej. id. *

11 Paykulii. Rossi. Dej. Italia.

12 Dejeanii. (Ziegler.) Dej.id.

13 Lacertosus. Dej. Sicilia.

14 Siculus. Dej. · id.

15 Oberleitneri. Dej. Sardinia.

16 Cylindricus. Kollar. Sturm. Cat. id.

17 Angustiformis. Solier. id.*

18 Stultus. Duf. Dej. Hispania.

19 Politus. Dej. Lusitania.*

20 Patruelis. Duf. (Brosc.) Pyrenceis. ${ }^{*}$

\{ Navaricus. Latr. Dej. Hispania. 


\section{CHEPORUS. Megerle,}

Molops. Bonelli. Dej. Feronia. Dej.

1 Striolatus. Fabr.

2 Metallicus. Fabr. $\{$ Var.Burmeisteri.Heer.Helvetia.

\section{MOLOPS. Bonelli.}

Feronia. Latr. Dej. Garabus, Fabroliv.

1 Robustus. Dej

\section{Hungaria.*}

2 Dalmatinus. Dej. Dalmatia.

3 Alpestris (Megerl.) Dej. Hungaria. Melas. Sturm. id.

4 Elatus. Fab. Pan.Dej. Germania.* Gagates. (Scarites). Panzer. id.

5 Bucephalus. (Parreys.) Dej. Croatia.

6 Depressus. Parreyss.

7 Longipennis. $D e j$. Sturm. Cat. Dalmatia.*

8 Terricola. Fabr. Croatia. Gallia.* Madidus, Payk.

Piccus. Panzer. Suecia. Germania. Var. Montanus. Heer. Helvetia. - Punctatus. Dahl. Hungaria.

9 Græcus. Chaudoir.

10 Picipes. Megerle.

11. Rufipes. Chaudoir. Gracia. Austria.* Grocia.

Caspicus. Ménétriés. Russ. merid. Subtruncatus. Chaud. Helvetia. Spinicollis. Dej.

Pyr. orient.

MYAS. Ziegler. Dej.

1 Chalybeus. (Ziegl.)Palliardi. Dej. Hungaria.*

BROSCUS. Panzer.

Gephalotes. Bonelli. Dej. Harpalus. Gyll. Carabus, Fabr. Scarites, Oliv. 1 $\left\{\begin{array}{l}\text { Cephalotes. Linné... P.* } \\ \text { Vulgaris. Bonelli.Dej. }\end{array}\right.$ 2 Politus. Dej. Sicilia. Algiria.* 3 \} Nobilis. Dej. Oriente. $^{*}$ I Rufpes. Guérin. Constantin.
STOMIS. Clairville. Dej.

Carabus. Duftsch. Panz. Harpalus. Gyll.

1 Pumicatus. Panz. Dej. Gallia.*

2 Rostratus. Sturm. Dej. Alp. Gall.*

3 Italicus. Sturm. Cat. Alp. Ital.

PELOR. Bonelli.

Zabrus. Sturm. Garabus, Duftsch.

1 Rugosus. Ménétriés. Russ. merid.

2 Spinipes. Fabr. Austria. $\{$ Blapoides.Creutz.Dej.id.

3 Steveni. Fischer. Russ.merid. $\{$ Tauricus. Chaudoir. Tauria.

ZABRUS. Clairville. Dej.

Harpalus. Gyll. Carabus, Fabr. Duftsch. Blaps. Fabr. Eutroctes. Zimmerm.

1 Femoratus. Dej. Gracia.

2 Gravis. Dej. Hispania.

3 Dentipes. Zimmerm. id. \{ Silphoides. Dej. id.

4 Marginicollis Dej. . id.

5 Cuírtus. Latreille. $\quad \boldsymbol{P}^{*}{ }^{*}$

6 Curtoides. Chaudoir. Europ.merid.

7 Inflatus. Dej. Gall.mer.oc.

8 Obesus, Latreille. Pyrenais. ${ }^{*}$

9 Robustus. Zimmerm. Gracia.* Fontenayi.Dej. : id.

10 Græcus. Dej. … id.*

11 Punctatostriatus. Brul. id.* \{Puncticollis. Brullé. id.

12 Distinctus. Lucas. Algiria.*

13 Latus. Sturm. Cat. Gracia.

14 Rufipes. Sturm. Cat. Smyrna.

15 Nitens. Victor. St. Cat. Russ, merid.

16 Incrassatus. Germar. Dalmatia.

17 Pinguis. Hoffmsg. Dej. Lusitania.

18 Puncticollis. Dej. Algiria.* Globosus. Gory. id,

19 Pyrenæus. Chaudoir. Pyrenais.

20 Orsini. Géné. Italia.

21 Gibbosus. Ménétriés. Russ. merid. Var. Morio. Ménétriés. id. (Rufomarginatus. Mén.id.

22 Trinii. Fischer. id.

23 Gibbus. Fabr. Gallia.* Tenebrosus. Fabr. Dania. Madidus. Olivier. Gall.merid. 
24 Flavoangulus. Chevrol. $N$.

25 Piger. Dej. Gall. merid.* 1

26 Sublævis. Ménétriés. Constantiu.

27 Rotundicollis. Ménét. id.

(Eutroctes. Zimmerm.)

28 Aurichalceus, Adaims. (Zab.) Russ. merid. Adamsii. Fischer. id.

29 Mœstus, Erichson. . id.

\section{ACORIUS. Zimmermann.}

Amara, Dej.

1 Metallescens.Dahl.Dej.Sardinia.

AMARA. Bonelli.

(Megerle.) Zimm. Harpalus. Gyll. Carabus. Fabr. Percosia, Celia, Bradytus, Leyrus, Leiocnemis, Acrodon. Zimmerm.

(Percosia, Zimm.)

1 Sicula. (Dahl.) Dej. Sicilia. ${ }^{*}$

2 Pastica.Zimmermann. Russ. merid.

3 Patricia. Creutz. Duft.

Var. Zabroides, Dej. $\quad$ Gall. merid.

$\{$ - Mancipium. Duft. Germania.

- Plebeja. Duftsch. id.

Equestris. Duftsch. id.

Nobilis. Sturm. id.

4 Dilatata. Heer.

5 (Helopioides. Heer. id. Alpestris.Villa. Lombardia.

(Celia. Zimmermann.)

6 (Ingenua. Creutz. Duft. Gallia.*

Lata. Sturm. id.

Subanea.Sturm. Austria.

7 Ruficornis. Dej. Gall. merid."

8 Floralis, Gaubil. id.*

9 Complanata. Dej Dalmatia.

10 (Fusca. Sturm. Dej. Gall. merid.* Saxicola. Ménétriés. Russ. merid.

Var. Montana. Ménét. id.

11 (Municipalis. Duftsch. Germania.* \{Modesta. Dej. Erich. Gall. merid.

12 Fuscicornis.Zimmerm. Dalmatia.

13 Prosperans.Zimmerm. Austria.

14 Cursitans, Zimmerm. id.
15 Ambulans. Esch.Zim. Russia.

(Erratica. Duftsch. St. Germania.

Punctulata. Dej. Helvetia.

Septentrionalis.

Schiodte, Dania.

17 Melancholica. Schiodte, id.

18 Quenselii. Schönherr. Lapponia.*

19 Silvicola. Schmidt. Suecia.

$\{$ Quenselii, var. Dej. id. Metallifera. Andersch. id.

20 -Graculus, Heer Helvetia.

21 (Monticola. Zimmerm. Alp. Pedem.*

Montana. Dej. Pyrenais.

Var. Marginata. Heer. Helvetia.

22 Onsburgeri. Heer. id.

23 Saxicola. Ménétriés. Russia.

24 Infima. Duftsch. St. Gallia bor.*

Brevis. Sturm.

I Granaria. Dej.

Livida. Fabr. $\boldsymbol{P}^{*}$

Bifrons. Gyllenh. Dej. Suecia.

Brunnea. Sturm. Germania.

26 Taurica. Motschulsky. Tauria.

27 Maritima. Schiodte. Dania.

28 Affinis. Dej. Hispania.

29 Rotundata. Dej. id.

30 Brevis. Dej. id.

31 Simplex. Dej. . id.

32 Grandicollis, Dej. Cat. Gall. bor.*

Var. Seileri. Heer. Helvetia.

Rufocincta. Mannerh. Finlandia.

Orcophila. Imhoff. id.

Pallens. Sturm. id.

33 Lapponica. Mannerh. Lapponia.*

34 Zimmermannii. Heer. Helvetia.

35 Antennata. Zimmerm. Tirolis.

(Amara, Zimm.)

36 Striatopunctata. Dej. Gallia.* Homatapa. (Parr.) Corfou.

37 Rufipes. Dej. Gall. merid.*

Erythrocnema. (Parreyss.) Kollar. Corfou.

38 Concinna. Zimmerm. Germania.

39 Lepida. Zimmerm. id.

40 Tricuspidata.Stm. Dej. id.*

41 Strenua. Zimmerm. id.

42 Plebeja.Gyllenh. Dej. Gallia.*

43 Lapidicola, Heer. Helvetia.

44 Varicolor. Heer. id.

45 (Saphyrea. (Ziegl.) Dej. Hungaria.*

Domidua, Sturm. Cat, id. 
46 (Communis, Fabr. Gallia."

Similata. Gyll. Dej. Suecia.

Montivaga. Sturm. Germania.

47 Obsoleta. Duft. Dej. Gallia.* Pratensis. Sturm. Germania.

48 Acuminata. Payk, St. Gallia.*

Eurynota. Illiger. Dej Germania.

Vulgaris. Fabr. Dania.

49 Trivialis. Gyllenh. Dej. P.*

50 Spreta.Zimmerm.Dej. Gall. bor.*

51 Famelica. Zimmerm. Germ. media. 79

52 (Vulgaris. Linné. Dej. Gallia.*

Contrusa? Schiodte. Dania.

Assimilis. Chaudoir. Russia.

Lenticularis. Schiodte Dania bor.

Despecta. Sahlberg, Lapponia.

Contensa. Schiodte. Dania.

Curta. Dej. Lacord. Gallia.

Depressa. Zimmerm. Germania.

Enea. (Megerle.) Austria.*

Communis, Dej.St. Gallia.

Rufiventris. Germar. Germania.

Var. Atrocerulea. (Me-

$\{$ gerle.) St. id.

- Atrata. Heer.

- Alpina. Heer.

Ferrea. Sturm.

59 Poeciloides. Heer.

\section{Helvetia.}

id.

Austria.

Helvetia.

60 (Nitida. Sturm. Erich. Germania.* Var.? Formosa. Schdte.Dania.

61 Lunicollis. Schiodte. Dania.

62. Limbata. Schiodte. id.

63 Familiaris. Duft. Dej. Germania.* Vulgaris. Müll. var. $\beta$.

Schh. id.

Levis. Sturm. Zimm. id.

Cursor. Sturm. id.

Var. Atrata. Heer. Helvetia.

64 Perplexa. Dej.

65 Brunnicornis. Heer.

66 (Lucida. Duftsch. Volhynia.

Helvetia.

Gemina. Zim. Erich. id.

67 Tibialis. Paykull. Dej. Suecia.*

$\{$ Var. Viridis, Duftsch. Germania.

68 Sahlbergi. Zettersted. Dania.

69 Deserta. Krynicki. Russia.

\section{(Bradxtus. Zimm.)}

70 Consularis. Duftsch. Gallia.* \{ Lata. Schönherr. Gyll. Suecia.

71 Apricaria, Fabr. Schh, P.*

Lata. Fabr.

Analis. Fabr.

Germania. id.
72 Convexilabris Schmid. Galizia.

73 Patrata. Schiodte. id.

74 Aurichalcea. Gebler.

Germar. Russ, merid.

75 (Ferruginea. Linné. P.*

Fulva, de Geer. Schh. Suecia.

Pallida.Fabr. Dania.

76 Iridipennis. Heer. Helvetia.

77 Nigra, Chaudoir. Silesia.

78 Eneomicans. Chaud. id.

Cognata. Sturm.

Carniolia.

(LejRus. Megerle. Zimm.)

80 Spinipes. Linné. $\quad P^{*}$

Picea.Fabr.St. Dania.

Aulica.Illig.Gyll.Dej. Austria.

Bicolor. Paykull. Suecia.

81 Torrida, Kugel. Illig. id.*

82 Convexiuscula. Marsh.

Dej. Anglia.*

83 Alpina, Fabr, Dej. Suecia.*

84 Montana. Chaudoir. Russia.*

(LeIOCNEMIS، Zimm.)

85 Cardui Dej. Helvetia. *

86 Cordicollis. Ménétriẻs. Russia.

87 Pyrenæa.Dej. Pyrencis.

88 Puncticollis. Dej. Pyr. orient.*

89 Grenata. Dej. Gall. merid.

Elongata. Sturm. Dalmatia.

90 Alpicola. Dej. Styria. ${ }^{*}$

91 Cuniculina. And. Dej. id.

92 Sabulosa. Dej. Gallia.

93 Dalmatina. Dej. Dalmatia.

Castanea.Sturm. Cat. id.

94 Eximia. Dej. Gall. merid.*

Flavipes. Sturm. Cat. Gallia.

95 Glabrata. $D e j$. id.

96 Nobilis. Dej. Austria.

97 Latiuscula. Chaudoir. Styria.

(ACrodon. Zimmerm.)

98 Brunnea, Gyllenhal.

(Harpalus.) Suecia.

DAPTUS. Fischer, Dej.

Ditomus. Germar.

1 Vittatus. Fischer. Dej. Gall. merid." Vittiger, Germar, Algiria. 
BROSCOSOMA. Putzeys. Rosenhauer.

1 Baldense, Putz. Ros, Tirolis.

ACINOPUS. (Ziegler.) Lat. Dej.

Carabus. Duftsch. Harpalus. Duftsch. Scarites. Germar. Oliv.

1 Lepeletieri. Lucas. Algiria.*

2 Mauritanicus, Lucas. id.

3 Elonğatus. Lucas. id.*

4 Megacephalus. Rossi. Olivier. Gall. merid Bucephalus, Dej. id.

5 Gulturosus, Buq. Luc. Algiria.*

6 Tenebrioides. Duftsch. Gall. merid.* Megacephalus. Latr. \{ Dej. id.

Pasticus. Germar. id.

Sabulosus. Sturm. id.

7 Quadricollis. Solier. Gracia.

8 Picipes, Oliv. Brullé. Algiria.* Megacephalus, Fabr. id.

9 Clypeatus, Fischer. Russ, merid.

10 Quadricollis, Brullé. Gracia.

11 Rufitarsis. Fischer. Russ. merid.

12 Sabulosus. Olivier. Algiria.*

Megacephalus, Fabr. id.

Obesus, Schonh. Dej. id.

13 Ambiguus. Dej. Sicilia.* Rufipes. Dahl. id.

14 Giganteus, Dej. Hisp. merid.

15 Spinipes. Fischer. Russ, merid.

16 Ammophilus. Dej. id.

17 Rotundicollis. St. Cat. Gracia.

18 Patruelis. Sturm. id.

SELENOPHORUS. Dejean.

Harpalus, St, Dej. Cat. Carabus, Fabr.

1 Scaritides. Ziegl. Dej. Austria.

ANISODACTYLUS, Dejean.

Harpalus. Gyll. St, Dej. Cat. Cirabus. Fabr.

1 Heros. Fabr. Lusitania.*

2 Dejeanii, Buquet. Dej. Algiria.*

3 Pseudoæneus. Dej. Gall. bor.*

4 f Virens, Dej. \{ Var. Distinctus, Sol, id.
5 (Signatus, Illiger. P.*

Eschscholtzii. Gebler. Sibiria. Rusticus, Dahl. Hungaria.

6 Nonsignatus. Krynicki.Russia.

7 Intermedius. Dej. . Gall. merid.

8 Binotatus. Fabr. P.*

Var.Spurcaticornis. Zgl.Gallia.*

9 (Nemorivagus. Duftsch.id.*

Gilvipes. Dej. Germania.

Rufipes, Bonelli. Italia.

DIACHROMUS, Erichson.

Carabus. Linné, Fabr. Harpalus. Dej.

1 Germanus. Linné. Gallia.*

GYNANDROMORPHUS. $D e j$.

Carabus. Schönh. Panz. Harpalus. Sturm.

1 Rossii. Ponza. ${ }^{1}$ Pedemont.* Etruscus, Quensel in

Schh. Gallia merid.

2 Jonicus. Sturm, Cat. Corfou.

OPHONUS. Ziegler.

Harpalus. Gyllenh. Dej.

1 Columbinus. Germar. Gall. merid.

2 Longicollis. Chaudoir. Turcia.

3 Sabulicola. Panzer. P.* Azureus. Olivier. Gall. merid.

Obscurus. Duftschmid.Germania.

4 Obscurus. Fabr. Gall. orient.* Monticola. Dej. $\quad$ Gall. merid.

Viridanus, Ménétriés, Russ. merid.

$5 \int$ Diffinis. Dej. $\quad$ Gall. merid.*

T Turbidus. (Megerle.) Italia.

6 Rotundicollis.Dej.Cat.Gall. merid.* Obscurus. Dej. Iconog. Austria.

Var, Opacus. (Dahl.) St.

Cat. Hungaria.

Algiricus. Buquet. Algiria.

7. Quadricollis. (Dahl.)

Dej. Sicilia.*

8 Oblongiusculus. Dej. Gallia. ${ }^{*}$

9 Jitomoides. Dej. Gall. merid.*

1 Mémoire inséré dons le volume de l'Académie des sciences de Turin, pour les années r 805 I 808 . 
10 Silbermanni. (Gaubil.)

11 Incisus. Dej.

$$
\text { Nov. Sp. Algiria.* }
$$

Gall. merid."

12 Punctatulus. Duftsch. Gallia.*

Umbricola. (Dahl.) Germania.

Reptans. (Dahl.) Hungaria.

13 Similis. Sturm. Dej. Dalmatia.*

14 Chlorophanus. Zenk.

Dej. Gallia.*

Gall. merid.*

15 Azureus. Fabr.

Russ. merid.

$\left\{\begin{array}{l}\text { Cribricollis. Stev. Dej. Rus. } \\ \text { Tauricus. Godet. id. }\end{array}\right.$

Cordicollis. (Parreys.) id.

Punctatissimus. OEsk. Hungaria.

Cordicollis: $D e j$.

Subquadratus. Dej.

Meridionalis. $D e j$.

Pumilio. Dej.

Rotundatus. Dej.

Cordatus. Duftsch.

Porrosus. Germar. Dalmatia.

Var, Denigratus. St.

Cat. Gall. merid.

23 Rupicola. Sturm.

I Subcordatus. Dej.

Russ. merid.

Gall. merid.

id.*

Sicilia.*

Gall. merid.*

id.*

24 Puncticollis. Paykull.

$$
\text { Gyll. id.* }
$$

25 Sardeus. Sturm. Cat. Sardinia

26

Rufibarbis. Fabr. Germania.*

Foraminosulus. Mars. Anglia.

Brevicollis. Dej. Gallia.

Puncticollis. Gyllenh. Sahlb. Suecia.

Melletii.Heer.

Helvetia.

Parallelus. Dej.

Hispania.

Complanatus. Dej.

Styria.*

30 Maculicornis. Duftsch. Gallia.*

Interstitialis. St. olim. Austria.

31 Signaticornis. Duftsch. Gall. bor.*

32 Hirsutulus. $D e j$.

33 Planicollis. Dej.

34 \} Mendax. Rossi.

\{Reichenbachii. St.Cat. id.

Pyr, orient.*

\section{Sicilia.*} Gall. merid.*

Luteus. Sturm. Cat. Sardinia

36 Ochraceus. Stm. Cat. Dalmatia.

37 Minimus. Motschulsky. Russ, merid.

HARPALUS. Latreille.

Carabus, Fabr.

1 Hospes. Creutzer. Dej. Hungaria.
2 (Sturmii. Dej.

Hospes. Sturm.

3 Ruficornis. Fabr.

Pubescens. Müller.

4 Griseus. Panzer.

Luridus. Faldermann. Gallia.

5 Dispar. Dej.

Chloripennis. St. Cat. id.

Roseri. Sturm. Cat. id.

6 Neesi. Sturm. Cat. id.

7 Semipunctatus. Dej. Hisp. merid. ${ }^{*}$

8 Eneus. Fabr. Gallia.*

Proteus. Paykull. Suecia.

Var. Azureus. Panzer. Germania. - Confusus. Dej. P.

9 Oblitus. Dej.

10 Diversus. Dej.

Dalmatia.*

11 Distinguendus. Dufts. P.*

\{irens. Ménétriés. Russ. merid.

12 Mauritanicus. Gaubil.

Lucas, Algiria.*

13 Patruelis. Dej. Gall. merid, ${ }^{*}$

14 Fastiditus. Dej. Hispania.*

15 Euchlorus. Ménétriés. Constantin.

16 Auratus. Sturm. Cat. Sicilia.

17 Contemptus Dej. Hispania.

18 Metallicus. Ménétriés. Constantin.

19 Minutus. Dej. Hispania.

20 Lateralis. Dej. id.

21 (Cupreus. Dej. Gall. merid.*

Fastuosus. Dahl. Dalmatia.

Metallicus. Godet. Russ. merid.

22 Honestus. Duftschm. Gallia.*

Ignavus. Creutzer. Germania.

Nitidus. Sturm. id.

Gravenhorstii. Kollar. Hungaria.

Var. Confinis. Dej. Gallia.

23 Impressipennis. Dej. Hispania.

24 Alpestris. L. Redtenb. Austria.

25 Nigripes. Sturm. Cat. Hungaria.

26 (Sulphuripes. Grm. Dej. Gall. merid." Chalybeipennis. Stm.

\section{Cat. Dalmatia.}

27 Consentaneus. Dej. Gall. merid. ${ }^{*}$ Desertus. Steven. Russ. merid.

Sardeus.Dahl.St.Cat.Sardinia.

28 Pygmæus. Dej. $\quad$ Gall. merid.

Brunnicornis. St. Cat. Hungaria.

29 Varians. (Dahl.) Stm.

30 Goudotii. Dej.

31 Pumilus. Dej.

Cat. id.
Gall. merid.*

id.* 
33

$\left\{\begin{array}{r}\text { Neglectus. Dej. Gall. occid. } \\ \text { Piger. Creutzer. Strm. } \\ \text { Gyll. Austria. } \\ \text { Capucinus. Schönher. Lusitania. }\end{array}\right.$

34 Decipiens. Dej. Gall. merid.

35 (Discoidens. Fabr. Er. Gall, bor.* Smaragdinus, Dufts. Austria. Perplexus. Gyll. Dej. P.

Var. Petifii. Duftsch. Austria.

Duftschmidtii. Sturm. Germania.

36 Saxicola. Dej. Illyria.

37 Siculus. $D e j$.

38 Incertus. Dej. Sicilia.*

39 Punctatostriatus. Dej. id.* Gentilis. Parr. Dej. Sturm. Cat. id.

40 Calceatus. Dufts. Dej. P.*

41 Ferrugineus. Fabr. Gall. bor,*

42 Fulvus, Dej. Algiria.*

43 Gilvicornis. Stm. Cat. Italia.

44 Flavicornis. Stm. Cat. id.

45. Hottentota. Duftsch. Gallia:

Deplanata. Godet. Russ. merid.

$\{$ Var.Subsinuata,Duft, Austria.

Ruficeps. OEskay. Hungaria.

Crinitus, Sturm. Cat. Germania.

46 Qudripunctatus. Dej. Gallia.

Seriepunctatus? Gyll.Suecia.

47 Fulvipes. Fabr. St, Gallia.*

Rufibarbis, Fabr. Dania.

Limbatus. Duft. Dej. Gallia.

Var. Erythrocephalus.

Fabr. Germania.

Flaviventris. Sturm. id.

48 Piceus. Sturm. Cat. Sardinia.

49 Maxillosus. Stév. Dej. Algiria.*

50 Puncticollis. Waltl.St.

Cat. Gracia.

51 Luteicornis, Duftsch.

St. Dej. Germania.*

Serotinus, Creutzer. Gallia.

52 Lævicollis. Duftsch, Gall,or, bor.* Satyrus. Knoch. Dej. Austria.

Var. Glabricollis. Dej. id.

Montanus. Sturm. Germania.

Var. Flavolimbatus.

Alpestris. Heer. Heer. He

Thoracicus. Heer. id.

53 Nitens, Heer. id.*

54 \} Solitaris. Eschsch. Dej. Lapponia.

I Var. Nigritarsis.Sahlb.id.

5ั5 Marginellus, Dej, Alp. Gall, *
56 Cuniculinna. Duftsch. Austria.

57 Rubripes. Duftschmid. P.*

Azurescens, Gyllenh. Suecia.

Azureus. Sturm. Germania.

Glabrellus, Stm.Ziegl, id.

58 Amøenus. Heer.

Helvetia.

59 Sobrinus. Dej. Pyr. orient.

60 Boristenicus. Krynicky. Russia.

61 Zabroides. Dej. Russ, merid. Latus. Stéven. id.

62 Hirtipes, Illig. Schh.

Dej. Germania.*

63 Semiviolaceus. Brong.

Var. Planicollis. San-

Dej. Gallia.

Corvus. Duftsch. vitale. Italia.

Depressus, Duftsch. id

Melampus. Duftsch. id.

Schreibersii. Duftsch. Dalmatia.

Crassipes.Duftsch. id.

Caspius. Stéven. Russ, merid.

Var. Vicinus. Dej. P. ${ }^{*}$

64 Hypocrita. Dej. Hispania.

65 Impiger, Duflschmid, Gallia.*

Autumnalis, Duftsch. Austria.

Inunctus. Sturm. Germania.

(Seriepunctatus. Stm. id.

66 Elatus. (Megerle) St.

67 Tenebrosus. Dej. Gallia. ${ }^{*}$

$\{$ Parallelus. Jenisson. Pyrencis.

68 Solieri. Dej. Gall. merid.

69 Melancholicus. Dej. P. ${ }^{*}$

Piciventris, Parreyss, Corfou.

70 Notatus. Cristofori. Lombardia.

71 Litigiosus. Dej. Gall. merid.*

72 Ineditus. Dej.

73 Hybridus. Dej. Russ. merid.

74 Coracinus. Sturm. Germania.

75 Tardus, Fabr. Panzer. P.*

Fuliginosus. Duftsch. Austria.

Lentus. Sturm. Germania.

Saginatus, Eschsch. Livonia.

76 Fröhlichii. Stm. Erich. Germania.*

Y Segnis. Dej. id.

77 Modestus. Dej. Styria.*

78 Chevrieri. Heer. Helvetia.

79 Rugulosus. Heer. id.

80 Serripes, Quensel. in

Schh. Gallia**

81 Taciturnus. Dej. Dalmatia.

82 Fuscipalpis.(Ziegl.)St.Austria.* 
83 Subcylindricus. Dej.

84 Kunzei. Sturm. Cat.

85 (Anxius. Duftsch.

Nigripes. Sturm. Cat. Germania.

Gracilis. Sturm. Gat. id.

86 Servus. Duftsch. Gallia.*

Complanatus. Sturm. Germania.

87 Flavitarsis. Dej. St. id.*

88 Vernalis. Fabr. Gallia.*

$\{$ Picipennis, Duftsch. Dej. Germania.

89

\section{STENELOPHUS. (Megerle.) Dej.}

Harpalus. Gyllenh. Carabus. Fabr. Acupalpus. Latreille.

1 ( Vaporariorum. Fabr. P. $^{*}$ \{ Nigriceps. (Ziegler.) Hungaria.*

2 Melanocephalus, Findel. Heer. Gallia.*

3 (Discophorus. Fischer. Gall. merid.* Centromaculatus.

(Dahl.) Hungaria.

4 Abdominalis. Géné. Sardin. Algir.

5 Hirticornis, Fischer.

6 Steveni. Krynicki.

7 Elegans. Dej.

8 Halophilus. Germar.

9 Chevrolatii. Gaubil.

10 Proximus. Dej.

11 ( Vespertinus. Illig. Dej. P

Ziegleri. (Megerle.)

12 Marginatus. Dej.

Russ. merid.

id.

Gall. merid.*

Germania.*

Pyr, orient. * Gall. merid.*

13

$\left\{\begin{array}{l}\text { Consputus. Duftsch. } \\ \text { Ephippiger. Duftsch. }\end{array}\right.$

Ephippium. Dej.

Gyll. Suecia.

15

16

17

Flavipennis. Lucas.

Russ. merid.* Algiria.

Suturalis. Ziegler. Dej. Dalmatia.

f Brunnipes, Duftsch.St. Germania.* Atratus. Dej.

18 Pallipes. Dej.

19 Dorsalis. Fabr.
Gallia.

Dalmatia.

Gallia.*
20 Meridianus, Lin. Fabr. P.*

Cruciger. Fabr. Germania.

21 Lucasii. Gaubil. in litt. Algiria.

Marginatus. Lucas. Expl. sc. id.

22 Flavicollis. Sturm. Germania.* Luridus. Dej. Gallia.

Fuscipennis. Sturm. Germania.

Liliputanus. (Ziegler.) Austria.

23 Nigriceps. Dej. Gallia.*

24 Exiguus. Dej. Germania.* Minimus. Mannerh.

(Tachys.) Sibiria.

BRADYCELLUS. Erichson.

Ophonus, Ziegl. Harpalus, Dej, Gyll. Acupalpus, Latr.

1 Pubescens, Paykull.

2 (Obsoletus. Dej.

(Harp.) Gyll. Gall. bor.*

Cerinus, Stéven Gall. merid.

Var. Dorsalis. Dej. Gall bor *

3 Chloroticus. Dej. Sicilia.

4 Pallidus. Dej.

5 f Stevenii. Dej.

\{ Sabulicola. Stéven. id.

6 Discicollis. Dej. id.

7 Rufithorax. Mannerh. Finlandia.

8 Cognatus. Gyll. Dej. Suecia.*

Deutschii. Sahlberg. Dania.

Alpinus. Schönherr. Lapponia.

9 Placidus. Gyllenh.Dej. Suecia.*

\{ Affinis. Sahlberg. Dej. Dania.

10 Lusitanicus. Dej.

11 Harpalinus. Dej.

Fulvus. Marsham.

Collaris. (Trechus.)

Lusitania.*

Gallia.*

Anglia.

Distinctus. Dej.

13 Collaris. Payluull.

14 Similis. Dej.

Sturm. Germania.

Gall. merid.*

Gaîl, bor.*

id.*

HISPALIS, Rambur,

Amblystomus, Erich. Acupalpus, Dej.

1 Mauritanicus. Dej. Hisp. merid. Alg. ${ }^{*}$

2 Metallescens. Dej. Gall. merid.* Albipes. Sturm. id.

3 Bifoveolatus. Rambur. Hisp. merid, *

4 Gracilis. Rambur. id.

5 Montanus. Rambur. id. 


\section{ANOPHTHALMUS. Sturm?}

1 Schmidtii. Sturm. Germania.

TRECHUS. Clairville. Dej.

Bembidum. Gyll. Garabus, Fabr. Aepus. 32 Leach.

1 (Discus, Fabr.

Unifasciatus, Panz. id.

Marice. Hummel. id.

2 Micros. Herbst. id.*

Rubens. Duftschmid. Austria.

Planatus. Duftsch. id.

Sericeus. Fleischer. Russia.

Crassicornis,Stm,Cat. Germania.

3 Quadricollis. Putzeys. Russia.

4 Longicornis. Sturm.

Brulle. Germania.*

Rubens. Duftschmid? Austria.

Littoralis, Dej. $\quad$ Gall.bor.

5 Ponticus. Motschulski. Russ. merid.

6 Paludosus. Gyllenhal.

Rubens. Fabr.? id.

St. Dej. Germania.*

Palpalis. Duftschmid. Austria.

7 Subnotatus. Dej. Gracia.

8 Rivularis. Gyll. Dej. Suecia.

9 Longhii. Comol. Putz. Pedemont.

10 Procerus. Putzeys. Transylvan.

11 Fulvus. Dej.

12 Rufulus. Dej.

Hispania.

13 Nigrinus. Putzeys.

Sicilia. *

14 Maurus. Putzeys. Carinthia.

15 Minutus. Fabr. Gallia.*

Tempestivus. Panzer. Germania.

Quadristriatus. Gyll. Suecia.

Rubens. Clairv. Dej. Helvetia.

16 Obtusus. Erichs. Putz. Gall. bor.

17 Castanopterus. Heer.

Putzeys. Helvetia.

18 Austriacus. Dej.

Austria.

19 Ochreatus. Dej.

Styria.

20 Pallescens. Redtenb. Austria.

21 Bannaticus. Dej. Bannat.

22 Montanus. Putzeys. Austria.

23 Palpalis. Dej. . id.*

24 Rotundipennis. Dft. St. Styria.*

Alpicola. Sturm. id.

Alpinus. Dej. $\quad$ id.

25 Latus. Putzeys.

26 Patruelis. Putzeys.

27 Croaticus. Dej.

Brevis. St. Cat.

28 Rotundatus. Dej.

29 Striatulus. Putzeys.

30 Piceus. Putzeys.

31 Pyrenæus. Dej. Pulchellus. Putzeys. Saxonia.* Glacialis. Heer. Putz. Helvetia.

34 Assimilis. Heer. Putz. id.

35 Profundestriatus. Heer. Putzeys, id.

36 Macrocephalus, Heer. Putzeys. id.

37

38

39

40 Pertyi. Heer. Putzeys. Helvetia.

41 Lævipennis. Heer. Pu-

42 Secalis. Paykull. tzeys, id.

$\{$ Testaceus. Fabr.

Gall. bor.*

Dania.

\section{(EPus. Leach.)}

43 Fulvescens. Leach. Anglia.

BEMBIDIUM. Latreille. St. Dej.

Cillenum. Leach, (Lymnaum, Leach. Blemus. Ziegl.) Tachys, Notaphus, Peryphus , Leja, Lopha, Tachypus. (Megerle.) Oxydromus. Frölich. Elaphrus. Duftsch. Carabus. Fabr.

\section{PREMIERE DIVISION.}

Cillenum. Leach.

1 Laterale. Curtis.

\{Leachii. Dej.

Gallia bor.* Anglia.

DEUXIËME DIVISION.

Lymnaum, Leach. (Blemus. Ziegl.)

2 Areolatum. Creutzer. Gall. merid.*

TROISIEME DIVISION.

Tachys, Megerle.

3 Fulvicolle. Dej.

Dalmatia.

4 I Scutellare. Dej. Gall. merid. ${ }^{*}$ Substriatum. Sturm. Germania.

5 Elongatum, Dej. Hispania.

6 Algiricum. Lucas, Algiria. 
7 (Bistriatum Duftsch. Gallia. ${ }^{*}$ Yar, Pallens, Dej. Hisparia. Angustatum. Dej. Cat. Austria. Pusillum, Dej. id.

* f Platypterus. Sturm. Gallia.* Rufescens. Dej. id.

9 (Quinquestriatum. Gyll. Suecia.* Pumilio. Duftsch. Dej. Gall. bor.

Obtusum. Sturm. Germania.

10 Silaceum. Dej.

11 Numidicum. Lucas, Algiria.

12 Nanum. Gyllenhal. Gallia.* Quadristriatum. Stm. Germania.

Minimum. Duftsch. id.

Var. Micros. Stéven. Russ. merid.

13 Quadrisignatum. Duft. Gall. merid. ${ }^{*}$

14 Lucidum. Sturm. Cat. Germania.

15. Gibberosum. Lucas. Algiria.

16 Sulcatum.Sturm. Cat. Germania.

17 Angustatum. Dej. Gall. merid.

18 Parvulum. Dej. id.*

$\{$ Var.Pusillum.Dej.Cat.Dalmatia.

Hæmorhoidale. Dej. Gall. merid.*

Guerinii. Gaubil.

Globulum. Dej.

Pulicarium. Dej. id.*

Hispania.

Gall. merid.

QUATRIEME DIVISION.

Notaphus. Megerle.

$\begin{cases}\text { Undulatum. Sturm. } & \text { Gallia. } \\ \text { Majus. Gyllenhal. } & \text { Suecia. } \\ \text { Varium. Gebler. } & \text { Sibiria. }\end{cases}$

24 Ustulatum. Lin. Fabr. Gallia.*

Majus, var, b. h. Gyll. Suecia.

Varium. Olivier. $P$.

25 Fossulatum. (Gaubil.) Gall. bor.*

26 Obliquum. Sturm.Dej. Gall. merid.*

IUstulatum. Gyllenhal. Suecia.

27 Fumigatum. Creutzer.

$\{$ Duft. Gallia.*

Exarticulatum. (Megerle.) Dahl. Austria.

28 Bifasciatum. Hope. St.

29 Dejeanii. Putzeys. Anglia.

30 Ephippium. Marsham. Gall. merid. Pallidipenne.Dej. Hispania.*

31 Venustulum.(Zieg.)Dej.Austria.

Prasinum. Duftsch. id.

Metallicum. Sturm. id.

Splendidum. Sturm. id.

32 Laticolle. Duftsch.Dej, id.
CINQUIEME DIVISION.

33 (Paludosum. Panz. Dej. P. *

$\{$ Littoralis. Olivier. id.

Var. Elegans. (Dahl.) Germania.

Corulescens. Krynicki.Russ. merid.

34 Chalybeum. Stm. Cat. Hollandia.

35 Argenteolum. Ahr. St. Germania.*

36 Impressum. Fabr. Gall. orient. ${ }^{\star}$

37 Foraminosum. Sturm. Germania.*

SIXIEME DIVISION.

38 Striatum. Fabr. Gallia. *

Clorophanum. Sturm. Germania.

I Orichalcicum. St. Dej.Germania.

39 Erosum. Erichson. id. ${ }^{*}$

\{ Striatum, Duft, Dej. id.

40 Ruficolle. Illiger. Dej. Surcia.*

41 Dives. Lucas. Algiria.

42 Pallidipenne. Illig.Dej.Gallia.*

\{ Andrea. Gyllenh. Dej. Suecia.

43 Mauritanicum. Lucas. Algiria.

44 Bipunctatum. Linné.

Fabr. Pyrenais."

Yar. Nivale. Godet. Helvetia.

- Sexpunctatum.

Heer. id.

45 Pulchellum. Lucas. Algiria.

46 Glaciale. Heer. Helvetia.

47 Rhælicum. Heer. id.

\section{SEPTIEME DIVISION.}

Peryphus. Megerle.

48 Eques. Sturm.

49 ( Tricolor. Fabr.

Gall. merid.*

Y Varicolor. Schönherr. Hispania.

50 Scapulare. Dej.

Y Var. Conforme. Dej. id.

51 Modestum. Fabr. Gall. orient. ${ }^{*}$

52 Ustum. Schönh. Dej. id.

53 Humerale. Nees. Tirolis. ${ }^{*}$

54 Lunatum. And. Duft. Germania.*

55 Binotatum. Stm. Cat. Sicilia.

56 Bisignatum. Ménét. Russ, merid.

57 Terminale. Lasserre. Helvetia. ${ }^{*}$

58 \} Rupestre.Illig.Dej.St.P.*

Humerale. (Megerle.) Helvetia.

59 Fluviatile. $D e j . \quad \therefore \quad P^{*}$

60 Concinnum. Stephens. Anglia.*

61 Andreæ. Fabr. Gall. merid.*

\{ruciatum.Dej. Gallia.

Signatum.Sturm,Cat,Germania. 


$\begin{cases}\text { Cursor. Fabr. } & \text { id. }^{\star} \\ \text { Obsoletum. Dej. } & \text { Gall. or. bor. } \\ \text { Testaceum? Duftsch. } & \text { Germania. }\end{cases}$

66

67

68

Hispanicum. Dej.

Hispania.

Lusitanicum. Putzeys. Lusitania.

\{ Femoratum. Gyll.Dej. P.*

Ustulatum. Olivier. Germania.

Cursor. Fabr. id.

Testaceum? Duftsch. Germania.

Bruxelense. Westmaet. Belgia.

Saxatile. Gyllenh.Dej. Suecia.*

Oblongum. Dej.

Gall. merid.*

Combustum. Ménét.

Russ, merid.

Præustum. Dej.

Deletum. Dej.

Gall, merid.

$\boldsymbol{P}^{*}$

Dentellum. Stév, Dej. Russ, merid. *

( Hastii. Sahlberg. Dej. Lapponia.

Punctiger. Germar. id.

Pfeiffii. Sahlherg. Dej. Suecia. *

(Prasinum. Sahl. Duft. Lapponia.*

Var. Olivaceum. Gyll, id.

Kolstromii. Sahlberg. id.

Felmanni. Mannerh, id.

Depressum. Ménétriés. Russ. merid.

Fasciolatum, Duft.Dej.Germania.*

Nigricolle, L. Redtenb. Austria.

Obscurum. L. Redtenb. id.

Cœruleum. Dej.

Complanatum. Heer.

Gall. merid.*

(Tibiale. Duftsch. Dej. Gall. orient. ${ }^{\star}$

Yar. Geniculatum.

Heer. Helvetia.

Cumatile. Schiodte Dania.

Affine, L. Redtenbach. Austria.

Simile. Sturm. Cat. Silesia.

Pareyssii. Sturm. Cat. Galicia.

Cyanescens.,Westmaël.Belgia.*

Nigrum. (Gaubil.) Alsatia. *

Decorum.Zenker.Pan-

Siculum. Dej.

zer. Dej. Gallia.*

Distinctum Sicilia.

Distinctum, Dej.

Picipes. Sturm.

Eneum Sturm Cat Helvetia.

Perplexum. Dej. Styria.

Fulvipes. Sturm. Austria.

Agile. Steph. St. Cat. Anglia.

Fuscicorne. Dej. Styria. ${ }^{*}$

(Rufipes. Illiger. Dej. Gallia.*

Brunnipes. Sturm. id.

Dalmatinus. Sturm. Dalmatia.

Striatus. Sturm.

(Elaph.)
Germania.
- Alpinum. Dej.
98 Sahlbergi. (Meg.) Dej. Finlandia. \{ Brunnipes.Sahlberg.id.

99 (Brunnipes. (Meg.) Dej.Gall. merid.* Rufipes. Duftsch.St. Germania.

Erythrocnemum.Parreys. id.

100 Stomoides. Dej. Pyr. orient.

10-1 Thalassinum. Erichs. Hungaria.

102 Grenatum, Dej. Germania.

Albipes, Sturm. id.

103 Dahlii. Dej. Sisilia. ${ }^{*}$

104 Elongatum. Dej: Gall. merid, bor.*

\section{HUITIEME DIVISION.}

LEJA. Megerle.

105 (Pygmæum,Fab.Erich,Gall, orient, ${ }^{*}$

Chalcopterum.(Zieg.)

Dej. Germania.

Orichalceum. Panz. id.

106 Ambiguum. Dej. Hispania.

107 Nigricorne. Gyll. Dej, Germania.

108 Velox. Erichson. id. ${ }^{*}$

109 Felixianum. Heer. Helvetia.

110 Celere. Fabr. Gallia.*

Tristis. Fabr. id.

Lampros. Herbst. Austria.

Pygmaum. Dej. Germania.

Rufipes, Olivier. $\quad P$.

111 Pyrenæum. Dej. Pyr. orient.*

112 (Sturmii. Panzer. Dej. Germania.*

Pictus. (Elaphrus.) Duftsch. id.

112 bis.Tenellum. Erichson. id.*

113 Maculatum, Dej. Gall. merid.

114 (Rivulare. Dej. id.*

Unicolor. Sturm. Germania.

115 Coracinum. Stm. Cat. Anglia.

116 Normannum. Dej. Gall. merid.*

117 Minimum. Fabr. Dania.*

Pusillum. Gyllenh. Gallia.

Atratum. Sturm. Germania.

Var, Kollari. Dej. Buchovina.

118 Gilvipes. Kollar.Stm.Germania.*

Var. Mannerheimii.

Sahlberg. Finlandia.

119 Arundinis. Stm. Cat. Germania.

120 Gracile. Sturm. Cat. id.

121 Tenellum. Erichson. Germania. *

122 (Pulchrum. Gyll. Dej. Finlandia.

Bellum. Suhlberg. id.

Humerale. Sturm. German. bor. 
123 Aspericolle. Germar. Gall. merid. ${ }^{*}$ I Lepidum, Dej. Germania.

124 Doris. Illiger. Dej. id.* Aquaticum. Panzer. var, id. Aquatilis. Illig, var, id. Minutum. Fabr, id.

Hypocrita. Dej.

Gratiosum. Dej.

Schuppelii. Dej.

Pyr. orient.*

Italia.*

Germania.

(Assimile. Gyllenhal. P.*

Yar. Dubium. Chevr. Helvetia.

129 Obtusum. Sturm. Dej.P."

Guttulum. Fabr.

Gallia.*

Immaculatum. Höpf. Germania.

Var, Bisignatum.Dej.

Cat. id.

Biguttatum. Fabr. $\quad P^{*}$

Var, Fuscipes, Dej. Gall. merid. ${ }^{*}$

Nigrocneum.St.Cat.Suecia.

Vulneratum. Dej.

Gall. merid.*

Vicinum. Lucas.

Algiria.

134

(Eneum. Germar.

Germania.

Marinum. Schiodte. Dania.

135 Bipustulatum. L.Redtenbacher. Austria.

136 Fenestratum. St. Cat. Finlandia.

137 Albofemoratum. Stm.

Cat. Corfou.

\section{NEUVIEME DIVISION.}

Lорна, Megerle.

138 Quadriguttatum.Pontopp. Fabr. Dej. P.*

139 Albosignatum. Stm.

Cat. Germania.

140 Laterale. Dej. . Gall. merid. *

141 Quadripustulatum. Fabr, Dej. Gallia. *

142 Quadrimaculatum. Lin. P.*

Subglobosum. Payk. Suecia.

Pulchellus. (Carab.)

Panzer. Germania.

Articulatum. Panzer. id. ${ }^{*}$

Subglobosum, Payk. var. $\beta$. Suecia.

Proila. (Lopha.) Dej. P.

DIXIEME DIVISION.

Tachypus. Megerle.

144 Picipes. Duftsch, Gallia. ${ }^{*}$
145 Pallipes. Duftsch.

146 Flavipes. Linné. Dej. P.*

Gallia.*

\section{FAMI, DYTISCI.}

\section{DYTISCUS, Linné.}

Fabr. Oliv. Dyticus. Geoff. Leach. Erich.

1 Latissimus, Lin. Fabr. Gall. bor. * Amplissimus. Müller. Germania.

2 Dimidiatus, Bergst. It-

$$
\text { liger, Gallia.* }
$$

3 Punctulatus. Fabr. P.*

Semi-sulcatus, Müller. Germania.

Punctatus,Oliv.Erich.Gallia.

Laterali-marginalis.

de Geer. Suecia.

Porcatus. Thunberg. id.

4 Marginalis. Linné. $\boldsymbol{P}^{*}$

Semistriatus, Linné. Suecia.

Tota-marginalis, de Geer. id.

Var. Circumductus.

(Ziegl.) Dej. Cat. P.

- Conformis. Kunze.

5 lPisanus, Aubé. Aube, Gall. bor. merid.

$\{$ Hispanicus, Dej. Cat. Hispania.

6 Circumcinctus, Ahr, St. Gall. bor.*

Marginalis, Ahr.var. Germania.

Var. Dubius. Gyllenh. Suecia.

$\{$ - Circumscriptus.

Dej. Cat. Lacord. P.

Angustatus, Stephens, Anglia.

Flavocinctus. Humm. Germania.

7 (Perplexus, Dej. Cat.

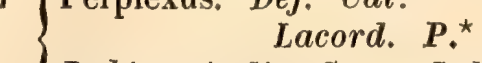

Dubius. Audin. Serv. Gall. bor.

8 Circumflexus. Fabr, Gallia*

Flavo-scutellatus. La-

treille, id.

Flavo-maculatus.Cur-

tis. Anglia.

9 Lapponicus. Gyllenh. Lapponia.

10 Septentrionalis, Gyll. id, 
ACILIUS. Leach, Erichson.

Aubé. Dytrscus, Linné. Fabr. Oliv.

1 Sulcatus, Linné. Fabr. P.*

Cinereus, Rossi. Müll. Italia.

Var. Tomentosus. Motschulski, Russia.

2 $\left\{\begin{array}{c}\text { Brevis. Aubé. Hispania. } \\ \text { Canaliculatus. Illig. } \\ \text { Dej. Cat. id. }\end{array}\right.$
$\left\{\begin{array}{c}\text { Fasciatus. de Geer. } \\ \text { Erich. Suecia. } \\ \text { Canaliculatus. Nico- } \\ \text { lä̈. Aubé. Germania. } \\ \text { Sulcipennis.Sahl.Zet. Suecia. } \\ \text { Caliginosus. Curtis. Anglia. } \\ \text { Dispar.(Ziegl.)Lacord.Gall. bor. }\end{array}\right.$

EUNECTES. Erichson.

Dytiscus, Linné. Fabr, Olivier. Eretes. Laporte. Nogrus. Eschsch. Dej. Cat.

1 Griseus. Fabr. Gall. merid. $\{$ Sticticus, Lin. Erich. id. Helvolus. Klug. id.

\section{HYDATICUS, Leach. Erich.}

Dytiscus. Linné. Fabr. Olivier.

\section{Stagnalis. Fabr.} Germania. $^{*}$

2 Grammicus. Germar. Lombardia.* Lineolatus. Falderm. Russ, merid. Strigatus, Dej. Cat. Lombardia.

3 l Leander. Rossi. Gall. merid.* \{ Distinctus. Dej. Cat. Italia.

4 Hybneri. Fabr. $\boldsymbol{P}_{\text {. }}^{*}$

5 Transversalis. Fabr. Pontopp. id.*

6 (Cinereus, Lin.Dej,Cat. id.* Treniatus, Rossi. Italia.

7 Austriacus, Dej. Cat.

. Sturm. Austria.*

8 Bilineatus. de Geer. Gall. bor.*

9 Zonatus. Hoppe. Panz. id. *

10 Verrucifer. Sahlberg. Finlandia.
GYBISTER. Curtis.

Dytiscus. Oliv. Fabr. Latr. Trogus. Leach. Trochalus, Eschsch. Dej. Cat.

1 Røselii. Fabr. : $P^{*}$

Virens? Müller. Germania.

Dispar. Rossi. Italia.

Dissimilis, Rossi. id.

2 Africanus. Lap. Aubé. Algiria.

YMeridionalis. Géné. Italia.

3 Senegalensis. Aubé. 'Algiria.

4 Immarginatus. Fabr.

5 Bivulnerus, Aubé.

COLYMBETES. Clairville, Aubé.

Meladema, Laporte. Dytiscus. Linné. Fab, Oliv. Scutopterus. Dej. Cat.

1 Coriaceus. Hoffmsg.

Laporte. Dej. Cat. Gall. merid.

2 Pustulatus. Rossi. Italia.

3 (Paykullii. Erichson. Germania.* Striatus. Payk. Clairville. St. Suecia.

4 (Striatus. Linné. Erich. Germania.* Bogemanni. Gyl.Eric. St. Aubé. id.

5 jFuscus. Linné. Gallia.* \{ Striatus,Dej.Cat.Mül.P.

6 Dolabratus, Paykull. Suecia.

7 Conspersus. Gyll.Aub. Gallia.* Notatus. Lacordaire. $P$. Pulverosus, Knoch. Sturm. Aubé. Germania.

8 (Notatus. Fabr. Gyll. Gallia.* Punctatus. Hoppe. Germania. $\{$ Suturalis, Lacordaire. Dej. Cat. P.

Roridus, Müller. Germania.

9 Notaticollis. Aubé. id. ${ }^{*}$

10 Bistriatus. Bergstr. $\left\{\begin{array}{r}\text { Erich. id. } \\ \text { Agilis, Payk, Gyl.Aub. id. }\end{array}\right.$

11 Collaris, Paykull. Germania.* Exoletus, Forster. id.

Adspersus, Panz.Lac.

Ramb. $P$.
Var. Consputus. Lintz.
St. Heer. Helvetia. 
12 Adspersus. Fabr. $P^{*}$ \{ Agilis. Lacord. Ramb. id.

13 Grapii. Gyllenh. Aubé. Germania.*

\section{ILYBIUS. Erichson. Aubé.}

Drtiscus. Linné. Fabr. Olivier. ColyuBETES. Clairville, Dej. Cat.

1 Ater. de Geer. Erich.

Aubé. Gallia.*

Fenestratus. Paykull. Suecia.

Obscurus, Marsham. Anglia.

2 Quadriguttatus. Dej.

Cat. Lacord. $P^{*}$

Fenestratus, var. $c$.

Var. Sexdentatus?

Gyll. Suecia.

Schiodte. Dania.

3 Fenestratus. Fab. Oliv.

Gyll. Erich. Gallia.*

Lacustris. Fabr. Dania.

Eneus. Panzer. Germania.

4 Subæneus. Erichson. id.

5 Nitidus. Fabr. id.

\{ Picipes. Ziegler. Austria.

6 Prescotti. Mannerh. Russia.

7 Guttiger. Gyll. Sahlb.

Erich. Suecia.*

8 Angustior. Gyllenhal. Germ. bor.* Guttiger, var. Erich. id.

9 Fuliginosus. Fab. Eric.

$\left\{\begin{aligned} \text { Aubé. } & \text { Gallia. } \\ \text { Lacustris, Panzer. } & \text { Germania. } \\ \text { Fœtidus, Müller. } & \text { id. }\end{aligned}\right.$

10 Meridionalis. Dej. Cat. Aubé. Gall. merid. ${ }^{*}$

AGABUS, Leach. Aubé.

Dytiscus. Linné. Fabr, Olivier. ColymBETES. Clairville. Dej. Cat.

1 Serricornis. Paykull. Suecia.* $\{$ Paykullii. Leach. id.

2 Agilis. Fabr. Germania.*

Hamorrhoidalis, Fab, Dania.

Oblongus. Fabr. Illig.

(Ranthus. Dej. Cat.) $\boldsymbol{P}$.

3 Arcticus. Payk. Erich.

Aubé. Suecia* *

4 Fuscipennis, Paykull. Erich. Aubé. Germania.* Fossarum, Germar. id.
5 Uliginosus, Lin, Erich.

6 Aubé. Gallia.*

7 Reichei. Aube. Gall. bor.

7 Assimilis, Stm. Aubé. Germaria.

8 Pygmæus. Frywaldsky.

St. Cat. Hungaria.

9 Femoralis, Payk, Er.

10 Congener. Payk. Gyll.

$$
\text { Aubé. Gallia.* }
$$

11 Sturmii. Schönherr.

$$
\text { Er. Aubé. Germania.* }
$$

12 Chalconotus, Kugel. Gyll, Aubé. Gall.bor *

Concinnus. Marsham. Anglia.

13 Maculatus. Lin. Oliv.

$$
\text { Fabr. Gallia.* }
$$

14 Pulchellus. Heer. Helvetia.

15 Albreviatus. Fab. Oliv.
$\left\{\right.$ Lacord. $\boldsymbol{P}^{*}$
Quadripunctatus.Muil.Germania.
16 Dydimus. Oliv. Lacord.P.*
$\{$ Vitreus, Paykull. Suecia.
Abbreviatus, var.Illig. Germania.

17 Brunneus, Fabr. Gall. merid.* \{ Castaneus. Schönherr.id.

18 Paludosus, Fabr, Lac, Gallia.

Congener. Illiger. Germania.

19 (Bipunctatus, Fab. Er, Gallia.*

Nebulosus. Forster.

Sleph. Anglia.

20 ? Frigidus. Schiodte. Dania.

21 Subnebulosus: Steph.

22 Confinis. Gyll. Sahlb.

$$
\text { Aubé. P.* }
$$

\section{Erich. Suecia.}

23 Nigricollis. Zoubkof. Sicilia.*

Affinis. Dej. Cat. id.

24 Binotatus. Géné. Sardinia.

25 Goryi. Aubé. Gracia:

26 Guttatus. Payk. Lac. Gall. bor.*

Fenestratus, Panzer. Germania.

27 (Dilatatus. Brullé.Aubé, Gracia.*

Aquilus, Dej. Cat. Gall.merid.

Guttatus, var, b, Gyll. Suecia.

28 Nitidus. Fabr. Germania.

Biguttatus. Olivier. Gallia.

29 Melas. Aubé. Gracia.

30 Adspersus. Mannerh. Finlandia.

31 Hoffneri. Mannerh. Suecia.

32 Wasatjernæ. Sahlberg. Lapponia.

33 Opacus. Mannerheim.

Aubé. Finlandia. 
34 Affinis. Payk. St. Er. Suecia.* Guttulus. Illiger. Germania.

35 Elongatus. Gyll. Aubé. Lapponia. Angustus. Dej. Cat. id.

36 Vittiger. Gyll. Erich. German. bor.

37 Striolatus, Gyll.Sahlb. Suecia.

38 Melanarius, Aubé. Russia.

39 (Bipustulatus, Lin. Oliv. $\left\{\begin{array}{c}\text { Fabr. }{ }^{*} \\ \text { Carbonarius. Fabr. } \\ \text { Gyll Schh. Suecia. }\end{array}\right.$

40 Alpestris. Heer.

Helvetia.

41 Solieri. Aubé.

Gall, orient.

12 Subtilis. Erichson. Germania.*

43 Splendens. Cristofori. Italia.

44 Neglectus. Erichson. Germania.

LACCOPHILUS. Leach. Aubé.

Dytiscus. Fabr. Dej. Cat.

1 Hyalinus. de Geer. Er. Germania.*

Minutus, Marsh. Gyll.
Obscurus. Panzer. Gallia.

2 Minutus. Linné. Fabr.
Erich. Gallia.*
Interruptus. Panzer.
Aubé. id.
Hyalinus, Marsh, Stm. Germania.

3 Testaceus. Aubé. Gall. merid.*

4 Variegatus. Knoch. St. Aubé. Gallia.*

\section{NOTERUS, Clairville, Aubé.}

Dytiscus. Fabr. Oliv. Dej. Cat.

1 Crassicornis, Müller. Gallia.*

Capricornis. Herbst. Germania.

Geeri. Leach. Anglia.

Semipunctatus. Stm.

Cat. Germania.

2 Sparsus. Marsh. Aubé. Anglia.*

Crassicornis. Lacd. St. P.

Semipunctatus. Fabr.

Erich. Germania.

3 Lævis, Dej, Cat. St.

Aubé. Gall. merid.*

13

12

$\begin{array}{ll}\text { Aubé. Hispania. } \\ \text { Polonicus. Aubé. } & \text { Polonia. }\end{array}$

3 Marginicollis, Dej. Cat.

Aubé. Helvetia.

4 Sansii. Solier. Aubé. Hispania.

5 Affinis. Géné, Aubé, Sardinia.*

6 Fenestratus. Escher.

Aubé. Sicilia.

7 Luctuosus. Aubé. Gall. merid.

8 Schaumei. Aubé. Sicilia.

9 Figuratus, Gyllenh.St.

10 Carinatus. Dej. Cat.

Cat. Lapponia.

$\left\{\begin{array}{l}\text { o Alpinus. Paykull, Suecia.* } \\ \text { o Bidentatus. Gyll. }\end{array}\right.$ Aubé. id.

Borealis. Gyllenhal. id.

Davisii. Curtis. Aubé. Anglia.*

Borealis, Aubé. Styria.

Minimus. Duftschmid. id.

Septentrionalis. Heer. Helvetia.

14 Frater.Kunze.Zetterst.German. bor.

Assimilis. Sturm. id.

15 Hyperboreus. Gyllenh. Lapponia.

Affinis. Sturm. id.

16 Septentrionalis. Gyll. id.*

Fluviatilis. Sturm. Germania.

Rotundatus, Knoch. id.

Striolatus. Dej. Cat. Anglia.

Rotundatus. Miiller. Illyria. 
18 Assimilis. Payk. Aubé. Helvetia. Var.Sanmarkii.Sahlb.Suecia.

19 Rivalis. Gyllenh. Aubé, Germania.* Var. Fluviatilis. Steph.Anglia.

20 Halensis. Fabr. Aubé. Gall. merid.* Areolatus, Duftsch. Austria.

21 Fuscitarsis. Géné.Aub.Sard.Gal.md.*

22 Canaliculatus.Dej.Cat.

Lacord. Gall. merid.

23 Griseostriatus. de Geer. Gallia.*

Halensis. Paykull. Suecia.

Quadristriatus. Esch. Russia.

24 Margineguttatus.(Dhl.) St. Cat. Sardinia.

25 Frontalis. (Dahl.) St. Cat. id.

26 Ceresyi. Aubé.

27 Picipes. Fabr.

Gall. merid.

Ovalis. Thunberg. Suecia.

Punctatus. Marsham. Anglia.

28 Alternans. Kunze. Gallia.*

Lincellus. Gyll. Aubé. id.

(Picipes o. Erichson. Germania.

29 / $\sigma^{\top}$ Parallelogrammus.

Áhrens, Gall. merid.*

Distinctus, Dej. Cat. id.

o Consobrinus.Kunze.

Aubé. Germania.

오Nigrolineatus. Kze. id.

Lineatus. Marsham. Anglia.

o Parallelogrammus.

\section{Erichson. Germania.}

30

Semirufus, Germar. Berolinensis.

Lautus. Schaum.

id.

32 Schönherri. Aubé. Lapponia.

Nigrolineatus? Gyll. id.

Consobrinus? Zetterst. id.

33 Parallelus, Aubé. Russ. merid.

34 Lapponum. Gyllenh. Lapponia.*

Marginatus? Steph. id.

35 Dorsalis. Fabr. Gyll. Gallia bor, ${ }^{*}$

Var.Figuratus, Gyll. Suecia.

36 Elevatus. Sturm. Cat. Germania.

37 Opatrinus. Germar. Gall. merid.

38 Platynotus. Germar. Saxonia.*

Murinus. Sturm.

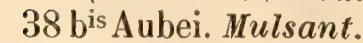

39 Ovatus. Sturm.

40 Sexpustulatus. Fabr. Litturatus. Panzer.

41 Palustris. Linné. Litturatus. Fabr.

Proximus. Stephens.

Gall. merid.*

id. ${ }^{*}$
42 Erythrocephalus. Fabr.

Gyll. Gallia.*

43 Rufifrons. Duft. Gyll.

Aubé. Gallia.*

44 (Deplanatus. Gyl.Steph.Germania.*

Erytrocephalus o

Erich. Gallia.

45 (Planus. Fabr, Gyllenh. id.*

Rufipes. Olivier. $\quad \boldsymbol{P}$.

Holosericeus. Steph. Anglia.

Var, Flavipes, Fabr, Germania.

46 Pubescens, Gyllenhal. Gallia.

Piceus. Sturm. Erich, Germania.

47 Vittula. Erichson. id. *

Ambiguus. Aubé. Gallia.

48 Marginatus. Duft. St. Austria.

Seglectus. Dej. Cat. Gall, merid.

49 Litturatus, Dej. Cat.

Brullé. id.

Humilis. Klug. Gracia.

Neglectus, Dej. Cat, Gall.merid.

Confusus. Lucas, Algiria.

51 Limbatus. (Dahl.)Aubé.Sardinia.

52 Analis. (Dahl.) Aubé. id.

Marklini. Gyll. Aubé. Suecia.

54 Obsoletus. Dej. Cat.

5̋6 Castaneus. Aubé. P.

57 Memnonius. Nicolaï.

$\begin{cases}\text { Erich. Berolini. } & \text { Germania. }\end{cases}$

Piceus. St. Step. Aubé. Gallia.*

59 (Scopularis. Gyllenhall.

$\left\{\begin{array}{r}\text { Schiodte. Dania. } \\ \text { Pubescens? var. b. } \\ \text { Gyllenh. Suecia. }\end{array}\right.$

60 Incertus.Dej.Cat. Aub.Gallia.

61 Melanarius. Stm.Erich. Germania.

62 Nigrita: Fabr. Gyll. Gallia.*

63 Gyllenhalii. Schiodte, Dania.

Melanocephalus. var. a. b. Gyll. Suecia.

64 Nivalis. Heer. Helvetia.

65 Foveolatus, Heer. id.

id. 66 Brevis. Sahlb. (Ferd.) Aubé. Finlandia.

67 Glabriusculus. "Sahlb. Aubé. Lapponia.

68 Tristis, Paykull, Gyll, Gallia. ${ }^{*}$

69 Elongatus. Höpf. Stm. Germania. *

Melanocephalus. Gyll.

Aubé, id. 
70 Angustatus.Stm.Erich.Germania.*

Tristis, Lacord. Aubé. P.

71 Melanocephalus.Marsh.

St. Schiodte. Dania.

Melanocephalus. var.

c. Gyllenh. Suecia.

72 Neglectus. Schaum. Germania.*

73 Terminatus. (Parreys.)

St. Cat. Corfou.

74 Quadrilineatus (Parr.)

$$
\text { St. Cat. id. }
$$

75 Obscurus. Stm. Erich. Gallia.

\{ Var. Tristis. Gyllenh. Suecia.

76 Umbrosus. Gyllenhal. Gallia.*

77 Striola. Gyllenh. Aubé. Suecia.

78 Notatus. Sturm. Erich.Berolini.

79 Pygmæus. Stm. Erich. id.*

80 Lineatus. Fabr, Gallia.*

Uvatus. Fabr. Dania.

Ovalis. Stephens. Anglia.

Var. Pygmaus, Fabr. Germania.

81 Flavipes. Olivier.

82 Meridionalis. Aubé.

83 Genei. Aubé.

Gall. merid.* 108

84 Sexguttatus, (Dahl.)

Aubé. id.

85 (Granularis. Lin. Gyll. Gallia.*

$\{$ Unistriatus. Schh. Suecia.

Minimus. Scopol. Carniolia.

86 Bilineatus. Stm. Erich. Gallia.*

Minimus. Stephens. Anglia.

87 Varius. Dej. Cat. Aubé,Gall. merid.*

88 Geminus, Fabr. id.*

Pusillus. Fabr. Dania.

Trifidus. Panzer. Germania.

Pygmaus. Olivier. $\boldsymbol{P}$.

Monolocus. Drapiez. id.

89 Hamulatus. Gyllenhal.Suecia.

90 Minutissimus. Dej. Cat.

Germar. Gall.meria.*

91 Delicatulus. Schaum. Austria.*

92 Unistriatus. Illig. Oliv. Gallia. ${ }^{*}$

Parvulus,var, a.Payk. Germania.

93 Goudotii, Lap. Aubé. Sicilia.

94 Pumilus. Dej. Cat.

Aubé. Gall. merid.

95 (Bicarinatus, Clairville. id.*

Costatus. Gyllenhal, Suecia.

Crispatus. Germar. Germania.

Cristatus. Dej. Cat. Gall. merid.

96
97 (Fasciatus. (Dhl.) Aub. Italia.

Yar. Crux. Fabr. id.

98 Rufulus. (Dahl.) Aub. Sardinia.

99 (Lepidus, Oliv. Gyll. Gall. merid.* \{ Scytulus. Stephens. id.

100 Ferrugatus. (Dahl.)

St. Cat. Sardinia.

101 Formosus. Chevrol.

102 Escheri. Aubé. Sicilia.

103 (Nigrolineatus. Stéven.

Aubé. Russ. merid.

Enneagrammus, Ahr. Austria.

Blandus. Germar. Germania.

104 Confluens. Fab. Oliv. Gallia,

105 Pallens. Mann. Aubé. Lapponia.

106 Decoratus. Gyll.Curt.Gallia.*

107 (Cuspidatus. Kunze.

$\{$ Germar. Aubé. id.*

Var. Bicolor. (Dahl.) Dej. Cat. Germania.

Inæqualis. Fabr. Gallia.*

Parvulus, Fabr. Dania.

Affinis? Stephens. Anglia.

109 Reticulatus, Fab, Gyl. Gallia.*

Obliquus. Fabr. Dania.

(Var. Collaris. Panz. Germania.

110 Quinquelineatus, Zet. Lapponia.*

111 Semirufus. Germar. Germania.

112 Biscruciatus. Germ. id.

113 Ferrugineus. Lucas, Algiria.

114 Salinus. Joly. Gall. merid.

\section{HYPHIDRUS. Illiger.}

Dytiscus. Linné. Hydrachma. Fabr. Hydroporus. Clairville.

1 Grandis. Klug.

2 (Ferrugineus, Lin. Lat. P.*

Ovatus, Linné.

Gibbus. Fabr.

Spharius. de Geer. Germania.

Ovalis. Gyllenhal. Suecia.

Grossus. Müller. Germania.

3 Variegatus. Illig. Dej.

Cat, Aubé. Gall. merid.*

HALIPLUS. Latreille.

Dytiscus,Linné, Fab, Oliv, Cnemidotus. Illig. Hoplitus, Clairville.

1 Elevatus. Panzer, Gallia, German.* 
2 (Equatus. Dej. Cat. Glabratus. Villa. id.

3 Obliquus. Fabr. Panz. P.*

Confluens.Fabr. Germania.

Marmoreus. Olivier. Gallia.

4 Pictus. Mannerheim. Finlandia.

5 f Lineatus. Aubé. $\quad$ P. $^{*}$

Confinis? Stephens. Anglia.

6 Impressus. Fab. Erich.

Schiodte. $P^{*}{ }^{*}$

Ferrugineus, Schönh.

Gyll. Aubé. Suecia.

Flavicollis. Sturm. Germania.

Subnubilus, Babingt, Anglia.

7 Fulvus. Fabr. St. Er. Gallia.*

Interpunctatus, Mars, Anglia.

Ferrugineus. var. $\beta$.

Schönh. Suecia.

Idem,var. b. Gyllenh. id.

8 (Badius. Dej.Cat.Aubé.Gall. merid.* Parallelus. Babingt. Anglia.

9 Guttatus.Dej.Cat.Aub.Gall, merid.* Rubicurdus, Babingt. Anglia.

10 Variegatus. Erich. St.

11 Cinereus. Aubé. Aubé. P.*.*

12 Fulvicollis. Erichson. Germania.*

13 Ruficollis. de Geer.Er. Suecia.*

Impressus. Schh. Gyll.

St. Lacord, Er. P.

Marginepunctatus.

Panzer. Schönh. Germania.

14 "Fluviatilis. Aub.Erich. P.*

15 Lineolatus. Mannerh. Firlandia.

16 Lineatocollis. Marsh. Anglia.

Bistriolatus. Duftsch.

Lacord. Gallia.

Trimaculatus. Drap. id.

\section{CNEMIDOTUS. Illiger.}

Aubé. Erich. Schiodte. Dytiscus. Duftsch.

Haliplus. Latr. Dej. Cat. Hoplitus. Clairv.

1 Cœsus. Duft. Aub. Er. P.*

Impressus. Panzer. Germania. Quadrimaculatus.

2 Rotundatus. Dej. Cat.

Drapiez. Gallia.

Aubé. Gall. merid.*

239

\section{FAII, GYRINI.}

GYRINUS. Geoffroy.

Dytiscus, Linné. Grrinus, Fabr, Oliv.

1 Strigipennis. Suffrian. Germania.*

Striatus. Aube. Gall, orient.

2 Striatus. Fabr. : Gall. merid.

Strigosus, Aubé. id.

Limbatus, Solier. id.

3 Minutus. Fabr. Gall, bor.*

Bicolor. Olivier. id.

4 Urinator, llliger. Gall. merid.*

Lineatus, Lacordaire. $P$.

Gracus. Brullé. Gracia.

Var, Variabilis.Solier, Gall. merid.

5 Natator. Linné. Suecia.*

Mergus, Ahrens. Suff. Gallia.

Natator. Gyll. Sahlb.

Aubé. Suecia.

Var. Marginatus. Ger-

mar. Germania.

올ejeanii. Brullé. Grocia.

Substriatus. Steph. Anglia.

6 Gercurus. Schiodte. Dania.

Natator? Ahrens. Germania.

7 Bicolor. Paykull. Gallia.*

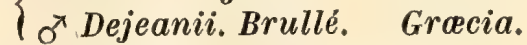

8 Caspius. Ménétriẻs. Russ, merid.

9 Angustatus. (Dahl.) Dej. Cat, Aubé, Gall. merid.*

10 Rivularis. Steven. Russia.

Distinctus. Suffrian. Germania.

11 Colymbus. Erichson. id.

Distinctus. Aubé. Gallia.

\{ Var. Elongatus.(Dahl.)

Dej. Cat. Aube. Gall. merid.

12 Marinus. Gyllenhal. Suecia.

Var. Dorsalis, Gyllenh. Aubé. id.

13 Lembus. Schiodte. Dania.*

\{Marinus.Sturm.Erich. Germania.

14 Opacus. Sahlb. Suff. id.

15 Eneus. Steph. Aubè. Gall. merid."

16 Celox. Schiodte. Dania.

ORECTOCHILUS. Eschscholtz.

Lacordaire. Aubé. Grrinus. Fabr.

1 f Villosus. Fabr.

P.*

Modeeri. Marsham. Anglia.

17 


\section{马. FAM, HYDROPHILI.}

\section{SPERCHEUS, Kugelann. Fabr.}

Dytiscus, schaller. Hydrophilus, Fabr.

1 Emarginatus. Schaller.

$\left\{\begin{array}{c}\text { Fabr. Gall. bor.* } \\ \text { Sordidus. Marsham: } \\ \text { (Hydrophilus.) Anglia. }\end{array}\right.$

HELOPHORUS, Fabr. Latr.

Silpha. Linné. Dermestes. Geoffroy. Hydrophilus, de Geer.

1 Rugosus, Olivier, Gallia.*

2 Nubilus. Fabr. Linné. id.* Cinereus. Marsh. Anglia.

3 Griseus. Brullé. Gracia.

Intermedius. Dej. Cat.

Mulsant. Gallia. ${ }^{*}$

4 Aquaticus. Linné. Oliv, id.*

Eneus. Illiger. Germania.

Flavipes, Herbst. id.

Stagnalis. Marsham. Anglia.

Grandis. Illiger. Austria.

5 Granularis. Linné. Gallia.*

Var. A. Arcuatus, Muls, Gall, bor.

- B. Obscurus. Muls. Gallia.

Aquaticus. Fabr. id.

Bipunctatus. Ullrich.

in litt. Germania.

Var. C. Granularis.

Linné. Suecia.

Flavipes, Fab.St.Oliv.id.

Var. D. Minutus, Oliv. Gallia.

Elegans, Ullr. in litt. Germania.

Emulus. Schüp, in litt. id.

Griseus. Herbst.

Austria.

Affinis. Marsham.

Anglia.

6 Borealis. Sahlberg.

7 Elegans. Knoch.

8 Dorsalis. Marsham.

9 Pumilio. Erichson.

10 Alpinus, Heer.

Finlandia.

Germania.

Gall. merid.*

Helvetia.

id.

11 Glacialis. Villa. Heer, id.

12 Nanus. Schüppel. in litt. Mulsant. $P .^{*}$

13 Fennicus, Paykull. Finlandia.

14 Sulcatus. (Dahl.) Hungaria.

15 Tuberculatus. Gyllenh.Suecia.

16 Costatus, Schönherr, Russ, merid.
17 Frigidus, Gralls. Hispania.

18 Arvernicus, (Rey.)Muls, Gall, orient.

HYDROCHUS, Gormar, in litt, Leach.

Latr. ELophorus, Fabr.

1 Brevis. Herbst, Curtis. $P$.*

2 Carinatus. Germar. id.*

Costatus. Dej. Cat. id.

3 Elongatus. Schaller. id.*

4 (Angustatus. Müller. in

litt. Germar. id.*

Elongatus. Olivier. id.

Crenatus. Stephens. Anglia.

Bicolor. (Dahl.) Austria.

5 Nitidicollis, Dej. Cat.

Mulsant. Alp. Gall.*

6 Gemellatus, Illiger. St, Germania.

7. Crenatus. Fabr. St, id.

8 Fuscipennis. Sturm, Hungaria.

\section{OCHTHEBIUS, Leach Latr.}

Elophorus, Fabr, Enicocerds, Curtis.

1 Granulatus. Dej. Cat.

Mulsant. Alp. Gall.*

2 Exculptus. (Mïl.)Germ.Gallia.*

$\sigma^{\top}$ Var.A. Viridi-aeneus.

Curtis, Anglia.

Var, B, Sulcicollis.

(Lintz.) Sturm. Germania.

of Gibsonii. Curtis. Anglia.

or Tristis.(Gibs.) Curt, id.

3 (Gibbosus. (Mül.) Germ. Alp. Gall.

Y Var. Lacunosus, Stm. Germania.

4 Margipallens. Latr. Gallia.*

\{ Obscurus. Dej. Cat. Gall. merid.

5 Marinus. Paykull. Gallia. *

Meridionalis, Dej, Cat, Gall, merid.

Pallidipennis. Fri-

I waldsky. in litt. Hungaria.

Var, Pallidus, Dej.Cat.P.

6 Pygmæus. Fabr. Gallia.*

Minimus. Fabr. Germania.

Riparia. Illiger. (Hy-

dræna.) Austria.

Impressus. Marsham. Anglia.

7 (Bicolor. (Kirby.) Germ. Gall. merid.*

Var. Rufo-marginatus.

Erich. Germania.*

Impressicollis.Dej.Cat.id.

8 Exaratus, Mulsant. Gall.merid. 
9 Pellucidus. Mulsant. $P_{\text {. }}^{*}$

10 Fossulatus, Mulsant, Sicilia.

11 Foveolatus. (Müller.) Germar. Gallia.*

12 Metallescens, Rosenh. Germania.

13 Punctatus. Stephens. Gall. merid. Nobilis. Villa. Lombardia.

Impressifrons. Dej.

Cat. Germania.

14. Hybernicus, Curtis, id.

15 Sulcicollis. Lintz. id.

\section{HYDRANA、Kugelann.}

Elophords, Fabr, Gyllenh.

1 Testacea, Curtis, Gall, orient. Margipallens. Chevrier, in litt. Heer. Helvetia.

2 Rugosa. Mulsant. Gallia.*

3 (Nigrita. Müller, in litt.

Germar. Gall. bor.*

Pusilla. Stephens, Anglia.

4 Intermedia. Rosenh. Germania.

5 Riparia, Kugelann. Gallia.*

Minimus, Payk. (Helo-

phorus.) Suecia.

Longipalpis, Marsh. Anglia.

Kugelanni.Leach. id.

Var. B.Spurcatipalpis.

\section{Heer. Helvetia.}

6 Angustata. Dej. Cat.

Mulsant. Gall. merid.

7 Angulosa. Mulsant. Germania.

8 Gracilis. Müller.Germ. Alp. Pedem.*

Elongata? Curtis. Anglia.

Var. Crassipes, Motsch.Tirolis.

9 Flavipes. (Lintz.) Stm. Gall.bor.

10 (Pulchella. Müller, Stm, Germania.

$\{$ Elegans. Dej. Cat. id.

11 Palustris. Erichson. id. ${ }^{*}$

12 Sieboldii. Rosenhauer, id.

\section{LIMNEBIUS, Leach. Solier.}

HydrophiLus. Marsh. Herbst. Thumberg.

1 Truncatellus. Thunb. Gallia.* Parvulus. Herbst, Austria.

Affinis? Stephens. Anglia.

2 Papposus. Mulsant. Gallia.*

Mollis? Marsham. Anglia.

Truncatellus, Gyllenh. Suecia.
3 (Nitidus. Marsham. Gall. orient* \{runcatellus? Steph. Anglia.

4. Atomus, Duftschmid. Gall. orient.* IMinutissimus, Germar, Germania.

BEROSUS. Leach, Latr.

Hydrophilus, Fabr, Olivier.

${ }^{\circ} 1$ Spinosus. (Serville.)

Schönherr. Gall.merid. ${ }^{\star}$

2 (Ericeps. Curtis. Gallia.*

Luridus. Olivier. $\quad P$.

Signaticollis, Charp. Russia.

3 Luridus, Linné, Fabr. Gallia.*

Fuscus, de Geer. Suecia.

Globosus? Curtis. Anglia.

4 (Affinis. Brulle. Muls. Gallia.*

Luridus, Olivier. P

Murinus, Küster. Germania.

Punctatissimus. Dej.

Cat. id.

๖ Suturalis. Küster. Germania?

\section{HYDROPHILUS. Geoffroy.}

Fabr. Oliv. Dytiscus. Linné. Hydrous. Leach.

1 Piceus, Linné. Gallia.

\{ㅇ Ruficornis. de Geer. Germania.

2 Aterrimus, Eschsch, id.*

Morio. Dej, Cat. Stm, id.

3 Inermis, Lucas. Algiria.*

HYDROUS, Linné, in litt. Brullé.

Hydrophilus. Fabr.

1 Caraboides. Linné. Gallia.*

Scarabaoides. Schrk. Austria.

Nigricornis, de Geer. Suecia.

(Var.Intermedius, Muls, Gall, merid.

2 (Flavipes. (Stév.) Schh. id.*

Scrobiculatus. Dej.

Cat. id.

HYDROBIUS, Leach.

Hydrophilus, Fabricius.

1 Convexus, Illig. Brul, Gall, merid.*

2 Oblongus. Herbst. Gallia.

\{ Picipes, Dumeril. $\quad P$. 
3 /Fuscipes. Linné. Gallia. *

Aquaticus. Linné. Suecia.

Scarabaoides. Fabr. Germania.

Var. Chalconotus?

Curtis. Anglia.
Eneus. Solier.
Var. Subrotundatus.

4 Bicolor. Paykull. Gallia.*

Stephens, Anglia.

Atricapillus, (Marsh.)

Steph. Anglia.

5 Eneus. (Stév.) Germ. Gallia.*

6 Globulus. Paykull. id.*

Limbatus. Fabr. Germania.

Minutus, Olivier. Gallia.

Bipustulatus, Marsh. var. Anglia.

Similis. de Castelnau. $\boldsymbol{P}$.

\section{LACCOBIUS. Erichson.}

Chrysomela. Linné. Hydrophilus, Fab. Limnebius. Solier. Brachypalpus, de Castelnau.

1 Minutus. Linné. Brullé. Gallia.* Chrysomelinus, Mül. Germania. Coccinelloides. Schrk. Austria. Marginellus. Herbst. id. $\{$ Bipunctatus. Fabr. Marsh. Anglia. Striatulus. Fabr, var. Germania. Colon. Stephens. Anglia.

Pallidus. de Casteln. Gallia.

2 Globosus. Heer. Helvetia.

\section{HELOCHARES. Mulsant.}

Hydrophilus. Fabr. Oliv. Geoff. Latr. Phylidrus. Solier, de Castelnau.
/Lividus, Forster. Gallia.* Obscurus. Müller. Dania. Griseus. Fabr. id.

Var. Erythrocephalus. Fabr. Germania.

Variegatus, Herbst, Austria. Var, Fulvus, Marsh. Anglia. Griseus, Duftschmid. Austria. Bicolor. Brulle. $\boldsymbol{P}$.

Var. Pallidus. Rossi. Italia.

Chrysomelinus. Panz. Germania.

2 Melanophthalmus, $D u$ four. Hispania.

\section{PHILHYDRUS. Solier.}

Limnebius, Leach. Latr. Hydrophilus. Fabr, Oliv, Geoff.

1 Melanocephalus. Oliv. Gallia. * Var. A. Testaceus. Fab. Germania. Torquatus. Marsham. Anglia. Quadripunctatus.

Herbst.
Grisescens.Sturm.Dej. Cat. Germania.

Bicolor, Fabr. id.

Fulvus. Stephens. Anglia.

Var. B. Melanocephalus. Gyllenh. Suecia.

Minutus, Paykull. id.

Ochropterus, Marsh. Anglia.

Marginatus, Duftsch. Austria.

Dermestoides, Marsh. Anglia.

Testaceus, Erichson, Germania.

2 (Marginellus. Fabr. Gall, orient.* Affinis.Payk. Gyllenh. Suecia.

Var. Minutus, Fabr. id.

3 Frontalis. Erichson. Germania.

4 Marginatus. Dej. Cat. Hispania.

๖ Salinus, Märkel.

Germania. *

\section{CYLLIDIUM, Erichson.}

Hydrophilus, Fabr. Ciloetarthria, $\boldsymbol{W} a$ terhouse. Coelostoma. Brullé. de Castelnau.

1 Seminulum, Paykull, Gallia.* Nigrinus. Marsham.

$\left\{\begin{array}{c}\text { (Dermestes.) Anglia. } \\ \text { Hemisphæricus. Dej. } \\ \text { Cat. Germania. }\end{array}\right.$

GYCLONOTUM. Dej, in litt. Erich.

Hydrophilus. Fab. Coelostoma, Brullé. de Castelnau.

$1\left\{\begin{array}{l}\text { Orbiculare. Fabr. Gallia.* } \\ \text { Var. Allabroix. de Cas- } \\ \text { telnau. } P .\end{array}\right.$ 
SPH ERIDIUM, Fabricius.

1 Scarabæoides. Linné. Gallia.* Quadrimaculatum.

Schrank. Austria.

I Var. Lunatum. Fabr. Germania.

Bipustulatum. Herbst. Austria.

2 Striolatum. Heer. Helvetia.

3 Bipustulatum. Fabr. Gallia.*

Hamorrhous. Schrank. Germania.

Testudinarium. Fourc, $P$.

Marginatum. Scriba. id.

Scarabaoides, Laich. Anglia.

Semistriatum.de Cast.P.

Quadrimaculatus.

(Dermestes.) Marsham.

Daltoni.Stephens. Anglia.

4 Testaceum. Heer. Helvetia.

\section{CERCYON, Leach.}

SpHeridium. Fabr.

1 Obsoletum, Gyllenhal, Gallia.*

Lugubre? Olivier. id.

Atomarium. Paykull. Suecia.

2 Hæmorrhoidale. Fabr. Gallia.*

Melanocephalum. var.

B.Illiger. Austria.

Impressum. Sturm. Germania.

Obsoletum.de Casteln.P.

Piceus. Marsham. (Der-

mestes.) Anglia.

3 Hæmorrhoum. Gyll, Gallia.*

Hamorrhoidale, Fab. Germania.

Melanocephalum. var.

$$
\text { ß. Illig. id. }
$$

4 Laterale. Stephens. Gallia.

5 Unipunctatum. Linné, Gallia.*

Cordiger. Fuessly. Germania.

Dispar. Paykull. ㅇ. Suecia.

Quisquilium. Steph. \&.Anglia.

6 Quisquilium. Linné. Gallia.*

Minimus? Socopol.

(Scarabæus.) Carniolia.

Xanthopterum. Laich. Anglia.

Unipunctatum. Fabr. Germania. Melanocephalum. var. Herbst. id.

Dispar, Paykull. ఠ઼. Suecia.

Var. Flavum? Steph. Anglia.

7 Centrimaculatum. Stm. Gallia.*

IPygmoum. Gyllenhal, Suecia.
8 (Pygmæum. Illiger. Gallia.*

Conspurcatum. Stm. Germania.

Ferrugineum? Herbst. id.

Merdarium. Stm. var, id.

9 Littorale. Gyllenhal. Gall. merid.*

10 Rufum. Sturm. Germania.

11 Plagiatum. Erichson. id.

12 Aquaticum. Stephens. Gallia.*

13 Flavipes. Fabr. Gallia.*

Hamorrhoidale. Stm. Germania.

Melanocephalum.Gyll. Suecia.

Var, Picinum. Steph. Anglia.

14 Erythropterum. Megl.

Mulsant, Sicilia.

15 Melanocephalum. Lin. Gallia.

16 Minutum. Fabr. Gall. bor.*

Triste. Illiger. Austria.

17 Suturale, Stephens. Anglia.

18 Granarium. Erichson. Sicilia.*

19 Castaneum. Heer. Helvetia.

20 Lugubre. Paykull. P.*

21 Pulchellum. Heer. Helvetia.

22 Triste. Gyllenhal. Suecia.

23 Anale. Paykull. Erich. Gallia.*

Flavipes. Thunberg. Suecia.

Terminatum.Marsh. Anglia.

Var.Marginellum.Pay-

kull. Suecia.

PELOSOMA. Mulsant.

1 Lafertei. Mulsant. Gallia.* \{icolor. Dej. Cat. Dalmatia.

MEGASTERNUM. Mulsant.

Dermestes. Marsh. Cercyon.de Castl.

1 Boletophagum, Erich. Gallia.*

\section{CRYPTOPLEURUM. Mulsant.}

Spheridium, Fabr, Gercyon, de Castln.

1 Atomarium. Fabr. Gallia.* Crenatum. Panzer. Germania.

Minutum. Paykull. Suecia.

Var. Sordidum, Marsh. Anglia. 


\section{FAII, SPHERII.}

SPH ERIUS, Waltl.

1 Acaroides, Waltl. Germania. *

PARNUS, Fabricius, Leach.

Dryops. Oliv, Latr. Leach.

1 Prolifericornis. Fabr.

Panzer. Germania.*

Auriculatus, Olivier. $P$.

Sericeus, Leach. Anglia.

Var. Impressus. Curtis.id.

- Bicolor. Curtis. id.

Niveus. Heer. Helvetia.

2 Algiricus, Lucas, Algiria.

3 Striatopunctatus. Dej.

Cat. L. Redtenb. Gall. merid.

4 Impressus. Géné. Villa. Lombardia.

I Striatus. Sturm. Cat. id.

5 Griseus, Erichson. Berolini.

6 Luridus, Erichson. Silesia.

7 Viennensis. (Dhl.Cat.)

Heer. Germania.*

Obscurus, Duftsch. Austria.

Punctulatus, Müller.

St. Cat. Germania.

Murinus,Waltl. in litt.id.

Punctatus, (Hoffmsg.) id.

8 Pilosellus, Erichson. Austria.

9 Auriculatus. Ilg. Panz.

10 Nitidulus, Heer. Erich. id.*

$$
\text { Lat. Germania.* }
$$

Villosus.(Bonelli.) id.

Rufipes. (Dahl.) Dej.

Cat. Hungaria.

Auricomus. (Waltl.) id.

11 Substriatus. Mül. Illig. Austria *

Dumerilii. Latreille, Gall. merid, Longipes, L. Redtenb, Austria. 11

8. FAM. ELMIDES.

POTAMOPHILUS. Germar. Erich.

Parnus. Fabr.

1 Acuminatus. Fabr, $\quad \boldsymbol{P}$.*

LIMNIUS. Müller. Erich.

Elmis. Latr.

1 Tuberculatus, Miiller.

Steph. Germania.*

\section{ELMIS. Latreille.}

Liminus. Illig. Pylidrus. Duftschm. Dytiscus, Hellwig. Panz. Chrysomeza. Marsh.

1 f Eneus. Steph. Müller. P.* Megerlei. Duftschmid. Germania.

2 Mangetii. Latreille. Gallia.*

3 Obscurus. Miiller. (Limnius.) Germania.*

4 Volkmari. Lat, Panz. P.*

与 Germari.(Märk.)Erich.Germania.*

6 Opacus. Muiller. (Lim-

7 Mulleri. Erichson. $P$.

8 Parallelopipedus. Stephens. Heer. id.*

9 Angustatus. Mül.Germ. Gallia*

10 Pygmæus. Müll. (Lim-

11 Cupreus, Steph. Miiller. nius.) Germania.*

12 Subviolaceus. Heer. Heer.
(Nees.) Gallia.*

13 Sodalis, Erichson. Germania.

14 Nitens, Müller. (Lim$\left\{\begin{array}{l}\text { Nius.) Germar. Germania.* } \\ \text { nius. }\end{array}\right.$ Cupreus, Gyllenhal. Suecia. Orichalceus. Gyl.Heer.id.

15 (Dargelasii. Latreille. $P$.* Tuberculatus. Mül. St. Germania. Variabilis. Leach, Anglia.

16 Troglodytes. Schönh. Suecia.* Tuberculatus. Gyll. id.

17. Confusus. de Casteln. P.

18 Rufipes, Dej.Cat. Gallia. 
STENELMIS, Léon Dufour.

Limnius. Gyllenhal.

1 Canaliculatus. L. Duf. Gallia,* Bituberculatus. (Bon.) id.

2 Sulcicollis. Gyllenhal. $P$.

MACRONYCHUS, Muiller, Latr.

1 Quadrituberculatus.

Miiller, Illig, Gallia.*

23

\section{FAI, HETEROCERI.}

HETEROCERUS, Bosc, Fabr. Latr.

1 Parallelus. (Fisch.) Geb. Germania.*

2 Fossor. Kiesenwetter, id.*

3 Femoralis. (Ullrich.)

Kiesenwetter, id.*

4 Obsoletus. Curtis. Austria,

\{Marginatus. Marsham. Anglia.

5 Marginatus. Fabr. P.*

6. Intermedius. Kiesenw.

7 Hispidulus. Kiesenw.

8 Lævigatus, Fabr. id.* Germar. Germania.*

Pusillus. Waltl. id.

Fenestratus. Thunb. Suecia.

9 Fusculus. Kiesenwett. Germar. Germania.* (o Pusillus. Waltl. id.

10 Pulchellus. Kiesenwet. Germar. id.

11 Sericans. Kiesenwett. Germar. id.*

12 Murinus.(Rosenhauer.) Kiesenwetter, id.

Flavidus. Rossi.

Italia.

Obliteratus. Kiesenw. Germania?

Hamifer. Géné.

Sardinia.

Nanus. Géné. id.

\section{FAM. SILPHA.}

\section{NECROPHORUS, Fabr.}

Latr. Oliv. Silpha. Linné. Sulzer.

1 Germanicus. Linné. Gall. bor.*

2 Speculifrons. Fischer, Russ. merid.

3 Humator. Fabr. Gall. bor.*

4 Stygius. (Dahl.) Dej.

Cat. Styria.

5 Vespillo. Linné. Fabr. Gall, bor.*

6 Basalis. Dej. Cat. Gallia.*

7 (Interruptus. Dej. Cat.

Steph. Gall. merid.*

Var. Cadaverinus. Dej.

Cat.de Castelnau. $P$.

Investigator. Mac-Leay. Anglia.

8 (Vestigator. Herschel. P.*

Sepultor, Gyllenh. Suecia.

Vespillo. var. Herbst.

9 Fossor. Erichson. id.*

Panzer. Germania.

10 Ruspator. Erichson. id.

Investigator, Zetterst. Suecia.

Vestigator. Gyllenh, id.

11 Sepultor. Charpentier, Gallia.

Abruptor. Erichson, Germania.

12 Sepulchralis. Heer, Helvetia.

13 Corsicus. Dej. Cat. de Castelnau. Corsica.

14 Funereus. Géné. Lombardia.*

15 Mortuorum. Fabr. Gall, bor.*

\section{NECRODES, Wilkin.}

Silpha. Linné, Fabr. Olivier.

1 Littoralis, Lin, Fabr, P.*

Clavipes. Sulzer. Germania.

Var, Lividus. Fabr. id.

Simplicipes. Dej. Cat. P.*

SILPHA, Linné, Fabr.

Oiceoptoma, Leach, Phosphuga, Leach.

1 Thoracica, Lin, Fabr. P,

2 Tuberculata. Dej. Hispania.

3 f Rugosa. Linné. Fabr, P.*

Y Paramariboa. Herbst. Germania.

4 Tuberculata. Lucas. Algiria.

$\fallingdotseq$ Lapponica. Herbst. Lapponia. * 
6 (Sibirica. Eschscholtz. Sibiria. Homorrhoidalis, Par. Russ, merid.

7 Subsinuata. Dej. Cat. Austria.

8 Sinuata. Fabr. Oliv. P.*

9 Dispar. Herbst. Gall. bor.*

10 Opaca. Linné. Fabr. Germania.*

11 Quadripunctata.Linné. Fabr. $P^{*}$

12 Reticulata.Fabr.Panz.id.*

13 Granulata. Olivier. Gall. merid.*

14 Puncticollis. Lucas. Algiria. \{ Hispanica. Dej. Cat. Hispania.

15 Tristis, Illiger. Gallia.*

16 Carinata. Illiger. P.* $\{$ Var. Lunata. Fabr. Germania. Opaca.Herbst. Austria.

17 Italica. Sturm, Cat. Italia.

18 Atropurpurea. St. Cat. Russia.

19 Obscura. Linné. Fabr. Gallia.* Var. Maura. (Ziegler.) Dalmatia. - Carniolica. Hoppe.

St. Cat. id.

- Sublinearis. (Dhl.) Hungaria.

- Granulosa. Besser. Podolia.

- Costata.Ménétriess. Russ, merid.

20 Terminata. Hummel. id.

21 Græca. Sturm. Cat. Gracia.

22 Turcica. Sturm. Cat. Constantinp.

23 Nigrita, Creutzer. Gall. merid.*

24 Alpina, Bonelli. Alp, Gall.*

25 Montana. Findel. St.

Cat. Hungaria.

26 Oblonga. (Dhl.) St. Cat. id. Alpestris. Friwaldsky. id.

27 Cribrata. Faldermann. Russ. merid.

(Рнosphuga, Leach.)

28

$\begin{cases}\text { Polita. Sulzer. } & \text { Gallia. } \\ \text { Lavigata, Fabr. } & \text { id. } \\ \text { Var. Gibba. Megerle. } & \text { Gall. merid. }\end{cases}$

29 Orientalis. Dej. Cat. Küster. Gracia.

30 Atrata. Linné. Fabr. P.* Var. Cassidea. (Dahl.) Hungaria.

$\{$ - Pedemontana.Fab. de Castel. Pedemont. (- Hyberna. (Luczot.) Gall.bor.

31 Souverbii, Fairmaire, Pyren, sup.

NECROPHILUS, Latreille.

Silpha. Illiger.

1 Subterraneus, Illig, St, Helvetia,
PTEROLOMA, Gyllenhal.

Holocnemis, Schilling. Adouus. Esch.

1 Forströmii, Gyllenhal. Silesia.

Brunneus, Eschsch. id.

Gravenhorstii. Schill. id.

SPHERITES, Duftschmid.

Hister. Fabr.

1 Glabratus, Fabr. Duft.

St. Germania.*

AGYRTES. Frœlich.

Mycetophagus. Fabr, Panzer.

1 Castaneus. Frolich. $P_{\text {. }}^{*}$

2. Spinipes. Panzer. Germania.

3 Subniger. Dej. Cat, Belgia.

4 Glaber. Paykull. (Tritoma.) Lapponia.

CATOPS. Fabricius.

Cistela, Fabr. Lupenus. Frolich. GhoLeva. Latr. Ptomophagus. Knoch. Illig. Helops. Panzer.

1 Angustatus. Fabr. P.* Agilis, Fabr. Germania. Elongatus, Paykull. Suecia. Rufescens, Illiger. Austria. Oblongus. Latreille. $P$. Cisteloides, Frolich. Germania.

2 Agilis. Illiger. Berolini.* Fuscus. Gyllenhal. Suecia. Testaceus, Latreille. P.

3 Castaneus. And. St. Germania.

4. Spadiceus. (Dahl.) St.

5 Fuscus. Panzer. $\boldsymbol{P}^{*}$ Sericeus. Paykull. Suecia. Rufescens. Fabr. Germania. Festinans.Spence.Gyl.Anglia.

6 Umbrinus. Erichson. Germania.

7 Longipennis. Chaud. Russia.

8 Marginicollis. Lucas. Algiria.

9 Picipes. (Hydrophilus.) $\begin{cases}\multicolumn{1}{c}{\text { Fabr. }} & \text { Gallia.* } \\ \text { Sericeus. Spence. } & \text { Anglia. } \\ \text { Striatus, Duftschmid. Austria. } \\ \text { Blapoides. Germar, }\end{cases}$ 
10 Nigricans. Spence. Berolini.

11 Abdominalis. Rosenh. Tirolis.

Grandicollis. Erich. St. Germania.*

Tristis. Panzer. $\quad$ id. ${ }^{*}$

Celer. Lucas. Algiria.

Chrysomeloides. Panz.

Latr. Gallia.*

Montivagus. Heer. Helvetia.

Alpinus. Gyllenhal. id.

(Nigrita. Erichson. Germania.*

Tristis. Gyllenhal.

Morio. Paykull. id.

19 Fuliginosus. Erichson. Germania.*

20 Scitulus. Erichson. id.

21 (Morio, Spence, Erich. id.*

Dissimulator. Spence. Anglia.

Var. Morio. Fabr. Germania.

22

(Fumatus. Spce. Erich. id.*

Agilis. Fabr. $\boldsymbol{P}$.

Nigricollis. Sturm. Germania.

23

24

Ambiguus. Heer.

Helvetia.

Velox. Spence. Erich. Germania.*

Præcox.Erichson. id.

Badius. (Megerle. St.) Helvetia.

Sericeus. Fabr. Panz. Germania.*

Truncatus. Illiger. Austria.

Villosus, Latreille. $\boldsymbol{P}$.

Picipes. Kugelann. Germania.

28 Varicornis. Rosenh, Germania.

29 Sericatus. Chaudoir. Russia.

30 Brunneus. (Knoch.) St. Austria. ${ }^{*}$

31 Rufipennis. Lucas, Algiria.

32 Anisotomoides. Spce. Germania.*

33 Caliginosus. Erichson. id.*

34 Brevicornis. Paykull. Suecia.

35 Longulus. Kellner. Germania.

36 Rotundicollis. Kellner. id.

37 Coracinus, Kellner. id.

38 Subfuscus. Kellner. id.

39 Transversostriatus.

Dej, Cat. Lusitania.

40 Luridus, Dej. Cat. Hispania.

41 Flavescens, Dej. Cat, id.

42 Minutus. Dej. Cat, Dalmatia.*

\section{COLON. Herbst.}

Miloechus, Latr, Catops, Sahlb, Gyll.

1 Claviger. Herbst. St. Germania.

2 Viennensis. Herbst. Austria.

3 Appendiculatus. Sahl. Finlandia.

4 Calcaratus. Erich. St. Germania.
5 Dentipes.Sahlb.St.Er.Germania."

6 Bidentatus. Sahlb. St. id.*

7 f Serripes. Sahlberg. St. Finlandia.

\{revicornis. Sturm. Germania.

8 Affinis. Sturm. Cat. Russia.

9 Angularis. Erichson. Germania.

10 Rectangulus. Chaud. Russia.

11 Brunneus. Spence, Lat. Gallia.*

12 Pubescens, Lucas. Algiria.

13 Fusculus. Erichson. St. Germania.*

14 Languidus, Erichson. id.

15 Pygmæus. Erichson. id. ${ }^{*}$

16 Nanus, Erichson. id. ${ }^{*}$

17 Sinuatus. Chaudoir. Russia.

18 Subdepressus. Chaud. id.

\section{LEPTINUS, Müller.}

Germar. MAgAz.

1 Testaceus, Müller. Helvetia. 115

\section{FAII. SCAPHIDII.}

SCAPHIDIUM. Olivier.

1 Quadrimaculatum. $O l$. P.*

SCAPHIUM. Kirby.

Scaphidium, Oliv, Dej. Cat.

1 Immaculatum. Oliv. $P_{.}^{*}$

SCAPHISOMA. Leach.

Scaphidium, Dej. Cat. Silpha, Linné. Dermestes. Scopol.

1 Agaricinum. Linné. P.*

Pulicarium. Rossi. Italia.

2 Boleti. Panzer. Germania.

3 Assimile.Schïp. Erich. id.

4 Limbatum.(Dhl.) Erich.Hungaria. * 


\section{FAH, TRICHOPTERYX.}

\section{TRICHOPTERYX. Kirby.}

Heer, Allibert. Pтilium. Schüpp. LatriDivs. Herbst.

1 Atomaria. de Geer.

Gallia.*

Flavicornis, Waltl. Germania.

2 (Fascicularis. Herbst. P.

Minutissima. Marsh. Anglia.

Lata. Motschulsky. Russia.

Grandicollis. Erich. Germania.

3 Picicornis. Mannerh. Finlandia.*

$\{$ Brevipennis, Erich. Germania.

Clavipes. Gillmeister. id.

4 (Intermedia. Gillmeist. id.

\{randicollis. Märkel, Saxonia.

Fascicularis, Erich. Germania.

5 Thoracica, Gillmeister. id.

6 Chevrolatii. Allibert. P.*

Pygmaa. Erichson. Germania.

Parallelogramma.

Gillmeister. id.

7 (Depressa. Sturm. id. *

Sericans. Heer. Helvetia.

$\{$ Volans. Motschulsky. Russia.

Acuminata. Motsch. id.

Bovina. Motschulsky. id.

8 Montandonii. Allibert. $P^{*}$

Sericans. (Schüppel.) Germania.

$\{$ Rivularis, Allibert. P.

Pumila. Erichson. Germania.

Longicornis. Motsch. Russia.

9 Abbreviatella. Heer. Helvetia.

10 Foveola. Mannerh. Finlandia.

11 Pulchella. Allibert. P.

12 Chevrieri. Allibert. id.*

13 Guerinii. Allibert. id.*

14 Alpina. Allibert. Gall. merid.

15 Myrmecophyla Allib. $P^{*}$

16 Brachyptera. Allibert. Gall. merid.

17 Fuscicola. Allibert. id.

18 Melanaria, Allibert. id.

PTILIUM. Schüppel. Erich.

Trichoptenyx. Heer, Allibert. Gillmeist.

1

$\begin{cases}\text { Suturale. Heer. } & \text { Helvetia.* } \\ \text { Corticale. Schüppel. } & \text { Germania. } \\ \text { Flavescens. Motsch. } & \text { Russia. } \\ \text { Bicolor, Motschulsky. id. } \\ \text { Fuscum. Motschulsky, id. }\end{cases}$

2 Limbatum. Heer. Helvetia.

$\{$ Var. Testaceum. Heer, id.

3 Ratisbonense, Gillm. Germania.

4 Gracile. Gillmeister. id.

5 J Apterum. Guérin. Gallia. Y Pallidum. Dej. Cat. P.

6 Microscopicum. Gillm. Germania. Tenellum. Erichson. id.

7 Foveolatum. Allibert. P.* Excavatum. Märk. Gil. Germania.

Limbatum. Motsch. Russia.

Gallicum. Motsch. Gallia.

Minimum. Herbst. Germania.

8 Angustulum. Spence. Gillmeister. id.*

9 Minutissimum. Weber.

$\left\{\right.$ (Elophorus.) $\boldsymbol{p}^{*}$

\{risulcatum. Aubé. id.

Lavicolle.Waltl. Germania.

10 (Exaratum. Allibert, P.*

Minutissimum. Heer. Helvetia.

Canaliculatum. Märk.

Gillm. Germania.

11 Latum. Gillmeister, id. \{Casum. Erichson. id.

12 Discoideum. Gillm. id.

13 Affine. Sturm. id.

14 Boudieri. Allibert. $P$.

Transversale. Erich. Germania.

Aterrimum. Motsch. Russia.

Picipes, Motschulsky. id.

15 Fuscum. Gillmeister. Germania.

16 Spencei, Allibert. $P$.

Angustatum. Spence, Anglia.

Rugulosum. Allibert, P.

Oblongum. Gillm.Er. Germania.

17 Kunzei. Heer. Helvetia.

Longicorne. Märkel. Germania.

Nanum. Stephens. - Anglia.

18 Ferrari. L. Redtenb, Austria.

19 Saxonicum. Gillmeist, Saxonia.

20 Punctatum. Gyllenhal.

$\left\{\begin{array}{c}\text { (Scaphid.) Suecia. } \\ \text { Alutaceum. Gillmeist. Germania. }\end{array}\right.$

PTENIDIUM. Erichson.

Anisarthria. Steph. Trichopteryx. Heer. Allibert. Gillmeister.

1 Pusillum. Gyllenhal. $\left\{\begin{array}{l}\text { (Scaphid.) Suecia.* } \\ \text { Nitidum. Heer. Helvetia. } \\ \text { Quadrifoveolatum.Alib.P. }\end{array}\right.$ 
2 Corpulentum. Allibert. Algiria.

3 Lævigatum. Gillm. Er. Germania.

4 Apicale. Sturm. id.

Elongatulum. Motsch. Russia. Myrmecophyla. Mot. id.

5 Fuscicorne. Erichsor, Germania.

6 Gressneri. Erichson. id. 44

\section{FAI, ANISOTOIE.}

TRIARTHRON. Markel, Er.

1 Mærkelii. Schmidt. Germania.

\section{HYDNOBIUS. Schmidt.}

\section{Anisotoma. Sturm.}

1 Punctatissimus. Steph. Germania. \{Tarsale, Riehl. Schm, id.

2 Punctatus. Erichson. id.

3 ( ${ }^{\top}$ Spinipes. Gyllenh. Finlandia. o Punctatum. Sturm. Germania. Edentatum.Sahlberg. Finlandia.

4 Strigosus, Schmidt. Germania.

\section{ANISOTOMA. Knoch. Schmidt.}

\section{Dej. Cat. Tetratoma, Panzer.}

1 Cinnamomea. Panzer. Gallia bor.*

2 Oblonga, Erichson. Germania. Ferruginea. Illiger. id.

3 Rugosa. Stephens. id. Armata. Stm. Schm. id.

4 Triepkii. Schmidt. id.

5 Rotundata. Erichson. id.

6 Rhætica.(Heer.) Erich. Helvetia.

7 Picea. Illiger. Germania.* I Consobrina.Sahlberg. Finlandia.

8 Obesa. Schmidt. Saxonia.* Picea. var. b. Gyllenh. Suecia.

Armata. Paykull. id.

Ferruginea. Gyllenh. id,
9 Dubia. Illiger. Var. 1. Rufipenne.

Paykull, Suecia.

Berolini. ${ }^{\star}$

- 2. Bicolor. Schm. Germania.

- 3. Ferruginea.St.id.

Longipes. Schmidt. id.

Var.4. Pallescens. Sch. id.

10 Flavescens. Schmidt. Pomerania.*

11 Furva. Erichson. Germania.

12 Giliaris, Schmidt. id.

13 Pallens. Sturm. id.

14 Ovalis. Schmidt. Pomerania.

Y Var. Brevipes. Schm. id.

15 Nigrita. Schmidt. Saxonia. *

16 Rubiginosa. Schmidt. Germania.

17 Scita. Erichson. id.

18 Calcarata. Erichson. Gallia. *

Ferruginea. Schmidt. Germania.

19 Brunnea. Sturm. id.* $\{$ Sylvicola. Schmidt. id.

Var. Nemoralis. Schm. id.

20 Nitidula. Erichson. id.

21 Badia. Sturm. id.

$\{$ Lavicollis. Sahlberg. Finlandia.

22 Hybrida, Erichson. Germania.

23 (Parvula. Sahlberg. id.*

Brunnea. Gyllenhal. Suecia.

Gyllenhalii. Stephens. Anglia.

Badia. Schmidt. Germania.

24 ? Livida. Dej, Cat. Lusitania.

CYRTUSA. Erichson.

Anisotoma. Schmidt.

1 Subtestacea. Gyllenh. Germania.

2 Minuta. Ahrens. Schm. id.*

Femorata. Schmidt. id.

3 Latipes. Erichson. id.

4 Pauxilla. Schmidt. id.

\section{COLENIS, Erichson.}

Anisotoma, Schmidt.

1 Dentipes. Gyllenhal. Saxonia.* Aciculata. Stephens. Anglia.

Immundum. (Sphæridium.) Sturm. Germania.

\section{AGARICOPHAGUS, Schmidt.}

1 Cephalotes, Schmidt. Germania.

2 Conformis, Erichson, id. 
LIODES. Erichson.

Leiodes. Latr. Anisotoma. Knoch. Dej. Cat.

1 Humeralis. Kugelann. Gall. bor.* Var. 1. Globosa.Payk. Suecia.

- 2. Clavipes, Hbst.Austria. Armata. Stephens. Anglia,

2 Axillaris. Gyllenhal.' Gall. bor.* Bipustulata. Ahrens. Germania.

3 Glabra. Kugelann. Gallia bor.* Abdominalis, Payk. Suecia.

4 Serricornis. Gyllenhal. id. Signata. Waltl. Germania.

5 Castanea. Kugelann. Gallia.* Axillaris. Stephens. Anglia. Var. Globosa. Herbst. Germania. Orbicularis. Kugelann.Germania. ${ }^{*}$ $\{$ Var. Seminulum. Stm.

Schm, Suecia,

\section{AMPHICYLLIS, Erichson.}

Spharidium. Fabr. Agathidium. St. Dej. Cat.

1 Globus. Fabr. P.* Ruficollis. Olivier. St, id.

Var. Staphylacum. Gyllenh. Suecia.

2 Globiformis. Sahlberg. id.*

\section{AGATHIDIUM, Illiger.}

Silpha, Linné. Anisotoma. Gyll.

1 Nigripenne. Kugelann. Panz. St. $P^{*}$

2 Atrum. Payk. (Sphæri-

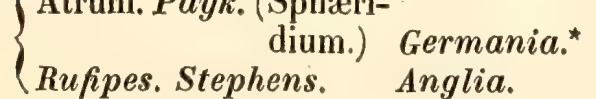
Atratum. Sturm, Algiria.

4 Seminulum. Linné. $\boldsymbol{P}^{*}{ }^{*}$

5 Badium. Ziegler. id.*

6 Lævigatum. Erichson. Germania.* Orbiculare. Stephens. Anglia.

7 Mandibulare. Sturm. Germania.

8 Plagiatum. Gyllenhal. id.

9 Piceum. Erichson. id.

10 Rotundatum. Gyllenh. id.*

11 Varians. Beck, Erich, id.

12 Nigrinum. Sturm. id.
13 Discoideum, Erichson. Germania.

14 Marginatum. Sturm. Austria.* Orbiculatum. Gyll. Suecia.

15 Hæmorrhoum. Erich. Germania.

16 Affine. Sturm. Cat. Italia.

17 Minutum. Sturm. Austria.*

18 Thoracicum. Dej. St. Cat. Germania.

19 Flavicorne, Andersch. St. Cat. Austria.

20 Carbonarium. St. Dej.

21 Atomarium. Sturm. Germania.

22 Punctulum, Beck. id.*

23 Erythrocephalum. Melsheimer. id.

\section{CLAMBUS. Fischer.}

Dermestes. de Geer. Scaphidium. Gyll. Cyrtocephalus? Audouin. Dej. Cat.

1 Pubescens. Redtenb. Austria.*

2 Armadillus. de Geer. Gyll. id.*

Cephalotes? Dej. Cat. P.

CALYPTOMERUS. Redtenbacher.

1 Alpestris. Redtenbach. Austria.

\section{PITOPHILUS, Heer.}

1 Atomarius, Heer, Gallia.

MISCROPHERA. Redtenbacher.

1 Corticalis. Redtenb. Austria.

72

\section{FAVI, PHALACRI.}

PHALACRUS, Paykull.

1 ? Aterrimus. Dej. Cat. Hispania.

2 Corruscus. Panzer, P.*

Fimetarius, Fabr, Germania.

Ater. Herbst. Austria.

Var. Picipes.Stephens, Anglia.

3 Grossus, Erichson. Germania. 
4 (Substriatus. Gyllenh. Suecia. Millefolii. Stephens. Anglia. Trichopus. Waltl. Germania. Punctato-striatus.

Waltl. id.

5. Caricis. Sturm. id. Millefolii. Gyllenhal. Suecia. Punctulatus. Dej.Cat.P.

6 ? Granulatus. Lej. Cat. Gall. merid.

7 ? Cardui. Bes. St. Cat. Austria.
6 Dalmatinus, Dej. Cat.

Caricis. Stephens. Anglia.

Rubicundus. Dej. Cat.

Har, Junci. Stephens, Anglia. Pallidus. Heer. Helvetia.

8 Testaceus. Dej, Cat. Gall. merid.

9 Affinis, Heer. Helvetia.

\section{OLIBRUS. Erichson.}

Spheridium. Fabr. Phalacrus. Payk. St, Dej. Cat. Anısotoma. Panz. Fabr.

1 Corticalis. Schönherr. P.*

Stercoreus, Fabr. Germania.

2 (Eneus. Illiger. Fabr. P.*

Ovatus. Marsham. Anglia.

Cognatus. Stephens. id.

3 Bicolor. Fabr. P.*

Var. Flavicornis. Stm. Germania.

4 Liquidus. Erichson. id.

Ovatus. (Hoffmanseg.) id.

5 Affinis. Sturm. id.

6 Millefolii, Paykull. Suecia. ${ }^{\star}$

Ulicis, Gyllenhal. Germania.

7 Pygmæus, Sturm, id.*

8 Striatipennis, Lucas, Algiria.

9 Geminus. Illiger. Austria. *

Testaceus.Illiger. Gyl.Suecia. ${ }^{*}$

Consimilis, Marsham. Anglia.

10 Piceus. (Knoch.) Steph. Germania.*

11 Oblongus, Erichson. id.*

18 .

\section{FAI. NITIDULE.}

CERCUS, Latreille, Erich.

Dermestés, Fáb. Payk. Cateretes. Heer.

1 Pedicularius. Lin. Fab, P.*

Truncatus, Fabr. Germania.

Spirec. Stephens. Anglia.

2 Barbarus. Lucas. Algiria,

Bicolor, Lucas. id.

4 Bipustulatus. Paykull. Germania.

5 (Sambucii. Erich. id.* ơ Solani. Heer. Helvetia.

(q Seutellaris, Heer, id.
BRACHYPTERUS. Kugelann, Schneid.

Dermestes, Fab. Catenétes. Illig. Panz.

Cercus. Latr.

1 Quadratus. Creutzer. Hungaria.

2 Gravidus. Illiger. Germania.

Pulicarius. Gyllenh. Suecia.

Linaria. Stephens. Anglia.

Scutellatus, Kugel. Germania. Agaricinus. Herbst. id.

3 Cinereus. Heer. Helvetia.

$\{$ Pulicarius, Latreille, $\boldsymbol{P}$.

4 Pictus. Heer. Helvetia.

כ (Pubescens.Erich.Luc.? Germania. *

Urtica, var.Illg. Duft.Austria. Glaber. Newmann. Suecia.

6 Urticæ. Fabr. Germania.

Abbreviatus. Herbst. Gallia.

Scutellatus. Panzer. Germania.

$6^{\text {bis }}$ Rubiginosus. Erich. id.

7 Fulvipes, Erichson. Sardinia.

8 Labiatus. Erichson. Tergest.

9 Fulvus. Erichson. id.

CARPOPHILUS. Leach, Steph.

Erich. Ips, Heer. Nitidula. Fabr.

1 Rubripennis. Heer. Helvetia.

Castanopterus, Erich. Germania.

2 Immaculatus. Lucas. Algiria.

3 Hemipterus. Linné. Gallia.*

Flexuosus. Paykull. Suecia.

Bimaculatus. Olivier, Gallia.

Cadaverinus, Fabr. Germania.

Ficus. Fabr.

Var. Quadratus. Fabr. id. Dania.

- Dimidiatus. Heer. Helvetia.

4 Bipustulatus. Erich. id.*

5 Sexpustulatus. Fabr. Germania.

Abbreviatus. Panzer.

(Lyctus.) id. 
6 Quadrisignatus. Erich, Sicilia.

7 Humeralis, Fab.Erich. Algiria.

\section{IPIDIA. Erichson. Germar.}

IPS, Fabr. Erich.

1 Quadrinotata. Fabr. Austria.*

\section{EPURAA, Erichson.}

Nitidula, Fabr. Heer.

1 Decemguttata, Fabr, Gallia. * 2 Rubiginosa. Heer. Helvetia.

3 Silacea. Herbst. Germania.

4 (Estiva. Linné. P.

Depressa. Gyllenhal. Suecia.

Obsoleta. Herbst. Austria.

Villosa. Stephens, Anglia.

Ochracea. Erichson. Germania.

Var.Bisignata. Sturm.id:

5 Melina. Erichson. id. ${ }^{*}$ Depressa. Illiger. Germania.

6 Deleta. Dej. Cat.Erich. Austria,* Silacea. Heer. Helvetia.

7 Immunda. Erichson. Germania. *

8 Variegata, Herbst. Austria.

9 Bipunctata. Heer. Helvetia.

10 Castanea. Duftschmid. Austria.

11 Neglecta. Heer. St. Helvetia.

12 Obsoleta. Fabr. $\boldsymbol{P}^{*}$

13 Distincta, Grimm, Er, Germania.

14 Parvula. Sturm. Er. id.

15 Angustata. Erichson, id.

16 Boreella. Zettersted. id.

17 Pygmæa. Gyllenhal. id.

18 Pusilla. Illiger. id.*

19 Oblonga. Herbst. Austria.

20 Nigrita. Lucas. Algiria.

21 Longula. Erichson. Germania.

22 Florea. Erichson. id.*

\{Estiva, Illig, Er, St. id.

23 Enea. Fabr. Algiria.

24 Melanocephala. Marsh. Anglia.*

Truncata. Stephens, id.

Discolor. Waltl. Germania

Ferruginea, Heer. Helvetia.

Var, Brunnea. Heer. id.

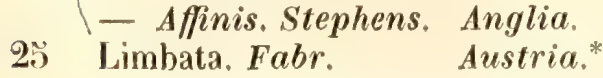

NITIDULA. Fabricius,

- Silpha, Linné.

1 Bipustulata. Linné, $P .{ }^{*}$

2 f Flexuosa, Fabr. Gall. merid.* f Flavomaculata. Rossi. Italia.

3 Obscura. Fabr. $\boldsymbol{P}_{\text {. }}^{*}$ Rufipes. Stephens. Anglia.

4 Quadripustulata. Fabr. $\boldsymbol{P}_{\text {. }}^{*}$ Carnaria. Schaller. Germania. Guttalis. Herbst. Austria. Var. Variata, Steph. Anglia. - Flavipennis. Heer. Helvetia.

SORONIA. Erichson.

Nitidula. Fabr. Heer. Dej. Cat. Silpha. Linné.

1 Punctatissima. Illiger. Gallia.*

2 Grisea.Linné. Suecia.

Var. Varia. Fabr. Germania.

Yariegata. Olivier. Gallia.

AMPHOTIS, Erichson.

Nitidula, Fabr, Heer, Dej, Cat.

1 Marginata. Fabr, P. Biloba,Herbst. Austria.

OMOSITA, Erichson.

Silpha, Linné, Nitidula, Fabr, Heer. Dej. Cat.

1 Depressa. Linné. Germania* Sordida. Fabr. Dania.

Colon. Herbst. Austria.

Varia. Olivier. $\boldsymbol{P}$.

Immaculata, Olivier. id.

2 Colon. Linné. id. ${ }^{*}$

Hamorrhoidalis, Fab, Germania.

3 Discoidea, Fabr, $\quad$. $^{\star}$

4 Cincta.Heer, Helvetia.

PRIA. Kirby, Erich.

Nitidula. Fab. Dej. Cat. Laria. Scopol.

1 (Dulcamaræ. Illiger. Germania.* Truncatella. Steph. Anglia.

$\sigma^{\top}$ Mandibularis. de Castelnau, $P$.

2 Pallidula, Erichson, Sicilia. 
MELIGETHES, Kirby. Erich.

Nitidula, Fabr, Dej, Cat.

1 Pyrenæicus. Laporte, Pyrenais.

2 Rufipes. Gyllenhal, Germania.*

3 Lumbaris. Stm. Erich, id.

\{ Rufipes, var, b. Gyll, Suecia.

4 Hebes. Erichson. Germania.

Olivaceus. Heer. St. Helvetia.

5 Eneus. Fabr, Er, Luc.

(Epurea.) P.*

Psyllius. Herbst. Austria.

Urticc. Stephens. Anglia.

Subtilis. Waltl. Germania.

Alpestris. Heer. Helvetia.

Var. Cœruleus. Herbst. Austria.

6 Nigritus. Lucas.

Suecia.

Algiria.

7 f Viridescens. Fabr. P. ${ }^{*}$

$\{$ Eneus. var. $\gamma$. Schönh. Suecia.

8 Coracinus. Sturm, Er. Germania.*

9 Azureus. Heer. Helvetia.

10 Pumilus. Erichson. Germania.

11 Subæneus.Stm. Erich. id

12 Corvinus, Erichson. id.

13 Subrugosus. Gyllenh, id.

14 Substrigosus. Erich. id.

15 f Symphyti. Heer, St. Helvetia.*

Convexus. Schüppel. Germania.

16 Ochropus. Sturm. Er. id.*

Ochropodus. Schüp. id.

Difficilis, Heer、St. Helvetia.

18 Kunzei, F.Schm.Er. Germania.

19 Memnonius. Erichson. id.

20 Morosus. Erichson. id.

21 Brunnicornis. Stm. Er. id. ${ }^{*}$

22 Viduatus, Schüpp. St. id.*

23 Pedicularius, Gyllenh. Gallia.*

24 Assimilis. Sturm. Er. Germania.

25 Serripes, Gyllenhal, id.

26 Umbrosus. Sturm. Er. id.*

27 Maurus. St, Er. id.*

28 Incanus. St. Er, id.

29 Tristis. St. Er. id.

30 Murinus. St, Er. id.

31 Seniculus. Erichson. id.

32 f Planiusculus, Heer, Er. Helvetia.

(Sericeus. (Waltl.) Germania.

33 Nanus. Erichson. id.

34 Møstus. Erichson. id.

35 Brachialis. Erichson. id.

36 Fuliginosus, Erichson, id.
37 Fibularis. Erichson. Germania.

38 Ovatus. Sturm. Erich. id.

39 Flavipes. Stm. Erich. id.

40 Picipes. Sturm. Erich, id.*

41 Discoideus. Erichson. id.

42 Lugubris. St. Erich, id.

43 Gagathinus. Erich. id.

44 Egenus, Erich. id.

45 Obscurus. Erich. id.

46 Distinctus, St, Erich, id.

47 Palmatus. Erich. - id.

48 Fumatus. (Ullrich.) Er.id.

49 Erythropus. Gyllenhal.id.*

50 Ruficornis. Heer. Helvetia.

51 Exilis, Sturm. Er. Germania.*

52 Fuscus. Rossi. id. ${ }^{*}$

53 Solidus. Ill. St. id.

54 Denticulatus. Heer. Helvetia.

55 Brevis. Sturm. Erich. Germania.

THALYCRA. Erichson.

Strongylus, Erich, Dej. Cat.

1 Sericea. Sturm. Er. Germania.* Fervida. Gyllenhal. Suecia.

\section{POCADIUS. Erichson.}

Nitidula. Fabr. Er. Spheridium. Panz. Strongruds, Herbst. Steph. Dej, Cat. Cxchramus, Heer.

1 Ferrugineus. Fabr. Germania. Estivus. Herbst. Austria. Striatus, Olivier, Gallia.

\section{CYGHRAMUS. Kugelann, Erich.}

Spheridium, Fabr, Panz. Strongrlus. Herbst, Dej, Cat. Nitidula. Illig.

1 Quadripunctatus, Hbst.
$\left\{\begin{array}{l}\text { Colon. Fabr. } \\ \text { Fungicola. Heer }\end{array}\right.$
Er. Germania.*
Quadripunctata.var.
Helvetia.
3 Luleus. Fabr.
b. Gyll. Suecia. 


\section{CYBOCEPHALUS, Erichson.}

Anisotoma. Sahlberg.

1 Exiguus. Erich. Germania.

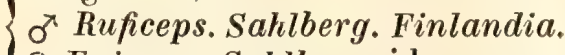
오 Exiguus. Sahlberg. id.

2 Festivus. (Betti.) Erich, Germania,

3 Pulchellus. Erichson. id.

\section{CYLLODES, Erichson.}

Strongyus, Herbst. Nitidula. Gyll.

$$
1 \begin{cases}\text { Ater. Herbst. } & \text { Gall. bor. } \\ \text { Morio. Kugelann. } & \text { Germania. } \\ \text { Var. Ruficollis. (Dahl.) Hungaria. }\end{cases}
$$

\section{CRYPTARCHA. Schuck. Erich.}

Nitidula, Fabr. Herbst. Strongylus. Herbst. Dej, Cat.

1 Strigata, Fabr. Undata. Olivier.

2 Imperialis. Fabr. Y Nebulosa. Marsham.

\section{$P^{*}$. \\ Gallia. Gall. bor.* Anglia.}

\section{IPS. Fabricius.}

Dermestes. Linné.

1 Quadriguttata. Fabr. Germania.* Var. Decemguttata. Quadripunctata Oliv. Gallia bor.

3 Oliv, id.*

3 Quadripustulata. Lin. id.* Quadripunctata, de Geer. Germania.

4 (Ferruginea. Linné. ( $\mathrm{Pi}-$ tiophagus.) Schuk. Austria. ${ }^{*}$ Dermestoides.Panzer. Germania. Linearis. Latreille. $P$.

\section{RHIZOPHAGUS. Herbst.}

Gyll. Lat. Lyctus, Fabr. Payk. Ips. Oliv. Synchita, Duftsch.

1 Grandis. Gyllenhal. Germania.

2 PPunctulatus. Dej.Cat, P.*

3 Depressus. Fabr. Austria.

4 Cribratus. Gyllenhal. Suecia.
5 Ferrugineus, Paykull. P, Suecia.

6 Perforatus, Erichson. Germania.

7 Parallelocollis, Gyll. Suecia. ${ }^{*}$

8 Nitidus. Fabr. Germania.* \{ Erythrocephalus.Fab. id.

9 (Dispar. Paykull. Suecia.* Elongatus. Olivier. Gallia.

|Bipustulatus, var. $\beta$.

10 Unicolor. Lucas. Algiria.

11 (Bipustulatus. Fabr. Suecia.

Dispar. var, b. Payk.

c. Gyll. id.

Bipunctatus, Herbst. Germania.

12 Politus, Hellwig. $\boldsymbol{P}^{*}$

$\{$ Depressus, var. $b$. Payk. Suecia.

13 ?Affinis. Dej. Cat. $P$.

14 Coeruleus. Waltl. Austria.

$\{$ Nitidulus. Duftsch. id.

15 Parvulus. Paykull. Suecia.*

NEMOSOMA. Latreille.

Colydium. Panzer, Erich.

1 Elongata. Linné. Latr. P.* $\{$ Fasciata. Herbst. Austria.

2 ?Cylindrica, Dej. Cat, Russ, merid.

\section{TEMNOCHILA. Erichson.}

Trogosita, Fabr. Duftsch, Erich, Oliv.

1 Coerulea. Olivier. Gall. merid. ${ }^{*}$ \{ Virescens, Rossi. Italia.

\section{TROGOSITA. Olivier.}

Tenebrio, Linné. Fabr. Panzer. Erich. (Platycerus. Geoff.)

1 Mauritanica. Lin. Oliv. Gallia.* \{ Caraboides. Fabr. P.

\section{PELTIS. Geoffroy.}

Silpha, Linné, Fabr. Oliv.

1 Grossa. Linné. Panz. Alp. Gall.* Lunata. Fabr. Germania.

2 Ferruginea. Linné. id.* Rubicunda. Laichart. Tirolis.

3 Oblonga, Linné. $\quad$ Germania ${ }^{*}$ 
4 Dentata. Payk. Fabr. Suecia:* \{ Scabra. Thunberg. Germania.

THYMALUS. Latreille. Cassida. Linné. Peltis. Fabr.

1 (Limbatus. Fabr. P. \{Brunneus. Paykull. Suecia. 159

\section{FAM. COLYDII.}

\section{SARROTRIUM. Illiger.}

Orthocerus. Latreille.

1 (Clavicorne. Linné. Gallia. Muticum. Linné, Fabr. $P$. Hirticornis, de Geer. Suecia.

2 Tereticorne. Erichson. Germania.

3 Crassicorne. Erichson. Austria.

CORTICUS. Dej. Cat. Latreille.

Sarrotrium. Germar. St. Cat.

1 Celtis. Germar. Dalmatia.

2 (Tuberculatus. (Dahl.)

Bolitoph. Hungaria.

Tauricus. (Parreyss.) Tauria.

3 Hispidus. Sturm. Cat. Helvetia.

4 Foveolatus. (Erichson.)

Fairmaire. Germania.

DIODESMA, (Megerle.) Erichson.

1 Subterranea. (Zgl.) Er. Gall. orient. ${ }^{*}$ $\{$ Crevulata. (Creutzer.) Austria.

C.OXELUS. (Ziegler.) Latreille.

Bolitophagus, Sturm.

1 Pictus. Sturm.

Gall. orient.*

2 Piceus. Sturm. Cat. Germania.

\section{DITOMA. Illiger.}

Biтома. Herbst. Srychita, Duftsch. Ips. Oliv. $\begin{cases}\text { Crenata, Fabr: } & \boldsymbol{P}_{.}^{\star} \\ \text { Var. Rufpennis, Fabr. Germania. } & \\ \text { Picipes, Olivier } & \text { Gallia. }\end{cases}$
LANGELANDIA Aubé.

1 Anophthalma. Aubé. $P_{.}^{*}$

COLOBICUS. Latreille.

Monotoma. Duftsch.

1 Emarginatus. Latr. P. Axillaris. Duftsch. Germania. Hirtus. Brullé. Grocia.

SYNCHITA. Hellwig, Schneid.

Lyctus. Elophorus, Fabr. Monotoma. Herbst. Duftsch.

1 Juglandis. Fabr. P* Striata. Herbst. Germania. Humeralis. Fabr. id.

2 Mediolanensis. Vil. Er, Lombardia.

3 Obscura. Ferrari.Redt. Austria.

GICONES, Curtis, Erich.

Synchita. Hellwig. Schneid. Cerylon. Germar.

1 Variegatus. Hellwig. $\left\{\begin{array}{cl}\text { Schneid. } & P .{ }^{*} \\ \text { Carpini. Curtis. } & \text { Anglia. }\end{array}\right.$

2 Pictus. Erichson. Hungaria.

AULONIUM. Erich. Redtenb.

Trogosita. Fabr. Ips. Oliv. Colydium. Duftsch. Herbst. Dej. Cat.

1 Sulcatum. Olivier. $P_{*}^{*}$ Bicolor. Fabr. Germania.

2 (Bicolor. Herbst. Austria.* Ruficornis. Oliv, Latr. Gall. merid. Ustulatum, Dej. Cat. id.

COLYDIUM. Fabr.

Latr, Panz, Er, Dej. Cat. Ips, Oliv, Rossi. Tritoma. Thunb.

1 Elongatum. Fab, Oliv. P.* \{Linearis, Rossi. Gallia.

2 (Filiforme. Fabr, Germania.* Elongatum. var. $\beta$. Payk. Suecia. 
TEREDUS, Dej, Cat. Schuck.

Teredosoma. Curtis, Lyctus. Fabr. Panz.

1 Nitidus, Fabr. $\quad P_{\text {. }}^{*}$ Cylindricus, Olivier, Gallia.

OXYLAMUS, Erichson.

Lyctus. Panzer. Synchita. Duftsch.

1 Cylindricus, Panzer. Germania.

2 Cœesus. Erichson. id.

AGLENUS. Erichson.

Anommatus. Schuck. Monopis. Redtenb. Dej. Cat.

1 (Brunneus. Gyllenhal. P.* Obsoletus. Schuck. Anglia.

(Rufescens. Dej, Cat. Gallia.

ANOMMATUS, Westmael.

Lyctus, Mül. Germar, Cervlon, Dej, Cat.

1 Duodecimstriatus. Mül.

$\left\{\begin{aligned} & \text { Germar. Gallia.* } \text { Terricola. Westmael. Belgia. } \\ & \text { Perforatum. (Chevrl.) P. }\end{aligned}\right.$

PHILOTHERMUS. Aubé.

1 Montandoni, Aubé. P.

\section{GERYLON. Latreille.}

Rhyzophagus et Monotoma. Herbst. Ips. oliv. Lyctus, Fabr. Panz. Synchita. Duftsch.

1 Trisulcatus. (Spinola.)

$$
\text { Germar. Italia. }
$$

2 Sulcicolle. Kollar. Hungaria.

3 (Histeroides, Fab. Latr.

Pilicornis. Marsham. Anglia.

4 Angustatum. Erichson. Germania.

5 Impressum. Erichson. Alsatia.

6 Deplanatum. Gyllenh. id.*

7 Ferrugineum,Dej.Cat. Hispania.
8 Loricatum, Dej. Cat. Gall, merid.

9 Fagi. Motschulsky. P.*

RHYSODES. Illiger, Newmann.

Latr. (Dahl.) Chinidnum, Kirby. Ips. Oliv. Cucusus, Fabr.

1 Sulcatus. Fabr. Pyrenais.*

Exaratus, Dalman. Germania.

Europaus. Ahrens, Austria.

2 Exaratus. Illiger. id.

Aratus, Newmann. id.

BOTHRIDERES, Dej, Cat. Erich.

Lyctus. Fabr. Bitoma. Herbst. Synchita. Duftsch.

1 Contractus. Fabr. P.*

\{Bipunctalus. Herbst. Austria.

PYGNOMERUS. Erichson.

Ips, Oliv. Lyctus. Fabr, Cerylon, Latr. Dej. Cat.

1 Terebrans, Olivier, $\boldsymbol{F}, \boldsymbol{P}$.

XYLOLFMUS, Dej, Cat.

1 Fasciculosus, Schönh. Suecia.

APEISTUS, Motschulsky, Redtenb.

Monotoma, Villa. Rhopalocerus. W. Redtenb.

1 Rondani. Villa. Lombardia.

\section{FAII. CUCUJI.}

\section{PROSTOMIS. Latreille.}

Trogosita, Fab. Megagnathus. Dej, Cat.

1 Mandibularis. Fabr, Germania.* Maxillosus. Muiller. id. 
CUCUJUS, Fabr, Dej, Cat.

Cantharis, Linné.

1 Sanguinolentus. Linné. Austria. $\{$ Depressus. Fabr. id.

2 Hæmatodes. Erichson. id. Depressus. Herbst. id. Punicus. Germar. id.

LEMOPHLAUS. Dej. Cat. Castelnau.

Erich. Cucujus, Fabr. Payk. Gyll. Latr. Creutz, Brontes, Duftsch.

1 Monilis. Fabr. Gall. merid.*

Bipustulatus. Panzer. Germania. Bimaculatus, Olivier, $\boldsymbol{P}$.

2 Muticus. Fabr. Suecia. Piceus. Olivier. Gallia.

Labiatus. Kugelann. Germania.

3 Castaneus. Rosenh. Er. id.

4 Bimaculatus, Paykull, P.

Unifasciatus. Latr. ${ }^{-}$id.

5 Testaceus. Fabr. id. ${ }^{*}$

6 Duplicatus. Waltl. Er. Austria.

7 , Pusillus. Schönh. Oliv. id.

Minutus. Olivier. id.

Testaceus. Stephens, Anglia.

Crassicornis. Waltl. Tirolis.

Exilis. Dej. Cat. Germania.

8 (Ferrugineus. (Creutz.)

Erich. id.*

(Testaceus. Payk. Gyll. Suecia.

9 Ater. Olivier. Spartii. Curtis.

Rufus. (Waltl.) Gall. merid.*

$\{$ Striatus. (Schmidt.) id.

Capensis. Waltl. in

Silbermann. Hispania.

Sturmii. Rosenhauer. Austria.

10 Alternans, Erichson. Berolini.

11 Clematidis. (Chevrier.)

Erich. Gall. merid.*

Denticulatus. Weber. Germania.

Nigricollis. Lucas.

Rufipes. Lucas.

Algiria.

Suberis, Lucas.

id.

Elongatulus. Lucas, id.

$\{$ Corticinus. Erichson. Germania.

Ferrugineus.Stm. Cat.id.

18 Fracticornis.(Chevrol.) Alsatia.*

19 Dufourii. Laboulbène. Gall. merid.
PEDIAGUS, Schuckard, Erich.

Cucujus. Fabr, Gyll. St, Cat. Biophlaus. Dej. Cat.

1 Depressus. Herbst. Germania.

2 Dermestoides. Fabr. Austria.*

3 f Fuscus. Erichson. id.

\{ Dermestoides. Schönh. Germania.

\section{LATHROPUS, Erichson.}

Trogosita? Müller. Germar.

1 Sepicola. Müll. Germ. Germania.

BRONTES. Fabr. Casteln.

Cerambex. Linné. Cucujus. Fabr. Herbst.

Uleiota. Steph. Latr. Schuck.

1 (Planatus, Lin. Herbst. Gallia.

$\begin{cases}\text { Flavipes. Fabr. } & \text { Germania. } \\ \text { Pallens, var. Fabr. } & \text { id. }\end{cases}$

DENDROPHAGUS, Schönh. Erich.

Cucujus. Payk. Fabr.

1 Crenatus. Paykull. Gallia.* 28

\section{FAII. CRYPTOPHAGI.}

SYLVANUS, Latreille, Gyll.

Dermestes. Linné. Lyctus. Fabr. Leptus. Duftsch. Golydium. Payk. Herbst.

1 Frumentarius. Fabr. Gallia.* Sexdentatus. Fabr. Germania.

Surinamensis. Linné. id.

2 Elongatus, Gyllenhal. Austria.*

3 Bicornis. Rosenh. Er. Tirolis.

4 (Bidentatus. Fab. Duft. Gallia.* Sulcatus. Fabr. Germania.

5 Unidentatus. Fabr. P.* Planus. Herbst. Germania.

6 Similis. Vesmael, Dej. Cat. Erich. Gallia.* 
7 (Advena. (Kze.) Waltl. in Silbermann. Gallia. Americanus. Dej. Cat. America. Ferrugineus.Stm.Cat.Germania.

8 Asperatus. Dej, Cat. Lombardia.*

9 Denticollis. Dej. Cat. Gallia.*

10 Pini. (Gaubil.) Alsatia.*

11 Populi. (Chevrolat.) $P^{*}$

\section{PSAMMOECIUS. Latreille.}

Psammoecus. Boudier. Dermestes. Fabr. Anthicus. Fabr.

1 Bipunctatus. Fabr, P.*

2 Boudieri. Lucas. Algiria.

PHLOEOSTICHUS. Redtenbacher.

1 Denticollis. Redtenb. Alp. Gall. ${ }^{*}$

DIPHYLLUS. Redtenbacher.

Dermestes, Fabr. Bitoma, Gyll. BiphylLUs. Dej. Cat.

1 Lunatus, Fabr.

Suecia.

\section{LYCTUS, Fabricius.}

Bitoma. Herbst. Ips. Oliv.

1 (Canaliculatus. Fabr. P.

Oblongus. Oliv. Latr. id.

Unipunctatus, Herbst. Germania.

2 Barbatus. (Chevrolat.) Algiria.*

3 (Pubescens. Panzer. Gall. orient.* \{ Subarmatus. (Megl.) Austria.

4 Angulatus. Sturm. Cat. Livonia.

5 Bicolor. Perrond. Comalli, Gall, merid.

6 f Colydioides, Dej. Cat, P.* \{lycyrrhiza. Chevrol, id.

7 Destructor. Dej. Cat. id.

8 Impressus. Dej. Cat, Gall. merid.* Glabratus.Villa. Lombardia.

\section{TELMATOPHILUS. Heer.}

Ips, Oliv, Cryptophagus, Gyllenh. Limnesius. Dej, Cat, Er. Typhea. Kirby.

1 Sparganii. Stm. Heer. $P$.

2 Typhæ. Fallen. Heer. id.*

3 Caricis. Olivier. id. ${ }^{*}$
ANTHEROPHAGUS. Knoch, Latr.

Mycetophagus. Fabr. Ips, Herbst. CrypTOPHAGUS. Gyllenhal.

1 Nigricornis. Fabr. P. Silaceus, Gyllenh. Suecia.

2 Silaceus. Herbst. Austria.

3 Pallens. Gyllenh. Gallia.*

EMPHYLUS, Erichson.

Croptophagus. Gyll. Antherophagus. Steph.

1 Glaber. Gyllenh, St. Alsatia.*

MYRMECINOMUS. Chaudoir.

1 Hochanthii. Chaudoir. Russia.

\section{CRYPTOPHAGUS, Herbst.}

Schh, Latr. Dej. Cat. Dermestes, Linné. Fabr. Panz. Iss, Oliv.

$1\}$ $\left\{\begin{array}{l}\text { Fycoperdi. Fabr. Gallia. } \\ \text { Fungorum. Panz. St. Germania. }\end{array}\right.$

2 Schmidtii. St. Erich. Austria.*

3 Setulosus. St. Erich. id.*

4 Pilosus. Gyllenhal, St. Germania.* \{Cellaris,var.Dej.Cat. id.

5 Laticollis, Lucas. Algiria.

6 Baldensis, Rosenh. Er. Germania.

7 Saginatus. Schüpp. St. id.

8 Umbratus. Erichson. id.

9 Scanicus. Linné. id. ${ }^{*}$

Cellaris. Fabr. Payk. id.

Humeralis, Stephens. Anglia.

Var. Cellaris. Sturm. Germania.

Patruelis. Sturm. id.

10 Quadricollis. Dej. Cat. Gall. merid.

11 Badius. Sturm. Erich. Austria.*

12 Fuscicornis, St, Erich. Germania.

13 Scutellatus. Newm. Suecia.

14 Labilis, Erichson. Germania.

15 Affinis. Sturm, Erich, id.

16 Cellaris. Scopoli. Er. id.

\{renatus. Herbst. St, Austria.

17 Acutangulus. Gyllenh. Germania.*

Uncinatus. Stephens. Anglia.

Cellaris. Dej.Cat. Gallia.

18 Fumatus, Gyllenhal. Germania.* 
19 (Dentatus. Herbst. Stm. Austria.*

$\{$ Var. Pallidus, Sturm, Germania.

20 Distinguendus. Sturm.

Erich. id.

21 Bicolor. Sturm. Erich. id.

22 Bimaculatus. Gyllenh.

$$
\text { Panz. id. }
$$

23 Dorsalis, Sahlb, Gyll.

$$
\text { St. id. }
$$

24 (Subdepressus. Gyll. Suecia.* \{ Cellaris, var. Dej.Cat. id.

25 Vini. Panzer. Heer. Austria.

26 Crenulatus. Erichson. id.

Crenatus. Gyllenhal. Suecia.

27 Pubescens. Sturm. Er. Austria. *

28 Angustatus. Lucas. Algiria.

29 Puncticollis, Lucas, id.

30 ? Maurus. Lucas. id.

31 ? Gibberosus, Lucas. id.

PARAMECOSOMA. Curtis, Er.

Dermestes. Payk. Lathridius. Gyllenh.

Cryptophagus, St. (Dej.) Erich.

1 Abietis Payk, Gyll. Austria.

2 Elongata, Erichson. Germania.

3 Pilosula. Erichson. id.

4 Melanocephala.Herbst. Austria.*

Fungorum. Gyllenh. Suecia.

Bicolor. Curtis. Anglia.

5. Serrata. Gyllenh. St. Germania.

ATOMARIA, Kirby, Heer.

Erich. St. Dermestes, Fabr. Cateretes. Herbst, Cryptophagus, Gyll. Dej. Cat.

1 f Ferruginea. Sahlberg. Austria.* Wolfi. (Waltl.) id

2 Fimetarii. Fabr. Hbst. Germania**

Parallelopipedus.

Waltl. id.

3 Fumata. Erichson. Austria.

4 Nana. Erichson. id.* Fimetaria. Heer. Helvetia.

5 J Umbrina. Gyllenh. St. Germania.* Fuscata. Heer. Helvetia.

6 Diluta. Erich. Sturm. Austria.

7 Badia, Erichson. Germania.

8 Prolixa. Erichson. id.

9 Procerula. Erichson. id.

10 Pulchra. (Märkel.) Er. id.
11 Elongatula. Erich. St. Germania.

12 Linearis. Stephens. Anglia.* Pygmaa. Heer. Helvetia. Dumetorum. Dej. Cat. St. Gallia.

13 Unifasciata.Sturm. Er. Austria.

14 Contaminata, Erich. Germania.

15 Mesomelas. Herbst.Er. id.

Dimidiata. Marsham. Anglia.

Var. Guttula.Mannerh. Finlandia.

16 Carbonaria. Stephens. Anglia.

17 Dimidiatipennis. Mannerheim. Finlandia.

18 Gutta. Stephens. Anglia.

S Sellata. Kunze. Germania.

19 Fuscipes. Gyllenh. St. id.

Concolor. Märkel. id.

20 Munda. Erichson. Austria.

21 Impressa. (Märkel.) Er.id.

22 Bicolor. Erichson. Germania.

23 Nigripennis. Payk. Gyl. Suecia.*

Ruficollis. Panzer. Germania.

Rubescens. Illiger. Gallia bor.

Basalis. Erichson. Austria.

Cognata. Erichson. id.

Atra. Herbst. Gyll. id.*

28 Gibbula. (Ullrich.) Er. id.

29 Fuscata. Schönherr. Suecia.

Var. Atra. Panzer. Germania.

Rufus. Waltl. id.

30 Apicalis. Erichson. id.

31 Gravidula. Erichson. id.

32 Nigriceps. (Märk.)Er, Saxonia.*

33 Pusilla. Payk. Gyll. Suecia.

Y Phoogaster. Marsh. Anglia.

34 Turgida. Erichson. Germania.

35 Analis. Schüppel. Er. Austria.*

36 Terminata. (Dhl.)Heer.

Er. id.*

37 Versicolor. Erichson. Germania.

38 Contracta. Géné. Sardinia.

\section{EPISTEMUS. Erichson.}

Ephistemus. Westwood. Stephens. PsyCHIDIUM. Müller. Heer.

1 Exiguus. Erich. (Ullr.) Tirolis.

2 Dimidiatus. (Phalacrus.)

$\left\{\begin{aligned} \text { Sturm. } & \text { Austria. } \\ \text { Confinis. Stephens. } & \text { Anglia. } \\ \text { Globulum. Heer. } & \text { Gallia. }\end{aligned}\right.$


3 Globulus. Paykull. Suecia.* Gyrinoides. Mannerh. Gallia.

4 Globus. (Cryptoph.)

๖ Ovulum. Erich. St. Gallia.

MYCETAA. Stephens.

Silpha. Marsh. Dermestes. Fabr. GrypTophagus. Gyllenhal. Dej. Cat.

1 Hirta. Marsham. Gallia.* \{ Subterranea. Fabr. Germania.

SYMBIOTES. Redtenbacher.

1 Latus, L. Redtenb. Austria.

ALEXIA. Stephens. Redtenbacher.

Tritoma. Panzer, Müll. Germar. Phalacrus. St. Hygrotophila. (Chevrolat.) Dej. Cat.

1 Globosa. Sturm. Germania.*

2 Pilifera. Müll. Germar. Gallia.*

Piligera. Germar. Germania.

3 Pilosa. Panzer. id.*

\section{LITOPHILUS. Frölich. Dej. Cat.}

Bolitophagus. Duft. Tritoma. Panz.

1 Connatus. Panzer. Austria.* Ruficollis. Frölich. id.

ORESTIA. (Chevrolat.) Dej. Cat. Redtenb. LYCOPERDINA. Germar.

1 Alpina. Germar. Styria.

LEIESTES. (Chevrolat.) Dej. Cat. Redtb. Cryptophagus. Gyll.

1 Seminigra. Gyllenhal. $\boldsymbol{P}^{*}$

ENGIS. Paykull.

IPS. Fabr. Dehmestes. Gyll. Dacne. Latr. Erotrlus, Oliv.

1 Bipustulata. Fabr.

2 f Rufifrons. Fabr. Iseparata. Parreyss. Croatia.
3 (Sanguinicollis. Fabr. Germania.* Quadripustulatus.

Panzer. Austria.

4 Humeralis, Fabr. P.*

$\{$ Scanicus. Panzer. Germania.

TRITOMA. Fabr, Payk.

Triplax. Oliv, Latr. Dej. Cat. DermesTES. Marsh.

1 Bipustulata. Fab, Oliv, Gallia.*

Humeralis. Marsham, Anglia.

Incerta. Rossi. Italia.

(Dimidiata. (Megerle.) Germania.

TRIPLAX. Paykull, Lacord.

Silpha, Linné. Ips, Fabr. Cryptophagus. Herbst.

1 Enea, Paykull, Fabr. Germania.*

2 (Russica. Linné. Payl. Suecia.*

$\{$ Nigripennis. Fabr. Gallia.

Castanea. var. Marsh. Anglia.

3 Elongata. Dej. Cat.

Lacord. Austria.

4 Ruficollis, Dej. Cat.

$\left\{\begin{array}{r}\text { Lacord. } \\ \text { Dimidiata. (Chevrol.) } \\ \text { id. }\end{array}\right.$

5 Melanocephala. Dej.

Cat. Lacord. Hispania.

6 Nigriceps. Dej. Cat.

Lacord. Gallia.

Collaris? Fabr. Austria.

Melanocephala? Latr. Gallia.

7 Scutellaris. Toussaint-

Charpentier. Hungaria.

8 Bicolor. Marsh. Gyll. Styria.*

9 Rutipes. Payk. Fabr. Gallia.* Collaris. Schaller. Germania.

10 Clavata. Lacordaire. Hungaria.

11 Capistrata.Lacordaire.Gallia.

AULACOCHEILUS, Chevrolat, in litt. Lacord.

Triplax. Germar, Dej. Cat.

1 Violaceus. Germar, Croatia.

2 Chevrolati. Lucas. Algiria.*

TETRATOMA, Fabricius, Schneid.

1 Fungorum, Fabr, P.* 
2 Desmarestii. Latreille. $\boldsymbol{P}$.

3 Ancora. Fabr. Austria.

4 Variegatum. Dej. Cat. Croatia.

SPHINDUS. (Megerle.) Chevrolat.

Germar. Nitidu LA, Gyllenhal.

1 Gyllenhalii. Dej. Cat.

Chevrolat. Alsatia.*

(Dubia. Gyllenhal. Suecia.

141

\section{FAI. LATHRIDII.}

\section{MONOTOMA. Herbst. Latr.}

Aubé. Gerroon. Gyll.

\section{Picipes. Herbst. Gallia.*}

2 Conicicollis. Guérin. Gallia bor.*

3 Angusticollis. Gyllenh. id.*

Formicetorum. Chevr. $P$.

4 Longicollis. Gyllenh. Gallia.*

5 Punctaticollis. Aubé, Gall. bor. ${ }^{*}$

6 Brevicollis. Aubé. P.

7 Blaivii. Guérin. Gallia.

8 Spinicollis. $A u b e ́$. $\quad \boldsymbol{P}^{*}$

9 Quadrifoveolata. Aubé. id. ${ }^{*}$

10 Quadricollis. Aubé. id.*

Angustata. Stephens. Anglia.

Picipes. Gyll.var. b. Suecia.

Bicolor.Villa.Dej.Cat.Lombardia.

Pallida. Stephens, Anglia.

11 Scabra. Märkel.

12

12

13

\section{HOLOPARAMEGUS, Curtis. Steph.}

Westwood. Calyptobium. Villa. Aubé. Redtenb.

1 ( Villæ. (Porro.) Aubé. Difficile. Villa.

Lombardia.

2 Caularum. Aubé. P.

Pankouckii. Guérin. id.

3 Niger. (Chevr.) Aub. Sicilia.

4 Kunzei. Aubé, Gallia.

\section{MYRMECHIXENUS, Chevrolat.}

1 Subterraneus, Chevrol.P.*

2 Vaporariorum. Guérin. id, *

\section{LATHRIDIUS. Herbst. Illig.}

Erich. Mannerh. Corticaria. Marsh. Ips. Oliv. Dermestes. Fabr. Payk. Panz. Tenebrio, Linné. de Geer.

1 Lardarius. de Geer. Germania.* Acuminatus. Kugelan.id. Quadratus. Herbst. id. Rugicollis. Marsham. Anglia.

2 Angusticollis, Schüpp. Gallia. ${ }^{*}$

3 Variolosus. Mannerh. Finl, orient.

4 Angulatus. Motsch. Saxonia.

5 Lapponum. Motsch. Lapponia.

6 Alternans. Mannerh. Finlandia.

7 Rugicollis, Olivier. Gallia.

8 Volgensis. Motsch. Russ. merid.

9 Carinatus. Gyllenhal. Bavaria.

10 Incisus. Mannerheim. Germania.

11 Constrictus. Gyllenhal.Suecia.* Ruficollis. Marsham. Gallia.

12 Elongatus. Curtis. Anglia.* $\{$ Angustatus. Stephens. Gallia.

13 Chlatratus. (Daht.) Austria.*

14 Liliputanus. Villa. Lombardia.* $\{$ Minutissimus, Cristof. id.

15 Exilis. Dej. Cat.Manh. Gallia.* Liliputanus. Motsch. P.

16 Gollaris. Mannerh. Gallia.* $\{$ Ruficollis? (Chevrol.) P.

17 Nanulus. Mannerh. Austria.

18 Concinnus. Schüppel. Saxonia.

19 Hirtus. Schüppel. Finlandia.* $\{$ Hirsutulatus. Steph. id.

20 Rugosus. Herbst. Gallia.

21 Rugipennis. Mannerh. Austria. $\left\{\right.$ Rugosus. $\begin{array}{l}\text { (Sehüppel.) } \\ \text { Mannerh. id }\end{array}$

22 Planatus. Motschulsky, id.

23 (Transversus. Olivier. Gallia.* Sculptilis. Hum. Gyll. Germania. Transversalis, Dej.

Cat. Gallia. Asperatus, Megerle. Germania. Crassicornis. Kirby. Anglia.

24 Minutus, Linné. $P$. 
25 (Porcatus, Herbst. Germania.

Marginatus, Paykull. Suecia.

Pullus. Marsham. Anglia.

Var. Ferrugineus.

Marsham. Anglia.

26 Anthracinus. Mannerh. Austria.

Minutus. Illiger. id.

27 Assimilis. Mannerh. Germania.

Collaris, Motschulsky. Finlandia.

28 Scitus. Motschulsky. Bavaria.

29 Consimilis. Mannerh. Suecia.

30 Gemellatus, Mannerh. Finlandia.

31 Parallelocollis. Mann. id.

32 Brevicornis.Schüppel. Gallia.*

33 Carbonarius. Chevrol. . $^{*}$

34. Filiformis. Dej. Cat.

\section{Mannerh. Gallia.*}

35 Parallelus. Schïppel. Austria.

36 Tantillus. Mannerh. Germania.

37 Nodifer. Westwood. Anglia.

Testaceus. Waterh. id.

\section{CORTICARIA. Marsham.}

Steph. Vils. et Duncan. Schuckard. DERMESTES. Linné. Fabr. Payk. LATRIdius. Herbst. Latr. Fabr. Payk.

1 (Pubescens. Illiger. P.*

Fenestralis. Fabr. Suecia.

Longicornis. Herbst. Germania.

Punctulata. Marsh. Anglia.

Var, Castaneipes. Meg. Austria.

2 Interstitialis. Mannerh Lapponia.

3 Crenulata. Schüppel. Bavaria.*

4 Saginata. Mannerh. Finlandia.

5 Denticulata, Schüppel, Alsatia.*

6 Impressa. Olivier. Gallia.*

7 Badia.(Meg.) Mannerh. Austria.

8 Fulva. Chevrol. in litt. Alsatia.*

9 Campicola. Mannerh. Russ. merid.

10 Serrata. Gyll. Payk. Gallia.*

11 Bella. L. Redtenb. Austria.

12 Laticollis. Mannerh. Suecia.

13 Sculptipennis. Motsch. Russ, merid.

14 Axillaris. Motschulsky. id.

Denticollis. Motsch. id.

15 Melanophthalma.Germ. Finlandia.

16 Formicetorum. Manh. id.*

17 Illæsa. Mannerheim. id.

18 Longicornis. Herbst. Suecia.

Rufipes. Schüppel. Germania.

Var. Ruficornis, Kug. id.
19 Cylindrica. Kunze. Saxonia.*

20 Umbilicifera. Mannerh.id.

Umbicilata? Beck. id.

21 Crenicollis. Mannerh. Gallia.

22 Lacerata. Mannerh. Germania.

23 Foveola. Beck. Suecia.

\{ Foveolata. Waterh. id.

24 Linearis. Paykull. Lapponia.*

25 Rubripes. Mannerh. id.

86 Fulva. Chevr. Mann. Helvetia.

27 Longicollis. Zetterst. Austria.

28 Lateritia. Mannerh. Finlandia.

29 Elongata. Schüppel. Germania.*

30 Ferruginea. Marsham. Suecia.

Fenestralis, Linné. id.

31 Subacuminata.Mannh. Suecia.

32 Curticollis. Mannerh. id.

33 Gibbosa. Herbst, Gyll, Austria.*

Minuta.Fabr, Germania.

Impressa.Marsham. Gall. bor.

34 Transversalis. Schüpp.

Gyll. Gallia.

35 Taurica. Mannerheim. Tauria.

36 Brevicollis. Chevr. Vil. Helvetia.

37 Hortensis. Motsch. Lithuania.

38 Parvula. Schüp. Mann. Austria.

39 Crocata. Motschulsky. Saxonia.

40 Trifoveolata. L. Redtb. Austria.

Fuscula.var.Manner, id.

41 Similata. Schïp. Gyll. id.

42 Subtilis. Mannerheim. Finlandia.

43 Suturalis. Motschulsky. Russ. merid.

44 Truncatella. Motsch. Helvetia.

45 Fulvipes. Motschulsky. Gallia.

46 Distinguenda. Chevrier.

Villa. Mannerh. id.*

47 Fuscipennis. Mannerh. Germania.

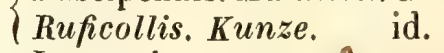

48 Lapponica. Zettersted. Suecia bor.

\{ Serrata. Zettersted. id.

49 Nigriceps. Waltl.Man. Bavaria.

50 Nigricollis.Zettersted. Lapp. merid.

51 Rufula. Zettersted. id.

52 Fuscula. (Meg.) Gyll, Austria.

53 Pallida. Marsham. Anglia.

\section{DASYCERUS. Brongniart.}

Müller. Germar, Redtenbacher.

1 Sulcatus, Müll, Germ. P.

2 Echinatus. Géné. Lombardia. 
LITARGUS, Erichson.

\section{FAI. MYCETOPHAGI.}

MYCETOPHAGUS. Hellwig. Fabr.

\section{Carabus, Chrisomera. Linné. Tritoma.} Geoff. Boletaria, Marsh.

1 Quadripustulatus. Lin. P.* Quadrimaculatus. Boleti. Herbst.

Hellwig. Germania.

Variabilis. Hellwig. Schneid. Germania. Lunaris. Fabr. id.

Sexpustulatus. (Ips.) Varia. Marsham. Anglia. Undulata. Marsham. id.

3 Decempunctatus. Fab. Alsatia.* 4 Atomarius. Fabr. Austria. 5 Múltipunctatus. Hellwig. Schneid. $P .^{\star}$ Similis. Marsham. Anglia.

6 Fulvicollis. Fabr. P.

7 (Populi. Fabr.Payk. Austria.* Brunneus. Panzer. Müller. id.

8 (4-Guttatus. Mïller. Germania. ${ }^{*}$ Pubescens. Stephens. Anglia. Tetratoma. Dej. Cat. P.

9 Salicis. (Chevrolat.) id.*

TRYPHILLUS. (Megerle.) Latr.

Dermestes, Fab. Thyphea. Schuck. Stephens. Mrcetophagus. Hellwig.

1 Punctatus. Hellwig. Schneid. P.* Pilosus. Herbst. Austria. Bicolor. Fabr. (Ips.) Dania. Humeralis. Marsham. Anglia.

2 Suturalis. Fabr. Germania. Ferruginea.Marsham. Anglia. Sparganii. Stephens. id. Obscurus, Dej. Cat. P.*
Mreetophagus. Fabr. Ips. Panz. Evgis. Fabr. Triphillus, Dej. Cat.

1 Bifasciatus. Fab. Hbst, P. Marginalis. Panzer. Germania. Var. Signatus, Panz. id. - Lunatus. Fabr.

(Engis et Ips.) id.

\section{TYPHAE, Kirby.}

Dermestes. Linné. Fabr. Mrcetophag. Gyll. MyceteA, Steph.

1 (Fumata, Linnẻ. Marsh. P.* Testacea. Fabr. Gallia.

Variabilis. Hbst. Payk. Germania. Tomentosa. Stephens. Anglia. Testacea. Stephens. id.

BERGINUS, Dej. in litt. Géné.

1 Tamarisci. Dej. in litt. Géné. Gall. merid.* 14

\section{FAII. DERHESTE.}

BYTURUS, Latreille.

Dermestes. Linné. Fabr. Geoff. Oliv. Ips. oliv.

1 Tomentosus. Fabr.

2 Fumatus, Linné. id. ${ }^{*}$

\section{DERMESTES, Linné.}

Fabr. Herbst, Duftsch. Oliv. Illig, Panz. Anthrenus. Fabr.

1 Vulpinus. Fabr. $\quad P^{*}$ Maculatus. de Geer. Suecia. Senex.(Dahl.) Germar.Germania. Lupinus. (Eschsch.) Er. Russ. merid.

2 Dimidiatus. Stév. Sch. id.

3 (Frischii. Kugn. Schnd. Austria. $\{$ Vulpinus. Illiger. id.

4 Murinus. Linné. Suecia. Nebulosus. de Geer. P. Catta. Panzer. id. Roseiventris.(Peir.) Cast. Pedèmont. 
5 (Carnivorus, Fab, Oliv, Austria.

Versicolor.Castelnau. id.

Humeralis. (Solier.) Buenos-Ayr.

6 Hirticollis. Fabr.

Thoracicus, Géné.

7 Pardalis. Schönherr. Gall, merid. Thoracicus. Dej. Cat, id.

8 Coronatus. Stéven. Russ, merid.

9 Undulatus. Brahm. Austria.

Tesselatus. Illiger. id.

Var. Vulpecula. Hbst. id.

Murinus. Olivier. Gall. merid.

10 Atomarius. (Ziegl.) Er. Austria.

11 Tesselatus. Fabr. $\quad$. $^{*}$

12 Mustelinus. Erichson. Germania.

Murinus. Dej. Cat. $P$.

Tesselatus. Olivier. id.

13 Laniarius, Illiger. Germania.*

Marcellarius. Herbst. Austria.

Murinus. Herbst. id.

Affinis. Gyllenhal. $\boldsymbol{P}$.

Catta. Duftschmid. Austria.

14 Ater. Olivier, Casteln. Gall. merid.*

15 f Fuliginosus. Rossi. Italia.

Ater. Duftschmid. Austria.

16 Lardarius. Linné. Gallia.*

17 Bicolor, Fabr. Herbst. Germania.*

18 ? Holosericeus. Bonel. Italia.

19 ?Signaticollis. Fischer.

St. Cat, Russ, merid.

\section{ATTAGENUS, Latreille.}

Dermestes, Fabr, Oliv, Duftschm. MegaTома. Herbst.

\section{Obtusus. Schönher.}

Hispania.

2 Trifasciatus, Fabr.

3 Repandus, Dej, Cat.

Gall. merid.*

4 Fallax. Géné.

Hispania.*

5 Serripes. (Chevrolat.) Algiria.*

6 Dalmatinus. Dej. Cat.

Kuister. Dalmatia.

7 Vigintiguttatus. Fabr. Germania.*

8 Sexguttatus. Stm. Cat, Sardinia.

9 Niseteoi. Dej. Cat. Dalmatia.

10 Bifasciatus. Rossi. Italia.*

11 Signatus, Dej. Cat. Russ, merid.

12 Megatoma. Fabr. Gall, merid.* Marcellarius. Duftsch. Austria.

13 Flavicornis. Dej. Cat. Gall. merid.* Bicolor, (Dahl.)
14 Pellio. Linné. $P^{*}$

Bipunctatus, de Geer, Suecia.

ơ Cylindricornis.

Schrank. Austria.

Schrankii. Kugelann. id.

Ater. Herbst. ... Germania.

I Marcellarius. Fabr. id.

15 Pantherinus. Ahrens. Germania.

16 Schæefferi. Herbst. id. ${ }^{*}$

17 Sordidus, Heer. Helvetia.

MEGATOMA. Herbst.

Dermestes. Linné. Attagenus, Latr.

1 б Undata. Linné. $\quad P^{*}$ 우 Undulata. Herbst. Germania.

HADROTOMA, Erichson.

Dermestes, Fabr, Payk, Gyll, GlobiCORNIS. Latr.

1 Marginata. Paykull. Suecia.* Emarginata. Gyll. Germania.

2 Nigripes. Fabr. P. Rufitarsis. Panz. Latr.

Guérin. Germania.

ORPHILUS. Erichson.

Anthrenus. Duftsch. Panz. Dej, Cat.

1 (Glabratus. Fabr. $\{$ Glaber. (Creutz.) Pz. |Austria. Niger. Rossi. Italia.

TRINODES. (Megerle.) Erich.

Anthrenus, Fabr.

1 Hirtus. Fabr.

Gall. bor.*

ANTHRENUS, Geoffroy.

Dermestes et Byrreus. Linné.

1 Scrophulariæ. Linné. $\boldsymbol{P}^{*}$

Var. Histrio. Fabr. Germania.

Verbasci. Herbst. Austria.

2 Pimpinellæ. Fabr. $\quad \boldsymbol{P}^{*}{ }^{*}$

3 Varius. Fabr. id.*

Verbasci. Olivier. id.

Tricolor. Herbst. Austria.

Pictus. (Megerle.) Germar, Geoff. id. 
4 Albidus, Dej. Cat. Gall. merid.* 3 Substriatus. (Chevrier.)

5 Signatus. (Ziegl.) Er. Austria.

6 Squamosus. Dej. Cat. Algiria.*

7 Museorum. Linné. $\quad P_{\text {. }}^{*}$

Verbasci. Fabr. Germania.

Fuscus. Olivier. Gallia merid.

Obscurus. Schönherr. Suecia.

Varius. Stephens. Anglia.

8 ( Claviger. Erichson. Germania.

Fuscus. Latreille. P.

9 Sertarius. Schmidt et Helfer. Smyrna.

10 Minutus. (Parr.) Erich. Sardinia.

\section{TROGODERMA. Latreille.}

Dermestes. Gyll. Anthrenus. Fabr.

1 (Versicolor. Creutzer.

Herbst. Austria.

(Elongatula. Duftsch, id.

2 (Elongatula. Fabr. Austria.*

Glaber. Herbst. id.

Ruficornis, Latreille. Gallia.

Versicolor. Illiger. Germania.

3 Nigra, Herbst. Austria.

Subfasciata. Gyllenh. Suecia.

Elongatula. Heer. Helvetia.

4 Villosula. (Meg.) Duft. Austria.*

\section{TIRESIAS. Stephens.}

Dermestes. Fabr, Duftsch. Megatoma. Dej. Cat. Atragenus. Latr. Citesias. Steph.

1 Serra. Fabr, Latr. P. ${ }^{*}$ $\{$ Viennensis, Herbst. Austria.

58

\section{FAM, GEORYSSI.}

\section{GEORYYSSUS. Latreille.}

Pimelia. Fabr. Trox. Panz. Byrrhus. Rossi. Cathamistes. 1llig.

$\begin{cases}\text { Pygmæus, Fabr. } & \text { P.* } \\ \text { Dubius. Panzer. } & \text { Germania. } \\ \text { Crenulatus, Rossi. } & \text { Italia. } \\ \text { Costatus, de Casteln. } & \text { Algiria. }\end{cases}$

Heer. Helvetia.

4 Striatus. Dej. Cat. : Gall. merid. ${ }^{*}$

5 ( Læsicollis. (Ull.) Germ. id.

\{ Vulneratus. (Ahrens.) Austria.

6 Bisulcatus. Motsch. Livonia.

7 Canaliculatus, Dej, Cat. Hispania.

8 Latreillei, Dufour. id.*

\{ Sulcatus. Dej. Cat. id.

9 Incisus, Motschulsky. $\boldsymbol{P}$.

\section{FAII, BYRRHI,}

\section{ASPIDIPHORUS, Latreille.}

Nitidula. Gyll.

1 Orbiculatus. Gyllenh. Gall. bor.*

Viennensis. (Ziegler.)

Dej. Cat. Austria.

\section{LIMNICHUS, Latreille.}

Dej. Cat. Byrrhos. Duftsch, Sturm.

1 | Versicolor.Walt. Heer. Austria. *

IRiparius. Dej. Cat. Gall. merid.

2 Sericeus. Duftschmid. Austria.

3 Pygmæus. Sturm. id.

Sericeus. Stephens. Anglia.

SIMPLOCARIA, Marsh. Steph.

Byrrhus. Fabr. Oliv.

1 Semistriata. Fabr. Gallia.*

Picipes. Olivier. id.

2 Metallica. Sturm.Duft, Austria. \{ Picipes, Gyll, Steph. Suecia.

3 Maculosa. (Märk.) Er. Austria.

4 Acuminata. Erichson. Alp. Austria.

SYNGALYPTA. Dillwyn. Steph.

Chetoponus. Kirby. Byrrhus. Rossi.

1 (Syinosa. Rossi. Austria.* Arenaria. Sturm. Germania.

Pusilla. Sturm. id.

Cretifera. Spce. Stef-

fahny. Austria. 
2

Setosa. Waltl.

\{ Setigera. Duftschmid. Austria.

Settigera.Heer. Helvetia.

3 Paleata. Erichson. Austria.

4 Setigera. Illiger. St. Germania.*

5 Striatopunctata. Dej.

Cat.Steffah. Hispania.

\section{CURIMUS. Erichson.}

Brrrhus. Dft. Vill. Heer.Steffah. Dej. Cat.

1 Erinaceus. Duftsch. Austria. \{Lariensis. Steffahny. Germania.

2 Lariensis, Villa. Heer. Helvetia.*

3 Hispidus. Erichson. Austria.

\section{NOSODENDRON, Latreille.}

Spheridium, Fab. Byrreus, Panz, Oliv.

1 Fasciculare.Fab.Panz. Gallia.*

\section{BYRRHUS, Linné.}

Dermestes, Linné. Gistela. Geoff.

\section{Gigas. Fabr.} Austria*

2 Scabripennis. Steffah. Styria.* \{Alpinus. Dej. Cat. Alp. Gall.

3 Pyrenæus. Dufour. Pyrenais. *

4 Inæqualis. Erichson. Tirolis.

5 Festivus. Sturm. Cat. Hungaria.

6 Signatus. Steffahny. Gall. bor.* Diana. Panzer. Germania. Var, Signatus, Panz. id.

7 Ornatus. Panzer. Germ. merid. * Glabratus. Heer. Helvetia.

Striatus, var, Märkel. Saxonia.

8 'Luniger. Germ. Panz. Austria.* Coronatus, Illig, Brul, id.

Cinctus. Heer. Helvetia.

Var. Lineatus, Panzer, Germania.

9 Picipes. (Meg.) Duft. id.

10 Dennii. Curtis, Berolini.

11 Pilula. Linné. Illig. P.*

Var. b. Oblonga.Stm. Germania.

- c. Aurato-fasciatus. Duft. Austria.

Albopunctatus. Fabr. id.

Var. d.Arietinus.Steff.id. ${ }^{*}$

- f. Flavocoronatus. (Waltl.) id.

- h. Ater. Illiger. id.

- g. Argenteo-fasciatus. Duft. id.
12. Fasciatus, Fabr. Gallia.*

Var, a. Diana. Fabr. Germania.

- b. Cinctus. Stm. id.

- d.Dorsalis. Panz.id.

13 Dorsalis. Fabr. Gallia.

Var. a.Ater.Fab.Oliv. Germania.

- b.Morio.Il.Panz.id.

- c.Fasciatus.Hbst. Austria.

- f. Rufipennis. Ill. id.

14 Regalis. (Dahl.) Steff. id.

15 Arietinus. Germar. Germ. merid.

16 Pilosellus. Heer. Helvetia.

17 Sulcatus. Zettersted. Lapponia.

18 Murinus. Fabr. Gallia. *

Rubidus. Kugelann. Germania.

Pulverulentus. Thunb. Suecia.

Undulatus. Kugelann.Germania.

19 Insignis. Steffahny. id.

20 Decorus. Steffahny. Hungaria.

21 Pulchellus. Heer. Helvetia.

\section{GYTILLUS. Erichson.}

Byrrhus, Fabr. Steffahny. Gistela. Fost.
1 Varius. Fabr.

Maculatus. Herbst.

Sericeus, Stephens.

Bicolor, Marsham.

Pilula. de Geer.

Var, a. Fuscus. Steph. Anglia.

- c.Aurieomus.Dft.Austria.

- d.Stoicus. Kugln. id.

\section{MORYGHUS, Erichson.}

Byrenus. Fabr. Illig. Payk. Panz. Steff.

1 Eneus. Fabr.

2 Nitens. Panzer. id.*

Nitidus. Schaller. id.

Punctatus. Germar. Germania.

Niger. Kugelann. id.

\section{PEDILOPHORUS. Steffahny.}

Byrnhus, Duftsch.

1
Eneus. Olivier. Gallia. 


\section{FAII. THROSCI.}

\section{MYRMECOBIUS. Lucas.}

1 Agilis. Lucas.

Algiria.

THORICTUS. Germar.

Xrlonotrogus. Motschulsky. PlatydeRUs. Dej. Cat.

1 Mauritanicus. Lucas. Algiria.*

2 Grandicollis, Germar, Orient.

\{Laticollis? Motschuls. Russ, merid.

3 Puncticollis. Lucas. Algiria.*

4 Germari. Lucas. id.

\section{CEUTOCERUS. Germar.}

1 Advenus. Germar. Gall. merid.

\section{THROSCUS, Latreille.}

Elater, Linné, Oliv, Dermestes. Payk. Trixagus. Kugelann.

1 (Dermestoides, Linné. Gallia. $\{$ Clavicornis, Olivier. Austria. Adstrictor. Fabr. Germania.

2 Elateroides. Heer. Helvetia.

3 Pusillus, Heer. id.

\section{FAII, HISTRI.}

\section{HOLOLEPTA. Paykull.}

Hister. Fabr.

1 Plana. Fabr. Payk. Carniolia.*

PLATYSOMA, Leach. Erich.

Hololepta. Payk. St. Cat. Hister. Fabr. Oliv.

1 Frontale. Paykull. Germania.

2 Oblongum. Fab. Payk. Alsatia.*

3 f Depressum. Fabr. Gallia.*

Y Var.Deplanatum. Gyl.Suecia.
4 (Lineare. Erichson.

Germania.*

Oblongum. Illiger. Austria.

Angustatum. Payliull. var. Gyll. Suecia.

5 Angustatum. Ent. Hft. Germania.

6 Algiricum. Lucas, Algiria.*

7 Filiforme. Erich. Lusitania.

HISTER, Linné.

Fabr. Payk. Oliv, Latr. Erich.

1 Major. Linné.

2 Inæqualis. Fabr.

Lavis. Panzer.

3 Grandicollis. Illiger. Lusitania.

Var. Gibbus. (Dahl.) Sardinia.

4 Quadrimaculatus. Lin. Payk. Gallia. ${ }^{*}$

Lunatus. Fabr. $P$.

Var. Gagates. Illiger. Austria.

5 Ethiops. Heer. Helvetia.

6 Quadrinotatus. Scriba. $P_{\text {. }}^{*}$

Quadrimaculatus.

Fabr, Germania.

7 Pustulosus. Géné. Sardinia.

8 Unicolor. Linné. Fabr. P.*

9 Barbarus. (Chevrolat.) Algiria.*

10 Punctifer. Paykull. Suecia.

11 Fimetarius. Herbst. Germania.* Sinuatus. Fabr. Austria.

Interruptus. Fischer. Russ. merid. Bipustulatus. Illiger, Austria.

12 Amplicollis. Erichson. Algiria.*

Graecus. Dej. Cat. Grocia.

13 Neglectus. Germar. Germania.*

14 Terricola. Germar. id.*

15 Merdarius. Ent. Heft. id.*

16 (Cadaverinus. Ent. Heft.P.*

Brunneus. Illiger. Germania.

Impressus. Fabr. id.

17 Distinctus. (Megerle.) Austria.

18 Carbonarius. Ent.Heft.P.*

Striatus. Illiger. Austria.

Var. Nigellatus. Germ. Germania. Duodecimstriatus. Ill. Austria.

19 Marginatus. Erichson. Germania.

20 Purpurascens. Payk. Gallia. ${ }^{\star}$

21 Binotatus. Dej. Cat.

Erich. Gall. merid.*

22 Stercorarius. Ent.Heft. Gallia.* 
23 Sinuatus, Paykull. Gall. merid.

Uncinatus. Illg. Erich. Austria.

Fimetarius. Herbst. id.

Illigeri. Duftschmid. id.

Velox. Ménétriés. Russ, merid.

24 Bipunctatus, Paykull, Gall. merid.

25 Politus. (Dahl.) Hungaria.

26 Nigerrimus. Dej. Cat. Gall. merid."

27 Sepulchralis. Erichson. Hungaria.

28 Mæreus. Erichson. Istria.

29 Funestus. Erichson. id.

30 (Bisexstriatus, Paykull, Gallia.*

12 -striatus. var. $b$. Sturm. Germania.

31 Puncticollis, Heer. Helvetia.

32 Corvinus. Germ. Gallia.*

$\{$ Bisexstriatus, var.

Payk. Er. Germania.
Vicinus. Besser.

33 Bimaculatus, Linne. Gallia.*

34 Scutellaris. (Dahl.) Sicilia.

I Var. Bifoveolatus. Kol. Gracia.

35 (Duodecimstriatus. Pay-

$\left\{\begin{array}{l}\text { kull. Gallia. } \\ \text {. }\end{array}\right.$

(Var.Bisextriatus,Illg. Germania.

36 Quatuordecimstriatus.

Gyllenh. Suecia.

HET ERIUS, Godet. Erichson.

Haterius. Dej. Cat. Hister, Oliv. Kugelann.

1 Quadratus. Ent. Heft. Gallia.* $\{$ Ferrugineus. Olivier. id.

\section{EPIERUS. Erichson.}

Hister. Paykull.

Retusus. Illiger.

Austria.

2 Comptus. Illiger.

id.

Italicus. Paykull.

id. ${ }^{*}$

4 Fulvicornis. Paykull, id.

\section{TRIBALUS. Erichson.}

Hister. Auct.

1 Scaphidiformis. Illiger. Austria.

2 Minimus. Rossi.

Ital, Algiria.
DENDROPHILUS. Leach. Erichson.

Hister. Linné.

1 Punctatus. Paykull. Gallia.*

\{ Pygmaus, Fabr. Germania.

2 (Pygmæus. Linné. $P_{\text {. }}^{*}$

Formicetorum. Aubé. id.

Scheppardii. Curtis. Anglia.

PAROMALUS, Erichson.

Hister, Hololepta. Paykull.

1 Troglodytes, Paykull. Gall merid.*

2 Complanatus. Paykull. Gallia.*

3 f Parallelopipedus, Hbst.id.*

Picipes. Sturm. Germania.

4 Flavicornis. Paykull. Gallia.*

5 Brunnipes. Stm. Cat. Gall. merid.

SAPRINUS, Erichson.

Hister. Payk, Dej. Cat.

1 Rotundatus. Illiger. Gallia.*

\{ Conjugatus, Illiger. Austria.

2 Maculatus, Rossi. Italia.*

Personnatus. Fischer, Russ, merid.

3 Cruciatus. Paykull. Algiria.*

4 Ornatus. Fischer. Russ merid.

Interruptus, Fischer, id.

5 Externus. Fischer. id.

6 Biguttatus. Stéven. id.

7 Interruptus. Paykull. Orient.

8 Semipunctatus, Fabr. Gall. merid.

Corulescens, Ent. Hft. id.

Cyaneus. Rossi. Italia.

Caspius. Ménétriés. Russ. merid.*

9 Intricatus, Latreille. Gall. merid. ${ }^{\star}$

10 Nitidulus. Fabr, Panz. Gallia.*

Acuminatus. Fabr. id.

Semi-striatus, Ent.

11 Furvus. Erichson. Gall. merid.

Massiliensis. Dej. id.

12 Detersus. Illiger. Austria?

13 Immundus. Gyllenh. Germania.*

14 Rugifer. Gyllenhal, Suecia.

15 Chalcites. Illig. Erich, Algiria.*

\{Affinis. Paykull. Gall. merid.

16 Speculifer, Lat, Payk.

Er. Gallia.*

Pulcherrimus, Weber. Germania.

Personnatus, Illiger, Austria. 
Cribellatus. Steven.

Eneus. Fabr.

Russ, merid. Gallia.*

Opacus. Sturm. Cat. Germania. Mediocris. Mac-Leay. Algiria.*

Ruficornis, Dej. Cat. id. ${ }^{*}$

\{ Virescens. Paykull. Gallia.*

Viridis. Duftschmid. Austria.

Lautus. Erichson.

Piceus. Paykull.

Emulus. Illiger.

Sabuleti. Rosenhauer. Germania.

Conjungens. Paykull. Gallia.*

Rufipes. Paykull. Gall.merid.

Ahenus. Sturm. Cat. Hispania.

Arenarius. (Dahl.) Austria.

Amœenus. Erichson. $\quad N$.

Antiquulus. Illiger. Austria.

Granarius. Erichson. $N$.

Spretulus. Erichson. id.

Impressicollis. (Gaub.) Algiria. *

Algericus. Paykull. Hisp. merid. ${ }^{*}$

Metallescens, Erich. Sardinia.

Ereus. (Megerle.) Italia.

Immundus. Gyllenh. Germania.

Mauritanicus, Lucas. Algiria.*

Vipidescens. Sturm. Gall. merid.

Metallescens, Dej. Cat. id.

Virens. (Dahl.) Hungaria.

Rubripes. Erichson. Lusitania.

Quadristriatus. Ent.

Heft. Alsatia.*

Apricarius. Erichson. Sicilia.

Metallicus. Fabr.Hלst. Germania. ${ }^{*}$

Pullus. Rosenhauer. id.

Rugifrons. Ent. Heft.

Payk. id. *

Metallicus, Ent. Heft.

Payk. St. id

Curtus. Rosenhauer. id.

Crassipes, Dej. Cat.

Erich. Hispania.*

Dimidiatus. Illiger, Gall. merid.* Latipes. Dej, Cat, Italia.

Modestus. Sturm. Cat. Hungaria.

Ovatus, Sturm. Cat. Germania.

\section{TERETRIUS, Erichson.}

Hister, Fabr.

1 Picipes, Fabr.

Germania.*
ONTHOPHILUS, Leach, Erich.

Hister. Fabr.

1 Striatus. Fabr. P.*

\{ Sulcatus. Olivier. id.

2 Affinis. L. Redtenbach. Austria.* Catenulatus. (Dahl.) id.

3 Sulcatus. Fabr. Gall. merid. ${ }^{*}$ \{Globulosus, Olivier. id.

4 Exaratus, Illiger. Sardinia.

PLEGADERUS. Erichson.

Abreus. Leach. Hister. Fabr.

1 Cæsus. Fabricius. Germania.*

2 Saucius, Erichson. Austria.*

Vulneratus, Gyllenh. Suecia.

3 Vulneratus. Panzer, Germania.*

4 Discisus. Erichson. Austria.

5 Dissectus. Erichson. Germania.

6 Pusillus. Rossia. Payk. Italia.

\section{ABREUS, Leach. Latr.}

Hister, Fabr, Oliv.

1 Globulus. Creutzer. Germania.*

2 Globosus, Ent. Heft. id.*

3 Granulum. Erichson. id.

$4\left\{\begin{array}{r}\text { Nigricornis. Ent. Heft. } \\ \text { Payk. id. }\end{array}\right.$

Minutus. Payk. Suecia.

5 Atomarius, $A$ ubé, $\boldsymbol{P}$.

6 Punctum. Aubé. Italia.

7 Parvulus. Aube. $\quad \boldsymbol{P}_{\text {. }}^{*}$

8 Rhombophorus. Aubé. Gallia.

9 ? Scaber. Fabr. Algiria.* 131

\section{FAII. SCARABEI.}

Divisio 1. Lucanida. Mac-Leay.

PLATYGERUS. Geoffroy. Latr.

Lucanus. Linné. Fabr. Geruchus. Mac-Leay.

1 Caraboides. Linné. Gallia.*

Var. A. Virescens. Mul, id.

- B. Viride-aneus.

Mulsant. id.

- C. Rufipes. Muls, id." 
CERUCHUS. Mac-Leay.

Tarandus. (Meg.) Platycerus. Latr. Lucanus. Fabr. Panz.

1 (Tarandus. Panzer, Alp, Gall.* Piceus. Bonsdorff. Russia.

Chrysomelinus. Hohw. Suecia.

Tenebrioides. Fabr. id.

Var. A. Sylvicola.Mul.Alp. Gall.

- Silesiacus? (Meg.) Silesia.

\section{LUCANUS. Scopoli. Linné.}

1 Cervus. Linné. Gallia.*

I Inermis. Marsham. Anglia.

Var. A.Microcephalus.

Mulsant. Gallia.

- B. Dorcas. Panz. Germania.*

Capreolus. Sulzer. id.*

Hircus, Scheven in

Fuessly. Austria.

Capra. Olivier. Gallia.

Var. Turcicus. Sturm. Constantinp.

2 Tauricus. Motschulsky. Tauria.

3 Maxillaris. Motsch. id.

\section{HEXAPHYLLUS. Mulsant.}

1 Pontbriantii. Mulsant. Gall. orient.*

\section{DORCUS, Mac-Leay.}

\section{Lucanus. Fabr.}

1 Parallelopipedus, Lin, Gallia.*
ㅇ Infractus. Bergstr. Germania.
- Dama. Müller. id.
- Capra. Panzer. id.
$\{$ - Bipunctata. Schrk. Austria.
- Tuberculatus.Mac-
Var. A. Immaturus.
Leay. Anglia.
Mulsant. Gallia.

2 Oblongus. Charpent. Pyrenceis.

3 Musimon. Géné. Algiria.*

\section{ESALUS, Fabricius.}

Lucanus, Panzer.

1 Scarabæoides. Fabr.

$$
\text { Panz. Alsatia.* }
$$

\section{SINODENDRON. Fabricius.}

Scarabeus. Linné. de Geer.

1 Cylindricum. Lin. Fab. Gallia.* Yar. A. Juvenilis. Mul, id.

\section{Divisio 2. Geotrupida. Mac-Leay.}

\section{GEOTRUPES. Latreille.}

Scarabeus, Linné. Fabr.

1 Stercorarius, Linné. Gallia.* o Spiniger. Marsham. Anglia.

Var. A. Puncticollis.

Stephens. id.

- B. Exaratus,Muls, Gallia.

- C. Foveatus. Mars. Anglia.

- F. Subrugulosus.

Mulsant. Gallia.

- G. Subviolaceus.

Mulsant. id.

- H. Chalybeus.Mul.id.

- I. Virescens. Muls. id.

- J. Juvencus, Muls, $1 \mathrm{~d}$.

- Octomaculatus.

(Gaubil.) Gall. merid.

2 (Putridarius. (Eschsch.)

Erich. Gallia.*

Stercorarius, de Geer. Gyll. Steph. id.

Punctatostriatus? Stephens. Anglia.

3 Mutator. Marsh. Steph. Gallia.*

Stercorarius. Herbst. Germania.

Politus. Malinowski. id.

4

Douei. Gory. Algiria.

Dentifrons. Mulsant. id.

Siculus, Dej. Cat. Er, Sicilia.

5 Purpureus. Stm. Cat, Bosporus.

6 Dalmatinus. Stm. Cat. Dalmatia.

7 Hypocrita. (Schneider.)

Lep. de St Farg. Gall. merid." Stercorarius, var, $b$. Rossi. Italia.

Sublavigatus. Steph. Anglia.

Var. A. Lavicollis. Mul. Gall, orient.

- B. Substriatus. Mulsant, id.

- C. Subvirescens. Mulsant, id. 
8 Sylvaticus. Panzer. P.*

Stercorosus. (Hartm.)

Scriba. Carniolia.

Var. A. Nigrinus.Muls, Gallia.

- B. Monticola.Heer. Helvetia.

- C. Amothystinus.

Mulsant. Gallia.

D. Juvenilis. Muls. id.

9 Vernalis. Linné. Gallia. *

Lavis, Curtis. Anglia.

Var. Autumnalis.(Zgl.) Helvetia.

- A. Obscurus. Muls. Gallia.

- B.Violaceus. Muls, id.

- C. Varians. Muls. id.

Vernalis. Heer. Gall, orient.

Var.D.Splendens.(Zgl.)

- G. Politus. Muls. Gallia.

- F. Pyrenceus.

Charpent. Pyrenais.

- Alpinus. (Hagenb.

St.) Charp. Alp. Lomb.*

10 Corruscans. Chevrol. Lusitania.*

THORECTES. Mulsant.

Geotrupes. Latr. Scarabeus. Linné. Fabr.

1 Rotundatus, Lucas. Algiria. ${ }^{*}$

\{ Cyslonotus. (Dejean.) id.

2 Puncticollis. Lucas. id.*

3 Latus. Rambur. Hispania.*

4 Hemisphæricus. Oliv. Álgiria. *

5 Lævigatus. Fabr. Gall. merid. ${ }^{\star}$

Var. A. Lineicollis.Mulsant, id.

- B. Desjardini. (Gory.) Mulsant, id.

- C. Subgeniculatus. Mulsant. id.

- D. Subrugulosus.

Mulsant. id.

- E. Simplicidens.

6

Mulsant. id.

Hoppei. Hagenbach.

Rugulosus Charpent. Tergest.

Glabratus. Dej.Cat. Corsica.

7 Sardeus. (Eahl.)Erich. Sardinia. *

8 Distinctuș. (Gaubil.) Algiria.*

9 f Germinatus. Dej. Cat. Corsica.*

I Pastor. Géné.

10 Globosus, St. Cat, Sicilia.
CERATOPHYUS, Fischer.

Geotrutes, Latr. Dej. Cat. Scarabeus. Lin. Fabr.

1 Ammon. Pallas.

Dispar. Fabr.

Russ, merid.

id.

2 Hoffmanseggii: Dej.Ct.Lusitania.*

Dispar. Sturm. Cat. id.

3 Fischeri. Zwick. Fisch. Russ, merid.

Monoceros.(Dahl.) Ger-

I mar. Italia.

Dispar. Rossi. id.

4 Typhæus. Linné. Gallia.

Vulgaris. Leach. Anglia.

ơ Var. A. Pumilus.

Mulsant, id.

우 - B. Pusillus.

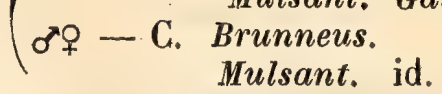

๖ Fossor. Friwaldsky. Waltl. Gracia.

6 Subarmatus. Dej. Cat.

7 Momus. Fabr.

Fairmaire. id.

8 Hispania.

Sardinia.

BOLBOCERAS. Kirby. Serville et Lep.

Dej. Cat. Mulsant. Odonteus. (Meg.) Erich. Scarabaus. Fabr.

(Odontaus. Erich.)

1 ( $\%$ Mobilicornis, Fabr. Gallia.*

$\left\{\begin{array}{c}\text { o Bicolor. Fabr. id. } \\ \sigma^{\star} \text { Armiger. Hochen- } \\ \text { warth. Laich. Tirolis. } \\ \text { Var. A. Recticornis. } \\ \text { Mulsant. Gallia. }\end{array}\right.$

o V Var. B. Obliteratus.

Mulsant. id.

ơㅇ - C. Fulvus.Mul. id.

- D. Testaceus.

Fabr. id.

(Bolboceras, Kirby.)

2 Gallicus. Mulsant. Gall. merid.*

ơ Var. A. Provincialis.

Mulsant. id.

우 - B. Cunjunctus.

Mulsant, id. 
3 Quadridens. Fab.Panz.Austria.*

$\{$ Unicornis. Schrank. id.

Eneas, Panzer. id.

4 Lusitanicus. Dej. Cat. Algiria.*

5 (Bocchus, Erichson. id.*

Fissicornis, Mulsant. id.

\section{LETIIRUS, Fabricius.}

Bolboceras. Vet, Clunipes. Hochenw.

1 Cephalotes, Fabr. Hungaria.* Var. Podolicus. Fisch. Podolia.

Scarabooides. Hochw. id.

2 jLongimanus. Fischer, Rus, mer, or. * \{ Eversmanni. Falderm.id.

\section{Drvisio 3. Coprida.}

\section{SCARABAUS. Linné. Oliv.}

Ateuchus, Web. Fabr, Copris, Geoff. Actinophords. Creutz, Panz. HelioCANTHARUS. Mac-Leay.

1 Sacer. Linné. Gall. merid.* Crenatus. de Geer. id.

Var. A. Inermis. Muls, id.

- B.Edentulus. MIul.id.

- C. Pius, Illiger. Austria.

- D. Punctulatus.

Mulsant. Gall, merid.

- E. Subsulcatus. Mac-Leay. Anglia.

2 Striatus. Dej. Cat. Hispania.

3 (Puncticollis. Dej. Cat.

Latreille. id. ${ }^{*}$

(Armeniacus. Ménétr. Armenia.

4 Semipunctatus. Fabr. Gall. merid.*

Variolosus. Olivier. id.

Var. A. Substriatus.

- B. Subinermis.

Mulsant. id.

Mulsant. id.

5 Variolosus. Fabr. Dalmatia. *

6 Cicatricosus. Dej. Cat.

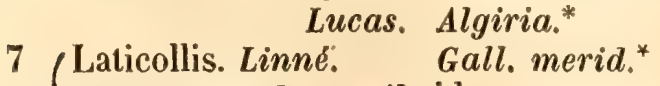

Hottentota. Dumeril. id.

Serratus. Fourcroy. id.

Var. A. Lovicollis.

Mulsant, Gall, orient.

\section{GYMNOPLEURUS, Illiger.}

Scarabeus. Pallas. Ateuchus, Fabr. Copris. Fourcroy. Actinophorus. Geoff. Duftsch.

1 /Flagellatus. Fabr. Gall. merid. ${ }^{*}$ Coriarius. Herbst. Austria.

Var, A. Clypeolatus.

Mulsant. Gall. merid.

- B. Rugulosus. Mul.id.

- C. Suturalis. (Chevrolat.) Mulsant.id.

- D. Asperatus.

$$
\text { (Stev.) Mulsant. id. }
$$

- E.Confusus. Muls, id.

2 Serratus. Fischer. Russ. merid.*

3 Mopsus. Pallas. id.*

Pillularius. Fab. Muls. Gallia.

Geoffroyæ. Sultzer. Germania.

Sinuatus. Fourcroy. $\quad \boldsymbol{P}$.

Var. A. Tuberculatus.

Mulsant. Gallia.

- B.Lavifrons.Mul. id.

- C. Laviusculus. Mulsant, id.

- D. Dorsalis. Muls, id.

- E. Indistirctus. Mulsant, id.

- F. Glabriusculus. Mulsant. id.

- G.Bidentatus. Mls, id.

4 (Sturmii. Mac-Leay. Tergest.

Pilularius. Stm. Cat. id.

Cantharus. Duftschm, id.

5 Cantharus. Erichson. id.

\section{SISYPHUS. Latreille.}

Scarabeus, Ateuchus. Fabr. Scarabaus. Linné. Copris. Oliv. Geoff. AcTINOPHORUS. Duftsch.

1

Schæfferi. Linné. Gallia. Longipes. Scopoli.

Schrank. Carniolia.

Arachnoides. Fourcr. $P$.

Var. A. Boschnoki.

- B. Subemarginatus. Mulsant. id.

- C. Subinermis. Mulsant. id. 
COPRIS. Geoffroy. Fabr.

Scarabeus. Linné.

1 (Paniscus, Fabr.

Hispanus. Scuckow. var, $\beta$. Russ, merid.

o Var. A. Sinuatus.

Mulsant Gall, merid.

B. Hispanus.

Linné. Hispania.

Hispanicus, Poiret. id.

ơ Var. C. Retusus. Mls. Gall, merid.

- - D. Tridens, Mls. id.

- - E. Lavicollis. Mulsant. id.

Lunaris, Linné. Gallia.*

Quadridentatus. de Geer, id.

Emarginatus. Olivier, id.

Lunus. Schrank.

Belisama. Schrank. id.

\& Var. A. Obliteratus.

Mulsant. id.

B. Cornicula-

tus. Muls, id.

우 - C.Deletus. Mul. id.

ơ - D. Castaneus. Mulsant. id.

\section{BUBAS. Mulsant.}

Scarabeus, Linné. Onitis, Fabr. Castelnau.

1 , Bison. Linné.

ơ Var. A. Brevicornis. Mulsant. id.

- - B. Dentifrons. Mulsant. id.*

ㅇ - C. Lineiformis. Mulsant. id.

ơ - D. Castaneus. Mulsant. id.

Bubalus. Olivier. id.*

ơ Var. A. Integricornis. Muls, id.*

8

1 Olivieri. Illiger. Sphinx. Olivier. of Var. A. Planifrons. Mulsant. id.

ONITIS, Fabricius.

Scarabeus. Olivier.

- - B. Inermicrus.

$\left\{\begin{array}{c}\text { Mulsant. id. } \\ \text { - C. Subtubercu- } \\ \text { latus, Muls. id. } \\ \text { - D. Subcostalis. } \\ \text { Mulsant. id. }\end{array}\right.$

- E. Fuscus. Mul. id.

2 Furcifer. Rossi.

Italia. *

3 Inuus. Fabr.

Algiria. ${ }^{*}$

4 Chevrolatii. Lucas, id.

5 Numida.de Castelnau, id.

6 Strigatus, Erichson, id. *

7 Irroratus. Rossi. Italia. *

Var. Clinias. Fabr. Hungaria.

- Lophus. Fabr. Algiria.

- Amynthas, de Cas-

telnau. Russ, merid.

- Melybous. Muls. Gall, merid.

- Tityrus. Mulsant. id.

- Alexis, Mulsant, id.

Ion. Olivier. ' id. *

Vandelli. Fabr. id.

ơ Var. A. Granulatus.

\&o - Bulsant. id. Mulsant. id.

9 Damon. Dej. Cat. Hispania.*

10 Mœris, Pallas. Russ, merid.

Mopsus. Sturm. Cat. id.

11 Damætas, Stéver. id.*

12 Pamphilus. Dej. Cat. id.

13 Schreibersii. Dahl.Cat, id.

ONTHOPHAGUS, Latreille.

Scarabeus, linné. Copris, Ateuchus. $\mathrm{Fabr}$.

1 Lucidus. Fabr. Hungaria. ${ }^{*}$

2 Austriacus, Panzer. Austria.* 
ơ ${ }^{\prime}$ Nuchicornis. Linné. Gallia. ${ }^{*}$

o Amyntas, Olivier. id.

- Juvencus. Scriba. Carniolia.

ㅇ Hybneri. Fabr. Gall. merid.

ơ Alces. Fabr.

id.

ㅇ Gibbosus. Scriba. Carniolia.

ণ Vittulus. Scriba. id.

- Var. A. Difformis.

Mulsant. Gall. merid.

† - B.Dubius. Mul.id.* latus. Muls. id.

- D. Sycophanta. Mulsant. id.

- E. Umbrinus. Mulsant. id.

- Subviolaceus.

Ménétriẻs. Caucasus.

4 Crocatus. (Chevrolat.) Algiria *

$4^{\text {bis }}$ Trachymenus, Kolen. Caucasus.*

5 Lemur. Fabr. Gallia.

ơuadrituberculatus.

Laichart. Tirolis.

o10-Punctatus.Schal.Germania.

ơ Tar, A. Curvicine-

tus. Muls. Gallia.

- B. Lineolatus.

Mulsant. id.

C. Mutabilis.

Mulsant. id.

- D. Grandicol-

lis. Muls. id.

- - E.Egenus.Mul.id.

6 Camelus. Fabr. Austria.

Vitulus. Olivier. id.

Var. Bicuspis, Stéven. Russ, merid.

7

Maki. Illiger. Gall. merid.

Var. A.Strigatus, Muls.id.

- B. Variabilis.

8 Analis. Lucas.

Mulsant, id.

9 Hirtus, Illiger.

Algiria.

Lusitania.

10 Leucostigma. Pallas, Russ, merid.

11 Pallidipennis. Sturm.

Cat. id.

12 Cruciatus, Ménétriés, id.

13 Tricornis, Fischer, id.

14 Maurus, Lucas. Algiria.*

15 Fiscicornis. Stéven. Gracia. ${ }^{*}$

16 (Curvicornis. Olivier. Orient.

Circumscriptus, Dej.

Cat. id.

Var. Caspicus, Ménét. Russ, merid.
Planicornis. Linné. Austria.

$?$ Acornis. Fourcroy. $\boldsymbol{P}$.

or Var, A. Xiphias.

Fabr. Gallia.

B. Trituberculatus. Schrk. Germania.

- C.Indistinctus.

Mulsant. Gallia.

D. Dillwynii.

Stephens. Anglia.

E. Immacula-i

tus. Muls. Gallia.

F. Vulneratus.

Mulsant. id.

G. Rubripes.

Mulsant. id.

18 Fracticornis, Preyssl, id.*

Nuchicornis, Olivier, id.

ㅇ Herbstii. Brahm. Germania.

Assimilis. Hoppe. id.

Xiphias? Panzer. id.

ot Var. A. Subrecticornis. Muls. Gallia.

- B. Sublaminatus. Muls. id.

C.Similis.Serb, Carniolia.

오 - E. Nasutus: Mulsant. Gallia.

- - F.Pauperatus. Mulsant. id.

- - G. Marginatus. Mulsant. id.

Nutans. Fabr. Gall. bor.*

Verticornis. Laichart. Tirolis.

8 Var. A. Distinguen-

dus. Muls. Gallia.

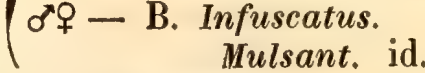

20 Viridis, Ménétriés. Russ, merid.

21 / $0^{7}$ Cœnobita. Herbst. Gall. orient. ${ }^{*}$

Tenuicornis. Preyss-

ler, Helvetia.

Fulgens. Brahm. Germania.

Nuchicornis. Fuessly. id.

ơ Var. A. Tricuspis.

Mulsant. Gallia.

- B.Cuspidiuscu-

lus. Muls, id.

으- C. Subprominulus. Muls, id.

22 Dorcas. Schmidt.

Helfer. Smyrna. 
23 / 9 Vacca, Linné.

Gall, merid.*

ơ Nuchicornis. Oliv, id.

Conspurcatus, Fourc, $\boldsymbol{P}$.

o Var. A. Affinis.Stm. Gall. merid.*

B.Vicinus. Mul.id.

C. Difficilis.

Mulsant. id.

D. Medius. $\mathrm{Ku}$ gelann. id.*

E.Intermedius. Mulsant. id.

F.Propinquus. Mulsant. id.

ơ

- - H.Sublineatus.

Mulsant. Gallia.

Taurus. Linné.

ơ Illyricus. Scopoli. Illyria.

ㅇ Rugosus. Scopoli. Carniolia.

- Quadrum? Kugln. Germania.

Corniger. Fourcroy. $P$.

Cruoreus? Schrank. Austria.

o Var. A. Bos. Villa. Lombardia.

- B. Borillus. Mulsant, Gallia.

Capra. Fab. Oliv. Lat. $P$.

ot Var. C. Recticornis.

Leske. Germania.

- D. Capreolus. Mulsant. Gallia.

- - E. Feminus. Mulsant. id.

ㅇ - F.Mendax. Mls.id.

ơ - G.Nigrovirens. Mulsant. id.

- - H.Fuscipennis. Mulsant. id.

- - I. Rufipes. Mul.id.

- K.Piliger.Mul.id.

25 /Schreberi. Linné. id.*

Homorrhoidalis.Four. $P$.

$\sigma^{T}$ Var. A. Bidentatus.

Mulsant. Gallia.

- - B. Mixtus. Mul. id.

ㅇ - C.Indistinctus.

ơo - D. Obscurus. Mulsant. id.

- E. Bimaculatus. Mulsant. id.

- - F.Rubripes.ML.id.

_ - G.Juvenilis.Ml.id.
26 Anthracinus, Dej, Cat, Russ, merid.

27 (ơ Semicornis, Panz, Gall. merid.* ơ Var, A. Angulicornis. Muls. id.

† - B. Descipiens. Mulsant. id.

28 Quadrituberculatus. Chevrolat, Algiria.*

29 ơo Furcatus, Fabr. Gall. merid, ${ }^{*}$ ơ Vitulus, Laichart. Tirolis.

- Var. A. Bicornutus. Mulsant. Gall, orient.

- B. Bidentatus. Mulsant. id.

- C. Laminiger. Mulsant. id.

- - D.Degener.Mls.id.

ợ-E. Rubellus.Ml.id.

30 Punctulatus. Illiger. Algiria.

31 Picitarsis. Sturm. Cat. Dalmatia.

32 Nigellus. Illiger. Lusitania.*

33 Ovatus. Linné. Gallia.*

$\left\{\sigma^{\tau}\right.$ Var. A. Fucatus.

Mulsant. id.

34 Emarginatus. Mulsant. Gall, merid.

35 Orcas. Helfer. Constantinp.

36 Mundus. Helfer. id.

ONITICELLUS. Lepelletier de SaintFarg, et Serv.

Scarabeus, Ateuchus, Copris. Fabr.

1 Festivus, Stéven, Russia merid.

2 Concinnus. Géné. Sardinia.*

Pallipes. Mulsant. Gall. merid.

Var. A. Subdeletus.

Mulsant, id.

3 Flavipes. Fabr. Gallia. *

Thoracocircularis, Laichart, Tirolis.

Fulvus. Fourcroy. $\quad P$.

Var. A. Subcornutus.

Mulsant. Gall, orient.

- B. Fulvicollis.Mulsant, Gallia.

- C.Maculatus.Mulsant. id.

- D. Fulvipterus.

Mulsant, id. 
Divisio 4. Aphodida.

\section{COLOBOPTERUS. Mulsant.}

Scarabeus. Linné. Aphodius, Illig.

1 (Erraticus. Linne.. Gall. merid.* Var. A. Submaculatus. Mulsant. Gall, orient.

- B. Nebulosus. Mls. id.

- C.Fumigatus, Mls. id.

\section{COPROMORPHUS, Mulsant.}

\section{Scarabeus. Linné. Aphodius. Illig.}

1 Scrutator. Herbst. Gall. orient.* Brevicornis, Panzer. Germania. Rubidus, Olivier. Gall. merid.

Var.A. Submaculatus. Mulsant. Gall. orient.

- B. Nigricollis. Mls, id.

- C. Brunnipes. Mls. id.

\section{EUPLEURUS. Mulsant.}

Scarabeus, Linné. Aphodrus, Illig.

1 Subterraneus. Linné. Gallia.* Var. A. Fuscipennis. Mulsant. id.

\section{OTOPHORUS. Mulsant.}

Scarabeus. Linné. Aphodius. Linné.

1 Hæmorrhoidalis. Lin. Gall, merid.* Alpinus. Scopoli. Granarius. Fabr. Carniolia.

Var. A. SanguinolenGermania.

tus. Herbst. Gall. orient.*

- B. Humeralis. Mls. id.

Bimaculatus. Kugln. Germania.

Var. C. Rubidus, Muls. Gallia.

2 Scolytoides. Lucas. Algiria.

\section{TEUCHESTES. Mulsant.}

Scarabeus. Linné. Aphodius. Illig.

\footnotetext{
1 Fossor. Linné.

Var.A. Brunneus.Muls, id:

Gall. orient.*

- B.Sylvaticus. Mls, id.
}

APHODIUS. Illiger.

Scarabeus. Linné, Fabr. CGopris. Oliv.

1 Scybalarius. Fabr. Gallia.* Conflagratus. Herbst. Germania.

Conspurcatus. Müller. Dania. Coprinus, Marsham. Anglia.

Var. A. Nigricans,Mls, Gallia. - B. Argilicolor. Mls, id.

Fimetarius. Linné. Suecia. Fatidus. Herbst. Germania.

Var. C. Pallipes. Muls, Gallia.

2 Napolitanus. Spence. Italia.

3 (Conjugatus, Panzer. Gall. orient.*

Fasciatus. Fabr. Germania.

(Var. A. Fasciatus. Mul. Gall, orient.

4 Sulcatus, Fabr. Germania.

5 Fotens. Fabr. Gall. orient.*

Var. A. Nigricollis. Mls, id.

- B. Vaccinarius.

Herbst. Germania.

Fimetarius. Schrank. id.

Var. C. Sanguinipennis. Mulsant. Gall, bor.

- D. Fuscipes. Muls. id.

Scrutator. Marsham. Anglia.

6 Cribricollis. Lucas. Algiria.*

7 Fimetarius. Lin. Oliv. Gallia.*

Pedellus. de Geer. id.

Bicolor. Fourcroy. $\boldsymbol{P}$.

Var. A. Bicolor. Muls, Gallia.

- B. Maculipes. Mls, id.

- C. Punctulatus.

Müller, Dania.

- D. Subluteus. Mls, Gallia.

- E.Hypogyalis.Mls.id.

- F. Imperfectus.

Mulsant, id.

8 J Lapponum. Schönherr. Lapponia.*

$\{$ Var. Rhenonum. Zett. id.

9 Rubens.Dej.Cat.Muls.Alp. Gall.*

Var. A. Carthusianus. Mulsant, id.

- B. Rupicola. Muls. id.

10 Ornatus. Findeli. Hungaria.

11 Montanus. Sturm. Cat. Austria.

12 Orophilus, Charpent. Russ. merid.

13 Alpicola. Mulsant. Alp. Gall.*

Yar. A. Orobius. Muls. id.

14 Vernus. Mulsant. id.*

Var. A. Martialis.Muls. id.

15 Mauritanicus. (Gaubil.) Algiria.* 
16 Dilatalus. Schmidt. Germania. 30 Parallelus. Rey.inlitt.

17 Schmidtii. Heer.

18 Fotidus. Fabr.

Putridus. Herbst.

Var. Sus. Kugelann. Germania.

19 Serotinus. (Creutzer.)

Sturm. id.

Minutus. Herbst. Austria.

Zenkeri.Germar. Germania.

21 Biguttatus. Germar. Austria.

22 Hypocophus. Jan. St.

$$
\text { Cat. Italia. }
$$

Ater. de Geer. Muls. Gall. orient.* Terrestris, Fabr. Germania.

Obscurus. Marsham. Anglia. Ater. Illiger.

Var. A. Terrenus. (Kir-

by.) Steph. Gall. orient.

Pusillus. Marsham. Anglia.

Affinis. Lucas. Algiria.

25 Granarius, Linné. Oliv, Gallia.*

Hamorrhoidalis, de

$$
\text { Geer. id. }
$$

Niger. Creutzer. Austria.

Inquinatus. Illiger, id.

Carbonarius, Sturm. Germania.

$\sigma^{x}$ Var. A. Parcepunctatus. Muls. Gallia.

우 - B. Cribratus. Mulsant. id.

- C. Mastus.

Schmidt. Germania.

- D. Concolor.

Mulsant. Gallia.

- E. Rugosulus.

$$
\text { Mulsant. id. }
$$

Cylindricus. Dej.

Hispania.

(Bimaculatus. Fabr. Gallia.*

Terrestris. Illg. var. B. Austria.

Varians. Duft. var. $\beta$. id.

Var. A. Ambiguus, Mls, Gallia.

- B, Punctatellus.

Mulsant. id.

jPlagiatus. Linné. - Germania.*

$\{$ Var. A. Niger. Hliger. Gall, bor.

Quadrimaculatus, Lin. Gallia.*

Quadripustulatus.Fbr.Germania.

Sanguinolentus. Pnz. id.

Var. A. Sanguinolens.

Mulsant. Gallia.

- B. Caudatus. Mls. id.

- C. Prolongatus.

Mulsant. id.

\section{Mulsant.}

Gall. merid.

Dichrous. Schmidt.

32 Griseus. Schmidt.

33 Gilvus. Schmidt.

34 (Tristis, Panzer. Germania.

id.

id.

Var. A. Vicinus, Muls. Alp, Gall.

- B.Pellucidus. Mls. Gall. merid.

- C.Scapularis.Mls.id.

- D. Fallax. Muls. id.

- E. Mirandus. Mls. id.

35 Exilis. Schmidt. Germania.

36 Cœnosus. Panzer. id.

37 Exiguus. Mulsant. Alp. Gall.

38 (Pusillus. Herbst. Schh. Gall. orient.* Granarius. Fabr. Illig. Germania.

Granum. Gyllenhal. Suecia.

Yar.A.Cœcus, Muls, Gall, orient.

- B. Rufulus. Muls. id.

Cœnosus? Panzer. Germania.

Var. C. Macularis.Mls. Gall. bor.

39

40

41

42

43

44 Piceus. Gyllenhal. id.*

Inquinatus. var. Fab. id.

45 Brunnipennis.Dej.Cat.Gracia.

46 Borealis. Gyllenhal. Lapponia.

47 Hydrochæris. Fabr. Gall. merid.*

Var. A. Coloratus,Mls, id.

- B. Dissimilis. Mls. id.

- C. Germanus.Mls, id.

48 Sordidus. Fabr. Oliv.

Var. A. Limbatellus. Illig. Gall, orient.*

Mulsant. id.

- B. Bipunctatellus. Mulsant. id.

- C. Quádripunctatus. Uddm. id.

Conspurcatus.de Geer, id.

Var.D. Aurantiacus.

Mulsant, id.

- E. Rufus. Molli. Austria.

Rufescens. Schmidt. id.

Var. F. Rufescens, Fab, Gall, orient.

- G. Hypocyphthus.

Mulsant. id.

- H.Arcuatus,Molli, id.

Fotens. Olivier. id.

Var, I. Melanotus, Mls, id. 
49

Var. A. Indecorus. Mls, id.

- B. Emarginalis.

50 Immundus. Creutzer. Gallia.*

Var.A.Melinopleurus.

Mulsant. id.

- B.Fulvicollis.Mls. id.

51 Cognatus. Dej. Cat. Algiria.*

52 Nitidulus. Fabr. Gallia.*

Ictericus? Molli. Germania.

Merdarius. Panzer. id.

Castaneus? Marsham. id.

53 Suturalis. Lucas. Algiria.*

54 Merdarius. Fabr. Gallia.*

Quisquilius, Schrank, Austria.

Var. A.Atricollis.Muls, Gallia.

$\{$ - B.Ictericus. Laich.Tirolis.

Foriorum. Panzer. Germania.

Gelbinus. Schrank. id.

YVar, C.Melinopus. Mls. Gallia.

55 Ferrugineus, Mulsant. Gall. merid.

56 Rufus, Fabr. Germania.

Vinaceus. Sturm, Cat. id.

Ferrugineus. (Dahl.) id.

57 Lividus. Olivier. Gallia.*

Vespertinus, Panzer. Germania. .

Biliteratus. Marsham. Anglia.

Anachoreta. Sturm. Germania.

Var. A. Limicola.Pnz. id.

- B. Anachoreta.

Fabr. id.

58 Circumcinctus. Schm. id.

\{imbatus. (Ziegler.) id.

59 (Lineolatus. Illiger. Gall. merid.*

Var. A. Fuscicollis.

Mulsant, id.

$\{$ - B. Deletus, Muls, id.

- C. Conjunctus.

Mulsant. id.

- D. Vittatus. Muls, id.

60 /Melanostictus. (Schüp.)

Schmidt. id.
Conspurcatus. Fabr. Germania.
Var. A. Egenus. Muls. Gallia.
- B. 6-maculatus.
Mulsant. id.
- C. 7-maculatus.
Mulsant. id.
- D. Catenatus.Mls. id.
- E. Subannulatus.
Mulsant. id.
Hirtipes, Fischer. Russ, merid.

Distinctus, Merbst.

Gallia.*

Dania.

Vaginosus. Fuessly. Germania.

Conspurcatus. Schrk. id.

Tessulatus? Laichart. Tirolis.

Attaminatus. Marsh. Anglia.

Var. A. B. Fumosus.

Pauper.Mls. Gallia.

- C.D.Baseolus. Hemicyclus. Muls, id.

- E. F. Scutellaris. Cunatus. Mls, id.

- G. H. Ophthalmicus, Auctus, Mls, id.

- I. K. Subcinctus. Interruptus.Mls, id.

Fœdatus, Marsham. Anglia.

Var. L. Interruptus.

Mulsant. Gallia.

- M. Centrolineatus.

Panzer. Germania.

- N. Anxius. Muls. Gallia.

63 Pictus. Sturm. Gall. orient.*

Inquinatus, Creutzer. Austria.

Yar. A. Flavidus. Mls, Gallia.

- B. Brunalis. Muls. id.

- C.Indigena.Muls. id.

64. Tessulatus. Creutzer, Gall.or,bor.*

Irquinatus. Olivier. id.

Var. A. Irregularis.

Mulsant. id.

- B. Connexus. Mls, id.

- C. Amplificatus.

Muls, id.

- D. Appendicula-

tus. Muls, id.

- E. Dilatatus. Mls, id.

Contaminatus. Panz. Germania.

Var. F. Scutellatus.

Muls. Gall, or. bor.

- G.Intricatus. Mls. id.

- H. Umbrosus. Mls. id.

Maculatus.Stm.Schm.Germania.

65 Sticticus. Panzer. id,*

Nemoralis. Panzer. $\boldsymbol{P}$.

Prodromus, Fabr.? Germania.

Var. A. Clypeolatus.

Mulsant. Gall. or. bor.

- B. Pallescens. Mls. id.

- C.Striolatus. Mls.id.

- D.Prolongatus. Ml.id.

- E. Ocellatus. Mls. id.

- F. Confusus, Mls, id. 
66 Nigrolineatus. Rosenh. Sardinia.

67 Consputus. Creutzer. Gall,or.bor.* Prodromus, Duftsch. Austria.

Var. A.Mendicus.Muls, Gall. or. bor.

- B. Metallescens.

Mulsant. id.

C. Impunctatus.

Mulsant. id.

68 Quadrignttatus. Hbst. Gallia.* Quadrimaculatus.

Fabr. Germania.

Quadripustulatus.

Duftschmid. Austria

- B. Cruciatus. Mls. id.

$69\left\{\begin{array}{l}\text { Sericatus.Ziegl.inlitt. } \\ \text { Schmidt. Gall. merid.* }\end{array}\right.$

Var. A. Immaturus.

Mulsant. id.

70 Thermicola. Sturm. Germania Var. A. Meridionalis. Mulsant. Gall. merid."

71 Lutarius. Fabr.

Immundus, Fabr.

Putridus? Brahm. Germania.

Anachoreta. Panzer. id.

Turpis. Marsham. Anglia.

Var. A. Homorrhoideus, Muls. Gallia.

- B. Ruficrus. Muls. id.

Villosus. Gyllenhal. Germania.

Hirtipennis. Lucas. Algiria. ${ }^{*}$

Dalmatinus. (Parreyss.)

Schmidt. Dalmatia.

76

Lunulatus, Ferrero. Italia.

Punctatissimus. Dej.

Cat. Gracia.*

78

Sphacelatus. Gyllenh. Russia.

79 Esuricus. Helfer. Sicilia.

80 Castaneus. Illiger. Hispania. ${ }^{*}$

81 Unicolor. Lucas. Algiria.

\section{ACROSSUS. Mulsant.}

Scarabeus. Linné, Aphodus. 1llig.

Bipunctatus. Fabr. Carniolia. ${ }^{*}$ (Discus. (Jurin.) Schm. Pyrencis. ${ }^{*}$ Var. Cyclocephalus.

Mulsant. Alp. Yedem.

3 Carpetanus, Gräells. Hispania.*
4 Rutipes. Linné. Gallia.* oblongus. Schrank. Germania. Var.A. Oblongus. Scpl. Carniolia. Capitatus. de Geer. Gallia. Var. B. Juvenilis. Muls. jd.

5 Luridus, Fabr. id. ${ }^{\star}$ Rufipes. Illiger. Austria. Nigripes. Schönherr. Suecia. Var. A. Nigro-sulcatus.

Lividus. Walck. $\boldsymbol{P}$. Marsh. Anglia.

Var. B.Interpunctatus. Herbst. Austria.

- C. Informis, Muls. Gallia.

- D. Intricatus. Mls. id.

- E. Connexus. Mls, id.

- F. Variegatus.

Varius. Gemel. Herbst. Austria.

Var. G. Apicalis, Muls, Gallia.

- H. Lateralis. Mls. id.

- I. Gagatinus. Fourcroy. $P$.

Gagates. Müller. Dania.

Arator. Herbst. Austria.

Var.J. Bipaginatus. Mulsant, Gallia.

- K. Rufitarsis, Lat. P.

6 Depressus. Kugelann. Gall.or.bor.* Nigripes. Duftschmid. Austria.

7 (Pecari. Fabr. Gall. $m^{\mathrm{d}}$. or. ${ }^{*}$ Satellitius. Herbst. Germania. Affinis.Brahm. id. Decipiens? Schrank. id. Var. A. Planus. (Dahl.) Schmidt. id.

8 Menetriesii. (Dej.) Russ, merid.

\section{MELINOPTERUS. Mulsant.}

Scarabeus. Linné, Aphodius. Illig.

1 Obliteratus. (Heyden.) $\left\{\begin{array}{c}\text { Panzer. Germania.* } \\ \text { Insubidus. Germar. id. } \\ \text { Var. A. Fulveolus. Mls. Gallia or. }\end{array}\right.$

2 Contaminatus. Herbst, Gallia.* Conspurcatus. Oliv. id.

Var. A. Incoloratus. Mulsant, id.

- B. Miser. Mulsant. id.

- C. Indistinctus. Mulsant. id. 
3 Prodromus. Brahm. Gallia. Contaminatus.Herbst.Austria. Consputus. Duftsch. id. Sphacelatus. Zetterst. Suecia. Conspurcatus, Laich. Tirolis. Var.A. Restrictus. Mls. Gallia.

- B. Marginalis.Stephens. Anglia.

- C. Flavogriseus. Mulsant. id. Fimetarius, de Geer. id. Var. D. Griseolus. Mls. id.

- E. Angustatus. Mulsant. id.

- F.Seminulus. Mls, id.

- G. Obliquus. Muls. id. - H. Extensus. Mls. id.

Punctatosulcatus. St. Germania.

4 Pubescens. Sturm. id. ${ }^{*}$

5 Ciliaris. Marsham. Anglia. Affinis. Panzer.

Germania.

\section{TRICHONOTUS. Mulsant.}

Aphodius. Illiger.

HEPTAULACUS. Mulsant.

Aphodius. Illiger.

1 Sus. Herbst.

Pubescens, Olivier. P.

Quisquilius? Schrank. Germania.

2 Nivalis. Mulsant.

\{Sus? var. Gyllenhal. Suecia.

3 Testudinarius, Fabr. Gallia.*

\section{AMMOECIUS. Mulsant.}

Scarabedes, Fabr. Aphod, lllig.

1 Elevatus. Fabr. Var. A. Edentulus. Mls, id. - B. Fusciventris. Mulsant. id.
PLAGIOGONUS. Mulsant.

Scarabeus, Oliv. Aphod. Illig.

1 Arenarius. Olivier. Gallia.* Pusillus. Preyssler. Germania. Rhododactytus. Mars. Anglia. Var. A. Sabuleti. Muls. Gallia.

OXYOMUS. Eschsch, in litt, de Casteln.

1 Porcatus, Fabr. Gallia.* Sylvestris. Scopol. Carniolia.

Fenestralis. Schrank. Germania.

Foveolalus. Molli. id.

Var. A. Foveolatus.

Molli. id.

PLATYTOMUS. Mulsant.

Oxyomus, Dej. Cat.

1 Sabulosus. Dej. Cat. Mulsant. Gall. merid.*

\section{PLEUROPHORUS. Mulsant.}

Scarabesus. Panz. Oxyomus. Dej. Cat.

$1\left\{\begin{array}{l}\text { Cœsus. Panzer. Gallia.* } \\ \text { Var. Elongatus. Muls. id. }\end{array}\right.$

\section{RHYSSEMUS. Mulsant.}

Ptinus, linné. Scarabeus. Fabr. AphoDIUS. Illig.

1 Germanus. Linné. Gallia.* Asper. Fabr, Mulsant. id. Var. Rufpes. Mulsant. id.

2 Verrucosus. Mulsant. Gall. merid.*

3 Godartii. Mulsant. id.

4 Algiricus, Lucas. Algiria.*

\section{DIASTICTUS. Mulsant.}

Scarabeus. Fab. Aphodius. Illig. PsamModius, St. Cat. Redtenb. Oxyomus. Dej. Cat.

1 Vulneratus, Stm. Gyll. L. Redt. Germania.* Sabuleti. Mulsant. Gall. bor, or. Semipunctatus.Bonel. Pedemont. Var, Latitans, Muls, Gall, or, bor. 
PSAMMODIUS, Gyllerhal.

Scarabedus. Payk. Aphodius. 1llig.

1 Sulcicollis. Illig. Asper. Paykull. Var. Canaliculatus.

Mulsant. Gall, orient.

2 Porcicollis. Illiger. Gall. merid.* $\{$ Var. Rugosulus. Mols. id.

DIvisıo 5. Trogida. Heer.

\section{EGIALIA. Latreille.}

Scarabeus. Linné. Aphodius. Illig.

1 Arenaria. $\mathrm{Fabr}$. Gall. merid.* Globosa. Kugelann. Austria.

(Var. Globosa.Mulsant. Gall, orient.

TROX. Fabricius.

Scarabeus. Linné.

1 Morticini. Pallas. Russ. merid.*

2 Cadaverinus. Illiger. Austria. ${ }^{*}$

3 Granulatus. Fabr. Hispania.*

4 Perlatus. Scriba. Gall. orient.*

$\{$ Subterraneus. Fourc. $P$.

Sabulosus. Olivier. Gall.merid.

5 Clathratus. Dej. Cat. Corsica.

6 Hispidus. Laichart. Gall. $m^{\mathrm{d}}$ or."

Luridus. Rossi. I alia.

Niger. Rossi. id.

Arenarius. Paykull. Suecia.

Arenosus. Gyllenhal. id.

7 Græcus. (Dej.)

8 Sabulosus. Linné. Hispidus. Olivier.

9 Scaber, Linné. Arenosus. Gmel. Arenarius. Fabr.

Barbosus. Laichart. Tirolis.

Hispidus, Paykull. Suecia.

10 Setosus. Sturm. Cat. Hungaria.

11 Cribratus. Géné. Sardinia.

HYBOSORUS. Mac-Leay.

Scarabeus. Linné, Geotrupes. Fabr.

1 Arator, Fabr.

Gall, merid. ${ }^{\star}$
GEOBIUS, Brullé.

Copris, Fabr, Egialia, Latr, Dej, Cat.

1 Dorcas. Fabr. Gall. merid.* Barbarus. de Casteln. Algiria. Cornifrons. Guérin. id.

2 Cornifrons. Brullé. id.*

3 Tricornis. Lucas. id.

OCHODOEUS. (Megerle.) Latreille.

Dej. Cat. Melolontha. Fabr.

1 Chrysomelinus. Fabr. Gall.orient.

Y Var. Scymnoides. Muls.id.

DIvisio 6. Dynastida. Mac-Leay.

Xylophil. Latr.

\section{ORYCTES. Illiger.}

Scarabeus. Linné. Geotrupes, Fabr.

1 Grypus. Illiger. Gall. merid.*

Nasicornis. Laichart. Tirolis.

$\sigma^{7}$ Var. A. Simus. Mls. Gall. merid.

( - B. Nasutus, Mls. id.

2 Nasicornis. Linné. $\quad P_{0}{ }^{*}$

Var. A. Aries. Fablsk. Germania.

Corniculatus. Villa. Lombardia.

Nasicornis. Heer.

var. $\beta$. Helvetia.

Var. B. Tuberculatus.

Mulsant. Gallia.

\section{PHILLOGNATHUS, Eschscholtz.}

Mulsant. Scarabeus. Linné. Geotrupes. Fabr, ORYCTES. Latr.

\section{1}

Silenus. Fabr. Gall. merid.*

Excavatus. Forster, id.

Var. A. Curvicornis. Mulsant. id.

우 - B. Gibbicornis. Mulsant. id.

o? - C. Bacchus.

Mulsant. id. 
PENTODON. (Kirby.) Hope.

ANOXIA. Laporte, de Castelnau.

Scarabeus, Fabr. Geotrupes. Fabr. St, Mulsant. Erich, Catalasis, Dej. Cat. Heer. cat.

1 Monodon. Fabr.

Idiota. Herbst.

Gall. merid.*

Punctatus, Latreille. Gall, merid.

Var. A. Brunneus. Mls. id.

2 Punctatus. de Villers. id.*

Algerinus. Herbst. Algiria.

Monodon.Duftschmid. id.

Var. A. Castaneus. Mls. Gall. merid.

CALICNEMIS. de Castelnau.

Muls. Colorhinus. Erich. Pachypus. Dej. Cat.

1 Latreillei. de Casteln. Gall. merid.* \{Truncatifrons.Dej.Ct.Algiria.

\section{Divisio 7. Melolonthida. Heer.}

\section{PACHYPUS, Latreille.}

Geotrupes. Fabr. Scarabeus. Petagna. Melolontha, Oliv. Coelodera, Géné.

1 (Impressus. Erichson. Algiria.* Excavatus.Guér.Géné.Corsica. Candida? Petagna. Sicilia.

2 Cornutus, Oliv, Erich. Corsica. Excavatus, Feistham. id.

3 Excavatus. Fabr. Sicilia. Candida. Petagna. id.

4 Coesus. Erichson. Sardinia.* \{ Candidoe. Mulsant. Gall.merid.

\section{POLYPHYLLA. Harris. Erich.}

Melolontha, Fabr. Oliv. Scarabeus. Linné.

1 Fullo. Fabr. $\left\{\begin{array}{l}\text { Var.? Boryi. Brulle. Gall. orient.* } \\ \text { - ? Hololeuca. Pall. Russ. merid. } \\ \text { - A. Luctuosa. Muls. Gallia. } \\ \text { - B. Marmorata. }\end{array}\right.$ Mulsant, id,

\section{Scarabeus. Pallas.}

1 Testaceus. Pallas. Russ, merid. Anketeri. Herbst. id.

2 Orientalis. Laporte. Austria.

3 Australis. Schönh. Mls. Tirolis."

Var, a. Occidentalis. Fabr, Casteln. Lusitania.

- b. Matudinalis.

Lap. Muls. Gall. merid.*

- A, Occitania. Mls. id.

4 Scutellaris. (Chevrol.)

$\{$ Mulsant. Pyr. orient.*

Lanuginosa. (de Je-

5 (Villosa. Fabr. nisson.) id.

Pilosa. Heer.Muls. Helvetia.

6 Pilosa. Fabr. Panz. Austria.*

$\{$ Villosa. var. Herbst. id.

7 Thoracica, Krynicki.

Fisch. Russ, merid.

\section{MELOLONTHA. Fabricius.}

\section{Scarabeus. Linné.}

1 (Vulgaris, Linné. Fabr. Gallia.* Majalis. Molli. Germania.

Var. A. Lugubris, Muls, Gallia.

- B. Discicollis. Mls. id.

- C. Ruficollis. Mls. id. ${ }^{*}$

2 Extorris. Erichson. Russ. merid.

3 Albida,Dej.Cat. Castl. Gall. merid. * Pectoralis. Germar. Austria.

Rhenana. Bach. id.

Var. Pulverea, Muls, Gall. merid.

4 Hippocastani. Fabr. Gallia. $^{*}$

Vulgaris, var. Oliv. id.

Var. Nigripes. Comol. Lombardia.

- Pectoralis. (Meg.) Austria.

- Tibialis. Mulsant. Gallia.

- Coronata. Muls. id.

5 Aceris. (Ziegl.) Erich. Austria.*

6 Candicans. Friwaldski. Turcia.

7 Papposa. Illiger. Lusitania.

8 Hybrida. Charpentier. Russ. merid.

9 Præambula, Kolenati. id.

10 Hesperica. Rambur.

Dej. Cat. Hisp. Merid. 
11 Fucata. Hoffmansegg. Hisp. merid.

12 Mauritanica, Lucas. Algiria.*

DASYSTERNA. Dej. Cat. Rambur.

1 Barbara. Dej. Cat. Rambur. Barbaria. *

2 Reichii. Rambur. Gracia.

\section{ARTIA. Rambur.}

1 Cartaginensis. Ramb, Barbaria.

\section{ELAPHOCERA. Géné.}

Melolontha, Illig. Leptopus, Dej. Cat.

1 Bedeaui. (Dufour,) Er, Hisp, merid.

2 Mauritanica. Ramb. Algiria.*

3 Malaceensis. Rambur. Hisp. merid.

4 Numidica. Rambur. Algiria.

5 Longitarsis. Illiger. Lusitania.

6 Hiemalis. Erichson. Constantinp.

7 Obscura. Géné. Erich.

Dilatata, Erichson. id Ramb. Sardinia.

Granatensis. Rambur. Hisp, merid.

Barbara. Rambur. Algiria.

Denticornis. Dufour. Hisp.merid. Sardea, Rambur. Sardinia.

Hispalensis, Rambur. Hisp. merid.

Bysantica. Rambur. Turcia.

Churianensis. Ramb. Hisp.merid.

Carteiensis, Rambur. id.

Rubripennis. Lucas. Algiria.

\section{PHLEXIS, Erichson.}

1 Wagneri. Erichson. Algiria.

\section{RHIZOTROGUS, Latreille.}

Scarabedes. Linné. Melolontha, Fabr.

1 Porulosus. Fischer.

2 Pulvereus. Knoch.

Russ, merid.

Vulpinus. Schönherr. id.

Aquinoxialis, Fabr. Austria. ${ }^{*}$

Vernus. (Meg.) Germ. id.

Tauricus. Stéven.

Russ, merid.

7 Caucasicus. Gyllenhal.id.
8 (Estivus, Olivier.

Gallia.*

Inanis. Brahm. Germania.

우 Var. A. Incertus, Mls, Gallia.

- B. Subvittatus.

Mulsant. id.

- D. Lividigaster. Mulsant. id.

9 Thoracicus, Dej. Cat.

Mulsant. Gall. orient.*

Maculicollis. Heer. Helvetia.

Var. A. Collaris. Muls. Gall. merid.

- B.Vitticollis.(Perroud.) Muls. id.

- C. Pallidifrons. (Laferté.) Mls. id.

- D.Lineicollis. (Laferté.) Muls. id.

10 (Cicatricosus. Mulsant. id.*

$\{$ Meridionalis? Dej. Ct. id.

(Var, A. Rubidus. Muls. id.

11 Marginipes. (Chevrol.)

Mulsant. id.*

Var. A. Pallidus, Muls, id.

- B. Signatus. Muls. id.

12 Vicinus. Dej. Cat. Muls. id."

Insularicus. Cristof. Lombardia.

Lutescens.Sturm. Cat. Italia.

13 Fraxinicola. Hagenb.

St. Er. Tergest.

14 Torulosus, Friwaldski. Graecia.

15 Lusitanicus.Schönherr.Lusitania.

16 Dispar. Buquet. Gory.

(Geotrugus. Guérin.) Algiria.*

17 Magagnoscii. (Geotrugus.) Guérin. id.*

18 Angusticollis. (Chevrolat.) Nov, sp. id."

Gerardii, Buquet. id. ${ }^{*}$

20 Amphytus, Buquet. id.

21 Euphytus, Buquet. id.*

22 Tusculus. Buquet. id.*

23 Barbarus. Lucas. id.

24 Numidicus. Lucas. ... id.*

25 Obesus. Lucas. id.*

26 Truncatipennis. Lucas, id.

27 Serraticollis. Lucas. id.

28 Scutellaris, Lucas. id.*

29 Inflatus, Buquet. id. ${ }^{*}$

30 Gabalus, Buquet. id. $^{*}$

31 Hirticollis. Lucas. id.

32 Carduorum. Erich. id.

33 Tenebrioides. Pallas. Russ. merid.

34 Friwaldskii. Ménétriés. Constantinp. 
35 Volgensis. Fischer. Russ, merid.

36 Caninus. Eschscholtz. id.

37 Costulatus. Friwalds. Turcia.

38 Flavicans. Dej. Cat. Hispania.

39 Naculicollis. St. Cat. Russ, merid.

40 Brunneus. St. Cat. - id.

41 Villicollis. St. Cat. Hungaria.

42 Autumnalis. Dej. Cat. Illyria.

43 Nomaticus. Friwaldsk.

St. Cat. Hungaria.

44 Faldermanni. Dej.Cat, Russ, merid

45 Monticola. Rambur. Hisp. merid.

46 Subemarginatus. Dej.

Cat. id.

47 Fuliginosus. Dej. Cat. id.

48 Siculus. Dej. Cat. Sicilia.

49 Carbonarius. Dej. Cat. Oriente.

50 Hispanicus. Dej. Cat. Hispania.

51 Menetriesii. Dej. Cat. Russ. merid.

52 Fuscatus. Dej. Cat. Croatia.

53 Oblongiusculus. Dej.

Cat. Russ. merid.

54 Flavescens. (Dahl.) St.

55 Flaveolus. St. Cat. Italia.

56 Pallescens. St. Cat. Austria.

57 Etuensis. Jan. St. Ct. Sicilia.

58 Verticalis. Schmidt et

Helf. Smyrna.

59 Flaviventris, St, Cat. Turcia.

60 Obtusus. St. Cat. Sicilia.

\section{AMPHIMALLUS. Latreille.}

Melolontha, Fabr, Oliv.

(o Ater. Herbst. Gallia*

$\{$-Fusca.(Melolontha.)

$\left\{\begin{array}{l}\text { Oliv. } \text { P. } \\ \text { - Var. A. Fuscus. Mls. Gallia. }\end{array}\right.$

o Perplexus. Dej. Cat. id.*

2
Pini. Olivier.
Gall. merid.
Spini. de Castelnau. id.
Yar. A. Bicolor. Muls. id.
- B. Ustulatipennis. Mulsant. id.

3 \{ 9 Pygialis. Mulsant. Pyr. orient.* \{Fulvicornis? (Dej.Ct.) Hisp. merid.

4 Solstitialis. Lin. Fabr. Gallia. Autumnalis. Fourc. P.

Tropicus. Schh. Heer, Helvetia.
5 Ochraceus. Knch. Heer.

Fallenii. Gyll. Schh. id.

Var.Tropicus. Muls. id.

- A. Lateralis. Mls, Gall, orient.

- B. Fulvicollis. Mls. id.

- C. Suturalis. Mls. id.

- D. Aurantiacus.

Mulsant. id.

6 Ruficornis. Fab.Scrib. id.*

Marginatus. Hbst.Mls. Germania.

Paganus. Olivier. $P$.

Castaneus? Herbst. N.

7 Assimilis. Hbst. Knoch. Germania.*

Apritinus, Duftsch. Helvetia.

Fulvicollis. (Ullrich.) id,

Castaneus. Schönherr. Germania.

8 Rufescens. Latr. Heer. Gallia.*

Semirufus. Gyll. Schh. Suecia.

9 Pilicollis. Schönherr. Hungaria.

APLIDIA, Kirby. Hoppe.

Rhizotrogus. Heer. Melolontha. Fabr.

1 Transversa. Fab. Duft.

Heer. Helvetia.*

ANOMALA. Köppe.

Mulsant. Euchlora. Mac-Leay. Mulsant. Melolontha, Fabr.

$\left\{\begin{array}{c}\sigma^{\top} \text { Auricollis. (Dahl.) } \\ \text { Laporte. id. } \\ \text { Errans, var. 1-3. Illg. }\end{array}\right.$

Oliv. Hungaria.
Profuga. Erichson. Lusitania.

Errans. var. 5. Illig. id.

2 Junii. (Creutz.) Duft. Austria.*

Etrusca. Dej. Cat. Etruria.

Signaticollis. (Dahl.) id.

Frischii. Panzer. Gall. merid.

Var. A. Thoracica. Mls, id.

- B.Scutellaris.Mls. id.

- C. Doublieri, Mls. id.

3 Vitis. Fabr. Oliv. id."

4 Ausonia. Erichson. Sicilia.

5 Luculenta, Erichson. Kuss, merid.

6 (Vagans. Illiger. Hisp. merid.

Errans.var. 4. Ilg. Ol. Lusitania.

Confusa. Dej. Cat. Hisp. merid.

Signaticollis. Dej. Cat.id. 
7 Devota, Rossi, Muls. Gall, merid.

Var. A. Apicalis. Muls. id.

- B. Versicolor.

Mulsant. id.

8 Donovani. Stephens. Anglia.

9 Solida. Erichson. Austria.

10 Oblonga. Fabr. Tirolis.*

Dubia. Scopoli. Carniolia.

Yar. Janthina. Leske. Gall. merid.

(Julii. Duftschmid, Austria.

11 Frischii. Fabr.

Julii. Paykull. Sueciamerid.

Var. Frischii. Herbst. Austria.

- Julii. Fabr. Germania.

- Dubius. Herbst. id.

- Eneus. de Geer. id.

- Micans. Mulsant. Gall. merid.

- Viridi-cuprea.

Mulsant. id.

- Rubro-cuprea.

Mulsant. id.

ANISONGHUS, Dej, Cat.

1 Atriplicis. Fabr. Ictericus. Dej. Cat. id.

\section{PHYLLOPERTHA. Kirby. Mulsant.}

Erich. Melolontha, Latr. Anisoplia. Laporte. Dej. Cat.

1 Campestris, Latreille. Gall, merid." Succincta. Laporte. id. ơ Var.A. Maculata. Mulsant, id.

- B. Abbreviata. Mulsant. id.

C. Cruciata. Mulsant. id.

D. Paupercita. Mulsant. id.

E. Occidentalis. Muls. id.

F. Circumdata. Mulsant. id.

- G. Sabulosa. Mulsant. id.

- - H. Arenaria.de Castelnau. id.
2 Horticola. Linne. P.

Var.A. Cyanocephala.

- B. Ustulatipennis.

Mulsant, Gallia.

- C. Macularis. Mls. Gallia.

- D. Adiaphora.

Poda. id.

Viridicollis, de Geer. id.

Var. E. Perrisii. Muls, id.

ANISOPLIA. (Megerle.) Lepelletier et Serv.

Scarabeus. Linné. Melolontha, Fabr.

1 Lencaspis. Stéven.

2 (Fruticola. Fabr. б Campestris Hustria. P Segetum. Herbst. id.

3 Velutina. (Parreyss.)

4 Straminea. Brullé. Gracia.

5 Hypocrita, Dej. Cat, Sardinia.

6 Agricola. Fabr. Gall. merid."

Graminicola.var.C.D.

Latr. id.

+ Var. A. Obscura.Mls, Gallia.

- B. Curvipunctata. Muls. id.

- C. Subarcuata.

Mulsant. id.

D. Trimaculata.

Mulsant. id.

- E. Punctum.

Mulsant, id.

- F. Defectiva.

Mulsant, id.

- - G. Quadrata.

Mulsant. id.

o’ Fruticola. Walk.

- Var. H. Sycophanta.

$$
\text { Latr. id. }
$$

Mulsant: id.

- I. Unicolor.

Mulsant. id.

7 Monticola. Erichson. Italia.

8 Depressa. Erichson. Gall. merid. Agricola. Illiger. Lusitania. Fruticola. Illiger. id.

9 Bætica, Erichson. Hisp, merid. 
10 Arvicola? Oliv, Muls, Gall. merid.* Agricola? Linné. Mls, id. of Var. A. Disjuncta. Mulsant. id.

- 13. Bifasciata. Mulsant. id.

- C. Fallax. Mls. id.

- - D. Unifasciata.

$$
\begin{gathered}
\text { Mulsant. id. } \\
\text { - E. Bipunctata. } \\
\text { Mulsant. id. }
\end{gathered}
$$

- F. Funerea. Mulsant. id.

- - G. Laeta. Muls. id.

- - H. Apicalis.

Floricola, $\mathrm{Fabr}$. Mulsant. id.

f Bromicola, Germar. Tirolis merid. Senticola. (Dahl.) Tergest.

13 Villosa. Besser. Fald. Russ, merid.

14 Aprica. Erichson. Dalmatia.

15 Pallidipennis. (Trichius.) Gyll. id.

16 Campicola, Esch. Fald. Russ, merid.

17 Lanuginosa. Erichson. Smyrna.

18 Austriaca. Herbst. Austria.

Floricola. Panz, Duft. id. Agricola. Schrank. Hungaria.

19 Tempestiva. Erichson. Gall. merid.* Austriaca. Mulsant. id.

(o Var. Agricola.Steph. id.

20 Crucifera. Herbst. Germ. merid. $\{$ Agricola. Schk.Laich. Fisch. Tirolis.

Cyathiger. Scopoli. Carniolia.

21 Dispar. Dahl. Cat, Er. Hungaria.

22 Adiecta. Erichson. Pedemont.

23 Lata. Erichson. Austria. ${ }^{*}$

24 Zwickii. Fischer. Russ, merid.

25 Deserticola. Fischer. Tirolis merid. Depressicollis. Dej. Ct. Hungaria.

26 Rumeliaca. Friwalds. Turcia.

27 Lineolata.Dej.Ct.Fsch.Russ, merid.

28 Lineata. Latreille. Gracia.

29 Variabilis. Dahl. Cat. Sicilia.

30 Puncticollis. Dej. Cat. Oriente.

\section{SERICA. Mac-Leay.}

Scarabeus, Linné. Melolontha. Fabr. Omaloplia. (Megerle.) Dej. Cat.

1 (Brunnea. Linné. Fulva. de Geer. Fulvescens. Fourcroy. $P$.
OMALOPLIA. Stephens.

(Megerle.) Dej. Cat. Scarabet Geoff. Melolontha. Herbst. Serica, de Castelnau. Heer.

1 Holoscericea. Scopoli. Gallia." Sultzeri. Fuessly. Germania.

Pellucida. Sultz. id.

Chrysomeloïdes. Schk. id.

Lamellata.Fourcroy. $P$.

Berolinensis. Herbst. Austria.

Variabilis. Oliv. Fabr. $P$.

Var. A. Fuca. Mulsant. Gall. orient. - B.Pellucida.Sulz. Germania.

2 Mutata. Schönherr. Hispania.

3 Carbonaria. Dej. Cat. Dalmatia.

Sigra. (Dahl.) Hungaria.

4 Erythroptera. (Dahl.)

Dej. Cat. id.*

\section{BRACHYPHYLLA. Mulsant.}

Scarabaus. Linné. Melolontha, Fabr. Omaloplia. Steph. Serica. de Casteln.

1 Ruricola. Fabr.

Marginata. Fuessly. Fourcroy. $\boldsymbol{P}$.

Floricola. Laichart. Tirolis.

Nigro-marginata.

Herbst. Heer, Austria.

Var. A. Immarginata.

Mulsant. Gallia.

- B. Obscura. Muls, id.

- C. Humeralis.Fab.id.

- D. Disca.Mulsant. id.

- E. Atrata. Fourc. $P$.

2 Barbara. Lucas.

Algiria.*

TRIODONTA. Mulsant.

Omaloplia, Dej. Cat. Serica, de Casteln.

1 Aquila. Dej. Cat. de

Castelnau. Gall. merid.* Var. A. Noctus. Muls. id.

2. Alni. Géné.

Sardinia.

3 Levaillantiï.Gaubil. in

litt. Algiria.*

4 Nitidula, Rossi Tirolis.

Sericea. Bonelli. Lombardia.

Sericans. Schönherr. id. 
HYMENOPLIA, Eschscholtz. Muls.

Hymenontra. Eschsch. Dej. Cat. Omaloplia. St. Er. Serica. de Castelnau. Triodonta, Er. Melolontha. Fabr.

1 (Strigosa. Illiger. Austria.* Bifrons. Eschscholtz, Lusitania.

2 Chevrolatii. Mulsant. Gall. merid. Strigosa. de Casteln. id.

(Var,Lugdunensis. Mls, Gall, orient.

3 Rugulosa. Rambur. Hisp. merid.

4 Cinerea. Rambur. id.

5 Ochroptera. Erichson. Algiria.

6 Proboscidea. Fabr. Africa bor.

7 Unguicularis. Erichson. id.*

8 Morio. $\mathrm{Fabr}$. id.

9 Cinctipennis. Lucas, id.*

10 Aterrima. (Chevrolat.) Lucas, id.

11 Puberula. Erichson. Sicilia.

CHASMATOPTERUS, Lepell, et Serville. Melolontha. Illig.

1 Villosulus. Illiger.

2 Pilosulus. Illiger.

3 Hirtulus, Illiger.

4 Hispidulus. Graells. id.

\section{HOPLIA. Illiger.}

Decamera. Mulsant, Melolontha, Fabr. Scarabeus. Linné. Poda.

(Decamera. Mulsant.)

1 (Brunnipes. Bonel. Mls. Gall. merid.* Carinthiaca. (Dahl.) Carinthia.

Pusilla. (Ziegler.) Tirolis.

(Var.Pauperata.Muls. Gall. merid.

2 Philanthus. Sulz. Hbst. Gallia.

Argentea, Fabr. Oliv.

Panz.Duftsch. Germania.

Pulverulenta. Illiger.

Schh. Muls. Austria.

Var. Varians. Muls. Gallia.

3 Praticola. Duftschmid. Gallia bor.*

오? Palustris. Heer. Helvetia.

(Var, Ripicola. Muls. Gall. bor.

(Hoplia, lllig.)

4 Bilineata, Fabr.

Hisp, merid. ${ }^{*}$
כั Aulica. Linné.

Regia. Fabr. id.

Citrina. Buquet. id.

6 Sulphurea, (Chevrol.)

Lucas. id.*

7 Chlorophana. Erich. Hisp. merid. Aulica. Illiger. Lusitania.

8 Pubicollis. Dej. Cat. Géné. Corsica.

9 , Cœrulea. Drury. Hbst.

Muls. Gall. merid.

Farinosa. Fabr. id.

Formosa. Latreille. id.

Argentea. Fourcroy. id.

Squamosa. de Villers. id.

10 Farinosa. Linné. Dej.

Schk. Oliv. Gall, orient. ${ }^{*}$

Squamosa. Fabr. Pnz.

Payk. Ill. Schm. Germania.

Argentea. Poda. Scop.

Herb, Rossi. Muls. Gallia.

Rorida. (Ziegler.) Helvetia.

Var.A. Deflorata. Mls, Gall, orient.

- B. Rufolutea. Mls. id.

- C. Sedicolor. Muls. jd.

- D. Sublutea.Muls.id.

- E. Micans. Muls. id.

- F. Viridula. Muls. id.

- G. Glauca. Muls. id.

- H. Ambigua.Muls.id.

11 Kunzei. Schmidt. Turcia.

12 Caucasica. Kal. Melet. Russ, merid.

13 Flavipes, Dej. Cat.

14 Minuta. Panzer. Italia.*

Pulverulenta. Schm. id.

Lepidota. Illiger. Austria.

15 Pilicollis, Böb. in litt.

$\left\{\begin{array}{l}\text { Erich. Volhynia. } \\ \text { Pollinosa.Böb. in litt. id. }\end{array}\right.$

16 Dubia. Illiger, Rossi.

17

Schmidt. Italia.

7 (Pollinosa. (Ziegl. Dej.)

Erich. Germania.

Minuta. Illiger.

Schmidt, id.

18 Pulvifera. Andersch. Dalmatia.

\{ Pulvisera. Dej. Cat. id.

19 Graminicola. Fab. Pnz.

Duftsch. Germania.

Pulverulenta. Fabr.

Herbst. id.

Farinosa, Herbst, id. 

$\left\{\begin{array}{c}\text { Nuda, (Ziegler. Dej.) } \\ \text { Erich. Austria. } \\ \text { Graminicola.Schmidt. } \\ \text { var. d.e. id. }\end{array}\right.$ Pubera. Dupont. Dej.

Cat. Oriente.

\section{Divisio 8, Cetonida. Leach.}

\section{MelitophiLI, Latr.}

\section{VALGUS. Scriba.}

Scanabeus. Linné. Trichius. Fabr.

Hemipterus. Linné. Gallia.* $\{$ Variegatus, Scopoli. Carniolia. Squamulatus. Müller. Dania.

\section{TRICHIUS, Fabr.}

Scarabeus. Linné. Melolontha. Herbst.

1 /Fasciatus. Lin. Fabr. Gallia.* Succinctus. Latreille. $\boldsymbol{P}$. Var. A. Dubius. Muls. Gallia.

- B. Interruptus. Mulsant. id.

- C. Obliquus. Muls, id.

- D. Prolongatus. Mulsant. id.

- E. Divisus, Muls, id.

- F. Abbreviatus. Mulsant. id. Gallicus. Dej. Cat. Heer. Abdominalis. Dej. Cat. Heer. Schmd, id. Fasciatus. Latr. Oliv. id. Succinctus, de Castel, id. o Var. A. Dorsalis. Mulsant. id. ơo - B.Intermedius.

$$
\left\{\begin{array}{c}
\text { Mulsant. id. } \\
\text { - C. Bivittatus. } \\
\text { Mulsant. id. }
\end{array}\right.
$$
Mulsant. id.

- E. Dentatus. Mulsant. id.

- F.Abdominalis. Dej. Cat. Muls. id.

3 (Zonatus. Germ. Géné, Sardinia.* frasciolatus. Géné. Algiria.
OSMODERMA. Lepell, et Serville.

Scarabeus. Linné. Cetonia et Trichius. Fabr. Gymnodus. Kirby.

1 Eremita. Scopoli. Gallia.* Coriarius. de Geer. id. (Eremitica.Knch, Gyll. Germania.

GNORIMUS. Lepellet. et Serville.

Gory et Perch. Mulsant. Erich. Trichios. Fabr. Scarabeus. Linné.

1 Variabilis. Linné. Gallia.* Albopunctatus, Pill.

et Mitt, id.

Cordatus. Gmel. Suecia.

8-punctalus. Behst. Germania.

Cordatus. Fabr. id.

Var.A. 8-punctatus.

- B. Angularis. C. Nigricollis. Mls. id.

- D. Cordatus, Fab, id.

- E. F. Ambiguus, Juvencus. Mls, id.

2 Subcostatus. Ménétr.

Fald. Russ, merid.

3 Decempunctatus. Helfer et Schm. Sicilia.

4 Nobilis. Linné. Fabr. Gallia.*

Variabilis, Linné. Suecia.

Viridulus. de Geer. id.

Auratus. Rosel. Germania.

Cuspidatus, Fabr. id.

$\{$ Var. A. Cupricollis.

Mulsant. Gallia.

- B. Rubrocupreus. Mulsant. id.

- C. Immaculatus. Mulsant. id.

\section{CETONIA. Fabr.}

Scarabeus. Linné.

(Athiessa. Burmeister.)

1 Floralis. Fabr.

2 Barbara. Gory et Perch.id.*

Var. Doguereau. Gory et Perch. id.

- Aupickii. GoryetP. id.

- Floralis, Burm. id. 
3 Refulgens. Schaum. Italia, *

Squamosa.Mulsant. Gall.merid.

Var. Refulgens. Hbst. Italia.

- Tenebrionis. Gory

et Perch. id.

- Elongata. Gory et

Perch. Algiria.

- Deserticola.Waltl. Hispania.

- Funerea. Mulsant, Gall, merid.

- Lefebvrei. Muls. "id.

- Dolorosa. Muls. id.

4 Inhumata. Gory et $\boldsymbol{P}$. Oriente.

Ethiops. Burmeister. id.

Leucospila. Burmeist. id.

Mesopotamica, Burm. id.

\section{(Oxythyrea. Mulsant.)}

丂 (Cinctella. Burmeister. Russ, merid." Variegata. Gory et Percheron. id.

6 Stictica, Linné, Fabr. Gallia.* Funesta. Poda. Fabr. Tirolis. Albopunctata.de Geer. Germania. Yar. A. Deleta.Muls.'Gallia. Grunii. Donovan. Germania. Var. Pantherina. Gory et Perch. Barbaria.

7 Græca. Brullé. Burm. Gracia." Quadrata. Gory et P. Algiria.

8 Feralis. Erichson. id.*

\section{(EPicometis. Burmeister.)}

9 |Femorata. Illig. Burm. Hispania.* ( Hispanica. Gory et $P$. id.

10 Crinita. Charpentier. Gall. merid. * Reyi. Mulsant. id.

Hirtella.Heer. Helvetia.

Var. Pilosa. Brullé. Gracia.

- Hirta.var.Gory et Perch. id.

Vulpina. (Megerle.) Germania.

Var.Submaculata.Mls.Gallia.

- Luctuosa. Muls. id.

11 Hirtella. Linné. Muls. id.*

Hirta. Fabr, Laich. Tirolis.

Var. Squalida. Linné. Germania.

- Subfasciata.Mls. Gallia.

- Nigrina. Mulsant. id.

12 Tonsa. Burmeister. Gracia. ISenicula? Ménétriés. Russ. merid.
(Cetonia. Burmeister.)

13 Funesta. Ménétriés. Russ. merid.

14 Libani. Gory et Perch. Oriente.

15 Sardea. Géné. Sardinia.

Tenebrionis. Gory et Perch. id.

16 Tincta. Germ. Burm. Sicilia.

17 Oblonga. Gor.et Perch. Gall. merid." Var. Luctifera. Muls. id.

- Mauritanica. (Gau-

bil.) Algiria.

18 Vidua. Gory et Perch. Oriente.

Excavata. Falderm. id.

Melancholica. Zoubk. id.

19 Afflicta. Gory et Perch. id.*

Var. Leucogramma.

Gory et Perch. id.

Osmanlis. Gory et $\boldsymbol{P}$. id.

Lefebvrei. Dej. Cat. id.

20 Purpurea. Burmeister. Buchara.

21 Trojana, Gory et Perch. Algiria.*

Albilatera. Falderm. Oriente.

Var. Godetii. Gory et

Perch. id.

_. Circumdata. Fald, id.

22 Viridis, Fabr. Hungaria."

Hungarica. Herbst. id.

Viridana, Brullé. Gracia.

Var.? Sibirica. Gebler. Sibiria.

Atrocarulea. Waltl. id.

Armeniaca. Ménétriés, Armenia.

23

Adspersa. Waltl. "Turcia. *

Asiatica. Falderm. Asia.

Exclamationis. Burm. Turcia.

24

Aurata. Linné.

Gallia."

Smaragdula. de Geer. id.

Nobilis. Schrank. Germania.

Variabilis. Fuessly. id.

Var. Pallida. Drury. Gallia.

- Lucidula. Casteln.

Heer. Helvetia. ${ }^{\star}$

- Valesiaca. Heer. id.*

- Carthami. Gory et Perch. id.

- Funeraria.Gory et Perch. Algiria. ${ }^{*}$

- Asiatica. Gory et Perch. Asia.

- Praclara. Muls. Gallia.

- Piligera. $(Z g l$.$) Mls. id.*$

- Cuprifulgens. Mls. id.*

- Meridionalis. Mls. Gall, merid." 
25 Morio. Falr. Olivier. Gall, merid.* 33 Fuliginosa. Scopoli. Carniolia. Var, Quadripunctata. Muls. Gallia.

26 Metallica, Payk. Duft. Gallia.* Var.a. Floricola. Hbst. Germania.* - b. Obscura. Andch.

\section{Hopp. id.}

- Metallira. Gory et Perch. Gallia.

- c.Albiguttata.Andersch. Germania. Enea. Fieb. id. Obscura. Gory et Perch. Gallia. - d.Cuprea. (Ziegl.) id.

- Nigra. Duftsch. Austria.

- e. Metallica. Fieb. id. - $\alpha$. Volhynensis. Go$r y$ et $\boldsymbol{P}$. Volhynia.

- ß. Metallica. Illig, Lusitania.

- . Cuprea. (Dahl.) Corsica.

- S. Metallica. Fabr. Italia.

- Florentina. Herbst, id.*

- . Ignicollis. Gory et $P$. id.

Marmorata. Fab. Panz.

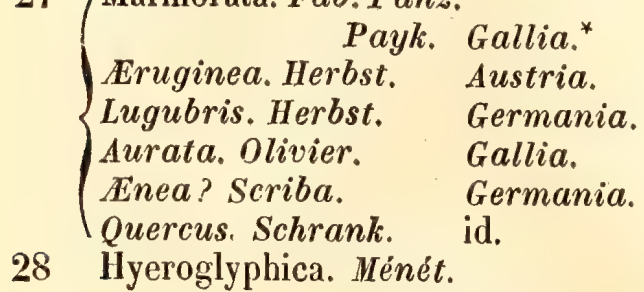

Fald, Russ. merid.

29 Excavata. Gory et $\boldsymbol{P}$. Grocia.

30 Opaca. Fabr. Gory et P. Burmeist. Hispania. * Cardui, Gyllenhal, in

Schh. Muls, Gall. merid. Morio. var. Illiger, Lusitania.

31 (Angustata, Germ. Gory.

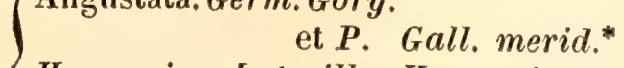
Hungarica. Latreille. Hungaria. Vicina.Schönherr. Gall. merid. Var. Nasuta. Germar. Italia.

32 Affinis. And. Pnz. Dft. Gall. merid.* Enea. Illiger. Austria. Quercus. Bonelli. Fastuosa. Dumeril. Var, Mirifica. Muls.
Lombardia. Gall. merid. id.
Speciosissima. Scopoli. Muls. Alsatia.* Superba. de Villers. Gall orient. Smaragdus, Brahm. Germania. Aurata. Olivier. Gall.merid. Fastuosa, Fabr, Panzer. Duftsch. Austria. Eruginosa. Drury. Italia. Nudiventris. Germar, id. Var.? Venusta. Ménét. Russ. merid. - Auro-cuprea. Mls, Gall, orient. Speciosa.Adams. Fald.Russ, merid. Psittacina. Ménétriés, Russ. merid. Var.? Jousselini. Gory et $\boldsymbol{P}$. id.

\section{PSILODEMA. Blanchard.}

Amphicoma, Latr. Melolontha, Fabr. Oliv.

1 Ciliata. Ménétriés. Constantinp. Mustela. Friwaldjski. in litt. Waltl. id.

$2 \int$ Melis, Fabr. $\{$ Var. Cyanipennis, Fab.id. Algiria.*

\section{AMPHICOMA '. Latreille.}

Scarabeus. Linné. Pallas, Melolontha. Fabr. Oliv. Eulasia. Truqui, in litt.

1 Vittata. Fabr. Strigata. Waltl. Constantin.* Cyanipennis. (Friw.) id. Lineata. de Castelnau. Oriente. Var.Strigata.Dej.Cat.id.

- Smyrnensis. de

Castelnau. Grocia.

- Flavicans. (Friw.) Turcia.

- Lineata. Olivier.

Dej. Cat, Gracia.

2 Goudotii. de Casteln. Hisp. merid. Saltzmanni. Sturm. id. Taniata. Dej. Cat. Barbaria.

3 (Lasserrei. (Parreyss.) Ahrens. Gracia.* Parreyssii, Brullé. id.

4 Bombylius, Fabr. Algiria.*

5 Aretos, Pallas. Russ, merid.

1 U.ne monographie de ce genre a été publićc par M. le docteur Truqui (Eugène), de Turin, dans ses Studii entomologii. 
6 Bombyliformis. Pallas. Russ. merid. Var. Ochraceipennis. Ménétriés. id.

- Patruelis. Stm. Ct. Gracia.

- Testacea. (Banon.) id.

- Rufipennis. deCastelnau. id.

7 Chrysopyga. (Stéven.) Falderm. Russ, merid.

8 Martes.(Friw.) Truqui. Turcia.

9 Maki. Truqui. id.

10 (Bicolor. (Friw.) Waltl. id. Distincta. Burmeist. id.

11 Vulpes. Fabr. Russ. merid.*

우 Hirta. Fabr. id. Alopecia. Pallas. id. 오 Distincta, Falderm. id.

Var.Vulpecula.(Friw.) Turcia.

- Purpuricollis. (Friwaldjski.) Waltl. id. Chrysonota. Brullé. Gracia.

Var. Bilotrichius. (Parreys.) Waltl. id.

Anemonina. Brullé. id.

Distincta ơ. Falderm. Russ, merid.

Var.Psilotrichius.Fld.id.*

Scutellata. Brullé. Gracia.

Var. Hirsuta. Brullé. id.

- Humeralis, Brullé. id.

-_ Apicalis. Brullé. id.

\section{ANTHIPNA. Eschscholtz.}

Dej. Cat. Melolontha. Fabr. Oliv. AniSOPLIA. Sturm.

1 Abdominalis. Fabr. Italia. Alpina. Olivier. id.

2 Romana. Duponchel. id. Erea.Ziegler. id.

\section{GLAPHYRUS. Latreille.}

Melolontha, Fabr, Oliv. Scarabeus. Linné.

Serratulæ, Latreille, Algiria.*

2 Viridicollis. Lucas, id.

3 j Maurus. Linné.

Cardui. Fabr. id.

4 Equestris. Dej. Cat. Oriente.

5 Globulicollis. Ménétr. Constantinp.
6 Festivus, Ménétriés. 7 Varians, Ménétriès. id.

Constantinp.

\section{FAH, BUPRESTI.}

JULODIS. Eschscholtz. Solier.

Buprestis. Fabr.

1 Pubescens. Olivier. Gracia.

2 (Pilosa. Fabr, Barbaria.

Pilosula, Herbst. id.

3 Onopordi. Linné. Hisp. merid.

Onopordinis. Fabr. Algiria.

Var. Algirica. de La--

porte et Gory. id.

- Albopilosa.Chevr. id.

- Mauritanica. Dej.

4 Fidelissima. Hoffman-

Cat. id.*

Onopordii. Olivier. id.

5 Olivieri, de Laporte et

Gory. Grocia.

6 Brullei. de Lap, et Gor. id. Onopordinis, Brullé. id.

7 Setifensis. Lucas. Algiria. ${ }^{*}$ \{ Cyanitarsis? Dej. Cat. id.

8 Ivenii. Bartels. Gracia.*

9 Manipularis. Fabr. Barbaria.

10 Kœnigii. Dej. Cat.

Mannerh. id.

11 Tingitana. Audouin. id.

12 Barbara. Gory et de.

Laporte. id.

ACMAODERA. Eschsch. Solier.

Buprestis. Fabr.

$1\left\{\begin{array}{l}\text { Treniata. Fabr. Austria.* } \\ \text { Flavofasciata. Pill. et }\end{array}\right.$

Miterp. id.

Hirta. de Villers. Gall. merid.

2 Quadrifasciata, Rossi. Italia.*

Var, Mutabilis.Spin. id.

3 Octodecimguttata.Hbt. Hispania.

4 Multipunctata. Lucas. Algiria.*

$\fallingdotseq$ Pulchra. Fabr. Hisp. Alg.*

6 Ottomana, Spinola. Constantinp.

7 Saxicola. Spinola. Romelia. 
8 Octodecimpunctata.

Gory et de Lap. Sardinia.

9 Feisthameli, Gory et de Lap. Pedemont.

10 Sexpustulata. Gory et de Laporte. Italia. *

11 Flavopunctata. Lucas. Algiria.

12 Flavonotata, Lucas. id.

13 Bipunctata. Oliv. Hbst. \{ap. et Gory. Gall. merid. * Var. Vaillantii, Spin. Algiria.

14 Adspersula. Illig. Gory et de Laporte. Gall. merid.* $\{$ Dermestoides, Solier, id. Variegata, Dej. Cat. id. Pedemontana.Dej.Ct.Pedemont.

15 Rubromaculata. Lucas, Algiria.

16 Affinis. Lucas, id. *

17 Discoidea, Fabr, Lap. et Gory. Sicilia.*

18 Lanuginosa. Schönh. Hispania.

19 Flavolineata, Lap, et G. Gracia.

20 Pilosellæ. Bonelli. Pedemont.*

21 Rufomarginata, Lucas. Algiria.

22 Flavovittata. Lucas. id.*

23 Barbara, Gory et Lap. id.

24 Trifoveolata. Lucas, id.

25 Coarctata: Lucas. id.

26 Cylindrica. Fabr. Hispania.* $\{$ Var.Hirsutula. (Dahl.) Sicilia.

27 Boryi. Brullé. Grasia.

28 Cuprifera, Lap, et Gor. id.

29 Melanosoma. Lucas, Algiria. ${ }^{*}$

30 Tristis. Lucas, id. ${ }^{*}$

31 Mauritanica. Lucas, id.*

32 Cyanipennis. Lucas. id.

33 Acuminipennis: Lap, et

34. Hirsutula. Dej. Cat.

$$
\text { Gory. id.* }
$$

Gory et Lap. Hispania.

35 Morio. Gory et Lap. Barbaria.

36 Dorsalis, Dej. Cat. Lap.

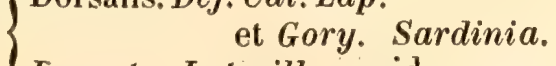

Dorsata. Latreille. id.

37 Lugens. Lap. et Gory. Constantinp.

38 Flavoguttata.Stm. Cat.Romelia.

PTOSIMA. Solier.

Serv. Dej. Cat. Gistl. Acmaodera. Lap.

1 (Novemmaculata. Fabr. Gall, merid. $\{$ Var. Sexmaculata. de Villers, id.

AURIGENA. Gory et Laporte.

Perotis. Megerle, Dej. Cat. Spinola. Buprestis, Eschsch. Laporte.

1 Lugubris. Fabr. Italia.* \{ Laportea. Brullé. Grocia.

2 Tarsata. Fabr. Algiria. * Unicolor, Olivier. id.

CAPNODIS. Eschscholtz.

Dej. Cat. Spinola. Latipalpis, Solier. Buprestis, Laporte. Argante. Gistl.

1 Cariosa. Fab

Bruteia. Petagna. id

Tenebrionis. Cyrille. id

2 Porosa. Klug. Constantin.*

3 Tenebrionis. Linné. Gall. merid.* Tenebrioides. Pallas. Russ. merid. Navia. Linné. id.

Tenebrion. Latreille, Gall. merid.

4 Erea. (Dahl.) Gory et

Lap. Russ.merid.

5 (Tenebricosa. Fabr. Gall. merid.* Tenebrionis. Rossi. Italia.

CYPHOSOMA. Mannerheim.

Crphonota. Dej. Cat. Coeculus. Gory et Lap.

1 Lauvsoniæ. Chevrolat. Algiria.*

\{ Var. Gravidus. Gory el

Lap, Ëgypt.

Buquetii. Gory et Lap. Algiria.

BUPRESTIS, Linné. Solier. Laporte.

(Galcophora. Serville. Sol.)

1 Mariana. Linné. $\quad$ Gall. merid.* Hiulca. Pallas. Russ. merid.

Massiliensis. deVillers. Gall, merid.

Var. Florentina. (Dhl.) Algiria.*

2 Fabricii. Rossi. Italia.*

(Dicerca. Eschsch. Solier.)

3 Pisana. Rossi. Plana. Olivier.

Italia.*

4 Mosta. Fabr. Austria.

5 Quadrilineata, Herbst. id. 
6 Enea. Linné, Lacord, Gall, merid. Reticulata. Fabr. Austria.

Subrugosa. Paykull. Suecia.

Austriaca. Schrank. Austria.

Oxyptera. Pallas. Russ. merid.

Cuprea. Rossi. Italia.

Gigantea. Scopoli. Carniolia.

Var. Carniolica, Fabr, id.

7 Berolinensis. Fabr, Gall, merid.* Mariana. de Geer. id.

Calcarata. Fabr. Gallia.

Fritillum. Ménétriés. Russ, merid.

8 Fagi. (Megerle.) Lap.

et Gory. Austria.*

9 Acuminata. Fab. Pall. Suecia.*

10 Scabrosa, Escher. Zoll. Russ. merid.

(Lampra, Megerle, Dej. Cat.)

11 Plebeja. Fabr.

Rustica. Herbst.

Gall. merid.*

Variolosa. Paykull. Suecia.

Tenebrionis. Panzer. Germania.

Conspersa. Gyllenhal. Suecia.

12 Rutilans. Fabr, Lac. Gallia.*

Rustica. Schrank. Austria.

Fastuosa. Jacquin. Gallia.

Aruginosa. Herbst. Austria.

13 Solieri. Lap. et Gory. Barbaria.

14 (Festiva. Linné. Gall. mérid.

Decempunctata.Fabr. id.

(Ancylocheira. Dej. Cat.)

15 Cupressi.Dej.Cat,Lap.

16 Rustica. Linné. et Gory. Dalmatia.

Violacea. de Geer. Gall. merid.*

Latus. (Elater.) Sultz. Germania.

Rusticus. (Cucujus.)

Fourcroy. $P$.

Rustica. (Mordella.)

Scopoli. Carniolia.

17 Punctata. Fabr.

Gall. merid. ${ }^{*}$

Rustica. var, Gyllenh. Sueciamerid.

Homorrhoidalis, Hbt: Austria.

Quadristigma.Herbst.id.

Var. Bicolor. Fabr. Germania.

Barbara. Olivier. Algiria.

Barbarica. Linné. id.

18 Hilaris. Klug.

19

(Octoguttata. Linné. Gall. merid.* Albopunctata. de Geer,id.

20 Mauritanica. Lucas. Algiria.*
21 Flav̌omaculata, Fabr. Gall, merid.* Octoguttata. Olivier. id.

Octomaculata.Pallas, Russ, merid.

Maculosa. Linné. Gallia.

Flavopunctata. de

Geer, Germania.

Novemmaculata. Lin. Sueciamerid.

Tetrastichon. Linné. id.

Douei. Lucas. Algiria. ${ }^{\star}$

Levaillantii. Lucas. id.

(Eurythyrea. Serville. Lej. Cat.)

24 (Micans. Fabr.

Marginata, Olivier, id.

Aurulenta. Rossi. Italia.

25 Austriaca, Linné. Lac, Gall. bor.*

Quercus. Herbst. Austria.

Aurulenta. Rossi. Italia.

Aurata. Pallas. Russ. merid.

Inaurata. Linné. Algiria.

Var. Carniolica. Hbst. Carniolia.

Marginata. Herbst. Austria.

\section{CHRYSOBOTHRIS, Eschscholtz.}

Solier. Laporte et Gory. Ambuss. Gistl.

1 Affinis, Fabr. Gallia.*

Congener. Paykull. Suecia.

Chrysostigma. Herbst. Austria.

2 Solieri. Lap. et Gory. Gall. merid.

Consentanea, Dej,Cat. id.

Pini. Klingelhöffer. Austria.

3 f Chrysostigma. Fabr. Gallia.*

\{Chrysostigmata. Latr, id.

\section{AGRILUS, Curtis.}

Buprestis. Linné. Fabr.

1 Guerinii. Boisd. et Lac. P.*

2 Chrysopterus, Chevrolat. in litt. Alsatia.

3 Sexguttatus, Herbst, Gall. merid. Biguttatus. Rossi. Italia.

4 Biguttatus, Fabr. Gallia.*

\{ Panonica, Pill. et Mit. Germania.

5 Faldermanni. Lap. et Gory. Petropolis.

6 Sinuatus. Olivier. Gallia.*

7 Distinguendus. Lap. et Gory. $P$.

8 Aubei, Lap, et Gory. id, 
9 Viridipennis. Lap. et Gory. id.

10 Auripennis. Solier. \{ Coryli. (Dahl.)

11 Cinctus. Olivier.

12 Viridis. Linné. Elongatus. Herbst.

Linearis. Schrank. Rosana. Scopoli.

Solieri. Lap. et Gory. Gall. merid.* Derasofasciatus.Boisd. et Lacord. Gallia.*

Graminis, Panzer. Illyria.

Cyaneus. Olivier. Gallia.*

Cyanescens? Ratzeb. Germania.

Viridis, var, Schönh. Suecia.

Amabilis. Gory et Lap. Saxonia.

Olivaceus. Gyllenhal. Suecia,

Linearis, Paykull. id.

Angustatus. Illig. Ménétriès. Gallia.

Laticornis, Illiger. Gall. merid.*

$\{$ Roberti. Chevrolat. Alsatia. ${ }^{*}$

Linearis. Fabr.

Germania.

\section{CORREBUS. de Laporte et Gory.}

Buprestis. Fabr.

1 Rubi. Linné, Lacord, Gall. merid." Y Nebulosus, Scopoli. Carniolia.

2 Undatus. Fabr. Lac. Gallia.* Pruni. Panzer. Germania. Quadrifasciatus. Ross, Gall. merid.

3 Bifasciatus, Olivier. id.*

4 Amethystinus. Olivier.

5 (Episcopalis, Dej. Cat. Solier. id.* $\left\{\begin{array}{r}\text { Mannerh. Dalgurans, Parreyss. id. } \\ \text { Fuld }\end{array}\right.$

6 Purpurens. Gory et

Lap. Hungaria.

7 Elatus. Fabr. Gall. merid.*

8 Metallicus. Solier. id.

9 Crlindraceus, Solier. id.

10 Eneicollis, de Villers. id.*

11 Granulatus, Lap. et Gory. Barbaria.*

12 Fulgidicollis, Lucas. Algiria.*

MELANOPHILA, Eschscholtz.

Mannerheim. Phenops. Meg. Dej. Cat. Gistl. Apatura. Lap, et Gory.

1 Decotisgma. Fabr. Austria.* Chrysostigma. Fabr. id.

Silphoides. Schrank. id.

Quatuordecimguttata.

Olivier. Gall. merid.

2 Appendiculata. Fabr. Suecia.* Morio. Paykull. id. Acuminata. de Geer. Corsica.

3 Tarda, Fabr. Gall. merid. Cyarea. Fabr. Austria.

Clypeata. Paykull. Sueciamerid.

4 Equalis. Mannerh. Algiria.*

\section{ANTHAXIA, Eschscholtz,}

Solier, Dej. Cat. Buprestrs, Linné. Fabr.

1 Auricolor. Herbst. Austria.* Aurulenta. Fabr. id.

Deaurata, Linné. id.

Senicula. Schrank. id.

Parmensis, Cristofori, Italia. 
Manca. Fabr. Gallia*

Elegantula. Schrank. Germania.

Bistriata. Fabr. Dania.

Rubinus. (Cucujus.) Fourcroy.

3 Candens. Fabr.

$P$.

Fulminans. Schrank.

Fulminatrix. Herbst. id.

4 Passerinii, Pecchioli. Italia.

5 Salicis. Fabr. Gallia.*

6 ( Bicolor. Faldermann. Russ. merid.

\{ Nitidula, var.?. Schk, id.

7 Salicetti. Illiger. Sardinia. *

8 Viminalis. Ziegl. Lap. et Gory. Italia.*

9 Parallela. Lap. et Gory. Gall. merid.

10 Crœsa. de Villers. id.

11 Nitens, Fabr. jd.*

Nitida. Rossi. Lacord. $P$.

Lucidula. Fabr. Germania.

Foveolata. Herbst. Austria.

Nitidula, Paykull. Suecia.

Fulgurans. Schrank. Austria.

Fulgens, Herbst. id.

Formosa. Towns. id.

Bipunctata. Olivier. id.

12 Dorsalis. Stéven. Russ. merid.

13 Brevis. Gory et Lap. Hispania.

14 Azurescens. (Dahl.) Lap. et Gory. Hungaria.

15 Gramica. Lap, et Gory, Dalmatia.

16 Gichorii. Olivier. Gall. merid.*

Millefolii. Fabr. id.

Umbellatorum. var.

Schar. iu.

17 Inculta. Germar. id.*

Chamomilla. (Dahl.) Austria.

18 Chlorocephala, Lucas, Algiria.

19 Læta。Fabr. Austria.

Nitidula.Herbst. id.

20 Scutellaris. Lucas. Algiria.

21 Fulgidipennis, Lucas. id.*

22 Hypomelæna. Illiger. Gall. merid.*

23 Signaticollis, Krynicki, Russ, merid. ${ }^{*}$

24 Nitidicollis, Dej. Cat. Lap, et Gory. Hispania.

25 Nitidula. Linné. Gall. merid.*

26 Discicollis. Friwaldks. Iap. et Gory. Romelia.

27 f Ferulæ, Géné. Sardinia.*

Vittaticollis. Lucas. Algiria.

28 Cyanescens. Dej. Cat.

Lap, et Gory, Hispania.*
29 Umbellatorum. Fabr, $\boldsymbol{P}_{\text {. }}^{*}$

30 Rugicollis. Lucas. Algiria.

31 Luctuosa. Lucas. id.

32 Quadripunctata. Linné. Gall. merid.*

33 Godeti. Lap, et Gory. Russ. merid.

34 Morio. Fabr. Dalmatia.

Acuminata, de Geer. id.

35 Chevrieri. Ullrich.Lap.

et Gory. Helvetia.

36 Istriana. Rosenhauer. Istria.

37 Pecchioli. Gory et Lap. Sardinia.

38 Bannatica, Dej. Cat. Lap. et Gory. Hungaria.

39 Funerula. Illiger. Hispania.

40 Confusa. Dej.Cat. Lap. et Gory. Gall, merid.*

41 Sepulchiralis. Fabr. id.*

42 Pygmæa. Brullé. Gracia.

43 Spinolæ. Gory et Lap. Italia.

44 Cyanipennis. Dej. Cat. Lap. et Gory. Dalmatia.*

\section{SPHENOPTERA. Dej. Cat. Solier.}

Laporte et Gory. Chinsodera. Gistl. BuPRESTIS. Fabr.

1 Glabrata. Ménétriés. Arnachantce. Godet.

Russ. merid. Tauria.

2 Orichalcea. Pallas. Russ. merid. \{ Dejeanii. Zoubkoff. id.

3 Coracina. Stéven. id.

4 Antiqua. Illiger. Sicilia.

5 Hypocrita. Rambur. Hisp, merid.

6 Iridiventris, Lap, et Gory. Gall, merid.

7 Inæqualis. Stéven. Russ, merid.

8 Dianthi. Tauscher. id.

9 Dilaticollis. Lap, et

Gory. Algiria.*

10 Bassii. Lap. et Gory. Sicilia.

11 Rauca. Fabr.

IBanonii. Dej. Cat. id.

12 Similis. Lap, et Gory, id.

13 Vittaticollis, Lucas. id.*

14. Gemellata. Dej. Cat. Lap, et Gory. Gall. merid.*

15 Cardui, Chevrolat. id.*

16 Conica. Gory et Lap. Italia.

17 Ardua, Dufour. Lap.

et Gory. Gall, merid.

18 Lapidaria. Brullé. Gracia.*

19 Cupriventris, Lap et G. Algiria. 
20 Smyrnensis. Gory et

21 Lineata. Fabr. Lineola. Herbst. Geminata. Illiger.

22 Metallica. Fabr.

23 Parvula. Lap. et Gory. id.

24 Rotundicollis. Gory et Lap. Algiria.

25 Bravaisii. Gory et Lap. id.

26 Chrysostoma. Gory et

27 Celtiberica. Gory et

$$
\text { Lap. Grocia. }
$$

Lap. Lusitania.

28 Scabra, Gory et Lap. Algiria.

\section{CRATOMERUS. Solier.}

Mannerh. Buprestis, Linné, Fabr. MorDELLA. Scopoli.

1 'Cyanicornis, Fabr. Gall. merid.* Femoratus. Villers. id.

우 Trochylus, Fabr. id.

Elegantulus, Schrank, Austria.

Stephanelli. Petagna. Italia.

Hungarica. Lin. Scop. Hungaria.

Diadema. Fischer. Russ, merid.

TRACHYS. Fabricius.

Solier, Dej. Cat, Laporte. Gistl. BuPRESTIS. Fabr.

1 Imperialis. Villa.

2 Reflexa. Géné.

3 Pygmæa. Fabr. Austriaca. (Megerle.) Austria.

Viridis. Samouel. id.

4 Pumila. Illiger. id.*

Minuta. Rossi. Italia.

Corrusca. Drapiez. id.

5 Intermedia. Rambur. Hispania.

6 Nana. Fabr. Gallia.*

7 Enea. Mannerheim. Gall. merid.*

8 Troglodytes. Schrank. Austria.

Triangularis. Boisduv. et Lacord. $P$.

\section{APHANISTICHUS, Latreille.}

Solier. Lacord, Casteln. BuPRESTIS, Fab. Germar.

1 Emarginatus, Fabr, Gallia.*
2 Augustatus. Lucas, Algiria.

3 Pusillus. Olivier. Gall. merit.*

4 Pygmæus, Lucas. Algiria.

5 Elongatus. Villa. Lombardia.

6 Lamotei, Guérin. Gallia.

\section{FAII. ELATERES.}

\section{MELASIS. Olivier.}

Lacordaire, Castelnau. Erater. Linné.

1 Buprestoides. Linné. Gallia.* Flabellicornis. Fabr. Germania.

(Elateroides,var.Illig. Austria.

\section{CEROPHYTUM. Latreille.}

Lacordaire. Castelnau. Guérin. Redtenb.

1 Elateroides, Latreille. $P$.

PHYLLOCERUS, Latreille.

$$
\text { Dej. Cat. }
$$

1 Flavipennis. Latreille.

2 Spinolæ. Guérin. id. Dej. Cat. Dalmatia.

DRAPETES. (Megerle.) Dej. Cat.

Eschscholtz. L. Redtenbacher. ELater. Fabr. Panz.

1 Equestris. Fabr. Austria.* Cinctus. Panzer. Germania.

\section{EUCNEMIS, Ahrens.}

Mannerh. Schönherr. Lacord. Redtenb.

1 Capucinus. Ahrens. P.*

2 Rugulosus. Dej. Cat. Lombardia.

Capucinus. Villa. id.

3 Feisthameli. Gräels. Hispania.

4 Pygmæus, Schönherr, P.* 
XYLOBIUS. Latreille. L. Redtenbacher.

Xylophilus. Mannerh. Xyloecus. Serv. Ctenonychus. Stephens. Elater, Fabr. Dej. Cat. Elater. Fabr.

1 Alni. Fabr. P. Testaceus. Herbst. Germania.

MICRORHAGUS. Eschscholtz.

Chevrier. L. Redtenbacher. Eucnemis. Marnerh. Elater, Paykull.

1 Pygmæus. Paykull. Austria.* 2 Sahlbergi. Mannerh. Finlandia. 3 Lepidus. Rosenhauer. Tirolis.

NEMATODES, Latreille, Dej. Cat. Eucnemis. Mannerh. Elater. Fabr.

1 S Procerulus. Mannerh. Suecia.

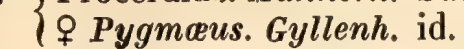

2 Elaterinus. Villa. Lombardia.

3 Flavescens. Dej. Cat. Styria.

4 Nigriceps. Mannerh. Finlandia.

\section{HYLOCHARES. Latreille.}

Hrppocalus, Eschsch.

1 Cruentatus. Mannerh. Finlandia.

HYPOCELUS. Eschscholtz. Dej. Cat.

1 Buprestoides. Rossi. Italia. Alticollis. Rondanni. id. |Franciscanus. (Eucne-
2 Filum. Fabr. mis.) Jan. id.
Austria.

THAROPS. Laporte. Redtenbacher.

Isorhipis. Lacord. Dej, Cat, Nematodes. L. Redtenbacher.

1 (Melasioides. Laporte. $P$.*

Lepaigei. Dej. Ct. Lac. id.

Strepens. L. Redtenb. Austria.

DIMA. Ziegler. Dej. Cat. Charpentier. Erich. L. Redtenbacher.

1
$1 \begin{cases}\text { Filiformis, Fabr. } & \text { Gallia.* } \\ \text { Cinereus. Illiger. } & \text { Austria. } \\ \text { Unguliserris. Schönh. } & \text { Suecia. }\end{cases}$

MONOCREPIDIUS. Eschscholtz. L. Redtenbacher.

1 Fulvus. W. Redtenb. Austria.* Cuneiformis.Natterer.id.

2 (Acuticornis. L. Redtb. id. \{ Nigellus. (Ampedus.)

Dej. Cat. id.

\section{MELANOTUS. Eschscholtz.}

L. Redtenbacher. Cratonychus. Dej. Cat .

1 Niger. $F a b r$. Iterrimus. Olivier. P.

2 Brunnipes. Germar. Gallia.*

3 Monticola. Ménétriés. Russ, merid.

4 Tenebrosus. Erichson. Italia.*

5 (Dichrous. Erichson. Gall. merid.* Bicolor. Schönherr. id.

6 Alpinus. (Gaubil.) Alp. Gall.*

7 Mauritanicus, Lucas, Algiria.

8 (Castaneipes. Paykull. Gall, merid.* Fulvipes, Gyllenhal. Suecia. Obscurus. Fabr. $\boldsymbol{P}$.

9 Rufipes. Herbst. Germania.* Fulvipes, Herbst. Gall.bor.

Var, Bicolor. Fabr. id.

10 Emulus. Erichson. Germania.

11 Grassicollis, Erichson. Gallia.

\{ Brunnipes, Lacord. $P$.

12 Fuscipes. Schönherr. Austria.

13 Aquilus. Dej. Cat. Dalmatia.

14 Menetriesii. Dej. Cat. Russ. merid. \{ Sobrinus. Ménétriẻs. id.

15 Cinerascens, Dej. Cat. Gall. merid.*

16 Dalmatinus. Stm. Cat. Dalmatia.

17 Hæmatopus. Stm. Cat. Græcia.

\section{AGRYPNUS. Eschscholtz.}

Dej. Cat, L. Redtenbacher. Erater. Fabr.

1 Atomarius. Fabr. Gall. merid.* Carbonarius. Rossi. Italia.

Cat. Dalmatia. 


\section{ADELOCERA, Latreille.}

L. Redtenbacher. Agrypuds. Eschscholtz. Dej. Cat. Elater. Fabr.

1 (Lepidoptera, Gyllenh. Gall, merid. Chrysoprasa. Herbst. Austria.

2 Conspersa. Gyllenhal. Suecia.

3 Fasciata. Linné. Gall, merid.* Incequalis, de Geer. Germania.

4 Varia. Fabr. quercus. Herbst. Gall, merid.* Austria.

LACON. Laporte. L. Redtenbacher.

Agrypnus, Eschsch. Dej. Cat, Elater. $\mathrm{Fabr}$.

1 Murinus, Linné. Gallia.*

2 Crenicollis. Ménétriẻs. Russ, merid.

\section{CALAIS. Laporte.}

Alaus, Eschsch. Dej. Cat. Elater, Guér.

1 Parreyssii. Stéven.

Tauria.

\{ Goryi. Guérin. id.

\section{ATHOUS, Eschsch. L. Redtenbacher.}

\section{Elater. Fabr.}

1 Rufus, Fabr.

2 Angusticollis. Megerle. Dej. Cat. id,

3 Rhombeus, Olivier. $P^{*}$

4 Undulatus. de Geer.

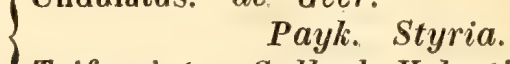

Trifasciatus, Gyllenh. Helvetia.

5 Bifasciatus, Gyllenh, Lapponia.

6 Puzosii. Dej.Cat. Pyrenais.

7 Tenebricosus.Dej.Cat.Gall. merid.

8 Dejeanii. Yvan. id.*

9 Tauricus. Dej. Cat. Tauria.

10 Coarctatus, Dej. Cat. Gall. merid.

11 Scrutator. Herbst. Suecia.*

Testaceus. Paylkull. id.

Luridipennis. Besser. Volhynia.

12 Rufocinctus, (Chevrol.) Alp. Gall.*

13 Hirtus. Herbst. Austria.*

Aterrimus. Fabr, id.

Niger, Olivier. id.

14 Mutilatus. Rosenh, Germania.

15 Læsus. Germar. Tauria.

16 Mutabilis. Mus, Berol. Germania.*

17 Affinis, Paykull. Austria.

18 Obscuripennis. Sturm.

Cat. Russ, merid.

19 Picicornis. Stm. Cat. Italia.

20 Piciventris. Stm. Cat. id.

21 Rubens. Sturm, Cat. Dalmatia.

22 Sulphuripennis. Stm.

23

24

(c)

33

34

35

36

(3)

西

Ater. Dej. Cat. Cat. Italia.

Vestitus, Dej. Cat. Gall. orient.* Cervinus. Dej. Cat. Pyrenais.

Procerus. Illiger. Gall. orient.

Hispania. Marginalis. (Dahl.) Italia. Longicollis. Fabr. P. ${ }^{*}$ Marginatus, Paykull. Germania. Marginellus. Herbst. Austria. Distinguendus. Sturm.

\section{Cat. Tergest.}

Angusticollis. Stm. Ct. Suecia.

Trifasciatus. Herbst. Austria. (Difformis. (Ziegler.) Gallia.* $\{$ Var. Distinctus. (Ullr.) Illyria. (Circumductus. Ménét. Russ. merid. Donei. (Gaubil.) Algiria.* Narbonnensis. (Gaub.) Gall. merid.* Quadrimaculatus. Fab. Austria.

(Hæmorrhoidalis, Fabr. Germania.* Ruficaudis, Gyllenhal. Austria. Analis. Herbst. Gallia. Fuscipennis, Stm, Cat. Italia. Fuscescens. Stm. Cat. id. Leucophæus, Dej. Cat, Gallia. Mauritanicus. (Gaubil.) Algiria.* Crassicollis. Dej. Cat. Gall. bor.* (Vittatus. Fabr. P. ${ }^{*}$ Subfuscus, Gyllenh. Gallia. Stricticollis. Panzer. Germania. jDilutus, Kugelann. id.* Subfuscus, Gyllenh. id. Perangustus. (Gaubil.) Algiria.* Ochraceus. Stm. Cat. Germania. Eratus. Sturm. Cat. Suecia. Jucundus. Tischer. St.

Cat. Germania.

Compressicornis. St. Cat. Russ, merid.

49 Cinereomicans. St. Cat. Germania.

50 Bivittatus. St. Cat, Italia.

51 Imbellis. Stév. St. Cat, Tauria.

52 Tenigratus, Stm. Cat, id.

53 Nigritus. (Zgl.)St. Cat. Croatia. 
54 Picipennis, Stm. Cat. Austria.

55 Pustulatus. Stm. Cat. Hungaria.

56 Parvulus. Panzer. Austria.

\section{CAMPYLUS, Fischer.}

Exophthalmus. Latr. Hammionus. Megerle. ELATER, Fabr, Latr.

1 Denticollis. Fabr. Austria.*

$\left\{\begin{array}{l}\text { Rubens. Panzer. id. } \\ \text { Pyrophorus. Fabr. ol. id. }\end{array}\right.$

2 Linearis. Fabr. Gall. bor.

Var. Mesomelas. Fabr. id.*

or Dispar. Paykull. Suecia.

Denticollis. Fischer. Russ, merid.

Var.Livens, Fabr. Germania.*

Bicolor. Panzer. id.

\section{LIMONIUS. Eschscholtz.}

Redtenbacher. Elater. Fabr. Oliv.

1 Rubripes. Germar. Germania.

2 Violaceus. Germar. Austria.

3 Corulescens. Dej. Cat. id.

4 Cyaneus.(Megerle.) Dej.

$$
\text { Cat. id. }
$$

5 Russus. Dej.Cat. Hispania.

6 Cylindricus. Paykull. P. ${ }^{*}$

7 Mœstus. Stéven. Tauria.

8 Murinus. Sturm. Cat. Germania.

9 Nigripes. Gyllenhal. id, ${ }^{\star}$

10 Fumosus. Sturm. Cat, Gracia.

11 Serraticornis.Paykull. Germania.* \{ Serricornis. Herbst. id.

12 Tereticollis. Stm.Cat. id.

13 Minutus. Linné. P.* Nitidicollis. (Megerle.) Germania.

14 Minusculus. Dej. Cat. Cróatia. Y Variolosus, Parreyss. id.

15 Lythrodes. Germar. Gallia. ${ }^{*}$ Flavoangulus. Sturm. Germania.

16 Radiolus. Germar. id.*

17 Bructeri. Fabr. Panz. id,* $\{$ Minutus. Paykull. Suecia.

18 Bipustulatus. Lin. Fab. P.*

19 Dorsalis. Sturm. Cat. Italia.

20 Nigritarsis. Stéven. St.

21 Parvulus. Panzer.

Cat. Russ. merid. Mus. Illiger.
CARDIOPHORUS, Eschscholtz.

L. Redtenbacher, Dej. Cat. Calonerus. Steph. Castelnau. Elater. Fabr. Oliv. Latr.

1 Thoracicus. Fabr. P.*

2 Discicollis. Herbst. Austria.

3 Ruficollis. Linné. Gallia.*

4 Collaris. Erichson. Sicilia.

5 Ulcerosus. Géné. Sardinia.*

Corsicus. Dej. Cat. Corsica.

6 Argiolus. Géné. Erich. Sardinia.* S Sardeus. Dej. Cat. id.

7 Anticus. Erichson. id.

8 Submaculatus.Dej.Cat.Gall, merid.

9 Pictus, Castelnau. id.

10 Ornatus. Dej. Cat. id.

11 Sexpunctatus. Illiger. Lusitania.

\{ignatus, Olivier. Hispania.

12 Biguttatus, Fabr. Gall. merid.

13 Sexmaculatus, Lucas, Algiria.*

14 Bipunctatus. Fabr. Italia.

15 Boticus. Rambur. Hisp. merid.

16 Bisignatus. Dej. Cat. Hispania.

17 Eleonoræ. Géné. Sardinia.*

18 Rutipes. Fabr. $\boldsymbol{P}^{*}$

19 Vestigialis. Erichson. Lusitaria.

Rufpes. Illiger. $\cdots$ id.

20 Tibialis. Erichson. Corfou.

21 Nigerrimus. Erichson. Germania. *

22 Atramentarius. Erich. id.

23 Ebeninus. Germar, Lusitania. ${ }^{*}$

\{ Advena. Fabr. Gall. merid.

24 Melampus. Illiger. Germania.

25 Asellus, Erichson. Berolini.

26 Exaratus.Dej.Cat.Castelnau. Gall. merid.

$\begin{cases}\text { Cinereus. Herbst. } & \text { German } \\ \text { Pilosus, Paykull. } & \text { Suecia. }\end{cases}$

Equiseti. Herbst. Austria. Weberi. Waltl. id.

28 Incanus, Erichson. Germania.

29 (Equiseti. Herbst. Gall. merid.

Filiformis. Rossi. Italia.

Pilosus. Herbst. Austria.

30 Rubripes. Germar. Gallia.*

Albipes. (Megerle.) Austria.

(q Pollux. Germar. Germania.

31 Crassicollis, Erichson. id.

32 Testacens. Fabr. Tirolis.

Fugax. Fabr. Styria.

33 Geminatus, Erichson. Germania. 
34 Siculus. Dej. Cat.

Germania.

35

36

37

38

39

40

Luridipes. Dej. Cat. Gallia.

Bivittatus. Dej. Cat. Sicilia.

Obsoletus. Sturm. Cat. Austria.

Nigritus. Sturm. Cat. Gracia.

Signaticollis. Stm. Cat. Sicilia.

Rufoangulatus. Sturm.

Cat. Sardinia.

Patruelis. Sturm. Cat. Croatia.

\section{EOLUS. Eschscholtz.}

1 (Crucifer. Rossi. Cruciger. (Dahl.)

Rossii. Godet.

Italia.

id.

Russ. merid.

\section{ELATER. Linné. Eschsch.}

Ampedus. (Megerle.) Dej. Cat.

1 Sanguineus. Linné. Gall. bor.*

2 (Lythropterus. Germar. Alsatia.*

Sanguineus, Stephens. Anglia.

Semiruber. Hoffmans, Germania.

3 Ephippium. Fabr. Gallia.*

Sanguinolentus. Schk. Germania.

Var.Sanguineus. Pay-

4 (Præustus. Fabr. Alp. Gall.*

$\{$ Var. $\beta$. Sanguineus.

5 Pomorum. Geoffrayk. Suecia.

Ferrugatus, Dej. Cat. id.

Elongatus.Zettersted. Suecia.

6 Crocatus. Geoffroy. Gallia.*

7 Balteatus. Linné. id.*

8 Ochropterus. Dej. Cat.

Küster. Russ. merid
Fabr. Alsatia*

Glycereus, Herbst. Austria.

Praustus, Stephens, Anglia.

10 Elegantulus. Schönh. Gall. merid.* Adustus. Eschscholtz. Austria.

Austriacus, Dej. Cat.

$$
\text { Cast. id. }
$$

11 Tristis. Linné. Suecia.

12 Sinuatus. (Zgl.) Germ. Austria.

13 (Erythrogonus. Müller. Dania.

Arrogans. Dej. Cat. Austria.

Auritus. Schönherr. Suecia.

14 Megerlei. Dej. Cat.

Germar. Gall, orient.

15 Foveicollis. Dej. Cat. Styria.
16 Brunnicornis, Germar. Gallia.

Athiops. Frælich. Germania.

17 Scrofa. Ahrens. Gall. merid. ${ }^{*}$ Morio. (Ziegl.) Germ. Germania.

18 Obsidianus. Germar. id.* Y Nigerrimus. Dej. Cat. P.

19 Nigrinus, Paykull. Suecia.* Pilosulus. Herbst. Germania.

20 Subcarinatus. Germar. Gallia.* \{Tibialis. Dej. Cat. id.

21 Lugens. Will. Redtb. Austria.

22 Carbonarius. Stm. Cat. Germania.

\section{ISCHNODES. Germar.}

Ampedus. Dej. Cat. Ctenicerus. Steph. Elater. Germar.

1 (Sanguinicollis, Panz. Austria. $\begin{cases}\text { Niger. Vet. } & \text { id. } \\ \text { Ruficollis. Donovan. } & \text { id. }\end{cases}$

GRYPTOHYPNUS, Eschscholtz.

Redtenb. Dej. Cat, Elater, Linné. Fabr.

1 Hyperboreus, Gyllenh, Lapp. bor.

2 (Riparius. Fabr. Alsatia.*

Eneus. Marsham. Anglia.

Littoreus. Herbst. Austria.

3 Rivularis. Gyllenhal. Suecia.*

Riparius. Panzer. Alsatia.

4 Pulchellus. Linné. Gallia.*

Var. Trimaculatus.

Fabr. Germania.

5 (Quadripustulatus. Fab. id.*

Agricola. Zettersted. Suecia.

Quadrum. Gyllenh. id.

6 (Tetragraphus. Germar. Gall. bor.*

Quadripustulatus.

7 Dermestoides. Herbst. Gall. merid. $\{$ Quadripustulatus.

$\left\{\begin{array}{l}\text { Gyll. var. Succia. } \\ \text { Qull. }\end{array}\right.$

Minimus. Dej. Cat. Gall. merid.

8 Lapidicola. Westerh. Germania.*

\{Exiguus. Dej. Cat. Gall. merid.

9 Minutissimus. Germar. Gall. orient. ${ }^{*}$

10 Liliputanus, Chevrier. Sisilia.

11 Curtus. Erichson. Gall. merid.* $\{$ Troglodytes. Dej. Cat. Pedemont.

12 Elongatus, L. Redtb. Austria. 
OOPHORUS. Eschscholtz.

1 Algiricus. Lucas. Algiria.

DRASTERIUS. Eschscholtz.

Dej. Cat. Redtenbach. Elater. Fabr.

1 Bimaculatus. Fabr, Gall. merid.*

2 Caucasicus. Godet. Dej.

3 Rossii. Stéven. id.

MACRODES. Dej. Cat.

1 Strictus. Dej. Cat. Hisp, merid.

\section{LUDIUS. Latreille.}

Steatoderus, Eschsch. Dej. Cat. ElaTER. Linné.

1 Ferrugineus. Linné. $P_{.}^{*}$

2 Theseus. Germar. (Ectinus. Dej.) Dalmatia.

\section{CORYMBITES. Latreille.}

Ludius. Dej. Cat. Creniceras. Latr. Elater. Linné. Fabr, Oliv.

1 Hæmatodes. Fabr. Gallia.* $\{$ Purpureus, Herbst. Germania.

2 Hæmopterus. Illiger, Lusitania.

3 Castaneus. Linné. $\quad \boldsymbol{P}$.*

4 Sulphuripennis. (St.)

$\{$ Germar. Italia. Apicalis. (Ziegl.) Dej.

5 Aulicus, Panzer. Helvetia*

Incequalis. Fabr. id.

Yar. Cupreus. Herbst. Austria.

Signatus, Panzer. Germania.*

Castaneus. Scopoli. Carniolia.

6 Cupreus. Fabr, Germania.*

7 Aruginosus. Fabr. id."

Var. Cupreus. Panz. id.

8 Heyeri.Saxesen. Harcynia.

9 Pyrenæus. Charpent. Pyrenais.

10 Pectinicornis. Linné. Gall. orient.*

11 Croaticus. Germar. Croatia.

12 Tessellatus. Linné. Alsatia.*

Yar. Assimilis. Gyll, Gallia.

13 Affinis. Paykull.
14 Geniculatus. (Gaubil.) Alsatia.*

15 Quercus. Gyllenhal. Suecia.* \{ Pallipes. Paykull. Lapponia.

\section{DIACANTHUS. Latreille.}

Ludius. Latr, Dej. Cat. Elater. Linné. Fabr.

1 Holosericeus. Fabr. P.*

2 Montanus.(Chevrolat.) Nov. sp. Alsatia.*

3 Chrysocomus. (Dahl.) Germar, Hungaria.

4 Nubilipennis. Germar. Iberia.

5 ) Metallicus. Paykull. Gallia.*

I Nigricornis. Panzer. Germania.

6 Impressus. Fabr. Suecia.

Aruginosus. Olivier, id.

7 Cinctus. Paykull. Germania.*

8 Guttatus. Dej.Cat.Germar. Styria.

9 Cruciatus. Linné. Gallia bor.*

10 Costalis, Paykull. Lapponia.

11 Latus. Fabr. Gallia.*

Germanus, Olivier. id.

Saginatus. Falderm. Russia.

12 Gravidus. Germar. Germania.

13 Milo. Hoffmsg. Germ. Germania.*

\{ Pulverulentus, Kunze, id.

14 Globicollis. Fisch. Ger-

mar. Iberia.

15 Amplicollis. Germar. Pyrenais.* Pyrenceus. Dej. Cat. id.

16 Melancholicus. Fabr. Germania.* \{ Scabricollis. Eschsch. Gall. orient. *

17 Longulus. Gyllenhal. Gallia.*

18 \{ Rugosus. Bonel. Germ. Gall, merid.* Confluens. Ledebour. id.

19 Eneus. Linné, Gall. orient.* Var. a. Impressus.

$\{$ Marsham. Anglia.

- b. Germanus. Lin. Suecia.

- c. Cyaneus. Marsh. Anglia.

\section{PRISTILOPHUS. Latreille.}

Ludius. Dej, Cat. Elater. Fabr.

$1\left\{\begin{array}{l}\text { Insitivus. Germar. Hungaria. } \\ \text { Depressus. Germ. (Dej. } \\ \text { Cat.) Volhynia. }\end{array}\right.$

Helvetia. ${ }^{\star} 2$ Famulus, Germar. Sicilia. 
AGRIOTES. Eschscholtz.

Dej. Cat. Citenonychus. Steph. Elater. Fabr. Oliv.

1 Pilosus. Fabr. p* Obscurus. Olivier. Gallia. Vilis, Illiger. Austria.

2 Fuscicollis. Parreyss. Dej. Cat. Gall. merid.

3 Inonatus. (Gaubil.) Algiria.*

4 Fulvescens. Dej. Cat. Gall. orient. Ruficollis. (Dahl.) Italia.

5 Gallicus. Dej. Cat. Lac.P.*

6 Graminicola. L. Redtb. Austria.* Fusculus. (Meg.) Dej. Cat. id. \{Gilvellus. (Ziegl.) Dej. Cat. $P$. Sputator. (Ziegler.) id. Blandus. Germar. Germania.

7 Rusticus. Dej. Cat.

8 (Segetis. Bierk. Gyll. Lineatus. Linné. Striatus. Fabr. Gall. merid.* Suecia.* Germania. $P$.

9 Obscurus. Linné. Gyll. Suecia.* $\{$ Variabilis. Payk. Fab. P.

10 Marginipennis. (Gaub.) Algiria. ${ }^{*}$

11 Sputator. Linné. Gyll. P.*

12 Flavicornis, Panzer. Austria.

13 Rufulus. Dej. Cat. $\quad P$.

14 Litigiosus. Rossi. Italia.

ANELASTES. Kirby.

Silends, Latr.

1 Barbaras. Lucas. Algiria.

SERICOSOMUS. Serville.

Eschsch. L. Redtenb. Dej. Cat. EcAter. Fabr. Linné.

1 Tibialis. Castelnau.

(Agriotes. Meg.) Austria.

2 Brunneus. Linné, Fabr.Germania.*

3 Fugax. Fabr. Gallia.*

DOLOPIUS. (Megerle.) Eschsch.

Redtenb. Dej. Cat. Elater. Linné, Fabr.

1 Marginipennis, Lucas. Algiria.
2 Marginatus. Linné. Lateralis. Olivier. Dorsalis. Paykull. Tenellus. Beck. Sticticus. Panzer.

3 Rufipennis. Dej. Cat. Gall. merid. $P .{ }^{*}$ Gallia. Suecia. Germania. id.

\section{ECTINUS, Eschscholtz.}

L. Redtenb, Dej. Cat. Elater, Linné.

$\left\{\begin{array}{l}\text { Aterrimus. Linné. } \\ \text { Obscurus. Olivier. } \\ \text { Atratus, Illiger. }\end{array}\right.$

Atratus. Illiger. Germania. Nigrinus. Herbst. Austria.

2 Xanthodon. Märkel. Germania.

3 Volhynensis. (Zgl. Dej.

Cat.) Volhynia.

4 Subæneus. L. Redtenb. Austria.

5 Jucundus. Märkel. Germania.

ADRASTUS. (Megerle.) Eschsch.

Redtenb. Dej. Cat. Elater. Linné, Fabr.

1 Terminatus. (Dahl.)

Erich. Dalmatia.*

2 Dimidiatipennis. (Gau-

- bil.) Algiria.*

3 Rutilipennis. Illiger. Lusitania.

4 Axillaris. Erichson. Germania.

5 Limbatus. Fabr. P. $^{*}$

6 Pallens. Fabr. Gallia.*

Limbatus.Herbst. Austria.

Pusillus. Herbst. id.

7 Bicolor. Lucas. Algiria.

8 Luteipennis, Erichson. Italia?

9 Lacertosus, Erichson. Iberia.

10 Humilis. Erichson. Austria.

11 Lateralis. Herbst. Germania.

12 Pusillus. Fabr. Gallia. ${ }^{\star}$ Nanus. Herbst. Austria.

13 Quadrimaculatus, Fab. Gall. merid.* $\{$ Bisbimaculatus. Schh. Austria.

14 Umbrinus. Germar. P.*

15 (Styriacus. Dej.Cat. Gall. merid.* Alpinus. (Dahl.) Illyria. (Var.Pustulatus. (Dhl.) Hungaria.

16 Pygmæus, Fabr. Hbst. Austria. 


\section{FAM, CEBRIONES.}

CEBRIO. Olivier.

Tibesia, Leach. Hammonia, Latr.

1 Gigas. Fabr. Longicornis, olivier. id.

I Brevicornis. Oliv. id. * Premulus. Leach. id.

2 Fabricii. Leach. id.* Xanthomerus. Hoffmg.id.

$\{$ 올elanocephala. Leach. id.

3 Morio. Dufour. Hispania.

4 Abdominalis. Cast.Luc.Algiria.*

5 Carrenii. Graëlls. Hispania.

6 Barbarus, Lucas, Algiria.*

7 Dimidiatus. Lucas. id.*

8 Attenuatus. Lucas. id.

9 Melanocephalus. Luc. id.*

10 Guyonii. Guérin.Luc, id.

11 Numidicus. Lucas. id.*

12 Nigricans, Lucas, id

13 Nigricollis. Buquet. Oriente.

14 Ustulatus. Dej. Cat. Hispania.

15 Ruficollis, Fabr. id.

Testaceus. Dej. Cat. id.

16 Siculus. Dej. Cat. Sicilia.

17 Strictus. Géné.

17

\section{FAI, GYPHONES.}

\section{ATOPA. Paykull.}

\section{Dascillus. Latr. Chrysomela. Linné.}

1 Cervina. Fabr. Linné. Gallia bor.*

2 Cinerea. Falsr. id.*

Cervina. Olivier. id.

\section{ELODES, Latreille.}

Cyphon. Payk. Fabr. Crioceris et CryptocephalUs. Marsh. Galleruca. Fabr. Gistela. Fabr. Lampyris. Linné.

Gallia.*

$\boldsymbol{P}$.

Suвcia. Anglia.

id.

id.

id.
2 Bohemanni. Mannerh. Finlandia.

3 Variabilis. Thunberg. Gallia.

Pubescens. Fabr. Gyll. Suecia.

Minuta. Olivier. $P$.

Dorsalis. Marsham. Anglia.

4 Coarctata. Paykull. Suecia.*

Livida. Fabr. Germania.

Grisea. Fabr. id.

Concolor. Marsham. Anglia.

Fuscescens. Latreille. $\boldsymbol{P}$.

Var.Variabilis. Thunb. Suecia.

5 Ochracea. Sturm. Germania.

6 Fusca. Sturm. id.

7 (Padi. Linné. $\quad$ Gallia.*

Var. Coarctata. Payk. Suecia.

$\{$ Discolor. Panzer. Germania.

Pusilla. Dej. Cat. Dalmatia.

Ater. var.? Stephens, Anglia.

8 (Marginata. Fabr. …

Var. Pallida.Paykull. Suecia.

Circumfusa. Marsh. Anglia.

Nimbata. Panzer. Germania.

Var. Limbata.Dej.Cat.P.

9 Serricornis. Müller. Germania.

Serraticornis, Gyll. Suecia.

Chrysomeloides. Stph. Anglia.

Testacea. Dej.Cat. P.

10 Paykulii. Guérin. Gallia.

11 Unicolor. Illiger. Austria.

12 Deflexicollis. Müller. Germania.*

Pini. Curtis. Anglia.

Nigricans. Dej. Cat. Dalmatia.

Pellucens, Melsheimer. Germania.

13 Pallida, Fabr. Oliv. Gallia.*

Melanura. Fabr. Suecia.

Var. Leta. Panz. Stph. Germania.

Minuta. Linné. Suecia.

14 Genei. Guérin. Corsica.

\{Flavicollis, Dej. Cat. Sardinia.

\section{SCIRTES. Illiger. Latr.}

Elodes, Latr. Cyphon. Payk. Fabr. Chrysomela. Linné.

1 Hemisphæricus.Linné. Gallia.*

2 Orbicularis, Panzer, Germania.

EUBRIA. (Ziegler.) Dej. Cat. L. Redtenbacher.

Crphon, Germar.

1 Palustris. (Zgl.) Germ. P.* 
EUCINETUS, Schüppel. Germar.

Nycteus, Latr. Amaxobium. Duftsch.

1 Hæmorrhoidalis. Germ. Austria.*

Hamorrhoum. Duft. Germania.

Mordelloides. Germar.

(Scaphid.) id.

2 Meridionalis, Eschsch. Gall, merid. \{Hispanicus? Dej. Cat. Hispania.

3 Testaceus. Dej. Cat. id.

22

\section{FAM, TELEPHORI.}

LYGISTOPTERUS, Dej. Cat, L, Redtenb. Lvcus, Fabr.

1 Sanguineus, Fabr. Gallia.*

\section{DICTYOPTERUS, Latreille.}

Lycus. Fabr, Latr. LAMPYRIS. Linné.

1 Aurora. Fabr.

Gallia.*

Coccinea. Linné. Germania.

2 Rubens, Megerle. Dej. Cat. L. Redtenb. Gallia.*

3 Discicollis. Stm, Cat. Germania.

4 Erythropterus. Dej. Ct. Russ. merid.

5 Flavescens, L. Redtb. Austria.

6 Minutus. Fabr. Gall. bor.*

7 Maculicollis. Dej. Cat. Hungaria. \{ Aurora. (Megerle.) Styria.

8 Merckii. Mulsant. Germania.* Cosnardi, Guérin. P.

PYROPTERUS. Mulsant.

Lycus, Payk, Gyll. Dictyopterus, Dej. Cat.

1 Affinis, Paykull.

Alp, marit.

OMALISUS. Geoffroy. Oliv. Fabr, Latr.

1 Sanguinipennis. Dej.

Cat. Costa. Küst. Dalmatia. Taurinensis? Bonelli.

2 Suturalis, Fabr.

in litt. Lombardia.

$P$.*
LAMPYRIS, Linné. Geoff, Fabr. Latr. Dej. Cat.

1 Noctiluca, Lin, Fabr. $\boldsymbol{P}_{\text {.* }}$

2 Splendidula, Lin. Fab.

Oliv. Gall. merid. ${ }^{*}$

3 Zenkeri. Germ. Brullé. Graecia.

4 Antiqua. Brullé. id.

5 Senki. de Villaret. Gallia.

6 Mauritanica, Lin. Fab. Gall. merid.*

PHOSPHOENUS. Laporte.

Geopyris, Dej. Cat. Lampyris, Fabr.

1 Hemipterus, Fabr. $\boldsymbol{P}^{*}$

\section{LUCIOLA. de Laporte.}

Colophotia. Dej. Cat, Lampyris, Linné.

1 Italica. Linné.

2 Græca. Laporte. Gracia.

3 Lusitanica. Charpent. Hispania.* Italica. Fabr. Lusitania.

Var. Mechadiensis.

(Dahl.) Hungaria.

- Illyrica. Dej. Cat,

Küster, Dalmatia.*

4 Pedemontana. Bonelli. Italia bor.*

5 Suturalis. Ménétriés. Constantinp.

DRILUS, Olivier, Latr.

Ptilinus, Fabr, Panz. Geoff.

1 Flavescens, Fabr. P.*

q Vorax. Mielzinsky. (Cochleoctonus.) id.

2 Mauritanicus, Lucas, Algiria.

3 Fulvicollis. Dej. Cat. Audouin. Dalmatia.

4 (o Ater, Dej. Cat, Aud. Germania.

Var. Floralis. St. Cat. id.

Pectinatus, Schh. (Dasytes.) id.

5 Niger. Sturm. Cat Austria.

6 Fulvitarsis, Stéven. Russ, merid.

MALACOGASTER, Bassi.

Ctenidion. Dej. Cat.

1 Passerinii.Bassi. Luc. Sicilia. *

Ruficollis. Hoffmsg. Algiria.

Thoracicus, Dej,Cat, id. 
PODABRUS, Fischer.

25

Dej. St. Cat. Telephonus. Oliv, CanthaRIs. Payk. Gyll.

1 Alpinus. Payliull, Suecia.* $\{$ Var. Thoracicus. Fisch. Sibiria. Annulatus. Fischer. id.

2 Schönherri. Dej. Cat. Lapponia. Pilosa. var. b. Gyll.

(Cantharis.) id.

3 Lapponicus. Gyllenh.

$$
\text { St. Cat, id. }
$$

4 Bannaticus. Rosenh. Hungaria.

5 Nigriventris, Fischer. Volhynia.

6 Lulunatus. Fischer. Russ. merid.

\section{TELEPHORUS, Geoffroy.}

De Geer. Oliv. Cantharis, Fab, Dej, Cat.

1 Oculatus. Gebler.

Russia.

2 Ammaris, Ménétriés. Constantinp. \{ Pupillatus, Friwalds, id.

3 Illyricus. Dej. Cat. Dalmatia.

4 Fuscus. Linné.

Anticus. Märkel. Dej.

$P$.

Cat. Germania.

5 Rugifrons. Castelnau. id.

6 Immaculicollis. Castl. id.

7 Hispanicus. Dej. Cat. Hispania.

8 Rusticus. Fallen, Gyll. Austria.

9 Varipes. Dej. Cat.

10 Dispar. Fabr.

\{Lividus. Illiger.

Gall. merid.

$P^{*}$

Germania.

11

12

13

14

15

Pellucidus. Fabr. Gyll, Gall. bor.*

Hospes, Rosenhauer. Hungaria.

Fuscipennis. Dej. Cat. Dalmatia.*

Violaceus. Paykull. Suecia. ${ }^{*}$

Cyanipennis. (Ziegler.)

$$
\text { Dej. Cat. Styria.* }
$$

Occipitalis. Rosenh. Tirolis.

Collaris. Dej, Cat. Russ, merid.

Abdominalis. Fabr. Gall, merid, ${ }^{*}$

Tristis, Fabr. id. ${ }^{*}$

(Nigricans, Fabr. Gyll. Germania.

Var. Albomarginata.

St. Cat. id.

Albomarginatus. Stm.

$$
\text { Cat. id.* }
$$

Obscurus, Fabr.Linné. $P^{*}$
Pagana, Rosenhauer. Hungaria.
(Humeralis. St.Dej.Cat.

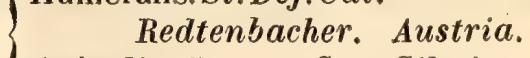
(Apicalis. Sturm. Cat. Silesia.

61 Signatus. Germar. Germania.* Fumigatus. (Ziegler,) Dalmatia.

Rufiventris. Stm. Cat, Germania.

Varicornis, Stm, Cat, id.

Atricornis. Stm. Cat. id.

Lateralis. Linné. Gallia.*

Discicollis. (Ziegler.) Dej. Cat. id.

Marginellus. Dej. Cat. Lusitania.

Affinis. Dej. Cat. Hispania.

Silloides. (Allibert.) Alp. Gallia. ${ }^{*}$

Nitidus, Rambur. Dej. Cat. Hispania.*

Flavilabris. Gyllenh. Suecia.

Opacus. Germar. Germania.

Pulicarius, Fabr. Oliv. Italia.*

Italicus. Dej. Cat. id.

Lætus. Fabr. id.

Coronatus. Gyllenhal. Hispania.

Palliatus. Gyllenhal. Suecia.

Menetriesii. Dej. Cat. Russ, merid.

Lividus. Fabr. $\quad \boldsymbol{P}^{*}{ }^{*}$

Translucidus, Dej, Cat. Styria.*

Obsuricornis. St. Cat. Italia.

Scutellaris. Lucas, Algiria.

Mauritanicus. Lucas. id. ${ }^{*}$

Colona. Erichson. id.*

Fossulatus, Lucas, id.

Angustus. Mus. Berol. Germania.*

Fuscipes. Sturm, Cat, id.

Rufus, Linné. Gallia bor.*

Melanogaster.Stm.Cat.Germania.

Basalis. Sturm. Cat. id.

Bicolor. Fabr. P.

Unicolor. Falderm. Russ. merid.

Lineatus. Suffrian. Germania.

Vicinus, Dej. Cat. Russ, merid.

Proximus, Dej. Cat. id.

Consentaneus. Dej. Cat. Italia.

Pugionatus. (Chevrol.) Algiria.*

Præcox. Géné. Sardinia.

\{Pilosus. Paykull. Gyll. Suecia.*

I Rufotestaceus. Letzn. Germania.

Lapponicus, Gyll. Dej.

\section{Cat. Lapponia.}

Alpestris. Dej. Cat. Styria.

Styriacus, Dej. Cat. id.

Marginipennis. Dej.

Cat. Russ, merid. 
70 Assimilis. Payk. Gyll. Austria.

$\{$ Pectoralis. Stm. Cat. Illyria.

71 Dilatatus. L. Redtenb. Austria.

72 Rufilabris. Sturm. Cat. id.

73 (Nigricornis. (Megerle.) Styria.

Lividipennis. Sturm.

Cat. Germania.

74 Lituratus. Gyllenhal. Austria."

75 Ochraceus. Stm. Cat. Suecia.

Signaticollis. Sturm.

Cat. id.

76 Melanocephalus, Creu-

$$
\text { tzer. Austria. }
$$

77 Fulvipennis. Germar. Germania.

78 Clypeatus. Illig. Gyll. Austria.* \{ Niveus. Panzer. Germania.

79 Axillaris.(Meg.)St.Cat.Illyria.

s0 Tauricus. Sahlberg. St.

Cat. Tauria.

81 Discoideus. Ahrens. Germania.*

82 Ramburii. Dej. Cat. Hisp. merid.

83 Intermedius. Dej. Cat. Styria.

84 Nigrifrons. Dej. Russ. merid.

85 Pallidipennis.Dej.Cat. Gall. merid.

86 Sulcicollis, Dej.Cat. Gracia.

87 Elongatus. Fallen. St.

Cat. Germania.*

88 Lusitanicus. Schmidt. Lusitania.

Opacus. Dej. Cat. id.

89 Rufitibius. OEsk. Dej.

Cat. Hungaria.

90 Algiricus. (Gaubil.) Algiria.*

91 Maritimus. (Chevrol.) Gallia.*

RAGONYCHA. Eschscholtz.

L. Redtenbacher. Cantharis, Linné. Fab. Dej. St. Cat. Telephorus, Geoffroy.

1 Fulvicollis. Fabr. Germania *

Thoracicus. Olivier. $P .{ }^{*}$

Nivalis. Germar. Austria. .

2 Melanura.Fabr. Panz. Gallia.*

3 Terminalis. L. Redtenb, Austria.

\{ Prausta? Dej. Cat. Styria.

4 Testacea. Linné, Gyll. Gall. bor.**

5 Fuscicornis. Oliv. Gyll. Austria.*

6 Nigriceps. Waltl. id.

7 iPallida. Fabr. Gallia.* (Pallipes. Fabr. Ill. Germania.*

8 (Nigripes. L. Redtenb. Austria.* \{ Barbara? Fab. Erich. Gall. merid.

9 Geniculata, Lucas, Algiria.
10 Femoralis. (Ziegl.) Dej. Cat. L. Redtenb. Gall, merid. Tristis. Bonelli. Pedemont.

11 Paludosa. Fallen. Gyl. Austria.*

12 Atra. Linné. Gyll. Germania.*

SILIS. Meg. Dej. Cat. Latr. de Casteln.

1 (Nitidula. Fabr. P. $\int$ Spinicollis. (Meg.) Dej.

o Lampyroides. Zenk. id. Atra. Besser.

Volhynia.

\section{MALTHINUS. Latreille.}

Dej. St. Cat. Schh. de Cast. Telephorus. Oliv. Gantharis. Linné. Nectdalis. Geoff.

1 Minimus. Olivier. P.*

Flavus, Latreille, id.

Flaveolus, Paykull. Suecia.

2 Flaveolus. Herbst. Germania.*

3. Apicalis. Sturm. Cat. id.

4 Fasciatus. Olivier. P.*

Imperialis. Gysselen. Austria.

5 Angusticollis. Dej.Cat. Pyr. orient.

6 Thoracicus. (Megerle.) Italia.

7 Modestus. Sturm. Cat. Silesia.

8 Biguttulus. Paykull. Suecia.*

9 Pallidipennis. St. Cat. Silesia.

10 Longicornis. OEskay.

Dej. Cat. Hungaria.

11 Biguttatus, Lin.Oliv. $P_{\text {. }}^{*}$

12 Aquestris. Fischer. Russ. merid.

\{ Equestris. Motschulski.id.

13 Longipennis. Lucas. Algiria.*

14 Marginatus. Latreille. $P$. $^{*}$

Minimus, Gyllenhal. Suecia.

15 Tenellus. Illiger. Austria.*

16 Rubricollis. Dej. Cat. Gall.orient.

17 Similis. Sturm. Cat. Silesia.

18 Maurus. (Ziegl.) Cast. Austria.*

19 Sanguinicollis. Schönh.P.*

Sanguinolentus. Gyll, Germania.

Var. Maculicollis.Mannerheim. Suecia.

Pallidicollis. Gysselen.Austria.

20 Dispar. Germar. id.

21 Cephalotes. Dej. Cat. Dalmatia.

22 Longiceps. Dej. Cat. Gall. merid.

23 Nigricollis, Dej, Cat. Germania. 
24 Laticollis. Schüppel. Illyria. \{Marginicollis.Dej.Cat.Dalmatia.

25 Discicollis. Dej. Cat, Gall. merid

26 (Sulcifrons. Dej. Cat. Styria. Sanguinolentus, var.

b. Gyll. Suecia. Pulchellus. Lucas. Algiria. Pusillus. Duftsch. Austria.

29 Obscurellus.Schüppel. Germania.*

30 Piceus. Sturm. Cat. Silesia.

31 Bimaculatus. Stm. Cat. Austria.

32 Brevicollis. Paykull. Suecia.

33 Pulicarius.St.Cat.Red.Austria.

34 Exilis. Nees, St, Cat. Germania.

35 Dimidiatocollis: Rosh. Tirolis,

175

\section{FAM, MALACHII.}

APALOCRUS. Erichson.

Malachius. Fabr, Dej. St. Cat.

1 Variegatus. Erichson. Russia.

2 Pectinicornis. Erich. Russ. merid.

3 Femoralis. Erichson, Austria.

\section{MALACHIUS, Fabricius.}

Gantharis. Linné. Telephorus, de Geer. Ceratipes. Fischer.

I Flavilabris.-Waltl, Er, Hisp. merid.

2 Longicollis. Erichson. Sardinia.

3 Cyanipennis. Dej. Cat.

4 Ruficollis. Fabr. Panz.

$$
\text { Erich. Hispania. }
$$

Terminatus. Ménét. Russ. merid.

5 Rubricollis. Fall. Gyll.

$\left\{\begin{aligned} \text { Erich. } & P_{*}^{\star} \\ \text { Ruficollis. Olivier. } & \text { Gallia. }\end{aligned}\right.$

6 Marginalis. Dej. Cat. Erich. $\boldsymbol{P}^{*}$

7 Pulicarius. Fabr. Oliv, id.*

8 Angusticollis. Lucas. Algiria.

9 Affinis. Ménétriés. Hungaria.

10 Cœruleus. Erichson. Lusitania.

11 Spinosus. Dej. Cat. Er. Gall. merid.

12 Parilis. Erichson. Sardinia.

13 Spinipennis. Germar.

Dej, Cat. Gall, merid.*
14 Narbonnensis. (Gaub.) Gall. merid. *

15 Elegans. Olivier. $\quad P^{*}$

16 Geniculatus. Germar. Gall. merid.* Annulatus. Gebl. Le--

debour. Sibiria.

Marginellus. Fab, Oliv. P.*

18 Insignis, Buquet. Algiria.*

19 Rufus. Fabr. Oliv. Gall. merid.*

20 (Viridis. Fabr. Oliv. Gallia.*

Bipustulatus, var. $\delta$.

Illig. Austria.

Dilaticornis. Dej. Cat.

Mauritanicus. Lucas, Algiria.

Marginicollis. Lucas, id. ${ }^{*}$

Sardeus. Erichson. Sardinia.

Faustus. Erichson. Russia.

Cornutus. Gebl. Fisch. Tauria.

Dentifrons. Dej. Cat.

Erich. Gall. merid.*

28 Lusitanicus, Erichson. Lusitania.

29 Bipustulatus. Lin. Fab. P.*

30 Rubidus. Ziegler. in litt. Erich. Gall.merid.*

31 Carnifex. Erichson. Constantinp.

32 Coccineus. Erichson. id.

33 \{ Scutellaris. Erichson. Austria.*

\{ Eneus, var. $\gamma$. Illig. id.

34 Patruelis. St. Cat. Germania.

35 \& Aneus. Fabr. $\quad \boldsymbol{P}^{*}$

Y Var. Purpuratus. Ull. Germania.

ATTALUS, Erichson.

Malachius, Fabr, Dej. Cat.

1 Lusitanicus. Erichson. Lusitania.

2 Erythroderus, Erich. Sardinia.

3 Luxurians, Erichson, id.

4 Dalmatinus. Dej. Cat.

Erichson. Dalmatia.

5 Sicanus, Erichson. Sicilia.

6 Maculicollis. Lucas. Algiria.

ANTHOCOMUS. Erichson.

Malachius, Fabr, Dej. Cat. Cantharis. Linné.

1 Sanguinolentus. Fabr。

2 (Equestris, Fabr. Oliv. id.*

Fasciatus. var. $\gamma$. Ill. Austria.

(Quadripustulatus. Ill, id. 
3

$\left\{\begin{array}{l}\text { Fasciatus, Fabr. Oliv. Austria. } \\ \text { Gouani. (Meloe.) Lin. Suecia. }\end{array}\right.$

Var. Regalis. Charp. Germania.

4 Cardiaceus, Payk. Lin. Suecia.

Pedicularius, Linné.

(Fabr. S). id.

5 Festivus. Wilh Redt Austria.

6 Lateralis, Dej. Cat. Er. Gall. merid.

Minima. (Cantharis.)

Rossi. Etruria.

7 o Jocosus, Erichson. Sardinia.

8 을. Emulus. Erichson. id.

9 오 Sericans, Erichson. id.

10 Parietariæ. Erichson. Sicilia.

11 Lobatus. Olivier. $P_{\text {.* }}$

12 ơ Coarctatus. Erich. Austria.

13 Constrictus. Erichson. Sardinia.

14 Ulicis. Erichson. Lusitania.

15 Amictus, Erichson. id.

16 Analis. Panzer. Austria.

17 오 Labilis. Erichson. Sardinia.

18 ㅇ Pallidulus. Erichson. Lusitania.

\section{EBAUS. Erichson.}

Malachius.Fabr. Dej. Cat.

1 Pedicularius. Schrank. Germania.* \{Praustus. Gyllenhal. Suecia.

2 Flavicornis, Erichson. Germania.

3 C Cœrulescens. Erich. Austria.* \{Coruleus, Dej.Cat. Dalmatia.

4 Appendiculatus. Erich. Hungaria.

5 Thoracicus, Fab. Oliv, P.*

6 Tristis. Lucas. Algiria.

7 Humilis. Erichson. Sardinia.

8 Affinis. Lucas. Algiria.

9 Collaris. Erichson. Gall. merid.

10 Flavicollis, Erichson, Austria.

11 Albifrons, Fabr, Oliv, P.*

12 (우 Flavipes. Fabr. Ahr. Germania.*

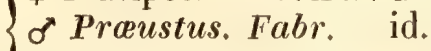

(o Productus. Olivier. Gallia.

13 Balteatus. (Chevrolat.) Gall. merid.*

\section{CHAROPUS. Erichson.}

Malachius. Fabr. Dej. Cat.

1 Pallipes. Olivier. P. $\{$ Flavipes, Paykull. Suecia.

2 Concolor. Fabr. Erich. Sardinia.* Furcatipennis? Villa. Lombardia.
3 Rotundatus. Erichson. Sardinia.

4 Punctatus, Erichson. id.

5 Graminicola. Andersch. Finlandia.

\section{ATTELESTUS, Erichson.}

Malachios, Dej. Cat.

1 Hemipterus. Dej. Cat.

Erichson. Gall, merid.

2 Erichsonii. Küster. Dalmatia?

TROGLOPS, Erichson.

Malachius, Fabr. Dej, Cat, Cantharis. Linné.

1 (Albicans. Linné. Germania.* Angulatus, Fabr. id.

Cephalotes. Olivier. id.

2 Silo. Erichson. Sardinia.

3 Capitatus. Erichson. id.

4 Verticalis. Erichson. Hisp, merid.

5 Marginatus. Waltl. Er. id.

6 Brevis, Erichson. Sardinia.

\section{COLOTES. Erichson.}

1. Trinotatus, Erichson. Gall. merid.*

2 Obsoletus, Erichson. Germania.

DASYTES. Fabricius.

Melyris, Oliv, Ill. Lagria, Panz.

1 Scutellaris. Fabr. Hispania.

2 Villosus. Hoffmanseg. id.*

3 Pilosus. Rambur. Hisp. merid.

4 Hirtus. Linné. Algiria. ${ }^{*}$

Ater. Olivier. $\quad$ Gall. merid.

5 Armatus. Lucas. Algiria.

6 Ereus. Rambur. Hisp. merid.

7 (Ramburii. (Gaubil.) id.

Hirtus. Rambur. id.

8 Maculicornis. Rambur.id.

9 Hispidus. Rambur. id.

10 (Pulverulentus.Dej.Cat.Dalmatia.

\{ Carbonarius. (Dahl.) Hungaria.

11 Bipustulatus. Fabr. Italia.*

12 Variegatus, Lucas. Algiria.

13 Mauritanicus. Lucas. id.

14 Nigromaculatus. Luc. id.

15 Cruciatus. Dej. Cat. Sicilia.

16 Quadripustulatus, Fab. Gall, merid. 
17 Hæmorrhoidalis, Fabr, Algiria, Cyaneus, Olivier.

5 5

Thoracicus, Dej. Cat. Gall. merid. Curtus. Dej, Cat. Dalmatia.

Rubidus. Gyll, in Sch. Hungaria. Floralis, Oliv, Gyll.

(Aplocnemus. Stph.) Suecia.*

Bæticus, Rambur. Hisp. merid.

Tarsalis. Gyllenhal. Austria.

Serricornis, Parreyss. Corfou.

Nigricornis. Fabr. Suecia.*

(Pini. L. Redtenbacher. Austria.

Metallicus. Dahl. id.

Serraticornis. Géné, Algiria.*

Semicornis, Chevrolat. in litt. id.*

Sardeus. Géné. Sardinia. ${ }^{*}$

(Cylindricus, Dej. Cat. Gall. merid.

Metallicus. Schönherr. Lusitania.

(Var. Virens, (Dahl.) Hungaria.

(Serratus, L. Redtenb. Austria.

Serrarius. (Megerle.) id.

Metallicus? Fabr. Algiria.

Nitidus. Sturm. Cat. Sicilia.

Morio, Gyllenhal. Algiria.*

Algiricus, Lucas. id. ${ }^{*}$

Pruinosus. (Chevrol.) Gallia.*

Brunneus. Sturm. Cat. Hungaria.

Chlorosoma. Lucás. Algiria.

Cribrarius, Dej. Cat, Gracia.

Antiquus, Schönherr, Gall, merid. *

Pecticornis, Lucas.

Affinis. Dej. Cat.

Pulchellus. Dej. Cat, id.

Elegans. (Parreyss.) Corfou.

Smaragdinus. Dej. Cat.

\section{Lucas, Sicilia.*}

Gall, merid.* id.

Ciliatus. Graëlls.

Protensus. Géné.

Distinctus. Dej. Cat.

Coarctatus. St. Cat.

Coeruleus. Fabr.

Hispania.*

Sardinia**

Styria.

Sardinia.

$\boldsymbol{P}^{*}$ *

\{ Obscurus. Gyllenhal. Suecia.

Var, Nitens. (Megerle.) Austria.

Aurarius. Hellwig. St.

$$
\text { Cat. id. }
$$

Niger. Fabr. Gyll. Germania.*

Yillosus. Olivier, Austria.

Nigrinus, Dej. Cat. Lusitania.

(Maurus. Dej. Cat. Gall. merid. *

$\{$ Var.Pauperulus.(Meg.)Austria.

(Setosus. (Parreyss.) Corfou.
56 Asphaltinus. (Megerle.) Germania.

57 Marginatus. Latreille. Hungaria. \{ Rubidus. Koy. St. Cat. id.

58 Atratus. Dej. Cat, Dalmatia.

59 Variolosus. (Dej.) Hispania.

60 Antennatus, Stm. Cat. Germania.

61 Subæneus. Schönherr. P. ${ }^{*}$

Eneus, Olivier. Gall, merid.

Rigidus. (Meg.) Dahl.

Cat, Austria.

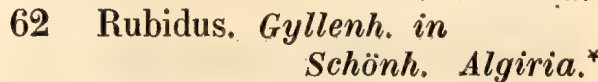

63 Scaber. Müller, Germania.*

64 Flavipes. Fabr. $P$.

65 Fusculus, Illiger, Gyll. Austria.

66 (Rufipes. Sturm. Cat. id.

Tibialis, Stürm. Cat. id.

67 Gracilicornis. St. Cat. id,

68 Indutus. Schmidt et

Helfer. Smyrna.

69 Pilicornis. St. Cat. Germania.

70 Alpinus. Dahl. Cat. id.*

71 Plumbeus. Olivier. $\boldsymbol{P}$.

Femoralis, Gysselen. Austria.

Nitidus. (Megerle.) Dalmatia.

Gracilis. St. Cat. Germania.

72 Hirtellus. Sturm, Cat, id.

73 Fuscipes. Dej. Cat. id.

74 Filiformis, Dej. Cat. Croatia.

75 Cøerulescens. St. Cat. id.

76 Imperialis. Géné. Sardinia.*

77 Distinctus. Lucas. Algiria.*

78 . Præcox. Schmidt et

79 Cinctus. Géné. Helfer. Smyrna.

Sardinia

80 Flavescens. Géné, id.

Pallipes. Illiger. $\quad \boldsymbol{P}^{*}$

Vulpinus. (Dahl.) Germania.

Flavipes. Panzer. id.

Lividus. Fabr. id.

\section{DOLICHOSOMA. Stephens.}

\section{L, Redtenbacher. Tincos, Creutzer.}

1 Linearis, Fabr, Gyll, P.*

Filiformis, Creutzer, Austria.

AMAURONIA. Westwood.

Aplolocnemis. Stephens.

1 Subænea, Westwood, Anglia. 
PHLOIOPHILUS. Waterhouse.

1 Edwarsii. Waterhouse. Anglia.*

2 Blondelii. Chevrol. in litt. $\boldsymbol{P}$.

ZYGIA. Fabr. Latr.

1 Oblonga. Fabr.

Gall. merid.*

MELYRIS, Fabr, Oliv, Latr.

Opatrum. $F a b r$.

1 Granulatus. Fabr. Hisp. merid.*

2 Andalusiaca. Waltl.

3 Rubripes. Lucas. Algiria.*
33. FAII. CLERI.

CYLIDRUS, Latreille.

Clenus. Fabr. Tillus. Charpentier.

Denops. Stéven.

1 Albofasciatus. Charp. Russ, merid.* Longicollis. Stéven. id.

Personatus, Géné.Spi-

$\begin{array}{ll} & \text { nola. Sicilia. } \\ \text { Agilis. Lucas. } & \text { Algiria. }\end{array}$

TILLUS. Fabricius.

Oliv. Latr. Panz. Kirby. Chrysomela. Linné.

1 Elongatus. Fabr. P.* Var. Ambulans. Fabr. Curtis. id.* - Hyalinus. Sturm. Germania.

2 Unifascialus, Latreille. P. $^{*}$ Y Var. Tricolor. Dej. Cat. Gallia bor.

3 Transversalis. Charp. Hisp. merid. * \{Myrmecodes. Hoffmsg. id.

THANASIMUS. Latreille.

Clerus. Fabr, Dej. Cat.

1 Mutillarius, Fabr, Lat. P.*
2 Formicarius. Fabr. P.*

$\{$ Var.Femoralis. Dej.Ct, id.

3 Quadrimaculatus. Fab.

Panz. Germania."

\section{OPILUS. Latreille.}

Illig. Steph. Notoxus, Fabr, Spinola. Clerdus. Oliv. Dermestes. Schrank. Attelabus. Linné.

1 Dorsalis. Lucas. Algiria. ${ }^{*}$

2 Domesticus. Sturm. Germania.* Mollis. Spinola. $\quad \boldsymbol{P}$.

3 Mollis. Lin. Fab. Oliv. Algiria.* Pallidus. Olivier. Gall. merid. Centromaculatus.

Cristofori. Italia.

4 (Cruentatus. (Dupont.) Charpentier. Turcia. Syriacus. Solier. Spin. id. Thoracicus. Friwald. id.

5 Germanus. Chevrolat. German.bor.

\section{CLERUS, Fabricius.}

1 Brevicollis, Kunze. Hungaria.

TARSOSTENUS, Spinola.

Clerus. Fabr. Notoxus. Dej. Cat.

1 Univittatus. Ros. Schh.

Dej. Cat. Gall. merid.*

TRICHODES, Fabricius.

Clenus. Fabr. Oliv.

1 Octopunctatus. Fabr. Gall. merid.*

2 Umbellatorum. Dej.Ct.

Schh. Algiria. ${ }^{*}$

3 Dahlii. Dej. Cat.Spin. Sicilia. \{Affinis (Dahl.) Sardinia.

4 Alvearius. Fabr. Schh. Dej. Cat. P. ${ }^{*}$

5 Apiarius. Fabr. Schh. id.* Var. Apicita.(Ziegler.) Austria.

- Arcuatus.Beaudet. Lafarge. Gall. orient.

- Panonicus.(Ziegl.) Germania.

- Interruptus. (Meg.) Austria.

- Substrifasciatus.

Sturm. Hungaria.

- Corallinus. Fald. Russia.

- Elegans. Dej, Cat. Italia. 
6 Crabroniformis. Fabr. $\left\{\begin{aligned} \text { Schh. Gracia. } & \text { Corfou. } \\ \text { Gulo. (Parreyss.) } & \text { Cor }\end{aligned}\right.$

7 Sanguineosignatus. $\mathrm{Du}$ pont.Dej. Cat.Spin. Grocia.

Var. Affinis. (Dupont.) id. $\{$ - Distinctus, Dej.

Cat. id.

- Illustris. Falderm. Russ. merid.

- Variabilis. (Dup.) Grocia.

8 Favarius, Fabr. Illig.

Var. Senilis. Kollar.

Schh. Styria.

Mannerh. Russ, merid.

- Punctatus. Dej. Ct. id.

- Illustris. Stéven. id.

- Obliquatus. Brul. Gracia.

- Phedinus, Godet. Russ, merid.

- Hispanicus. (Dup.) Hispania.

- Vicinus. Dej. Cat. Oriente.

- Axillaris. (Dup.) id.

- Affinis. (Dupont.) id.

- Elegantulus. (Dupont.) id.

- Latifasciatus,Spinola. id.

9 Leucopsideus. Schönh.
Latr. Gall. merid.
Var. Cerarius, Hoff- manseg. Hispania.
Fuscicornis. Spin. Gall. merid Ammios. Fabr. Schh. Hispania.* Var. Arthriticus. Chev, id.

- Dauci. Villa. Lombardia.

- Flavocinctus.(Dhl.) Sicilia.

- Smyrnensis.(Dup.)Smyrna.

- Visnaga. Friw. id.

- Omoplatus. (Dup.) Graecia.

- Sypilus. Fabr. id.

- Subfasciatus. Faldermann. Russ, merid.

- Quadriguttatus. Ménétriés. id.

- Quadripustulatus. Dej. Cat. Oriente.

- Quadripunctatus. Friwaldski. id.

11 Viridifasciatus. Chevrolat. Algiria.*
ENOPLIUM. Fabricius.

Latr. Trllus. Oliv, Fabr. Dermestes. Rossi. Orthoptevra. Spinola.

1 Serraticorne, Latreille.Gall. merid. Dentatus, Rossi. Dalmatia.

2 Sanguinicolle. Fabr. Gall. bor.* Weberi. Latreille. Germania. Dulce. Ledoux. $\quad \boldsymbol{P}$.

NECROBIA. Latreille. Spinola.

Corynetes, Fabr, Laporte. Dej. St. Cat.

1 Rufipes. Fabr, Latr. Schh, Dej. Cat. Gall. merid. ${ }^{*}$

2 Ruficollis. Fabr. Latr. Schh. Dej. Cat. id.*

3 f Violacea.Lat.Dej. Cat. $P^{*}$ I Angustata. Falderm. Russ. merid.

4 (Defunctorum. Waltl. Spinola. Hisp. merid. Carbonarius.Dej,Cat.id.

5 ) Bicolor. Laporte. id. Thoracica. Dej. Cat. id.

6 Rufitarsis. Chevrolat. in litt. Algiria.*

CORYNETES. Paykull.

1 f Violaceus. Paykull. 'Suecia.* \{Chalybeus. Dej. Cat. P.

OPETIOPALPUS. Spinola.

Conynetes. Panzer. Schh. Dej. Cat.

1 Scutellaris. Panzer. Austria.

LARICOBIUS, Rosenhauer.

1 Erichsonii. Rosenh. Tyrolis. 39

\section{FAM. PTINI.}

HEDOBIA. (Ziegl.) Latreille.

Prinus. Linné. Fabr, Panz, Duftsch.

1 Pubescens. Fabr. \{ Vulpes. (Dahl.)
Austria. id.

lis, Hope.) Chevrol. id. 
2 Imperialis. Lirné. $\quad P$.*

3 Regalis, Duftsch, Austria.

\section{PTINUS. Linné.}

Bruchus, Geoff.

1 \{ariegatus. Rossi. $\quad \begin{array}{ll}\text { Austria.* } \\ \text { Ornatus. (Dahl.) } & P .\end{array}$ (Pulchellus. (Ziegler.) Dalmatia.

2 Mauritanicus. Lucas. Algiria.*

3 Quadriguttatus. Dej.

Cat. Gall. merid.

4 Sexpunctatus. Panz. Germania.*

5 (Fur. Linné. Gyllenh, P.*

Germanus, Fabr. id.

Clavipes. Panzer. St, Germania.

6 Rufus. Lucas. Algiria.*

7 Lusitanicus. Illiger. Lusitania.

8 Fossulatus. Lucas. Algiria.

9 Italicus. (Chevrolat.) Aragona. Italia.*

10 Carinatus. Lucas. Algiria.

11 Gibbicollis, Lucas. id.

12 Spitzyi. Villa. Italia.

13 Lepidus. Villa. id.

14 Atratus. Sturm. Cat. Hispania.

15 (Pallipes. Duftsch. St. Austria. $\{$ Pallidus. (Megerle.) id.

16 Raptor. Sturm.

17 Bicinctus. (Dahl.)

18 Rufipes. Fabr. Oliv. Elegans. Fabr.

19 Fuscus, Dej. Cat. Germania.* $^{*}$ Austria.

$P_{\text {. }}^{*}$

Germania.

$P$.*

20 Pusillus. Sturm. Cat. Germania.

21 Latro. Fabr. id.

22 Nitidus. Duft. Sturm. Austria. Signaticollis. (Dahl.) id.

23 Rotundicollis. Lucas, Algiria. *

24 Obesus. Lucas. id.*

25 Hirticollis. Lucas. id.*

26 Crenatus. Fabr. Gyll, $\boldsymbol{P}_{\text {. }}^{*}$

27 (Minutus, Duft, Redtb, Austria. Pallipes? Illiger. id. Pinicola. (Ullrich.) id.

28 Pygmrus. Dej. Cat. Gall, orient.

29 Quercus, Gyllenh. P.*

30 Museorum. Doué. id.*

\section{GIBBIUM. Scopoli.}

Ptinus, Fabr, Oliv, Bruchus, Geoff.

1 Scotias. Fabr.

$P$.
MEZIUM. Curtis.

Ptinus. Fabr.

1 Sulcatum. Fabr, Lusitania.

2 Affine. (Gibbium.) Ullr. Germania.

$\{$ Sulcatum. Sturm. id.

Sulcicolle. Stm. Cat. id.

MASTIGUS. Hoffmanseg, Illig, Latreille.

1 Palpalis. Hoffmanseg.

37

\section{FAII, ANOBII.}

ANOBIUM, Fabricius.

1 Pusillum, Gyll. (Dryo-

philus. Chevrolat.) Germania.

2 Denticolle. Pnz. Duft, id.*

3 Crenatum, Dej. Cat. Gallia.*

4 (Paniceum. Fabr. Gyll.

$\begin{cases}\text { St. } & \text { P.* } \\ \text { Var. Ireos. Villa. } & \text { Lombardia. }\end{cases}$

5 Nitidum. Fab. Gyll.St, Germania.*

6 f Rufipes. Fabr. id. ${ }^{*}$

Elongatum. Paykull. Suecia.

7 Substriatum. (Chevrol.) Algiria. *

8 Striatum. Illiger, Gyll. P*

Pertinax. Panzer. Germania.

9 Fulvicorne. Stm, Redt, Austria.*

10 (Pertinax, Lin. L. Redt. id.* $\{$ Striatum. Gyllenhal. Germania.

11 Emarginatum.Duft.St.Austria.

12 Tesselatum. Fab. Gyll.

$$
\text { Panzer. } P \text {. }
$$

13 Plumbeum. Illig. Stm. Germania.*

Politum. Duftschmid. Austria.

Eneicolle. Dahl. Cat. Sicilia.

14 Molle. Fabr. Hbst. St. Germania.*

15 Abietis. Fabr, Duftsch.

Panz, Austria.*

16 Gentile. Rosenhauer. Tyrolis.

17 Nigrinum. (Erich.) St. Germania.

18 Politum. (Meg.) Redtb. id.

19 Vestitum. Dej. Cat. Gall. merid.*

20 Brevicorne. Ratzeb. Germania.

21 Morio. Villa. Lombardia.* 
22 Fucatum, Dej. Cat. Germania.

23 Tomentosum.Dej.Cat. Gall. merid.

\section{Villosum. Bonelli. Italia.}

25 Cylindricum. Dej. Cat. Germania.

26 Variabile. Dej. Cat. id.

27 Cinnamomeum. Sturm. id.

Castaneum. Herbst. Austria.

28 Pini. Erichson. Germania.

29 Abietinum, Gyllenhal. Suecia. ${ }^{*}$

30 Longicorne. Stm. Cat. Germania.

31 Bucephalum.Stm. Cat. Lusitania.

32 Angusticolle. Ratzeb. Germania.

33 Filiforme. Höpfner. id.

34 Oblongum. (Ziegler.) Gall. merid.

35 Minutum, Fabr. Germania.

36 Chevrieri. Kunze, St. Cat. Italia.

37 Castaneum. Olivier. P.*

38 Tricolor. Olivier. id.*

39 Latreillei. L. Dufour. Gall. merid.

40 f Exile. Gyllenhal. St. Germania.*

Immarginatum. Germ, id.

41 Sericeum. Gyllenhal. P.*

42 Costatum. Géné. Italia.

\section{TRYPOPITYS. Redtenbacher.}

Anobium. (Megerle.)

1 Serricornis. Duftsch. Austria.*

\section{OLIGOMERUS. L. Redtenbacher.}

Aловичм. St. Duftschmid.

1 Brunneus, Olivier. Gall. merid.*

OCHINA. (Ziegler. Dej. Cat.) L. Redtenb.

Xyletinus. Latr. Germar. Anobium. Sturm, Pricinus. Germar.

\section{1}

2

Hederæ. Germar. P.*

$\{$ Carpini. Herbst. Austria. \{ Anobioides, Dej. Cat. Germania.

3 Sanguinicollis. Duft. Austria. Latreillei. Bonelli. Lombardia.

\section{ENDECATOMUS, Mellié.}

Anoвium. Herbst. Creutz, Fabr, de Castelnau. Dyctialotus. L. Redtenbacher.

1 Reticulatus. Herbst. Austria. ${ }^{*}$
XYLOGRAPHUS, Dej, Cat. Mellié.

Cis, L. Dufour. Waltl, Lucas. Prinus. Marsh.

1 (Bostrichoides. L. Duf, Gall. merid.* Punctiger. Waltl. id. Cribratus, Lucas. : Algiria. Var, Aubei. Mellié. Sardinia.

\section{(Ropalodontus, Mellié.)}

2 Perforatus. Gyllenhal, Gallia.*

CIS. Latreille.

Gyll. Germar. Dermestes. Scopol. Fabr. Anobidm. Fabr. Herbst. Bostrichus. Kugel. Ptinus. Marsh.

1 Boleti. Scopoli. Gallia.* Variabilis, Fabr, Germania.

Picipes. Herbst. Austria.

Bidentulatus? Oliv. Gallia.

Caucasicus? Ménétr. Russ, merid. Var. Ubliteratus, Mell. Gallia**

- Striatus. Mellié. id.*

- Setiger. Melliẹ. id.

- Minor. Mellié. id.

2 (Rugulosus. (Mannerh.)

Mellié. id.*

Var, Rubiginosus. Mel. id.

- Pyrocephalus.Mel.id.*

3 (Setiger. (Chevrol.) Mel.id.*

Villosus? Marsham, Anglia.

Var, Minor. Melliẻ. Gallia.

4 Fissicollis. (Schh.) Mell. Gall, bor.

5 Tomentosus. Dej, Cat. Mellié. Hispania.

6 Micans, Herbst, Payk, Gallia.

\{Villosus? Marsham. Anglia.

7 Olivieri. Mellié. Gallia.

8 Hispidus. Paykull, id.*

Y Var. Minor. Mellié. id.

9 Striatulus, Mellié. id.

10 Flavipes, Lucas. Algiria.*

11 Comptus, Gyllenhal. Gallia.*

12 Quadridens. (Chevrier.) Mellié. id.

13 Elongatulus, Gyllenh. Suecia.

14 Bidentatus. Olivier. Gallia.*

$\{$ 을 Inermis, Marsham, Anglia.

15 Dentalus. (Gacogne.)

Mellié, Gallia. 
16 Nitidus. Herbst

17 Nitidulus. (Reich.)Mell. Lombardia.

18 Glabratus, (Dej.) Mell. Gallia.*

19 Minutissimus, Mellié, N.

20 Lineato-cribratus. (Chevrier.) Mellié. Gallia.

21 Laminatus. (Er.) Mell. id.*

22 Alni. Gyllenhal. id.* (Punctulatus. Lucas. Algiria.

23 Oblongus. (Schönh.)

24 Punctifer. Mellié. Helvetia.

25 - Punctulatus, Gyllenh. Gallia.

26 Sericeus. Mellié. id.

27 Alpinus. Mellié. . id.*

28 Muriceus. Mellie. $\quad N$.

29 Festivus. Panz. Kugel. Gallia.*

$\{$ Pygmaus. Marsham. Anglia.

30 Castaneus. Melliẻ. Gallia*

31 Fuscatus. Mellie. id.

32 Vestitus. Mellié. jd.*

33 Laricis. (Reich.) Mellié. id. Caricinus. Mellié. id.

34 Bicornis. (Guillebeau.) Mellié. id.

35 Bidentulus, Rosenh. Tyrolis.

36 Concinnus. Marsham. Anglia.

37 Flavus. Stephens. id.

38 Rhododactylus. Marsh. id.

39 Nigricornis. Marsham, id.

40 Ruficornis. Marsham. id.

41 Piceus. Marsham. id.

42 Fagi. Waltl. Germania. \{ Flavipes. Motschulsky. Russia.

43 Betulæ. Zettersted. Suecia.

44 Pallidipennis, St. Cat. Italia.

45 Substriatus. Gysselen. Austria.

\section{ENNEARTHRON. Melliẻ.}

Apate. Panz. Gis. Gyll. L. Dufour. EnTYPUS. L. Redtenbacher.

1 Cornutum. Gyllenhal. Gallia.*

2 Affine. Gyllenhal. id.*

3 Fronticorne. Panzer. id.*

\section{OCTOTEMNUS. Mellié.}

Cis. Gyll. Orophius. L. Redtenbacher.

1 Mandibularis. Gyllenh. Suecia.* Incequidens, Chevrol, Gallia.

2 Glabriculus, Gyllenh. id.*
DORGATOMA. Herbst.

Fabr. Brdchus, Fabr. Dermestes, Panz. Serrocerus, Kugelann.

1 Rubens. Ent. Heft. St. Gall, bor.*

2 Flavicornis, Fabr. St. Austria.

3 Dresdensis, Herbst. P.*

Bistriatum. Paykull, Suecia.

Serra. Panzer.

4 Bovistæ. Ent. Heft. St. id.

5 Zusmeshausensis. Beck, id.

6 Puberula. Dej. Cat. Russ. merid.

7 Chrysomelina, Sturm. Germania.*

8 Striatopunctata, de Castelnau. $P$.

9 Meridionalis, de Castl, Gall. merid.

XYLETINUS, Latreille.

Ptilinus, Fabr. Panz. Anobium. Illig. XYlmurinds. Sturm.

1 Pectinatus, Fabr. : Germania.*

2 Ater. Panz. Duftsch. Austria.*

3 Laticollis. Duftsch. St. id.

4 Testaceus. Duftsch.St. Hungaria. *

Flavescens. (Dahl.) id.

5 Hederæ. L. Dufour. Gall. merid. Lavis. Latreille. id.

Cardui. Dej. Cat. id.

Niger. Germar. Austria.

Murinus. Sturm. Germania.

Flavicornis, Melsh. id.

Lavis. Illiger. Austria.

6 Cylindricub. Germar. Dalmatia.

7 Pallens. Stéven. Russ. merid.

8 Hæmorrhous. Stéven. Dej. Cat. id.

9 Limbatus.Stv, Dej.Ct. id.

10 Flavipes. Dej. Cat. Styria.

11 Tibialis. Dej. Cat. Dalmatia.

Niger. Stéven. Russ. merid.

12 Subrotundatus. (Ziegl.)

Dej. Cat, Gall, merid.

13 Nigripes, Dej. Cat. id.

14 Flavescens, Dej. Cat. Hisp. merid.

15 Tomentosus. Dej. Cat. Dalmatia.

PTILINUS. Geoffroy.

Prinus. Linné.

1 Pectinatus, Linné. P.* $\{$ Var, Elongatus. (Par.) Croatia. 
2 Costatus. Gyllenh. St. P.*

Flabellicornis, Dej.

Cat. id.

Pectinicornis, Illiger, Austria.

APATE. Fabricius.

Bostrichus, Olivier.

1 (o Francisca. Fabr. Algiria.* ㅇ Carmelita. Fabr. id. Monacha. Fabr. Brasilia.

Rufiventris. Lucas, 'id.

\section{XYLOPERTHA. Guérin.}

Bostrichus. Olivier.

1 Sinuata. Fabr. Gall. orient.*

2 Chevrieri. Dej. Cat.

3 Picea. Olivier. Algiria.*

4 Humeralis. Dej.Cat. Lucas. id. *

5 Appendiculata. Dej. Cat. Lucas. id. ${ }^{*}$

6 ? Capillata. (Dahl.) St.

Cat. Hungaria.

7 ? Asperata. Stm. Cat. Tyrolis.

\section{SYNOXYLON. Duftschmid.}

Sinodendron. Fabr. Panz. TrypoclaDUs. Guérin.

1 Muricatum. Fabr. Pnz. Austria.* I Bispinosum. Olivier. Illyria.

2 Sexdentatum. Olivier. Gall. merid.*

\section{RHIZOPERTHA. Stephens.}

Guérin. Sinodendron. Fabr.

1 Pusilla. Fabr.

Algiria.*

BOSTRICHUS. Fabricius.

Dermestes. Linné.

1 Luctuosus. Olivier. Gall. merid.*

2 Anthracinus. (Chevrl.) Algiria. ${ }^{*}$

3 Nigriventris, Lucas. id.*

4 Capucinus. Linné. Fab. Gallia.*

5 ) Dufourii. Latreille. id.*

\{ Gallicus. Panzer. id.
6 Bimaculatus, Fabr. Gall. merid.

7 Dactyliperda. Panzer. Germania.*

DINODERUS. Stephens.

Guérin. Apate. Payk. L. Redtenbacher.

1 fo Substriatus. Paykull.Suecia.

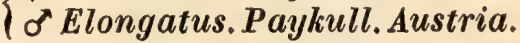

PSOA. Herbst. Fabr, Latr.

1 Viennensis. Hbst. Pnz. Austria.*

2 Italica. Dej. Cat. Italia.*

3 Herbstii. Klug. Grocia?

149

\section{FAII. LYMEXYLONES.}

HYLECOETUS, Latreille.

Lymexylon, Fabr, Oliv.

1 Dermestoides, Fabr. Alsatia.* o Proboscideus. Fabr. Germania.

2 Morio: $\mathrm{Fabr}$. Marci. Olivier. id.

Barbatus. Panzer, Germania.

Dermestoides. (Cantharis.) Linné. id.

LYMEXYLON, Fabr.

Gantharis, Linné.

1 Navale. Linné. Fabr, P.* 3

\section{FAII. BOSTRICHI.}

PLATYPUS. Herbst.

Bostrichus, Fabr, Panzer, Cylindra, Duftsch.

1 f Cylindrus. Fabr.Panz.P.* Bimaculatus. Fabr. Germania. 
TOMICUS, Latreille.

Dermestes. Linné. Bostrichus. Fabr. 27 Bostrychus. L. Redtenbacher.

1 Typographus, Linné. $P_{.}^{*}$ Stenographus, Fabr. Germania.

2 Stenographus. Duftsch, Gallia.* Typographus. Fabr. Germania.

3 Cembræ. Heer. Helvetia.

4 Laricis. Fabr. Germania. *

5 Geminatus. Sahlberg. Finlandia.

6 (Suturalis, Dej. Cat.

Ratzeburg. Germania. o Nigritus? Gyllenhal. Suecia.

7 Calcaratus. Dej. Cat. Dalmatia.

8 (Acuminatus. Gyllenh. Ratzeb. Germania. Iconographus, Kugln, id.

9 (Bispinus. (Meg.) Ratzb. id. ${ }^{*}$ o Sculptor. (Dahl.) id. Retusus, Olivier. Gallia.

10 Curvidens, Germ.Rtzb. Germania. of Psilonotus. Germar. id. ơ Calligraphus. Duft. Austria. ? Orthographus, Duft. id.

11 Monographus. Fabr. Ratzeb. Gallia.*

12 Populi. Duft. Ratzeb. Germania.

13 Chalcographus. Linné. id.

14 Bidens, Fabr, Ratzeb, Alsatia.* o Bidentatus. Herbst. Germania. \{ Chalcographus. Pay. kull, Suecia.

15 Sexhamatus. Chevrol. Gallia?

16 o' Cornutus. Chevrolat. Lombardia. o Diodon. Schüppel. id.

17 Modestus. Chevrolat. Gallia.

18 Robustus. Chevrolat. id.

19 Parvus. Chevrolat. id.

20 (Autographus. Knoch. $\left\{\begin{array}{c}\text { Ratzeb. } \\ \text { Villosus. Gyllenh. }\end{array}\right.$

21 Cryptographus. Knoch. Ratzeb. Germania.

22 Dactyliperda. Fabr. Austria.* Castaneus, Stm. Cat. id.

23 Villosus. Fabr. Ratzeb, Germania.

24 (Bicolor. Hbst. Ratzeb. id.* Fuscus, Gyllenhal, Suecia. Retusus, Dej. Cat. Austria.

25 Obscurus, Dej, Cat. Styria.
26 Dispar. Hellwig. Fabr. Alsatia.* Brevis, Panzer. Germania. Thoracicus. Panzer. id.

Dryographus. Erich.

Ratzeb. id.*

28 Saxonii. Ratzeburg. Saxonia.*

29 Eurygraphus. Erich. Germania.*

30 Pfeilii. Ratzeburg. id.

31 Longicollis, Gyllenhal. Suecia.

32 Aphodioides. Villa. Lombardia.

33 Denticulatus, Chevrol. P.* Micrographus. Oliv. id.

34 Cucullatus. Chevrolat, id.

35 Olivieri. Chevrolat. Alp, Gall.* Melanographus. Oliv. Algiria.

36 Fuscus. Marsham. Austria.

\section{GRYPHALUS, Erichson.}

de Castelnau. Redtenb. Apate, Fabr. Panz.

1 Tiliæ. Fabr, Ratzeb. Germania.*

2 Fagi. Fabr. id.

3 Piceæ. Ratzeburg. id.

4 Binodulus. Weber. $R a$ tzeburg. Alsatia.

5 Asperatus. Gyll. Ratz. Germania.

6 Abietis. Ratzeburg. id.

7 Granulatus, Ratzeb. id.

8 Serraticollis. Ullrich. id.

9 Carinatus. Waltl. Tirolis.

10 Pini. Villa. Lombardia.

\section{HYPOBORUS. Erichson.}

de Casteln. Bostrichus. Dej. Cat.

1 Ficus. Erich. de Cast. Gall, merid. Fici. Dej. Cat. _ id.

\section{GRYPTURGUS. Erichson.}

Bostrichus. (Dej.) Gyllenh.

1 Cinereus, Hbst, Ratzb. Germania.*

2 Pusillus. Gyll. Ratzb. id.*

3 (Troglodytes. Dej. Cat. Croatia.

\{ Pusillus. Stéven. Russ, merid.

4 Porcatus. Dej. Cat. Dalmatia.

5 Melancholicus. Chevrl. Gallia.

6 (Pytiographus. Ratzeb. Germania.* Micrographus. Gyll. Suecia.

Lichtensteini, Ratzeb. Germania.

7 Exculptus. Waltl. id. 
HYPOTHENEMUS. Westwood. Casteln.

1 Eruditus. Westwood. Anglia.

\section{XYLOTERUS, Erichson.}

de Casteln. Dermestes. Linné. Bostrichus. Gyll. Apate, Fabr. TrypodénDRON. Steph.

1 Domesticus. Linné. Gall. bor.* $\{$ Limbatus, Fabr. Austria.

2 (Lineatus. Gyll. Ratzeb. Germania.* Signatus. Fabr. Austria. Marginicollis. (Dahl.) id.

$$
58
$$

\section{FAII. HYLESINI.}

\section{SCOLYTUS. Geoffroy.}

Eccoptogaster. Herbst. Coptogaster. Duft. Ips, Ratzeb. HrLesinus, Duft.

1 (Destructor. Oliv. Duft. Gallia.* $\begin{cases}\text { Betula. Guérin. } & P . \\ \text { Scolytus, Ratzeburg. } & \text { Germania. }\end{cases}$

2 Pygmæus. Fabr. Duft. Gallia.*

3 Multistriatus. Marsh. Germania.*

4 Intricatus, Koch, Ratz. Alsatia.*

\{Pygmous. Gyllenh. Suecia.

5 Ulmi. L.Redtenbacher. Austria.

6 Pruni. Ratzeburg. Germania.*

7 Pyri. Ratzeburg. id,

8 Rugulosus. Knoch. Ratzeb. id.*

9 Carpini, Erichson. id.*

10 Castaneus, Koch. id.

11 Noxius. Andersch. id.*

12 Armatus. Chevrolat. P*

13 Pomorum. Chevrolat. id.*

14 Flavicornis. Géné. Sardinia.*

\section{POLYGRAPHUS, Erichson}

Hylesinus. Fabr. Dermestes. Linné.

1 Pubescens, Fabr. Gallia.* Polygraphus, Linné. Germania.
PHLOEOTRIBUS, Latreille.

Hylesinus. Fabr. Scolytus, Oliv.

1 Oleæ. Fabr.

Gall, merid.*

\section{HYLESINUS, Fabricius.}

Anthribus, Fabr.

1 Crenatus. Fabr. Duft. Gallia.*

2 Oleiperda. Fabr. Gall. merid.

3 Varius, Fabr, Oliv. Suecia. ${ }^{*}$

Fraxini. Fabr. Duft. Gallia.

Melanocephalus. Fab, Algiria.

Pubescens. Fabr. Gallia.

4 Luridus, Dej. Cat. id.

5 Sordidus, Dej, Cat. id.*

6 Betulæ. (Chevrolat.) P.*

7 (Serraticornis. Dej. Cat. Dalmatia.

\{ Cornutus. Ullr. (Cis.) Illyria.

8 Vittatus, Fabr. Duft. Austria.

9 Impressus. Olivier. $\quad P$.*

10 Sus. (Chevrolat.) Lombardia.*

DENTROCTONUS. Erichson.

Hylesinus, Fabr. Dej. Cat.

1 Micans. Kugelann. in

$\left\{\begin{array}{c}\text { Schneid. Germania.* } \\ \text { Ligniperda. } \\ \text { Ratzeb. Austria. }\end{array}\right.$

2 Pilosus. Knoch, Ratzb. Germania.

3 Minimus. Fabr, Ratzb, id.

4 Hederæ. Schmidt. id.*

HYLURGUS. Erichson.

Dermestes. Linné. Hylesinus. Fabr.

1 Ligniperda. Fabr. Germania.*

Flavipes, Panz. Ratz. id.

Elongatus, Herbst. Austria.

2 f Piniperda. Linné. Germania.

Testaceus. Fabr. id.

3 Minor, Hartig. id.*

HYLASTES. Erichson.

Bostrichus, Payk. Hylügus. Latr. Dej. Cat. Hylesinus. Gyllenh.

1 Decumanus. Erichson. Germania. \{ Paykulii? Duftsch. Austria. 
2 Palliatus. Gyllenhal. Germania.* $\{$ Angustatus. Herbst. id. Marginatus, Duftsch. Austria.

3 Attenuatus. Erich, Ratzeburg. Germania.*

4 Angustatus, Herbst. id.*

5 Crenatulus. Duftsch. Austria.

6 Ater. Paykull. Alsatia.* \{ Chloropus. Duft.Ratz.Germania.

7 Cunicularis, Erich. Ratzeburg. id.

8 Rhododactylus. Marsh, id.

9 Opacus. Illiger. id.

10 Linearis, Ratzeburg. id.

11 Brunneus. Ratzeburg. id.

12 |Trifolii. Müller, Erich. id.*

\{Crenatus, Olivier, Gallia.

13 Glabratus. Zettersted. Suecia.

14 Gyllenhalii. Schmidt. Germania.* \{ Angustatus. Gyllenh. Suecia.

15 (Socius. (Chevrolat.) $P$. \{Affinis. Dej. Cat. id.

16 Brevicollis. Dej. Cat. Lombardia.

17 Ferrugineus. Stm. Cat, Germania.

18 Sericeus, Dej. Cat. $P$.

19 Juniperi. (Chevrolat.) Helvetia.

20 Chevrieri. Solier. id.

21 Abietis. (Chevrolat.) id.

22 Cylindricus. Dej. Cat. Gall. merid.

55

\section{FAII. CURCULIONES,}

\section{DRYOPHTHORUS. Schüppel.}

Schh. Latr. Boisd. (Dej. Cat.) Stéven. Bulbifer. (Megerle. St. Dej. Cat.) CAhandra. (Dej. Cat.) Cossonus. Oliv. Gyll. Lixus et Calandra. Fabr. Rhynchophorus et Curculio. Herbst.

$1 \begin{cases}\text { Lymexylon. Fabr. } & \text { Gallia.* } \\ \text { Corticalis. Paykull. } & \text { Suecia. }\end{cases}$

RHYNCOLUS. Creutzer. Germar.

(Megerle. Dej. St. Cat.) Latr. Steph. Stév. Schh. Cossonus. Germ. Gyll. Hylesinus. Fab. Gurculio. Linné. Payk. H'st.

1 Cylindricus. Schh. Dalmatia.* \{ Longicollis. (Chevrol.) id.
2 Chloropus. Fabr.

Ater. Linné.

Linearis. Schrank.

Germ. bor.*

id.

Exaratus. Linné. id.

3 Elongatus. Gyllenh. id.

Planirostris. Panzer. Suecia.

4 (Porcatus. Müll. Germ. Pyrencis.*

Crassirostris. Dej.Cat.P.

Puncticollis. (Ziegler.) Germania.

5 Culinaris. (Reich.) Ger-

mar. Gallia.*

6 Exiguus. Schh. Bavaria.

7 Submuricatus, Schh. Gallia.*

8 Truncornm. Gyl. Schh. id.*

9 Cylindrirostris. Oliv. id.

Lignarius. Marsham. Anglia.

10 Reflexus. Schh. $\boldsymbol{P}^{*}$

11 Punctatulus. (Ziegl.)

Schh. Gallia.*

12 ? Populi. Dej. Cat. Gall. merid.

13 ? Exaratus. Dej. Cat. id.

14 Cribricollis. (Chevrol.) Algiria.*

15 Ventricosus. Villa. Pedemont. ${ }^{*}$

16 Strangulatus. (Chevrl.) Pyr. orient. ${ }^{\star}$

17 Ovalis. (Marietté.) Lombardia.*

18 Cellaris. Perris. Gall. merid. *

\section{COSSONUS. Clairville.}

Fabr, Latr, Germ, Gyllenh. Oliv, Cuv. Illig. Thumb. Zett. Steph. Walk. Schh. Gurculio. Linné. Herbst. Payk. HyleSINUS. Fabr.

1 (Linearis, Fabr. Herbst. Gallia.*

$\{$ Var. Ferrugineus. Dej.

2 (Cylindricus. (Dej. Cat.)

Cylindricus. (Dehh. id.*

Linearis. Payk. Gyll. Suecia.

Parallelipipedus. Hbt. Austria.

Molleri. Waltl. Germania.

Corticollis. (Stm.Cat.) id.

Linearis, var. b. Gyll. Suecia.

3 Ferrugineus. Clairv. Helvetia.*

Linearis. Paykull. Suecia.

Parallelipipedus. Hbt. Austria.

Ferrugineus. var. $b$.

Gyllenh. Suecia.

4 Angulatus. Stm. Cat. Hungaria.

5 Ilicis. Mac-Leay. (Dej.

Cat.) Anglia. 
6 Longirostris. (Megerle. Dej. Cat.) Lombardia.

7 Scabricollis. (Dej. Cat.) Gall. merid.

\section{PHI_OEOPHAGUS. Schönherr.}

Cossonus. Oliv. Gyll. Rhyncolus. Steph. Dej. Cat. Gurculo. Herbst. Marsham.

1 Eneopiceus. Kirby. Anglia.*

Truncorum. Stephens, id.

2 Turbatus, Schh. Suecia.

3 (Spadix. Schh. Herbst. Gall. merid.* Velutinus. Dej. Cat. id.

Globulipennis. Chevrolat. in litt. Algiria.

4 Sculptus. Schh. Suecia.*

5 Uncipes. Chevrl. Schh. Italia.

6 Lignarius. Marsham. Anglia.*

\section{MESITES. Schönherr.}

Calandra et Cossonus. (Dej. Cat.)

1 Tardii. Vigors.

Anglia.

2 Pallidipennis. Schh. Tauria.*

\{alandroides.Dej.Cat.Gall. merid.

3 Cunipes. Solier, in litt,

Schh.
4 id.
Rarus. Chevrolat,
Algiria.

SITOPHILUS. Schönherr.

Rhyncophorus, Herbst. Schh. Galandra. Clairv. Fabr. Illig. Oliv, Germ. Gyll. Steph. (Dej. St. Cat.) Stév. Curculo. Linné.

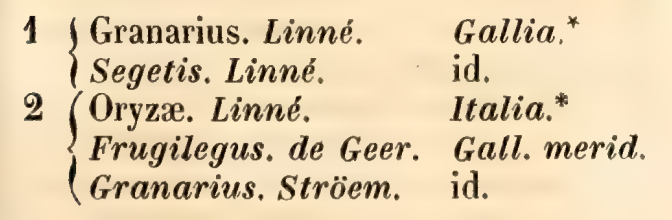

\section{SPHENOPHORUS, Schh.}

Rhynchophorus. Herbst. Schh. GalanDRA. Clairv. Fabr. Illig. Oliv. Germ. Latr. (Dej. St. Cat.) Curculo. Linné. Pallas.

1 Piceus, Pallas.

Gall, merid.*
2 Abbreviatus. Fabr. Decurtatus Linné. Suecia. Brachypterus.Olivier. Gallia. o Scotina. Germar. Germania. Porcula. Fabr. id.

3 Parumpunctatus. Schh. Gall. merid.

4 Opacus. Schh. Italia. Maurus. Meg. in litt. id.

5 (Mutilatus. Laicharting. Gall. merid.* Abbreviatus. Herbst. Austria. Fimbriatus. Linné. id.

6 Meridionalis. (Dej. Cat.) Schh. id.*

\section{NANOPHYES. Schönherr.}

Cronus. Clairv. Germ. Oliv. Nanodes. Schh. Spharulus. (Megerle.) Stephens. Orobitis. (St. Dej. Cat.) Rhynchanus. Fabr. Gyll. Zett.

1 Siculus. Schh. $\{$ Globulus.(Desjeardin.) id.

2 Annulatus. (Chevrol.) Géné. Italia.

3 Hemisphæricus, Oliv. Sicilia.

4 Lythri. Fabr. P.* Salicarice. Olivier. id. Pygmaus. Herbst. Austria. Fasciatus. Villers. Gallia. Leucozonius. Linné. id. Transversus. Olivier. id.

5 Chevrieri. Germar. Helvetia.

6 Gracilis, Redtenbach. Austria.

7 Lateralis. Rosenhauer. Tyrolis.

8 Globulus. Germar. Germania.

9 Brevis. Schh. Helvetia.

10 Ulmi. (Megerle.) Schh. Austria.

11 (Languidus. Schh. Sicilia.*

Tamarisci. Helfer. in litt. id.

12 Nitidulus. Hoffmanseg. Schh. Lusitania.

13 Tamarisci. (Dej. Cat.)

14 Pallidus. Olivier. Lusitania. ${ }^{*}$

15 Pallidulus. Gravenhst. Gall, merid. S Bimaculatus. Dej.Cat. id.

Sahlbergi. Schh. Finlandia.

Marmoratus, Fourcroy. Gallia.

18 Duriœi. Lucas. Algiria.

19 Obtusus. Chevrolat. Gallia.

20 Posticus. (Dej. C'at.) Gall. merid. 


\section{MECINUS. Germar.}

Schh, Latr. Steph. (Dej. St. Cat.) Ahrens. 14 Rhynchanus. Gyllenh. Neptaphilus, 15 (Megerle.) Macipus. Stéven.

1 Pyraster. Herbst. Gallia. ${ }^{*}$ Semi-cylindricus. Gyl. Suecia. ơ Cerasi. Paykull. id. Var. Hamorrhoidalis. Herbst. Austria.

Denigratus. Linné. Suecia.

2 Barbarus. Schh. Algiria.

3 Longiusculus. Chevrolat. Schh. Corsica.

4 Teretiusculus. Schh. Lusitania.

5 Collaris. Germar, Austria. Cinctus. Rossi. Italia.

6 Janthinus. Germar. Gallia. Violaceus. (Dej. Cat.) id.

7 Circulatus. Marsham, id.* Marginatus. Germar. id. Homorrhoidalis, Stph, Anglia.

8 Comosus. Schh. Lusitania,

9 Fimbriatus. Germar. Germania.

\section{GYMNETRON. Schönherr.}

Steph. (Dej. Cat.) Gronus, Germ. Oliv. Mianus. Schh. Steph. Rhinusa. Kirby. Steph. Cleopus. (Megerle. Dej. St. Cat.) 36 Rennceanus. Fabr. Latr. Gyll. Zett. 37 Sahlb. Curculio. Linné. Herbst.

1 Sascuorum. Gyllenh. Gallia. \{ Nanus. Dej. Cat. id.

2 Bicolor. Schh.

3 Melas. Schh. Gall. sept.

4 Villosulus. Schh. \{ Dorsalis. Olivier.

5 Veronicæ. Germar. Beccabunge, var, b.

Gyllenh. id.

6 Beccabungæ. Linné.

Gallia. Gall. merid. Germania.

7 Concinnus. Schh.

8 Ictericus, Schh.

9 Labialis. Herbst. Tricolor. Gyllenhal. (Obiquus. (St. Cat.)

10 Rostellum. Schh.

id.*

Volhynia.

Hungaria. Gallia.*

Suecia.

Germania. id.

11 Melanarius. Germar, id. Intaminatus. Steph. Kirby. Anglia.

12 Perparvulus. Schh.

Saxonia.

13 Stimulosus. Germar.

Germania. Rotundicollis. Schh. Tauria.

Teter, Fabr.

Crassirostris. Lucas. Algiria.

Comosus. Chevrolat.

Vulpes. Lucas. Schh. Gracia.

f Asellus. Gravenhorst. Germania.

Polonicus. Schh. Polonia.

Nasutus. Rosensch.

Schh. Gracia.

Plagiatus. Gyllenh. Volhynia.

Plagiellus. Schh. Odessa.

Fuscescens. Schh. Tauria.

Antirrhini, Germar. Gallia.*

f Noctis. Herbst. id.*

Antirrhini. Gyllenhal. id.

Fuliginosus. Rosenh. Hungaria.

Collinus. Gyllenhal. Gallia.

Netus. Germar.

Borussia.

Pilosus. Besser. Sch. Podol. Germ.

Amictus, Germar. Lusitania.

Vestitus. Germar. id.

Cylindrirostris. Gyll. Gall. merid.* Thapsicola, Müll. Schh. Bavaria.

(Spilotus. Germar. Gallia.*

Bipustulatus. Rossi. Italia.

Ellipticus, Dej. Cat. Gall, merid.

Hæmorrhous. Rosenh. Hungaria.*

(Linariæ. Panzer. Germania.*

Teter. Panzer. id.

Orontii. Hellw. in litt.id.

38 Longirostris.(Dej.Cat.) Schh. Gall. merid.*

Graminis. Gyllenh.

Plantarum. (Dej. Cat.)

Germar. Gall. merid.*

41 Campanulæ. Linné. Gallia."

Distinctus. Chevrier.

\section{Schh. Helvetia.}

\section{Micros. Germar.}

Germaria.

Hispidus. St. Cat. Italia.

Verbasci.Ros.Dej.Cat.Gall. merid.* Rufirostris Stm. Cat. Germania. Scolopax. Dej. Cat. Gall, merid. Hæmorrhoidalis, Dej. Cat. id.

Brevirostris, Dej. Cat, id.

Angustatus. Dej. Cat. P.

Minutus. Dej. Cat. Hispania.

Herbarum, Dej, Cat. P.* 
CIONUS, Clairville.

Latr. Germ. Oliv. Illig. Cuvier. Schh. Zett. (Dej. St. Cat.) Steph. Stév. Oken. Lamarck. Guérin. Gleopus. (Megerle.) Stephens, Rhynchenus, Fabr.

1 Scrophulariæ. Linné. Gallia.* Blattaria. Voet. Germania. Verbasci. Voet. id.

2 Verbasci. Fabr. Gallia.* $\{$ Scrophularia, var, $d$. Latr.'id.

Tuberculosus. Scopoli. Carniolia.

3 Olivieri, Chevrol. Schh, Gall. merid.* Thapsus. Olivier. id.

4 Thapsus. Fabr. Gallia.* Thapsi. Germar. Germania.

Scrophularia. Latr. Gallia. Hortulanus, Fourc. $\boldsymbol{P}$. Afinis. Harrer. id.

5 Ungulatus. Germar. Gallia. fo Ocellatus, Hoffrisg.

(Pictus. (Dahl.) litt. id.

6 Hortulanus. Marsham. Gallia.* Thapsus, var. (Dej. (Cat.) id.

7 Clairvillei. Schh. id.

8 Simplex. Rosenschöld.

9. Olens. Fabr. Gallia.*

10 Blattariæ. Fabr. Germania.* Bipustulatus, Marsh. Anglia.

11 Pulvereus. (Parreyss.)

Schh. Dalmatia.

Fœtidus. Fabr.

Geeri. Linné. id.

Var.Rectangulus, Hbt.Germania.

13 Pulchellus. Herbst. id.*

Solani. Gyllenhal. Suecia.

Immunis, Marsham. Anglia.

14 Solani. Fabr. Germania.*

Setiger. Germar. id.*

Setosus. Rossi. Italia.

Spinosulus. Megerle.

Coll. Austria.*

15 Mixtus, Dej. Cat. Schh. (Phytonom.) Schh, P.*

16 Trinotatus, Fischer. Russ, orient.

17 Ocellatus. Illig. (Dej.

$$
\text { Cat.) Lusitania. }
$$

OROBITIS. Germar.

Latr. Steph. (Dej. Cat.) Stév, Rнумснжnus. Gyllenh. Sahlb. Atrelabus. Fabr. Panz. Curculio, Linné. Herbst.

1 $\begin{cases}\text { Cyaneus. Linné. } & \text { Gallia.* } \\ \text { Globosus. Linne. } & \text { Suecia. } \\ \text { Giganteus. Meg. Coll. } \text { Germania. } \\ \text { Hypoleucus, Quensel. } \text { Suecia. } \\ \text { Croceus. Fabr. } & \text { id. }\end{cases}$

RHYTIDOSOMUS. Schönherr.

Ceutorhynchus.' (Dej. Cat.) Germar. Rhynchenus. Gyllenh.

1 Globulus. Herbst. Gallia.*

TAPINOTUS. Schönherr.

(Dej. Cat.) Atrelabus. Fabr. CeutoRHYNCHUS. Germar.

1 Sellatus. Fabr. Gallia.*

Lysimachia, Olivier, id.

Ephippiger. Schh. Germania.

\section{ACENTRUS. Chevrolat. Schh.}

1 Histrio, Schönherr. Gall. merid.*

POOPHAGUS. Schönherr.

Rhynchanus, Fabr, Oliv, Gyll. ChutoRHYNCHUs, Dej. Cat. Schh.

1 Sisymbrii. Fabr. Gallia.*

2 Olivaceus. Schh. id.

3 Nasturtii. Spence. id. ${ }^{*}$

Seniculus, Dej, Cat. id.

4 Nebulosus. Stm. Cat, id,

\section{RHINONCUS. Schönherr.}

Campylinhynchus. (Megerle. Dej. St. Cat.) Stev. Geutorhynchus. Germar. Schh. Rhynchenus, Fabr. Oliv, Gyll. Zett.

1 Inconspectus, Herbst. Gallia. Suturalis. Olivier. id.

Crassus. Marsham. Anglia. Occipitrinus. Reich. Austria. Canaliculatus, Steph. Anglia. 
2 Castor, Fabr. Gallia.*

Pericarpius. var. Lin. Suecia.

Fructiculosus. Herbst. Austria.

Seniculus. Gravenh. id.

4-cornis. Dej. Cat. Gallia.

Var. Flavipes. Stéven. Russia.

Leucostigma. Marsh. Anglia.

Scabratus. Fabr. Germania.

Rufipes. Stephens. Anglia.

Interstitialis. Steph. id.

3 Bruchoides. Herbst. Germania.*

\{ Var. Leucogaster. Gyll. Suecia.

Rufescens. Stephens. Anglia.

4 (Pericarpius. Fabr. Gallia.*

Spartii.Kirby. Anglia.

Gramineus. Germar. Germania.

5 Gramineus. Fabr. id. ${ }^{*}$

6 Subfasciatus. Gyllenh. Gallia.* \{tibialis. Stephens. Anglia.*

7 Guttalis. Gravenhorst. Germania.*

Scutellatus. Stm. Cat. id.

Erythrocnemus, Beck. id.

Perpendicularis, Rch. id.

8 Denticollis. Schh. Italia.

9 Albicinetus. Schh. Gallia.

10 Grypus. Herbst. Germania.

11 Canus. Dej. Cat. Anglia.

12 Granulipennis. Chevrl, Gallia.

13 Nigrescens. Stm. Cat. Germania.

14 Obsoletus. Erichson. id.

15 Brunnipes. Stm. Cat. id.

16 Parocnlus, Dahl. Cat. id.

\section{GEUTORHYNCHUS. Schüppel.}

Germar. Latr. Dej. Cat. Schh. Nedyos. 30 Steph. Falciger. Megerle. St, Cat. 31 Stév. Dej. Rhynchenus. Fabr, Oliv. 32 Gyll. Zetterst.

1 Topiarius. Germar. Hungaria.

Tessullatus. Stm. Cat. id.

2 Albovittatus. (Dahl.)

$$
\text { Schh. id.* }
$$

3 Macula-alba. Herbst. Germania.* Cardui. Olivier. Gallia.

4 Suturalis. Fabr. id.*

5 Flavomarginatus, Luc, Algiria.*

6 Alboscutellatus. Che-

7 Seriatus. Schh. $P$

8 Steveni, Schh. Tauria.
9 Arator. Schh. Germania.

Cinerascens. Nees. id.

10 Syrites. Germar. Gyll. Gallia. Abstrictus. Stephens. Anglia. Affinis. Panzer. Germania. Assimilis. Dej, Cat. Austria. Alauda. Fabr. Gallia.

11 Assimilis. Payk. Gyll. id.* Abstrictus. Marsham. Anglia. Alauda.Herbst. Germania.

12 Austerus. Schh. $\quad$. $^{*}$

13 Melanarius. Kirb.Schh.Anglia.*

14 Hepaticus. Schh. Gallia.

15 Consputus. Germar. id. Agrotus. Schh. Germania.

16 Depressicollis. Gyll. Suecia.* \{ Nigrinus, Marsham. Anglia.

17 Erysimi. Fabr. Panz. Germania.*

18 Rubescens. Schh. Austria.

19 Coerulescens. (Dej. Cat.) Schh. Gallia.

20 Marginicollis, Chevrolat. in litt. Gall, merid.*

21 Contractus. Marsham. Germania.* Minutus. Sturm. Cat, id.

22 Atomus. Germar. Illyria.*

23 Atratulus, Gyllenh. Gallia.

24 Setosus. Märkel. Schh. Saxonia.

25 Cochleariæ。 Gyllenh. Gallia.*

26 Cyanopterus. Redtenb. Austria.

27 Quercetti. Gyllenh. Suecia mer.

28 Apicalis. Gyllenh. Germania.*

29 (Terminatus. Herbst. Gallia.

Sii. Chevrolat. id.*

Homorrhoidalis. Dej.

Cat. id.

Pumilio. Gyllenhal. Suecia.

Asperulus. Aubé.Schh. P.*

Posthumus. Illiger. Germania.*

33 Floralis. Payk. Gyll. Gallia.*

Thypha. Herbst. Austria.

Sulculus. Kirby. Anglia.

Monostigma. Marsh. id.

34 Pulvinatus. Gyll. Schh. Gallia.*

35 Constrictus. Marsham. id.

36 Fallax. Schh. Tauria.

37 Convexicollis. Schh. Germania.

38 Pyrrorhynchus. Marsh. Gallia.

Erythrorhynchus. Sch. id.

Phoorhynchus. Marsh. Anglia.

(Var. Ruficrus. Marsh. id.

39 | Achillæ. (Hellw.) Schh. Germania.

I Millefolia. Hoffmsg. id. 
\{ Rufirostris. Dej. Cat. i

45 Albosetosus. Schh. id.

46 Sphærion. Märk. Schh. Saxonia.

Globosus. St. Cat. id.

47 Variegatus. Olivier. Gallia.

48 Tuber. Reich. Schh. Germania.

49 Fossarum. Reich. Schh. id.

50 Minutus. Reich. Schh. id.

51 Pultiarius. Fourcroy. $\boldsymbol{P}$.

Pygmaus. Olivier. Gallia.

Oculeatus. Linné. id.

52 Echii. Fabr. Panz. id.*

Glyphicus. Schaller. Germania.

Geographicus. Villers. Gall, orient.

53 Glaucinus. Waltl.Schh. Bavaria.

54 Radula. Schüp. Germ. Germania.

รัว (Horridus. Fabr. (Ull-

$\left\{\begin{array}{r}\text { rich.) Panz. } \text { Gallia. }^{*} \\ \text {. }\end{array}\right.$

(Spinosus. Linné. id.

56 Viduatus. Gyllenh. Suecia. ${ }^{*}$

57 Raphani. Fabr. Germania.*

Symphyti. de Heyden. id.

Andreopiginis. Koch. id.

58 Borraginis. Fabr. Gallia.*

59 Lineatus. Schh. Tauria.

60 Abbreviatus. Fabr. Germania.*

Invasor. Herbst. Austria.

Variegatus. Gravenh. Germania.

Atomarium. Ziegler. in litt. id.

61 Crucifer.Oliv. Gyllenh.

Herbst. id.*

Trimaculatus. Gyll. id.

Quadrimaculatus.

Germar. id

62 Aubei. Schl. P.

63 T.-Album. Schh. Hungaria.

64 Andreæ. Germar. Germania. Ornatus. Schh. Volhynia.

65 Peregrinus. Chevrol.

66 Uroleucus. Chevrolat. Schh. Sicilia.

Sehh. Sardinia.

67 Litura, Fabr. Gill. Gallia. Cruciger. var. Herbst. Germania.

Ovalis. Marsham. Anglia.

Leucomelas. Kirby. in litt. id
68 Trimaculatus. Fabr.

Oliv. Germania.

69 Trisignatus. Schh. Tauria.

70 Albosignatus. (Dahl.) Schh. Gallia.*

71 Melancholicus. Schh. Tauria.

72 Asperifoliarum. Kirby. Gyll. Germania. *

Intersectus. Knoch. Anglia.

Congener. Schüppel. Austria.

Leucostigma. Germar.

in litt. Germania.

Oleacus. Scopoli. Carniolia.

73 Lepidus, Schh. Alsatia.

74 Uliginosus. Walton. Anglia.

75 Urticæ. Walton. id.

76 Abruptestriatus. Schh. Tauria.

77 Signatus. (Dahl.) Schh. Germania.

Lamii. Germar. in litt. id.

78 Sahlbergi. Schh. Finlandia.

Lamii, Sahlberg. id.

79 Campestris. Zgl. Schh. Bavaria.*

$\left\{\begin{array}{r}\text { Var.Variegatus. Waltl. } \\ \text { in litt, id. }\end{array}\right.$

80 Decoratus. Schh. Tauria.

81 Molitor. Schh. Sicilia.

82 Chrysanthemi. Germar.

\{ Gyll. Germania.*

Rugulosus.var.c.Gyl.Suecia.

83 Figuratus. Schh. Sueciamerid.

84 (Rugulosus. Herbst. Gallia.*

Quercicola. var. b. .

Payk. Suecia.

Melanostigma. Marsh. Anglia.

Cinereus. Marsham. id.

Scutellatus. Stephens. id.

85 Gallicus. Chevrolat.

Schh. P.

86 Mendosus. Chevrolat.

Schh. Gallia.

87 Occultus. Schh. Gall. merid.*

88 Concinnus. Schh. P.

89 Arcuatus. Hbst? Schh. Germania.

90 Melanostictus. Marsh. id.

\{ Rugulosus, Stephens, Anglia.

91 Triangulum. Märkel.

Schh. Saxonia.

92 Lycopi. (Chevrl.) Schh. P.*

93 Perturbatus, Schh. Gallia.

94 Quadridens. Panzer. Germania.*

Borraginis. Gyllenh. Suecia.

Quercicola. Marsham. Anglia.

Pallididactylus. Mars, id. 


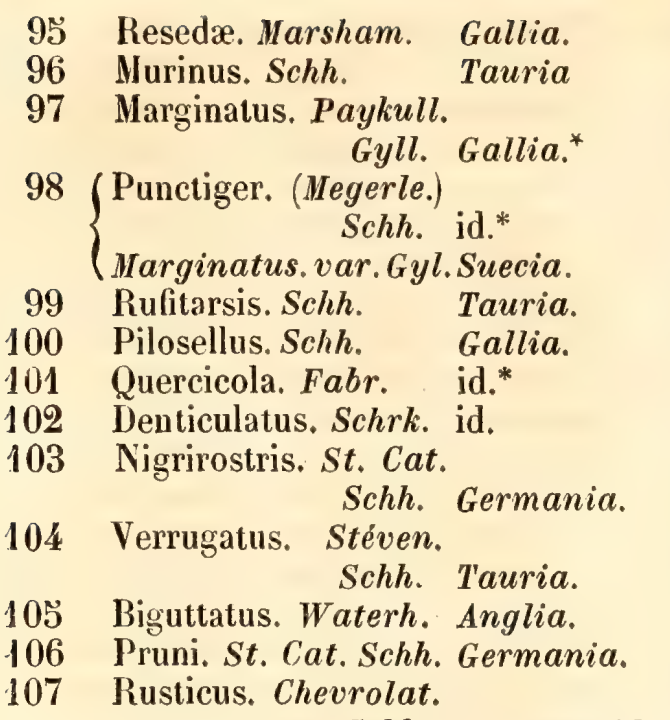

108 Pollinarius. (Chevro- Gall. merid. lat.) Schh. Gallia. Dentatus. Marsham. Anglia. Glaucinus, Dej. Cat. Gallia. Caliginosus, Steph. Anglia.

109 Angulosus. Germar. Saxonia.

110 Obsoletus. Germar. Germania.

111 Frculentus. Schh. Gallia.

112 Picitarsis. Stév. Schh. Helvetia.

113 Tibialis. Schh. Germ. merid.

114 Sulcicollis. Gyllenh. Suecia.* Alauda. Fabr. Gallia. Affinis. Steph. Schh. Anglia.

115 (Rapæ. Gyllenh. Gallia.* Napi. Dej. Cat. id. Sulcicollis, var, Gyll. Suecia.

116 Waltoni. Schh. Anglia. $\{$ Ruftarsis. Kirby. in

117 Roberti. Chevrl.Schh. Belgia.

118 Napi. Koch. Schh. Germania.* Assimilis, Olivier. id.

119 Inaffectatus. Schh. $P$.

120 Glabrirostris. Schh. id.

121 Sophiæ. Stév. Schh. Tauria.

122 Neutralis. Schh. Borussia.

123 Obtusicollis. Schh. Tauria.

124 Cyanipennis. Illiger. Austria.*

125 Eneicollis. (Meg.) Germar. id.

126 Scapularis. Schh. $P$.

127 Obscurecyaneus. Sch. id.

128 Melanocyaneus. Chevrolat. Schh. Gall, merid.
129 Ignitus. Germar. Germania. $\{$ Viridanus. Gebler. Russia.

130 Barbareæ. Suffrian. Germania.

131 Gyanipennis. Illiger. Suecia. $\{$ Sulcicollis. Paykull. id.

132 Tarsalis. Märk.Schh. Saxonia.

133 Suturellus. Schh. Tauria.

134 Carinatus. Gyllenh. id.

135 ( Chalybeus. Germar. Gallia. \{ Subcyaneus. Schüpp. Germania.

136 Hirtulus. Schüp.Schh. id.

137 Troglodytes. Fabr. Gallia.* $\{$ Var. Spiniger. Hbst. Austria.

138 Ureus. Hoffmansegg. Schh. Lusitania.

139 Validirostris, Schh. Tauria.

140 Uniguttatus, Marsh. Anglia.

141 Rumulosus. Germar. Germania.

142 Mostus, Fabr. (Cio-

143 Virgatus Schh. Tauria.

144 Nubilosus. Schh. id.

145 Interstinctus. Schh, Austria.

146 Vilis. Schh. Tauria.

147 Coarctatus. Schh. id.

148 Camelinæ. Chevrolat.

149

(Pubicollis. (Dahl.) Schh. $P$.

Signatus, Creutzer. in litt. Austria.

150 Signatellus. Schh. Tauria.

151 Dubitabilis. Schh. id.

152 Lineola. Sturm. Cat. Germania.

153 Cinerascens. Nees. St. Cat. id.

154 Coruleus, Dej. Cat, Styria.

155 Smaragdinus. Dej. Cat. Gall. merid.

156 Stigmulus. Illig. St. Cat. Germania.

157 Parvulus, Dej. Cat. id.

158 Subtilis, Illg. St. Cat. id.

159 Atomarius. St. Cat. id.

160 Brunnipes. St. Cal. id.

161 Scabrosus, Parreyss, Croatia.

162 Aulicus. Dej. Cat. Germania.

163 Iota. Dej. Cat. P.*

164 Erinaceus. Dej. Cat. Dalmatia.

163 Hirsutulus, Dej, Cat. Hungaria.

166 Fulvitarsis. Stm. Cat. Germania.

167 Arvicola, Gyllenh.St. 
168 Nigrirostris. St. Cat. Germania. 169 Mirabilis. Villa. Lombardia.

\section{SCLEROPTERUS, Schönherr.}

Gryptorhynchus, Germar.

1 Serratus, Eschscholtz.

2 Offensus. Schh. Germar. Livonia. Carinthia.

ACALLES, Schönherr. Steph.

Tylodes. Guérin. (Dej. Cat.) Rhynche- 36 nus. Fabr, Gyll. Gryptorhynchus. (Sturm. Cat.)

1 Roletti. Kunze. Schh. Sicilia.

2 Barbarus, Lucas. Algiria.*

3 Dromedarius. Hoffmsg.

Schh. Lusitania.

4 Fasciculatus. Schh. Sicilia.

5 Pyrenæus. Chevrolat.

Schh. Pyrenceis. ${ }^{\star}$

6 Denticollis. Germar. Hung. merid.

7 Circumflexus. (Gaub.) Algiria.*

8 Punctaticollis. Lucas. id.*

9 Impressicollis. Lucas. id.*

10 Sulcicollis. (Dej. Cat.) Pyr. orient."

11 Tuberculatus. (Gaub.) Gall. merid.*

12 Tristis. (Gaubil.) Algiria. ${ }^{\star}$

13 Dioclectianus, Germar, Gall. merid.*

14 Teter. Kunze. Schh. Sicilia.

15 Aubei. Schh. Gallia.

Wolfi. Waltl. Coll. id.

16 Hypocrita, Creutzer.

Schh. Styria. Alp.*

17 Lemur. Müller. Schh Germania.

18 Quercus. (Par.) Schh. Styria.

19 Camelus. Fabr. Berolini.*

20 Abstersus. Schh. N P.*

Roboris. Curtis, Anglia.

21 Navieresii. Chevrolat.

$$
\text { Schh. } P^{*}{ }^{*}
$$

22 Variegatus. Schh. Sicilia.

23 Ptinoides. Marsham. Gallia.*

Echinatus. Megerle. id.

Nocturnus, Chevrolat. $P$.

24

iTurbatus. Schh. Gallia.

Ptinoides. Schh. id.

25. Echinatus. (Megerle.)

Schh. Carniolia.
26

Misellus. Schh.

Gallia.

Ptinoides. Gyllenh. Suecia.

Picirostris. Ull. Coll. Germania.

Variegatus. Stephens, Anglia.

27

Parvulus. Schh.

Gallia.*

28 Sulcatus. Schh.

$\boldsymbol{P}$.

Fallax. Schh.

id.

30 Contractus. Dej. Cat, Gall. merid.

31 Scabratus. Dej.Cat. Dalmatia.

32 Griseus, Sturm. Cat. Gall. merid.

33 Porcatus. (Ziegl.) Dej.

\section{Cat. Styria}

34 Inæqualis, Dej. Cat. id.

35 Squalidus. Dej, Cat. Gall, merid. Porcinus. (Ziegl.) Lej.

Cat. Dalmatia.

37 Meticulosus. Dej. Cat. Gall. orient.

38 Globatus. Dej. Cat. Gall. merid.

\section{MARMOROPUS, Schh.}

1 Besseri, Schh.

Polonia.

\section{MONONYCHUS. Schüppel. Germar.}

1 Pseudacori. Fabr. P. ${ }^{*}$

Punctum-album. Hbt. Germania.

2 Superciliaris. Hoffmsg.

Schh. Lusitania.

3 Algiricus. (Gaubil.) Algiria.*

4 Salviæ. Hoffmansegg. $P_{\text {. }}^{*}$

\section{CELIODES. Schönherr.}

Ceutorhynchus. Schüppel. Germar. Lat. Steph. Schh. (Dej. Cat.) Rhynchenus. Fabr. Gyll. Oliv. Zett, Falciger. (Megerle. Sturm. Dej. Cat.)

1 Quercus. Fabr. Gallia.* \{Pallens. Marsham. Anglia.

2 Ruber. Marsham. id.* Quercus. Olivier. Gallia.

Rufrostris. Spence. Anglia.

3 Rubricus. Schh. Gracia.

4 (Rubicundus. Payk. Germania.* Quercus.var. B. Payk. Suecia. Melanocephalus. Mars. Anglia.

5 Epilohii. Paykull. Germania.

6 Guttula. Fabr. Gallia.* Carduelis. (Megerle.) Austria.

7 Fuliginosus. Marsh. Anglia. Subcostatus. Dej. Cat. Austria. Ruficornis, Stephens, Anglia. 
8 Umbrinus. Sch .

Tauria.

9 Canaliculatus. Schh. Volhynia.

10 Mannerheimii. Schh. Suecia.

11 Schüppelii. Schh, Ragusa.

12 Subrufus. Herbst. Gallia.

Cinctus. Rossi. Italia.

Tricinctus, Walcken. Germania.

Erythroleucus. Linné. Suecia.

Var. Subrufus, Gyll. id.

13 Didymus. Fabr. $P^{*}$

Urticarius. Clairville. Helvetia.

Viduus. Panzer. Germania.

Albopunctatus. Linné. Suecia.

Bipunctatus. Linné. id.

Tripunctatus, Foure. P.

ơ Oleraceus. Scopoli. Carniolia.

Var. Gibbipennis. Ger-

mar. Germania.

Urtica. Marsham. Anglia.

14 Lamii. Herbst. : Germania.*

\{Lucampyx. Dej. Cat. Austria.

15 Punctulum. Germar. Germania.

16 Geranii, Paykull. id.* \{ Affinis. Paykull. Suecia.

17 Exiguus. Olivier. Gall. aust.*

18 Asperatus. Schh. Tauria.

19 Hæmorrhoidalis. Stph. Anglia.*

20 Granulicollis. Schh. id.

21 Subrufus. Stephens. id.

22 Hæmaticus. Dej, Cat. Gall, orient.

23 Rimulosus. Germar. Germania.

24 Zonatus. Germar. Austria.

GASTEROCERUS, de Laporte et Brullé.

Latr. (Dej. Cat. Chevrolat.) Curculyo. Fabr. Herbst.

1 Depressirostris. Fabr. Gallia.

Plicatus. Herbst. Austria.

Anthriboides. Chevrolat. Coll. $P$.

Dumerilii. de Lap. Coll. id.

Oblitus. Dej. Cat. id.

CAMPTORHINUS. Schönherr.

Ruynchanus, Fabr. St. Cat.

1 Statua. Fabr.

Gallia. $^{*}$

\section{CHRYPTORHYNCHUS, riliger.}

Germar. Latr. Steph. Schh. (Dej. St. Cat.) Rhynchenus. Fab. Oliv. Herbst. Gyll. Gurculio. Lirné.

1 Lapathi. Linné. $\quad P_{\text {. }}^{*}$ Albicaudis, de Geer. Gallia. Carbonarius. Scopoli. Carniolia.

Trimaculatus. Vol. Germania.

\section{BARIDIUS. Schönherr.}

Baris. Germ. Latr, Steph. (Dej. St. Cat.) Stév. Bilberg. Murhorhy nchus et Stenorhynchus. (Megerle.) Rhynchenus, Fabr, Oliv, Gyll. Calandra. Fab.

1 (Sellatus, Chevrl.Schh. Algiria.* Balteatus. Chevrolat. in litt. id.

2 Subsignatus. Schh. id.

3 f Nitens. Fabr. Gall. merid. ${ }^{*}$ \{ Timidus, Ol. Dej. Cat. id.

4 Carbonarius. Stéven.

5 Luczotii. Chevrolat. Schh. Russ, merid. Schh. Gall, merid.

6 (Exasperatus. St. Cat. Schh. Austria.
Melana. Sturm. Cat. id.
$\left\{\begin{array}{r}\text { Artemisiæ. Dej. Cat. } \\ \text { Schh. Gallia.* } \\ \text { Melcena. Sturm. Cat. Germania. }\end{array}\right.$

8 Convexicollis. Schh. Russ. mérid.

9 Spoliatus, Dej. Cat.

10 Atronitens, Chevrolat.

11 Ornatus, (Gaubil.) id. * in litt. Algiria.*

12 Quadraticollis. Schh. Gall. interm.

13 /Picinus. Germar. Gallia.* Nitens. Herbst. Austria.

Glaber. Herbst. id.

$\{$ Laticollis. Marsham. Anglia. Artemisia. Olivier. Gallia. Atriplicis. Fabr. Germania. Interpunctatus.Steph. Arglia.

14 Atramentarius. Schh. Hungaria.

15 Analis. Olivier. Gall.merid.

16 Scolopaceus. Germar. Hungaria. Coloratus. Schh. Tauria. Parvulus. Schh. id. 
17 Pallidicornis. Schh.

18 Melas. Stéven. Schh. id.

19 Cuprirostris, Fabr. Gallia.*

Viridissimus, Dej. Cat.id.

Viridis. Fourcroy. P.

Virens, Olivier. Gall.merid.

Gramineus. Linné. Germania.

20 Siculus, Schh.

21 Prasinus. Schh.

22 Violaceus. Schh.

23 Janthinus. Schh.

24 Chloris, Fabr.

$\{$ Parisinus. Thunberg. $P$.

25 Cœrulescens. Scopoli. Gallia.*

Nitidulus. Müller. Germania.

Var. Chloris. Germar.

Schh. id.

26 Chlorodius. (Parreyss.)

Schh, Illyria.

27 (Chlorizans. Muill. Schh. Germania.* Chloris. Olivier. Gallia.

(Virescens. Dej. Cat. id.

28 Lepidii. Müller. Schh. id.*

Picicornis. Marsham. Anglia.

Chloris.Dej. Cat. Gallia.

Chlorodicus. Meg. Cat. Austria.

Var. Artemisia. Hbst. Germania.

29 Punctatus, Dej. Cat.

30 Pulchellus. Lucas, Algiria. *

31 Abrotani. Müller. Gallia.

\{ Picirostris. Marsham. Anglia.

32 Concinnus. Schh. Tauria.

33 | Villæ. Dej. Cat. Schh. Lombardia,

\{Violaceus, Villa, Cat. id.

34 Rufus, Schh. Sicilia.

35 Armeniacæ. Olivier. Gallia.

36 T. Album. Linné. id. ${ }^{*}$

Atriplicis, Olivier. id.

Pilistratus. Stephens. Anglia.

Funerus. Herbst. Austria.

Nigrinus. Herbst. id.

Hypoleucus. Marsham. Anglia.

Porolosus. Linné. Germania.

Atriplicis. Latreille. $P$.

Pusio. Schh. Sicilia.

Morio. Schh.

$P$.

Crocopelinus. Schh. Russ, merid.

Punctulatus. Dej. Cat. id.

Godetii. Dej. Cat. id.

Punctatissimus. Dej.

Cat. $P^{*}$

13 Episcopalis, Dej. Cat. id.
44 Amethystinus. Dej.Cat.Hispania.

45 Cyanellus. Dej. Cat, Gall. merid.

46 Lucidus. Dej. Cat. Austria,

47 Albomaculatus, Dej.

Cat. Hispania.*

\section{IERELOMUS. Schönherr.}

1 Chamæropis. Fabr, Algiria.*

2 Subcostatus. Schh. Sardinia.

BAGOUS. Germar. Schk.

Latr. (Dej. St. Cat.) Stephens. Stéven Rhynchenus. Gyll, Oliv. Curculio. Fabr.

1 Orientalis. Friwaldsky. Hungaria.

2 Elegans, Fabr. Germ. Germ. sept.

3 (Binodulus. Herbst. Germania,

Atrirostris. Olivier. $\boldsymbol{P}$.

Binodosus? Guérin. id.

4 (Nodulosus. Schh. Germania.

Binodulus. Stm. Cat, id.

Sibiricus, Gebler. Sibiria.

כ Argillaceus. Schh. Tauria.

6 Rotundicollis. Schh. Germania.

7 Inceratus. Schh. Berolini.

8 Encaustus. Chevrolat.

9 Linnosus. Gyllenh. Suecia.

10 Subcarinatus. Schh. Gallia. ${ }^{*}$

Frit. Dej. Cat. id.

11 Hæmatopus, Schönh. Caucasus.

12 Petrosus. Herbst. Gallia.*

Laticollis, Schh. Germania.

13 Collignensis. Herbst. id.*

14 Bi-impressus. Schh. Etruria.

15 Chorinæus. Chevrolat.

Schh. P.

16 Frit. Herbst. Germania.

17 Halophilus, L. Redıb. Austria.

18 Mundanus. Schh. Helvetia.

19 Claudicans. Schüppel.

Schh. Germania.

20 Brevis, Schh. Suecia.

21 Curtus. Schh. id.

22 Biglyptus. Germar. Saxonia.

23 Lutulosus, Gyllenhal. Gallia.

24 Tempestivus. Herbst. id.

25 Cnemerythrus. Marsh. id.

26 Convexicollis. Schh. Saxonia.

27 Lutosus, Gyllenhal. Gallia** 
28

$\begin{cases}\text { Tibialis. Stephens. Anglia. } \\ \text { Binotutus. Stephens. id. }\end{cases}$

29 Puncticollis. Schh.

30 Validitarsus, Schh.

31 Validus, Rosenhauer.

32 Tibialis. Schh.

33 Glabrirostris. Herbst.

$$
\text { (Dej. Cat.) id. }
$$

34 Elongatus. Dej. Cat. id.

35 Cryptocephalus. Dej.

Cat. Hispania.

\section{LYPRUS, Schönherr.}

Lixus, Ahrens. Bagous. (Dej. St. Cat.) Rhynchenus. Gyllenhal.

1 Cylindricus. Paykull. Gallia.* \{ttenuatus. Dej. Cat. Suecia.

MYORHINUS. Schönherr.

Apsis, Germ. Latr. (Dej, St. Cat.) Curculo. Fabr. Olivier.

1 Stevenii. Schh. Tauria.

Incrassatus, Stm. Cat, id.

2 Albolineatus. Fabr. Hungaria.

\{ Complicatus. Germar. id.

TRACHODES. Schïppel. Germar.

Rhynchenus. Gyllenh. Curculio.Linné. Paykull.

1 Cicatricosus. Sturm.

Schh. Hungaria.

2 Costatus, Schh. Bavaria.

3 Hystrix. Schh. Tauria.

4 Hispidus. Linné. Gallia.*

$\{$ Squamifer.Gyllenhal. Suecia.

Acanthion. Beck. Germania.

5 Exculptus. Germar. Sicilia.

\section{STYPHLUS. Schönherr.}

Orthochetes. Müller, Germar. Comasinus. (Megerle. Dej. Cat.)

$1\left\{\begin{array}{l}\text { Penicillus. Schh. Gall. merid. } \\ \text { Var. Anotolicus. Che- }\end{array}\right.$

$$
\text { vrolat. Smyrna. }
$$

2 Sulcipennis. (Megerle.)

Schönherr. Austria.

3 Setiger. Beck. Sch, Gall, bor.

\{ Setosus. Dej. Cat. Austria.

4 Museorum. Fairmaire. Pyren, sup.

ORCHESTES. Illig. (Dej. St, Cat.)

Rhynchenus. Clairville. Latreille. RamPUUS. Thunberg.

1 Quercus. Linné.

Viminalis. Fabr. Lam. P.

Saltator Ulmi, de Geer, id.

Setosus. Müller. Germania.

Alni. Herbst. Austria.

Var, Depressus, Steph. Anglia.*

2 Scutellaris. Fabr. Suecia. ${ }^{*}$

Rufus. Schrank. Germania.

Viminalis. Besser. Russia.

Alni, var. v, Paykull. Suecia.

Stellaris, var. b. Gyll. id.

Flavus. Sturm. Cat. Germania.

Testaceus, Müller. id.

3 l Carnifex. Germar. id.

Y Viminalis. Schrank. Austria.

4 (Rufus. Olivier. Gallia.*

Betuleti.Panzer. Germania.

Homaticus, Germar. id.

5 J Semirufus. Gyllenhal. Gallia.

I Rufpennis. Stm. Cat. Anglia.

6 Melanocephalus. Oliv. Gallia.

Ferrugineus. Marsh. Anglia.

Nigricollis. Stephens. id.

Atricapillus. Marsh. id.

7 Alni. Linné. P.*

Inquinatus. Voet.Pnz. Germania.

8 Ilicis. Fabr. P.*

Segetis. de Geer. Gallia.

Pilosus. Fabr. Germania.

9 (Pubescens. Schrank. id.*

Pilosus. Latr. Gallia.

Calceatus, Germar. Germania.

Calcar, var, Payk. Suecia.

Pilosus, var. c. Gyll. id.

10 Fagi. Linné. id.*

Fragaria. Fabr. Latr. $P$.

Calcar, Oliv, Latr. Gallia.

Subater. Müller. Germania.

Rhododactylus. Marsh. Anglia.

Rhodopus. Marsham. id.

11 Pratensis. Germar. Germania.*

S Segetis, Herbst. id. 
12 Tomentosus. Voigt.

Schh. Austria.

13 Iota. Fabr. Gallia.

Rosa.Herbst. Germania.

14 Subfasciatus. Schh. Tauria.

15 f baniceræ. Fabr. Germania.

IXylostei. Clairville. Helvetia.

16 Sparsus. Fabr. id.

17 Populi. Fabr. P.*

Fagi. Paykull. Suecia.

18 Signifer. Creutzer. Germania. Salicis, Latreille. Gallia.

Hortorum. Latreille. $\boldsymbol{P}$.

19 Fœdatus. Schh. Gallia.

20 Rusci. Herbst. Germania.* Bifasciatus, Gyllenh.

Sahlb. Suecia.

Salicis. Schrank. Austria.

Decoratus. Stephens, Anglia.

Affinis. Stephens. id.

21 Erythropus. Müller. Gallia.

22 Cinereus, Chevrolat.

Schh. Dalmatia.

\section{TACHYERGES. Schönherr.}

Orchestes et Rhynchenus, Auctorum.

1 Salicis, Linné.

Scapularis, Beck. Germania.

Caprece, Latreille. $P$.

2 Rufitarsis. Dej. Cat.

Schh. Gallia.

3 Decoratus. Schüppel. id.*

Confinis. Dej, Cat. Austria.

Salicis, Beck.

Affinis. Stephens.

4 (Stigma. Germar.

Iota. Gyllenh.

Confondatus. Schk. id.

Alboscutellatus. Dej.

Cat. $P$.

Rufitarsis. Stephens, Anglia.

Aterrimus, Stm. Cat. Germania.

כ) Saliceti. Fabr. Anglia.*

Foliorum. Müller. Suecia.

Scutellatus.Stph.var. Anglia.

6 Crinitus, Chevrol.Schh.P.*

7 Suturalis. Zettersted. Lapp. merid.

8 Ruficornis, Zetterst. id.

9 Monedula, Herbst. Germania.
ANOPLUS. Schüppel. Schönh.

(Dej. Cat.) Rнyмchanus. Gyllenhal. Orchestes. Stéven.

1 Plantaris. Nezen.Schh. Gallia.*

Roboris. Suffrian. Germania.*

Brevis. Marsham. Anglia.

Fitidulus, Steph, var. id.

Atratus, Steph, var, id.

Armeniace. Fabr. Armenia.

PHYTOBIUS, Schmidt. Schönh.

Hydaticus. Schh. Pachyrhinus. Kirby. Steph. Geutorhynchus. Fabr. RhynCHENUS. Gyll. GampyLiRHYNCHUS (Dej. Cat.)

1 Granatus. Schönherr. Helvetia.

2 Waltonii. Schh. Anglia.

3 Velaris. Gyllenh. Suecia.

4 Notula. Schüpp. Schh. Gallia.*

5 ) Quadrinodosus. Gyll. Suecia.*

Mucronulatus. Germ. Germania.

6 Comari. Herbst. Gallia.

7 Quadrituberculatus. Fabr. Suecia.

Quadricornis. Payk, id.

Ribis. Stroëm. Anglia.

Quadridentatus. Stph.id.

8 Canuliculatus. Kirby. Schh, Hungaria.

9 Quadricornis. Gyllenh. Gallia.*

4-Tuberculatus. var.

Herbst. Germania.

Rufescens. Stephens, Anglia.

10 Bruchoides.(Dhl.Cat.) Hungaria.*

LITODACTYLUS, Redtenbacher.

Rhynchanus. Beck. Gyll. Curculio. Marsh. Рнутовius. Schönh.

1 Velatus. Beck. Germania.* Myriophylli. Steph. Anglia.

2 Leucogaster. Marsh. id.* \{Mriophylli, Gyllenh. Suecia.

\section{ACALYP'TUS. Schönherr.}

Ellescus. (Megerle. Dej. Cat.) Sibinia

(St. Cat.) Ruynchenus, Gyllenhal.

1 Carpini. Herbst.

Germania.* 
2 Sericeus. (Meg.) Nej.

Cat. L. Redtenb. Austria.

3 f Rufipennis. Schh.

\{ Alpinus. Villa.
Gallia.* $^{*}$

Lomb. Alp.
SIBYNES. Schönherr.

Sininia. Germar. (Dej. Cat.) RhynchaNUS. Fabr.

1 C Canus. Herbst.

Gallia.*

$\{$ Pelluscens. Scopoli.

2 Viscariæ. Linné. Ajuga. Herbst.

3 (Vulpinus. (Meg.) Schh, id.*

Lineata. St. Cat. id.

4 Fugax. Schüpp. Schh. Berolini.

5 Zebra. Stéven. Schh. Russia.

6 Attalicus. Schh. Italia.

7 Femoralis. (Meg.) Schh. Austria.

8 Aurifer. Sturm. (Ellescus.) Cat. Schh. Russ. merid.

9 (Potentillæ. Koch. Schh. Germ.Borus. * \{Villosus. Marsham. Anglia.

10 Vibex. (Gaubil.) Algiria."

11 Virgatus. (Gaubil.) id.*

12 Tibiellus. Schh. * Gall. merid.

13 Phaleratus. Schh. Germania.

I Dorsalis. St. Cat. id.

14 Arenariæ. Steph.Schh. Anglia.*

15 Primitus, Herbst. Austria.

\{ Signatus, Gyllenh. Suecia.

16 Unicolor. Schh. Hungaria.

17 Variatus. Chevrl.Schh. Gallia.

18 Sodalis. Schüppel. id.

19 Sellatus, lucas. Algiria.

\section{SMICRONYX. Schönherr.}

Ellescius. (Dej. Cat.) Gurculio, Reich. Micronyx. Schönherr.

1 Cyaneus.Dej.Cat.Schh.Gall.merid.*

2 Jungermannix. Reich.

\{

Atomarius. St. Cat.

(Rhynch.) id.

3 Reichii. Schh.

Politus. Schh.

Gall.bor.

5 Variegatus, Dej. Cat.

1

Conspersus.

Schh. Gall. merid.*

Cicus. Schh.

Chevrol.

in litt. id.

id.
6 Fortirostris. Kunze.

Schh. Germania.

7 Coecus. Reich. Schh. id.

8 Pygmæus. Stm. Schh. Austria.

Heptaphilus. (Megerle.) Sibinia. Germ. Trchius. Steph.

1 Capucinus. Kze. Schh. Sicilia.

2 l Cuprifer. Panzer. Austria.* Ulmi. Dej. Cat. Gallia.

3 (Picirostris, Fabr. $\boldsymbol{P}_{\text {* }}^{*}$

Cinerascens, Gyllenh. Suecia.

Fuscirostris. Payk. id.

4 Posticinus. Schh. Gallia.

5 f Lineatellus. Schh. Anglia.

I Lineatus. Stephens, id.

TYCHIUS. Germar. Schh.

Rhychenus. Fabr. Gyll. Curculio. Linné. Herbst. Sibinia. Germar.

1 /Quinquepunctatus, Lin, Gallia.* క̌-Maculatus, Linné. Suecia.

Quinquenotatus, Man, id.

Var. Quadrimacula-

tus. Müller. Germania.

2 Fuscolineatus, Lucas. Algiria.

3 Gravidus. Chevrolat.

4 Venustus, Fabr. Gallia.*

Parallelus, Olivier. $\boldsymbol{P}$.

Pegaso. Herbst. Austria.

Vernalis, Reich. id.

Var. Nervosus. Marsh. Anglia.

Vittatus, Schüppel. Germania.

5 Rufirostris. Schh.

6 iPolylineatus. (Meg.)

Schh. Austria. ${ }^{*}$

Lineola. Sturm. Cat. id.

7 Siculus. Schh. Sicilia.

8 Carinicollis. Lucas. Algiria.*

9 Striatulus. Dej. Cat.

Schneideri. Herbst. Gallia.

11 Genistæ. Märkel, Schh. Helvetia.

12 Hæmatopus, Schh. Tauria.

13 Thoracicus, Kze. Schh. Sicilia.

Suturalis. St.Cat.Schh.Austria.

Elongatus. Schönherr. Algiria.

Aurichalceus. Schh. Lusitania.*

Sorex. Schh.

Russia. 
18 (Tomentosus. Herbst. Gall. merid.* Picirostris, Gyllenh. Suecia. Stephensi.Schh. Anglia.

19 Junceus, Reich. Schh. Germania.* Suturalis. Meg. Cat. id.

20 Virescens, Zsch. Schh. id.

21 Canescens, Marsham. Anglia.

22 Meliloti. Kirby. in litt. Steph. Schh. Germania.

23 Flavicollis. Kirby. in litt. Steph. Schh. Anglia.

24 Strumarius. (Hoffmsg.) Schh. Lusitaria.

25 Hæmatocephalus.Schh.Helvetia.*

26 Auricollis. Schh. Tauria.

27 Sparsutus. Olivier. Gallia. ${ }^{*}$

28 Fraxini. (Dahl.) Schh. Hungaria.

29 Obesus, Chevrol. Schh. Helvetia.

30 Tibialis. Schh. Gallia.

31 Pernix. Creutzer. Hungaria.

32 (Squamosus. (Dej. Cal.)

$\{$ Schh. Gall. merid.*

Hordei. (Chevrolat.) id.

33 Lineatulus, Kirby. Anglia.

34 Signatus, Vogt. Schh. Germania.

35 Centromaculatus. Villa.

\section{Schh. Lombardia.}

36 Tæniatus. Dej. Cat. Gall. merid.

37 (Lineolatus. (Ziegler.)

Dej. Cat. Styria.

Var. Lineatus, Gyssel. Austria.

38 Vicinus. Dej. Cat. Gall. merid.

39 Asperatus. Dej. Cat. id.

40 Vestitus, Dej. Cat. id,*

41 Lateralis. Dej. Cat. id.*

42 Suturaalba. (Chevrol.) Gallia.*

43 Laneirostris.(Chevrol.)id.*

\section{CORYSSOMERUS. Schh.}

Poecilma. Germar. Rhynchenus. Beck.

1 Gapucinus, Beck. Germania.* Var. Ardea. Germar. id.

AMALUS. Schönherr.

Ceutorhynchus. Germar. RhyncheNUS. Gyllenhal.

1 Scortillum. Herbst. Gallia.*

Homorrhous. Herbst. Austria.

Agricola. Paykull. Suecia.

Rubicundus. Panzer. Germania.
BALANINUS, Germar.

Rhy nchanus. Fabr. Gurculio. Linné.

1 Elephas. And. Schh. Gall. merid.* Gulosus, Latreille. Hispania.

Nucum. var. Olivier. id.

Hispanus? Dej. Cat. id.

2 Pellitus. Schh. Gallia.

3 f Nucum. Linné. id.*

Gulosus. Germar. Germania.

4 Glandium. Marsham. Gallia.*

\{Venosus. (Dahl.) Gall. merid.

5 Turbatus. Gyllenh. Gallia."

Nucum. Germar. id.

Pusillus. Sturm. Cat. Austria.

6 Cerasorum. Herbst. Germania.*

7 Rubidus. Dej.Cat.Sch. P.*

8 Villosus. Herbst. Gallia. *

Esuricus. Fabr. var. id.

Cerasorum. Oliv, var. $\boldsymbol{P}$.

Tenuirostris, Fab.var, Germania.

Cordifer. Fourcroy. $\boldsymbol{P}$.

9 (Crux. Fabr. Gallia.*

Salicis. Panzer. Germania.

Iota. Panzer. id.

10 Brassicæ. Fabr. Gallia.*

Salicivorus. Gyllenh. Suecia.

Macropus, Olivier. Gallia.

Arcuatus. Marsham. Anglia.

Cinerascens. Linné. Suecia.

Scutellaris. Stephens. Anglia.

Var. Intermedius $\sigma$.

Stephens. id.

11 Ochreatus. Dej. Cat.

12 Pyrrhoceras. Marsham.Gallia.*

o Curvatus. Marsham. Anglia.

Salicivorus, var. $b$.

Gyllenh. Suecia.

Intermedius. Marsh. Anglia.

13 Brunneus. Marsham. id.

ANTHONONUS. Germar.

Schh. Rhynchenus. Fab, Gurculio, Lin.

1 Ulmi. de Geer. Gallia.*

Pedicularius. Linné. Suecia.

Avarus. Latreille. $P$.

Clavatus. Dej. Cat. id.

Bavarus. Schrank. Germania.

Var. Fasciatus, Steph. Anglia.

Pomona. Germar. Germania. 
2 Undulatus. Schh.

3 Pyri. Chevrolat.

Germania.

$P$.

4 Spilotus. L. Redtenb. Austria.

5. Elongatulus. Schh. Helvetia.

6 Pomonæ. Linné. Gallia.*

Clavatus. (Ziegl.) St.

\{ Cat. Germania.

Incurvus. Stephens. Anglia.

7 Incurvus. Panzer. Germania.

Pomarum. var.b.Gyl. Suecia.

Avarus. Dej. Cat. Gall. merid.

Var. Humeralis. Panz. Germania.

- Cratogi. Chevrol. P.*

8 Pubescens. Paykull. Suecia.*

9 Varians. Paykull. id.*

Beccabungce. Fabr. id.

Var. Melanocephalus.

Fabr. id.

10 Sorbi. Germar. Germania.

11 Dorsalis. Sturm. Cat. Schh. Austria.

12 Rufus. Schh.

13 Rubi. Herbst. Clavatus. Marsham. Anglia.

Perforatus. Herbst. Austria.

Melanopterus. Marsh. Anglia.

Obscurus. Stephens. id.

Ater. Stephens. id.

14 Druparum. Linné. Gall. bor.*

$\{$ Tesselatus, Fourcroy. $P$.

BRADYBATUS, Germar. Latr.

Rhinodes. (Dej. Cat.) Pissodes. Stéven.

1 (Creutzeri. (Meg.) Schh. Gallia.* $\{$ Var. Elongatus. Chev. id.

BRACHONYX. Schönherr.

Rhynchenus. Gyllenhal. Rhinodes. (Dej. Cat.)

\section{1.} $\begin{cases}\text { Indigena. Herbst. } & \text { Gallia. }^{*} \\ \text { Malva. Herbst. } & \text { Austria. } \\ \text { Pineti. Paykull. } & \text { Suecia. }\end{cases}$

\section{LIGNYODES. Schh.}

ELLescus. (Dej. Cat.)

1 (Enucleator. Panzer. Gallia.* I Bicolor, Germar. Germania.
ELLESCUS. (Megerle. Dej. Cat.) Schh.

Hypera. Germar. Rhynchenus, Fabr.

1 Scanicus, Linné. Gallia.*

Rubicundus. Herbst. Austria.

Y Placidus. Herbst. id.

Pallidesignatus. Hbst. Germania.

2 (Bipunctatus, Linné. id.*

$\{$ Unipunctatus. Oliv. Gallia.

3 Sericeus. (Meg.) Schh. Austria.

4 Alpinus. Villa. Schh. Italia.

5 Carpinus. Solier. Gall. merid.

\section{HYDRONOMUS. Schh.}

Rhynchenus. Gyllenh. Bagous. (Dej. Cat.)

1 Alismatis. Marsham. Germania.* Productus. Illig. Dej. Cat. id.

2 Erythrorhynchus. St.

Cat. Schh. id.

\section{GRYPIDIUS. Schh.}

Grypus. Germar. Rhynchenus, Fabr.

1 Equiseti. Fabr. P.* Nigro-gibbosus. de Geer. id. Gibbosus, Latreille. id. Var. Atrirostris. Fabr. Dej. Cat. Germania.

2 Brunnirostris, Fabr. P.* Obliteratus. Herbst. Austria. Obsoletus. Stm. Cat. Germania.

\section{ERIRHINUS. Schönherr.}

Rhynchenus. Fabr. Curculio, Linné. Latr. Herbst.

1 Bimaculatus. Fabr. P.*

2 (Scirpi. Fabr. Gallia.* Rhamni.Herbst. Austria.

3 Acridulus, Linné.. P. ${ }^{*}$ Punctum. Fabr. Germania. Rigidus. Marsham. Anglia.

4 Märkelli. Schh. Saxonia.

5 Stevenii. Schh. Russia.

6 (Ethiops. Fabr. Suecia. $\{$ Holomelanus. Herbst. Austria. 
7 Petax, Stéven. \{ Barbirostris. Stm. Cat. id.

8 Pilumnus. (Dahl.) St. Cat. Schh. Germania.

9 Sparganii. (Dhl.) Schh. Austria.

10 Festucæ. Herbst. Germania.* Caricis. Thunberg. Suecia.

Carecti. Hoppe. Germania.

11 Inquisitor. Herbst. Austria. ${ }^{*}$ Nereis. Paykull. Suecia. (Thypha. Ahrens. Austria.

12 Albofasciatus. Dahl. Cat. Schh. Sardinia.

13 Scirrhosus. Schh. P.*

\section{DORYTOMUS. Germar.}

Rhynchenus. Fabr. Gyll. Curculio. Linné. Nataris. Germar.

1 Vorax. Fabr. o Ventralis. Dej. Cat. id.

Cursor. Paykull. Gallia. Longipes, Laichart. Tyrolis. Longimanus. Forst. id.

2 Macropus. Hellwig. in litt. L. Redtenb. Austria. 3 Tremulæ. Paykull. Gallia.* Toniatus. Herbst. Austria. Fumosus. Rossi. Italia.

4 Variegatus. (Megerle.)

Schh. Austria.

6 Costirostris. Schh. Gallia.* \{ Tremula. Dej. Cat, id.

7 Affinis. Payk. id.* $\{$ Var. Tremula, Payle. Suecia. Flavipes. Olivier. Gallia.

8 Suratus. (Meg. Dej. Cat.) Schh. Austria.*

9 Validirostris. Schh. Gallia.*

10 Capræ. Chevrolat. Nov.

Waltonii. Schh. $s p . P . *$ Tæniatus. Fabr. Gall. bor.* Bituberculatus. Zett. Schh. Scandinavia.

Occalescens. Schh. Dalmatia. Juratus. Dej. Cat. id.

15 Flavipes. Panzer. Germania.

16 Salicinus. Gyllenhal. id. Tariatus.var. c. Gyll. Suecia. Parvulus, Zetterst. id.
17 Agnathus, Dahl. Cat. Schh. P.*

18 Tenuirostris. Schh. Gallia.

19 Majalis. Paykull. Suecia.* Flavipes. Fabr. Gallia.

20 Pectoralis. Panzer. id.* Fructum, Mannrrh. Suecia. $\{$ Rubellus. Marsham. Anglia. Var. Melanophthalmus. Payk. Suecia. - Arenatus. Fabr. Austria.

21 Nebulosus. Schh. Gallia.

22 Clitellarius. Schh. Finlandia.

23 Minutus. Dej. "Cat. Schh. Austria.

24 Villosulus. (Meg.)Schh. Gallia.

25 Puberulus. Schh. Dalmatia.

26 Infirmus. Herbst. Germania.*

27 (Tortrix. Linné. Gallia.*

Fulvus. de Geer. id.

Rubigineus. Fourcroy. $P$.

Arcuatus. Stephens. Anglia.

28 Filirostris. Schh. . P. . $^{*}$

29 Punctator. Herbst. Germania.

30 Flavus. Dahl. Cat.

31 Dorsalis. Fabr. Germania. *

32 Sulphureus, Stm. Cat.

Schh. Anglia.

\section{MAGDALINUS. Germar.}

Tamnophilus. Schönherr. Rhinodes. Schh. (Dej. St, Cat.) Rhina, Oliv. Curculo. Linné.

1 Violaceus. Linné. Gallia. * Assimilis. Herbst. Austria. Affinis. Linné. Germania.

2 Frontalis. Gyllenh. Helvetia. ${ }^{*}$

3 Duplicatus. Germar. Germania.* Y Var. Violaceus. Zett. Suecia.

4 Phlegmaticus. Herbst. Germania.*

5 (Cyanipennis. Sturm.

Cyanescens, Stm, Cat, id.

6 Cerasi. Linné. Gallia.* Armeniaca. Fabr. Germania. Nassata. Germar. id. \{ Carbonarius. Panzer, id. Striatulus. Linné. Suecia. Barbicornis. Steph. Anglia. Rhina. Gyllenh, Suecia. 
7 Nitidus, Gyllenh.

8 (Linearis. Gyllenh. Yirescens. Germar.

9 Carbonarius. Fabr.

10 Cyaneus.Dej.Cat. Srh. Helvetia.

11 Gracilis. Erichson. Germania.

12 Asphaltinus. Germar. Germania. Cerasi. Fabr.

13 Stygius. Gyllenh. Gallia.* Aterrimus. Germar. Germania. Cerasi. Olivier. Gallia. Asphaltinus. Steph. Anglia. Atramentarius.Marsh.id.

14 Atramentarius. Germ. Gallia. Atratus. Gyllenh. Suecia. Aterrimus. Herbst. Austria. Cerasi ${ }^{7}$. Paykull. Suecia. Carbonarius ‥ Linné. id.

15 Atrocyaneus. Schh. Westrogoth.

16 Rufus. (Meg.) Schh. Germania.

17 Barbicornis, Latreille. Gall. bor.* Var. Rhina. Gyllenh. Suecia.* Trifoveolatus, Gyll. id.

18 Pruni. Linné. Gallia. Erythroceros. Herbst. Austria. Incognitus. Herbst, id. Ruficornis, Linné. Suecia.

19 Flavicornis. Schh. P.

20 Scutellaris. Dej. Cat. Gallia.*

21 Nitidipennis. Erichson. Helvetia. $\{$ Brevirostris. Chevrol. Gallia.

\section{PISSODES. Germar.}

Rhynchenus. Fabr, Gunculio, Linné.

1 f Piceæ. Illiger. Schh. $\{$ Pini. Panzer.

Austria. ${ }^{*}$

2 Pini. Linné. Bohemia.

3 Fulvofasciatus. Schmd. Gall. bor.*

4 Notatus. Fabr. Schh. Carniolia.

Pini. Olivier. Gall. bor.*

Castaneus. de Geer. Palmes. Herbst. id. id. Brunneus. Panzer.

Austria. Germania.

5 Validirostris. Gyllenh. Gall. bor.*

6 Juniperi. (Ziegler.) Algiria.*

7 Gyllenhalii. Schh. Germania. Hercynia. Gyllenh. id.

8 (Harcyniæ. Herbst. id. 4-Notatus. Panzer. id. Interstitiones, Sahlb. Suecia.

9 Strobyli. L. Redtenb. Austria. 10 Piniphilus. Herbst. Germania. 11 Fabricii. Leach. Anglia.

\section{RHINOCYLLUS. Germar.}

Rhinobatus, Dej. Cat. Larinus. (St. Cat.) Lixus. Illig. Cunculo. Herbst: Rossi.

1 Antiodontalgicus. Ger$\left\{\begin{array}{r}\text { bi. Schh. Italia: } \\ \text { Thaumaturyus. Rossi. id. }\end{array}\right.$

2 Latirostris, Latreille. Gall. merid.* Thaumaturgus? Stph. id. Odontalgicus, Dej. Ct. id. Sulcifrons. Dej. Cat. id. Conicus. Frœhl. Austria.

3 jOlivieri. (Meg.) Schh. id.*

Odontalgicus. Oliv. P.

4 Planifrons. Dej. Cat.

Schh. Dalmatia.*

5 Inquinilus. Gyllenh. Finlandia.

\section{LARINUS. Schüppel. Germar.}

Rhinobatus. Germar. Lixus. Olivier. Rhynchenus, Fabr. Curculo. Auct.

1 Cinaræ. Fabr.

2 Costirostris, Schh.

3 Cardui, Rossi.

Cinara. Olivier.

Triangularis. Petag. Calabria. Subcostatus. Dej. Cat. Dalmatia.

4 Cirsii. Steven.

5 Teretirostris. Schh. id.

6 Glabrirostris. Schh. id.

7 (Buccinator. Olivier. Hispania.* Costirostris. Escher. Zollikoffer.

8 Inquinatus. Olivier. Algirin. Major. Herbst. id.

9 Onopordinis. Fabr. id.* Onopordi, Olivier. id.

10 Tubicenus. Schh. Hispania.

11 (Maculatus. Fald. Schh. Lusitania. Var. Obesus. Chevrol. id.

Guttiger. Germar. Russ, merid.

12 Maculosus. Bess. Schh. Gall. merid.*

13 Albarius, Schh. Sicilia.

14 f Scolymi. Olivier. Gall. merid.* iochreatus. Olivier. id. 
15 Timidus. Schh.

Planus. Herbst.

Cat.

Germ. Gall. merid.* 50

Dalmatia.

17 Virescens. Dej. Cat.

$$
\text { Schh. id. }
$$

18 Olivasceus, (St.) Schh, Gracia.

Gall. merid.*

Jacea. Herbst. Austria.

Fringilla, Sahlberg. Suecia.

Hercynice. (Dahl.) Dalmatia.

20 Conspersus. Dej. Cat.

Schh. Gall. merid.* 53

Pollinis, Laicharting. Dalmatia.

\section{Maurus. Olivier. \\ Sicilia.}

Stellaris. Stéven.

Russ, merid. 55

Carenirostris. Schh

Sicilia.

Variabilis. Kollar.

Russ, merid.

Lineatocollis. Schh.

Jaceæ. Fabr.

Guttiger, Schh. id.

Tenuirostris. Sturm.

Rusticanus. Schh.

Immitis. Schh.

Gall. merid.*

Sicilia.

Gracia.

Sardinia.

Longirostris. Schh.

Cylindrirostris. Dej. Cat. Schh. id. *

Schh. id.*

35 (Canescens. St. Cat.

Cinerascens. St. Cat. id.

36 Crassirostris. Dej. Cat.

Schh. Gall. merid.* 66

(Concretus, Dej. Cat. Schh. Podolia.

(Brevis. (Ziegler.) Styria.

38 Intermedius, Dej. Cat.

Schh. Gallia.

3
$\{$ Planus. Fabr.
Teres. Herbst.
Germania.* id.

40 Obtusus. Sturm. Cat. Schh. Aust

41 Brevis. Schh.
Ferrugatus. Schh. Ovatus. (Chevrolat.) id." Bombycinus. Buquet. id. Morio. Schh.

\{ Carlinæ. Olivier.

Gall. merid. id. * ZEbencus. Marsham. Anglia. Rugicollis, Schönherr. Algiria. Carinifer, Schh.
Sicilia.
Acanthiæ. Hoffmansegg. in litt. Schh. Lusitania.

Oblongus. Dej. Cat. $\left\{\begin{array}{r}\text { Schh. Gall. merid. } \\ \text { Carthami. Latreille. id. }\end{array}\right.$

51 Hypocrita. Dej. Cat. Schh. id.

$\begin{cases}\text { Ursus. Fabr. } & \text { id.* } \\ \text { Vittatus. Fabr. } & \text { id. } \\ \text { Canaliculatus. Oliv. } & \text { id. } \\ \text { Stolatus. Linne. } & \text { id. } \\ \text { Trivius. Dej. Cat. } & \text { id. }\end{cases}$

Albicans. Chevrolat. in litt. Lucas. Algiria.*

Genei. Schh.

Sardinia.

Helsleucus. Motsch. Russ, orient.

Marginicollis. (Dahl.) Schh. Sicilia.

57 Confinis. Dej. Cat. $\left\{\begin{array}{r}\text { Schh. Gall. merid. } \\ \text { Var. Toeniolatus, Duf. Hispania. }\end{array}\right.$

58 Crinitus. Schh. Russ, merid.

59 Senilis. Fabr. Brevis. Herbst. Dalmatia.* Austria.

60 Reconditus. Schh. Gall. merid. *

61 Biguttatus. Stm. Cat.

62

63

64

65

Idoneus. Schh. Schh. Germania.

Bicolor. Fischer, Schh. id.

Siculus. Schh.

(Chevrolatii. Helfer, in

litt. Schh. id.

Albovittatus. Chevrol.id.

Nanus. Lucas. Algiria.

67 Villosus. Schönherr, Russ. merid.

68 Hirtellus. Helfer. Constontinp.

69 Cardopatii. Lucas. Algiria.

\section{LIXUS. Fabricius.}

\section{Curculio. Linné.}

1 (Paraplecticus. Linné. P.*

Patruelis. Dej. Cat. id.

$\{$ Gracilis, Dej.Cat. id.

Phellandrii. de Geier. id.

Productus. Stephens. Anglia.

2 Turbatus. Gyll. (Dej.
$\begin{cases}\text { Cat.) Gall. merid. } & \\ \text { Paraplecticus, Panz. } & \end{cases}$
Dumeril. Germania.
Pulverulentus. Fabr. Austria.

3 Connivens, Gyllenhal, Russ. merid. 
4 Gemellatus. Gyllenhal.Germania.*

$\{$ Paraplecticus. Steph. Anglia.

5 Geminatus. Schh. Corsica.

6 Incarnatus. Schh. Dalmatia.

Rubiginosus. Dahl. in

litt. id.

Umbellatorum. Fabr. id.

7 Canescens. Stév. Schh. Tauria.

8 Siculus.Dej.Cat.Schh. Sicilia.

9 Chevrolatii. Schh. id.

10 Acuminatus. Dej. Cat. Schh. Algiria.

11 Tenuirostris. Schh. Sicilia.

12 Anguinus. Linné. Italia.*

Mucronatus, Fabr. Algiria.

Bufo. Fabr. Sicilia.

13 Anguiculus. Schh. Gracia.

14 Inops. Schh. Algiria.*

Var. Cachrydis. Rambur. Dej. Cat. Hisp. merid.

Venustulus. Dej. Cat. Cylindricus. Fabr. Gall. merid. ${ }^{\star}$ Lefebvrei. Schh.

Barbarus. Schh.

Augurius. Schh.

Sicilia.

Algiria.

id. *

Mucronatus. Olivier. Schh, id.

20

Nanus. Sehh. Algiria.

Brevirostris. Chevrol.

Schh. Hispania.

Albomarginatus. Schh, Hungaria.

Ochraceus. Stm. Cat.

Schh. Dalmatia.

23

Rosenschœldi. Schh. Lusitania.

24 Ascanii. Linné.

25 (Wagneri. Lucas.

Ascanoides? Villa. Gall. merid. ${ }^{*}$ Algiria. Lombardia. Algiria.

26

27

28

29

30

31

32

33

34

Affinis. Lucas.

Bimaculatus. Lucas.

id.

Brevicaudatus. Lucas, id.

Palpebratus. Schh. id.

Sanguineus. Rossi. Italia.

Superciliosus. Schh. Lusitania.

Ruficornis. Schh. Gall. merid.

Acutus. Dej. Cat.Schh.id.*

Elegantulus. (Dahl.)

Schh. Hungaria.

35 Myagri. Olivier. $P$.

Diloris. Germar. Gall. merid.

Sabulosus. Dej. Cat. id.

Pisanus. Schneider. in

litt, Italia.
36 Punctirostris. Schh. Hungaria.

37 f Fallax. Schh. Gall. merid.

Lineatus. Sturm. Cat. id.

38 Linearis. Olivier. Grocia.

39 Subulipennis. Schh. Podolia.

40 Angustatus. Fabr. Gall. merid. ${ }^{*}$ Var. Ferrugatus. Latr, id.*

Pulverulentus. Rossi, Italia.

Algirus. Linné. Algiria.

41 Cribricollis. Dej. Cat.

Schh. Gallia."

Ferrugatus, Olivier, id.

Acetorella. Chevrolat. in litt. var. $P . *$

42 Lycophæus. Schh. Russ. merid.

43 Spartii. Olivier. Gall, merid.* Scoparii. Dej. Cat. id.

44 Varicolor.(Dahl.) Schh.Dalmatia.*

45 Juncii. (Dahl.) Schh. Sardinia.

46 Circumdatus. Schh. Germania.

Var. Ascanii. Latreille. id.

47 Bicolor. Olivier. Gall. merid.* Ferrugatus. Stm. Cat. Austria. Lateralis. Stephens. Anglia.

48 Abdominalis. Schh. Sicilia.

49 Punctiventris. Stéven.

Schh. Russ. merid.

50 Gultiventris. Germar. Sicilia.

Var, Affinis.Chevrolat.

in litt. id.

51 Orbitalis. Schh. Tauria.

52 Nigritarsis. Schh. Europ. mer.

53 Sardiniensis. Schh. Sardinia.

54 Vilis. Rossi. Italia.

55 Lateralis. Panzer. id.

Aretii. Panzer. id.

56 Inquinatus. Stév. Schh. Russ, merid.

57 Subtilis. Sturm. Cat.

Sehh. Hungaria.

58 Sulphuratus. Schh. Sicilia.

59 Flavescens, Schh. Hungaria.

60 Pollinosus. Germar. Gall. merid.* Filiformis. Ménét. Cat. Russ. merid.

Cardui, Olivier. Gall, merid.

Tigrinus.Megerle. Dej.

61 Striatulus, Fabr. Algiria.*

62 Filiformis. Fabr. Gall. merid.* Hamotoceras. Germ. Austria.

Angustus. Herbst. id.

Var. Bardance. Panz. Germania.

63 Trivittatus. Chevrolat. Gall, merid."

64 Pardalis. Schk. Lusitania. 
65 Constrictus, Schh. Tauria.

66 (Rufitarsis. Schh. Filiformis. Dej. Cat. id.

Angustatus, Dahl. in litt. Sardinia.

67 Coarctatus, Lucas, Algiria.

68 Acicularis. (Hoffmsg.) Schh. Lusitania.

Immeritus, Schönherr, Russ, merid. Steveni. Schönherr. id.

\section{NASTUS. Schönherr.}

1 Goryi. (Parreyss.) Sch. Tauria. 2 Humatus, Germar. Russ, merid.

Brunnitarsis. Stm.Cat.id.

\section{CHLOEBIUS. Schönherr.}

3 Albopunctatus, Lucas, Algiria.*

4 Albomarginatus. Luc, id.

\section{ELYTRODON. Schönherr.}

1 Bidentatus. Stév. Schh,Tauria.

2 Bispinus. (Ziegl.) Schh. Hungaria.

3 Inermis, Schh. Europ, mer.

\section{TYLODERES, Schönherr.}

Brius. (Dej. Megerle. Sturm. Cat.)

1 Megerlei. Fabr. Austria.

2 Chrysops. Herbst. Dej. $\begin{cases}\text { Cat. } & \text { Styria. } \\ \text { Dejeanii, Schh. } & \text { id. }\end{cases}$

\section{OTIORHYNCHUS. Germar.}

Pachygaster. Stéven. Germar. BrachyRhinus. Latreille. Cunculio. Linné. Fabr, Gyll., etc.

1 (Ragusensis, Dej. Cat. Schh. Dalmatia.* Cat. id.

2 Gœrzensis. Herbst. ․ Austria.*

3 Spalatrensis. (Parr.)

4 Planatus. Herbst. Hungaria." Sensitivus, Scopoli. Styria.

Cardiniger. Hoffmsg. Austria.

5 Caudatus. Rossi. Italia.*

6 Pyrenæus. Dej. Cat. Pyrenæis.*

7 Sabulosus, Find. Schh. Hungaria.

Cupreipennis, Dahl. in litt. id.

8 Griseopunctatus. Dej.

Cat, Schh. Pyrenøis, *

9 Hungaricus. (Megerle.)

Angusticollis. Chevrol, Gall, merid.*

10 Cœcus. (Meg.) Schh. Carniolia.*

11 Orientalis. Schh. Orient. Sicil.

12 Sulphuripes, Fabr. Austria.*

Friulicus. Herbst. id.

13 Carinthiacus. Germar, Carinthia.*

$\left\{\begin{array}{c}\text { Bisulcatus. Germar. } \\ \text { Latr. } \\ \text { Cinifer, Germar. } \\ \text { Var. Vochinensis. } \\ \text { Schmidt. Carniolia. }\end{array}\right.$

14 Istriensis. (Dahl.)Schh. Istria. 
15 Longicollis, Andersch.

(Plumipes. Germar. Illyria.*

Flabellipes. Dej. Cat. Carniolia.

17 Fortis. Rosenhauer. Tyrolis.

18 Cribrosus. Germar. Illyria. $\{$ Hirtipes. Dej. Cat. Carniolia. Erythropus. Stm. Cat. id.

19 Dalmatinus. Dej. Cat. Schh. Dalmatia.

20 Aurifer. (Dahl.) Schh. id.

21 Lefebvrei. Dej. Cat. Sch. Europ. mer. (Pulverulentus. Germ. Carniolia.* Pubescens. Dej. Cat. Illyria. Interstitialis. (Meg.) Dalmatia. Punctorum. Schrank. Styria. Brunnipes. Olivier.

Lombardia.*

24 Nigripes. Schh.

25 Niger. Fabr. Latr. Alsatia. ${ }^{\star}$ Scrobiculatus. Schh. Germania. Var. Villosopunctatus. (Ziegl.) Dej. Cat. Styria.*

26 Rugipennis. Schönh. Sicilia.

27 Fuscipes. Olivier. Gall. merid. ${ }^{*}$ Pedemontanus. Dej.

Tenebricosus. Sturm. Cat. Germania.

Ater. Stephens. id.

28 Adumbratus. Dej. Cat. Austria."

29 Hæmatopus.Meg.Schh.Helvetia.

30 Erythropus. Chevrol. Schh. Gallia.

31 Subsulcatus. Dej. Cat. Pyrenceis." 32 Tenebricosus. Herbst.

$\begin{cases}\text { Schh. } & \text { Gallia.* } \\ \text { Morio. Paykull. } & \text { Suecia. } \\ \text { Clavipes. Oliv. Latr. } & \text { Gallia. } \\ \text { Niger. Marsham. } & \text { Anglia. } \\ \text { Var. Maritimus. Do- } & \\ \text { novan. } & \text { id. }\end{cases}$

33 Lugdunensis. Chevrol.

Schh. Gall. bor.

34 Fagi. Chevrolat. Schh. Gall. merid.

Rufpes. Sturm. Cat. id.

(Var.Tenebricosus.Gyl. id.

35 Substriatus. Schh. Alsatia.*

36 Auropunctatus. Dej. Cat. Schh. Pyr, orient.

37 Vehemens. Schh. Sicilia. ${ }^{*}$

38 Latipennis. Dej. Cat. Sch. Illyria.
39 Armadillo. Rossi. Nigrita. Rossi. Orbicularis, Olivier. Gall. merid. Sulphurifer. Herbst. Helvetia.

40 Scabripennis. Schh. Gallia.*

41 Lafertei. Chevrolat. Pyrenceis. ${ }^{*}$

42 Multipunctatus. Fabr. Germania.*

43 Femoralis. Chevrolat.

Schh. Gall, merid.

44 Inflatus. (Dahl.) Schh. Austria.

4 Morulus. Schh. Sicilia.

46 Obsitus. Schh. Illyria.

47 Irritans. Herbst. Austria.*

\{Multipunctatus. Fabr. Silesia.

Adspersus. Dahl. Cat. Hungaria.

48 Perforatus. L.Redtenb. Austria.

49 Mastix. Olivier. id. *

50 Pruinosus. Germar. Dalmatia.

51 Turgidus. Germar. id.

52 Scabricollis. Germar. Illyria.

53 (Geniculatus. (Megerle.)

$\left\{\begin{aligned} \text { Schh. } & \text { Germania." } \\ \text { Irroratus. Ziegler. } & \text { Austria. }\end{aligned}\right.$

54 Consentaneus. (Parreyss.) Schh. Dalmatia.

55 Periscelis. Schh. Illyria.

56 Insubricus. Villa. Lombardia. ${ }^{*}$

57 Scabrosus. Marsham. Anglia.*

Crispatus. Dej. Cat. Gallia.

58 Sulcirostris. Schh. Dalmatia.

59 Meridionalis. Dej. Cat.

60 Fossor. Schh.

Schh. Gall. merid.*

61 Parvicollis. Schh.

Pyrenceis.

62 Histrio. Stéven.

63 Lævigatus. Fabr.

Algiria.

64 Concinnus. Schh.

65

Giraffa. Germar.

Corruptor, Jacqu. Sicilia.

Var. Crysescens. Dahl.

in litt. Hungaria.*

66

Turca. Stéven.

Turcia.

67

$$
\text { Armatus. Dej. Cat. }
$$

$\left\{\begin{array}{c}\text { Schh. Dalmatia. } \\ \text { Irroratus, Dahl. in } \\ \text { litt. id. }\end{array}\right.$

68 Romanus. Chevrolat.

Schh. Roma.

69 Polycoccos. Schh. Constantinp.

70 Krattereri. Stentz.Germar. Schh. Turcia.

Gemellatus. (Parreyss.

Dej. Cat.) Volhynia. 
71

(Lavandus. Koy. Schh. Austria.

Metallifer. Dahl. in litt. Hungaria.

72

73

Roscidus. Schh. id

Affinis. L. Redtenb. Austria.
Sulcifrons. Schh. Illyria.

Aurosparsus. Germar.

Russ, merid.

75 Squameus. God. Schl. Hungaria.

76 Psegmaticus. Schh. Tyrolis.

Metallifer. Dahl. in litt. id.

77

78

79

80

81

82

83

84

Brachialis. Schönherr. Russ. merid.
Vilis. Stéven. Schh. Tauria.

Alutaceus. Germ. Sch. Dalmatia.

Punctatissimus. (Ziegler.) Schh. id.

Vittatus. Kunze.Schh.id.

Memnonius. Gyllenh.

Schh. Germania.

Morio. Fabr. Gallia.

nicolor. Hbst. Schh. id.*

Morio. Schh. Sturm. Germania.

Tenebricosus, Oliv. Gallia.

85

Wagneri. Chevrolat. Algiria."

Ebeninus. Fabr. Germania.*

Corticalis. Lucas. Algiria.

Malefidus. Schh.

Imus. Schh.

Gall. merid.

Helvetia.

90 Honoratii.Dej.in litt. Alp. Gall.*

91 Sanguinipes. Chevrolat. Schh. Italia.*

92

93

94

95

96

97

98

99

100

101

102

103

10

10

106

107

Clavipes. (Peyroleri.)

Argutus. Schh. Schh. Helvet. Alp. ${ }^{*}$ Europ. mer.? 134 Dalmatia. $\quad 135$

Sabus. Schh.

Densatus. Schh. Helvetia.

Lanuginosus. Chevrolat. Schh. Italia.

Pilosus. Schh.

Tauria.

Repletus. Schh.

Scitus. Schh.

Ostentatus. Schh.

Polonia.

Constantinp. 140

Vestitus. Schh.

Germania. id.

Chrysonus. (Dahl.)

Schh. Hungaria.

Reticulatus. Chevrolat. in litt. Alp. Gall.*

Lasius. Germ. Schh. Illyria.

Frigidus. Rambur. Hisp. merid.*

Montanus. (Parreyss.)

Schh. Styria.
108

109

110

111

112

113

114

115

116

117

118

119

120

121

122

123

124

125

126

127

128

129

130

131

Cribricollis. Dej. Cat.

Schh. Gall. merid.*

Numidicus. (Gaubil.) Algiria. *

Reticollis. Germar. Ragusa.*

Concavifrons. Schh. Orierite.

Comparabilis. Schh. Dalmatia.

Striatosetosus. (Helfer.) Schh. Sicilia.

(Dulcis. Germar. Schh.Dalmatia.

Yar. Mastix, Dej.Cat. Styria.

Stomachosus. Schh. Gall. merid.

Pubeus. Chevrolat.

Schh. id.

Grandicollis. Schh. Oriente.

Intercalaris. Schh. Hungaria.

Perdix. Olivier. : Austria.*

Squamifer. Schh. Gracia.

Affaber. Schh. Italia. *

Pubifer, Klug. Schh. Oriente.

Velutinus. (Megerle.) Germar. Schh. Hungaria.

\{ Humilis. Germar. Russ, merid.

Godetii. Besser. id.

Brunneus. Stév.Schh. Tauria.

Crispus. Schh. _ Russ. merid.

Vitellus. Schh. Gallia.

Scabridus. Kirb.Schh.Anglia.

Crenatus. Luczot. in

litt. Gall. merid."

Tomentifer. Schh. Tauria.

Infernalis. Germar.

Schh. Illyria.

132 Scalptus. Chevrolat.

Dej. Cat. Or. Sardin.*

Acatium. Schh. Tauria.

Impressipennis. Brul. Gracia. ${ }^{*}$

Rugosus. Hum. Schh. Petropoli.

Rhæticus. Heer. Helvetia. ${ }^{*}$

Agnatus. Stm. Schh. Hungaria.

Obsidianus. Germar. Gallia.

139 Asphaltinus. Germar. T'auria.

Tauricus. Dej. Cat. id.

Crenatostriatus. Chevrolat, in litt. Alp. Gall.*

141 Alpicola. Esch. Schh, Gall, orient.*

142 Ecoffeti. Chevrolat. in litt. Gall. merid.*

143 Aterrimus. Schmidt. Schh. Carniolia. ${ }^{*}$

144 Geminatus. Fabr. Styria.*

Religiosus. Schrank. Hungaria.

Var. Squamiger. Oli-

vier. Illyria. 
145 Chlorophanus. (Meg.)

146 Schh. Austria.*

146 Dives. (Dahl.) Schh. Hungaria.*

147 (Cymophanus. Germ. $\left\{\begin{array}{l}\text { Schh. id. } \\ \text { Dives. Dej. Cat. id. }\end{array}\right.$

148 Nigrita. Fabr, Gall. bor. ${ }^{*}$

Eneopunctatus. Gyl. Suecia.

Sulcatus. Paykull. id.

Cupreus. Laichart. Tyrolis.

Tristis. Scopoli. Carniolia.

Lugubris. Linné. Suecia.

Salicis. Stroëm. id.

149 Lepidopterus. Fabr. Austria.*

(Squamiger, Fab.Lat. Norwegia.

150 Inductus. Sturm. Cat.

Schh. Croatia.

151 Pupillatus, Schh. Sicilia.

152 Transversempressus. Chev.Schh. in litt. Algiria.*

153 Signatipennis. (Dahl.)

$\left\{\begin{array}{c}\text { Schh. Croatia. } \\ \text { Aureolus. } \\ \text { Schs. } . \text { id. }\end{array}\right.$

154 Eremicola. (Miller.) Rosenhauer. Tyrolis.*

155 Maxillosus. Dej. Cat. Schh. Austria.

156 Squamosus, Dej, Cat. Styria.

157 Gibbicollis. Schmidt. Schh. Carniolia.*

158 Squamulatus. Dej.Ct. Styria.*

159 Gracilis. Dej. Cat.

Schh. Gall.merid.

160 Elegantulus. (Dahl.) Schh. Carniolia.

161 Bicostatus. (Friwaldszky.) Schh. Tauria.

162 Orbicularis. Herbst. Schh. Austria. ${ }^{*}$ Tenebricosus. Oliv. Gallia.

163 Petrensis, Germ.Schh.Illyria.

164 Navaricus. Dej. Cat. \{Schh. Pyrenceis. ${ }^{*}$ q Catelunatus. Schh. id.

163 Sulcogemmatus. Chevrolat. Schh. Hispania.

166 Maurus. Gyllenh. Suecia.* Bructeri.1lliger. Austria.

Demotus. Schh. id. Wiesurii. Parreyss. id. Silesiacus. Besser. Silesia.

167 Comosellus. Gyl. Sch. Hungaria.
168 (Pauper. (Ziegl.) Schh. Austria. ${ }^{*}$ Ventricosus, Dahl. in litt. id.

Hortensis, Megerle.

in litt. id.

Pustulatus. Ullrich.

169 Brevicornis. Sch. Constantinp.

170 Atroapterus. de Geer. Pomerania.* Rotundatus. Linné. Suecia.

Y Var. Arenosus. Mac-

Leay. Anglia.

171 Monticola. Germar Germania.

172 Lævigatus. Gyllenh. Suecia.* Arcticus. Fabr. id. Monticola. Dej. Cat. Schh. Pyrenceis.

173 Blandus. Schh. Lapponia.

174 Obcrcatus. Schh. Russ, merid.

175 Denigrator. Schh. Hungaria.

176 Angusticollis. Schh. Buckaria.

177 Poricollis. Schh. Russ. merid.

178 Puncticornis. Schh. Tauria.

179 Lithanthracius. Schh. Austria.

180 Chrysocomus. (Meg.)

Schh. Hungaria.

181 Impexus. Schh. Russ. merid.

182 Juvencus. Dej. Cat.

Schh. Gall. merid.

Vellicatus. Germar. id.

Lanuginosus. Dej.Ct. id.

183 Tomentosus.Dej.Cat.

Schh. id.

184 Ovatulus. Schh. Gall. merid.

185 Juvenilis. Schh. Russ, merid.

186 Fulvipes, Dej. Cat.

187 Pusio. Schh. Schh. Gall, merid.

188 Conspersus. Herbst, id.

\{ Chrysostictus. Stẻv. id.

189 Obtusus. Germ. Schh. Illyria.

190 Picipes. Fabr. Gallia.* Singularis. Stephens. Anglia.

Squamiger. Marsh.' id.

Notatus. Bonsd. Germania.

Asper. Marsham. Anglia.

Granulatus. Herbst. Austria.

isingularis. Schrank. id.

191 Dentatus. Heer. Schh. Petropolis. *

Marquardtii. Fald. id.

Notatus. Stephens. id.

Vastator. Marsham. id.

192 Chevrolatii. Schh. Belgia. 
193 (Duinensis.(Dahl.)Sch.Illyria. \{ Singularis. Dej. Cat. Styria.

194 Confusus. Schh. Illyria. Duinensis. Dej. Cat. Styria. Singularis. Dej. Cat. id.

195 Singularis. Linné. Lusitania

196 Hirticornis. Herbst. Schh. Austria.*

Chrysoleucus.Stenz. Suecia.

Variegatus. Schh. Helvetia.

Depubes. Schh. Alp. Helvet.

Pellicus. Schh. Oriente.

Cremieri. Chevrolat. Schh. Italia.*

gerle. St. Cat.) Austria.*

202 Septentrionis. Herbst.

Schh. Germania.

Setosus. Sahlberg. Suecia.

203 (Porcatus, Hbst. Schh, Austria.*

Costatus. Fabr. id.

Senex. Olivier. id.

204 Raucus. Fabr. P.*

Luctuosus. Latreille. Gall. bor.

Var. Tristis. Latr. id.

Arenarius. Herbst. Austria.

Fulvus. Fabr. Germania.

205 Mandibularis. L. Redt. Austria.

206 Ligneus. Olivier. Gall. merid. ${ }^{\star}$ Rufipes. Dej. Cat. id.

Gallicanus. Schh. Hispania.

Var. Setosus. Megerle. in litt. id.

Planithorax. Chevrolat. Schh. Algiria.

208 Foraminosus. Germ. Schh. Helvetia.

209 Hystrix. Schönherr. Russ. merid.

210 Granulosus. Küenburg. Schh. Hungaria.

211 Uncinatus. Germar.

Setifer. Schh. Schh. Germania.*

Ligustici. Linne. Gallia.

Rugosus, Schrank. Austria.

Monopterus. Fourc. $P$.

Levistici.Müller. Germania.

Mulleri. Linné. . id.

Var. Collaris. Fabr.

$$
\text { Latr. } P \text {. }
$$

Agnatus. Sturm. Cat. Germania.

214 Auricapillus. (Meg.) Germar. Schh. Carniolia.
215 Granicollis. Sturm.

Cat. Schh. Hungaria.

216 Helvetius. Chevrolat.

Schh. Helvetia.

217 Granulatus. Dej. Cat. Sicilia**

218 Sulcatus. Fabr. Gall, merid.*

Griseopunctatus. de

$\left\{\begin{array}{l}\text { Geer. id. } \\ \text { Strictus. Linné. }\end{array}\right.$

219 Bisulcatus. (Ziegler.

220 Auricomus, (Megerle.)

221

\{ Funicularis. Schh. Illyria.

Schh. Carniolia.

Infaustus. Schh. id.

222 Nubilus, Esch. Schh. Helvetia.

223 Populeti. Friw. Schh. Hungaria.

224 (Clathratus. Germar.

$\{$ Schh. Illyria.

Ruficapillus. Dej. Ct. Austria.

225 Cancellatus. Schh. Italia.

Clathratus. Chevrol. in litt. id.

226 Punctiscapus, Schh. Germ. austr. \{ Rugosus, Dahl, in litt, id.

227 Partitialis, (Zollikofer.j Schh. Gallia.

228 Mostus. Dej. Cat.

Schh. Gall, merid.

229 Mostificus. Schh. Russ. merid.

230 Strigirostris. Schh. Italia.

231 Montivagus. Schh. Tyrolis.

232 Kollari. Germ. Schh. Bucovina.

233 Austriacus. Fabr. Austria.*

234 Subquadratus. Rosen-

hauer. Tyrolis.

235 Carinatus, Stm. Schh. Illyria.

236 Erifer. Schüp. Schh. Germania.*

Elaboratus. Schh. Saxonia.

237 Subsignatus. Schh. Russ, merid.

238 Zebra.Fabr. Austria.*

$\{$ Carinatus. Paykull. Suecia.

Fulla. Schrank. Austria.

239 Varius. Schh. Helvetia.

240 Pauxillus, Rosenh. Tyrolis.

241 Cratægi. (Dahl.) Schh. Istria.

242 (Rugicollis. Germar.

Sulcicollis Sch. Dalmatia.*

243 Aureolus.(Par.)Schh. Croatia.

244 (Rugifrons. Gyllenh. Suecia.*

Dillwyanii. Kirby. Anglia.

Var. Scaber, Steph. id. 
245 Impoticus.Chevrolat.

246 Pinastri. Hbst. Schh. Styria.*

Pertusus, Dej. Cat. id.

247 Glabellus. Rosenh. Tyrolis.

248 Frescasti. Helf. Schh. Italia.*

249 Segnis. Schönherr. Germania.

250 Verticosus. Schönh. Gallia.

251 Globus. Waltl. Schh. Hungaria.

252 Ovatus. Linné. Gallia.*

Rosa. de Geer. Suecia.

Rufipes. Scopoli. Carniolia.

Scopolii. Linné. Suecia.

253 Desertus. Rosenh. Tyrolis.

254 Clemens. Schönherr. Russ. merid.

255 Arenarius. (Melet.) Helvetia.*

256 Lasius. Germ. Schh. Dalmatia.

257 Pimeloides. Olivier. Gall. merid.

258 Lirus, Schönherr. Gallia.

\{ Lavigatus, Olivier. Gall. merid.

259 Lima. Marsham. Anglia.

260 Ambiguus. Schönh. id.

\{ Rugicollis. Steph. id.

261

262

263

264

265

Pubescens. Schönh. Russ, merid.

Fraxini.(Dahl.) Schh, Illyria.

(Turbatus. Schönherr. Italia.

Nigrita. Olivier. Gall. merid.

Anthracinus. Scopoli. Carniolia.

(Corrugatus. Gmelin.

Rugosissimus.Villers.id.

266 Metallescens. Lucas. Algiria.

267 Prolixus. Rosenhauer.

(Erich.) Tyrolis.

268 Cisalpinus. Dej. Cat. Ital. sup.

269 Apenninus. Dej.Cat. id.

270 Epidaurensis.Dej. Ct. Dalmatia.

271 Gilvipes. (Dahl.) Dej.

Cat. id.

272 Biturigensis. Dej. Cat.Gallia.

273 Parcipilis. Imhoff. St.

Cat. Helvetia.

274 Italicus, Sturm. Cat. Italia.

275 Nobilis. Dej. Cat. Carinthia.

276 Subcarinatus. St.Cat. Sicilia.

277 Elongatus. Dej. Cat. Gall, merid.

278 Rorulentus. Rosenh.

279 Atomarius. (Dahl.)

St. Cat. Hungaria,

Dej. Cat. Austria.

280 Tigrinus. St. Cat. Sicilia.

281 Nebulosus. St. Cat. Dalmatia.

282 Submaculatus, St, Ct. Illyria.
283 Subplanatus. St. Cat. Gall. merid.

284 Costatus. Sturm. Cat. Grocia.

285 Subcostatus. Waltl.

St. Cat. Dalmatia.

286 Testaceus. Stm. Cat. Styria.

287 Rhamni. Sturm. Cat. Austria.

288 Melanarius. Dej. Cat. Bucovina.

289 Gemellatus. Parreyss. Dej. Cat. id.

290 Aulicus. Sturm. Cat. Bannat. A Aurifer. Sturm. Cat. id.

291 Opulentus. Kuenbg. Austria.

292 Ethiops. Dej. Cat. Styria.

293 Aurulentus. Dej. Cat. Bucovina.

294 Comptus. Dej. Cat. Styria.

295 Gallicus. Sturm. Cat. Gall. merid.

296 Conspurcatus. (Parreyss.) Dej. Cat. Croatia.

297 Punctatostriatus. Stm.

Cat. Constantinp.

298 Terreticollis. St. Cat. Germania.

299 Lævistriatus. St. Cat. id.

300 Tristis, Dej. Cat. Gall, merid.

301 Corvinus. Dej. Cat. Anglia.

302 Morosus. Dej. Cat. Gall. merid.

303 Carinærostris, Dej.

304 Ereus.(Ziegler.) Dej.

305

Carmagnola. Villa.St.

$$
\text { Cat. id. }
$$

306 Genei. (Ziegler.) St. Cat. Lombardia.

307 Costipennis. Dej. Cat. Lombardia.

308 Alpestris. (Dahl.) Dej.

Cat. Hungaria.

309 Hispidulus. Dej. Cat. Austria.

310 Costulatus. (Ziegler.) Dej. Cat. id.

311 Patruelis. Dej. Cat. Gracia.

312 Modestus. Stm. Cat. Italia.

313 Rotundicollis. Sturm.

Cat. Gall. merid.

314 Lineatus. Sturm.Cat. id.

315 Lugens. Germar. Dej.

Cat, Dalmatia.

316 Rugipennis. (Dahl.)

Dej. Cat. Dalmatia.

317 Hottentota. Dej. Cat. Styria.

318 Dahlii. Dej. Cat. Hungaria.

\{ Chrysocomus. (Dahl.) id.

319 Parreyssii. Dej. Cat. Russ, merid. Longicollis. (Parr.) id. 
320 Costatopunctatus.Dej. Cat. Austria.

321 Bidens. Sturm. Cat. Sicilia.

322 Rugulosus. (Dahl.)

Sturm. Cat. Carniolia.

323 Pulvereus. Dej. Cat. $P$.

324 Striatopunctatus. Dej.

Cat. Gall. merid.

325 Gazella. (Dahl.) Stm. Cat. Hungaria.

326 Terricola. (Dahl.) St. Cat. Bannat.

327 Cuprifer. (Dahl.) St. Cat. id.

328 Calcaratus. Dej. Cat. Dalmatia.

329 Asperipennis, Sturm.

Cat. Gall. merid.

\section{CHILONEUS. Schönherr.}

1 Siculus, Schönherr. Sicilia.

\section{PHOLICODES. Schönherr.}

Brachyderes. Dej. Cat.

1 Plebejus. Schönherr, Russ. merid. 2 Trivialis. Schönherr. id.

3 Inauratus. Schönherr, id.

\section{LAPAROCERUS. Schönherr.}

Eremnus. Schönherr.

1 Morio. Schönherr. Lusitania. \{Barthelotti. Guérin. id.

2 Piceus. Schönherr. id.

\section{HOLCORHINUS. Schönherr.}

1 Seriehispidus. Schönh. Algiria.

2 Querulus. Schönherr. id.

\section{PERITELUS. Germar.}

\section{Curculio. Sparm.}

Griseus. Olivier. Gallia.*

Spharoides. Germar. Germania.

Inquinatus. Illiger. Austria.

2 Sphæroides. Creutzer. Hungaria.*

3 Setulosus.(Chevrol.) Gall. merid.*

4 Necessarius. Schönh. id.*

5 Elegans, (Gaubil.) Algiria.
6 Encaustus. (Chevrol.) Pyr. orient.*

7 Rusticus. Chevrolat.

8 Schœnherri. Chevrol.

Schh. Gall. merid.*

9 Famularis. Schönherr. Tauria.

10 Familiaris. Schönherr. Italia.

11 Noscius. Chevrol. Sch. id.*

12 (Senex. Dej. Cat. Schh. Gall, merid.* Spharoides.Dej. Cat. Hungaria.

(Sentinus. (Schönherr.) Gall. merid.

13 Albolineatus. Germar. Germania.

14 Leucogrammus. Germ. id.*

15 Lithargyreus. (Meg.) Austria.

16 Fuscipes. (Megerle.) id.

17 Lanuginosus. Creutz. id.

18 Virens. Sturm. Cat. Italia.

19 Rudis. Schönherr. Etruria.

20 Globulus. (Dahl.) Schh. Hungaria.

21 Oblongus. Dej. Cat. Gall. merid.

22 Canus, Dej. Cat. id.

23 Elegans. (Gaubil.) Algiria.*

\section{OMIAS. Schönherr.}

Gurculio. Fabr. Peritelus, TrachyPHLAUS. Germar.

1 (Seminulum. Fabr. Hungaria.

Globulus. Olivier. Germania.

Globosus. Sturm. id.

2 Glomeratus. Schönh. Tauria.

3 Globosus. Stéven. id.

4 Rotundatus. Fabr. " Podolia.*

Ovatus. Olivier. Austria.

5 Rufipes. Schönherr. Volhynia.

6 Verruca. Stéven. Schh. Hungaria.

7 Puberulus. Stév. Schh. Tauria.

8 Bohemanii. Schönherr. Saxonia.

9 Strigifrons. Schönherr, Russ. merid.

10 Ruficollis. Fabr. Germania.

\{ Holosericeus, Fab.Lat. id.

11 Mollinus. Ahrens. Schh. id.

12 Gracilipes. Panzer. id.

13 Rugicollis. Schönherr. Austria.

14 Hirsutulus. Fabr. Germania. * Scabriculus. Herbst. Austria.

Scaber. Müller. id.

Var.Echinatus. Bonsd.

Latr. Gallia.

15 Villosulus. Germar. Austria.

16 Pruinosus, Chevrolat.

Schh. Dalmatia. 
17 Indigens. (Hoffmsgg.)

Schh. Lusitania.

18 Brunnipes. Olivier. $P^{*}$

Gracilis. Beck. Germania.

Piceus. Germ. Steph. id. Araneiformis, Schrk. Austria.

Mollicomus. Ahrens. Saxonia.*

20 Punctirostris. Schönh. Hamburgi.

21 (Pellucidus. Chev.Schh. P.*
$\{$ Piceus. Leach.
Anglia.

22

Brunneus. Dej. Cat. P.*

23 Chevrolatii. Schönh. Dalmatia.

24 Subnitidus. Schönherr. Gallia.

25 Ebeninus. Chevr.Schh. Gall. bor.

26 Nitidus. Stéven. Schh. Tauria.

27 Sericeus. Schönherr. Illyria.

28 Parvulus. (Ullr.) Schh. Austria.

29 Concinnus. Schönh. Gallia.

30 Oblongus. Schönherr. Gall. merid.*

31 Sulcirostris. Schönh. Pyr. orient.*

32 Companyonis, Chevrol.

$$
\text { Schh. id. }
$$

33 Forticornis. Germar, Germania.*

34 Sulcifrons. Schönherr, Anglia.

35 Pectoralis. (Gaubil.) Algiria.*

36 Barbarus. (Gaubil.) id.*

37 Orbicularis. Stm. Cat. Hurgaria.

38 Picipennis. Stm. Cat. Russ, merid.

39 Squamosus. Stm. Cat. Hungaria.

40 Provincialis. Dej. Cat. Gall. mérid.

41 Castaneus. (Chevrol.) id.*

42 Porcinus. (Meg.) Dej.

43 Velutinus, Dej. Cat. Austria.

44 Lusitanicus. Dej. Cat. Lusitania.

45 Trichopterus. (Chevrl.) Gall. bor. ${ }^{*}$

46 Validicornis. Germar. Germania.

\section{STOMODES. Schönherr.}

Oмias. Schönh.

1 Totularius. Schönh. Tauria.

2 Gyrosicollis. Schönh. Dalmatia.*

3 Rudis. Schönherr. Constantinp.

\section{CATHORMIOCERUS. Schönherr.}

Trachyphloevs, $S c h h$.

1 Horrens. Schönherr. Hispania.* Erinaceus, Fabr. Dej. Cat. id.

2 Socius. Schönherr. Anglia.
TRACHYPHLOEUS, Germar, Schh.

Curculio. Iinné. Fabr. Gyll.

1 Erinaceus. Schönh.? L.

Redtenb. Austria.

2 Ventricosus. Germar. Hungaria.

3 Scabriculus. Linné. $\quad \boldsymbol{P}_{\text {. }}$

Bifoveolatus, Beck. Germania.

Viverra. Herbst. Austria.

Var. Hispidulus, Hbst. id.

4 Squamosus. Schneider.

Schh. Gallia.

Var. Confinis. Steph. Germania.

5 Waltoni. Schönherr. Anglia.

6 Aristatus. Schönherr. Gallia.*

Stipulatus.Hoffmann.Germania.

7 Setarius. Schönherr. Germania.* Scabriculus. Herbst.

Germar. Austria.

8 Squamulatus. Olivier, Gallia.

9 Scaber. Linné. $\quad$ id.*

Echinatus, Dej, Cat. P.

Var. Alternans, Schh. Suecia. ${ }^{*}$

Spinimanus. Steph. Anglia.

10 Camomillæ, Dahl. St.

Cat. Hungaria.

11 Spinosulus. Dej. Cat. Hispania.

12 Spinimanus. Germar.

$\left\{\begin{array}{r}\text { Spinimanus. Germar. } \\ \text { Lucas. Algiria.* }\end{array}\right.$

(Scabriculus. Olivier. id.

13

14

15

16

17

18

Spurcus. Dej. Cat.

Aquilus. Dej. Cat.

Hispania.

Metallicus, Dej. Cat. id.

Digitalis. Gyllenhal. Suecia.

Lanuginosus. Mannerh. Podolia.

Inermis. Germar. Germania.

\section{PTOCHUS. Schönherr.}

Peritelus. Germar, Sitona. St. Cat.

1 Porcellus, Schönherr, Tauria.

2 Setosus. Schönherr. Russ, merid.

3 Perdix. Schönherr. Tauria.

4 Rufipes. Schönherr. Russ. merid. Fasciculatus, Gebler, id.

5 Bisignatus. Germar. Hungaria.

$\{$ Grandicornis.Dej.Cat. Dalmatia.

6 Subsignatus. Schönh. Tauria.

7 Gremieri, Chevr. Schh. Gallia.* 
8 Maculatus. Stm. Cat. Tauria.

9 Bimaculatus. Stm. Cat. Istria.

10 Setulosus, Stm. Cat. Tauria.

11 Pulchellus. Stéven. St.

12 Flavipes. Germar. Volhynia.

\section{PHYLLOBIUS. Schönherr.}

Germar. Curculio. Linné. Fabr.

1 Calcaratus, Fabr. Gallia.*

Pyri. Illig. Dej. Cat. Germania.

Var. Glaucus. Scopoli. Carniolia.

Carniolicus, Olivier. id.

2 Atrovirens. Schönherr, Gall, orient. ${ }^{*}$

3 Pyri. Linné. Gallia.*

Eruginosus, Latreille. $P$.

Cœsius, Marsh. Steph. Anglia.

Argentatus. Laich. Tyrolis.

Alneti. Illiger. Germania.

Var. Cœlestinus. Scop. Carniolia.

Prasinus. Oliv, Latr. P.

4 Pomaceus. Schönherr. Sueciamerid.

5 Canus. Schönherr. Tauria.

6 Feculentus. Schönh. id.

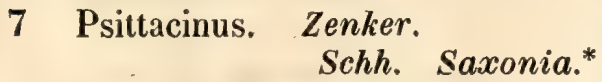

8 Longipilis. Germar. Sicilia.

9 Pellitus.Chevrol.Schh.Sardinia:

10 Fulvipes. Schönherr. Tauria.

Fulvago. Stéven. Schh. id.

Piliferus. Schönherr. Russ. merid.

Verecundus. Schönh. id.

(Argentatus, Linné. Gallia.*

Sericeus. Piller et Mit-

terps. id.

Var, Arborator, Hbst. Austria.

15

Pineti. L. Redtenb. Austria.

Maculicornis. Germar, Germania.*

Viridans. Schönherr, Bavaria.*

Acuminatus. Schönh. id.

Virens, (Stm.) Schh. Germania.

Contemptus, Dej, Cat.

\section{Schh. Tauria.}

Oblongus. Linné. Gallia.*

Floricola. Herbst. Austria.

Pruni. Scopoli. Carniolia.

Querneus. Fourcroy. P.

Fuscus, Laichart. Tyrolis.

Var. Rufescens. Marsh. id.
24 Mus. Fabr.

Cinerascens, Germar, Germania.

Canescens. Germar, id.

Cinereus. Dej. Cat, $\quad P$.

25 Sinuatus. Fabr. Germania.

26 Vespertinus, Fabr. Gallia.*

Mali. Gyll. Dej. Cat.

$\{$ Latr. P.*

Fulvipes. Fabr. Latr. id.

Var. Erythropus, Lat, id.

27 Mutus. Schönherr. Volhynia.

28 Aurifer. Schönherr. Hungaria.

29 Ginereus. Hartl. Schh. Silesia.

30 Subdentatus. Germar. Sicilia.

31 Incanus. Schönherr. Austria.

32 Scutellaris. L.Redtenb, id.

33 Pictus. Stéven. Schh. Tauria.

34 Pallipes. Schönherr. Gracia.

35 Betulæ. Fabr. Gallia.*

36 Ruficornis. Dej. Cat.

L. Redtenb. Gall, merid. ${ }^{\star}$

37 Trivialis. Schönherr. Russ. merid.

38 Tereticollis. Schönh. Volhynia.

39 Cylindricollis. Schönh. Russ, merid.

40 Brevis. Schönherr. Tauria.

41 Uniformis. Marsham. Gallia.*

Var. Parvulus, Gyll. Suecia."

Fulvipes. Paykull. id.

Var. Pomonce. Olivier. P.*

Argentatus. Bonsd. id.

Viridiareis, Laichart. Tyrolis.

Cinereipennis. Schönh. Tauria.

Viridicollis. Fabr. Germania.*

Sulcirostris. Schönh. Volhynia.

Dispar. L. Redtenb. Austria.

Planirostris. Schönh. Tauria.

Suratus. Schönherr. Russ, merid.

48 Seriehispidus. Schönh. Tauria.

49 Albidus. Stephens. Anglia.

50 Minutus. Stephens. id.

\section{RHYTIRHINUS. Schönherr.}

1 Nodifrons. Schönherr. Hispania.

2 Crispatus. Chevrolat.

Schh. Gall, merid.

3 Impressicollis.Dej.Cat.

Schh. id.*

4 Dentipennis, (Javet.)

$$
\text { Schh. id.* }
$$

5 Variegatus. Lucas,

6 Humilis. Lucas.

7 Annulipes. Lucas.
Algiria.

id.*

id. * 
8 Impressicollis. Lucas. Algiria.

9 Horridus, Lucas, id. *

\section{CONIATUS. Germar. Schh.}

Hypera, Ger, Gurculio, Fabr.

1 Tamarisci. Fabr.

Y Vanus. Herbst.

2 Repandus. Fabr.

Var. Tamarisci. Oliv. id.

3 Splendidulus. Fabr. Russ, merid.

4 Suavis. Schönherr. Sardinia.*

5 Chrysochlora, Lucas, Algiria.

\section{LIMOBIUS. Schönherr.}

Phyronomus. Schh. Dej. St. Cat. Curculio. Hbst.

1 Dissimilis. Herbst. Gallia.*

\section{PHYTONOMUS, Schönherr.}

Hypera. Germar. St. Cat. Phinthos. Latr. RhyNChenus. Fabr, Oliv. Gyll. Gurculio. Herbst.

1 Philanthus. Olivier. $\boldsymbol{P}^{*}$

\{Limbatus. (Dahl.) Sicilia.

2 Turgidus. Dej. Cat. Algiria.*

3 Punctatus. Fabr. Germar. Gyll. Gallia.*

4 Circumvagus. (Chev.) Algiria.*

5 f Fasciculatus. Herbst. Gallia.*

Danei. Olivier.

6 Fuscatus. Schönherr, id.

7 Anceps. Schönherr. Russ. merid.

8 Rufus. Schönherr. Helvetia.

9 Viennensis, Herbst. Austria.

10 Cyrtus. Germar. Dalmatia.

11 Intermedius. Schönh. Helvetia.

12 Velutinus. (Parreyss.)

Schh. Hungaria.

13 Turbatus. Schönherr. id.

14 Latipennis. Schönh. Italia.

15 Salviæ. Schrank. Germ. merid. Cyrtus, Germar. Italia.

16 Elegans. Dahl. Cat. Schh. Hungaria.

17 Palumbarius. Germar. Carniolia.

18 ,Comatus. (Meg.) Schh. Austria. $\{$ Palumbarius.Dej.Cat.Etruria.

19 Crinitus, Dej. Cat.Sch. Gall. merid.

20 Socialis. (Helf.) Schh. Sicilia.
21 Maculatus. L. Redtenb. Austria.

22 Tesselotus. (Meg.) Sch. Germania.

23 Contaminatus. Herbst. id. *

24- Oxalis. Herbst. id.*

25 Circumvagus. Schönh. Gall. merid.

26 Dapalis. Schönherr. Algiria.

27 Elongatus. Paykull. Germania.

Oblongus. Schönherr. Sicilia.

29 Fuscescens. Schörh. P.

30 Ovalis. Schönherr, Gallia.*

31 Arundinis, Fabr. Germania.*

32 Rumicis. Linné. $\quad \boldsymbol{P}_{\text {. }}^{*}$

Acetosa. Panzer. Germania.

33 Pollux. Fabr. $\boldsymbol{P}_{\text {* }}^{*}$

Adspersus. Fabr. Germania.

Commaculatus. Hbst. Austria.

Interruptus. Marsh. Anglia.

Fasciolatus. Villers. Gallia.

34 Ignotus. (Chevrier.)

Schh. Helvetia.

35 Histrio. Schönherr. Austria.

36 Suspitiosus. Herbst. Gallia.*

Miles. Gyllenh. Payk. Suecia.

Bitcmätus. Marsh. Anglia.

37 Sejugatus. Schönherr, Gall, orient.

38 Lubenculus. Schh. Algiria.

39 Setosus. Schönherr. id.

40 Signatus. Schönherr. id.

41 Viciæ. Gyllenhal. Germania.*

42 Tigrinus. Dej.Cat.Sch. Gall. bor.*

43 Pastinacæ. Rossi. Gallia.

44 Malarhynchus, Olivier Lusitania.

45 Farinosus. Schönherr. Russ, merid.

46 (Plantaginis. de Geer. Gall. bor.*

Novius. Linné. Suecia.

Spartii. Hoppe. Germania.

47 Brunnipennis. Schönh. Sicilia.

48 Murinus. Fabr. $\quad \boldsymbol{P}$.

Incidiosus. Bohemann.

Schh. Suecia.

Elongatus. Stm. Cat. Germania.

Var. Melancholicus. Fabr. Latr, $P$.

49

(Variabilis. Herbst. Austria.*

Yar. Murinus. Germar.

Dej. Cat. Gallia.

50 (Suturalis. L. Redtenb. Austria.

\{Elongatus? Gyllenh. Suecia.*

51 Polygoni, Fabr. P**

Fasciatus, de Geer. id.

Striatus.Herbst. Austria.

Cinereus, Olivier. Gall, merid.

Var. Arator, Linne, Suecia. 
52 Kunzii. Ahrens. Gallia.* Lineatus. Herbst. Austria.

53 Striatus. Sturm. Schh. Sardinia.

54 Hispidulus. Dej. Cat.

5 Meles. Fabr. Schh. Gall. merid.* Trifolii - Herbst. Trivialis. Herbst. id.

Var. Roselii. Linné. Suecia. Pallidus. Dej. Cat. $\quad P$. Stramineus. Marsh. Anglia. Griseus. Müller. Germania.

56 Posticus. Gyllenhal. Gallia.* Bimaculatus. Marsh. Anglia.

57 Parcus. Schönherr. Gallia.

58 Incomptus. Schönherr. Lusitania.

59 Mixtus, Dej.Cat.Schh. Gallia.*

60 Defloratus. (Chevrol.) P.*

61 Constans. (Ziegl.) Schh. id.*

62 Plagiatus. L. Redtenb. Austria.

63 (Nigrirostris. Fabr. Gallia. ${ }^{*}$ Virescens, Quensel. Suecia. Var. Variabilis, Fabr. Germania.

64

65

66

67

68

69

70

71

72

73

74

Balteatus. Chevrolat. Lusitania. ${ }^{*}$

Liliputanus. Lucas. Algiria.

Austriacus. (Megerle.) Austria. ${ }^{*}$

Gravidus. Dej. Cat. Gall. merid.

Lateralis. Dej. Cat. id. ${ }^{*}$

Tumidus. Dej. Cat. Hispania.

Griseus. Dej. Cat. Gall. merid.

Melancholicus. Illiger. Austria.

Canus. Sturm. Cat. Germania.

Globosus. Dej. Cat. Gall. merid.*

Proximus. Carmagno-

la. Villa. Italia.

75 Varius. Sturm. Cat. Grocia.

76 Palustris. (Dahl.) St.

Cat. Bannat.

Flavicans. (Megerle.) Dej. Cat. Austria.

78

79

80

81

82

83

84

85

86

87

88

89
Maculosus. Dej. Cat. Styria.

Maculipennis. Dej.Cat. Hispania. ${ }^{*}$

Canescens. Stephens. Anglia.

Picicornis. Stephens. id.

Sublineatus. Kirby. id.

Nebulosus, Stephens, id.

Villosulus. Stephens, id.

Picipes. Stephens. id.

Fulvipes. Stephens. id.

Phacopus. Stephens, id.

Rufipes. Stephens. id.

Rotundatus. Chevro-

lat. Gall. merid.*
ADEXIUS. Schönherr.

1 Scrobipennis. Schönh. Helvetia.

PLINTHUS. Germar. Schh.

Curculio, Fabr, Herbst, Panz, Rhynchenus. Oliv, Fabr. Lixus, Fabr. LIparus. Oliv.

1 Gerlii. (Parr.) Schh. Croatia.

2 Findelii. (Dahl.) Schh. Hungaria.

3 - Megerlei. Panzer. Austria. ${ }^{*}$

4 Schmidtii. Sturm. Cat. Carniolia.

5 Tischeri. Germar. Germania.

6 Anceps. Schönherr. Silesia.

7 Styriacus. And. Schh. Styria.

8 (Sturmii. (Megerle.) Germania.

$\{$ Porculus. Sturm. Cat. id.

9 Illigeri. (Dahl.) Schh. Carniolia.

10 Granulifer. Schh. Europ.merid.

11 Parreyssii. Schh. . Illyria.

$\{$ Creutzeri? Dej. Cat. Carinthia.

12 Silphoides. Herbst. Russ. merid.

Geometra. Olivier. id.

Leucographus. Bœber. id.

Vittatus.Motschulsky. id.

13 Tigratus. Rossi. Italia.

14. Granulatus, Schönh. Alp. Helv.

15 Mucronatus. Rösenh. Tyrolis.

16 Schalleri. Germar. Carniolia.

17 Porculus. Fabr. Hungaria.*

$\{$ Porcatus. Panzer. Austria.

18 Caliginosus. Fabr. Gallia.*

19 Humilis. Findeli. Stm.

Cat. Italia.

\section{TROPIPHORUS. Schönherr.}

Barynotus. Germar. Curculio, Fabr. Brachyrivus. Latr.

1 Micans.(Friwaldszky.)

2 Mercurialis. Fabr. Suecia.

Schh. Hungaria.

Lapidarius. Paykull. id.

Lepidotus. Herbst. Germania.

Ubtusus, Bonsd, Latr. id.

Suecicus, Linné. Suecia.

Attenuatus. Dej. Cat. Styria.

3 Carinatus, Miiller, Sch. Germania.

Suturalis. Linné. id.

Mercurialis. Dej. Cat. Styria. 
4 Cinereus. Schönherr, Hungaria.

\{ Cuprifer. (Dahl.) id.

5 Globatus. Herbst.Schh. Gallia.

6 Ochraceosignatus. Sch. Austria.

\{cuprifer? (Megerle.) id.

\section{MOLYTES. Schönherr.}

Rhynchanus. Fabr. Gyll. Curculo. Linné. Marsh. Panz. Clairv.

1 Coronatus, Latreille, $P_{\text {.* }}^{*}$ Germanus, Fabr. Germania. Anglicanus, Marsh. Anglia. Teutonus. Illiger, Austria.

2 Germanus. Linné. Germania.* Fusco-maculatus, Fab.id.

YVar. Carincerostris. (Megerle.) Schh. Carniolia.

3 Illyricus. Ullrich. Schh. Illyria.

4 Glabratus, Fabr. Austria.* Dirus, Olivier. Gallia.

5 Dirus. Herbst. Germania.

6 Lævigatus. Stẻv. Schh. Tauria.

\section{TRYSIBIUS. Schönherr.}

MoLYTes. Schh. Dej. St. Cat.

1 Tenebrioides, Pallas. Russ, merid.* \{ Besseri. Schönherr. id.

2 Intermedius. (Friw.)

Schh. Constantinp.

3 Olivieri. Schönherr. Oriente. Tenebrioides, Olivier.

Schh. Grocia.

4 Punctipennis. Brullé. id.*

\section{ANISORHYNCHUS. Schönherr.}

Molytes. Schh. Dej. St. Cat. Liparus. Oliv, Rhynchenus. Fabr.

1 Bajulus. Olivier.

Gall. merid.*

2 Sturmii. Schönherr Sicilia.* \{ Bajulus, Olivier. Algiria.

3 Costatus. (Dahl.) Schh. Sardinia.*

4 Barbarus. Schönherr. id.*

5 Ferus, Erichson. Algiria.

6 Siculus. Schönherr. Sicilia.

7 Monachus.Germ. Schh. Sardinia.*

8 Aratus. Germ. Schh. Lusitania.
LEIOSOMUS. Kirby.

Molytes. Dej, St. Cat. Schh.

1 Ovatulus. Clairville. $P^{*}$

Punctatus. Marsham. Anglia.

Deflexus. Panzer. Germania.

Crassicollis. Dej. Cat. Austria.

2 Cyanopterus, L. Redtb, id.

3 Atritus. (Gaubil.) Algiria.*

4 Oblongus. Schönherr. Helvetia.

5 Impressus. Schönherr. Germania.

Deflexus. Panzer. id.

6 Gribrum. (Meg.) Schh. Austria.*

HYLOBIUS. Schönherr.

Liparus, Oliv, Rhynchenus, Fabr, Gyll. Curculio. Fabr. Rossi.

1 Arcticus. Paykull. Suecia sept.

2 Pineti. Fabr. Suecia. ${ }^{*}$

Confusus. Paykull. id.

Excavatus, Laichart. Tyrolis.

Inaccessus. Schrank. Sicilia.

Var. Piceus. de Geer. Suecia.

3 (Abietis. Linné. Gallia bor.*

Pini. Marsham. Anglia.

Juniperi. Stroëm. Germania.

Excavatus. Schrank. Austria.

Norvegicus. Petit. Norvegia.

Tigrinus, Fourcroy. $P$.

4 Pinastri. Gyllenhal. Suecia.*

5 Rugicollis. Mannerh. Gallia.

6 Rugulosus. Schönherr, Gall. merid.*

7 Fatuus. Rossi. Gallia.*

Var. Excavatus. (Lai-

( chart.) Tyrolis.

8 Albopunctatus. Stéven.

Schh. Russ, merid.

TANYSPHYRUS. Germar.

Rhynchenus. Fabr.

1 Lemnæ. Paykull.Fabr. $\left\{\begin{array}{r}\text { Gyll. } \text { Gallia. } \\ \text { Inspectatus, Herbst. }\end{array}\right.$

PROCAS. Stephens.

Schh. Curculio, Marsh.

1 Stevenii. Schonherr. Russ, merid. 
$2\left\{\begin{array}{l}\text { Picipes. Marsham. Anglia. } \\ \text { Pyrrhodactylus.Mars- } \\ \text { ham. id. }\end{array}\right.$

\section{LEPYRUS. Germar. Schh.}

Lipards, Oliv. Rhynchenus. Gyll. Fabr. Gunculio, Fabr, Herbst, Payk.

\section{Colon. Linné.}

Palustris. Scopoli. Germania.

Bipunctatus, Fourc, $P$.

2

Bimaculatus. Oliv. P

Semicolon. Herbst. Austria.

Capucinus. Schaller. id.

Coloniformis. Schrk. id.

Derasus. Panzer.

Germania.

3 Canus. Sturm. Cat. id.

4 Herbichii. Zawadsky.

\section{St. Cat. Galicia.}

\section{MINIOPS. Schönherr.}

Plinthus. Germar. Liparus. Oliv. Curculio, Fabr. Rossi. Meleus. St. Cat.

1 Carinatus, Linné. Gall. merid. Senex. Rossi.

Funereus. Herbst. Rugosus. Linné.

Italia.

Austria.

Scabrosus. Villers. Gall. merid.

Rugosostriatus, Schrk. Austria.

2 Perlatus. Sturm. Cat, Hungaria.

3 (Variolosus. Fabr. Austria.*

Carinatus. Olivier. $P$.

4 Scrobiculatus. Gyllenh.Tauria.

5 Sinuatus. Schönherr. Sicilia.

6 Costalis. Friwaldszky.

Schh. Austria.

7 Costatus. Friw. Schh. Turcica.

8 Minutus. Friw. Schh. id.

\section{BARYNOTUS. Germar.}

Schh. Gastrodus. Dej. Cat. Cunculio. Fabr.

1 Margaritaceus. Germ. Helvetia.* Tardus. Dej. Cat. Italia.

2 Parnassius. Imhoff. St. Cat. Helvetia.

3 Maculatus. Waltl. Sch.Alp.St.-Bern.
4 (Obscurus. Fabr. Murinus, Bonds. Honorus. Herbst. Pilosus. Marsham.

Vagus. Laichart.

Suecia.*

id. Austria. Anglia.

Tyrolis.

5 Carinatus. Müller. Styria.

6 Mœrens. Fabr. Saxonia. Bohemani. Gyllenhal. Suecia.

7 Mercurialis. Fabr. Styria.*

8 Alternans. Schönherr, Gallia.

9 Pyrenæus. Dej. Cat. Pyrenais.

10 Squalidus. Schönherr. Gallia.

11 Goryi. (Parreyss.) Dej.

Cat. Russ. merid.

12 Schœnherri. Boheman. Norvegia.*

13 Cuprifer. (Dahl.) St. Dej. Cat. Austria.

14 Squamosus. Dej. Cat.

Schh. Pyrenais.

15 Tesselatus. Stm. Cat, Russ. merid.

16 Trisulcatus. (Gaubil.) Alp. Gall.*

17 Lævigatus, Dej. Cat. Gall, merid.

\section{LIOPHLÆUS. Germar.}

Schh. Gurculio. Fabr.

1 Nubilus. Fabr. $\{$ Tesselatus. Latreille. id.

Var. Maurus. Marsh. Anglia.

2 Chrysopserus. Friw.

Schh. Hungaria.

3 Geminatus. Chev. Sch. $P$.

4 Obsequiosus. Schh. Volhynia.

5 Herbstii. (Meg.) Schh. Germania.*

6 Pulverulentus. Dej.Cat.

\section{Schh. $P$.}

7 Lentus. Germar. Hungaria.

8 Gibbus. (Meg.) Schh. id.

9 Schmidtii. Schönherr, Alp. Bohem.

\section{GEONEMUS. Schönherr.}

Geophilus. Schh. Banynotus. Germar. Meriones. Sturm.

1 Murinus. Schönherr. Algiria.

2 Schœnherri. (Gaubil.) id.*

3 Illætabilis. Schönh. Gall. merid.

4 Flabellipes. Olivier. id. ${ }^{*}$

Tergoratus. Germar. id.

Terrenus. Schönherr. id. 
ALOPHUS, Schönherr.

Curculio, Fabr. Lepyrus. Germar.

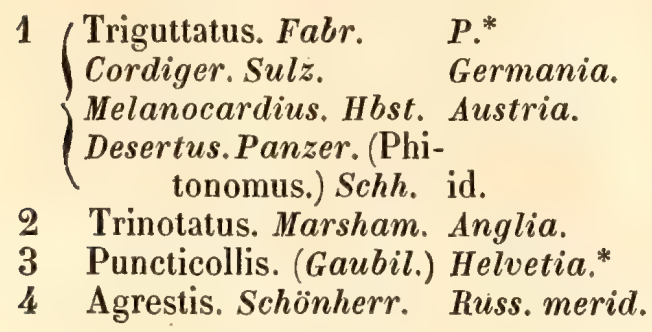

\section{GRONOPS. Schönherr.}

Bagous. Germar. Aulacus. (Megerle.) Curculo. Fabr. Oliv. Herbst. Rhy NCHENUS. Gyll. Ahrens.

$1 \begin{cases}\text { Lunatus. Fabr. } & \text { P.* } \\ \text { Testaceus. Gyll. Stév. } & \text { Suecia. } \\ \text { Amputatus. Olivier. } & \text { P. } \\ \text { Percursor. Herbst. } & \text { Austria. } \\ \text { Rubricus. Ahrens. } & \text { id. } \\ \text { Elevatus, Fabr. Schh. } & \text { Germania. }\end{cases}$

2 Sulcatus. Fisch. de Waldh. Russ, merid.

PACHYCERUS. Gyllenhal. Schh.

Cleonus. Schh. Lixus. Fabr. Oliv. Curculio. Herbst.

1 Mixtus. Fabr.

Algiria. Menetriesii. Schönh. Russ. merid. Atomarius. Schönherr.id.

2 Varius. Herbst. Gall. merid.

3 (Segnis. Hoffmansegg. $\left\{\begin{array}{r}\text { Schh. Sicilia. } \\ \text { Segnis. Ho/fmans. }\end{array}\right.$ Cordiger. Germar. Hungaria. Faldermanni.Dej.Cat.Podolia.

4 Scabrosus. Dej. Cat.

5 Planirostris. Schönh, id.

6 Albarius. Cevrol. Schh. Gallia.

7 Rugosus, Lucas. Algiria.

8 Corsicus. (Gaubil.) Corsica.*

\section{CLEONUS. Schönherr.}

Cheonis. Meg. Lixus. Illig. Germar, Latr. Oliv. Epimeces. Bilb. Curculio. Fabr. Oliv. Pall.

1 Sparsus, Schönherr. Italia.
2 Marmoratus. Fabr. Gallia.

Tigrinus. Panzer. Germania.

Marmoreus. Schrank. id.

Dalbatus. Linné. Suecia.

'Rodirus. Voet. id.

3 Interstinctus. Schönh. Russ. merid.

4 Morbillosus. Fabr. Gall. merid.* Tigrinus, Oliv.(Lixus.) Hisp, merid.

5 Testaceus. Schönherr. Etruria.

6 Achates. (Friw.) Schh. Hungaria.

7 Nebulosus. Linné. Gallia.* \{ Carinatus. de Geer. Germania.

8 Guttulatus. Schneider.

Schh. Suecia merid.

9 (Turbatus. Schönherr. Gall. bor.* \{ Glaucus. Dej. Cat.Sch. Suecia.

Nebulosus, Oliv. Hbst, Germania.

10 Vittiger. (Friu.) Schh. Tauria.

11 Bisulcatus.Sturm. Cat. Hungaria.

12 Pruinosus. Schönherr. Russ. merid.

13 Ophthalmicus. Rossi. Gall, merid.*

Distinctus, Fab. Steph. Dalmatia.

Quadripunctatus.

Schrank. Austria.

14 Ocellatus. Fahrous.

15 Concinnus. Schönherr. Podolia.

\{ Tetragrammus? Pall. Russ. merid.

16 Microgrammus. Stév.

17 Obliquus. Fabr. Gall. merid.*

Glaucus. Panzer. Germania.

18 Tabidus, Olivier. Gall, merid.

19 Megalographus. Schh. Sicilia.

20 Sulcicollis. (Parreyss.) Schh. id. ${ }^{*}$

21 Crinipes. Schönherr. Russ.merid.

22 Excoriatus. Illiger. Gall. bor.*

23 Fastigiatus. Erichson. Algiria.*

24 Ericæ. (Dahl.) Sch. Sicil.Gall.mer.*

25 (Trisulcatus. Herbst. Gallia.*

Madidus, Olivier. Dej.

Cat. $P$.

Adumbratus. Schönh. Suecia.

Hybridus. Germar. Germania.

Quinquelineatus.Hbst.id.

Altaicus, Gebler. Sibiria.

26 Ocularis. Fabr. Italia.

$\{$ Barbarus, Olivier. Algiria.

27 Roridus. Fabr. Austria.

28 Mœrens. Schönherr. Tauria.

29 Grammicus, Panzer. Germania.*

Bilineatus, Olivier, Gallia. 
30 f Costatus. Fabr. \{ Bilineatus, Rossi.

31 Cinereus. Schrank. Costatus. Herbst.

id.

Bicarinalus. Fischer, Russia.

32 Cunctus. Gyllenhal, Gall. merid.

33 Alternans. Olivier. id.*

Var. Lurcans. Herbst. Austria.

Incisuratus. Schh. id.

34 Conobita. Olivier. Gallia.*

Nanus, Schh. var. id.

Alternans. Schönherr. Austria.

Sisymbrii. Dej. Cat. id.

33 Misellus. Gyllenhal. Lusitania.

36 (Palmatus. Olivier. Gall. merid.*

Florentinus. Herbst, Italia.

Emarginatus. Fabr.

Latr. Saxonia.

37

Leucomelas, Chevrol.

$$
\text { Schh. Algiria.* }
$$

38 Surdus. Schönherr. id.

39 Sulcirostris. Linné. Gall, merid.

Var.Nebulosus. Knoch. Germania.

Fuscatus. Linné. Suecia.

Fasciatus, Villers. Gall. orient.

40 (Scutellatus. Schönherr. Gall. merid.* \{uadricarinatus, Meg. Austria. Firmus. Schönherr. Russ.merid.

42 Plicatus. Olivier. P. Algiria. *

Siculus. (Dup.) Schh. Sicilia.

4 (Sexmaculatus. Zoubk. Russ. merid.

Sollicitus. Schönherr. id.

Var. Squalidus, Schh. id.

45 Quadrivittatus. Esch.

$$
\text { Schh. Tauria. }
$$

16 Atomarius. Waltl. St.

$$
\text { Cat, Grocia. }
$$

\section{(BOTHYN ODERES. Schönherr.)}

(Candidatus. Pallas.

Russ, merid, ${ }^{*}$ Quagga. Herbst.

id.

Farinosus. Olivier. id. Strabus, Schönherr, id.

Pilipes. Schönherr. id.

Nubeculosus. Schönh. id.

Halophilus. Schönherr.id.

(Punctiventris. Germar, Austria.*

Stigma. Sturm. Cat. id.

Glaucus. Fabr. id.

l Carinatus. Zoublkoff. Saxonia.

Carinulatus. Schönh. Hungaria.

54 Conicirostris, Olivier. Gall merid.* 5ร Flavicans. (Parreyss.)

Schh. Illyria.

56 Carinicollis. Schönh. Russ. merid

57 Vexatus. Schönherr, id.

こ̊8 Volvulus. Schönherr, id.

59 Virgatus. Schönherr. id.

60 Mendicus. Schönherr. Gall. merid.

61 Brevirostris. Dej. Cat.

\section{Schh. id."}

62 Bartelsii. Schönherr. Russ, meria.

63 Albicans. (Ullr.) Schh. Austria,*

64 Orbitalis. Schönherr. Algiria.

65 Picipes. Stéven. Schh. Russ, merid.

66 Albidus, Fabr. Gallia. *

Niveus. Bonsd. id.

Bonsdoffi. Olivier. id.

Candidus. Herbst. Austria.

Affinis, Schrank. id.

67

Berolinensis. Linné. Germania.

Declivis. Olivier. Hungaria.*

68 Glaucus, Fabr. Dania.

69 Nomas. Pallas Russ, merid.

70 Tetragrammus. Pallas.id.

\{ Concinnus. Schönherr. id.

71 Fenestratus. Pallas. Russia.

72 Gicatricosus. Hoppe.

Schh. Germania.

73 Incanescens, Panzer. id.

74 Margaratiferus. Lucas, Algiria.*

\section{METALLITES. Schönherr.}

Polvdrusus. Sturm. Dej. Cat.

1 Mollis, Germar. Helvetia. *

2 Geminatus. (Chevrol.) Alp. Gall.*

3 Atomarius. Olivier. Germania.* Eratus. Gravenh. Dej.

Cat. id.

4 Elegantulus. (Parr.)

$$
\text { Schh. id. }
$$

(Ambiguus. Schönherr. Gallia.*

Fulvipes. Germar. Austria.

Flavipes, Panzer. Germania.

() Murinus. Dej. Cat.

Schh. Gall. merid.

7 Tibialis. (Ullrich.) Sch. Istria.

8 Globosus. Chevrolat.

Schh. Gall, merid.*

9 Cylindricollis. Schh. Illyria.

10) Globulipennis. (Allib,j Alp. Gall.* 
POLYDRUSUS. Germar.

Polpmesus. Schönh. Curculio. Auct. Dascillus et Murenus. Megerle.

1 Undatus. Fabr. Gallia*

Tereticollis. de Geer. Latr. $P$.

Albofasciatus, Herbst. Austria. Cinereus. Schaller. Germania. Selenus. Marsham. Anglia.

2 Intermedius. Zetterst. Suecia.

3 Fulvicornis. Fabr. id.

Ruficornis. Bonsd. id. Fasciatus. Müller. Dania. Undulatus. Linné. Suecia.

4 Ornatus, Stéven. Schh. Tauria.

5 Ferrugineus, Schönh. id.

6 Viridicinctus. Schönh. Hungaria. I Aurifer. Dej. Cat. Austria.

7 (Planifrons. Dej. Cat. Schh. P.* (Argentatus, Olivier, Gallia.

8 Armipes. Schönherr, Sicilia.

9 Impressifrons. Dej.Cat. $\left\{\begin{array}{c}\text { Schh. Gallia. } \\ \text { Var. Sericeus. Germar, Germania. }\end{array}\right.$

10 (Flavipes. de Geer. Gallia.* $\{$ Var.Argentatus, Bons, Germania. Ochropus. Linné. Suecia.

11 Pterygomalis. Schönh. Gallia.*

12 (Corruscus. Müll. Schh. Germania.* Herbeus. Schönherr. Russ. merid. Squamosus. Knoch. Germania.

13 Flavovirens. Schönh. Austria.* Sericeus. Herbst. id.

14 Xanthopus. Schh. Lusitania.

15 (Cervinus. Linné. Gyll. Suecia.* Messor. Herbst. Austria.

Var. Maculatus, Hbst. id. -Atomarius.Dej.Cat.P.* Grisocneus, de Geer. Gallia.

16 Astutus. Schönherr. Tauria.

17 Vilis. Stéven. Schh. id.

18 (Chrysomela, Olivier. Alsatia.* Perplexus, Dej. Cat. P.

19 Sparsus, Dahl. Cat.

20 Picus. Fabr. Schh. Italia. Ornatus. Herbst. id.

21 Sericeus. Schall.Schh.Germania.* Smaragdinus.Dej.Cat. $P$. Squamosus. Germar. Austria. o Splendens, Herbst. id.
22 Thalassinus. Schönh. Varsovia.

23 Lateralis. (Meg.) Schh. Italia.

24 Dorsualis, Schönherr, Corcyra.

25 Leucaspis. Chev. Schh. Sardinia.

26 Micans. Fabr. Germania. $\{$ Argentatus. Fourcroy. $P$.

27 Squalidus. Schönherr. Austria.

28 Vittatus, Dahl. Cat. Schh. Illyria.*

29 Amœnus. Germ. Schh. Germania.*

30 Rubi. Gyllenhal. Suecia.

31 Pallipes. Lucas. Algiria.

32 Albomaculatus. Stm.

Cat. Istria.

33 Aurulentus. (Megerle.)

St. Cat. Austria.

34 Virens. Sturm. Cat. Germania.

35 Scutellaris. Stm. Cat. Italia.

36 Holosericeus. Stm.Cat.Austria.

37 Gilvipes. Sturm. Cat. Germania.

38 Amaurus. Marsham. Anglia.

39 Confluens. Kirby. id.

40 Marginatus. Stephens. id.

41 Pulchellus. Stephens. id.

42 Melanotus. Stephens. id.

\section{CHLOROPHANUS, Dalman.}

Schönherr. Germar. Redtenbacher. CURculio. Linné, Fabr. Gyll. BrachyrhiNus. Latr.

1 Viridis. Linné. Alsatia.* Flavocinctus, de Geer. Germania. Brevicollis. Gyllenh. Suecia. Inermis. Schönherr. id.

2 Rugicollis. Schönherr. Pyr. orient.*

3 Pollinosus, Fabr. Austria.*

4 Salicicola. Germar. Silesia.

5 Nobilis. Dahl. Cat.

Schh. Hungaria.

6 Voluptiferus. Schönh. Austria.

7 (Graminicola. (Megerle.)

Schh. Hungaria.* Pollinosus. Oliv. Stév, id.

Flavescens, Herbst. Austria. Var. Viridis. Dej. Cat. Gallia.

8 Vittatus. Schönherr. Russ. merid.

9 Excisus. Fabr. Moldavia.

10 Caudatus. Stév. Schh. Russ, merid.

11 Micans. Stév. Schh. id.

12 Piliferus. Schönherr, id. 
13 S Sellatus. Fabr. Latr. Russ. merid. 21 I Dorsalis. Dej. Cat. id.

14 Fallax. Illig. (Phænodus. Schh.) Hungaria.

\section{SCYTHROPUS. Schönherr.}

Thenmecus, Dej. Cat. Gurculio. Herbst.

1 Mustela. Herbst. Schh. Germania.* Villosus. Herbst. Squamosus. Dej. Cat. id.

2 Septifrons. (Chevrol.) Gall. merid.*

\section{MESAGROICUS. Schönherr.}

1 Piliferus. Schönherr. Russ, merid.

2 Obscurus. Stẻv. Schh. id.

\section{SITONES. Schönherr.}

Sitona. Germar. Gurculio, Linné. Schh. oliv.

1 (Tesselatus. Schönherr. Algiria.*

Var. Ambulans. Chevrolat. Schh. Gall. merid.*

2 Latipennis. Schönherr. Lusitania.

3 Griseus. Fabr. Gall. merid.* Palliatus, Olivier. id.

Fuscus. Stephens. Anglia.

Var. Gressorius. Germar. Schh. Gall, merid. ${ }^{*}$

Tennis. Rosenhauer. Tyrolis.

Callosus. Schönherr. Tauria.

Cinerascens, Schönh, $P$.

Cribricollis, Schh. Austria.

8 Constrictus. Germar. Saxonia.

9 Regensteinensis. Hbst. P.*

10

Globulicollis. Schönh. Gallia,

Mauritanicus. Chevrol.

$$
\text { Schh. Algiria. }
$$

Obscuripes, Schönh, Russ, merid.

Chloroloma. Géné.Sch. Sardinia.

Virgatus. Schönherr. Sicilia?

(Lineellus. Bonsdorff. Germania.*

Ocator. Herbst. Austria.

Var. Lineatus, Payk. Suecia.

Tibialis. Herbst.

Gallia.*

Geniculatus. Schh. $\quad \boldsymbol{P}$.

Brevicollis. Schönherr. Germania.

Ambiguus. Gyllenhal, id.

20
Sulcifrons. Thunberg. Suecia.*

Campestris, Olivier. Gallia.

Tibialis. Steph. Gyll. Anglia.

22 Languidus. Schönherr. Tauria.

23 Arciicollis. Schönherr, id.

24 Crinitus, Oliv.Dej.Cat. Gallia.*

Crinita. Dej. Cat. id.

Nanus. Besser. Germania.

Macularis, Marsham. Anglia.

25 Crinifrons. Olivier. $\boldsymbol{P}^{*}$

26 Variegatus. Dahl. Cat.

Schh. Sardinia.

Insulsus. Schönherr. Russ, merid.

Octopunctatıs. Germ. Gallia.*

Obsoletus. Linné. Suecia.

Caninus. Zett, Gyll. id.

Var. Flavescens. Mars-

ham. Steph. Anglia.

Lineatus. Bonsd. Gallia.

Subrufus. Linné. Germania.

29 Cylindricollis. Schönh. Gallia.

30 Lateralis. Schönherr. Tauria.

31 Medicaginis. Redtenb. Austria.

32 Atritus. Schönherr. Tauria.

33 Discoideus. (Meg.) Sch. Austria.

34 Promptus. Schönherr, Gall. merid. ${ }^{*}$

35 Viciæ. Stephens. id.

36 Longicollis. Schönh. Tauria.

37 Cachecta. Chevr, Schh. Hispania.

38 Lineatus. Linné. Gallia.

Squamosus. Linné. Suecia.

Intersectus. Fourcroy. $P$.

Caninus, Fabr, Latr.? id.

Chloropus, Lin, Latr.? id.

Rufitarsis. Marsham. Anglia.

39 Elegans. Schönherr. Saxonia.

\{ Var, Lineatus, Payk, Suecia.

40 Anchora, Schönherr. Tauria.

41 Argutulus, Schönherr. Italia.

Verecundus, Rossi. id.

42 Bicolor. Schönherr. Dalmatia.

43 Hispidus. Chevrolat? $P$.

44 Inops. Schönherr. Austria.

45 Trisulcatus. Schönh. Gall. merid. *

46 Hæmorrhoidalis. Schh. Austria.

47 Fœdus. Schönherr. Tauria.

48 Tibiellus. Schönherr, Austria.*

49 Hispidulus, Fabr. $\quad \boldsymbol{P}^{*}$

Crinitus. Herbst. Austria.

Hirtus. Linné. Suecia.

Tibialis, Olivier. Gallia.

In Turbatus. Schönherr. Anglia.

Griseus. Stephens. id. 
51 Decorus. Sturm. Cat. Austria.

52 Humilis. Sturm. Cat. id.

๖3 Ulicis. Kirby. Anglia.

54 Spartii. Kirby. id.

55 Femoralis. Stephens. id.

56 Pleuriticus. Kirby. id.

57 Pallipes. Stephens. id.

58 Nigriclavis. Marsham. id.

59 Puncticollis. Kirby. id.*

60 Longiclavis. Marsham. id.

61 Suturalis. Stephens, id.

62 Humeralis. Kirby. id.

63 Pisi. Stephens. id.*

64 Rotundicollis. (Chev.) Gallia.

65 Albescens. Kirby. Anglia.

66 Cambricus. Kirby. id.

\section{TANYMECUS, Germar.}

Curculio, Fabr, Oliv, Herbst, Marsh.
$P{ }^{*}$

Graminicola. Olivier. Gall. merid.*

Canescens. Herbst. Austria.

Diffinis. Marsham. Anglia.

Glis. Rossi. Italia.

2 Argentatus. Bartels.

Schh. Russ. merid.

3 Variegatus. Gebl. Sch. id.

4 Urbanus. Schönherr. id.

5 Simplex. Sturm. Cat. Austria.

6 Limosus. Rossi. Italia.

7 Vittiger. (Meg.) Dej. Cat. Hungaria.

\section{EUSOMUS. Germar.}

Thylacites, Olim. Eusomatus. Sturm. Cat.

1 Ovulum. Illiger.

\section{Gallia.*}

2 Virens. Schönherr. Hungaria.

3 Piliferus. Schönherr, Russ, merid.

4 Elongatus. Schönherr. Tauria.

5 Pilosus. Schönherr. Russ. merid.

6 Martini. Humm. Schh. Petropoli.

7 Affinis. Lucas. Algiria.*

\section{BRACHYDERES. Schönherr.}

'Thylacites. Germar. Gurculio. Linné. Fabr.

1 Lusitanicus. Fabr. Gall. merid.*
2 Gracilis, Schneider. Lusitania*

3 Opacus, Schönherr, Gall. occid.*

4 Illæsus. Schneider. Lusitania?

5 Incanus. Linné. $\quad \boldsymbol{P}^{*}$

6 Lepidopterus. Chevrolat. Schh. id.*

7 Pubescens. Dej. Cat. Schh. Gall. merid.*

8 Albidus. Schönherr. Russ, merid.

9 Quercicola. Dufour.

Dej. Cat. Hisp, orient.

10 Hispanicus. Dej. Cat. Hispania.

11 Linearis. Dej. Cat. id.

12 Subfurcatus. Dej. Cat. id.

13 Brevis, Rambur. Dej.

14 Pini. Chevrolat. P.*

15 Elegans. Dej. Cat, Lusitania.

16 Marginatus. Dej. Cat. id.

17 Ilicis. (Dahl.) St. Cat. Sardinia.

\section{PLATYTARSUS. Schönherr.}

Curculio. Linné. Fabr.

1 Setiger. (Meg.) Schh. Austria.

\section{SCIAPHILUS. Schönherr.}

Eusomus. Germar. Curculio, Fabr, Gyll.

1 Muricatus. Fabr. P. . $^{*}$

Planirostris. Linné. Suecia.

Asperatus, Latr. (Bra-

chycerus.) $P$.

Var. Lucidulus. Man-

nerheim. Suecia.

2 Meridionalis. Schönh. Sicilia.

3 (Barbatulus. Germar. Dalmatia.*

Smaragdinus. Dej.

Cat. (Thylacites.) id.

4 (Smaragdinus. Dej. Cat. Schh. id.

\{ Var. Barbatulus. Dej.

Cat. id.

5 Bellus. Rosenhauer. Tyrolis.

6 Scytulus. Germar. Germania. Crinitus. Sturm. (Eu-

$\left\{\begin{array}{c}\text { somus.) id. } \\ \text { Parvulus. Fabr. }\end{array}\right.$

7 Setosulus. (Dahl.) Sch. Illyria.

8 Hispidus, Redtenb. Austria.

9 Viridis, Chevrol. Schh. Italia. 
10 Oblongus. Dej. Cat.

Schh. Hispania.

11 Pusillus. Steph. Schh. Anglia.

12 Carinatus, Oliv. Dej. Cat. Lusitania.

Asferculatus. Germar. in litt. id.

13 Aurosus. Germar. Sicilia.

14 Aurarius. Dej. Cat. Styria.

15 Subsignatus. Dej. Cat. Dalmatia.

16 Fulvipes. Dej. Cat. Gall. merid.

17 Asscerulatus. Germar.

St. Cat. Germania.

18 Globosus. (Sitona.) $S t$.

Cat. Austria.

STROPHOSOMUS, Bilberg. Schönh.

Cinehorhinus. Schh. Dej. Cat. Thylacites. Germ. Dej. St. Cat. Cunculio. Fab.

$\begin{cases}\text { Coryli. Fabr. } & P^{*} \\ \text { Var. Affinis. Dej. Cat. } & \text { Gallia. } \\ \text { Truncatus, Latreille. } & \text { id. }\end{cases}$

2 Crinifrons. Chevrolat. Gall. merid.*

3 Alternans, Schönherr, Gall. merid.

4 Faber. Herbst. P.*

Pilosellus. Stephens.

Herbst. Gyll. Germania.

Limbatus.Dej. Cat. id.

5 Cristatus. Schönherr. Hispania.

6 (Squamulatus. Herbst.

Latr, Suecia.

(Griseus. Dej. Cat. Austria.

7 Hispidus. Schönherr. Gall. merid.

Porcellus, Schönherr, id.

Comatus. Dej. Cat. Dalmatia.

8 Squamosus. (Gaubil.) Algiria. ${ }^{*}$

9 Subsulcatus. Schönh. Italia.

10 Hirtus. Schönherr. Gallia.

11 Setulosus. Schönherr. Helvetia.

12 Planifrons. Dej. Cat. Gall. merid.

13 Monticola. Dej. Cat. Alp. Gall.

14 Laticollis. Dej. Cat. Hisparia.

15 Capillatus. Dej. Cat. Dalmatia.

16 Rufipes. Stephens. Anglia.

17 Asperifoliarum. Kirby. id.

18 Cognatus. Stephens. id.

19 Subrotundatus. Marsh. id.

20 Nigricans. Kirby. id.

21 Scrobiculatus, Marsh. id.

22 Nebulosus. Stephens. id.

23 Chætophorus, Steph. id.
CNEORHINUS. Schönherr.

Curculio, Fabr. Thylacites. $\boldsymbol{S} t$.

1 Barcelonicus. Herbst. Hispania.

Var. Incanus. Fabr. id.

2 Designatus. (Chevrol.) Algiria.*

3 Barbarus. (Gaubil.) id.*

4 Meridionalis. (Chevrol.) Gall. merid.

5 Narbonensis. (Gaubil.) id.

6 Argentatus. (Gaubil.) Algiria.*

7 Prodigus. Fabr. Barbaria.

Hispanus. Herbst. Hispania.

8 Ludificator.Schönherr.id.

9 Geminatus, Fabr. Gallia.*

Globatus, Latreille. $\quad \boldsymbol{P}$.

10 Albicans. Dej.Cat.Sch. Gall, occid.

11 Albinus. Schönherr. Russ. merid.

12 Plumbeus. Marsham. Anglia.

13 Exaratus. Marsham. id. ${ }^{*}$

14 Carinæirostris. Schh. Lusitania.

15 Pyriformis. Schönh. id.

16 Hypocyanus. Hoffgg.

Schh. id.

17 Amplicollis. Schönh. id.

\section{THYLACITES. Germar.}

Curculio. Herbst. Fabr.

1 Fullo, Erichson.

Algiria.

2 Cataractus. Schönh. Lusitania.

3 Comatus, Erichson. Algiria.

4 Mus. Herbst. Lusitania.

5 (Fritillum. Panzer. Gall. merid.

Lapidarius. Dej. Cat. id.

Canescens, Rossi. Italia.

Var. Hapfneri. Jeniss, id.

6 Tesselatus. Schönh. Lusitania.

Rotundicollis.Dej,Cat.id.

7 Barbarus. Schönherr. Algiria.*

8 Turbatus, Gyllenhal. Hispania.*

Sabulosus, Dej. Cat. id.

9 Chalcogrammus. Schh. id.

10 Umbrinus, Schönherr. Lusitania.

Fritillum. Fabr. id.

(Elongatus? Dej. Cat. Hispania.

11 Glabratus. Schönherr, Lusitania.

Robinice. Herbst. id.

Obtusus? Dej. Cat. id.

12 Lasius, Schönherr. id.

13 Vittatus, Chev, Schh, id.*

14 filosus. Fabr. Germania.

Licinus. Herbst. Austria. 
15 Variegatus, Lucas. Algiria.

16 Farinosus. Dej. Cat. Russ, mer. oc.

17 Atratus. Dej.Cat. Hispania.

18 Juvencus. Dej. Cat. Barbaria.

19 Asphodeli. Rambur.

Dej. Cat. Hisp. merid.

20 Hirtellus. Dej. Cat. Hispania.

21 Subterraneus. Dej.Cat.Gall. merid.

HOMALORHINUS. Schönherr.

1 Tristis. Schönherr. Russ, merid.

\section{PSALLIDIUM. 1lliger.}

Schh. Curculio, Fabr. Oliv. Hbst.

1 Maxillosum. Illiger. Austria. \{ Articulatum. Fabr. Tauria.

2 Sculpturatum. Schh. Constantinp.

3 Interstitiale. Schönh. Tauria.

4 Anatolicum. Chevrol.

Schh. Anatolia

5 Græcum. St. Cat. Gracia.

6 Vittatum. Friwaldszky.Turcia.

7 Gracile. Sturm. Cat. Gracia.

\section{BRACHYCERUS. Fabr.}

Curculio. Linné. de Geer. Rossi. Drury.

1 Transversus. Olivier. Algiria.*

2 Libertinus. Schönherr, Barbaria.

3 Rignus. Erichson. Algiria.*

4 Pterygomalis. Gyllenh. id.*

Serratus. Olivier. id.

Algirus. Herbst. id.

Barbarus. Thunberg. id.

5 Konigii. Dej. Cat. Sch. id.*

6 Lateralis. Gyllenhal. Lusitania.* \{ Barbarus. Linné. Algiria.

7 Mauritanicus. Olivier. id.* Barbarus. Latreille. id.

1 Var. Albidatus. Chev. id.*

8 Undatus. Fabr. Gall. merid.

Lacunatus, Latreille. id.

Barbarus. Dumeril. Algiria.

Incultus. Gyllenhal. id.

Crispatus. Fabr. id.

Algirus. Olivier. id.

9 Corrosus. Dej. Cat.

Schönh. Corsica.*
10 Albidentatus. Helf. Sch. Sicilia. Siculus, Dej. Cat. id. (Sardeus. (Dahl.) Sardinia.

11 Dentatus. (Chevrolat.) Algiria.*

12 Latro. Schönherr. id. ${ }^{*}$

13 Costicollis. (Chevrol.) id.*

14 Pupillatus. (Chevrol.) id.*

15 Distinctus. (Chevrol.) id. ${ }^{*}$

16 Besseri.Dej. Cat.Schh.Volhynia.* Var. Pisiferus. Thunb. Podolia.

17 Lutulentus. Schönherr. Italia. ${ }^{*}$ \{Junix. Herbst. id.

18 Siculus, Mannerheim.

Schh. Sicilia.

19 Chevrolatii. Helf. Schh. id.*

Var. Callosus. Schönh. id.

Variolosus. Thunb. id.

Rudis. Chevrolat. id.

20 Barbarus? Fabr. Algiria.

21 Semituberculatus. Chevrol. in litt. Luc. id. *

22 Plicatus. Schönherr. Hispania.

23 Algirus. Fabr. Gall. merid. * \{Mricatus. Olivier. id.

24 Rubiginosus. (Chevrl.) Algiria.*

25 Scutellaris. Chevrolat. in litt. Lucas. id.*

26 Tetanicus. (Chevrol.) id. ${ }^{*}$

27 Planirostris. Schönh. Italia.

$\{$ Var. Algirus. Dej. Cat. id.

28 Lutosus. Schönherr. Gall. merid. *

29 Fimbriatus. (Chevrol.) Algiria.*

30 Scirrosus. Schönherr. Sicilia?

31 Perodiosus. Schönh. Andalusia.

32 Superciliosus. Schönh. Russ. merid.

Var. Europaeus. Thun-

berg, id.

33 Muricatus. Fabr. Hungaria.

Foveicollis. Schönh. Austria.

Gemmatus. Thunb. id.

34 Hispanicus. Dej. Cat. Hispania.

35 f Dahlii. Dej. Cat: Sicilia.

\{Globosus. Dahl. Cat. id.

36 Sordidus. Ramb. Dej.

Cat. Hisp. merid.

37 Scabratus. Dej. Cat. Sardinia.

Tuberculosus, Dahl.

Cat. id.

38 Græcus. Sturm. Cat. Gracia.

39 Spinosus, Dahl. St.

Cat. Hungaria.

40 Nodipennis. Sturm.

Cat. Insul. Malla 
AMOPHOCEPHALUS. Sehh.

Arrhenodes. Stéven. Dej. Cat.

1 Coronatus. Germar. Italia.*

\section{RAMPHUS, Clairville.}

Rhynchenus. Gyll. Sahlb. Curculro. Herbst, Payk. Marsh.

1 f Flavicornis, Clairville.

$\left\{\begin{array}{c}\text { Dumeril. } \text { P.* }^{\star} \\ \text { Pulicarius. Stph. Cast. Anglia. }\end{array}\right.$

2 Tomentosus, Olivier. Helvetia.*

3 Eneus,Dej.Cat.Sahlb.Gall, merid.

\section{TANAOS. Schönherr.}

1 Fallax. Gyllenhal. Africa Austr.

2 Bicolor. Schönherr. id.

\section{APION. Herbst.}

Attelabus. Fabr. Payk. Apius. Bilb. Curculro. Linné, de Geer. Rhynomacer. Clairv. Oxystoma. Herbst. Dumeril. Steph. Pelamis. Meg.

1 (Pomonæ. Fabr. Steph. P.*

Caruleum. Kirby. Anglia.

Cyaneum. Panzer. Germania.

2 (Craccæ. Linné. Steph.

$\left\{\begin{array}{c}\text { Latr. P.* } \\ \text { of Ruficorne. Germar. } \\ \text { Sahlb. Germania. }\end{array}\right.$

3 Subulatum.Kirby.Germar. id.

4 Marshami. Stephens, Anglia.

5 Scrobicolle. Schönh. id.

6 Ochropus. Germar. Germania.*

Rufitarse. Dej. Cat. Styria.

7 Neglectum. Schönherr.Tauria.

8 Confluens. Kirb.Steph. Gallia.

9 Stolidum. Germar. Germania.* Confluens. Gyllenh. Suecia.

10 Betulæ. Chevrol. Schh.P.*

11 Vicinum. Kirby. Steph. $\left\{\begin{array}{c}\text { Dej. Cat. Gallia.* } \\ \text { Loti. Germar. } \\ \text { Incrassatum. Germar. id. }\end{array}\right.$

12 Glaucinum. Schönherr. Gallia.

13 Atomarium. Kirb.Stph.Anglia. Pusillum. Germ. Dej.

Cat, Gallia.
14 Oculare. Schönherr.

Tauria.
15

16

17

18

19

20

21

22

23

24

25

26

27

\section{8}

29

30

31

32

33

34

35

36

\section{7}

38

39

40

41

42

43

44

45

\section{6}

\section{7}

Acium. Schönherr. id.

Palpebratum. Schönh. id.

Cylindricolle. Schönh. id.

Hookeri. Kirby. Steph. $\left\{\begin{aligned} \text { Sahlb. } & \text { Anglia.* } \\ \text { Rotundicolle. (Meg.) } & \text { Helvetia. } \\ \text { Dispar. Gyllenhal. } & \text { Suecia. }\end{aligned}\right.$

Dispar. Gyllenhal. Suec
Sahlbergi. Schönherr, id.*

Penetrans. Germar. Germania.*

(Basicorne.Ill.Dej.Cat.id.

Brevicorne. (Meg.) Dej.

Cat. Gallia.

Tennæ. Kirby. Steph. id.*

Pubescens. Kirby. Stephens. Germar. Anglia. ${ }^{*}$

Nigrescens, Stephens, id.*

(Eneum. Fabr. Steph. Gallia.*

Yar. Chalceum. Marsh. Ménétr. Anglia.

(Radiolus. Kirby. Stph. Gallia.*

Aterrimum. Gyl.Sahlb.Suecia.

Var. Eneum. Paykull. Germania.

Curvirostre. Schönh. Russ, merid.

Onopordi. Kirb.Steph. Gallia.*

(Gibbirostre.Gyll.Shlb. Suecia.*

Carduorum. Stephens.

Dej. Cat. $P$.

Pecticorne. Dej. Cat. id.

Setiferum. Schönherr. id.*

Ulicicola. Perris. Gall. merid. ${ }^{*}$

Brunnipes. Schönh. Gallia.

Insculptile. Perris. Gall. merid.*

Lævigatum. Kirb.Stph.Anglia.

Bifoveolatum, Kirby.

Steph. id.

| Hydrolapathi. Kirby. Germania.*

(Coruleopenne. Steph. Anglia.

Chevrolatii. Schönherr.P.*

Tamarisci. Dej. Cat.

Schh. Gall. merid.*

Aciculare. Germar. German.P.*

Rugicolle. Germ. Stph. P.

Brevirostre. Hbst. Dej.

Cat. Gallia.*

Timidicolle. Märkel. Saxonia.

Longirostre. Olivier. Hungaria. ${ }^{*}$

Holosericeum.Dej.Cat.

Schh. Dalmatia.

Millum. Schönherr. Austria.

$\left\{\begin{array}{c}\text { Pallipes. Kirby. Steph. } \\ \text { Dej. Cat. Gallia. } \\ \text { Rufipes, Dej. Cat. }\end{array}\right.$ 
48 Geniculatum. Germar. Germania.

49 Flavimanum. Sahlberg.

Dej. Cat. Gall, merid.

Rufomanum. Dej. Cat. P.

50 Canescens. Dej. Cat. Algiria.*

¿1 Semivittatum. Schönh. Tauria.

52 Ulicis. Forster. Schh. Gallia.*

Ilicis. Stephens. Anglia.

33 Fuscirostre, Fabr. Dej.

Cat. P.*

Melanopum. Kirby.

Steph. Anglia.

54 (Difficile. Herbst. Helvetia. ${ }^{*}$

Corniculatum. Germ. Germania.

(Ruficorne.(Megerle.) Austria.

55 Genistæ. Kirby. Anglia. ${ }^{*}$

Astragali, Herbst. Austria.

56 Nigrirostre. Fabr. Africa austr.

57 Rufirostre, Fab.Steph.Gallia.*

58 Fulvirostre, Schönh. Tauria.

59 Pallydactylum. Schh. Sicilia.

60 Femorale. Fabr. Algiria.

61 (Flavofemoratum. Hbst.

$\{$ Dej. Cat. Gall. merid.*

Femoratum, Dej. Cat. Styria.

62 Russeolum. Schönherr. Africa austr.

63 Malvæ. Fabr.Steph. Gallia.*

64 Vernale. Fabr. Steph.

$\left\{\begin{array}{r}\text { Sahlb. P.* } \\ \text { Fasciatum. Latreille. id. }\end{array}\right.$

65 Rufescens. Gyllenhal. Lusitania.

66 Pallidulum. Schönh. Sicilia.

67 Atritarse. Schönherr. Tauria.

68 Viciæ. Payk. Steph.

Dej. Cat. P.*

Griesbachi. Stephens, Anglia.

Trifolii.Linné. Suecia.

69 Obscurum. Marsham.

Steph. Anglia.

70 Difforme. Germ. Steph.

$\left\{\begin{array}{c}\text { Dej. Cat. Gallia. } \\ \text { Compressicorne. Dej. } \\ \text { Cat. } P .\end{array}\right.$

71

Dissimile, Germar. Saxonia.*

72 (Varipes. Germ. Steph. Germania.*

Flavipes. Gyllenhal. Suecia.

Flavifemoratum.Kirb.Anglia.

73 Apricans, Hbst. Steph.

$$
\text { Sahlb. } \boldsymbol{P} \text {, }
$$

74 Fagi. Kirby. Steph, Anglia.

75 Ononidis. Gyllenhal. Suecia.*

76 Bohemanii, Schönh. Westrogoth

77 Lievicolle. Kirb.Steph. Anglia.
78 Schönherri. Waterh. Anglia.

79 Flavipes. Fab, Sahlb.

80 Estivum. Germar.

Sahlb. Steph. Germania.*

81 f Ruficrus. Germ.Steph.id.*

Estivum.var.b.Germ.id.

82 Assimile. Gyllenhal. id.*

83 Waterhousei. Schh, Anglia.

84 Angusticolle.Schönh. Odessa.

85 Nigritarse.Kirb. Sch. Helvetia.*

86 Leachii. Stephens. Anglia.

87 (Miniatum. Sahlb. Dej.

Cat. Helvetia.
Frumentarium.Stph. Anglia.

88 Frumentarium. Linné.

Gyll. Sahlb. Gallia.*

Hamatodes. Steph.

Dej. Cat. Anglia.

Purpureum. Latr. $\quad P$.

Ferrugineum.Dej.Ct. id.

89

Sanguineum. de Geer.

Sahlb. Suecia.*

Rubiginosum. Dej.

Cat. P

Hæmatodes. (Sturm.)

Schh. Germania. *

91 Gyllenhali. Kirb. Dej.

Cat. Gallia.

Unicolor. Kirby. Anglia.

Ethiops. Gyllenhal, Suecia.

Africanum.Schönh. Africa austr.

93 Mecops. Schönherr. Scania.

94 Incanum. Schönherr. Helvetia.

Salvia. Ullrich. id.

95 Seniculus. Kirby. ' $\boldsymbol{P}^{*}$

Tenuis. Dej. Cat. Suecia.

Plebejum. Germar. Germania.

Elongatum. Germar. id.

96 Trifolii. Lintz. Schh. Germania.

97 Civicum. Germ.Steph.id.*

98 Salicis. Chevrol.Schh.P.

99 Foraminosum. Germ. Germania.*

100 Lævithorax. Schönh. Gallia.

101 Validum. Germar. Germania.*

\{ Caruleum. Herbst, Austria.

102 Columbinum. Germ.

Steph. Gallia.*

103 Superciliosum. Gyll. Germania.*

104 (Tubiferum. Dej. Cat.

$\left\{\begin{array}{r}\text { Schh. Gall, merid. } \\ \text { Scolopax. IIoffmsgg. Lusitania. }\end{array}\right.$

105 Alcyoneum. Dej, Cat. Germaria. 
106 Eratum. Stephens. Anglia. Auratum. Stephens. id.

107 Ebeninum. Kirby.

Sahlb. Finlandia.* Nigrinum. (Ullrich.) Illyria.

108 Kunzei. Schönherr. Germania. Ebeninum. Kunze. in litt. id.

109 Angustatum. Kirby. P.* Modestum. Germar.

Sahlb. Dej. Cat. Germania. Meliloti.var. $\beta$. Kirb. Anglia.

Flavipes. Paykull. var, B. Suecia.

110 Languidum. Schüppel. Schh. Germania. Afer. Schönherr. $P$.

112 Furvum. Sahlb.Schh. Finlandia.

Oblungum. Sahlberg.

Schh. Tauria.

114 Morio.Germ. Dej.Ct. Gallia.*

115 Ononis, Kirby. Steph. Helvetia.* o Cinerascens. Germ. Germania.

116 Platalea. Germar. id.*

117 Coracinum. Schönh. P.

118 Cyanescens. Dej. Cat.

Schh. Gall. merid.

119 Ervi. Kirby. Sahlb. P. Y Var. Lythri. Kirby. Germania. Perplexum. Schonh. id. Nigrum. Olivier. id.

Validirostre. Schönh. P.

Loti. Kirby. Steph. Anglia. ${ }^{*}$

Scutellare. Kirby.

$$
\text { Steph. id. }
$$

Kirbyi. Germ. Steph. id.

Glabratum. Germar.

$$
\text { Steph, id. }
$$

Filirostre. Kirb.Stph. id. ${ }^{*}$ Meliloti. Kirb.Steph. Gallia.* Reflexum. Schönh. $\boldsymbol{P}$. (Virens. Herbst.Sahlb. id.* Eneocephalum.Gyll.Suecia.

Cyaneum. Dej. Cat. P. of Marchicum. Steph. Anglia. Simile. Kirby. Steph. id.

131 Punctirostre.Schönh. Tauria.*

132 Punctigerum. Germ.

$$
\text { Steph. Gallia.* }
$$

133
134 $\left\{\begin{array}{l}\text { Intrusum. Gyll.S } \\ \text { Var. Foveolatum }\end{array}\right.$

Kirby. Dej. Cat. Anglia.

135 Spencei. Kirb. Steph. Gallia.

136 Sulcifrons. Herbst.

137

Sahlb. Germania.*

\{

139

140

141

142

143

144

145

146

147

148

149

150

151

152

153

154

155

156

157

158

159

Pivi. Stephens.

Leptocephalum. Ger-
mar. $P$.

Ethiops. Hbst.Sahlb. Gallia. ${ }^{*}$

Subcœruleum. Steph. Anglia.

Marchicum. Gyllenh. Suecia.

Translaticeum. Schh. Germ. bor.*

Livescenum. Schh. Gallia.*

Gracilicolle. Schh. Lusitania.

Incisum. Schönherr. Helvetia.

Spartii. Kirby. Steph. Anglia.

Schmidtii. Märkel. Saxonia.

Curtisii. Stephens. Anglia.

Astragali. Paykull.

Steph. Germania.*

Elegantulum. Germ. Gallia.*

Facetum. Schönherr. Tauria.

Pullum. Schönherr. id.

f Vorax. Sahlb. Steph. Germania.

Villosulum. Marsh. Anglia.

(Pavidum. Germ.Stph. Germania.

Ervi.var. $\gamma$. Steph. Anglia.

Juniperi. Schönherr. Helvetia.

Plumbeum. Schönh. $\boldsymbol{P}$.

Orbitale. Schönherr. Gallia.

Amplipenne. Schh. Grocia.

Sundevalli. Schönh. Westrogoth.

Angulicolle. Schönh. Africa, aust.

Pallicorne. Schonh. Gallia.

160 Pisi. Fabr. (Megerle.

Dej. Cat.) Schh.
(Gravidum. Ohtoier.

Gravidum. Olivier. id.

Pasticum. Germar. Germania.

Punctifrons. Steph. Anglia.

161 Cyanipenne. Schönh. Germania.

162 Sorbi. Herbst. Sahlb. Gallia.

Virescens. Marsham. Anglia.

$\left\{\begin{array}{c}\text { Carbonarium. Ste- } \\ \text { - }\end{array}\right.$ phens. Germar. id.

163 Dispar. Germar. Germania.

161 Atratulum. Kirb. Ste-

$\left\{\right.$ phens. $P .{ }^{\star}$

Tumidum. Dej. Cat. Germania.

16.) Immune. Kirby. Stephens. Helvetia.*

Cyaneum. Gyllenhal.

Steph. Anglia.

Dej. Cat, Sueria.
166 Aquilinum. Schönh. Scania. 
167

Humile. Germ. Sahlb.

Steph. Gall. bor
Brevirostre. Dej.Cat. Suecia.

Curtirostre. Steph.

Germar. Germania.

Sedi. Gyllenhal. Suecia.

168 Interstitiale. Schönh. Gallia.

169 Sedi. Germar. Germania.

170 Simum. Germar. Gallia.*

Lineare. Dej. Cat. id.

171 Minimum. Herbst, Finlandia.*

Velox. Stephens. Anglia.

Crenatum.Dej. Cat. P.

172 Violaceum. Kirby.

$\left\{\begin{array}{c}\text { Sahlb. }{ }^{*} \\ \text { Hydrolapathi. Dej. } \\ \text { Cat. Germania. } \\ \text { Cyaneum. Olivier. Gallia. }\end{array}\right.$

173 Marchicum. Herbst.

Sahlb, P.*

\{Violaceum. Dej. Cat. Suecia.

Rumicis.Steph,Germ.Germania.

174 Affine. Kirby. Germ. id.

175 Limonii. Kirby. Anglia.*

176 Aterrimum. Kirby. Germania.

177 Chrysostoma. Kiesenwetter. id.

178 Germari. Walton. Anglia.

179 Albopilosum. Lucas. Algiria.

180 Apeticum. Märkel. Germania.*

181 Illustre. Géné. Pyr. orient.*

182 Gilvipes. Dej.Cat. Russ. merid.

183 Hæmatopus. Dej.Cat.Gall, merid.

184 Erythromerum. Dej.

Cat. Russ, merid.

185 Xanthocerum. Dej.

Cat. Dalmatia.

186 Globulipenne. Dej.

Cat. Hispania.

187 Puncticolle. Dej. Cat, Austria.

188 Cupreum, Dej, Cat. Gallia.

189 Misellum. Dej. Cat. Gall. merid.

190 Confusum. Dej. Cat. Anglia.

191 Violacipenne. Dej.

Cat. Dalmatia.

\section{AULETES. Schönher,}

\section{Rhynchites. Waltl.}

1 Tubicen, Schh, Germ, Dalmatia.

2 Basilaris. Germar. Hungaria. Nigrocyaneus. Waltl. id.

3 Politus. Stév. Schönh. Tauria.
DIODYRHYNCHUS. Germar.

1 Austriacus.(Meg.) Schh.Austria.*

NEMONYX. L. Redtenbacher.

Rhinomacer, Fabr, Oliv, Panz, Scht.

1 Lepturoides. Fabr. Gallia bor.

RHINOMACER. Fabricius.

Schh. Rhynchites. Gyllenhal. Germar. Anthribus. Oliv, Payk.

1 Attelaboides. Fabr. Gallia bor.*

RHYNCHITES. Herbst.

Attelabes. Oliv, Latr. Fabr. Rhinomacer. Clairv. Curculro, Linné, de Geer. Mechoris, Bilb.

1 Hungaricus. Fabr. Hungaria. ${ }^{*}$

2 Scalptus. Schönherr. Africa aust.

3 (Giganteus. (Meg.) Schh. Gracia.

Trojanus. Chevrolat.

in litt. id.

4 Auratus. Scopoli.Schh. Gallia.

Bacchus. Steph. Latr. Anglia.

Rubens. Dej. Cat. Austria.

Aurifer, Olivier. Gall, merid.

5 Rectirostris. Schönh. P.*

6 Bacchus. Linné. Gallia.*

7 Corruleocephalus.

Schaller. id.*

(Cyanocephalus. Hbst. Austria.

8 Equatus. Linné. Gallia bor.* Purpureus, Olivier. $\boldsymbol{P}$.

9 (Cupreus. Linné. Gallia bor. AEneus, Latreille. $P$.

Punctatus. Herbst. Germania.

10 (Obscurus. (Meg.) Schh. id.

$\{$ Punctatus, Olivier. Gallia.

11 Planirostris. Fabr. Austria.

Ethiops. Creutzer. Volhynia.

Niger. (Meg.) Dej. Cat. id.

Crassicornis. Olivier. Gallia.

12 Megacephalus. Germ. Saxonia.*

Angustatus, Dej. Cat. Gallia.

Alliaria. Stephens. Anglia.

Mannerheimii. Humm. Austria. 
13 (Conicus. Illiger. Germ. Austria. ${ }^{*}$ Alliarice. Fabr. Dej. Coruleus. Fabr. Germania. Germanicus. Herbst. Austria.

14 Pauxillus. Germar. Germania.*

15 Minutus. Herbst. Austria.* Arquatus. Dej. Cat, $\quad P$. (Viridis. (Megerle.) Austria.

16 (Fragariæ. Sturm.Schh. Germania.* Arquatus. Dej. Cat. Gallia. Eneovirens. Steph. Anglia. Virescens. (Ziegler.) Austria.

17 Nanus. Paykull. P.* Alliaria. Linné. Germania.

18 Populi. Linné. Fabr. Panz. $P .^{*}$

19 Betuleti. Fabr. Herbst. id.* Betula. Linné. Steph. Germania.

20 Sericeus. Herbst. Hungaria.* Pubescens. Latreille. Alsatia. Azureus. Dej. Cat. Germania.

21 Pubescens. Fabr. Hbst. Alsatia.* $\{$ Virescens. Thunberg. Suecia.

22 Cavifrons. Chevrolat. Schh. Gallia.*

23 Olivaceus, Schönherr. Gall. merid. 24 Cyanicolor. Gyllenhal. Schh. P.

25 Comatus. Dej. Cat.

26 Brevirostris. (Chevro-

$$
\text { Schh. id. }{ }^{*}
$$

27

$$
\text { Tristis. Fabr. Germania. }
$$

28 Tomentosus, Schönh. Gall. bor.

29 Constrictus. Waltl.

Schönherr. Bavaria.

$30\left\{\begin{array}{l}\text { Betulæ. Linné. } \\ \text { Femoratus. Latreille. Gallia. }\end{array}\right.$

31 Melas. Herbst. Austria.

32 Rhedi. Schrank. id.

33 Fulgidus. Fourcroy. $\boldsymbol{P}$.

34 Craccæ. Fabr. Alsatia.

35 Cyaneus. Fabr. Gallia. ${ }^{*}$

36 Cylindricus. Kirby. Anglia.

37 Lævicollis. Stephens. id. \{yaneopennis, Steph. id.

38 Ophthalmicus. Steph. id.

39 Interpunctatus. Steph. id.

40 Atroceruløus. Steph. id.

11 Præustus. Peyroleri. Dalmatia. Semiruber. Dej. Cat. id.

12 Luridus. Schönherr. Croatia.
ATTELABUS. Linné.

Klug. Fabr. Rhynchites. Illig. GurcuLo. de Geer. Chypus. Thunbery.

1 Curculionides. Linné. Gallia.* Var. Maculipes, Rondani. Italia.

2 ) Variolosus, Fabr. Algiria. * Cribratus. Olivier. id.

APODERUS, Olivier. Schh.

Attelabus. Linné. Fabr. Gyllenk. Gurculio. de Geer.

1 Coryli. Linnẻ. Gallia.* \{ Avellance.Lin. Panz. Germania.

2 Intermedius. Illiger. Austria. * \{ Erythropterus, Linné. id.

3 Submarginatus. Schh. Africa aust.

4 Cyaneus. Schönherr. id.

5 Morio. Bonelli. Lombardia.

\section{CHORAGUS, Kirby.}

Alticopus. Villa. Anthribus. Robert.

1 Scheppardii. Kirby. $\left\{\begin{array}{c}\text { Dufour. Anglia. } \\ \text { Galeazzii. Villa. Schh. Lombardia. } \\ \text { Pygmaus, Rob.Guérin.P. }\end{array}\right.$

ANTHRIBUS. Geoffroy. Fabr.

1 Albinus. Linné. Latr. Gallia.*

2 Cordiger. Schönherr. Africa austr.

PLATYRHINUS, Clairville.

Macrocephalus, Oliv. Anthribus. Fab. Panz. Gyll. Curculio. Linné.

1 Latirostris. Fabr, Gallia bor.*

CRATOPARIS. Schönherr.

1 Centromaculatus.

(Dahl.) Schh. Italia.

ENEDREYTES. Schönherr.

I Hilaris. Chevrol.Schh. Gallia.* 
TROPIDERES. Schönherr.

Anturibus, Fabr. Panz. Schh. Herbst.

Pratyrainus. Clairv. Amblycerus. Thunb.

1 Albirostris. Hbst. Schh. Gallia.

2 Dorsalis. Thunb, Gyll. Germania.

3 Undulatus. Panz.Schh. Gall. merid.* (Undatus. (Chevrolat.) P.

4 Edgreni. Schönherr. Suecia.

5 (Sepicola. Herbst.Schh. Gallia.*

Fuscirostris, Clairv. Helvetia.

Ephippium, var. Dej.

Cat. $\boldsymbol{P}$.

6 Pudens. Chevrol. Schh, Gallia.

7 f Cinctus. Payk. Gyll. Austria. Marchicus. Herbst. id.

8 Niveirostris. Fabr. Gallia.* $\{$ Brevirostris. Panzer. Germania.

9 Bisignatus. (Dahl.) Sch. Hungaria.

\section{BRACIYTARSUS. Schönherr.}

Anthribus. Fabr. Gyllenh. Schh. Cunculio, de Geer.

1 (Scabrosus, Fabr, Schh. Gallia.*

Y Var. Scapularis. Gebl. Sueciamerid.

2 Varius. Fabr. Schh. Gallia.*

3 (Tesselatus, Schönherr. Styria.

Nebulosus. Dej. Cat. id.

Sepicola. (Ziegler.) Austria.

4 (Bostrichoides. Müller. Pomerania.

\{ Piceus. (Schmidt.) id.

5 Areolatus.Waltl.Schh. Sicilia.

6 Pantherinus. Lucas. Algiria.*

\section{URODON. Schönherr.}

Bruchus, Fabr. Oliv. Anthribus. Fabr. Latr. Germar. Bruchella. (Megerle. Dej. Cat.) Clairv. Geoffroy.

1 Rufipes. Fabr. $\{$ Sericeus. Fabr.

$\boldsymbol{P} . *$

2 Concolor. Stév. Schh. Russ, merid.

3 Pygmæus. Hoffmansegg. Schh. Gallia.*

4 Suturalis. Fabr. Austria. $\{$ Var. Rufipes. Olivier. Gallia

5 Vermiculatus. Schönh. Africa aust.
SPERMOPHAGUS. Schönherr.

Bruchus. Olivier.

1 Rufiventris. Schönh. Tauria.

2 Cardui. Stéven. Schh. Gallia.* Cisti. Uliv, Latreille, $P$. Villosus. Dej. Cat. Gallia.

3 Variolosopunctatus.

Schh. Tauria.

4 Convulvuli. Schönherr, id.

5 Algiricus. (Gaubil.) Algiria. ${ }^{*}$

\section{BRUCHUS, Linné. Latr. Gyll. Oliv.}

1 Virgatus, Schönherr. Russ, merid.

2 Obscuripes. Schönh. Gall. merid.

3 Gilvus. Stéven. Schh. Tauria.

4 Biguttatus. Olivier. Gallia.

Creutzeri. Dej. Cat. Dalmatia.

5 Fulvipennis. Dej. Cat. Germar. id.*

6 J Variegatus, Dej. Cat. Germar, Gall.
Bimaculatus.Oliv.Lat. id.

7 Dispar. Chevrol.Schh. Gallia.

8 Fulviventris. Blanch. Sicilia.

9 Dispergatus. Schönh. $P$.

10 Decorus. Schönherr. Tauria.

Pacilus. Germar. id.

11 Marginellus. Fab. Guẻr. Gallia.*

$\{$ Marginalis. Latreille. id.

12 Astragali. Stév. Schh. Tauria.

13 Fischeri. Humm. Schh. id.

14 Lucifugus. Schönherr. id.

15 (Picipes. (Ziegl.) Germ. Dalmatia. Imbricornis. var. Dej.

Cat. id.

16 Microdon. Géné. Sicilia.

17 Inspergatus. Schönh. P.

18 Albolineatus. Blanch. Sicilia.

19 Tarsalis. Schönherr. Gallia.

0 (Galegæ. (Ziegl.) Schh. id.*.

$\{$ Var.? Imbricornis.

Dej. Cat. id.

21 Obscuricornis. Blanch. Sicilia.

22 Braccatus. Stév, Schh. Tauria.

Imbricornis. Dej. id.

23 Femoralis. Schönherr. Dalmatia.

$\{$ Var. Imbricornis. Dej.

Cat. id.

24 Varius, Olivier. Latr. Gallia.

Imbricornis, var, Dej, id. 
25 Grandicornis. Blanch. Sicilia.

26 Imbricornis. Panzer. Germania. Nebulosus. Latreille, Gallia. Galego. Rossi. Italia.

27 Basalis. Schönherr. Lusitania.

28 Albopunctatus.Blanch. Sicilia.

29 Siculus. Schönherr. Sicilia.

30 Laticornis. Blanch id.

31 Pusillus. (Meg.) Germ. Dalmatia.

32 Canus. Germar. Germania.*

Unicolor. Latreille. Gallia.

33 Debilis. Schönherr. id.*

34 Olivaceus, Germar. Saxonia.*

35 Virescens. (Stm.) Schh. Gallia.

36 Varipes. Schönherr. Dalmatia.

37 Tibiellus. Schönherr. $\boldsymbol{P}$.

38 Nanus. (Ziegler.) Schh. Dalmatia.

39 Oblongus. Blanchard. Sicilia.

40 Ginerascens. Chev. Sch. id.

41 Lutescens. Blanchard. id.

42 Misellus. Schönherr. Dalmatia.

43 Costatus. Blanchard. Sicilia.

44 Perparvulus. Schönh. Gallia.

45 Tibialis. Schönherr. Tauria.

46 Pauper. Schönherr. Odessa.

47 Pygmæus. Dej. Cat.

Schh. Dalmatia.

48 Foveolatus, Gyllenhal, id.

49 Miser. Schönherr. Gallia.

50 Sericatus. Stév. Schh. Tauria.

\{ Rufimanus, Dej. Cat. id.

51 Antennalis. Schönherr. id.

५\% Carinatus. Schönherr. id.

53 Anxius. Schönherr. Gallia.

54 Pisi. Linné.Latr. id.*

55 Rufimanus. Schönherr. Pomerania. ${ }^{*}$

Var. Bipunctatus.

(Ziegler.) Germania. *

- Granarius. (Dej. Cat.) id.

56 (Flavimanus.(Meg.) Sch.Gall. merid.* Affinis. Frölich. Austria.

57

58

Javeti. Chevrol. Schh, Gall. merid.*

8 (Nubilus. Dej. Cat. Sch. Gallia.*

Rufipes. Herbst. Austria.

Grararius.var. Panz. id.

Ervi. (Ziegler.) id.

Signatus. (Megerle.) id.

59 jGranarius. Linné. Latr. Gallia.*

$\{$ Var. Atomarius. Linné. Suecia.

60 Wasastjernii. Schh. id.

61 Troglodytes. Schönh. P.

62 Brachialis. Chev. Schh. Gallia.
63 Tristis. Dej. Cat. Schh, Gall, merid.

64 Tristiculus. Schh. Gall. occid.

65 Signaticornis. Dej.Cat. Schh. Dalmatia.*

66 Pallidicornis. Dej. Cat. Schh. id.

67 Luteicornis, Illiger. Gallia.*

68 Griseomaculatus. Chevrolat. Schh. $P$.

69 ( Nigripes. Dej. Cat. $\left\{\begin{array}{l}\text { Schh. Austria. } \\ \text { Vicia. Olivier. }\end{array}\right.$

70 Farhæi. Schönherr. Suecia.

71 Loti. Paykull. id.*

72 Lividimanus. Schönh. Gallia.

73 (Lentis. Koy. Schh. Austria.*

Y Variegatus. (Meg.) Germania.

74 Laticollis. Schönherr. P. Tauria.

75 Velaris. Schönherr. Italia.

76 Longicornis. Illiger. Lusitania.

77 Histrio. Schönherr. Hispania.*

Pictus. (Parreyss.) Lusitania.

78 Jocosus. Schönherr. Hispania.

\{ Serraticornis? Fabr. Lusitania.

79 Discipennis. Chevrolat.

Schh. Groecia.

80 Stevenii. Schönherr. Tauria.

Umbellatorum. Stév. in litt. id.

81

(Pubescens. (Ziegler.)

$\{$ Schh. Dalmatia.

Villosus. Fabr. Austria.

Tomentosus.(Megerle.) id.

82 Sutor. Géné.

83 (Cisti. Fabr.

Gallia*

Villosus, Fabr. St. Germania.

Ater. Marsham. Anglia.

84 Murinus, Stẻven. Schh. Lusitania.

85 Seminarius. Linné. Gallia.

86 Alni. Fahraus. Schh. $P$.

87 Nigritarsis. Schönh. Morea.

88 Bipunctatus. Fabr. Helvetia.

Quinquepunctatus.

Fabr. Barbaria.

90 Fasciatus. Olivier.

91 Lathyri. Kirby. Schh. Galh

92 Meleagrinus. Géné. Algiria.

93 Flavescens. Lucas. id.*

94 Plumbeus. Lucas. id.

95 Helvolus. Sturm. Cat, Italia.

96 Ruficornis. Dej. Cat. Gall. merid.

\{ Seminarius. Stéven. Russ, merid. 
97 Maculicornis. Dej.

Cat. $P$.*

98 Atomarius, Stm. Cat, Germania.

99 Caliginosus. Dej. Cat. Hispania.

100 Pectinatus, Dej. Cat. Gall. merid.

101 Oblongus. Dufour. Dej. Cat. Hispania.

102 Concolor, Stm. Cat. Italia.

103 Sertatus. Illiger. St.

Cat. Europ. mer.

104 Fulvimanus. Dej.Cat. Hispania.

105 Obscurus. Stm. Cat. Austria.

106 Calceatus. Dej. Cat. Lombardia.

107 Cinereus. Dej. Cat. Gall, merid.*

108 Trifolii. Schmidt. St.

Cat. Carniolia.

109 Genistæ. (Chevrolat.) Gall. merid. 2396

\section{FAM, CERAMBYCES.}

SPONDYLIS, Fabricius, Latr. Mulsant.

1 (Buprestoides. Fabr. Gall,or.bor.* $\{$ Var. Elongatus, Dej. Cat. Latr. id.

\section{MACROTOMA. (Dej. Cat.) Serville.}

Prinobius. Mulsant.

1 f 9 Scutellaris. Germar. Dalmatia.* $\{$ Germari.Dej.Cat.Mul.Gall.merid. (o Myardi. Mulsant. Corsica.

ERGATES. Serville. Mulsant.

Redtenbacher. Prionus, Fabr, Oliv.

1 (o Faber. Linné. Fabr. Gall. merid.* $\{$ Obscurus, Olivier. id.

Serrarius, Panz.Muls. Germania.

\section{EGOSOMA. Serville.}

Muls. Redtenbach. Prionus, Fabr. Oliv. Panz.

1 Scabricorne. Scopoli. Fabr. Gallia.*
AULACOPUS, Serville.

1 Serricollis. Motschulsk, Russ. merid.

TRAGOSOMA, Dej. Cat, Serville.

Mulsant. L. Redtenbach. Prionus. Fabr. oliv.

1 Depsarium, Linné. Alp. Gall.

\section{PRIONUS, Geoffroy.}

Fabr. Oliv, Lat, Serv, Muls. Cerambyx. Linné.

1 Coriarius. Linné. Fab. Gallia.*

2 Patruelis, Sturm. Cat, Grcecia.

CERAMBYX. Linné: Mulsant.

de Casteln. Hammatrcherus. (Meg. Dej. Cat.) Serville. L. Redtenbacher.

1 Heros. Scopoli. Fabr, Gallia.*

2 Nodulosus. Kaulfuss.

Germar. Illyria.

Welensii. (Dahl.) Dej.

Cat. id.

3 Miles, Bonelli, Muls, Gall, merid.* Procerus. Hoffmg. id.

Friulanus. (Dahl.) Dalmatia.

4 Velutinus, Dej. Cat. Brullé. Gall. merid.* Audax. Kollar. Dalmatia.

5 Mirbeckii. Lucas. Algiria.*

6 Nodicornis. Küster. $N$.

7 Carinatus. I Iister. id.

8 (Cerdo. Linné. Fabr. P.*

$\{$ Paludivagus. Lucas. Algiria.

9 Necrii. Erichson. id.* Mauritanicus. Buquet. id.

10 Levaillantii. (Cerambyx ?) Lucas, id.

11 Intricatus. Fairmaire. Apenninus.

PURPURICENUS. (Zieg. Dej. Cat.) Serv.

Mulsant. de Cast. Redtenb. Cerambyx. Fabr. Oliv.

1 Desfontaini. Fabr. Alg. Gracia.*

2 Dalmatinus. Sturm. Dalmatia.

3 Budensis. Gatze. Muls, Hung. Gall.* 
4 Kohleri, Fabr.

Wredii. Fischer.

Gallia.*

Russia.

Var. Cinctus. Villa. Gall. merid.

- Bilineatus. Muls. Gallia.

- Servillei. (Ziegl.)

Dej. Cat. Muls, $P$.

5 Etnensis. Bassi. Sicilia.

6 Globulicollis. Dej. Cat.

Muls. Gall. merid.

7 Affinis. Brullé. Gracia.*

8 Dumerilii. Lucas. Algiria.*

9 Barbarus. Lucas.

ANOPLISTES. Serville.

Purpuricenus. St. Cat.

1 (Ephippium. Schönh. Russ, merid. Eleagri. Stéven. id.

Holodendri, var, Gebl. Sibiria.

ROSALIA, Serville.

Muls, de Casteln. L. Redtb. Gallichroma. Latr. Cerambyx. Linné, Fabr. Oliv.

1 Alpina. Linné. Alp. Gall.*

AROMIA. Serville.

Mulsant, Redtenbach. Cerambyx, Fabr.

1 Moschata.Linné.Scop. Gallia.*

2 Suaveolens. Rambur.

(Dahl.) Hisp. merid.

3 Ambrosiaca, Stév, Mls, Pyr, orient. *

Var. Thoracica. Fisch. Russ, merid.

4 Rosarum. Dahl. Cat.

$\left\{\begin{aligned} \text { Lucas. } & \text { Sicilia.* } \\ \text { Var. Thoracica. Fald. } & \text { Russ, merid. }\end{aligned}\right.$

SAPHANUS, Megerle. Dahl. Dej. Cat.

L. Redtenbacher.

Callidium. Fabr. Ahr. Germar.

1 Spinosus. Fabr. Austria.

Piceus. Laichart. Tyrolis.

PHYMATODES. Mulsant.

Redtenb. Cinuludum. Fabr. St, Cat. GeRAMBYX, Linné.

1 Brevicollis. Schönherr.Suecia. *

Thoracicus, Dej. Cat.

Mls. Luc. (Callidium.) Gallia.

2 Variabilis, Linné.Schh. Gallia.*

Var. Nigrinus. Muls. id.

- Fennicus. Fabr. Finlandia.

- Nigricollis. Muls. Gallia.

- Proustus. Fabr. id.

- Testaceus. Linné. id.

3 Humeralis. Dej. Cat.

$\left\{\begin{array}{l}\text { Muls. id.* } \\ \text { Barbipes, Villa. }\end{array}\right.$

4 Analis, L. Redtenb. Austria.

LEIODERES. L. Redtenbacher.

1 Kollari, L, Redtenb. Austria.

\section{CALLIDIUM. Fabr.}

Cerambyx. Linné. Leptura. Geoff. Fabr.

1 Hungaricum. Fabr. Hungaria.

2 Dilatatum. Paykull. Gall. Alp.

Variabile, Olivier. id.

Cognatum. Laichart. Tyrolis.

Eneum. Herbst. Germania.

3 Patruele. Sturm. Cat. Hispania.

4 Coriaceum. Paykull. Gall. Alp.

5 Violaceum. Linné. id.*

6 Macropus. Ziegl. Dej.

St. Cat. Austria.

7 Sanguineum. Linné. Gallia.*

8 Rufipes. Fabr. id.

Amethystinum. Schh. Suecia.

9 Castaneum. Megerle.

L. Redtenb, Austria.

Gracile. Dej. Cat. Podolia.

10 Unifasciatum. Rossi. Gall. orient. *

11 Alni. Linné. Fabr. Gallia.*

12 Puncticolle. Dej. Cat. Hungaria.

Castaneum. Parreyss. Russ. merid.

13 Angustum. Kriechb. Tyrolis.

14 Cyaneum. Fabr. Hungaria.

15 Similare. Küster. Germania?

SEMANOTUS. Mulsant.

Redtenbacher. Gerambyx. Linné. GaleiDIUM. Fabr. Oliv.

1 Undatus. Linné. Gallia.*

2 Russicus, Fabr, Oliv, Austria.

RHOPALOPUS, Mulsant.

Redtb. Callidium, Fab. Cerambyx, Lin.

1 Femoratus, Linné. Gallia, 
2 Insubricus, Ziegl, in

3 (Clavipes. Fabr. Gallia.* litt. Germar. Alp. Gall. \{ Macropus. (Ziegler.) Austria.

\section{CRIOCEPHALUS. Mulsant.}

Redtenbacher. Dej. St. Cat. Callidium. Fabr. Gerambyx. Linné.

1 Rusticus. Linné. Gall. orįent.* Yar. Ferus. Dej, Cat. Solier. id.

- Pachymerus. Muls. id.

2 Elongatus. Dej. Cat. Gracia.

3 Morbillosus. Dej. Cat. Hispania.

4 Fulvus. Dej, Cat. id.

ASEMUM, Eschscholtz. Mulsant.

L. Redtenbach. Callidium. Fabr. GeramBYX. Linné.

1 Striatum. Linné. Gall. orient.* Yar. Agreste. Fabr. Germania.

NOTHORHINA. L. Redtenbacher.

Callidium. W. Redtenbach.

1 Muricata. Dalm. Schh. Austria.

CRIOMORPHUS. Mulsant.

IsARthron. Dej. Cat. L. Redtenbach.

1 Aulicus. Fabr.

Gall. orient.* Curialis. Panzer. Germania.

Var. Fulcratus, Fabr. Gall. orient. * Ruficrus. Schrank. Germania.

Var. Luridus. Linné. Gall. orient. ${ }^{\star}$ - Castaneus. Payk. id.

- Impressus. Payk. Alp. Gall.*

2 Femoralis. Ménétriés. Russ. merid. ${ }^{*}$

HYLOTRUPES. Serville.

Mulsant. L. Redtenb. Callidium. Fabr. Gerambyx. Linné.

1 Bajulus. Linné. Fabr. Gallia.*

Var. Lividus. Mulsant. id.*

- Puellus. Villa. id.

\section{DRYMOCHARES. Mulsant.}

1 Truquii. Mulsant. Alp. Marit.

1 Nodieri. Mulsant. Gall. merid.

STROMATIUM. Serville.

(Dej. Cat.) Mulsant. de Casteln. Solenophorus. Muls. Gallidium. Fabr. Oliv.

1 Strepens. Fabr. Hispania. ${ }^{*}$ Unicolor. Olivier. Gall. merid. Fulvum. Villers. id.

HESPEROPHANES. (Dej. Cat.) Mulsant. Gallidium. Fabr.

1 Sericeus. Fabr. Gall. merid.* Latreillei. Brullé. Grocia. Sericeus. Brullé. id. Rotundicollis.Dej.Cat.

2 Griseus. Fabr. id.* Tomentosus. Lucas. id.

3 Affinis. Lucas. id. *

4 Pulverulentus. Erich. id.

5 (Nebulosus. Olivier: Gall. merid. ${ }^{\star}$ Holosericeus. Rossi. Italia. Cinereus. Villers. Gall, orient.

6 fPallidus. Olivier. Gall. merid. * Mixtus. Fabr. id.

\section{GLYTUS. Fabricius.}

Leptura et Cerambyx. Linné. Calleidium. Oliv. Arhopalus. Serville. Platynotus et Plagionotus. Muls. AnagLYPTUS. Mulsant.

\section{(Plagionotus. Mulsant.)}

1 Detritus. Linné. Gall. bor.*

2 Arcuatus. Linné. Gallia*

Lunatus. Fabr, Linné. id.

Detritus. Woet. id. 
(Currus. Fabr.)

3 /Liciatus. Linné. Oliv. P.* Rusticus. Linné. Suecia

Hafniensis. Linné. $\quad$ id.

Confusus, Herbst, Austria.

Longipes, Villers. Gallia.

Atomarius. Fabr. Dania.

Omega. Rossi. Italia.

Oo. Schrank. Germania.

Maculatus. Linné. Suecia.

4 Bablayei. Brullé. Gracia.

5 Conspicuus. Stéven.St.

6 Siculus. Gory Cat. Tauria.

7 Græcus. Sturm. Cat. Gracia.

8 Scalaris.Dej.Cat.Brul.id.*

9 Floralis. Fabr.

Aulicus, Laichart.

Fasciatus. Herbst.

Gall. merid.*

Tyrolis.

Austria.

Arcuatus, Schrank. id.

Trilineatus. Woet. id.

Nigrofasciatus, Woet. id.

10 Pulchellus. Sturm. Cat.Russ, merid.

11 Dalmatinus. Stm. Cat, Dalmatia.

12

(Mucronatus. Fabr. P.

Tropicus. Schrank. Austria.

Maculosus. Linné. Suecia.

13

14

Arvicola. Olivier.

(Arietis. Linné.

$\{$ Quadrifasciatus. de

Gall. merid* 35

34
35

$\boldsymbol{P}^{\star}$

15 Geer. Suecia.

\{ Antilope. Schrank.

Arietis. Fabr.

16 Gazella. Fabr.

Temesiensis. Germ

Temesiensis. Germar. Germania.*

Hieroglyphicus. Drap. id.

17

Rhamni. Germar.

Trifasciatus. $\mathrm{Fabr}$.

Portugalus, Linné.

Dalmatia.

j Var. Ferrugineus. Du-

19

four. Mulsant.

Egyptiacus, $\mathbf{F a b r}$.

20 Sexguttatus, Dej. Cat.

21
22
23

Ruficornis. Olivier.

Lucas. Algiria.*

Nigripes. Brullé.

Gall, merid.*

(Cinereus. Gory et Cas-

Gall. merid.*

Lusitania.

Gall. merid.* Hungaria.

Duponti. Dej. Cat.

Mulsant. id.
25 (Semipunctatus. Fabr. Austria.* Speriosus. Schmidt. id. Figuratus. Herrer. id.

26 Ornatus. Fabr. Gall. merid.* Verbasci, Laichart. Tyrolis.

C.Duplex. Scopoli. Carniolia.

Venustus, Linné. Suecia mer.

Strigosus, Linné. id.

27 Verbasci. Fabr. Gallia.* Herbstii. Brahm. Germania.

28 Qnadripunctatus. Fab. Gallia.*

$\{$ Villosus. Rossi. Italia.

Navius. Linné. Suecia.

29 jGlaucus. Fabr. Algiria.*

$\{$ Griseus. Gory. Castl. Hispania.

30 f Capra. Germar. Tyrolis.

\{ Sibiricus. Dej. Cat, Sibiria.

31 Pelleteri. Gory. Gall, merid.*

32 Massiliensis, Fabr. P.*

Lineola. Scopoli. Carniolia.

Achilla. Brahm. Germania.

33 Plebejus. Fabr. Gallia.*

Funebris. Laichart. Tyrolis.

Rusticus, Razoun. id.

Figuratus. Scopoli, Carniolia.

Lamda. Schrank. Austria.

Arietis. Woet. Germania.

Leucozonia. Linné, Suecia.

Virens. Fabr. Barbaria.

Latifasciatus. Bul, des nat. de Moscou. Caucasus.

36 Zebra. Dalman. Russ, merid.

37 Cordiger. Géné. Italia.

38 Quinquepunctatus. Luc. Algiria.

(Anaglyptus, Mulsant.)

39 Gibbosus. Fabr. Gall. merid. ${ }^{*}$ Y Var.Scriptus. Mulsant,id.

40 Mysticus. Linné. Gallia.*

Quadricolor. Scopoli. Carniolia.

Var. Albofasciatus, de

Geer. Suecia.

- Hieroglyphicus.

$\begin{array}{ll}\text { Herbst. } & \text { Germania. } \\ \text { Rusticus. Scopoli. } & \text { Carniolia. } \\ \text { Litteratus. Linné. } & \text { Suecia. }\end{array}$

ANISARTHRON. Dej. Cat. Redtenbach.

1 Barbipes. (Dahl.) Dej.

Cat. Redtenbach. Austria.*

Molle. Sturm. Cat, Germania.

Pubescens. (Ziegler.) id.

Testaceum. Ullrich. id. 
GRACILIA. Serville.

Mulsant. L, Redtenbach. Callidium, Fab.

1 Timida. Ménétriés. Gall.merid. Fasciolata. (Ziegler.)

Dej. Cat.
Tatarica. (Parreyss.)
Russ. merid.

2 Pygmæa. Fabr.

Pusilla. Fabr.

Var. Vini. Panzer. id

\section{LEPTIDEA. Mulsant.}

Gracilia. Dej. Cat.

1 Minuta. Motschulsky. Russ. merid.

2 or Brevipennis.Dej.Cat. $\left\{\begin{aligned} & \text { Sol. in litt. Muls. Gall. merid.* } \\ & \text { o Thoracica. Solier. id. }\end{aligned}\right.$

AXINOPALPIS. Dej. Cat, Redtenbacher.

1 Gracilis. (Ziegler.) L,

Redtenbacher. Austria.

OBRIUM. Megerle. Dej. Cat. Latreille.

Mulsant. L. Redtenbach. SA PERda. Fabr. Cerambyx. Linné. Stenchorus. Schh.

1 Brunneum. Fabr. P.*

2 Cantharinum. Linné. Alp. Gallia.* Ferrugineum. Panzer. Germania.

CALLIMUS. Mulsant.

Callidium. Fabr. Stenopterus, L. Redtb.

1 f Cyaneus. Fabr. Austria. Bourdini. Mulsant. Gall, orient.

CARTALLUM. (Meg. Dej. Cat.) Mulsant.

Callidium. Fabr.
STENOPTERUS, Illiger.

Necydalis. Fabr. Molorchus. Schh. GarLIDIUM. Fabr.

1 (Rufus. Linné Dispar. Schönherr. Suecia.

2 Flavicornis. Dej. Cat. L. Redtenbach. Austria.

3 Præustus. Fabr. Gall. merid.* Var. Ater. Fabr. id. ${ }^{\star}$

4 Ustulatus. Dej. Cat. Mulsant. id. ${ }^{*}$

5 Mauritanicus. Lucas, Algiria.*

MOLORCHUS. Fabr. Mulsant.

Necydalis. Linné.

$1\left\{\begin{array}{l}\text { Dimidiatus. Fabr. } \\ \text { Minor. Linné. }\end{array}\right.$

Alp. Gall."

2 Umbellatorum. Linné. $\boldsymbol{P}_{\text {. }}^{*}$

NECYDALIS. Linné. Mulsant.

Molorchus. Fabr.

1 Major. Linné. Gallia.* Abbreviatus. Fabr. Germania.

2 Salicis. Dupont. in litt. Butner. $\boldsymbol{P}$.

ACANTHODERES. Serville. Muls.

Redtb. Acanthoderus. Dej. Cat. Lamia. Fabr.

1 Varius. Fabr.

Gall. merid.*

\section{LEIOPUS. Serville.}

1 \{ Ruficolle. Fabr.

Gall. merid.* Muls. L. Redtenb. Lamia. Panzer. Schk. $\{$ Var. Nigricolle.(Gaub.) Algiria."

Gyllenh. Cerambyx. Linné. Payk.

\section{DEILUS. Serville.}

Mulsant. L. Redtenb. Callidium. Fabr. oliv.

1 Fugax. Fabr.

Gall, merid.
1 Nebulosus. Linné. Gallia.*

2 (Punctulatus. Paykull. Alp. Gall.*

Cinereus. Muls. (Exocentrus.) id.

3 Illyricus. Sturm, Cat, Illyria.

4 Fennicus. Paykull. Fennia. 
EDILIS. Serville.

Astynomus. Dej, Cat. Redtenbach. AcanThocinus. Panz. (Meg.) Dej. Cat. LAmia. Fabr. Cerambyx. Linné.

1 Montana. Serv. Muls. Gallia.* $\{$ Edilis, Linné. Suecia.

2 Atomaria, Fabr. ơ Costata. Fabr.

3 Grisea, Fabr.

Carniolia.

4 Alpina. L. Redtenb. Austria.

EXOCENTRUS, Meg. Dej. Cat. Mulsant.

L. Redtenbach. Pogonocherus, Serville. Lamia. Schk. Cerambyx, Fabr, Linné.

1 Balteatus. Fabr. Gall. orient. |Balteus. Linné. Suecia. Crinitus. Panzer. Germania. Pubicornis. Schrank. Austria. Lusitanicus. Olivier. Gall. merid. 2 Adspersus. L. Rey. Mul. Gall. orient. 3 Besseri. Dej. Cat. Volhynia.

POGONOCHERUS. Meg. Dej. Cat. Latr. Serv.

Mulsant. Redtenbach. Lamia, Gyll. Schh. Cerambyx. Geoff. Fabr, Panz.

1. Ovalis. Gyllenh. Muls. Gallia.*

2 Fascicularis. Panzer. Gall. orient.*

3 Perroudi. Mulsant. Gall, merid.

4 Hispidus. Fabr, Oliv. Gall. bor.*

5 Pilosus, Fabr. Oliv.
Panz. Gallia.*
(Hispidus. Panzer. Germania.

6 Scutellaris. (Rey.)Muls, Gall.orient.

MONOHAMMUS. (Meg, Dej, Cat.) Muls.

Mon ochamus. Serville, L. Redtenbacher. Lamia, Fabr, Oliv. Cerambyx, Linné.

$\begin{cases}\text { Sutor. Linné. Oliv. } & \text { Gallia.* } \\ \text { Pistor. Germar. } & \text { Germania. } \\ \text { o Maculatus. (Ziegler.) id. }\end{cases}$

2 Sartor.Fabr. Panz. Alp. Gall.*

3 (Galloprovincialis, Oliv. Gall, merid.* Pellio. Germar. Germania.

Lignator, Dej. Cat. 'Gall, merid.
LAMIA. Fabr, Serv.

Muls. L. Redtenbach. Pachystola, Dej. Cat. Cerambyx. Linné.

1 Textor, Linné. Gallia.*

MORIMUS. Serville.

Dej. Cat. Muls, L. Redtenb, LAMia, Fabr.

1 Funestus. Fabr.

2 Lugubris. Fabr. Textor. Oliv.

3 Tristis. Fabr.

Gall. merid.* Gall, orient.* id.

Gall. merid.*

\section{STENIDEA. Mulsant.}

Stenosoma. Muls. Deroplia. Dej. Cat. Waltl.

1 (Genei.(Chevrolat.) Ara-

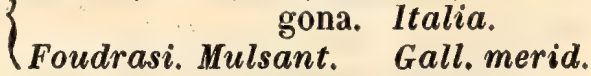

2 Troberti. Mulsant. Algiria.*

3 Obliquetruncata. Walt. Hungaria.

NIPHONA. (Zieg. Dej. Cat.) Mulsant.

1 (Picticornis. Mulsant. Gall. merid.* Saperdoides. (Ziegl.) Dej. Cat. id. Dalmatina. Dej. Cat. Dalmatia.

2 Nephele. Dalman. Barbaria.

\section{ALBANA. Mulsant.}

1 M-griseum. (Foudras.) Mulsant. Gall. merid.

MESOSA. (Meg. Dej.) Serville, Mulsant. Cerambyx. Linné. Oliv. Lamia. Fabr.

1 Curculionides. Linné. Gallia.*

2 Nubila, Olivier. Gall. orient. Nebulosa. Fabr. Serv.

L. Redtenb. id.

3 Myops. Schönherr. Finlandia.

PARMENA. (Meg. Dej. Cat.) Serville.

Mulsant. Cerambyx. Villers. Fabr.

1 \{ Solieri. Mulsant. \{ Pilosa. Solier.

Gall. merid.* id. 
2 Fasciata. Villers. Gall. merid.* 21

3 Pubescens, Schönherr, Dalmatia.

4 Algirica. de Casteln. Algiria.* Maura. (Dej.) id.

5 Unifasciata. Rossi. Italia. ${ }^{*}$

Balteata. Fabr. id.

(Var. Interrupta. Villa. Lombardia.

6 j Dahlii. Dej. Cat. Sicilia.

Cincta. Dahl. Cat. id.

7 Hirsuta. Küster. N.

\section{DORCADION, Dalman in Schh.}

Muls. Lamia, Fabr. Herbst. Gerambyx. Linné. Pallas.

1 Tomentosum. St. Cat. Küster. Gracia.

2 Parallelum. Stm. Cat. Küster. Turcica.

3 Glycyrrhizæ. Fabr. Russ, merid.*

4 Pigrum. Schönh. Pall. id.

Carinatum. Pallas. id.

Morio. Fischer. id.

5 Morio, Fabr. Oliv, Austria.*

6 Fulvum. Hbst.Scopoli. id.*

Morio. var. B. Schönh. id.

오 Canaliculatum. Fi-

scher. scher. id.

7 Rutipes. Fabr. Panz. Austria.*

8 Atrum. Illiger. Germania.*

9 Italicum. (Dej.) Kiist. Italia.

10 Caucasicum. St. Cat. Küster. Caucasus.

11 Nitidum. Victor, Küst. id.

12 Wagneri. Erichson. id.

13 Sturmii. Friwaldszky.

Waltl. Küster. Constantinp.

14 Scabricolle. Schönh. Russ, merid.

\{ Peregrinum. Ménétr. id.

15 Kindermannii.Friwlds.

Waltl. Küster. Constant.

16 Murrayi. Kollar. Küst. Hungaria.

17 Holosericeum. (Meg.)

Küster. Volhynia.*

18 Sericatum. Stéven. Russ, merid. Rubripes. (Ziegler.) id.

19 Pusillum. (Besser.) Küster. Podolia.

20 Septemlineatum. St. Cat. Küster. Constantinp.
1 Lineatum. Fabr. Panz. Hungaria.

Vittigerum, Panzer. id.

Vittatum.Stm. Cat. ㅇ. id.

22 Lemniscatum. St. Cat.

23 Abruptum. (Megerle.)

Küster. Dalmatia. *

24 Axillare. Küster. Italia.

25 Pedestre. Linné. $F$. Illyria.*

26 Striatum. Schönherr. Caucasus.

27 Cruciatùm. Fabr. Russ, merid.*

28 Sulcipenne. Küster.

29 Thracicum. Dej. Cat.

Küster. Constantinp.

30 Albolineatum. St. Cat. Kïster. id.

31

Divisum. Germar.

32 Crux. Schönherr. Turcica.

\{ Gracum. Dej. Cat. Grocia.

33 Dimidiatum. Victor.

Küster. Caucasus.

34 Fuliginator. Linné. $\quad \boldsymbol{P}^{*}$

$\{$ Var, Quadrilineatum. Chev. Muls, Gallia.*

Meridionale. Dej. Cat. Mulsant. Gall. merid.*

36 Quadrilineatum. St. Cat. Küster. Hispania.

Pyrenæum. Dej. Cat.

Germar, Pyr, orient.*

Striola. Dej. Cat. id.

Navaricum. Dej. Cat. Hispania.

Bilineatum. (Meg.) Ill. Hungaria.*

Inclusum. Dej. Cat. Constantinp.

Lineola. Illiger.

Donzelli. Mulsant.

Gall. merid.*

Hispanicum. Dej. Cat. Hispania.

Albicans. Dej. Cat. id.

Neapolitanum. Dej.Ct. Ital. merid.

Siculum. Dej. Cat. Sicilia.

Tauricum. Friwaldsk. Tauria.

\{ Nigritarse. Stéven. Russ, merid.

Sericeum. Sturm. Cat. id.

Convexifrons. Dej.Cat. Constantinp.

Friwaldszkii. Stm. Cat, id.

Velutimma. Stéven. Russ. merid.

Laqueatum. Klug. Turcica.

Vittatum. Sturm. Cat. Constantinp.

Vicinum. Sturm. Cat. id.

Fuscum. Sturm. Cat. Groccia.

(Seductor. (Dahl.) Dej.

$\begin{cases}\text { Cat. Dalmatia. } & \text { Dal. } \\ \text { Dispar. (Ziegler.) } & \text { id. }\end{cases}$ 
58 Apicale. Waltl.

59 Irroratum. Sturm. Cat. id.

60 f Vittigerum. Fabr. Italia. Molitor. Sturm, Cat, id.

61 Atrum. Illiger.

62 J Nigrum. Dej. Cat. Spinola. Schönherr. id.

63 Murinum. Dej. Cat. id.

64 Sulcatum. Dej. Cat. id.

\section{ANESTHETIS, Dej. Cat. Mulsant.}

L. Redtenbacher. Sapenda. Fabr.

1 Testacea. Fabr. Gall, orient.*

COMPSIDIA, Mulsant.

Saperda. Fabr. Cerambyx, Linné.

1 Populnea. Linné. Gallia.* 10-punctata. de Geer. Suecia.

ANEREA. Mulsant.

Saperda, Fabr. Cerambyx, Linné.

1 Carcharias. Linné. Gallia.* $\{$ Punctata, de Geer. Suecia. (Var. Grisescens. Muls. Gallia.

\section{SAPERDA, Fabricius.}

Oliv. Panz. Latr. Muls. Castel. L. Redtb. Gerambyx. Linné.

1 Bipunctata. Zoubkoff. Austria. \{ Biguttata. W. Redtb. id.

2 Phoca. Fröl. Charp. id.

3 (Scalaris. Linné. Gallia.* Yar. Estella. Mulsant, id.

4 Punctata. Fabr. id.*

5 (Tremulæ, Fabr. Gyll. id.* Octopunctata. Schrk. Austria. Tremula. Schönherr. Suecia.

6 Seydlii. Frölich. Gyll. Austria.

STENOSTOLA. Dej. Cat, Mulsant.

W. Redtb. Küster. Saperda, Fabr, Gyll. Panz.

1 Nigripes. Fabr. Alsatia.* Yar. Ferrea. Schrank, Germania.

2 Tiliæ. Küster.
TETROPS. (Kirby.) Stephens.

W. Redtb. Anetia. Dej. St. Cat. PolyopsiA. Muls.

1 Præusta. Linné. Gallia.

2 Gilvipes. Stéven. Russ, merid.

3 Muhlfeldii. Dej. Cat. Austria. Discoides. Megerle. id.

OBEREA. (Meg.) Dej. Cat. Mulsant.

W. Redtenb. Saperda. Fabr. Gerambyx. Linné. Schrank.

1 Linearis. Linné. Gall. bor.*

2 Erythrocephala. Schrk. Gall. merid.* Var. Euphorbia. Germ. Austria.

3 Pupillata. Gyll. Schh, Gall, orient.*

4 Ragusana. Dej. Cat.

Kïster. Dalmatia.

5 Xanthocephala. Dej. Cat. Russ. merid.

6 Mauritanica. Lucas Algiria.

7 Maculicollis. Lucas, id.

8 Oculata. Linné. Gallia.*

PHYT ECIA. Dej. St. Cat. Mulsant.

W. Redtb. Saperda. Fabr. Panz. CeramBXX. Schh.

1 Ephippium. Fabr. Gall. merid.

2 Argus. Panz. Fabr. Austria.*

3 Balcanica, Friwaldsk. Turcia.

4 Affinis. Harrer. Panz. Helvetia.*

5 Punctum. (Ziegl. Dej.

Cat.) Ménétriés. Gall. merid.*

6 Flavipes, Fabr. Algiria.*

7 Cephalotes. Küster.

8 Rubricollis. Lucas. Algiria.*

9 Lineola. Fabr. Gall. orient.

10 Virgula. Charpentier, Russ. merid.

11 Rufimana. Fabr. Austria.

Flavimana. Panzer. Germania.

Gilvimana. Stéven. Russ, merid.

12 Jourdani. Mulsant. Gall. orient.* Ophthalmica.Dej.Cat.id.

13 Puncticollis.Ménétriés. Russ. merid.

14 Azurea. Schönherr. id.

15 Prætexta. Stéven. Russ, merid.

16 Pulchella. Dej. Cat, Gracia.

17 Cylindrica. Linné. Alp. Gall.*

18 Scutellata. Fabr. Austria. 
19 Globulicollis. Dej. Cat. Dalmatia. Echii. Dahl. Cat. Hungaria.

20 Virescens. Panzer. Gallia.*

21 Flavescens. Mulsant. Gall. merid.

22 Malachitica. Dahl. Cat.

$\begin{array}{lll}23 & \text { Ferrea. Fabr. } & \text { Lucas. } \\ 24 & \text { Sicilia. } \\ 25 & \text { Higricornis. Fabr. } & \text { Alp. Gall. }{ }^{*} \\ 25 & \text { Hirsulula, Fabr. } & \text { Hungaria. }\end{array}$

26 Uncinata. W. Redtenb. Austria.

27 Vestita. Waltl. Grocia.

28 Brevicollis. Sturm. id.

29 Molybdæna.Dalm.Sch.Austria. *

30 Erythrocnema. Lucas. Algiria.*

31 Cyrtana. Lucas. id.*

32 Guerinii. de Brême. id.*

33 Warnieri. Lucas. id.*

34 Vittigera. Fabr. id.*

Maculesa. var. Muls. Gall. merid.

35 Græca. Sturm. Grocia.

\section{CALAMOBIUS. Guérin.}

Saperda, Fabr. Augapanthia. Serville.

1 | Marginellus. Fabr. i Gracilis. Creutzer.

Gall. merid.* Austria.

\section{AGAPANTHIA. Serville. Mulsant.}

Castelnau. W. Redtenbach. Saperna. Fabr. Cerambyx, Linné.

1 Irrorata, Fabr.

Hispania.*

2 (Verbasci. (Megerle.) Hungaria.* Kirbyi. Schönh.

3 Asphodeli. Latreille. Spencei. Gyllenhal.

4 Panonica. Creutzer. Dahlii. Koy. Decora. Stéven.

Hellespont. Gall. merid.* id.

Hungaria. id.

5 j Cynaræ. Germar. Russ, merid. Obscuricornis. Ullrich. Illyria.

6 Cardui. Fabr. Gall. merid.*

7 Angusticollis. Schönh. $P^{*}$

8 Maculicornis. (Dahl.) Hungaria.

9 Annularis. Olivier. Algiria.*

10 Squalis. Gyllenhal. Austria. Frenata. Dej. Cat. Hisp. merid.

11 Græca. Sturm. Cat.

12 Lixoides. Lucas.

13 Gerardii. Buquet.
14 Suturalis. Fabr. Gall. merid." Annulata. Fabr. id. Var. Marginalis. Muls, id. - Nigrocnea. Muls. id.

15 Violacea. Fabr.

Austria.

16
Micans. Panzer.

Germania.*

Smaragdina.Dej. Cat. Gall. merid.

Var. Corulea. Schh. id.

- Chalybea. Muls. id.

- ? Leucaspis. Stév. Russ. merid.

17 Alboscutellata. (Dahl.) Hungaria.

VESPERUS, Dej. Cat. Serville.

Muls. W. Redtenb. Stenoconus. Fabr. oliv.

1 Strepens. Olivier. Gall. merid.*

2 Luridus. Rossi. id.* YSolieri.Dej.Cat.Germ.id.

3 Xatartii. Dej, Cat. ㅇ. Muls. Pyr, orient. Melopennis. Dufour. in litt. Hispania.

RHAMNUSIUM. Meg. Dej. Cat. Latr.

Serville. Muls. W. Redtb. Rhagium. Fabr. Sch. Casteln. Ste nocorus. Oliv. Sch.

1 Salicis, Fab. Oliv. Sch. P.* $\begin{cases}\text { Ruficolle. Herbst. } & \text { Austria. } \\ \text { Etruscum. Rossi. } & \text { Italia. } \\ \text { Var. Glaucopterum. } & \text { Schall. Germania. }\end{cases}$

\section{RHAGIUM. Fabricius.}

Stenoconds. Olivier. Cerambyx. Linné.

1 Bifasciatum. Fabr. Gallia.* Var. Unifasciatum. Mulsant. Alp. Gall. - Ecoffeti. Mulsant. Gallia.

2 Indagator. Fabr. Gallia.* Inquisitor. Linné. Suecia.

Var. Minutum. Fabr. Dania. - Investigator. Muls. Gallia.

3 Mordax. Fabr. id.* Scrutator. Olivier. $P$. Sycophanta. Schrank. Germania. Var. Cephalotes. Muls. Gallia.

4 Inquisitor. Linné. id.* Mordax. Olivier. id. Bifasciatum. Schrank. Germania. 
3 Maculatum. Gysselen. Carniolia.

6 Rufiventris. Germar.

TOXOTUS, Meg. Dej. Cat. Serville.

Muls. Casteln. L. Redtb. Rhagium. Fabr. Cerambyx et Leptura. Linné. Leptura. Fabr. Payk. Pachyta. Panzer.

1 Cinctus. Fabr. Austria. Dentipes. Mulsant. Gall. or.

2 Cursor. Linné. Alp. Gall. Noctis. Linné. Oliv. Suecia.

Var. Verneuillii. \&. Mulsant. Alp. Gall.

3 Interrogationis. Linné. id. Var. Duodecimmaculatus. Fabr. id.

- Curvilineatus.Mul.id.

- Flavonotatus.Mul.id.

- Marginellus. Fabr. id.

- Bimaculatus.Muls.id.

- Ebeninus. Muls. id.

4 Meridianus. Linné. Gall, orient.* Var. Chrysogaster. Ol. id.*

- Lavis. Olivier. $\quad \boldsymbol{P}$.

- Sericeus. Olivier, id. ${ }^{*}$

- Ruficornis. Scop. Carniolia.

- Geniculatus. Four-

5 Humeralis. Fabr. Austria.*

6 Dispar. Panz.Mulsant.Gall. bor.

7 Dorsalis. Sturm. Cat. Russ, merid.

8 (Quadrimaculatus. Lin.

(Pachyta. Mulsant) Alp. Gall.*

(Var. Bimaculatus. Mls. id.

9 Spadiceus, Payk. Gyll. Austria.

10 12-Maculatus. Fabr. Alp. Gall.* Var. Interrogationis.

Linné. Suecia.

- Curvilineatus. Mls. Alp. Gall.

- Flavomaculatus.

Mulsant. id.

- Marginellus, Fabr. id.

- Bimaculatus. Mul. id.

- Ebeninus. Muls. id.

PACHYTA. Meg. Dej. Cat. Serville.

Muls. Casteln. L. Redtb, LePTURA, Fabr. Oliv.

Suecia.

2 Borealis. Gyllenhal. Lapponia.
3 Octomaculata, Fabr. Alp. Gall,*

Var. Decempunctata.

Olivier. id.

- Cerambyciformis.

Schrank. id.

- Quadrimaculata. Scopoli. id.

- Sexmaculata.Panzer. Germania.

- Sexpunctata. Muls. Gallia.

4 Sexmaculata. Linné. Germania.*

5 Trifasciata. Fabr. Suecia.

6 Signata. Sturm. Cat. Italia.

7 Clathrata. Fabr. Gallia.*

Var. Brunnipes. Muls, id.

- Reticulata. Fabr. id.

Signata. Panzer. Germania.

8 Strigilata. Fabr. Payk. Gall. merid." Y Var. Suturalis. Muls. id.

9 Smaragdula, Fabr. Lapponia.

Y Var. Alpina. Ménét. Russ. merid.

10 Marginata. Fabr. Lapponia.

Var. Morio. Fabr. Austria.

11 Virginea. Linné. Alp. Gall,*

Var. Violacea. de Geer. id.

- Nupta. Mulsant. Gall. merid.

- Vidua. Mulsant. Alp. Gall.

12 Collaris. Linné. Gallia.*

Thalassina. Schrank, Austria.

(Var, Nigricollis. Muls. Gallia.

STRANGALIA. Serville.

Muls. W. Redtb. Strangalia et Stendra.

Dej. Cat. Leptura. Linné. Fabr.

1 Septem-punctata. Fab. Austria.*

2 Nigra. Linné. Gallia.*

3 /ơ Villica. Fabr. id.*

Revestita. Linné. Germania.

Var. Rufomarginata.

Serville. $\boldsymbol{P}$.

- Ferruginea. Muls. Gall. orient.

- Vitticollis. Muls, P.

- Labiata. Dej. Cat. Mulsant. id.

- Fulvibarbis. (Perroud.) Mulsant, Gallia.

4 Atra. Fabr. id.*

5 Pubescens. Fabr. id.

Var. Obscura. Panz. id.

$\left\{\begin{array}{l}\text { Holosericea. Fabr. id. } \\ \text { o Aurifina. (Megerle.) Austria. }\end{array}\right.$ 
6 Aurulenta. Fabr. Gall. orient. Quadrifasciata. Rossi. Italia.

7 Árcuata. Panzer. Alsatia. Annularis. Fabr. Austria.

8 Quadrifasciata, Linné. Gall. bor.

9 Strangulata. Ill.Germ. Lusitania.

10 Alboscutellata. St.Cat.Caucasus.

11 Verticalis, Dej. Cat. Küster. Dalmatia.

12 Armata. Herbst. Gyll. Gallia.* Var.Impunctata.Muls.id. - Externepunctata. Muls, id.

- Binotata. Muls. id.

- Punctatofasciata.

Muls. id.

Elongata. Rossi. Italia.

Var. Subspinosa. Fab. Gallia.

- Undulata. Muls. id.

- Sinuata. Panzer. id.

13 Attenuata, Linné. id.*

14 Frgeri. Stéven. Caucasus.

15 Melanura. Linné. Gallia, \{ Sutura-nigra. de Geer. Suecia.

16 Cruciata. Olivier. Gall. orient.* Bifasciata. Schrank. Austria.

17 Distigma. Charpentier. Gall. merid. ${ }^{*}$

18 Quinquesignata, Küst. $N$.

LEPTURA. Linné. Fabr. Oliv. Latr. Muls. Casteln. W. Redtb.

1 Virens, Linné.

2 Rubrotestacea Illiger. . Iliger. Gallia.* Testacea. Linné. Suecia. Rubra. Linné. Germania.

Var. Occipitalis. Muls, Gallia.

3 Fontenayi. Mulsant. Gall. merid.* \{rythroptera.Dej.Cat,id.

4 Oblongomaculata. Buq. Algiria. *

5 (Rufa. (Dej. Cat.) Brul. Gall. merid.* Hamorrhoidalis. (Du. four.) Muls. Hispania.

6 Apicalis. Dej. Cat. Dalmatia.

7 (Rufipennis. Mulsant. L.

Redtenbacher. Austria.

(Rubens. Meg. Dej. Cat. id.

8 Scutellata. Fabr. Gallia bor.*

9 Melas. Lucas. Algiria.

10 Hastata, Fabr. Gall. merid,

11 (Tesserula. Charpent. Hungaria.

Bisignata. (Dahl.) Dej.

Cat. id.

12 Bisignata. Brullé. Gracia.

13 Affinis. Sturm. Cat. Turcica.

14 Tomentosa. Fabr, Gallia.*

Fulva, de Geer. Suecia.

15 Pilosa. Sturm. Cat. Hungaria.

16 Pallens. Dahl. Cat. id.

17 (Sanguinosa. Gyllenh. Sibiria.*

Vinicolor, Faiderm. id.

(Rubripennis. Dej.Cat. Austria.

18 Cincta. Gyllenhal. Alp. Gall.*

Sanguinolenta. Oliv.
Panz. Austria.

Var. Variabilis, Payk, Alp. Gall.*

- Notata. Olivier. Gall. bor.

- Dubia. Scopoli. id.

- Luctuosa.Mulsant. id.

I- Chamomilla? Fab. id.*

19 Sanguinolenta. Fabr.

$\{$ Gyll. Gall.bor.

Variabilis, de Geer. Suecia.

20 Maculicornis, de Geer. Gall. bor,

21 Livida. Fabr. Gallia.

Pastinaco. Panzer. Germania.

22 Unipunctata. Olivier. Gall. merid.*

Strangulata. Germar.

Charpentier. Pyr. or.

Var, Sublineata. Muls. id.

- Scapularis. Muls. id.

- Abbreviata. Muls, id.

- Fusciventris. Muls.id.

- Rufiventris. Muls. id.

- Luteipes. Mulsant. id.

- Varipes. Mulsant. id.

- Fuscipes, Mulsant. id.

\section{ANAPLODERA. Mulsant.}

Leptura. Fabr. Casteln. Dej. Cat. GramMOPTERA. L. Redtenb.

1 Sexguttata. Fabr. Gallia. *

Var. Exclamationis.

Fabr. id.

- Biguttata. Muls. id.

2 Rufipes. Fabr. Gall. orient.*

$\{$ Var. Fuscipes, Muls. id.

3 Lurida.Fabr. Gallia.*

Suturalis. Olivier, id.

GRAMMOPTERA. Serville.

Muls, Dej, Cat. Leptura. Fabr.

1 Spinosula. (Foud.) Mls. P.* 
2 Lævis. Fabr. Alp. Gall.* Tabacicolor, de Geer. Suecia.

3 Quadriguttata. Muls. Germania.* Var. Suturalis. Fabr. Gallia.

4 Holosericea.Fab. $n^{\circ}$ 68. Austria. Var. Velutina. Dahl. Cat. Hungaria. Nigra. (Dahl.) id.

\% Hæmorrhoidalis. Stm. id.

6 Analis. Panzer. Gall. orient. \{ Varians. (Meg.) Dej. Cat. Germania.

7 Rufimana. Dej.Cat, Grocia.

8 Ruficornis. Fabr.

9 Præusta. Fabr. $\boldsymbol{P}^{*}$ Splendida. Herbst. Gallia.* id.

10 Saperdoides. Stéven. Russ. merid. 404

\section{FAM. DONACIE.}

DONACIA. Fabricius.

Leptura. Linné. Prionus. Scopoli. StenCORUs, Geoffroy.

1

$\left\{\begin{array}{l}\text { Crassipes. Fabr. } \\ \text { Striata. Panzer. } \\ \text { Micans. }\end{array}\right.$

Hicans. Hoppe.

Aquatica. var, b. Lin. Suecia. Spinosa. de Geer. id.

2 Bidens. Olivier. Clavipes. Paykull. Cincta. Germar. Micans. Marsham. Aquatica. Martyn. Versicolor? Brahm. Dentata. Hoppe. Lac. Bidens, Dej. Cat. Gallia.* Germania. id. Gallia.* Suecia. Germania. Anglia. id.

Germania.

4 Angustata. Kunze. Lac. Lombardia.* Bidens, var. Dej. Cat. Italia. Eruginea. Dahl. Cat. id.

5 (Sparganii. Ahrens. Gallia. Bidens. var. Gyllenh. Suesia.

6 Polita. Kunze. Lacord. Hispania. Femorata. Dej. Cat. Italia. Elegans. Dahl. Cat. Sardinia.

7 Appendiculata. Ahrens, Gall. merid.* Reticulata. Schh. Dej,

Cat. Illyria.
8 Dentipes. Fabr.

Vittata. Olivier.

Fasciata. Hoppe. Lin. Suecia.

Nitida. Linné.

Gallia.*

$P$.

Marginata. Hoppe. Anglia.

Yittata. Panzer. Germania.

Limbata. Panzer. id.

Lateralis. Bonelli. Lombardia.

10 Simplicifrons. Lacor-

11 Sagitariæ. Fabr. Gallia. *

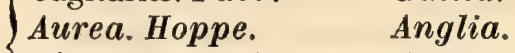

Bicolor, Linné. Suecia.

Var. A.Collaris. Panz. Germania.

12 Obscura. Gyllenhal. id.*

Impressa. Ahrens. Gall. bor.

13 Brevicornis. Ahrens. Suecia. P.

14 Thalassina. Germar. P. Germania. I Impressa, var.c. Gyll. Suecia.

15 Impressa. Paykull. Gall. merid.* Y Var, Antiqua, Kunze. Sicilla.

16 Menyanthidis. Fabr. Gallia.* Simplex. Payk. Marsh, Anglia.

Clavipes. Fabr. Dania. Crassipes. Linné. Germania.

17 Apricans. Lacordaire. Sicilia. ${ }^{*}$

18 Linearis. Hoppe. Gallia.*

Simplex. Fabr. Germania.

19 Typhæ. Brahm. Gallia.*

I Linearis, var. b. Gyll. Suecia.

20 Simplex. Fabr. Gallia.*

Semi-cuprea.Panzer, Germania.

Vulgaris. Linné. Suecia.

Var. Maura? Kunze. Germania.

21 Malinovskyi. Ahrens. id.*

22 Fennica. Gyllenhal. Suecia.*

Arundinis. Ahrens. Germania.

23 (Hydrocharidis, Fabr, P. Gallia.* Cinerea. Hoppe. Germania.

Var. A. Tarsata. Panz. id.

24 Tomentosa. Ahrens. id.

25 (Nigra. Fabr. Dalmatia.*

Abdominalis. Olivier. id.

Palustris. Herbst. Austria.

Violacea? Pallas. Sibiria.

Braccatas. Scopoli. Carniolia.

26 (Discolor. Hoppe. Germania.*

Nigra, Olivier. Gallia.

Rufipes. Olivier. id.

Consimilis et Assimilis.

Schrank. Germania.

Var.Variabilis. Kunze. id. 
Affinis. Kunze. Nigra, Paykull. Discolor. Gyllenhal. Enea. Olivier.

Rustica. Kunze.

Pallipes. Stm. Kunze. Planicollis. Kunze.

Besseri. Dej. Cat.

Fusca. Pallas.

28 Sericea. Illiger.

Var. A. Sericea. Linné. var. à Gyll. Suecia.

- B. Festucce. Fabr. Dania.

- C.Violacea.Hoppe. Germania.*

- D. Micans. Panzer. id.*

- E. Armata. Payk. Suecia.

- F. Nymphea. Fabr. Dania.

- G. Sericea. var. $f$. Gyll.var.d.Zett. Suecia.*

- H.?Violacea. Gyll, id.

HEMONIA. (Megerle.) Lacordaire.

Rhagium. Fabr, Payk. Donacia, Fabr. MaCROPLAa. Curtis.

1 Equiseti. Fabr.

Suecia.

Appendiculata. Panz. Germaria.

Mucronata. Hoppe. id.

Mutica. Payk.

2 Curtisii, Lacordaire.

Zostera? Curtis.

Helvetia.

Anglia.

id.

3 Chevrolatii. Lacord. Gallia.

4 Zosteræ. Fabr. Suecia.

Ruppix. Germar.' id.

Schiodtei. Guérin. id.

Multica. Fabr. id.

5 Gyllenhalii. Lacord. id.

Zostera.Gyll.Dej.Cat.id.

Equiseti. Fallen. id.

6 Sahlbergii. Lacord. Finlandia.

Zosterce. Sahlberg. id.

Intermedia. (Mannh.) id.

3.4

\section{FAM. GHRYSOMELE.}

\section{divisio 1. Lemidae.}

ORSODACNA. Latreille.

Galleruca. Fab. Crioceris. Fab. Lema.

Panz. Donacia. Ahrens.

1 Mespilli, Lacordaire. $P_{*}^{*}$
2 Nigricollis. Olivier. Gallia. Var. Marginella. Duft. Austria.

Picipennis? Duftsch. id.

3 Cerasi. Fabr. Gallia. *

Chlorotica. Gyll. Oliv. Suecia.

Fulvicollis.Payk.Pnz, id.

Var. Chlorotica. Latr.

Gyll. var. $\boldsymbol{P}$.

- Nigroculata. Moll. Germania.

- Lineola. Besser. Volhynia.*

- Melanura. Fabr. Suecia.

Limbata. Olivier. $\boldsymbol{P}$.

- Glabrata.Panzer. Germania.*

- Nigripennis. Dej.

Cat. Volhynia.

- Cantharoides.Fab.

Duftsch. Austria.*

4 Nigriceps. Latreille. Gallia.*

Cerasi.var. Dej. Cat. P.

| Var. Nigriceps, Duft. Austria.

- Lineola. Fab.Panz. Germania.

5 Humeralis. Latreille. Gallia. ${ }^{*}$

Lineola. var. F. G. E.

Duftsch. Germania.

Var.Cœrulescens. Duft.Austria.

Lineola. var. B. Duft. id.

Oxyacanther. Schott, id.

Violacea. Chevrolat. $\boldsymbol{P}$.

6 Nematodes, Lacord. Pedemont.

SYNETA. Eschscholtz. (Dej. Cat.)

Crioceris. Fab. Lema. Sch. Orsodachna. Gyll. Auchenia. Zettersted, Guérin.

1 Betulæ.Fab. (Dej.Cat.)Suecia.

ZEUGOPHORA. Kunze, Lacord.

Crioceris. Fab. Lema, Gyll. Auchenia. Marsh. Lema, Fab. Chyptoceph. Linné. Chrysomela, de Villers.

1 Scutellaris. Suffrian. Germania.*

2 Frontalis. Suffrian. Gall. orient. Subspinosa. var. b. Gyllenh. Suecia.

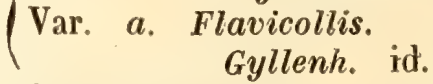

3 Subspinosa. Linné. Gallia. * Erythrocephala. Hbst. Austria.

Berilonensis. Linné. Germania. 
4 Flavicollis. Marsham. Gallia.

Subspinosa, var. $\beta$.

Schönh. Suecia.

Melanocephala. (Bo--

nelli.) Dej. Cat.Suff. Gall. merid.

LEMA. Fabricius, Redtenb.

Crioceris. Geoff. Auchenia. Marsh.

Chrysomela et Cryptocephalus. Lin.

(Chiocenis. Geoff. Lacord.)

1 Stercoraria. Linné. Pe-

tagna. Sicilia.*

Cicatricosa. Dej. Cat. Algiria.

2 Merdigera. Linné. … Gallia.*

Rubra-liliorum. de

-Geer Germania.

Lilii. Scopoli. Carniolia.

3 Brunnea. Fabr. P.*

Merdigera, Linné. Suecia.

Rubra-liliorum. var.

de Geer. id.

Var. Corineta. Fald. Russia.

- Collaris. (Dahl.)

Dej. Cat. Austria.

4 f Rufipes. Herbst. id. Unicolor. Panzer. id.

5 Suffriani? Schmidt. Germania.

6 Mediciana. Lacordaire. Pedemont. Abdominalis. Medici. id.

7 Alpina. Ferrari. $\boldsymbol{W}$.

Redtb. Austria.

8 (Quinquepunctata. Fab. Germania.* Quinquenotata.Linné.

Dodecatisma.(Ziegler.)

10 Duodecimpunctata.

Suffrian. Sicilia.*

Lin. Panz. Gyll. Suff. Austria.*

11 Quatuordecimpunctata.

Scopoli. Germania.*

12 Distincta. Lacordaire. Gracia.

13 Paracenthesis. Linné.

$\left\{\right.$ Touss, Charp. Gall. merid. ${ }^{*}$

Suturalis. Oliv. (Dej.

Cat.) id.

14 Dahlii. Dej. Cat. Lac. Sicilia.

15 (Asparagi. Linné. Gallia.*

Campestris.Fab.Laich.Tyrolis.

Var. Pupillata. Ahr. Austria.

Maculipes. Parr. Russ. merid.

16 Campestris. Linné. Sicilia.
(LEMA. $\boldsymbol{F} a b r$.)

17 (Rugicollis. Suffrian. P. German.* Puncticollis. Curtis. Anglia. Cyanella. Fabr. Gyll. Suecia.

18 Cyanella. Fabr. Gallia.* $\{$ Var, A. Obscura? Stph. Anglia.

19 Erichsonii. Suffrian. P. German.*

20 Flavipes. (Meg.) Suff. Hungaria.*

21 Melanopa. Linné. Suecia."

Hordei, Fourcroy. $P$.

Cyanipennis. Duftsch. Germania.

22 Rufocyanea. Suffrian. id.

23 Hoffmanseggii. Lacord. Lusitania.

Collaris. (Hoffmsgg.) id.

\section{DIvisio 2. Hispidae.}

\section{HISPA, Linné.}

1 Testacea. Linné.

2 Numida. Guérin.

3 Algeriana. Guérin.

4 Atra. Fabr.

5 Aptera, Bonelli.

Gall. merid.* Algiria.

id.

Gallia.*

Gall. merid.*

LEPTOMORPHA. Chevrolat.

1 Filiformis. Dahl. Sicilia*

\section{Divisio 3. Cassidae.}

\section{CASSIDA. Linné.}

1 Atrata, Fabr. Austria.

2 Equestris. Fabr. Gallia.*

Yiridis, Linné. Suecia.

3 Sardea. Sturm. Cat. Sardinia.

4 Hemisphærica, Herbst, Austria.*

5 Illyrica, Dej. Cat. Illyria.

6 Austriaca. Fabr. Austria.*

Speciosa. Brahm. id.

7 Alpina. Sturm. Cat. Alp. Lomb.

8 f Vittata. Fabr. Austria.

Ocellata. Herbst. id.

9 Murræa. Linné. Gallia.*

Maculata.Linné. Suecia.

10 Vibex. Linné. id. ${ }^{*}$

11 Rosea. Dej. Cat. Styria.

12 Rubiginosa. Illiger. Gallia.*

Viridis. Fabr. Coll. Germania.

Vibex. Fabr. Coll. id.

Prasina, Fabr, Coll. id. 
13 Sanguinosa, Creutzer, Germania.

$\begin{cases}\text { Prasina. Herbst. } & \text { id. } \\ \text { Rubiginosa. Gyllenh. Suecia. } & \text { Thoracica. Stephens. Anglia. }\end{cases}$

14 Nigra. Herbst. Austria.

15 Thoracica. Kugelann. Germania.*

16 Rufovirens. Suffrian. id.

17 Azurea. Fabr. Gallia.*

Ornata. Creutzer. Austria.

18 Globosa. Sturm. Cat. id.

19 Denticollis, Suffrian. Austria.

20 Chloris. Suffrian. Germania.*

21 Stigmatica. (Illiger.) Suffrian. id.

22 Sanguinolenta. Fabr. $\boldsymbol{P}^{*}$

23 Margaritacea.Schaller.

$$
\text { Fabr. id.* }
$$

24 Lucida. Suffrian. Germania.*

25 Viridula. Paykull. Suecia.*

26 Bella. Stéven. Caucasus.

27 Subreticulata. (Meg.)

Suff. Germania

Salicornio. Steph. Anglia.

29 Nobilis. Linné. Gall. merid.*

Pulchella. Panzer. Germania.

(Lavis. Herbst. Austria.

30 Berolinensis. Dej. Cat.

Suff. Germania.

31 Obsoleta. Illiger. Gall. merid.*

Nebulosa.Fabr. Germania.

(Exculpta. Charpent. Russ. merid.

32

33

34

35

36

37

38

39 Herbea. Bohem.

Lineola. Creutzer.

Austria. ${ }^{*}$

Meridionalis. Dej. Cat. Gall, merid.

Ferruginea. Fabr.

$P$.*

Modests. Sturm, Cat. Hispania.

Nebulosa. Linné. Germania.*

Mutabilis. Villa. Lombardia.*

f Testudo. Friw. Suff. Turcica.

Hablitzlia. Stéven.

40 Algirica. Bohem.

Russ, merid. Algiria.*

id. ${ }^{*}$

\section{DIvisio 4. Gallerucidae.}

\section{ADIMONIA, Laicharting.}

Meloe et Galleruca. Fabr.

1

$\left\{\begin{array}{l}\text { Brevipennis. Illiger. } \\ \text { Marginata. Fabr. }\end{array}\right.$

2 Barbara. Erichson.

Gall. merid.*

Italia.

3 Cariosa, Dej. Cat.

Barbaria
4 Lata. Chevrolat.

Algiria.*

5 Sardea.Dahl.Ct.Küist.Sardinia.*

6 Provida. Buquet. Algiria.*

7 Littoralis. Fabr. P.*

Var. Italica. Dahl. Dej.

Cat. Italia.

Gagatina. (Megerle.) Illyria.

Lineata. Ullrich. id.

8 Artemisiæ. Rambur. Hisp. merid.*

9 Tanaceti. Fabr. $\boldsymbol{P}^{*}$

10 Rotundicollis. Chevrl. Alp. Gall.*

11 Alpina. (Gaubil.) id. ${ }^{*}$

12 Monticola. (Gaubil.) Pyr. orient.*

13 Circumdata. Duftsch. Austria.*

14 Rustica. Schall. Fabr. P.*

15 Florentina. Dahl. Dej.

Cat. W. Redtb. Italia.*

Circumdata. Ullrich. Illyria.

Limbata. (Ziegler.) Austria.

16 Dispar. Chevrolat. Gall. merid.

17 Brachyptera. Küster. Lombardia. ${ }^{*}$

Yilla. Dej. Cat. Küst, id.

18 Interrupta. Geoff. Oliv.Gallia.*

19 (Rufa. (Meg.) Germar. Austria.*

Reticulata.(Ziegl.) Dalmatia.

20 Silphoides, Dalman. Russ. merid.

Rufa. Stéven. id.

21 Hæmatidea. (Megerle.)

Germar. Austria.*

22 Rubicunda. Dej. Cat. Hispania.

Fossulata. Dufour. id.

23 Aptera. Bonelli. Lombardia.

Melanocephala.Ponza.id.

24 Sanguinea. Fab. Payk, $P$.*

25 Pallidipennis. Küster. Germania.

26 (Capreæ. Linné. Gyll. P.*

Var. Polygonata. Lai-

chart. Tyrolis.

27 Violacea. Lucas. Algiria.*

28 Lucasii. Gaub. in litt. id.*

\section{GALERUCA. Geoffroy.}

\section{Chrysomela. Linné.}

1 Viburni. Paykull. Gall. bor.*

2 Xanthomelæna. Schrk. P.*

\{ Calmariensis, Fabr. Germania.

3 Geniculata. Dahl. Cat. Italia.*

4 (Sublineata. Dej. Cat. 
6 Nympheæ. Linné. Fab.

(Var. Sagittarice. Gyll. Suecia.*

7 Lineola. Fabr. Panz. $P^{*}$

8 Galmariensis. Linné. Germania.* Lythri. Gyllenhal. P.

9 Tenella. Linné. id.*

10 Flaviventris, Dahl. Cat. Hungaria.

RAPHIDOPALPA. Chevrolat.

1 Foveicollis. Dej. Cat.

Lucas. (Galleruca.) Dalmatia.* (Crioceroides. Dufour. Hispania.

MALACOSOMA. (Chevrolat.) Blanchard.

Chrysomela. Linné, Cistela, Fabr

1 Lusitanica. Linné. Gall. merid.* Nigripes. Olivier. id. Abdominalis. Schönh. id.

Testacea. Fabr. id.

2 Lepida. Dej. Cat. Podolia.

Fulvicollis. Gebler. Sibiria.

( Var. Fulvicollis, Latr, Syria.

AGELASTICA. Chevrolat. Dej. Cat. W. Redtb. Kuist.

Chrysomela. Linné. Galleruca. Fabr.

1 Halensis. Linne. Gallia.* Nigricornis. Fabr. Germania.

2 Alni. Linné. . Gallia.*

PHYLlobrotiCa. Chevrolat. Dej. Cat, W. Redtenb.

Galleruca, Fab. Crloceris, Fab, Duft. Panz.

1 Quadrimaculata. Fabr.

$\{$ Duftsch. P.*

Bimaculata. Panzer. Germania.

2 Trinotata, Dej. Cat. Russ, merid.

Tripunctata. Godet. id.

3 Adusta, Fabr. Creutz. Austria.
CALOMICRUS. (Dillw.) Stephens.

W. Redtb. Crioceris, Marsh. Duftsch. Altica. Duftsch. Entom. Hefte. Panz. LUPERUS. Ratzeb.

1 Circumfusus, Marsh. Austria. S Spartii, Ent. Hefte. Duftsch. id. Brassica. Panzer. Germania.

2 Pinicola, Duftsch. Austria.*

\section{LUPERUS. Geoffroy.}

Chrysomela. Linné. Crioceris. Fabr. Duftsch.

1 Rufipes. Fabr. Panz. P.*

2 Betulæ. Chevrolat. Alsatia. P. $^{*}$

3 Flavipes. Linné. Panz. $P$.

4 Pyrenæus, Dej. Cat.

Germar. Pyr. orient. ${ }^{*}$

5 \{ Flavipennis. Lucas. Algiria.

\{Flavus? Dej. Cat. Hispania.

6 (Pallipes. Dej. Cat. Austria.

Xanthopus. Besser. Volhynia.

Rufipes. Stéven. Russ, merid.

7 Pygmæus. Dej. Cat. Dalmatia.

8 Cyaneus. Dej. Cat. id.*

9 Xanthopus. Duftsch.

(Illig.) Austria.

10 Suturalis. Dej. Cat. Hispania.*

11 Umbraticus. Ullrich.

St. Cat. Austria.

12 Coerulescens. Duftsch. id.*

$\{$ Viridipennis.Dej.Cat. Styria.

MONOLEPTA. (Chevrolat.)

1 Terrestris. Dej. Cat, Pyr. orient. ${ }^{*}$

\section{LITHONOMA. (Chevrolat.)}

1 Marginella. Fabr. Hispania.

2 Andalusiaca. Rambur. id.

\section{ALTICA. Linné.}

Galleruca. Fabr. Chrysomela. Linné. Haltica. Illig. Redtenb. Graptodera, Crepidodera, Phyllotreta et AphTHona. (Chevrol.) Dej. Cat.

(Graptodera, Chevrolat.)

1 Oleracea, Fabr. Duft. Gallia.* 
2 Erucæ, Fabr, Duftsch. Austria. Lythri. (Chevrol.) Aub.P.

3 Vitis. Chevrolat. id.

4 Mercurialis. (Hellwig.) Fabr. id.*

(Podagrica. (Chevrolat.)

5. Ruficollis. Lucas. Algiria. ${ }^{*}$

6 Fuscipes. Fabr. $\quad$. $^{*}$

7 Malvæ. Illiger. Gall. merid. ${ }^{*}$

8 (Fuscicornis, Linné. Austria.* Fulvipes. Ent. Hefte, P.

Rufipes. Paykull. Suecia.

Malve. Fourcroy. P.

\section{(Crepidodera. Chevrolat.)}

9 Geminata. Fabr. Hispania.*

Lineata. Oliv, Rossi. Gall. merid.

10 Transversa. Marsham. $\boldsymbol{P}_{\text {.* }}^{*}$

11 Exoleta. Linné. id.

Ferruginea. Fourcroy. id.

12 Punctipennis. Lucas. Algiria.

13 Impressa. Fabr. Germania.

14 Rutipes. Linné. Gall. merid.* \{ Ruficornis. Ent. Hefte. Germania.

15 Peyroleri. Dej. Cat. Illyria. $\{$ Fuscipes. Peyroleri. id.

16 Femorata. Gyllenhal. Germania.*

17 Melanopus. Dej. Cat. Pyr. orient.* 44

18 Concolor. Dej. Cat. Gall. merid.

19 Cyanescens. Duftsch.

$$
\text { Villa? Austria. }
$$

20 Nigritula. Gyllenhal. Finlandia.

$\{$ Var. Coarctata. Dej.

21 Nitidula.Fabr. Cat. Illyria.

22 (Helxines, Fabr. Duft. P.*

Fulvicornis. Fabr. Germania.

Metallica. Duftsch. Austria.

23 Modeeri, Linné. $P$.

24 Melanostoma, L. Redt. Austria.

$25\left\{\begin{array}{c}\text { Rustica. Linné. (Bala- } \\ \text { nomorpha.) Chevr. Gall. merid. }\end{array}\right.$

(Semi-anea. Ent.Hefte.Austria.

26 Pubescens. Ent.Hefte. P.*

27 Nigriventris. Chevrolat. Dej. Cat, id.*

28 Subcyanea. (Dahl.) St. Cat. Austria.

29 Alpicola. Schmidt et Helfer. Smyrna.
(PhyllórReta. (Chevrolat.)

30 Armoriacæ. Ent. Hefte. Duftsch. Austria*

31 Cincta. Dej. Cat. id.* Y Cochlearia. Chevrol. $P$.

32 Amabilis. Dej. Cat. Pyr. orient.*

33 Parallela, Reiche. : P.*

34 Excisa. L. Redtenb. Austria.

35 (Brassicæ. Fabr. Germania.* Quadripustulata.Illy. - Ent. Hefte. id.*

36 Sinuata, Dej. Cat. L. Redtenb. id.

37 Flexuosa. Ent. Hefte. Panzer. id.*

38 Nemorum. Lin. Panz, $\boldsymbol{P}^{*}$

39 Vittula. (Schïpp.) L. Redtenb. Austria.

40 Antennata. Ent. Hefte. Duftsch. Gallia.*

41 Atra. Paykull. $\boldsymbol{P}^{*}$ $\{$ Melana. Gyll. Illig. Suecia.

42 Lepidii. Ent. Hefte. Gyllenh, $\boldsymbol{P}$.

43 Procera. L. Redtenb. Austria.* IErea? Dej. Cat.

Gall, merid.

45 Chalybea. Besser. St. Cat. Austria.

46 Euphorbiæ, Fab. Gyll. Gallia.* Gagatina. Besser. Volhynia.

47 Palustris. Chevrolat. $P$.

48 Erythropus. Dej. Cat. id.

49 Cœrulea. Paykull. id.* \{Hosciami. Gyll. Duft. Austria.

50 Campanulæ. L. Redtb. id. *

51 Lacertosa. Rosenh. Hungaria.

52 Janthina. Dej. Cat. Austria.

53 Megerlei. Dej. Cat. id.

54 Virescens. Dej. Cat. P.*

55 Tantilla. Dej. Cat, Austria.

56 Rugulosa. Dej. Cat. Gall. bor.

57 Rubi. Fabr. Germania.*

Y Var.Dumetorum. OEsk. Hungaria.

58 Rubivora. (Chevrolat.) $\boldsymbol{P}^{*}$

59 Divaricata. L. Redtb. Austria.

60 Salicarix. Paykull. Suecia.

\{ Striatella? Gyll.Illig. Austria. 
61 Ventralis. Illiger.

62 Tarda. Märkel.

Atropa, Märkel.

\section{(Bala Nomorpha. (Chevrolat.)}

64 Chrysanthemi.Ent.Hft.Germania. ${ }^{*}$

65 Declarata. Dej. Cat. Gall, merid.

66 - Eraria. (Chevrolat.) P.*

\section{LONGITARSUS, Latreille.}

Thyamis, Steph. Teinodactrla, Chevrol. Crioceris, Fabr. Chrysomela. Linné.

1 Consolidæ. Stéven. Russ. merid. 2 (Echii. Ent. Hefte. Illig. P.*

Pallipes. Besser. Dej.

Salvia. Géné.

Cat. Volhynia.

Salvia. Géné. Lombardia.

3 Flavipes. Dej. Cat. Hispania.

4 Eruginosus. Dej. Cat. id.

5 Punctatus. Dej. Cat. Dalmatia.

6 Etruscus, (Meg.) St.

Cat. Austria.

7 Anchusæ. Payk. Gyll. Germania.* Tristis. Dej. Cat. Suecia.

8 Analis. (Creutz.) Duft. Austria. ${ }^{*}$

9 Apicalis. Beck. Germania.

$\left\{\begin{array}{l}\text { Apicalis. Beck. } \\ \text { Praticola. Sahlberg. Finlandia. }\end{array}\right.$

10 Holsaticus.Linné.Ent. Hefte. Suecia.*

11 Quadripustulatus. Fab. $P$.* Quadrimaculatus.

Ent. Hefte. Germania.

12

Dorsalis, Fabr.

13 Sisymbrii. Fabr.

Thapsi? Marsham.

Suturalis. (Ziegler.)

Opalirans. Besser.

Brunneus. OEskay.

$P^{*}$.

id. *

id.

Styria.

Volhynia.

Croatia.

14 Verbasci. Panzer.Ent.

$$
\text { Hefte. } \boldsymbol{P}^{*}
$$

15 Lutescens, Gyll. (Aphthona.) Chevrol. id.*

16 Nigriceps. L. Redtenb.

$$
\text { Lej.? Cat. Austria. }
$$

17 Sanguinolentus. Dej.

$$
\text { Cat. Hispania. }
$$

18 Trilineolatus. Dej.Cat. Dalmatia.

19 Melanocephalus. Schh.

$$
\text { Gyll. } \boldsymbol{P} \text { ** }
$$

20 Ochrolencus, Marsh. id. *
21 Tabidus, Fabr, Illig.

22 Atricillus, Linné. Ent.

$$
\text { Hefte. id.* }
$$

23 Nasturtii. Fabr. Austria.*

Pratensis, var. Gyll. Suecia.

24 Pusillus. Gyllenhal. Austria.*

25 Pratensis. Panz. Gyll. P. $^{*}$

26 Ruficeps. (Ullrich. St. Cat.) Austria.

27 Luridus. Oliv. Rossi. id.*

28 Brunneus. Duftsch. id.

29 Femoralis. Marsh. Gyl. id.

30 Fusco-æneus. L. Redt. id.

31 Linnæi. Duftschmidt, id.

32 Parvulus. Payk. Gyll. id.*

33 Obliteratus. Rosenh. Hungaria.

34 Niger. Ent.Hefte.Gyll. Austria.

\section{PSYLLIODES. Latreille.}

Macrocnema. (Megerle.) Steph. ChrysoMELA. Linné. Marsh. GaLlerdGa. Payk.

1 (Melanophthalma. Duft. Austria.

\{Picina? Stephens. Anglia.

2 Gircumdata. L. Redtb. Austria.

3 Affinis. Paykull. id.* Atricilla. Panzer. Germania.

Exoleta. Illiger. $\quad \boldsymbol{P}$.

4 (Dulcamaræ. Ent.Hefte. $\left\{\begin{array}{c}\text { Gyll. id:* } \\ \text { Var. Chalcomera. Ill. Istria. }\end{array}\right.$

5 Hyosciami. Linné. Ent. Hefte. P.*

6 Ecalcarata, L. Redtb. Austria.

7 Cuprea. Ent. Hefte. Germania.

8 Attenuata. Ent. Hefte, Germania.*

9 Chrysocephala. Linné. Ent. Hefte. $P$.

10 Cyanoptera. Illiger. Hispania.* Elongata. Gyllenhal. Austria.

11 Picipes. L. Redtenb. id.

12 Ruficollis. Sturm. Cat. Podolia.*

13 Antica. Schönherr. Tauria.

14 Decipiens, Stéven. id.

15 Alpina. L. Redterb. Austria.

16 fucullata. Illiger. Germania.*

\{ Spergula. Gyllenhal. Austria.

17 Vicina. Dej. Cat. Gall. merid."

18 Patruelis, Dej. Cat. Silesia.

19 Anglica, Fabr, Gall, bor," 
20 (Luteola. (Chevrolat.) P.*

Affinis. var. Dej. Cat. id.

Pallida. (Ullrich.) Dalmatia.

Var. Striata. OEskay. Hungaria.

21 Kunzei. (Marietti.) Lombardia.*

22 Operosa. Dej. Cat. Gall. bor.

23 Lucidicollis. Dej. Cat. Oriente.

24 Fusciformis. Illiger. Austria.

25 Picea. L. Redtenb. . id.*

26 Rufilabris. Ent. Hefte.

27 Scerpili. Knoch. Germania.

28 Rapæ. Illiger. Austria.*

$\{$ Napi. Ent. Hefte. P.

PLECTROSCELIS. (Chevrolat.) Dej. Cat. L. Redtenb.

Ghactocnema. Stephens. Haltica. Ent. Hefte. Duftsch. Galleruca, Paykull.

1 Calcarata. Dej. Cat. Styriá.

2 (Semi - cœrulea. Ent.

$\left\{\begin{array}{r}\text { Hefte. Illig. Austria.* } \\ \text { Hefl. }\end{array}\right.$

Eneicollis. Dej. Cat. Germania.*

3 Meridionalis. Dej.Cat. Gall. merid.*

4 (Chlorophana. Duftsch. Austria.*

\{Viridissima? Dej. Cat, Gall. merid.

5 Dentipes, Ent. Hefte.

$$
\text { Gyll. } \boldsymbol{P}^{*}
$$

6 Obtusata. Gyll. (Balanomorpha. Chevrol.) Suecia.

7 Pumila. Dej. Cat, Gall. merid.

8 Insolita. Dej. Cat. id.

9 Schüppelii. (Ullrich.) id.*

10 Solieri. Dej. Cat. id.

11 Sahlbergi. Gyllenhal. Austria.*

12 Mannerheimii. Gyllenh. id.*

Eumolpus. Dej. Cat. Dalmatia.

(Rivularis. (Chevrol.) $P$.

13 Aridella. Paykull. id.*

14 Aridula. Gyllenhal. $P_{\text {.* }}^{*}$

15 Angustula. Rosenh. Tyrolis.

16 Palustris. (Dahl.) St.

Cat. Germania.

\section{DIBOLIA, Latreille.}

Haltica. Ent. Hefte.

1 Femoralis. (Ziegl. Dej.

Cat.) Redtenb. Austria. *

Punctatissima. (Chev.) Gall. merid.

Salvioe. Géné.
2 Rugulosa. L. Redtenb. Austria.

3 Occultans. Ent. Hefte. Germania.

4 Cryptocephala. Ent.

Hefte. Austria. ${ }^{\star}$

5 (Cynoglossi.Ent. Hefte. Germania.*

Ahenata. (Meg.) Dej.

Cat. id.

6 ) Eryngii. (Chevrolat.) $P$.

Femorata. Dej. Cat. id.

7 Orata. Dej. Cat. id. *

8 f Ovoides. Dej. Cat. Illyria.*

Ahena, (Chevrolat.) $P$.

9 Granaria. Dej. Cat. Dalmatia.

10 Maura. Dej. Cat. Gall. merid.

Chrysomela. Fabr. Haltica. Duftsch.

Spheroderma. Steph, L. Redtenb.

1 Ahrensii. Germar. Dalmatia.*

2 (Hemisphæricus. Duft. Austria.*

Globosus. Besser. Volhynia.

3 Cardui. (Kirby.) Gyll. P.*

4 Testaceus. Fabr. id. *

๖ Dorsalis. Fabr. Austria.

APTEROPEDA. (Chevrolat.) Redtenb.

Haltica. Oliv, Illig. Mniophica. Steph.

1 Giliata. Olivier. Gallia.*

Hedera. Illiger. Germania.

Globus, Duftsch. Austria.

2 Conglomerata. Illiger. id.*

Globosa. Panzer. Germania.

3 Orbicularis. (Ziegl.) Styria.*

Subglobosa.Dahl.Cat.Austria.

4 (Muscorum. Ent. Hefte. Germania.* Gibbium. Melsheimer. id.

Leodiensis. Wesmael. Belgia.

5 Caricis. Märkel. Germania.*

GYRTONUȘ. Dalman. Dej. Cat.

1 Rotundatus. Dej. Cat. Gall. merid.*

2 Coarctatus. Dej. Cat. Pyr.orient.

3 Dufourii. Dej. Cat.

Dufour. Gall. merid.*

(Eumolpus. Dufour. Hispania.

4 Nobilis. Dej.Cat. Lusitania.

5 Eumolpus. Hoffmsgg. Hispania.

6 Elongatus. Rambur. Hisp. merid. 
Divisio รั. Chrysomelidae.

TIMARCHA. (Megerle.) Latreille.

Chroysomela. Linné.

1 Grandis. Dej. Cat. Barbaria.

2 . Inæqualis. Dej. Cat. Algiria.*

Maura. Chevrolat, Barbaria.

3 Generosa. Erichson. Algiria.*

Chalconota. Dej. Cat. id.

Cuprea. (Chevrolat.) id.

4 Turbida, Erichson. id.*

5 f Rugosa. Fabr. Barbaria.

Confragoso, Buquet, Algiria.

6 Latipes. Olivier. id.*

\{ Banonii? Dej. Cat. id.

7 Punica. Lucas. id.*

8 Endora. Buquet. id.*

Scabripennis? Dej. Ct. id

9 Punctata, Leach. Barbaria.

10 Tenebricosa. Fabr. Gallia.*

\{ Lavigata. Duftsch. Germania.

11 Bætica. Rambur.

12 (Bicolor. (Ullrich.) Sicilia.

$\{$ Pimeloides. (Chevrol.) id.

Var. Blapoides. (Zieg.) id.

13 \} Viridis. Dej. Cat.

Balearica. Gory. id.

14 (Italica. Dej. Cat. Italia.*

Nicecensis. Villa. Pedemont.

Tenebricosa. Géné. Lombardia.

15 Lævicollis. Dahl. Cat. Sardinia.

16 Cribraria. Dej. Cat. Hispania.

17 Hispanica. Dej. Cat. id.

18 Parnassia. Rambur. Hisp. merid.

19 Rugosula. Rambur. id.*

20 Hesperica. Dej. Cat. id.

21 Lævis. Rambur. id.

22 Lævigata. Dej. Cat. Gall. merid.*

23

9

24

25

Prunneri. Géné.

Sardinia. ${ }^{*}$

Alpina. Dahl. Dej. Cat. Sicilia.

(Pratensis. (Megerle.) Dalmatia.

Rugosa. Germar. id.

26 Coriaria. Fabr. $\boldsymbol{P}^{*}$

Var. Rugosipennis.

(Luczot.) Gallia.*

- Asperata.(Luczot.) id.*

27 Globata. (Dahl.) Hungaria.

28 f Rugulosa. Dej. Cat. Volhynia.

Coriaria. Besser. id.

29 Geniculata. Hoffmsgg, Hispania.

30 Occidentalis. Hoffmsg. Lusitania.
31 (Lusitanica. Olivier. Lusitania.*

Punctatissima. Dej.

Cat. Hisp. merid.

Erea. Dej. Cat. Dalmatia.

Metallica. Fabr. Styria.*

34 (Globosa. (Meg.) Redtb. Austria.

\{ Var. Gibba. Stm. Cat. Carniolica.

CHRYSOMELA. Linné.

1 Inæqualis. Dej. Cat. Algiria.*

2 Cribrosa. Germar. Dalmatia.*

Reticulata. (Dahl.) Algiria.

3 Anibalis. Banon. Dej.

Cat. Barbaria.*

4 Metallica. Fabr. Algiria.

5 Affra. Erichson. id.*

6 Tricolor. (Gaubil.) id.*

7 Carbonaria. (Dahl.) Sicilia.

8 Nigra. Luczot. Gallia.*

9 Caliginosa. Olivier. Algiria.

10 Corulea. (Meg.) Duft. Austria.*

11 Gottingensis. Linné. Germania.*

\{ Hamoptera. Duftsch. Austria.

12 Hungarica. Dej. Cat. Hungaria.

13 Nigroænea. Stm. Cat, Germania.

14 Ethiops. Fabr. id.*

15 Morosa. Dej. Cat. Hisp. merid.

Tristis. Rambur. id.

16 Gennensis. Dej. Cat. Italia.*

17 Crassipes. Lucas. Algiria.*

18 Affinis. Fabr. id.

19 Femoralis. Olivier. Gall. merid.* \} Var. Lifficilis. Dufour. Hispania.

20 Femorata, Dej. Cat. id. Var. Femoralis. Dufour. id.

21 Bætica. Rambur. Hisp. merid.

22 Ulyssiponensis. Illig. Lusitania.

23 Zenkeri. (Dahl.) Illyria.

24 Orbiculata. Dahl. St.

Cat. Hungaria.

25 Erea. (Meg.) Duftsch. Alsatia.*

26 Globosa. Meg. St. Cat. Illyria.

27 Globata. Dahl. St. Cat. Hungaria.

28 Rufocuprea. Dej. Cat. Styria.

29 (Crassimargo. Germar.

$\left\{\begin{array}{c}\text { Duftsch. Hungaria.* } \\ \text { Dahlii. (Knoch.) Dej. } \\ \text { Cat. Austria. } \\ \text { Crassicollis. (Dahl.) }\end{array}\right.$

Dej. Cat. Hungaria. 
31

32

33

34

35

36

37

38

39

40

41

42

43

\section{Var. Cinctella. (Ziegl.) Styria. \\ $\{$ - Rubripennis. (Gau-}

$$
\text { bil.) Alp. Gall.* }
$$

65 (Striatopunctata. Gaub. Gall. merid. ${ }^{*}$ \{ Viridana.(Chevrolat.) id.

66 Analis. Linné. Gallia.

67 Schach. Fabr. Austria .

68 Aurata. (Meg.) St. Cat. id.

69 Consularis. Erichson. Algiria.*

Bicolor. Fabr. Regalis. Olivier. Egypt. Var. Orientalis. Klug. Gracia. ${ }^{*}$ Regia. Kollar. Sicilia.

Lusitanica. Gyllenh. Finlandia.

72 Heri. (Sturm.) Küster. Sicilia.*

73 Vernalis, Brullé. Gracia.*

74 Affinis. Lucas. Algiria.

75 (Banksii. Fabr. Gall. merid.* \{ Rubrocuprea. Fourc. id.

76 Erythromera. Dej.Cat. Lucas. Gall, merid.* (Obliquata. Chevrolat. Algiria.

77 Opulenta. (Dahl.) Silesia.

78 Ignita. Olivier. Hispania.

79 Maura. Dej. Cat. Podolia.

80 Lamina. Fabr. Gall. merid. ${ }^{*}$ Lavicollis. Olivier. id. Heteropunctata.(Meg.) Germania.

81 Cuprina. Duftschmidt. Austria. \{ Salvia. Dej. Cat. Illyria.

82 Rufornea. Dej.Cat. Hispania. ${ }^{*}$

83 Modesta. Sturm. Cat. Russ. merill.

84 Lapidicola.Märkel.St.

85 Lichenis. Märkel. St.

Cat. Germania. Cat. id.

86 Elevata. Sturm. Cat. Anglia.

87 Geminata. Payk. Oliv.

Brunsvicensis.(Knoch.) Gyll. Gallia.* Duftsch. Germania.

Var. Interpunctata. Gysselen. id.

Approximata. Germar. Dalmatia.

89 Duplicata. Germar. id.

90 fucata. Fabr. Oliv. P.* $\{$ Gemellata.Rossi.Duft.Germania.

91 Hyperici. (Kinderm.)

92

f Centaurei. Fabr. P.*

I.Eruginosa. Falderm. Sibiria. Varians. Fabr. Gallia.*

94 (Islandica. Andersch. Styria.* Ahenea. (Ziegler.) Dej. Cat. id.
Asclepiadis. Villa. Lombardia. Viridana. (Dahl.) Kïst. Germania. Chloris. Dej. Cat. Lu- 
98 (Fulgida. Fabr. Duft. Germania.*

Graminis. var. Man-

nerheim. Alsatia.

Aurolimbata. Besser. Volhynia. *

99 Graminis.Linné. Gyll.

Duftsch. Gallia.*

Mentha. (Schott.) Germania.

100 Tanaceti. Klingelhafer. id.*

101 Fastuosa. Lin. Panz. Gallia.*

102 Elegans. Géné. „ Italia.

Genei. Dej. Cat. id.

103 Smaragdula. Dej.Cat.Styria.

104 Menthæ. Schrank. Gallia.*

Metallica. Herbst. Austria.

105 Violacea.Fabr.Panz.

Duftsch. Germania.*

106 Mixta. (Ziegler.) Dej.

107 (Megerlei. Fabr. Cat. Gallia* ${ }^{\star}$ Germania.*

$\{$ Var. Alternans. (Meg.)

108 (Cerealis. Fabr. Panz. Austria.

Var. Erica. (Dahl.)

St. Cat. Germania.

- Ornata, Dej. Cat. id.*

109 Luxurians, Olivier. Gall. merid.

110 Americana. Linné. P.*

Rosmarini. Dufour, Hispania.

111 Staphylæa. Lin. Oliv. P.*

112 Reluscens. Rosenh. - Tyrolis.

113 Distincta. (Dej.) Kuist, Gallia.*

114 Polita. Linné. Oliv.

$$
\text { Duftsch, } P \text {. }^{*}
$$

115 Grossa. Fabr. Italia. Algir.

116 Chloromaura. Oliv.

Charpentier. Lusitania.*

- 117 Dichroa. Hoffmsgg. Gall. merid. ${ }^{*}$

118 Lucida. Olivier. Italia.

$\{$ Chloromaura. (Dahl.) id.

119 Sicula. Dej. Cat. Sicilia.

120 Lurida. Linvé, Fabr. P.*

121 Diluta.Hoffmansegg. Gall. merid.*

122 Egyptiaca. Olivier. Algiria.

\section{OREINA. Chevrolat.}

1 Phalerata. (Illig. Dej.

Cat.) Redtenb, Carniolia. (Somptuosa. (Ziegler.) $\{$ Redtenb. Styria.* Gloriosissima. (Meg.) Austria.

3 Monticola, Duftsch. id.
4 (Cacaliæ. Vliv. Duftsch.

Redtenb. Austria.*

Bifrons, Fabr.? Duft. id.

与 Diversa, Sturm. Cat, Istria.

6 Coruleolineata. Duft. Austria.

7 Speciosa. Linné. Gall. merid.*

8 Gloriosa. Fabr. Styria.*

Superba. Olivier. id.

Monticola. Villa. Lombardia.

Var.Pretiosa.Duftsch. Carniolia.

Basilea. Gebler. Sibiria.

9 Viridis. Duftsch. Styria.*

10 Nivalis, Heer. Helvetia.

11 Margarita. Latreille, Gall. merid.*

12 Ignita, Villa. Italia.

Splendens. Jurine. id.

13 Senecionis. Andersch, Germania.*

Y Var. Indigacaa. Chevrolat. Gall, merid.

14 Venusta, Dej. Cat. Gall. orient.*

Tristis. Olivier. Gall. merid.

Pyrenaica. Dufour. Pyrencis.

Alpicola. Sturm. Cat. Carinthia.

15 Luctuosa. Duft. Oliv. Pyrencis. *

16 Anderschii. (Ziegler.) Austria.

17 Cyanea, Sturm. Cat. Italia.

18 Tristis. Fabr. Gallia. *

Hamoptera. Panzer. Illyria.

Cyanea. (Megerle.) Carniolica.

Lugubris. (Dahl.) Hungaria.

19 Elongata. Stentz. . Illyria.*

20 Melanocephala. Duft, Austria.

Peirolerii. Bassi. Italia.

21 Helvetica. (Chevrolat.) Helvetia. *

22 Subrugosa. Sturm. Germania.

23 Bannatica. Sturm. Hungaria.

24 Punctatissima. Kollar. Buccovina.

Cupreoviridis. Koll. id.

Viridescens. Kollar, id.

25 Intricata. Germar. Germania?

26 Virgulata. Germar. id.?

LINA. (Meg. Dahl.) Redtenbacher.

Melasoma. (Dillw.) Steph. Chrysomela. Linné.

1 Populi. Linné.

2 Tremulæ. Fabr.

3 Cuprea. Fabr.

4 Enea. Linné.

5 ) Bulgharensis. Fabr.

$\{$ Lapponica, var.
$P{ }^{*}$

id. ${ }^{*}$

Gall. bor.*

Alsatia.*

Russia.*

Finlandia. 
6

7

8 Collaris, Linné. Fabr. Alsatia* Var. Salicis. Fabr. Suecia.

9 Zetterstedti. Dej. Cat. Norvegia.* \{ Alpina. Zettersted. Suecia.

10 Escheri. Heer. Helvetia.

ENTOMOSCELIS. (Chevrol.) Redtenb. Chrysomela. Fabr.

1 Jorsalis, Fabr. Austria. \{ Adonidis. var.Schönh. id.

2 Adonidis, Fabr. Gall. merid. ${ }^{*}$

PLAGIODERA. (Chevrolat.) Redtenb. Ghrysomera. Linné.

1 Armoriacæ. Linné. $P .{ }^{*}$ $\{$ Punctulata. Besser. Volhynia.

GASTROPHYSA. (Chevrol.) Redtenb.

Chrysomela. Linné. Fabr.

1 Polygoni, Linné. P. $\{$ Var. Distincta. (Chev.) Amer. bor.

2 Raphani. Fabr. Suecia.* Viridula. Olivier. $\boldsymbol{P}$.

3 Chalybea. Dej. Cat. Hispania.

\section{PHRATORA. (Chevrolat.) Redtenb.}

Chrysomela, Linné. Galleruca. Panz.

1 Vitelinæ. Linné. Panz. $P$ *

Yulgatissima. Linné. Suecia.

J Var. Betula. Besser. Volhynia.

Tibialis.Sturm. Germania.

PHEDON. (Megerle.) Redtenbacher.

Chrysomela, Fabr.

1 Carniolicus. Duftsch. Carniolia. $\{$ Var. Olivaceus. (Dahl.

2 (Pyritosus Rossit.) Hungaria. Sabulicola. Heer. Austria.

3 Betulæ. Linné. $\quad \boldsymbol{P}^{*}$ Cochlearia. Fabr. Germania.

Parvulus. Duftsch. Austria. Aquaticus, Godet. Podolia.
4 Graminicola. Duftsch. Austria.

5 Gramicus. Duftsch. id.

6 Neglectus. Dej. Cat. id. $\{$ Pyritosus. Dahl. Cat. id.

7 Egenum. (Ziegl.) Dej.

Cat. Germania.*

8 Chalybeus. Stm. Cat. Anglia.

9 Peregrinus. Parreyss. Corfou.

IPanonicus. Chevrol. id.

10 Videtur. Erichson. Germania.*

11 Triglochinis. Schaum. id.*

\section{HELLODES, Fabricius.}

Chrmsomela. Linné. Fabr. Crioceris. Panz.

1 Violacea. Fabr. $P_{\text {. }}^{*}$

Beccabungw. Paykull.

Panz. Germania.

2 Vicina, Lucas. : Algiria.*

3 Phellandrii. Linné. $\quad \boldsymbol{P}$.

4 Marginella. Linné. "Germania.*

5 Distincta. Lucas. Algiria.

6 Hannoverana. Fabr. Germania.*

7 Aucta. Fabr. P. $^{*}$

8 Chalybea. Dahl. Cat. Sicilia.

PALES. (Chevrolat.) Dej. Cat.

1 Ulema. (Meg.) Dej.Cat. Hungaria.*

\section{COLASPIDEA. de Castelnau.}

Dia, Dej. Chrysomela. Fabr.

1 Sphæroides. Dej. Cat. Etruria. \{ Aurata. (Dahl. Cat.) id.

2 Pubea. Dufour. Hispania.

3 Eruginea. Fabr. Gall. merid.

4 Nitida. Lucas. Algiria.

COLASPIDEMA, de Castelnau.

Chrysomela, Fabr. Colaphus. (Meg.) Dej. Cat.

1 Signatipennis.Dej.Cat.

Lucas. Algiria.*

2 Rufifrons. Olivier. id. *

3 Rumicis. Fabr. id.*

4 Erata. Hoffmansegg. Hispania.

5 (Barbara. Fabr. Gall. merid.* Atra, Olivier. id. 
6 Sophiæ. Schall, Fabr, Austria.*

7 Pulchella. Lucas. Algiria.*

8 (Caucasica. Dej. Cat. Caucasus. \{Violacea. Godet. Russ. merid.

Erythropa. Ménétr. id.

Höftii. Faldermann. Per, occid.

LAMPROSOMA. Kirby.

Lacordaire. Byrnhus. Sturm. OomorPhus. Curtis.

1 Concolor. Sturm. Germania.*

GONIOCTENA. (Chevrol.) Dej. Cat. Redt.

Spartophila. (Chevrol.) Dej. Cat. Chrysomela. Linné. Fabr. Paropsis. Oliv.

(Spartophila. (Chevrolat.)

1 Litura. Fabr.

Gallia. *

$\{$ Var. Flavicans, Oliv. $\boldsymbol{P}$.

2 Spartii, Olivier. Hispania.*

Variabilis, var, Oliv. Gall. merid. Unipunctata. Olivier. id.

Sexnotata. Fabr. Algiria.

오 Egrota. Fabr. Gall. merid.

Caprea. Illiger.: id.

$\backslash$ Plagiodera. (Chevrol.) id.

(Gonioctena. Chevrolat.)

3 Sexpunctata.Fab. (Dej.

Cat.) Austria.*

4 Dispar. Paykull. Gyll. Duftsch. Germania.

5 ) Rufipes. Paykull. Suecia.* Fulvipes. Duftsch. Austria.

6 Viminalis. Gyllenhal. Suecia.* Decempunctata.Fabr. Gall.bor.

Baaderi. Fabr. Germania.

Hamorrhoidalis. Fab. Austria.

Tibialis. Duftschmid. id.

Affinis. Gyllenhal. Germania.

7 Pallida. Fabr. Italia.

BROMIUS. Chevrolat. Dej. Cat. Redt.

Chrysomela, Linné. Eumolpus. Fabr.

1 Obscurus. Linné.

Gallia.*

2 Vitis. Fabr.
CHRYSOCHUS. (Chevrol.) Dej.Cat.L.Red.

EumoLpus. Fabr.

1 Asiaticus. Fabr. Russ. merid.

2 Pretiosus. Fabr. P.*

Dalmatinus. Villa. Dalmatia.

Exquisitus. Eschsch. id.

PSEUDOCOLASPIS. de Castelnau.

1 Setosa. Lucas. Algiria.

PACHNEPHORUS. Chevrolat. Dej. Cat. Redtenb.

EumoLpus. Fabr.

1 Cylindricus. (Hoffmsg.)

Lucas. Gall. merid.*

2 Villosus. Duftschmidt. Austria.*

3 Arenarius. Fabricius. id.*

$\{$ Var. Pusillus. Besser. Volhynia.

4 Troglodytes. Dej. Cat. Gall. merid.

5 Oblongulus. Dej. Cat. Corsica.

6 Tesselatus. Duftsch. Austria.

7 (Lepidopterus. (Ziegl.) id.

$\{$ Var. Sabulosus. Gebl. Sibiria.

\section{CLYTHRA. Laicharting:}

Chrysomela. Linné. Pallas. Labidostomis, Lachnaia, Coptocephala, Cheilotoma et Craniris. (Chevrol.) Dej. Cat. L. Redtenb. Lacordaire. Smaragdina. (Chevrol.) Lacordaire. Macrolenes. (Chevrol.) Lacord. Galyptorhina, Titubosa, Barathrea, GynandrophTHALMA, Lacordaire.

(Labidostomis et Lachnaia. Dej. Cat.)

1 Taxicornis. Fab. Payk. Gall. merid.* Similis. Schneider. Germania. Tridentata? Petagna. Sicilia.

2 Rubripennis. Lucas. Algiria.*

3 Tibialis, Lacordaire. Gall, merid. ${ }^{*}$ $\{$ Gallica. (Chevrolat.) Pyr. orient.

4 Meridionalis, Lacord. Gall. merid. Scapularis, Dej. Cat. id. Lusitanica. (Germar.) Lusitania.

5 Hybrida. Dej.Cat. Luc, Algiria.*

6 Propinqua. Falderm. Turcia.

7 Rufa. (Friw.) Lacord, Constantinp. 
8 Stevenii. (Friw.) Germar. Lacordaire. Turcia.

9 Sulcicollis. Lacord. Constantinp.

10) Pallidipennis. Gebler. Ledebours. Gall. merid. ${ }^{*}$ Longipennis. Dhl. Dej.

$$
\text { Cat. Tyrulis. }
$$

11 (Pilicollis. Dahl. Cat. $\left\{\begin{array}{c}\text { L. Redtenb. Hungaria. } \\ \text { Longipennis. Dej. Cat. Grocia. }\end{array}\right.$

12 Distinguenda. Rosenh. Tyrolis.

13 Cyanicornis. (Dahl.) Germar. Austria. Fulvipennis. (Besser.) Dej. Cat. Pedemont.

4 Tridentata. Linné. Gallia.* Viridicollis. Dej. Cat. Germania. Cyanicollis.Dahl.Dej.

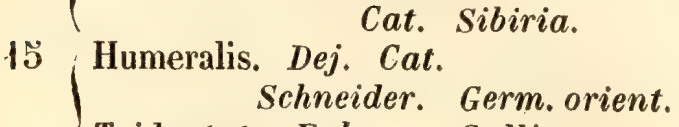
Tridentata. Fabr. Gallia. Impressihumera. 16 (Lucida. Germar. $\quad$ Gall. merid.* Notata. Gebler. Sibiria. Fulgida. (Dahl.) Italia. Axillaris. (Dahl.) id. Albipennis. (Mannerh.) Sibiria. Bisignata. Falderm. id. Axillaris. Dahl. Dej. Cat. Lacordaire. Austria. Laticollis, Dahl. Dej. Cat. id. Longimana, Lin. Fabr. Gallia.* Tridentata. Panzer. Germania. Pallida. Fourcroy. $P$. Dalmatina. Dej. Cat. Dalmatia.

19 Uralensis. Lacordaire. Caucasus.

20 Hispanica. Dej. Cat. Lacordaire. Hispania.

21 (Centromaculata.(Dahl.) $\left\{\begin{array}{l}\text { Géné. Sardinia. } \\ \text { Var. Syriaca.Dej. Cat. Corsica. }\end{array}\right.$

22 Ghilianii. Lacordaire. Hispania.

23 Guerinii.Bassi.Lacord.Sicilia.* \{ Terminata. Dej. Cat. Algiria.

24 Hordei. Fabr, Linné. Hispania.*

(Calyptonhina. Lacordaire. LabidostoMIs. Dej. Cat.)

25 Foncipifera, Dej. Cat. Lucas, Algiria. *
26 Chloris. (Dahl.) Dej.

Cat. Lacordaire. Hungaria.

(Macrolenes. Dej. Cat. Lacordaire.)

27

$\left\{\begin{array}{c}\text { Ruticollis. Fabr. Pedemont. } \\ \text { Octopunctata.Schnei- } \\ \text { der. Panzer. Tyrolis. } \\ \text { Var. Bimaculata.Rossi.Italia. } \\ \text { Dentipes. Olivier. Algiria. } \\ \text { Crassimana.Four- } \\ \text { croy. Barbaria. }\end{array}\right.$

(Titubea. Lacordaire. Macholenes. Dej. Cat.)

28 Illigeri. Lacordaire. Algiria.*

29 Sexmaculata.Fab. Lin. Russ, merid.*

30 Macropus. Illiger. Dej.

Cat. Gall, merid.

Var. Grandipes. Forsb. Hispania.

31 Parviceps. Lacordaire. Algiria.*

32 Sexpunctata. Oliv. Dej.

Cat, Gall. merid. ${ }^{*}$

Biguttata. Olivier. Italia.

Var. Novempunctata.

I. Dufour. Hispania.

33 Dispar. Dej. Cat. Luc. id.*

34 Octosignata. Fabr. Algiria.*

35 Laticollis, Olivier. id.

36 (Paykulii. Lacordaire. id.

Octopunctata. (Payk.

Klug.) Barbaria.

37

Octopunctata.Fab.Lin.Algiria. *

38 Salicariæ. Ménétriés. Russ. merid.

(Barathra, Lacordaire, Lachnaia, Dej. Cat.)

39 Cerealis, Olivier. Algiria.

40 Straminipennis. Lucas, id.

(Lachnaia. Lacord. Lachinaia et CampTOLENES. Dej. Cat.)

41 Paradoxa. Olivier.Dej. Cat. Sicilia.*

42 Vicina. Dej. Cat. Lac. Hisp. merid.*

43 (Palmata. Lacordaire. Gall. merid.* Rufpennis. Dej. Cat. id. Pubescens. L. Dufour. Hispania.

44 Macrodactyla. Dej. Cat. Lacordaire. id. 
45 Longipes. Fabr. Gallia.*

Sexpunctata. Scopoli. Carniolia.

Var. Brachialis. (Dhl.)

Küster. Germania.

46 Tripunctata. Fabr. Dej. Cat. Gall, merid.*

47 Tristigma.(Hoffmsgg.) Dej. Cat. Lacord. id.*

48 Variolosa. Linné. Fabr. Algiria.* Lentisci. Fabr. Hisp. merid.

49 Cylindrica. Dej. Cat. Lacordaire. Gall. merid.*

50 Puncticollis. Chevrol. Hispania. ${ }^{*}$

\section{(Chythra. Laichait.)}

51 Nigrocincta. Dej. Cat.

$\left\{\begin{array}{c}\text { Lacordaire. Constantinp. } \\ \text { Unifasciata. Ménétr. }\end{array}\right.$

Ovata. Klug.

L. Redienb. Oriente.

Algirica. (Gaubil.) Algiria.*

52 Quadripunctata. Linné.

Fabr. Gallia.*

Secunda. Schaffer. Germania.

Var. Quadrisignata.

Märkel. Schmidt. Gallia.

54 Læviuscula. Ratzeb. id.*

Quadripunctata. Lai-

charting. Tyrolis.

Prima. Schaffer. Germania.

らૅ, Appendicina. (Gysse-

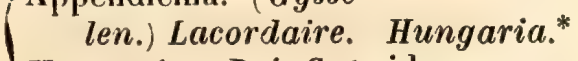

Hungarica. Dej. Cat. id.

Quadripunctata. var.

Schmidt. id.

56 Valerianæ. Ménétriés. Caucasus.

Yar. Stevenii.Dej.Cat. Constantinp.

- Tetrastigma.Schm. Hungaria.

57 (Novempunctata. Oliv. Gall. merid.

Elegans.Faldermann. Constantinp.

Aleppensis. L. Redtb. Styria.

58 Atraphaxidis. Fabr. Linné. Pall. Gall. merid. * 77

59 Maculifrons, Zoubkoff. Russ. merid.

(Gynandrophthalma, Lacordaire. Gyaniris et Smaragdina. Dej. Cat.)

60 Hypocrita.Stèven. Dej. Cat. Lacordaire. Russ, merid. Psittacina. Germar. id.

Var. llicis. (Parreyss. Germar.) id.
61 Bioculata Lacordaire, Gracia.

62 (Concolor. Fabr. Dej.

\{Enea. Germar. Cat. Gall. merid.

63 Amabilis, Lacordaire. Lusitania.

Terminalis. (Klug.) id.

64 Gratiosa. Lucas. Algiria.*

65 Limbata. Stévien, Dej.

Cat. Russ. merid.

Var. Dorsalis. Olivier. id.

66 Menetriesii. Falderm. id.

67 Ferulæ. Géné, Lacord. Sardinia.*

68 (Nigritarsis. (Chevrol.)

$\int_{\text {Lacordaire. }} \boldsymbol{P}^{*}$.

Virens. Rambur. Hisp. merid.

Var. Fuscitarsis. Dej.

Cat. Gall. merid.

69 Rufimana. Dej. Cat. Lacordaire. Algiria.

70 Thoracica.Lacordaire, Lusitania.

71 Cyanea. Fabr. P.*

IPersicaria. Schrank. Germania.

Saphirina. Linné. id.

Ruficollis. Herbst. Austria.

Salicina. Scopoli, Carniolia.

72 (Flavicollis. (Meg.) Dej.

Cat. Charpentier. Styria.*

(Var.Diversipes. Letzn. Hungaria.

73 Affinis. Illiger. Gallia.*

Musciformis. Schnei-

der. Gaze, Germania.

Collaris. Schrank. Austria.

74 Xanthaspis. Germar. Germania.

Collaris. Schneider.

Dej. Cat. Constantinp.

75 Aurita. Linné. Fabr. Gallia.*

Bicolor. Grimmer, Germania.

(Cheilotoma. (Chevrolat.) L. Redtenb.)

76 (Erythrostoma.Dej.Cat.

Faldermann. Russ, merid.

(Bucephala. Falderm. Persica occid.

Bucephala. Fabr. Dej.

Cat. Gall. merid.*

Musciformis. Linné. Austria.

Muscoides. Fourcroy. $P$.

(Coptocephala. (Chevrol.) L. Redtb.)

78 Melanocephala. Oliv. Algiria.*

Sexnotata.Fab.Dej.Ct. id.

Bistrinotata. Schönh. Barbaria.

Trinotata. Fæsberg. id. 
79 Cyanocephala. (Dahl.)

Dej. Cat. Lacord. Sardinia.*

80 Scopolina.Linné.Fabr. Gallia.*

Var. Tetradyma. Dej.

Cat. Kuister. Germania.

Rubicunda? Laichart. Tyrolis.

Rubra? Olivier. Gall. merid.

Melanocephala.Schall, Germania.

Bimaculata? Fabr.

Octopunctata. var,

Schneider. Austria.

Var. Plagiocephala.

Panzer. Germania.

Fabr. Oliv, Hispania.

Cyanocephala? Uliv. Gallia.

81 Quadrimaculata.Linné. Gall. merid. Scopolina. Rossi. Pedemont.

Unifasciata. Scopoli. Carniolia.

Femoralis. Küster. Austria.

82 Floralis. Olivier. Dej.

Cat. Hispania.*

$83\left\{\begin{array}{l}\text { Gebleri. Dej. Cat. Geb- } \\ \text { ler. Lacord. Russ. merid. }\end{array}\right.$

Quadrimaculata.Fab. Sibiria.

84 (Chalybea. Germar. Austria.

Corulea. Dej. Cat. id.

Hypocrita. Schönherr. id.

85 Unicolor, Lucas. Sicilia*

86 (Apicalis. Dej. Cat. La-
$\left\{\begin{array}{r}\text { cordaire. } \\ \text { Godetii Dej Cat. }\end{array}\right.$
Russ, merid.
Godetii. Dej. Cat. id,

\section{PACHYBRACHYS. (Chevrolat.)}

L. Redtenbacher. Cryptocephalus. Fab. Panz. Oliv. Gyllenh.

1 Azureus. Suffrian. Hispania.

2 Viridissimus.(Dj.)Suff.id.*

3 Piceus. Suffrian. Volhynia.

4 Scriptus. Duftsch. Italia. ${ }^{*}$

5) Lineolatus. Suffrian. Hispania.

(i) Cinctus. Géné. Sardinia.

7 Hippophaes. (Kunze.)

Suff. Austria.

8 Scripticollis. Falderm. Caucasus.

9 (Hieroglyphicus. Fabr. Germania.*

Histrio. Fabr. Gallia.

10 Histrio. Olivier. Algiria.

Hieroglyphicus.Schnd.Germania.

T'essulatus, Olivier. Gall. merid.

11 Tauricus. (Kze.) Suff. Tauria.

12 Maculatus. (Par.) Suff. Italia.
13 Limbatus. Ménétriẻs. Turcia.

14 Fimbriolatus. Müller. Germania.

15 Fulvipes. Suffrian. Hispania.

\section{STYLOSOMUS. Suffrian.}

Pachybrachys. (Chevrol.) Dej. Cat.

1 Tamarisci. (Jeniss.)

Suff. Gall. merid.*

2 Minutissimus. (Dej.)

Suff. Gallia.*

3 Illicicola, (Kze.) Suff. Gall, merid.

PROCTOPHYSUS. (Chevrol.) Dej. Cat.

L. Redtenb, Cryptocephalus. Fab. Suff.

1 ( ${ }^{7}$ Lobatus. Fabr. Gallia. ${ }^{*}$

Notatus. Schneider. Germania.

우 Hamorrhoidalis.

Fabr. Pedemont.

2 Cyanipes. (Dej.) Suff. Alp. Pedem.

HOMALOPUS. (Cievrol.) Dej. Cat.

Criptocephalus. Suffrian.

1 f 9 Loreyi. Sol.ơ.Roug. Gallia.* Major. Comolli. Italia.

\section{CRYPTOCEPHALUS. Geoffroy.}

Fabr. Chrysomela. Linné.

1 Cinaræ. (Frv.) Suff. Hisp. merid.

2 Curvilinea. Olivier. Sardinia.*

8-Punctatus. Schönh. Sicilia.

Ornatus. Herr.-Schff. id.

Dahlii. Guérin. Algiria.

3 6-Maculatus. Olivier. Gall. merid.

4 Tristigma, Charpent. Hisp. merid.* $\{$ Grandis. Dej. Cat. Algiria.

¿) Hirticollis. (Parr.) Suff.Italia. *

6 Ilicis. Olivier. Gall. merid. \{ Siculus. Herr. Schff. Sicilia.

7 Bæticus. Suffrian. Hisp. merid.

8 (Rugicollis. Olivier. Gall. merid.

Humeralis, Fabr. Hispania.

6-Notatus. Illiger. Pedemont.

9 Virgatus. Géné. Suff. Gall. merid.

6-Notatus. Fabr. Dalmatia.

Humeralis. Olivier. Sardinia.

10 Lætus. Fabr. Hungaria.

Mixtus. Schneider. Pomerania. 
11 Imperialis. Fabr. Pz, P.*

12 Pexicollis. Suffrian. Gall. merid.

13 Coronatus. (Kze.) Suff. Russ. merid.

14 Albolineatus. Suffrian. Tyrolis.

15 Bimaculatus. Fabr. Gall. merid. ${ }^{*}$

\{ Blockii. Rossi.

16 Informis. Suffrian.

Italia.

Tyrolis.

Lombardia.

Italia.

Tricolor. Rossi.

Gallia.*

18 Cordiger. Linné.

19 Distinguendus. Schnd. Pomerania.

Variegatus. Panzer. Germania.

20 Variegatus. Fabr. Lombardia.*

\{ Axillaris. Charpent. Pedemont.

21 Variabilis. Schneider. Germania. * Cordiger. Olivier. Gallia.

6-Punctatus.Herr.-

Schff. Hungaria.

22 6-Punctatus. Linné. F. Gallia. *

23 Interruptus. (Meg.) Suff. Germania.

6-Punctatus. Schneid. Austria.

Variabilis. Herr.-Schff. Hungaria.

Cribratus. Suffrian.

Constantinp. 58

25

26

27

Lævicollis. Gebler.

Turcia.

4-Punctatus. Olivier. Pyrenais. *

Fasciatus. (Dej.) Herr.-

Schff. Gall. merid.*

28 Carinthiacus. (Dahl.)

Suff. Carinthia.

29 Lusitanicus. Suffrian. Lusitania.

30 (क Coryli. Linné. Fabr. Gallia.* or Vitis. Panzer. Germania.

Chermesinus. Olivier. Hisp. merid.

31
(Coloratus. Fabr. Austria. $^{*}$

\{14-Maculatus. Schnd. Hungaria.

32 Halophilus. Gebler.

Russ. merid.

33 Nigritarsis. Suffrian. id.

34 Flexuosus. (Parr.)

$$
\text { Suff. id. }
$$

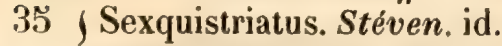
Ypsilon. Parreyss.
Finlandia.

36

Rubi. Ménétriès.

Caucasus.

37 Bœehmii. (Illig.) Germ. Hungaria.

38 Elongatus. (Ziegler.)

$$
\text { Germar. Austria. }
$$

39 Violaceus. Fabr.

Fuscipes. Olivier.

40 Virens. Suffrian. Germania.* $P$.

41

12

19

is

Concolor. Suffrian. id.*

Sericeus. Linné.

Gallia.*

45 Hypochæridis. Linné. Alsatia.* \{ Sericeus. Küster. Germania.

46 Globicollis. Suffrian. Hisp. merid. ${ }^{\star}$

47 Villosulus. (Meg.) Suff. Austria.

48 12-Punctatus. Fabr. Germania.*

49 Stramineus. Suffrian. Russ. merid.

50 Sulfureus. Olivier. Lusitania.

51 Lævigatus. Suffrian. Russ. merid.

52 Modestus. Erich. Suff. Orenburg.

53 Nitens. Lin. Gyllenh. P. Gallia.*

Flavifrons. Fabr. Germania.

Assimilis. Herbst. Hungaria.

54 Nitidulus. Gyllenh. Germania.*

\{ Nitens. Fabr. Tyrolis.

55 Marginellus. Olivier. Gall. merid.*

2-Pustulatus. var. $\beta$.

Rossi, Pedemont.

Flavipes. Fabr. P.*

Parenthesis. Sehneid. Germania.

Marginatus. Olivier. Italia.

Flavifrons. Olivier. Tyrolis.

57 4-Pustulatus. Gyllenh. Germania.

(4-Guttatus. (Koy.) Ger-

1 mar. Hungaria.

Nigribuccis. Gebler. Russ. merid.

2-Bipustulatus, Herr-

59 Creticus. Suffrian. Gracia.

\section{Schff. Hungaria.}

60 Flavoguttatus. Olivier. Austria.

Apicalis. Gebler. Russ. merid.

61 Ramburi. (Dej.) Suff. Hisp. merid.

62 Moræi. Lin. Fabr. P.*

63 Frenatus. Fabr. Helvetia.*

64 Signatus. Olivier. Gall, merid.

65 4-Signatus. (Dej.) Suff. id.*

66 10-Punctatus. Linné. Germania.*

67 Flavescens. Schneider. id.

68 Punctiger. Paykull. id.

69 Janthinus. Schüppel.

$$
\text { Suff. id. }
$$

70 Fulcratus. Germar. id.*

i1 Flavilabris. Paykull. id.*

72 (Marginatus. Fabr. id. ${ }^{*}$

Flavilabris, Rossi. Italia.

Terminatus. Germar. Germania.

73 Grohmanni. (Mus, B.)

Suff. Sicilia.

74 Biguttulus. Suffrian. Tauria.

Caucasus.
id.*

Germania.*

$P$.

$\begin{cases}\text { Salicis. Fabr. } & \text { Turcia.* } \\ \text { 3-Maculatus. Rossi, } & \text { Italia. } \\ \text { 6-Maculatus, Olivier. Gall, merid. }\end{cases}$

I Sericeus. Küster. 
2-Tripunctatus. (Crtz.)

Salicis. Oliv. Schh. id.

78 Cicatricosus. (Dej.) Lucas. Algiria.*

79 2-Punctatus, Linné. P.* Dispar,var, Gyllenh. Suecia.

80 Lineola. Fabr. Panz. Italia.* Sispar. Paykull. Suecia.

81 Anticus. Suffrian. Caucasus.

82 6-Pustulatus. Rossi. Germania.* 우 8-Punctatus. Schneid. id.

83 Gravidus. (Dej.) Suff. Gall. merid.* o 8-Guttatus, Fabr. Algiria.

84 Rossii. (Mus. B.) Suff. Gall. merid.

83 (Vittatus. Fabr. Gallia.* ơ Quadratus. Oliv. id. Suturalis. Olivier. id. o. Quadrum. Fabr. Germania.

86 Celtibericus. Suff. Hispania.

87 Tesselatus. Germar. Germania. \{ Elongatulus, Olivier, Gallia.

88 Bilineatus. Linné. id.*

89 Connexus, Illiger. Germania. Amanus. Charpent. id. Vittatus, Rossi. Italia.

90 Vittula. Suffrian. Germania.

91 Pygmæus, Fabr. $\quad \boldsymbol{P}^{*}$

92 Signaticollis. (Dahl.)

Suff. Dalmatia.*

93 Pulchellus. Suffrian, Sicilia.

94 Minutus. Fabr. $\quad \boldsymbol{P}^{*}$

Ochraceus. Stephens, Anglia.

95 Populi. (Dahl.) Suff. Germania.

96 f Pusillus. Fabr. . id. Minutus. Stephens. Anglia.

97 Gracilis. Fabr. $\boldsymbol{P}^{*}$

98 Hübneri. Fabr. Gallia. Homorrhoidalis.

Schneid. Germania.

99 Labiatus. Linné. id.*

100 Diagrammus. Suff. id.

101 Wasastjernii. Gyll. id.

102 (Geminus. (Megl.) Suff. id.*

Labiatus. Olivier. Gallia.

103 Mistacatus. (Hoffmg.) Suff. Lusitania.

104 Querceti. Erich. Suff. Germania. Labiatus, Fabr. Gallia.

105 Larvatus. (Hoffmgg.) Suff. Lusitania.

106 Scapularis, Suffrian, Sicilia.
107 Frontalis. Marsham. Germania.

Labiatus. Schneider. id.

108 Strigosus. (Ill.) Panz. id.

109 Wagneri. Kuister. Algiria.

110 Barbarus, Linné. ?

111 8-Guttatus. Linné. ?

112 Exiguus. Schneider. ?

113 Stragula. Rossi. Gall. merid.

114 Insignis. Payk. Suecia.

$\{$ Pallifrons. Gyllenh. id.

115 Nigripennis. Steph. ?

116 Punctulatus. Dej.Cat. Gall, merid.

117 Elegans. Dej. Cat. id.

118 Sinuatus. Stm. Cat. Helvetia.

119 Lateralis. Dej. Cat. Russ. merid.

120 Melanarius. (Ziegl.) Dej. Cat. Styria.

121 Signatifrons. Sturm.

Cat. Germania.

122 Furcatus. (Ziegl.) St. Cat. id.

123 Cyaneus. Dej. Cat. P.

\{ Punctipes. Dahl. Cat. Hungaria.

124 Exilis. Schüpp. St.

125

Cat. Germania.

Eneus. (Creutzer.) Charp. Austria.

DISOPUS. (Chevrol.) Dej. Cat.

L. Redtenbacher. Cryptocephalus.Suffrian. Chrysomela. Linné.

1 Pini. Linné. Gall, orient.

2 Abietis. (Kch.) Suff. Gall, merid. 799

\section{FAN. CLVPEASTRES.}

CLYPEASTER. Andersch. Dej, Cat.

Redtenbacher. Cossvphus. Gyllenhal.

1 Pusillus. Gyll.Germar.Austria.

2 Niger. Sturm, Cat. Croatia.

3 Ater.(Ziegl.) Dej. Cat. Styria.

4 Minutus. Sturm. Cat. Austria.

5 (Lividus. Dej. Cat. $\quad P^{*}$

$\{$ Pubescens. Schüppel. Illyria.

6 Bicolor. Sturm. Cat. Austria.

7 Luridus. Dej. Cat. Gall. merid. 
a) GRYPHINUS. L. Redtenbacher.

Cossyphus. Gyllenhal. Chypeaster. Dej. Cat.

1 Lateralis. (Meg.) Gyll. Austria.

2 f Piceus. (Kze.) Comolli. id. Obscurus. Dej. Cat. P.

b) CORYLOPHUS. Leach. Steph.

Dermestes, Marsh. Glypeaster. Schuck.

1 Cassidoides, Marsham. Austria. 10

\section{FAM. COCGINELLE.}

\section{CHILOCORUS, Leach.}

L. Redtenbacher, Mulsant. (Dej, St. Cat.) Coccinella. Linné.

1 Renipustulatus. Scriba.Gallia.* Similis. Rossi. Italia.

Medio-pustulata.(Coccinella.) Schrank. Austria.

Abdominalis. Westm. in Thunb. Suecia.

Cacti. Scopoli. Carniolia.

2 (Bipustulatus. Linné. Gallia.* Fasciatus. Müll.Hbst, Austria. Frontalis. Thunberg. Suecia.

\section{EXOCHOMUS. Redtenbacher.}

Mulsant. (ChIloconus. Dej. St. Cat.) CoccIN ELLA, Linné, Fabr.

1 Quadripustulatus. Lin. Gallia.* Var. Quadripustulatus. Linné. Suecia.* Lunulatus. Zschach. id.

Quadriverrucatus.

Fabr, Germania.

Cassidioides. Donov. Anglia.

Varius. Schrank. Austria.

Var. Meridionalis. (Dej. Cat.) Muls. Gall, merid.

Hamatideus, Chevrol. in litt. id.
2 (Auritus, Scriba. Testudinaris. (Coccinella.) Fourcroy. $P$. Humeralis. Townson. Hungaria. Specularis. Bonelli. Lombardia.

PLATYNASPIS. Redtenbacher.

Muls. (Scrmeus. Megerle. Dahl. Dej. Cat. Géné in litt.) Goccinelca. Fourcroy.

1 $\left\{\begin{array}{l}Q \\ Q \\ Q\end{array}\right.$

Villosa. Fourcroy. Gallia. ${ }^{*}$ Quadrimaculata.

Rossi. Italia.

Quadriguttata,Brhm. Germania.

Pubescens, Olivier. Gallia.

Quadripustulata. Kugelann. Germania.

Bisbipustulata. Illig. Payk. Austria.*

Bipustulata. Duméril. $P$.

Quadrinotata. (Meg.

Dej. Cat.) Dufour. id.

Var. Bisbipustulata.

Fabr. Redtb. Austria.

- Confluens. Gëné.

in litt. Sardinia.
Coadunata. Dej. in
Coll. id.

ANISOSTICTA. (Chevrolat.) Duponchel.

Redtenbach,Muls. Coccinelda. Linné.

1 Novemdecimpunctata.

Linné. Gallia.*

MICRASPIS. Chevrolat 1 .

Redtenb. Muls. (Dej. Cat.) CoccinelLA. Linné.

1 Duodecimpunctata.
Suturata. (Coccin.) Gallia. ${ }^{*}$
Octodecimpunctata.
16-Punctata. Fourc. P
11-Punctata. Gmelin. Gallia.
12-Punctata. Steph. Anglia.
Var.16-Punctata.Lin. Suecia.

1 Dict univ. d'hist. nat, t, IV, p. 43. 
2 Phalerata. (Dahl. Cat)

Lucas, Algir, Sicil.*

HYPERASPIS. Chevrolat ${ }^{1}$.

Redtenb. Muls. (Dej. Cat.) Coccinella. Linné. Fabr. Dej. Cat.

1 Quadrimaculata. $W$.

$\{$ Redtenbacher. Austria.

Tetraspilota. Mus. de Berlin. id

2 Hoffmanseggii. (Hell-

wig.) Mulsant. Gall. merid.* Marginella. Dej. Cat. id.

Simulata. Chevrolat. Dej. Cat. P.

3 Illecebrosa. (Chevrol.) Mulsant. Hispania.

4 Fabricii. Mulsant. Dania.

\{ Erythrocephala. Fabr. id.

5 Reppensis. Herbst. Gallia.*

o Bipustulata.Thunb. Suecia.

I Nigra. Zschach. Germania.

†Marginella.Quens. Suecia.

ơ Zanthocephala.

Quensel. id.

Margirella? Fab Dej.

Cat. Dania.
o Stigma. Olivier. Gallia. Apicalis, Dufour. Pyrenais.

6 Campestris. Herbst. Gallia. *

Frontalis. Schneider. Germania.

Lateralis. Panz. Stph. Anglia.

7 Concolor. Suffrian. Germania.

\section{NOMIUS. Mulsant.}

1 Cruentatus. Mulsant. Berolini.*

\section{SCYMNUS, Kugelann.}

Redtenb. Muls, (Dej, Cat.) Coccinella. Linné. Fabr. Panz. Tritoma. Panz. Spharidium. Herbst.

1 (Nigrinus. Kugelann. Gallia.*

Minimus? Müller. Germania.

Ater. Westman. in

Morio. Paykull. $\quad$ id.

I Dict. univ. d'hist, nat., t. IV, p. 43, et t. VI, 1). -80 .
2 Pygmæus, Fourcroy. P.* Flavipes. Panz. Fabr. Germania. Parvulus. Fabr. Redt. Dania. Sericeus. Kugelann. Germania. Collaris. Herbst. Austria.

Pubescens. Schiippel. Germania. Flavilabris. Olivier. Gallia.

3 Femoralis? (Kirby.) Gyllenh. Anglia.*

4 Redtenbacheri. Muls. Gall. orient.

Femoralis. W. Redtb, Austria.

5 Quadrilunulatus. Illig. Gallia. *

Bisbipustulatus. Pnz. Germania.*

Quadrimaculatus. $K u$ -

gelann. id.

Pulchellus, Schüppel. id.

Colon. Stephens. Anglia.

6 Biverrucatus, Panzer. Gall. orient. Bipunctatus. Kugeln. Germania.

Bipustulatus. Westm.

in Thunb. Suecia.

Affinis. Paykull. id.

Bimaculatus. Marsh. Anglia.

7 Frontalis. Fabr. Gallia.*

Quadripustulatus. Hbst.Austria.

Bisbipustulatus.Mars-

ham. Steph. Anglia.

Oblongopustulatus.P.

W. J. Muill. in Germ. Germania.

Bisbisignatus. L. Redt. Austria.

Frontalis. Suff.var. $\gamma$. Germania.

Quadriverrucatus. St.

Quadrivulneratus.

Cat, id.

(Erichson.) id.

Var. Altica. (Chrysomela.) Schrank. Austria.

- Humeralis. Panz. Germania.

- Bipunctalus. Kullberg. in Thunb. Suecia.

- Bimaculatus.Westman. in Thunb. id.

- Didymus. Herbst. Austria.

- Flavilabris. Payk. Suecia.

- Affinis. W. Redtb. Austria.

8 Marginalis. Rossi. Gallia.*

Interruptus. Fourcroy. $P$.

Bimaculatus. Herbst. Austria.

Frontalis. Panzer. Germania.

Rufipes. Fabr. id.

Morio. Fabr. id.

Var. Basalis. W. Redt. Austria

- Flavicollis, Redtb. id. 
9 Apetzii. Mulsant Gallia.* \{rontalis. Rossi. Italia.

10) Incertus? Mulsant . Gallia.

11 Ahrensii. (Küist.) Muls. Italia.* IMarginellus.vr. Rossi. id.

12 , Fasciatus. Fourcroy. . $^{*}$ Var. Pubescens. Payk. Suecia.

- Aurantiacus. Pnz. Germania.

- Minutissimus.

Schrank. Austria.

- Luridus. Dej. Cat, Gallia.

- Dorsalis Waltl. Germania.

- Quadrillum.W.

13

Redtenb. Austria.
Gassi.

Undatus. Dahl. Cat. Germania.

Variegatus. Dahl.Cat. id.

Signatus. Melsheimer.

Dej. Cat. id.

14 Capitatus. Fabr.

Auritus. Westman.

Parvulus. Illiger.

Gall. merid. ${ }^{*}$

Austria.

Fulvifrons. Stephens. Anglia.

15 (Ater. Kugelann. Gall. orient.* Minimus? Rossi. Italia.

Oblongus. Dej. Cat. P.

16 Analis. Fabr. . Gall. bor."

Ruficollis. Olivier. id.

Parvulus. Illig. Latr. var. e. $\boldsymbol{P}$.

Abdominalis. (Mus. Berl.) Austria.

17 Hæmorrhoidalis. Hbst. Gallia.

Analis. Rossi.L.Redt. Italia.

Parvulus, Illig.var. $\gamma$. Latr.var. $\boldsymbol{e}, \boldsymbol{P}$.

18 Discoideus. (Schneid.)

Suturalis. Westman. Illig. Gallia. * in Thunb. Suecia.

Limbatus.(Kirb.)Stph. Anglia.

Discipennis. St. Cat. Germania.

Var. Pusillus? Miill. id.

- Atriceps. Stephens. Anglia.

- Pini. (Byrrhus.) Marsh. id.

Rufipes. (Megerle.) Germania.

Plagiatus. Kullberg. in Thunb. id.

Pilosus, Herbst.

19 Abietis. Paykull. Austria. Gallia.*

20 Fulvicollis. (Dej. Cat.)

Mulsant. Gall. merid.
21 Minimus. Paykull. P.* Ater. Dej. Cat. $\quad$ id.

\section{RHIZOBIUS. Stephens.}

Muls. Nundina. (Dej. Cat.) L. Redtenb. Coccinella. Illig. Nitidula. Fabr.

1 Litura. Fabr. Illig. Gallia. Var. Hypomelanus.

(Dermest.) Marsh. Anglia.

- Aurora. Panzer. Germania.

- Coadunatus. Mars. Anglia.

Lividus. Olivier. $P$.

Chrysomeloides. Hbst, Austria.

Testaceus. Fabr. Dania.

Fasciatus, Fabr. id.

2 Discimacula. (Ziegl.)

Muls. Alsatia.

\section{COCGIDULA. Kugelann.}

Redtenb. Mulsant. Coccinella. Illiger. Chrisomela et Dermestes. Herbst. Nitidula. Fabr. Cacicula. Steph.

1 Rufa. Herbst. Gallia.* Pectoralis. Fabr. Illig.

Testacea. Kinmanson Payk. Germania.

Rosea. (Silpha.) Marsh. Anglia.

2 (Scutellata. Herbst. Gallia.*

Quinquepunctata.

Fabr. Herbst. Germania.

Melanocephala. Zsch, id.

Bipunctata. Gmelin. oliv. id.

Testacea. Zschach. id.

\section{HIPPODAMIA. Chevrolat 1 .}

Coccinella. Linné. L. Redtenb.

1 (Tredecimpunctata. Lin. Gallia. *

Trinacris. Fourcroy. $\boldsymbol{P}$.

Quatuordecimpuncta-

ta. Donovan. Anglia.

13-Punctata. Steph, id.

Var. 11-Maculata.

Schaffer. Germania. 
ADONIA. Mulsant.

Coccinella, Linné. Scriba.

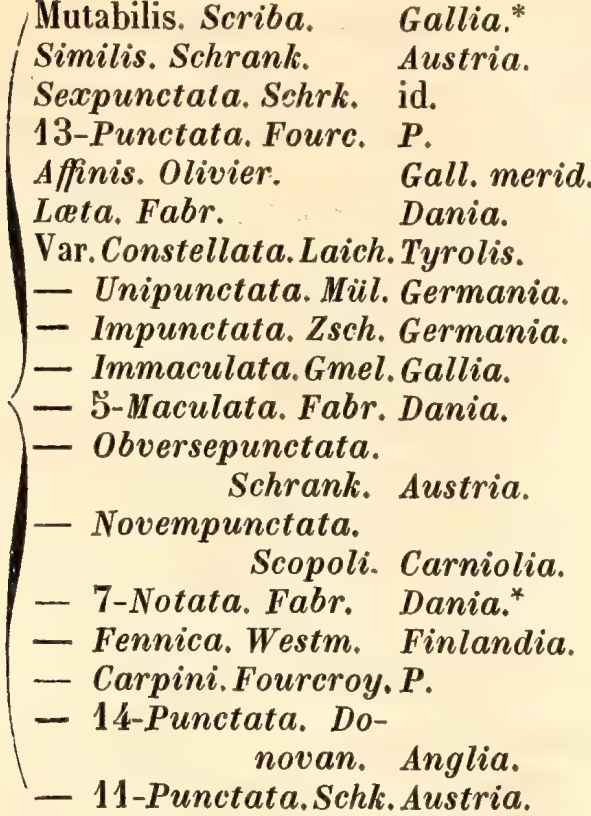

IDALIA. Mulsant.

Coccinella. de Geer. Linnë. W. L. Redt.

1 Livida. de Geer. Gallia**

Pallida.Thunberg. Suecia. Obsoleta. Schneider. Germania. Var, M-Nigrum. Illig. Redtenb. Austria. - Sexnotata.Thunb. Suecia. Bipunctata. Lin. Poda. Scopoli. Gallia. * Dispar. Schneid.Illig. Var. Quadripunctata. Donovan. Anglia.

- Unifasciata. Fabr. Gallia.

- Perforata. Marsh. Anglia.

- Annulata. Linné. Suecia.

- Hastata. Olivier. Gallia.

- Pantherina.Linné.Suecia.

- 8-Pustulata.Schff. Germania.

- 8-Guttata.Sultzer.id.

- 6-Pustulata.Serib. Carniolia.

-Varia. Schrank. Austria.

- 3-Pustulata. Zsch. Suecia.

- 7-Pustulata. Mars. Anglia.

- Cincta. Müller. Germania.

- L-Pustulata.Scop, Carniolia.
3 Bothnica. Paykull. Gallia.

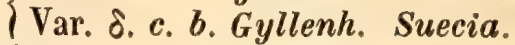

4 Alpina. Villa. W. Redt. Muls, Alp, Gall.*

Picta, Ziegl. Dej. Cat. Styria.

5 Undecimnotata. Schnd. Gall. merid.* Var.9-Punctata.Fourcroy. Oliv. $\boldsymbol{P}$.

- 11-Maculata.

Schneider. Germania.

- ơ Cardui. var. Brahm. id.

- 11-Punctata. Herrich-Schäff. id.

- Distincta. var. $W$. Redtenbacher. Austria.* Inquinata. (Luczot.) Mulsant. Gall. orient.* Hungarica. Dej.in Col.Hungaria.

\section{COCCINELLA. Linné.}

1 Undecimpunctata. Lin. Gallia.*

Bimaculata. (pour 11Maculata.) Westm. Suecia. Collaris. Paykull. id. Var. Tripunctata. Lin. id.

- 11-Punctata. Illg.

Schönh. Austria.

- 4-Maculata. Fabr. Dania.

- 9-Punctata, Linné. Fabr. Germania.

- Variegata. (Dahl. Parreyss.) id.

- Oculata. Westm.

- Nigrofasciata. in Thunb. Suecia.

- Triangularis. Rossi. Italia.

Westman. Suecia.

2 Quinquepunctata. Lin. Gallia.* $\{$ Var. Tripunctata. Ros, Italia.

3 Septempunctata, Lin. Gallia.* Var.7-Maculata.Tigny. id. - Divaricata. Oliv. id.

4 Labilis. (Erichson.) 7-Punctata? var. Mulsant. Gall, bor.* Schneider. Germania.

Var. Domiduca. Ziegl. Dej. Cat. Austria.

Magnifica. (Ziegl.) W. Redtenb. id. 
ร Variabilis, Illiger. Gallia.* 14-Punctata. Müller. Germania. 13-Maculata. Frst. Fb. id. Subpunctata. Schrk. Austria. 13-Notata. Thunb. Suecia. 12-Punctata.vr. Zsch. id. ${ }^{*}$

Var.Impunctata.Zsch. id.

- 10. Punctata. Hbst. Austria. ${ }^{*}$

- Lutea. Rossi. Italia.

- Marginepunctata.

Marsh. Anglia.

- 4-Punctata.Linné. Fabr. Suecia.* Obliterata. Linné. id. 6-Punctata. Miill. Germania. 8-Punctata. Laich. Tyrolis.

- 6-Maculata.Wstm. in Thunb. Suecia.

11-Punctata.Four.P. Varians, Olivier. id.

Ulmi. Olivier. id.

- 11-Notata. Marsh. Anglia.

- Conglomerata.Lin.Suecia.

- 10-Pustulata.d.G.id.

- Humeralis. Schall. Germania.

- Humerata. Villa. Pedemont. *

- 10-Guttata. Donv. Anglia.

- Guttata-punctata. Linné. Suecia.

- 10-Maculata.Scop. Carniolia.

- Pantherina. de G. Suecia.

- Similata.Westm. id.

- Bimaculata. Pont. Dania.

- Didyma.Müller. Germania.

- Bipustulata. Hbst. Austria.

- Austriaca. Schrk. id.

- 6-Pustulata.Schk. id.

- Marginata.Thunb. Suecia.

- Mutabilis. Gmelin. Gallia.

- Thunbergii. Gmel. id.

- Limbata. Zschach. Suecia.

- Unifasciata. Scrib. Carniolia.

- Lunigera. Brahm. Germania.

- Bipunctata, Hbst. Austria.

- Marginella. Wstm. Suecia.

- Dispar. Paykull. id.

- Varia. Schrank. Austria.

- Lununaris. Marsh. Anglia.

- Lunapustulata.

Haw. Steph. id.

- Bimaculata. Hbst. Austria.

- Biguttata. Fabr. Dania.

- Flava. Marsham. Anglia.
6 /Hieroglyphica. Linné. Gallia.* Var. Lineolata Marsh. Anglia.

- Sinuata. Marsh. id.

- Flexuosa. Fabr. Dania.

- Trilineata. Herbst. Austria.

- 4-Lineata. Gmelin. Germania.

- Sinuosa.Marsham. Anglia.

- Octopustulata.

Westman. Suecia.

- Marginemaculata.

Brahm. Germania.

- Impustulata.

Schneid. Illig. id.

- Ribis. Scriba. Carniolia.

- Curvipustulata.

- Bistriverrucata. Haw. Steph. Anglia.

Haw. Steph

Rdtb, Austria.

7 Quatuordecimpustulata.

Linné. Gallia.*

Leucocephala. Gmel. id.

14-Maculata. Poda. Gracia.

14 -Guttata. Donn-

dorff. Germania.

8 Agnata. Rosenhauer. Hungaria.

9 Hyperborea. Payk. Lapponia.

10 Trifasciata. Paykull. id.*

11 19-Notata. Dej. Cat. Russ. merid.

19-Maculata. Humm. Sibiria.

12 Lyncea. Olivier. Hispania.

13 Buphthalmus. Fischer, Bucharia.

HARMONIA. Mulsant.

Coccinella. Linné.

1 Impustulata. Linné. Gallia.* Rosea. de Geer. Suecia. 16-Punctata. Scopoli. Carniolia.

16 - Maculata. Fabr. Oliv. Dania.

Conglobata. Fourcroy. Oliv. $P$.

Var. Gemella. Herbst. Austria.

- 18-Maculata.Westman. Suecia.

- Flavipes. Westm. id.

- Vidua. Olivier. P.

2 Doublieri. Mulsant. Gall. merid.

3 Duodecimpustulata.

Fabr. Gallia** 
4 Marginepunctata.Schll.Gallia.*

Sedecimpunctata.Fab. Dania.

Var.4-Punctata.Pontoppidam. id.

- Marginella. Müll. Germania.

- Albida. Gmelin. Suecia.

- Notata Olivier. Gallia.

- 16-Punctata:

Rœnbeck. Germania.

\section{MYRRHA. Mulsant.}

Coccinelda. Linné.

1 Octodecimguttata.Lin. Gallia.*

Ornata. Herbst. Austria.

MYSIA. Mulsant.

Coccinelea. Linné.

1 Oblongogultata. Linné. Gall, orient.*

ANATIS. Mulsant.

Coccinelza. Linré.

1 Ocellata. Linné. Gall, bor.* Var. Baberi. Cederhielm. Germania.

- 15-Punctata. de Geer. Suecia.

SOSPITA. Mulsant.

Coccinelea. Linné.

1 ( Tigrina. Linné. Gallia. I Var. 20-Guttata. Lin. Suecia.

\section{CALVIA, Mulsant.}

Coccinella. Linné.

1 Quatuordecimguttata.

$$
\text { Linné. Gallia.* }
$$

2 Decemguttata. Linné. P.*

3 Septenguttala. Schall. Gall. orient. Marginata. Foureroy. $P$.

15-Guttata. Schaff. Germania. Bis-7-Guttata. Illig. Austria.* Var. 12-Gemmata. Herbst. id.
HAI,YZIA. Mulsant.

Coccinflea. Linné

1 Sedecimguttata. Linné. Gall. bor.* 16-Punctata. Shaw. Anglia.

VIBIDIA. Mulsant.

Coccinella. Linné. Poda.

1 Duodecimguttata. Pod. Gracia. Gal.* Bissexguttata. Fabr, Gallia.

PROPYLEA. Mulsant.

Coccinelea. Linné.

1 Quatuordecimpunctata.

Linné. Gallia.*

Var. Tessulata. Scop. Carniolia.

- 14-Macula. Fabr. Dania.

- Tetragonata. Laicharting. Tyrolis.

- Tesselata. Schneid. Germania.

- Conglomerata.

Paykull. Suecia.

- Conglobata. Illig. Austria.

- 12-Pustulata.Fab.Dania.

- Fimbriata. Sulz. Germania.

- Dentata. Casstr. Suecia.

- Leucocephala.Zsch. Germania.

- Bissexpustulata.

Fabr. id.

THEA. Mulsant.

Coccinelea. Linné.

1 Punctata. Linné. Algiria.

Vigintiduopunctala. Linné. Gallia.

Var. 20-Punctata. Fab. il.

- Bisdecimpunctata. Dumeril. $P$.

\section{EPILACHNA. Chevrolat.}

Redtenbacher. Coccintila. Linné. Fabr.

1 Argus. Fourcroy. P.*

11-Maculata. Fabr. Germania.

(11-Punctata. Brahm. id.

2 Chrysomelina. Fabr. Gall. merid." Var. Hieroglyphica.

Sultz. Germania.

- Elateri, Kossi. Italia.* 
LASIA, Hope. Muls.

Coccinella. Linné. Subcoccinella. Huber. Cyngetis. Steph. Epilachna. Redtenb.

1 Globosa. Schneider. Gallia.* 24-Punctata. Fabr. Dania. Gibbosa. Dumeril. P. 25-Punctata. Linné. Suecia. ơ Saponarice. Huber. Helvetia. Var. Impunctata. de

Geer. Suecia.

- Immaculata. Ross. Italia.

- Hemisphorica.

Schrank Austria.

- Livida. Herbst. id.

- Colon. Herbst. id.

- Punctum. Brahm. id.

- 4-Notata. Fabr. Dania.

- Meridionalis. (Parreyss.) Dej. Cat, Corfou.

- Palustris. (Dahl.)

Dej. Cat. Sicilia.

- 18-Punctata. Fab. Germania.

- 22-Punctata. Fab. Dania.

- 23-Punctata. Fab. id.

- 22-Punctata-obscura. de Geer. Suecia.

- Confusa. Ménétr. Dej. Cat. Russ. merid.

- Hamorrhoidalis.

Fabr. Dania.

CYNEGETIS. Chevrolat.

Redtenb. Coccinella. Linné. Lasia. Mulsant.

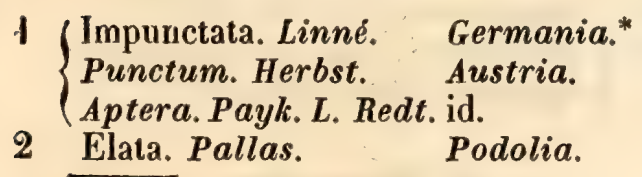

82

\section{FAH. LYCOPERDINE.}

\section{POLYMUS. Mulsant.}

1 Nigricornis. (Chevrol.)

Muls. Gallia.
ENDOMYCHUS. (Helwig.) Panzer.

Chrysomela, Linné. Galeruca. Fabr.

1 (Coccineus. Linné. de Gallia.* $\left\{\begin{array}{r}\text { Quadrimaculata. de } \\ \text { Geer. Suecia. }\end{array}\right.$

Var.Coccineus. Krynik.Russia.

2 Thoracicus. (Kollar.) Charpentier. Hunyaria.

\section{MYCETINA. Mulsant.}

Redt. Chrysomela. Schaller. Tenebrio. Panz. Opatrum. Gmelin.

1 Cruciata. Schaller, Alp. Gall.* \{Lithophilus. Gmelin. Suecia.

\section{GOLGIA. Mulsant.}

Silpha, Linné. Galeruca, Fabr. EndoMYchUS. Fabr. LycoperdiNa. Latr. LYCOPERdina. I. Redtenb.

1 $\left\{\begin{array}{l}\text { Succincta. Linné. Gall. bor. } \\ \text { Quadripustulata.Fab. }\end{array}\right.$
Panz. Payk. Germania.
Var. Fasciata. Fabr. Suecia.

\section{LYCOPERDINA. Latreille.}

W. Redt. Muls. Galeruca. Fabr. EndoмyснUS. Fabr. Muls.

1 Bovistæ. Fabr. Panz. Gall, bor, or.* $\{$ Lycoperdi. Latreille, $P$. Immaculata. Latr. id.

DAPSA. (Ziegler.) Latreille.

Redterb. Muls. Endomichus. Germar el. Ahrens.

1 (Trimaculata. (Kollar.)

$\left\{\begin{array}{l}\text { Motschulsky. Pyr. orient.* } \\ \text { Trim. }\end{array}\right.$

Trisignata. Dej. Cat. Gall. merid.

2 Denticollis. Germar. Austria.*

Trimaculata. W. Redt. id.

3 Barbara. (Dej. Cat.)

Lucas. Algiria.

4 Nigricollis, Dahl. Cat. Hungaria. 
COLOVOCERA. Motsehulsky.

1 Formicaria. Motschlsk. Algiria.

11

46. FAM, BLAPES.

\section{A. Frodites.}

ERODIUS. Fabr.

1 Bicostatus. (Dej.) Sol. Barbaria.

3 Barbarus. (Dej.) Solier, id.

4 Costatus, Klug. Sol. Algiria.*

5 Maillei. Solier. Smyrna.

6 Gibbus. Olivier. Algiria.*

7 Servillei. Solier. id.

8 Carinatus. Solier. Barbaria.

9 Brevicostatus. Solier. Gracia.

10 Wagneri. Erichson. Algiria.*

11 Bicarinatus, Erichson. id.* Dejeanii. Solier,

Barbaria.

Boyeri. Solier.

(Longus. Solier.

Orientalis.(Dej.)? Mus.

Oblongus. Solier.

Gory. Gracia.

Parvus. Solier.

id.* Algiria.* id.

Laticollis Solier.

Europæus. (Dej.) Sol. Hisp. merid.*
$\{$ Duponti. Solier.

Orientalis. (Dupont.) id.

20 Orientalis. Brullé.

Neapolitanus. Solier. id. * Latus. Solier. Barbaria.

Proximus. Solier. id.

Nitidiventris. Solier. id. ${ }^{*}$

Africanus. Solier. id. *

Subnitidus. Solier. : Algiria.

Subcostatus. Solier. Barbaria. ${ }^{\star}$ Tangerianus. Solier. id.

35 / Vicinus. Solier. $\{$ Lavigatus. Stm. Cat. id.

36 Nitidicollis. Solier. Algiria. \{ Var. Gibbus. Dej. Cat. Barbaria.

37 Subparallelus. Solier. id.

38

39

40

41

42

43

3 Gibbus? Latreille. Bilineatus. Peiroleri. id.

44 Mittrei. Solier.

Dispar. (Gaubit.)

\section{ZOPHOSIS. Latreille.}

\section{Erodius. Fabr, Oliv. Schh.}

1

\section{Barbara. Solier.} Inflata? Brullé?

Barbaria. Grocia.

2 Personata. Erichson. Algiria.*

3 Punctata.Brullé.(Dej.) Gracia,*

4 Curta. Solier.

5 Gibbicollis. Solier. id.

6 Algeriana. (Dupont.)

Solier. Algiria.

7 Maillei. Solier. Barbaria.

8 Errans. Dej. Cat. ? Sol. id.

9 Minuta. Latreille. id.

10 Suborbicularis. Solier. Hisp. merid.

11 Græca. Dej. Cat. Gracia.*

12 Ovata. Latreille. Russ. merid. Acuminata. Fischer. id.

13 Subcostata. Stm. Cat. Gracia.

14 Pusilla. Friwaldszky. Turcia.

15 Sicula. Villa. Sicilia.

16 Subglabrata. Stm. Cat. id.

17 Troglodytes. Stm. Cat. Hungaria.

\{ Pygmaea. Sturm. Cat. id.

DAILOGNATA. Stéven. Eschsch.

\section{B. Tentyrites.} Tentyria. Brullé.

Barbaria.

Smyrna.

Sicilia.

1 Hispana. Solier.

2 Variabilis. Solier.

$\{$ Lavigata? Brulté.

3 Rugata. Solier. 4 Impressicollis. Solier. id.

s. Carceli. Solier.
Hisp. merid. Graecia.* id. id.

Smyrna. 
6 Caraboides. Sol.Esch.?

(Gnathosia.Dej. Cat.) Grocia. ${ }^{*}$

7 Audouini. Solier. Constantinp.

8 Vicina. (Gnathosia.Dej. Cat.) Solier. Groecia.

9 Quadricollis. Dej. Cat. $\left\{\begin{array}{c}\text { (Gnathosia.) id. } \\ \text { Dardana. Stev. (Ten- } \\ \text { tyria.) id. }\end{array}\right.$

STENOSIS. Herbst.

1 Depressicornis. Fald.

(Gnathosia, Dej.) Russ, merid.

\section{CALYPTOPSIS. Solier.}

1 Emondi. Solier.

Gracia.

DICHOMMA. Solier.

1 Maillei, Solier.

Gracia.

HYPEROPS. Eschsch. Solier.

Hegeter. Gory. Dej. in Eschsch.

1 Tagenioides. Eschsch.

2

Solier. Algiria.

(Minuta.Tauscher. (Ten-

tyria.) Russ, merid.

Pusilla. Mannerheim, id.

Pygmaa. Falderm. id.

\section{LOPHOMA. Solier.}

Pachychila. Eschsch. Acisba, Dej. Cat.

1 Punctata. Fabr. Esch.

$\left\{\begin{array}{l}\text { Solier. Barbaria. } \\ \text { Cribripennis. Dej.Ct.? id. } \\ \text { Cribrosa. Sturm. Cat, id. }\end{array}\right.$

\section{PACHYCHILA. Eschsch. Solier.}

Acisba. Kollarir, (Dej.) Tentraia et Opatrum. Stéven. Microdera. Esch.

1 Suborata, Esch. (Kol-

2 Hispanica. Solier. Hisp. merid.

\{edinoides? (Dupont.

3 Nitens. Solier. Dej.) id. Barbaria.

4 (Pedinoides. Eschsch.

(Dej.) Sol. Barbaria
Brevis. Sturm.

5 Sulcifrons. Solier. Barbaria.

Pedinoides. (Dupont.) id.

6 Steveni. Solier. Algiria.*

.7 Salzmanni, Solier. Barbaria.

8 Kunzei. Solier. Algiria."

9 Impressifrons. Solier. id.*

\{ Glabra? Fabr.? Hbst.? id.

10 Subcylindrica. Solier. id.

11 Frioli. Solier. id."

12 Punctulata.Lucas. id.

13 Sabulosa, Lucas. id.

14. Germari. Solier. id.

15 f Tripoliana. (Dup.) Sol. id.

Bonniensis, Solier. id.

16 Brevicollis. Buguet. id.

17 Laticollis. Dej. Cat. Gracia.

Rotundicollis. (Dahl.) Sardinia.

18 Callina. Rambur. Hisp.merid.

19 Bifida. Rambur. id.

Glabra. Latreille. id.

20 Bimucronata. Rambur, id.

(Microdera. Eschsch.)

21 Sublunata. Solier. Ins.Baleares.

22 Servillei. Solier. Corsica.

23 Tarsalis. Fischer. Russ, merid.

24 Pygmæa. (Dahl. Pey. roleri.) Sardinia.

25. Gracilis. Eschsch. (Ten-

tyria.) Dej. Russ, merid.

26 Convexa. Esch. (Tentyria. Tausch. Stév.) id.

Acuminata, Erichson. Algiria.

\section{TENTYRIA. Latreille.}

Eschsch. Stéven. Heliodromus, Brullé. Akis. Fabr. Schönh. Prmelia. Oliv.

1 Mucronata, Stéven. \{ Glabra. Olivier.

2 Oblonga. Solier.

3 Nitida. Solier.

Ligurica. (Dej.) Solier, Corsica.

5 Subcostata. Solier. Barbaria.

6 Ramburi. Solier. Corsica.

7 Angusticollis. Solier. id.

8 Livicollis. Solier. id.

9 Maillei. Solier. id.
Gall. merid.

Barbaria.

Hispania.

Gracia? 
10 Interrupta. Latreille. Gall: merid.* Orbiculata. (Dej.) id.

11 Substriata.(Dej. Dup.)

$$
\text { Solier. Barbaria. }
$$

12 Marocana, (Dej.) Sol. id. $\left\{\begin{array}{l}\text { Orbiculata. (Dupont.) id } \\ \text { Bipun }\end{array}\right.$

14 Bipunctata. Solier. id.*

15 Affinis. Lucas.

16 Latreillei. Solier.

17 Sardea. (Dej.) Solier.

18 Barbara. Solier.

Sicula. (Dup.) Solier. Italia.

Grandis. (Dej.?) Sol. Sicilia.*

Tristis. Solier.

Dejeanii. Solier.

Barbaria.

Subdepressa. Solier.

Angulata. Solier.

Sicilia.

Italica. Solier.

Gracia.

id.

Salzmanni. Solier.

Italia.

Subrugosa. (Dej.) Sol. id.

Nomas. Pallas.

Var. Rugulosa. Germ. id.

- Sibirica. Dej. Cat.

\section{Solier. Sibiria.}

- Podolica. Besser. Russ. merid.

29 Cylindrica. (Dej.) Sol. Gracia.

30 Taurica. Tausch. Stév. Russ. merid.*

31 Fischeri. Solier. id.

Taurica. (Dupont.) id.

32 Bassii. Solier. Hispania.*

\{Curculionoides. (Dup.) id.

33 Peiroleri. Solier. Hisp. merid.?

34 \{ Levis. Solier. id."

\{ Platyceps? Eschseh. id.

35

36

37 Incerta. Solier. Goudotii. Solier. id.

Barbaria. Grossa. (Dej.) Solier. Gracia.* Solieri. Lucas.

\section{Algiria.*}

(Orbicollis. Solier. Rotundata. (Dup.) Brullé. id.

\section{Turcica. Sturm. Cat. Turcia.}

Excavata. (Dup.) Sol. \{ Scabriuscula. Olivier. id. YNodulosa. (Eupont.) id.

3 Scabripennis.(Dej.)Sol. Oriente.

Olivieri. Solier. id. Rugosa. Géné. Sardinia. Floresii. Géné. id. Cribrata. Géné. id.

49 Gaditana. Rambur. Hisp. merid.*
Glabrata. Illiger. Hisp. merid. ${ }^{*}$ 51 Strigosula. Rambur. id.

52 Lusitanica. Dej. Cat. " Lusitania.*

53 Dorsosulcata. (Chev.) Algiria.*

54 Emarginata. Rambur. Hisp. merid.* 55 Mauritanica. Dej. Cat. Barbaria.

56

57

58

Rugosostriata. Ramb. Hisp. merid. Arenaria. Rambur. id.

Grandicollis. Dej. Cat. Sicilia.

59 Corsica. Dej. Cat. Corsica.

60 Substriata. Dej. Cat. Lusitania.*

61 Frigida. Rambur. Hisp. merid.

62 Levigata, Rambur. id.

63 Exculpta. Buquet. Algiria.

64 Deserta. Tauscher. Russ, merid.

Dorsalis. Fischer. id.

65 Campestris. Stéven. id.

f Var. Convexa. Fald. Turcomania.

66 Inæqualis. Dej. Cat. Barbaria.

67 Tessulata. Tauscher. Russ. merid.

68 Besseri. Krynicky. id.

69 Kindermanni. Fischer, id.

70 Maura, Erichson. Algiria.

\section{HEGETER. Latreille.}

Eschsch. BuAps. Oliv.

1 Anıaroides. (Dup. Dej.)

Sol. Hispania.

\section{ANATOLICA. Eschsch. Sol.}

\section{Tentyria, Opatrum. Stéven.}

1 Lata. Eschsch. Stév. Russ, merid.

2 Tristis. Zoubk. (Dej.) id.

3 Eremita. Stéven. Russia.

4 Impressa.Esch.Tausch.id.

5 Postica. Mannerh. Russ, merid.

6 Aucta. Faldermann. id.

7 Macrocephala. Tausch. id.*

8 Subquadrata. Tausch. id. $\begin{cases}\text { Scutellata. Fischer. } & \text { Bucharia. } \\ \text { Angustata. Gebler. } & \text { Sibiria. }\end{cases}$

\section{RHOSTAX. Karelini.}

Anatolica. (Ménétriés.)

1 Menetriesii. Fischer. Russ. merid. $\{$ Elegans. Ménétriẻs. id. 


\section{Pimelites.}

MEGAGENIUS. Solier.

1 Friolii. Solier.

Algiria.*

ADESMIA. (Fisch. Dej.) Solier.

Pimelia, Fab. Oliv. Sch. Trachyderma. Fabr. Oliv.

$\begin{array}{lll}1 & \begin{array}{l}\text { Microcephala. Solier. Algiria.* } \\ \text { Rotundipennis. Dej. }\end{array} \\ 2 & \text { Doüei. Lucas. } & \text { Cat. id. } \\ 3 & \text { Solieri. Lucas. } & \text { id. } \\ 4 & \text { Biskrensis. Lucas. } & \text { id. } \\ 5 & \text { Faremonti. Lucas. } & \text { id. } \\ 6 & \text { Affinis. (Dej. Dup.) }\end{array}$

Solier. Barbaria.

\section{PLATYOPE. Fischer.}

Pimelia, Fabr, Oliv, Latr. Akis, Fabr.

1 (Lineata. Fabr. Oliv. Russ. merid.* Var. Leucographa.

Schönh. id.

\section{LASIOSTOLA. (Dej.) Solier.}

Pimelia. Schh. Tenebrio. Linné. Pallas.

1 (Pubescens. Pallas. Russ. merid. \{ Hirta. Fischer. (Dej.) id.

\section{TRACHYDERMA. Latreille.}

Pimela. Fabr, Oliv. Sch. Thryptera. Solier.

1 Hispida. Fabr. Latr. $\{$ Sch. Barbaria.* Var. Latreillei. Solier. Senegal. Elongata. Leach. Agypt. 2 Angustata. Solier. Algiria. 3 Genei. Solier. Barbaria.

\section{(Thryptena. Solier.)}

4 Varvasi. Solier.

ॐ Villosa. (Dej.) Géné. Solier. Barbaria.
PTEROCOMA. Solier.

Pimela. Fischer. Gebler.

1 (Sarpæ. (Dej.) Fischer,

Solier. Russ, merid.

ơ Gracilicornis. Sol. id.

Costata. Faldermann. id.

\section{PACHYSCELIS. Solier.}

Pimela. Latreille. Brulle.

1 Granulosa, Latreille. Gracia.*

2 Crinita. (Dup. Gory.)

Latreille. "Oriente.

4 Quadricollis. (Dej.)

Brullé. Sol. Grœcia.*

5 Obscura. Solier. id.

6 Porphyrea. (Dup.) Sol. id.

7 Granulifera. Stm. Cat. id.

8 Brevis. Sturm. Cat. id.

PIMELIA. Fabr. Oliv, Latr. Schönh.

1 (Suturalis. Fisch. (Podhomala. Solier.) Russ, merid. Torulosa. Zoubkoff. id.

2 Angulosa. Solier. Algiria.

3 Sericea. Olivier. Gracia.

Var. Latreillei. Solier. Algiria.

- Denticulata. (Dej.

Dup.) Solier. Oriente.

4 Granulata. (Dej. Dup.) Solier. Algiria.

Algerica. Solier. id.

כ Obsoleta. (Dej. Dup.)

Solier. Barbaria.

Grossa. (Desmarest. 6 (Interstitialis. Maille.) id. Grossa. (Mus. Turin.)
Fabr. id.

7 Inflata. Herbst. Sicilia.* Barbara. (Dej.) Solier. Algiria. Sicula. (Dej.) Sicilia. ) Var. Vestita. Dej. Algiria. - Grossa. Fabr.? Barbaria. - Aspera. (Ziegler.) Sardinia.

8 Latipes. Solier. Gracia.

9 Asperata. (Dej.) Sol. Oriente. 
10 Balearica. (Dej. Dup.)

Solier. Ins. Baleares.

11 Servillei. Solier. Algiria.

\section{2}

13

14

15

16

Subquadrata. Solier. id.*

Arenacea. Solier. id.

Depressa. Solier. id.*

Cribripennis. (Dup.) Solier. id.*

(Neglecta. Fischer. Mé-

nẻtriés. Russ.merid.

Schönherri. (Dej.) Sol. id.

17 Cephalotes. Pallas. iid.

18 Oxysterna. Solier. Barbaria.

19 Simplex. Solier. (Melanostola. Dej. Cat.) Algiria.*

20 Comata. (Dej.) Solier. Barbaria.

21 Variolosa. Solier. Hisp. merid.

22 Senegalensis. Olivier.

Schönh. Barbaria.

23 Claudia. Buquet. Rev.

24 (Dejeanii. Solier.

Interstitialis. Dej.Cat.Barbaria.

25 Mittrei. Solier. Gracia.

26 Radula. (Dej. Dup.) Solier. Barbaria.*

27 (Maura. (Dej. Dup.) Sol. Algiria.* Alutacea. Sturm. Cat. Hisp. merid.

Tuberculata. Panzer. Gory. id

28 Atlantis. Solier. Barbaria.

29 Mauritanica. (Dej. Dup.) Solier. id.*

30 f Ryssos. Hbst.? Solier. id.* Rugosa. Olivier. id.

31 Salebrosa. Solier. id.*

32 (Boyeri. Solier. Algiria.*

Var. Rugifrons. Sol. id.

Nervosa. (Dupont.) id.

33 Duponti. Solier. id.

34 Granifera. Solier. id.

3. Punctata. (Dej. Dup.) Solier. Hispania.

36 Rotundata. Solier. Hisp, merid.*

37 Hispanica. Solier. id.*

38 Cribra. (Dup.) Solier. Ins. Balear.*

39 Ruida. (Rambur.) Sol. Hisp. merid. *

40 (Sublævigata. Solier. Sicilia. \{ Aspera. (Dahl.) id.

41 Rugulosa. (Megerle.) Germar. id.

42 Bifurcata. (Cristofori.) Solier. id.
43 Payraudii. Latreille. Corsica.

Y Var. Rugatula. Sol. Sardinia.

44 Subscabra. (Dej.) Sol. Sicilia.*

45 Undulata. Solier. Sardinia.

46 Goryi. Solier. id.

Aspera. (Gory.) id.

47 Angusticollis. Solier. Corsica?

48 (Sardea.Mouxi-Deloche.Sardinia.*

Rugosa.(Dahl.) id.

Corrugata. (Ullrich.) Sicilia.

(Var. Corsica. Solier. Corsica.

49 Incerta. Solier. Hispania.

Muricata. Fab.? (Dup.

Gory.) Lusitania.

50 Hesperica. (Dej. Dup.

$\{$ Gory.) Solier. Hispania.

Var. Lineata. Solier. id.

- Gadium. Solier. id.

51 (Bætica. (Dej.) Solier, Hisp, merid.*

Var. Distincta. Solier, id.

52 Nitida. (Gaubil.) Corsica.

53 Brevicollis. (Dej.) Sol. Hisp. merid.

54 (Bipunctata. Fabr. Gall. merid.

Muricata. Olivier. id.

55 f Polita. (Dej.) Solier. Gracia.

I Hydropica. (Dupont.) id.

56 Mongeneti. (Dup.) Sol. id.

Var. Phymatoptera.

Solier. id.

57 Subglobosa, Lin. Sch. Russ, merid.

58 Verruculifera. Solier. Gracia.

\{ Var. Diffusa. Solier. id.

59 Græca. Stéven. Solier, id.

60 Asperata. Solier. id.*

61 (Exanthematica. (Dej.

Gory.) Solier. id.

Graca. Brullé. id.

62 Sericella. Latreille. id. ${ }^{*}$

Var. Calculosa. Sol. id.

( - Trachyderma. Sol. id.

63 Phymatodes. Solier. id.

64 Scabrosa. (Dej. Dup.)

Solier. Hisp. merid.*

65 Valida. Erichson. Algiria.

66 Fornicata, Herbst. Barbaria.

\{ Obesa. (Dej. Latr.)

Solier. Algiria.

67 Capillata. Solier. Barbaria.

68 Napolitana. Dej. Cat. Sicilia.*

69 Verrucosa. Hêrbst. Smyrna.

70 Callosa. Sturm. Cat. Gracia.

71 Famosa. Sturm. Cat. id.

72 Alternans. Sturm. Cat. Constantinp. 
D. Asidites.

ASIDA. Latreille.

Peatymotus. Fabr. Sch. Oliv. Pimelia. Oliv.

1 Morbillosa. Fabr. Grisea. Latr. Solier. id.

Var. Glabricostata. Solier. id.

- Lutosa. Stéven. Russ. merid.

2 Vicina. Solier. Gallia.

3 Pyrenæa. Dej. Cat. Pyr. orient.*

4 Helvetica. Solier. Gall. merid.

5 Reticulata. Solier. Hispania.

6 Dejeanii. Solier. Gall. merid.*

Sabulosa. Dej. Cat id . 48

7 Rugosa, Solier. Algiria. ${ }^{*}$

8 Ruficornis. Solier. Barbaria.

9 Grisea. Fabr. Oliv. Germania.

Variolosa. Panzer. id.

Var. Gibbosa. (Dahl.) id.

10

Bayardi. Solier.

Goryi. Solier.

Sicilia.

id.

Longicollis. Solier. Corsica.

Carinata. Solier. id.

Inæqualis. Solier. Algiria. ${ }^{*}$

Bigorrensis. Solier. Pyrenceis.

Fascicularis. Germar. Dalmatia.

\{ Sericea. Olivier.

Jurinei. Solier.

Gall, merid ${ }^{*} 60$

Italia.

Sinuatocollis. Solier. Algiria. ${ }^{*}$

Complanata, Lucas. $\quad$ id. $^{*}$

Genei. Solier.

Sardinia.

Exasperata. Buquet. Algiria.

Corsica. Laporte. Sol. Corsica.

Ramburi. Solier.

Hisp. merid.

Puncticollis. Solier. id.

Papulosa. (Chevrolat.) Algiria. ${ }^{\star}$

Chauveneti. Solier. id.*

Attenuata.(Chevrolat.) id.*

Lapidaria. Lucas. id.*

Servillei. Solier. id. ${ }^{*}$

Affinis. Lucas. id.

(o'Silphoides. Lin. Oliv.id.*

f Granulata. Fabr,

Schönh. id.

32 Serpiginosa. Erichson. id.

33 Subcostata. Solier. Barbaria.

34 Cariosicollis. Solier. id.*

35 jLævigata. Fabr. Algiria.*

o Variolosa. Fabr. id.

q Miliaris, Erichson. id.
36 Duodecimeostata.

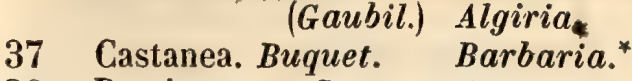

38 Brevicostata. Solier. Algiria.

39 Porcata. Solier. Hispania.

\{Costata. (Dej.)? id.

40 Asperata. Solier. Hisp.merid.

41 (Grossa. (Dahl.) Solier. Sicilia.

\{ Sicula. (Dej.) Solier. id.

42 Costulata. Solier. Lusitania.

Laevigata. Gory. id.

43 Tangeriana. Solier. Barbaria.

44 Goudotii. Solier. Hispania.

45 Ventricosa. Solier. id.

46 Parallela. Fabr. id.

47 Granifera. Solier. id. *

48 Hispanica. Solier. id.

49 Elongata. (Dej.) Solier. id.

50 (Lævis. Solier. id.

$\{$ Lavigata. (Dej.) id.

51. Depressa. Solier. Barbaria.

52 Solieri. Géné. Sardinia.

53 Planata. Dej. Cat. Ins.Baleares.

54 Elevata. Dej. Cat. id.

5y Montana. Rambur. Hisp.merid.

56 Variolosa. Dej. Cat. Lusitania.

57 Rugosula. Rambur. Hisp.merid.

58 Sulcata. Dej. Cat. id.

59 Quadricostata. Buq. Algiria.

60 Lineata. Dej.Cat. Barbaria.

61 Limbata. Dej. Cat. id.

62 Cristata. Dej. Cat. Corsica.

63 Hesperica, Dej. Cat. Hisp. merid.

64 Angustata. Dej. Cat. Corsica.

63 Tomentosa. Dej. Cat. id.

66 Perplexa. Dej. Cat. id.

67 Orientalis. Dej. Cat. Oriente.

68 Mosta. Dej. Cat. Sardinia.

Obscura. (Dahl.) id.

69 Gincta. Rambur. Hisp.merid.

70 Oblonga. Dej. Cat. id.

71 Inquinata. Rambur. id.

72 Pygmæa. Rambur. id.

73 Sordida. Dej, Cat. id.

74 Scaphidium. Hoffmsgg, id.

75 Opatroides, Dej. Cat. Ital. merid,

76 Turcica. Sturm. Cat. Turcia.

77 Acuticollis. Dej. Cat. Dalmatia.

78 Dahlii. Sturm. Cat. id.

\{ Acuticollis. (Dahl.) id.

79 Marginata. Dej. Cat. Italia.

80 Rugipennis. Sturm.

Cat. id. 
PACHYPTERUS. Solier, Dej.) Lucas.

1 Mauritanicus. Lucas. Algiria.* Pusillus. Dej. Cat. Corsica.

\section{E. Sepidites.}

\section{SEPIDIUM. Fabricius.}

1 Wagneri. Erich. Algiria.*

2 Tomentosum. Erich. id.*

3 f Aliferum. Erich. Mittrai. Solier.

4 Douei. Solier. Tricuspidatum. Oliv. id

5 Genei. Solier.

6 Uncinatum. Erich. Algiria. ${ }^{*}$

7 Fissum. Erich. id.*

8 Variegatum. Fabr. id.

9 Dufourii. Solier. Barbaria.

10 Barbarum. (Dup.) Sol. Algiria.

11 Servillei. Solier. Sicilia.

12 Maillei. Solier. Barbaria.

13 ? Siculum. Dej. Cat. Sicilia.

14 ? Hispanicum. Dej.Cat. Hispania.

15 ? Affine. Dej. Cat. id.

16 ? Albidentatum. (Chevrolat.) Algiria.*

\section{F. Akites.}

\section{ELENOPHORUS, Latreille.}

Акis, Fabr. Pimelia, Olivier.

1 Collaris. Fabr. Latr. Oliv. Gall, merid.

MORICA. Solier. (Dej. Cat.)

Akis, Fabr. Pimelia, Oliv.

1 Planata. Fabr. Inflata. Kollar. Hisp. merid.* id.

2 Octocostata. Sulier. Algiria.*

3 Obtusa. Latreille. Hispania.

AKIS. Herbst.

Pimela. Olivier.

1 Punctata. Thunberg. Gall. merid.* Reflexa. Schönh. Oliv. Fabr.? id.
2 Subterranea. (Dahl.)

Solier. Sicilia.

3 Acuminata. Fabr. Hispania.

4 Reflexa. Fabr. Algiria.*

5 Elongata. Brullé. Grocia. \{ Acuminata. (Dupont.) id.

6 Sansi. Solier. Hispania.

7 Discoidea. Quensel in Sch. id.

8 Salzei. Solier. id.

9 Algeriana. (Dupont.) Solier. Algiria.*

10 Planicollis. Solier. Sicilia.

11 Olivieri. Solier. id. Spinosa. Solier. Barbaria.

12 Nitida. Solier. id.

13 Hispanica. Solier. Hispania.

14 Spinosa, Fabr. Schh. id.* Angusticollis. (Dahl.) Sardinia. (Var. Genei. Solier. Hispania.

15 Trilineata. Herbst. Italia. Amplicollis. (Dahl.) id.

16 Bayardi. Solier. Lusitania.

17 Lusitanica. Solier. id.

18 Granulifera. Sahlberg. Hispania.

19 Barbara. (Dej.) Solier. Sardinia.*

20 Italica. (Dej.) Solier. Italia.*

21 Solieri. de Castelnau. Algiria.

22 Elegans. Charpentier. id. Carinata. (Dej.) Sol. id. Bilineata. (Sturm.) id.

23 Latreillei. Solier. id.

24 Goryi. Guérin. id.* Costata. (Dej) id.

25 Hybrida. Charpentier. Hispania.

26 Discoidea. Schönherr. id.

27 Terricola. Ménétriés. Constantinp.

\section{CYPHOGENIA. Solier.}

Akıs. Dej. Cat.

1 Aurita, Pallas.

Russ. merid.

\section{G. Adelostomites.}

ADELOSTOMA. Duponchel. Latr.

1 Sulcata. Duponchel. Hispania.* C Carinata. (Dej.) Algiria. 


\section{H. Tagenites.}

\section{MICROTELUS. Solier.}

Adelostoma. (Dupont.)

1 Asiaticus. (Dup.) Sol. Graecia.

\section{TAGENIA. Latreille.}

Stenosis, Herbst. Schönh. Akis, Fabr.

1 (Angustata, Hbst. Schh. Gall, merid.*

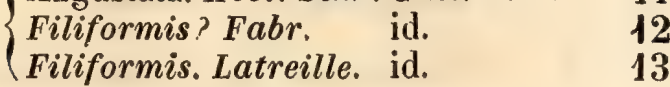

2 Intermedia. Solier. id.*

3 Sicula. Solier. Sicilia.*

4 Lævicollis. Solier. Algiria.*

5 Friolii. Solier. id.

6 Orientalis. Gory. Guér. Graecia.

7 Pilifera. Solier. id.

8 Hispanica. Solier. Hisp. merid.

9 Filiformis. Fabr.? Sol. Algiria.*

10 Webbii. Guérin. id.

11 Græca. Brullé.(Dej.) Gracia.

12 Hesperica. Ramb. Sol. Hisp. merid. Nigrita. Solier. Algiria.

13 Obliterata. Solier. id.

14 Smyrnensis. (Dup.) Solier. Smyrna.

15 Minuta. Lat. de Serres. Gall. merid.* Var. Pygmaea. Latr. id.

16 Corsica. Solier. Corsica* ${ }^{*}$ Algirica. Solier.

Pumila. Géné. Algiria.*

Punctata. Fischer. Sardinia. Russ. merid.

20 Subcostata. (Dej.) Sol. Hispania.

21 Puncticollis.Dahl. Cat. Sicilia.

22 Cylindrica. Dej. Cat. Hispania. 23 Italica. Dej. Cat. Italia.

24 Marginata. Stẻven. Russ, merid.

25 Taurica. Dej. Cat. Tauria.

\section{HYPEROPS, Eschscholtz.}

2 Tristis. Olivier. Calcaratus, Fabr.

3 Distinctus. Solier.

Gall. merid.*

Italia.

arbaria.

4 Rugulosus. Latr. (Dej.) Hispania,

5 Varvasi. Solier. Algiria.

6 Carinatus. Schmidt.

7 Porcatus. Erichson. Algiria.*

8 Sancti-Amandi.Solier. Barbaria.

9 Hespericus. (Dej.) Sol. Ins. Baleares.

10 Striatus, Fabr. Gall. merid.* Dubius. Solier. Algiria.*

12 Punctatus. Hbst. (Dej.) Gall. merid.*

13 Atratus, Fabr. id. *

$\{$ Var. Vicinus. (Dup.) id.

\section{CEPHALOSTENUS. Solier.}

Scaurus. (Dej.)

1 Dejeanii. Solier. Gracia. \{Elegans. (Dej.) Brull.? id.

\section{J. Blapsidae.}

GNAPTOR. (Megerle.) Fischer.

Tenebrio, Pallas. Blaps. Fabr.

1 Spinimanus. Pallas. Tauria.* Lavigatus. Fabr. Gracia.

Glabratus, Herbst. Hungaria. Spinipes. Fabr. id.

\section{BLAPS, Fabr, Latr.}

Tenebrio et Prmelia. Linné. Peltarium et Dica. Fischer.

1 Corrosa. Fischer. Russ. merid.

2 Superstitiosa. Erich. Algiria.*

Magica. Erichson. id.

3 Hians. Fischer. Podolia mer.

4 Magica. Erichson. Algiria.* \{ Superstitiosa. Erich. id.

5 Eymondi. Solier. id.*

6 Gages. Linné. Fabr. Gall. merid.* Gigas. Olivier. id.

Lusitanica. Herbst. Iusitania. Nitens. Castelnau. id. Piligera. Villa. Lombardia.

7 Sublevigata. Stm. Cat. Sardinia.

8 Producta.(Dej.) Fisch. Gall. merid."

9 Hispanica,Dej.Cat. Hispania.* 
10 Abbreviata. Friwalds.

Ménétriës. Constantinp.

11 Oculta. (Dahl.) Solier, Gall, merid.*

12 Stygia, Erichson. Algiria.*

13 Prodigiosa. Erichson. id.*

14 Lineata. (Dej.) Castel. id.

15 Mortisaga. Linné. Gall. merid.*

16 (latidica. (Creutzer.) $\left\{\begin{array}{l}\text { Sturm. Gallia. } \\ \text { Var. Similis. Latr. id. }\end{array}\right.$

17 Seriata. Fischer. Bucharia.

18 Depressa, Klug. Russ. merid.

19 Convexa. Fischer. Podolia.

20 Ovata. Solier. Hungaria.*

21 Australis. Dej. Cat. Italia. ${ }^{*}$

22 Pterosticha. Fischer. Podolia.

23 Marginata. Fischer. Russ. merid.

24 Brevis. (Stm.) Fischer. id.

23 Confusa. Fischer. Podulia.

26 Canaliculata. Fischer. Russ, merid.

27 Reflexicollis. (Ziegl.

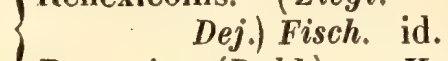

(Panonica. (Dahl.) Hungaria.

28 Longicollis. Stév. Fisch.Podolia.

Elongata. Fisch. (Dej.) Russ. merid.

29 Confluens. Fischer. Volhynia.

$\{$ Var. Glabrata. Besser. Podol.merid.

30 Cuspidata. (Megerle.)

St. Cat. Hungaria.

31 Intrusa. Fischer, Russ merid.

32 Pinguis. Dej. Cat. Grocia.

33 Brevicollis. Dej. Cat. Constantinp.

34 Parvicollis. Eschsch. Russ. merid.

35 Stricticollis. Villa. Lombardia.*

36 Microphthalma. Fisch. Pod. merid.

Rarepunctata. Fisch.

Olim. id.

37 Carbo.Stéven. Fischer. Russ. merid.

38 Turcomana. (Karelin.)

39 Fisch. Podolia.

39 Obtusa, Fabr. P.*

\{ Mortisaga. Herbst. Austria.

40 Dorsata. Fischer. Podol.merid. (Deplanata. (Besser.) id.

41 Krynicki. Fischer. Russ. merid.

42 Damascena. Fischer. Podol.merid. Punctata. (Besser.) id.

\section{(Peltarium. Fischer.)}

43 (Fischeri. (Gaubil.) Podol.merid. Ovata. Fischer. id.

44 Halophilum. Fischer, Russ, merid.
(DiLA, Fischer.)

45 Attenuata. Fischer. Russ. merid. \{ Cylindrica, Dej, Cat, id.

46 Cylindrica. Fischer. Podol.merid.

\section{TAGONA. Fischer.}

1 Acuminata. Fischer. Russ, merid.

2 Macrophthalma, Fisch, id.

\section{MISOLAMPUS, Latreille.}

1 Hoffmanseggii. Latr. Hisp. merid. Gibbula. Herbst. (Pimelia.) id.

2 Ramburii. Dej. Cat. id.

3 Goudotii. Guér, Erich, Algiria.*

\section{ACANTHOPUS. (Megerle.) Dej. Cat.}

Blaps. Germar. Helops. Panzer.

1 Caraboides. Germar. Dalmatia.* Dentipes, Panzer. Italia.

\section{PLATYSGELIS, Latreille.}

Blaps, Sturm. Pedinus. Fischer.

1 Hypolithos. Pallas. Russ. merid.

2 Melas. Fischer. Volhynia.*

3 Gages. Fischer. Russ. merid. Politus. Gebler. Sibiria.

\section{ISOCERUS, Latreille.}

Tenebrio. Linné. Blaps. Fabr. Helops. Oliv.

$1\left\{\begin{array}{l}\text { Ferrugineus. Fabr. Algiria.* } \\ \text { Purpurascens, Herbst. } \\ \text { (Tenebrio.) id. }\end{array}\right.$

\section{LANA. (Meg.) Redtenbacher.}

Küster. Helops, Fabr. Scaurus. Duftsch.

1 Pimelia. Fabr. Viennensis. Sturm. id.

2 Pubella. (Ziegl.) St.

Cat. Russ, merid.

3 Villosa. Sturm, Cat. Constantinp.
Austria * 
PEDINUS. Latreille.

Tenebrio. Linné. Blaps. Fabr.

1 Helopioides. Germar. Dalmatia.* Laticollis, (Ziegler.) id.

2 Femoralis. Fabr.

I Dermestoides, Fabr, Germania.

3 Meridianus, Dej. Cat, Gall, merid. ${ }^{*}$

4 Gracilis. (Ziegler.) Dalmatia.

5 f Tauricus. Dej. Cat.

Femoralis. Stéven.

6 Curvipes. Dej. Cat.

7 Cylindricus. (Parr.)

8 Oblongus. Schmidt et

9 Sericeicollis. Dahl. Helfer. Smyrna.

Cat, Italia.

10 Quadratus. Dej. Cat. Corfou.* Coarctatus. (Parr.) id.

11 Costatus. Chevrolat. Algiria.*

12 Punctatostriatus. Ull. Sicilia.*

13 Obesus. (Chevrolat.) Algiria.*

14 Ovatus. Sturm. Cat. Gracia.

15. Gibbosus. Dej. Cat. id.*

525

\section{FAM, OPATRI.}

DENDARUS. Latreille.

Heliopates. Dej. Cat. Redtenbacher. Opatrum. Fabr.

1 Gibbus. Fabr.

2 Picipes. Stéven.

Glaber. Ménétr. (Crypticus.) id

Fuscus. Faldermann.

(Pedinus.) Persia occ.

3 Impressicollis. (Gaub.) Algiria. ${ }^{*}$

4 Quadricollis. Solier? Gall. merid.*

5 Elongatus. Solier. Hisp, orient.

6 Subsulcatus. Dej. Cat. Hisp. merid.

7 Punctatissimus. (Che-

$$
\text { vrolat.) Algiria.* }
$$

8 Obscurus. (Gaubil.) id. ${ }^{*}$

9 Milleporus. (Chevrol.) Pyrenais.

10 Amænus. (Gaubil.) Algiria.*

11 Subvariolosus, Lucas, id.

12 Rotundicollis, Lucas, id.
13 Barbarus, Dej. Cat.?

Lucas, id.

14 Interstitialis.(Chevrol.)id.

15 Nivalis, Rambur. Hisp. merid.

16 Iners. Ménétriés. Russ. merid.

17 Ambiguus. Dej. Cat. Gracia.

18 Lusitanicus. Herbst.

(Tenebrio.) Lusitania.

19 Hispanicus. Dej. Cat. Hispania.

20 Montivagus. Rambur, id.

21 Pedatus. Hoffmansgg, Lusitania.

22 Hybridus. Latreille. Gall. merid.*

Variolosus, Olivier. Algiria,

PANDARUS. (Meg, Dej.) Küster.

Blaps, Fabr. Dendarus. St, Cat.

1 Gribratus. Klug. Constantinp,

2 Coriaceus. Dej. Cat. Barbaria.

3 Emarginatus. Fabr. Gall, merid.*

4 Tristis. Rossi. Italia.

5 . Dalmatinus. Dej. Cat. Dalmatia.*

Emarginatus. Germ. id.

(Var. Tristis.'Schönh. Italia.

6 Costatus. (Chevrolat.) Algiria.*

7 Lugens. Dahl. Cat. Ital, merid.

8 Crenatus. Sturm. Cat. Constantinp.

9 Rotundicollis.Dej.Cat.id.

10 Carbonarius. Dej. Cat. Corfou.

11 Græcus. Dej. Cat. Gracia.*

12 Stygius. Schmidt et Helfer. Smyrna.

13 Mœsiacus, Friwaldszk. Turcia.

14 Gravidus. Dej. Cat. Oriente.

15 Orientalis. Dej. Cat. id.*

16 Dardanus. Stéven. Hellespont.*

Foveolatus. Fischer. Russ, merid.

Crenulatus? Ménétr. id.

Cribratus. Falderm. Persia occid.

Punctulatus. Baber. Turcia.

17 Glabratus. Waltl. Gracia.

18 Angustatus. Stm. Cat. id.

19 Punctulatus. Friw. Constantinp.

20 Algiricus. (Gaubil.) Algiria.*

21 Opacus, Sturm. Cat. Hungaria.

\{ Glabratus.(Friwalds.) id.

22 Ovatus. Sturm. Cat. Groecia.

23 (Punctatus. Stéven. Russ, merid.

Laticollis. Sturm. : id.

Var. Odessanus. Stév. id.

24 Porcatus. Dej. Cat. : Corsica.*

25 Corcyricus. (Parreyss.) Corfou. 
OPATRINUS. Dej. Cat.

Opatrum. Fabr.

1 Oblongus. Stéven. Russ. merid.

2 Carinatus. Sturm. Cat. Barbaria.

PHILAX. (Meg. Dej.) Brullé.

Opatrum. Auct.

\section{Striatus. Latreille. id. \\ Crenatus? Schönherr. id.}

Laticollis, Rambur. Hisp. merid. Punctulatus. Dej. Cat. Gracia.* Nivalis. Géné. Italia.

Carbonarius. Stm. Cat. Sicilia. Maurus. Dej. Cat. Barbaria.

Striatus. Solier. (Dej.

$$
\text { Cat.) Hisp. merid. }
$$
$\left\{\begin{array}{l}\text { Crenatus. Dej. Cat. } \quad \text { Gall. merid.* } \\ \text { Gibbum.Bonelli. (Opa- }\end{array}\right.$

Miser. Rambur. trum.) Pedemont.

Torpidus. Buquet. Algiria.

Gnaphosus. Buquet. id.*

Costatipennis. Lucas. id.*

Variolosus. Lucas. id.*

Moreletti. Lucas. id.

Agricola. Dej. Cat, Hispania.

Barbarus. Erichson. Algiria.

Striatus. Solier. Hispania.

Latebricola. (Chevrol.) Algiria.*

Meridionalis. Dej. Cat. Gracia.*

(Ulyssiponensis. Hoff-

mansegg. Hispania.

Melancholicus. Dej.

Cat. id.

21 Levaillanti. (Gaubil.) Algiria.*

22 Distinguendus. (Gaub.) id. *

23 Atlanticus. (Gaubil.) id. ${ }^{*}$

24 Plicatus. Lucas. id.

25 Riehlii. (Gaubil.) id.*

\section{OPATRUM. Fabricius.}

Silpha. Linné. Tenebrio. Geoff. GonoCEPHALUM. Brullé.

1 Corsicum. Dej. Cat. Corsica.

2 Laticolle. (Chevrolat.) Algiria.*

3 Gemmatum. Solier. id. ${ }^{*}$

\& Brticum, Rambur. Hisp. merid.
ร) Dahlii. Dej. Cat.

Laticolle. Dahl. Cat. id.

6 Verrucosum. Germar. Dalmatia.*

7 Triste. Dej. Cat. Sardinia.

(Politum. (Parreyss.) id.

8 Salebrosum. Dej. Cat. Algiria.*

9 Emarginatum. (Solier.) Lucas. id.*

10 Granuliferum. Lucas. id.*

11 Montanum. Rambur. Hisp, merid.

12 Perlatum. Dej.Cat. id.

13 Pyrenæum. Solier. Pyr. orient.*

14 Sabulosum. Linné. $P_{*}^{*}$

15 Inquinatum. Sahlberg. $N$.

16 Terrosum. Germ. Küst. id.

17 Gibbum. (Parreyss.) Corfou.

18 Dardanum. Stéven. Turcia.

(Gonocephalum. (Brullé.).

Pusillum, Fabr. Gall. merid.*

Pygmæum. Dej. Cat. Gall, orient.*

21 Viennense. Duftsch. Austria.

Arenarium. Duftsch. id.

22 Pedestre. Rosenhauer. Tyrolis.

23 Assimile. Sturm. Cat. Sardinia.

24 Obscurum. Stm. Cat. Sicilia.

25 Pesthiense. Friw. Hungaria.

26 Geminatum. Brullé. Gracia.

27 Sordidum. Dej. Cat. id.?

28 Hespericum. Dej. Cat. Hisp. merid.

29 Vestitum. Dej. Cat. id.

30 Fuscum. Herbst. Dej.

Rusticum. Brullé.

Cat. Gall. merid.*

Parvulum. Lucas. Algiria. *

Angustatum. Dej. Cat. id. ${ }^{*}$

Famelicum. Banon.

Villa. Grocia.

Substriatum. Jan. Italia.

Barbarum. Erichson. Algiria.

Lugens, Dahl. Cat. Sardinia.

Lineare. Parreyss. Corfou.*

Lusitanicum. Stm. Cat, Lusitania.

Meticulosum. Buquet. Algiria.

Affine. Bilberg. Dej.

Cat. id.*

SCLERUM, Dej. Cat.

Opatrum. Fabr.

1 Foveolatum, Olivier, Barbaria.*

2 Armatum. Waltl. Hisp. merid. 
3 Lineatum. Dej. Cat. Hisp. merid.

4 Muticum. Sturm. Cat. Austria.

5 Scrobiculatum. Schm.

6 Algiricum. Lucas, Algiria. et Helfer. Smyrna.
Lucas.

LEICHENUM. Dej. Cat.

Opatrum. Fabr.

1 (Pulchellum. (Klug.)

Lucas. Gall.
Tigrinum. Dahl. Cat. Italia.

2 Pictum. Fabr. Austria.*

Tesselatum. Koy. Hungaria.

MICROZOUM. Dej. Cat. L. Redtenbacher. Opatrum. Fabr.

1 Tibiale. Fabr. $\quad$ P. $^{*}$

2 (Liliputanum. Lucas. Algiria.

Minutissimum? Dej.

Cat. Hisp. merid.

BOLITHOPHAGUS. Illig, Fabr.

Silpha. Linné. Opatrum. Panzer.

1 Reticulatus. Linné. Germania.*

Crenatus. Fabr. id.

Gibbus. Panzer. id.

2 Interruptus, llliger. Austria.

Gœdeni. Panzer. id.

3 Agricola. Fabr. $\quad P^{*}$

\{ Agaricola. Panzer. Alsatia.

4 Armatus. Fabr. Austria.

\section{FAMI. DIAPERLDES.}

OPLOCEPHALA, Laporte et Brullé.

Neomida. (Ziegl. Dej.) Ips et Diaperis. Fabr.

1 Bituberculatus. Oliv. $\boldsymbol{P}$.

2 Hæmorrhoidalis. Fabr.

Gyll. Styria.*

CHEIRODES, Dej. Cat.

1 Opatroides. Dej. Cat. Hispania.*

TRACHYSGELIS. Latreille.

1 Aphodioidis, Latreille, Gall. merid. .

2 Rufus. Latreille. id.

PHYLETHUS. (Meg. Dej.) L. Redtenb.

1 Populi.(Meg.) L. Redt. P.*

PENTAPHYLLUS. (Meg. Dej.) L. Redtenb.

Mycetophagus, Fabr.

1 Algiricus. (Gaubil.) Algiria.*

2 Melanophthalmus. Meg.

Dej. Cat. Gall. merid.*

Testaceus, Parreyss. Russ, merid.

3 Testaceus, Fabr. Geoff. $\boldsymbol{P}^{*}$

DIAPERIS. Geoffroy. Fabr.

Chrysomela. Linné.

1 Boleti. Lin. Oliv. Gyll. P.* Morio. Stentz. Styria.

2 Bipustulata. Dej. Cat.

Castelnau, Hisp, merid.*

SCAPHIDEMA. L. Redtenbacher.

Diaperis et Mycetophagus, Fabr. Scolytus. Panz. Neomida. Dej. Cat.

1 Bicolor. Fabr. Gallia.* \{ Enea. Fabr. Gyll. Suecia.

PLATYDEMA. Laporte et Brullé.

Mrcetophagus et Diaperis. Fabr. NeoMIDA. (Ziegler.) Dej. Cat.

1 Petitii. Perroud. Gall. merid.*

2 (Dejeanii. Laporte.Dej. Cat. Austria."

Cornigera. Dej.Cat. Hungaria.

Armata. Dahl. Cat. Styria.

3 Tristis. Stéven. Russ. merid.

4 Violacea. Fabr. Panz. P.*

PHALERIA. Latreille.

Tenebrio. Fabr.

1 Hemisphærica. Dej.Ct. Gall. merid.* Pallida. Dufour, Hisp, orient.

(Var.Arenicola.Dufour.id. 
2 Limbata. Dahl, Cat. Sardinia.

3 Cadaverina, Fabr. Gall. merid.*

Pellucida. Passerini. Etruria.

Maritima. Dufour. Hisp. orient.

Pontica. Besser. Russ. merid.

Var. Bimaculata. Dej.

Cat. Gall. merid.*

\section{ELEDONA, Latreille.}

Endophloevs. Dej. Cat.

1 Spinosula. Latreille, $\boldsymbol{P}$.*

2 Exculpta. (Parreyss.) Russ. merid.

21

\section{FAM. COSSYPHI.}

COSSYPHUS, de Castelnau, de Brême.

1 (Insularis. de Casteln.

de Brême. Algiria. *

(Siculus. Dej. Cat. Sicilia.

2 Tauricus. Stéven. Tauria.

Deplanatus, Schönh. id.

3 Moniliferus. Guérir. de

4 Hoffmanseggii. Herbst. Brême. Algiria.*

$\left\{\begin{array}{l}\text { Latr. id.* } \\ \text { Depressus. Fabr. }\end{array}\right.$

5 Ovatus. de Brême. id.

6 Barbarus. de Brême. id.*

7 Dejeanii. Rambur, de

Brême. Hisp. merid.

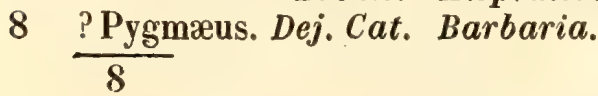

\section{FAM. TENEBRIONES.}

HETEROPHAGA. (Dej.) Lucas.

L. Redtenb. Helops. Panz. Tenebrio. Herbst. Fabr. Mycetophagus. Fabr. Alphitoвius. Steph.?

1 (Diaperina. Panzer. Germania.* Opatrina. Sturm. Cat. id. Opatroides. Dej. Cat. id.
2 Fagi. Panzer. Duftsch. Austria.* Oryza. Herbst. St. Cat.id. Mauritanica. Dej. Cat.

Steph.? Lucas, Algiria.

3 Chrysomelina. Fabr. Austria.

GATAPHRONETIS. Dej. Cat. Lucas.

1 Brunnea. Dej. Cat. Gall. merid.*

2 Levaillantii. Lucas. Algiria.*

3 Phaleroides. (Myria.

Ziegler.) Sardinia.

ULOMA. (Meg. Dej.) L. Redtenbacher.

Tenebrio, Linné. Phaleria. Duft. Gyll.

1 Culinaris. Linné. Duft. Gall. merid.*

PHTORA. Dej. Cat.

1 Crenata. Dej. Cat. Gall.mer.oc.*

TRIBOLIUM. Mac-Leay.

Stene. Steph. Colydium. Herbst. 'Trogosita, Ips et Lxctus. Fabr. Margus. (Dej.) L. Redtenb.

1 (Castaneum. Hbst. (Dej.) P.* Ferrugineum. Fabr.

Steph. Germania.

Testaceum. Fabr. Gallia.

2 Madeum. Charpentier. Austria. Obscurum. L. Redtenb. id.

CERANDRIA. (Dej.) Lucas.

Trogosita. Fabr, Phaleria, Latr.

1 Cornuta. Fabr. Hispania.*

2 Testacea. Dej. Cat. Gallia.*

\section{HYPOPHLEUS. Fabr.}

IPS. Rossi. Oliv.

1 Castaneus. Fabr. $\quad$ P. $^{*}$

2 Angustus. Lucas. Algiria.

3 Siculus. Dej. Cat. Sicilia.

4 Suberis. Lucas. Algiria.

5 Depressus. Fabr. $\boldsymbol{P}^{*}$.

6 Linearis. Fabr. Austria. *

7 Suturalis. Paykull. Suecia.

8 Longulus. Gyllenhal, id. 
9 Pini. Panzer. P.*

10 Rufulus. Rosenhauer. Germania.

11 Minutus. Dej.Cat. Dalmatia.

12 Fraxini. Paykull. Austria.

$\{$ Ferrugineus. Creutz. id.

13 Fasciatus. Fabr. Panz. Gall, bor.*

14 Bicolor. Fabr. P.*

\section{CALCAR, Latreille.}

Trogosita, Fabr.

1 Procerus. Schüppel. Gall. merid.*

Trogosita. Stéven. (Tenebrio.) Russ. merid.

Elongatus. Herbst. (Te-

nebrio.) Hispania. *

Calcar. Fabr. Algiria.

Variabilis. Fabr. id.

BIUS. Dej. Cat.

1 Thoracicus. Fab. (Trogosita.) Suecia.*

BOROS. Herbst.

1 Elongatus. Herbst. Germania. Boros. Fabr. (Hypophlæus.) id. Schneideri. Panz.(He-

(lops.) lops.) id.

2 Tagenoides

3 ? Rufipes. Lucas. id. *

\section{HEMEROPHYGUS. Dej, Cat.}

1 Asperatus. Dej. Cat. Gracia.

UPIS. Fabr.

Spondilis. Fabr. Attelabus. Linné.

1 Ceramboides. Fabr. Suecia. ${ }^{*}$

\section{ANTHRACIAS. Stéven.}

Bicornis. Stéven. Caucasus, ${ }^{*}$
TENEBRIO. Linné.

Upis. Fabr. Atrelabus. Linné. Boros. Herbst.

1 Gurvipes. Fabr. Gall. merid.* Cylindricus. Herbst. Austria.

2 Molitor. Linné. $\quad \boldsymbol{P}^{*}$

3 Obscurus. Fabr. id. ${ }^{*}$

4 Transversalis. (Meg.) Duftsch. Austria.

IPHTHINUS. Dej, Cat.

Tenebrio et Upis. Sturm.

1 Campiliensis. Spinola. Italia.*

Angustatus. Rossi. (Tenebrio.) id.

Italicus. Bonel. (Upis.) Pedemont.

2 Clypeatus. Germar.

41

(Blaps.) Lusitania.

\section{ॐ̈1, FAI. HELOPES.}

CRYPTICUS. Latreille.

Blaps. Fabr. HeLops. Oliv.

1 Obesus. Lucas,

2 Gibbulus. Herbst. id.*

3 Pruinosus. Dufour. Hispania. ${ }^{*}$

4 Variegatus. Dej. Cat. id.

5 Pusillus. Hoffmansgg, id,

6 Ferrugineus. Klug. Constantinp.

7 Alpinus. Géné. Italia.*

8 Glaber. Fabr. Gallia.

Laticollis, Panzer. Germania.

Quisquilius. Linné?

Payk. Suecia.

HELOPS, Fabricius.

oliv. Tenebrio. Linné.

1 Lacertosus. Dej. Cat. Smyrna.

2 Anthracinus. (Dej.) Kï-

ster. Sicilia.

3 Dilatipennis. Géné. Sardinia. ${ }^{*}$

4 Insignis. Lucas. Algiria**

5 Subdenticollis. (Gaub.) id. 
6 Coriaceus. Hoffmsgg. Hispania.* Anthracinus. Germar. id.

7 C Coruleus. Linné. Fab. Gall. merid.* Chalybeus. Olivier. Italia.

8 Elongatus. Stm. Cat, id.

9 Rossii Germar.

\section{(⿸丆口,}

Fischeri. Dej. Cat. id.

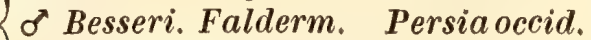

ơ Hegeteroides. Fald. id.

Nycterinoides. Fald. id.

25 Foveolatostriatus. Motschulsky. id.

26 Quadraticollis. Ménétriés. (Hedyphanes.) id.

Menetriesii. Fisch. (He dyphanes.) id.

Tentyrioides. Falderm. (Hedyphanes.) id.

Upioides.Faldermann. (Hedyphanes.) id. Subrugosus. Creutzer. Hungaria. ${ }^{*}$ Mauritanicus. (Gaub.) Algiria.* Lanipes. Fabr. $P{ }^{*}{ }^{\star}$ Picipes. Bonelli. Pedemont. Villipes. Hoffmansegg. Lusitania. Tuberculatus. Kollar. id. Villiger. Sturm. Cat. id. Congener. Dej. Cat. Tanger.* Serropalpus. Hoffmsg. Lusitania. Distinctus. (Gaubil.) Algiria. ${ }^{*}$ Mucoreus. Klug. Constantinp. Augustatus. Lucas. Algiria. Duponchelii. Dej. Cat. Gracia. Clypeatus. Andersch. Sicilia. f Exaratus. Germar. Dalmatia. Friulicus. Dahl. Cat. id. 14 Badius. (Dahl.) Redtb. Hungaria.

45 Convexicollis. (Chev.) Gall, merid.*

46 Meridionalis. Dej.Cat. id.

Oleæ. (Chevrolat.)

Assimilis. Dej. Cat. id. ${ }^{*}$

Ophonoides. Lucas. Algiria. ${ }^{*}$

Nitidicollis. Lucas. id.

Crassicollis. Rambur. Hisp merid.

Memnonius. Stm. Cat. Gall. merid.

Perforatus. Dej. Cat. Corsica.

$\{$ Perplexus. Dej. Cat. Russ. merid. $\{$ Arboreus. Godet. id.

马อ (Arboreus. Stéven. id. Brevicollis, Godet. id. (Gracilis. (Parreyss.) id.

56 Subcrenatus. Hoffmsg. Hispania.

57 Ecoffetti. (Chevrolat.) Gall. merid.

58 Harpaloides. Dej. Cat. id.*

59 Caraboides. Panzer. P.* Striatus. Olivier. Gallia. Var. Inflatus. Castelnau. id.

Dermestoides. Illiger. Germania.

60

61

62

63

64

65

66

67

\section{8}

Brevicollis. Stéven.

Quisquilius. Fabr. Egrotus. Dej. Cat, Ovalis. Dej. Cat. Curtus. Dej. Cat. Pinguis. Dej. Cat. Plebejus. Waltl.

f Convexus. Lasserre.

$\{$ Laticollis. Villa.

Grandicollis. Sturm.

Cat. Russ. merid.

Brevis. Sturm. Cat. id.

Testaceus. Dej. Cat. Gall. merid.* Pallidus. Curtis.

Pyrenæus. Dej. Cat. Pyr, orient.

(Subæneus. Dej. Cat. Hisp. merid.

Nitidus. Dufour. id.

Tenebrioides. Dahl.

Pallipes. Géné.

Cat. Etruria.

Monilicornis. Stéven. id.

Juncorum. Helfer. Sicilia.

(Pusillus. Dahl. Cat. Italia.

$\{$ Gracilis. Sturm. Cat. id.

Pumilus. Dej. Cat. Gall. merid. Parvulus. Lucas. Algiria.* Minutus. Dej. Cat. Dalmatia. Metallescens, Dahl.

Cat. Sardinia.

83 Rufescens, Friwaldsk. Turcia.

81 ?Atratus. (Chevrolat.) Algiria * 
NEPHODES. Dej. Cat.

1 (Villiger. Hoffmansegg. Hispania.* \{ Var. Algiricus. (Gaub.) Algiria.*

STENOTRACHELUS. Latreille.

Dryops. Paykull.

1 Eneus. Paykull. Suecia. 94

\section{¿2. FAM, CISTELA.}

\section{ALLECULA. Fabricius.}

Gistela. Oliv. Panzer.

1 Aterrima. Dej. Cat. Illyria.*

Saperdoides. (Upis.)

Ziegl. id.

Cisteloides (Upis.) Stm. Hungaria.

2 Morio. Fabr, Austria.*

\section{PRIONYCHUS. Solier.}

L. Redtenbacher, Amarignus, Dalman. Helops, Fabr.

Ater, Fabr.

Gallia.*

2 Mauritanicus. (Gaub.) Algiria.*

\section{CISTELA. Geoffroy.}

Fabr. Chrysomela, Linné.

1 Ceramboides, Linné, Gallia.*

2 Saperdoides. Dej. Cat. Hungaria.

3 Morio. Dahl. Cat. Sicilia.

4 Metallica. (Chevrolat.) Lombardia.

5 (Varians. Fabr. Oliv. Gallia.*

Fusca. Panzer. Germania.

6 Lævis. Jan.

7 Lugens. Dahl. Cat. Sicilia.

8 Fulvipes. Fabr. $\quad P^{*}$

9 Rufipes. Fabr. id.*

10 Lutea. Dahl. Dej. Cat. Hisp. merid.

11 Collaris. Dej. Cat. Hungaria.

Maculicollis, Stm, Cat. id.

12 Murina. Fabr. Gallia.*

YVariabilis, Dahl. Cat. Hungaria.

Reppensis. Herbst. Austria.

Var. Evonymi, Herbst. id.
13 Oblonga. Olivier. Italia.

Sericea, Dahl. Cat. id.

14 Antennata. (Parreyss.)

St. Cat. Hungaria.

15 Anthracina. Schmidt et Helfer. Smyrna.

16 Melanaria. Zentz. Lombardia.

17 Melanophthalma, Luc. Algiria.

\section{CTENIOPUS. Solier.}

Redtenbacher. Gistela. Geoff. Fabr.

1 Sulphureus, Linné. $\quad P^{*}$

2 Bicolor. Fabr. Panz. Gall, bor.*

3 . Sulphuripes. L. Redtenbacher. Hungaria.

\section{MEGISCHIA. Solier.}

L. Redtenb. Gistela. Fabr. OMophlus. (Meg.) Dej. Cat.

1 Nigrita. Fabr.

Dalmatic.,

2 Nigripennis. Fabr. Algiria.*

3 Erythrocephala. Sol. id.*

\section{OMOPHLUS, Solier. (Meg. Dej. Cat.)}

L. Redtenbacher. Cistela: Fabr.

1 Lepturoides. Fabr. Gall. merid.* YMajor Géné. Lombardia.

2 Elongatus. Dahl. Cat. Istria.

3 Pinicola. (Meg.) Redtb. Austria.*

4 Atripes. Sturm. Cat. Volhynia.

5 Armillatus. (Parr.) Corfou.*

6 Pallidipennis. (Meg.) Gall. merid.*

7 Picipes. Fabr. Germania.*

8 Curvipes. Dej. Cat. Gall. merid.*

9 Curtus. (Parreyss.) Corfou.

10 Coeruleus. Fabr. Algiria. ${ }^{*}$

Violaceus. Klug. id.

Cœrulescens. Olivier. id.

11 Ovalis. Castelnau. id. ${ }^{*}$

12 Marocanus. Lucas. id.

43 Distinctus, Castelnau. id.*

14 Abdominalis. Dej.? Cat. (Gaubil.) id.*

15 Erythrogaster. Lucas. id.*

Testacea. de Casteln. id.

16 Ruficollis. Fabr. Oliv. Hisp. merid. *

17 Distincticornis. (Gaub.) Algiria." 
MYCETHOCARES. Latreille.

Mycetophila. Gyll. Cistela. Fabr.

1 Flavipes. Fabr.

2 Linearis. 1llg. Schneid. id.*

$\{$ Barbatus. Latreille. Gallia.

Brevis.Panzer. Germania.

3 (Morio. (Ziegl.) L. Redt. Austria. ${ }^{\star}$

Rufipes. Friualdszky. Hungaria.

Estonica. Falderm. Russ. bor.

4 Brevis. Gyllenh. Austria.

Picipes. Panzer. Giermania.

5 Scapularis. Gyllenhal. Austria. ${ }^{\star}$

Humeralis. Panz. Dej.

Cat. Germania.

6 Bipustulata. Illiger. Austria.*

7 Axillaris. Paykull. Suecia.*

8 Quadripustulata. Dej.

Cat. Gall. merid.

Quadripunctata.Perrond. id.

9 Bimaculata. Mannerh. Finlandia. 53

53. FAM. SERROPALPI.

MELANDRYA. Fabricius.

Chrysomera. Linné.

1 Caraboides. Linné. P.* S Serrata. Fabr. Stm. Germania.

2 Canaliculata. Fabr. id.*

3 Flavicornis. Duftsch. id.* Barbata. Sturm. id.

4 Sulcata. Dej. Cat. P.

\section{PYTHO, Latreille.}

TENEBRIo. Linné.

1 Depressus. Linné. Germania.* Coruleus. Panzer. id.

Castaneus. Panzer. id.

Var. Festivus. Fabr. Hungaria.

\section{PHRYGANOPHILUS, Sahlberg.}

Dircas. Fabr. Melandrya. Gyll. st.

1 Ruficollis. Fabr.

Austria.
CONOPALPUS. Gyllenhal.

1 Flavicollis. Gyllenhal. P.*

2 Thoracicus. Dej. Cat. Lalmatia.

3 Collaris, Dej. Cat. Gall. merid.

SCOTODES, Eschscholtz. Germar.

Pelmatopus. Fischer.

1 Annulatus. Eschsch. Livonia.

$\{$ Humeralis. Fischer. Russ. merid.

HYPULUS. Paykull.

Dircea. Fabr. Helops et Notoxus. Panzer.

1 Bifasciatus. Fabr. Germania. Fasciatus. Panzer. id.

2 (Quercinus. Quensel.

(Elater.) Austria.

(Dubius, Fabr. Panz. Germania.

DIRCEA. Fabricius.

Xylita. Payk. Lymexylon. Panzer.

1 Quadriguttata, Fabr. Germania.

2 Lævigata. Hellenius. id.* Discolor. Fabr. id.

Buprestoides. Paykull. Suecia.

Rufipes. Besser. Volhynia.

3 Rufipes. Gyllenhal. Finlandia.

4 (Parreyssii. Dej. Cat. Russ, merid. Modesta. (Parreyss.) id.

5 Modesta. Dej. Cat. id.

6 Quadriguttata. Fabr. Croatia.

7 (Ferruginea. Paykull. Suecia. Sericeus. Sturm. (Helops.) id.

8 Variegata. Fabr. Gallia. ${ }^{*}$

9 Undulata, Dej. Cat. Gall. occid.*

10 Triguttata. Gyllenhal. Suecia.

11 Tenuis. Rosenhauer. Tyrolis.

MYCETOMA. Ziegl. Dej. Cat.

Dryors. Panzer.

1 Suturale. Panzer. Irungaria, \{ Phalerata. (Ziegler.) id. 
SERROPALPUS. Hellenius.

Dircea. Fabr.

1 Striatus, Hellenius. $\boldsymbol{P}$. Barbatus. Fabr. Austria.

2 Vaudoueri. Latreille. P.*

EUSTROPHUS. Illiger.

Mrcetophagus. Fabr.

1 Dermestoides. Fabr. Alsatia.*

HALLOMENUS. (Hellwig.) Illiger.

Dircea. Panzer.

2 Fuscus, Gyllenhal. Suecia.

Axillaris. Illiger. Austria.

Vr. Bipunctatus. Payk. Germania.

3 Flexuosus. Paykull. id.*

Undatus. Panzer. id.

Affinis. Paykull. Alsatia."

5 Marginicollis. (Chev.) $P_{\text {.* }}^{*}$

ORCHESIA. Latreille.

Hallomenus. Paykull.

1 Fasciata, Paykull, Suecia.

2 Micans. Paykull. P.*

3 Laticollis, L. Redtenb. Austria.

4 Sepicola, Rosenhauer. Tyrolis.

5 Grandicollis. Rosenh. id.

\section{7}

\section{כั4. FAII, MORDELLE.}

\section{SCRAPTIA, Latreille.}

Serropalpus. Illiger.

1 f Fusca. Latreille. Gallia.* (Sericea. (Dircæa.) Sch. Suecia.

2 Fuscula. Illiger. Austria. \{Minuta. Dej. Cat. P.

RIPIDIUS. Thunberg.

Synbius. Sundevall.

1 Blattarum. Sundevall. Austria.
EVANIOCERA. Guérin.

Pelecotoma. Fisch. Rhipiphorus. Payk. Ptilophorus. Dej. Cat.

1 Mosquensis. Fischer. Russia. Latreillei. Fischer. id.

2 Boryi. Lucas. Algiria.

3 Dufourii. Latreille. Gall. merid.* Friwaldszkyi. Sturm. Cat. Hungaria. Stevenii. Fischer. id.

\section{RHIPIPHORUS. Fabr.}

Redtenbacher. Metoecus. Dej. Cat. MonDELLA. Linné.

1 Paradoxus. Lin. Gyll. Gallia. \{ Angulatus. Weibehen. Germania.

EMENADIA, de Castelnau.

Rhipiphorus. Fabr.

1 Flabellata. Fab. Oliv. Gall. merid.* Y Var. Affinis. Sturm. Cap. Bon. Sp.

2 Bimaculata, Fabr. Gall. merid.* Vr.4-Maculatus. Schh. Hungaria. Carinthiaca. Panzer. Carinthia.

3 Fulvipennis. Dej. Cat. Dalmatia.

4 Bipunctata, Stm. Cat. Gall. merid.

5 Rutipennis. Stm. Cat. id.

\section{CTENOPUS. Fischer.}

1 Sturmii. Küster. Russ, merid.?

\section{MYODES. Latreille.}

\section{Rhipiphonus. Fabr.}

1 Subdipterus. Fabr. Gall. merid. Dortheni. Latreille. id.

\section{MORDELLA. Linné.}

1 Duodecimpunctata. Rossi. Oliv. Germania. Perlata, Herbst. Austria.

2 Maculosa. Nozen. id. Atomaria. Fabr. Germania. Guttata, Paykull. Suecia. 
3 Albosignata. Dej. Cat. Styria.

4 (Bisignata. (Ziegler.) L.

$\{$ Redtenbacher: Germania.

Fasciata. Gyllenhal. Suecia.

5 Picta. Besser. Volhynia.

6 Bipunctata. Dej. Cat. Germar. Dalmatia. *

7 Fasciata. Fabr. $\quad$. $^{*}$

8 Obsoleta. Dej. Cat. Dalmatia.

9 Guttatofasciatu. Fin-

del. Hungaria.

10 Sericea. (Ziegl.) Dej.

1 Micans. Dej. Cat. Aat. Austria.

12 Humerosa. Rosenh. Hungaria.

13 Aculeata. Linné. Oliv.

$\left\{\begin{array}{c}\text { Gyll. } P . \\ \text { Var. Femoralis. Gys- } \\ \text { selen. Austria. }\end{array}\right.$

14 Villosa. Schrank. id.

15 Tibialis. Dej. Cat. $\quad P$.

16 Grisea. Frölich. Gall. merid.

17 Pumila, Gyllenhal. Suecia.*

\{Elongata. Dej. Cat. P.

18 Pusilla. L. Redtenb. (Meg. Dej.) id.

19 Troglodytes. Manner-

20 Aterrima. Gysselen.

21 (Brunnea. Fabr.

Fusca. Schrank?

Neuwaldeggiana.

Panzer. Germania.

22 Detrita. Sturm. Cat. id.

23 Latreillei. Nees. jd.

24 Sexpunctata. Herbst, Austria.

25 Testacea. Fabr.

26 Angustata. Dej. Cat.

Auripennis. (Par-

27 Abdominalis. Fabr.

Smyrna.*

Gall. merid.*

Austria.

id.

id.

.

merid.

Olivier.

28 Ventralis. Fabr. Gyl-

29 Humeralis. Linné. lenhal. Suecia.*

30 Axillaris. Gyllenhal. \{ Collaris. Dej. Cat.

Germania.*

Suecia.

Styria.

31 Variegata, Fabr. P.

Lateralis. Olivier. Italia.

Dorsalis, Panzer. Germania.

32 Decora. (Chevrolat.)

33 Insidiosa. Lucas.
34 Flavescens. Marsham. Anglia.

$3 \check{ }$ Ferruginea. Gyllenhal. $P$.

\section{ANASPIS. Geoffroy.}

Mordella, Linné, Fabr, Oliv.

1 Frontalis. Linné. Panz. P.

Var. Pulicaria. Stm. Germania.

Atra.Fallen. Suecia.

2 Clypeata. Erichson. Germania.

3 Rufilabris. (Sturm.) Gyllenh. id.*

4 Pygmæa. Dej. Cat. Dalmatia.

5 (Nigra. (Megerle.) $\boldsymbol{P}^{*}{ }^{*}$

Depressa. Schüppel. Germaria.

6 Brunnea. Dej. Cat. P.*

7 Humeralis. Oliv. Fabr. id.*

\{Geoffroyi. Müller. Austria.

8 Bipunctata. Stm. Cat. Germania.

9 Flava. Linné. Panzer. P.*

10 Badia. Rosenhauer. Hungaria.

11 Quadriguttata. Latr. Gall. merid.

12 Quadrimaculata. Dej.

Cat. id.*

13 Phalerata, Erichson. Germania.

14 Maculata. Geoffroy. P.*

Obscura. Gyllenhal. Suecia.

Bipunctata. Bonelli. Italia.

15 Binotata. Fabr. Gallia.

16 . Bicolor. Gemelin. P.

\{uadrinotata. Heyd. Germania.

17 Obscura. Geoffroy. P.

18 Fasciata. Dej. Cat. Hungaria.

19 Pallida. Dej. Cat. id.

20 Quadripustulata. Mül-

ler. Germar. Germania.*

21 Lateralis. Fabr. Suecia.

22 Rubricollis. Dej. Cat. Dalmatia.

\{ruficollis. Stéven. Russ. merid.

23 Ruticollis. Geoff. Fabr.P.*

Fuscicollis. Stm. Cat. Germania.

24 Collaris. Dej. Cat. Gall. merid.*

25 Thoracica. Linné, Fab.

Oliv. $P_{\text {. }}^{*}$

26 Basalis. Erichson. Germania.

27 Exigua. Schmidt et

Helfer. Smyrna.

28 Arctica. Schönherr. Zett. Lapponia.

29 Submaculata,Dej.Cat.Gallia.* 


\section{כัכ̆. FAM. GANTHARIDES.}

ZONITIS. Fabricius.

Apalus, Oliv. Meloe. Livné.

1 Præusta. Fabr.

Nigripennis. Panzer.

Gall. merid*

Flava. Stéven.

Germania.

Russ. merid.

2 Nigricornis. Dej. Cat.

3 Nigripennis. Fabr.

4 Mutica. Fabr.

Hisp. merid.*

5 Lunata, Tauscher. Russ. merid.

6 Fulvipennis. Tauscher. id. ${ }^{*}$

7 Sexmaculata. Olivier. Gall. merid.*

8 Quadripustulata. Fabr. id.*

\{ Quadrimaculata.Stév. Russ, merid.

9 Bifasciata, Koy. Schh. Hungaria.

Fasciata. Tauscher. Russ. merid.

10 Caucasica. Pallas. Caucasus.

11 Humeralis. Dej. Cat. Hungaria.

12 Atra. Koy. Schönh. id.

YNigra. Tauscher. Russ. merid.

\section{LEPTOPALPUS. Guérin.}

ZoNitis. Fabr.

1 Rostratus. Fabr. Chevrolatii. Guérin. id.

\section{NEMOGNATHA. Latreille.}

\section{Zonitis. Fabr.}

1 Quadrinotata.Dej.Cat. Algiria.

2 Chrysomelina. Fabr. Gall. merid.*

\section{APALES. Fabricius.}

1 Bimaculatus. Linné.

(Meloe.) Fabr. Germania.*

2 Binotatus. Dej. Cat. Italia.

3 Bipunctatus. (Ziegler.)

Germar. Hungaria.

4 Necydalens. Pallas. Russia.

\section{SITARIS. Latreille.}

Necydalis. Fabr. Cantharis. Geoffroy.

1 Humeralis. Fab. Oliv.

\footnotetext{
Guérin. Gall. merid."

3 Rufipes. Gory.
}

4 Rufipennis, Dufour. Hisp. orient.

5 Abdominalis.Dej.Cat. Hispania.

6 Cyanea. Dej. Cat. Sicilia.

7 Adusta. (Ziegler.) Silesia.

8 Apicalis. Latreille. Gall. merid.*

9 Thoracica. Dej. Cat. id.

10 Melanocephala. Stév. Russ, merid.

\section{MELOE. Linné. Fabr.}

1 Proscarabæus. Linné.

P Punctata. Panzer. Fabr. Illig. Germania.

Brunsviscensis, Meyer, id.

Tectus. Leach. Anglia.

Atratus. Meyer. Germania.

Rugipennis. Mannerh. Suecia.

Tauricus. Dej. Cat. Russ. merid.

Volgensis. Tauscher. id.

Cyanellus. Dej. Cat. Styria.

Gallicus. Dej. Cat. Gallia.

2 Violaceus. Marsham.

Leach. Gyll. Anglia.*

Proscarabaus. Panz.

Rossi. Latr. Gallia.

Similis. Marsham. Anglia.

Aprilinus. Meyer. Germania.

3 Plicatipennis. Lucas, Algiria.*

4 (Autumnalis. Olivier. $P_{.}^{*}$

Glabratus. Leach. Anglia.

Punctatus. Marsham. id.

Cyaneus. Fabr. $P$.

5 (Tuccins. Rossi. Gall. merid.*

Punctatus. Fab. Leach. Italia.

6 Foveolatus, Guérin. Algiria.*

7 L Lavigatus. Olivier. id.

\{Majalis. Linné. id.

8 Luctuosus. Brandt et Erich. Sicilia.

9 (Brevicollis. Panzer.

$\left\{\begin{array}{c}\text { Hoppe. Gallia.* } \\ \text { Semipunctatus.Dj.Ct.Styria. }\end{array}\right.$

10 Parvicollis. (Megerle.) Lombardia.*

11 Scabriculus. Bæber. Germania.

Brevicollis, Dej. Cat. id. $P$.

Laticollis. Dej. Cat. id.

Brevicollis. Fabr. id.

12 Rugosus. Marshäm. Anglia.*

Autumnalis. Leach. Italia.

Rugulosus. Dej. Cat, Gall. merid.

Pullus. Hoffmansegg. Lusitania.

Globosus. Knoch. Germania. 
13 Murinus. Brandt et $\{$ Erich. Sicilia. . Dat. id.

14 Affinis. Lucas.

15 Variegatus. Donavan. Marty. Anglia. ${ }^{*}$

Scabrosus. Marsham. Germania. Majalis. Fabr. Panz. Majalis. Olivier. id

16 Cicatricosus. Leach. Anglia.* Reticulatus. (Ziegler.) Gallia.

17 Coriarius. Hoffmsgg. Germania.* $\{$ Reticulatus. Brandt et Limbatus. Fabr. Illig.

Germar. id

Hungaricus. Schrank. Tauria. Uralensis. Pall. Leach. Hungaria. Punctatus. Meyer. Austria. Glabratus. (Megerle. Ziegl.) Dej. Cat. Hungaria.

Decorus. (Creutzer.) Brandt et Erich. id.

21 Corallifer. Hoffmsgg. Brandt et Erich. Lusitania. Majalis. Brandt et Er. Gall. merid. ${ }^{*}$ Eneus. de Castelnau. Algiria. Maculifrons. Lucas. id.* Insignis. Charpentier. in Germar. Hispania.

26 Excavatus. Leach. $N$.

27 Pygmæus. L. Redtenb. Austria.

28 Nanus. Lucas. Algiria.

29 Corrosus. Dej. Cat. Sicilia.

30 Sulcicollis. Latreille. Tauria.

31 Sardeus. Géné. Sardinia.

32 Bilineatus. Géné. Italia.

33 Fascicularis. Géné. id.

\section{MYLABRIS. Fabr.}

Meloe, Linné, Gantharis, de Geer.

1 Oleæ, Chevrol. Castel. Algiria.* \{ Marocana. Dej. Cat. Barbaria.

2 Cincta. Olivier. Oriente.

3 Bimaculata. Olivier, Gracia.*

4 Trniata. Waltl. Constantinp. \{Militaris. Klug. id.

5 Interrupta. Olivier. Algiria. *

6 Lacera. (Megerle.) Küster. Italia.
7 Rubripennis. Chevrol. Algiria.* Var. Guerinii. Chevrol. id.

- Tricincta. Chev. id. - Litigiosa. Chev. id.

8 Mutans. Guérin. id. ${ }^{*}$ Melanura. Pallas. Russ. merid. Var. Quadripunctata.

Linné. Gall. merid. - Cichorii. Olivier. id.

9 Splendida. Pallas. Russ. merid."

10 Variabilis. Pall. Oliv. Gall. merid. Var. Fasciatopunctata. Mannerh. Russ. merid.

11 Fueslini. Panzer. Bilb. Austria. Cichorii. Rossi. Schrk. Hungaria. Tauscheri.var.Gebler. Russ. merid.

12 Spartii. Germar. Dalmatia.

13 Dahlii. Dej. Cat. id.* Australis. (Parreyss.) Corfou.

(Fuesslini.var. Stéven. Russ. merid. Minuta, var. Gebler. id.

14 Flexuosa. Olivier. Helvetia.* \{ Var. Alpina. Ménétr. Russ. merid. 15 Geminata. Fabr. Gall. merid.* Var. Grisescens. Schh.

Dej. Cat. Russ. merid. Centropunctata. Eschscholtz. id. Besseri. Mannerheim. id. Calida var. Gebler. Sibiria. 12-Maculata. Chevrol. Barbaria.

17 Ruficornis. Fabr. id.

18 Gilvipes. (Dej.) Chev. id.

19 Maura. Chevrolat. Algiria.*

20 Circumflexa. Chevrol. id." Var. Bissexpunctata. Latreille. id. - Distincta.Dej.Cat. Barbaria.

21 Goudotii. Laporte. id. $\{$ Variegata. Dej. Cat. id.

22 Affinis. Lucas. Algiria.* 23 Dejeanii. Schönherr. Hispania.* 24 Wagneri. Chevrolat, id. 25 Decempunctata. Fabr. id. 26 Impressa. Chevrolat. id.* 27 Paykulii. Bilb. Chev. id. 28 Trifasciata.Voet, Chev. id.

29 Tenebrosa. Laporte. \{hevrolat. Barbaria. Luctuosa. Dej. Cat. id.

30 Terminata. Chevrolat. Algiria. *

31 Silbermanni. Chevrol. id.

32 Curta. Chevrolat. id. 
33 (Præusta. Fab. Bilberg. Algiria.*

$\left\{\right.$ Var. Apicalis. Chev. id. ${ }^{*}$ - Contexta. Chev. id.

34 Scapularis. Chevrolat. id

35 Cingulata. Latreille. Oriente.

36 (14-Punctata. Bilberg. Russ. merid.

Combusta. Stm. Cat. id.

(Var.Famelica.Ménétr. id.

37 Sericea. Pallas. id. 14-Punctata. vr. Bilb. id. Gebleri. Eschscholtz. id.

38 Bivulnera. Schönherr.

39 Calida Pallas. $\begin{array}{ll}\text { (OEnas.) id. } & \\ \text { id. } & \end{array}$

Maculata. Bilberg. id.

Decora. Olivier. id.

10-Punctata.vr.Schh. id.

Signata. Faldermann. Sibiria.

Daurica.Mannerh. Dauria.

40 Cyanescens. Illiger. Gall. merid. ${ }^{*}$

41 Crocata. Pall. Bilberg. Hungaria.

12-Punctata. Tausch. Russ, merid.

42 Varians. Dej. Cat.

43 Adamsii. Fischer. Hispania.

Fasciatopunctata.

$\begin{array}{cc}\text { Adams. id. } \\ \text { Floralis. (Dej.) } & \text { id. }\end{array}$

44 Pusilla. Tauscher.

45 Græca. Ahrens.

46 Floralis. Pallas.

47

48

49

Minuta. Krynicki.

Turcia.

OEskayi. Fisch. St. Cat. Turcia.

fOlivieri. Bilberg. Russ. merid.

$\{$ Cohorens. Fischer. id.

50 Grisescens. Tauscher, Russ. merid.

51

Aulica. Ménétriés. id.

52 Conspicua. Helf. St.Ct. Constantinp.

53 Mutabilis. Dej. Cat. Hispania.

54 Cylindrica, Dej. Cat. id.

55

56

57

.58

59

Assimilis. St. Cat. Gall. merid.

Kindermanni.Stm.Cat.Constantinp:

Similio. Sturm. Cat. Tyrolis.

Sturmii.Stentz.St.Cat.id.

Brevicollis. Schmidt et

Helfer. Smyrna.

60 Decora. Friwaldszky. Turcia.

61 Decemmaculata.Waltl. Grœeria.

HYCLEUS, Latreille.

Dices. Dej. Cat.

1 Bilbergi. Schönherr. Gall. merid.* Clavicornis, Illiger. Hispania.
2 Distincta. Chevrolat. Algiria. *

3 Confluens, Klug. St. Cat. Constantinp.

LYDUS. (Megerle.) Dej. Cat.

Myeakris, Fabr. LytTa, Herbst.

1 Algiricus. Fabr. Algiria.*

2 Sanguinipennis. Chev, Barbaria.

3 Marginatus. Fabr. Algiria. ${ }^{*}$

4 Trimaculatus. Fabr. Hungaria.*

Var. Quadrisignatus.

Quadrimaculatus.

Tauscher. id.

5 Chalybeus. Tauscher. id.

OENAS. Latreille.

Meloe, Linné. Lytra, Fabr. Gantharis. oliv.

$1 \int \sigma^{x}$ Afer. Fabr.

O Uricolor de Castel Algia.

T. Algiria.

2 Crassicornis, Fabr. Hungaria. *

CEROCOMA. Geoffroy.

MeLOE. Linné.

1 Olivieri. Dej. Cat. Smyrna.

2 Schæfferi. Linné. Gallia.*

$\{$ Var. Afinis, Stéven. Russ. merid.

3 Muhlfeldii. Schönherr. Hungaria.

\{Micans. Faldermann. Russ, merid.

4 Vahlii. Fabr. Gall. merid.*

Var. Chalyboiventris.

Chevrolat. Algiria.

Festiva. Eschscholtz. Persia occid.

5 Wagneri. Küster. N.

6 Concolor. Dej. Cat. Barbaria.

7 (Stevenii. Fischer. Russ. merid. ${ }^{*}$

Prausta. Stéven. Dej.

Cat. id.

8 Kuntzei. Friwaldszky. Turcia.

CANTHARIS. Geoffroy.

Meloe, Linné. Lytra, Fabr.

1 Collaris, Fabr. Fisch. Russ, merid,*

2 Syriaca. Linné. Panz. Austria.*

3 (Myagri. (Zgl.)Dej.Cat. id.* Austriaca. (Megerle.) id. 
4 Menetriesii. Falderm. Russ. merid.

5 Lutea. Klug. Sturm.

Cat. Constantinp.

6 Vesicatoria, Lin. Panz.Gallia.*

7 Phalerata.Friwaldszk,Grocia.*

8 Dives. Brullé. id.

9 Vittata. Brullé. id.

10 Segetum. Fabr. Algiria.*

11 Viridissima Lucas. id.*

12 Scutellata. de Casteln. id.* Yar. Cœrulea. (Gaub.) id.*

13 Curtana. Lucas. id. ${ }^{*}$

14. Herbivora. Rambur. Hisp. merid.

EPICAUTA. Dej. Cat, Redtenbacher.

Lytta. Fabr. Brullé. Castelnau. CanthaRIs. Oliv. Latr.

1. Fulviceps. Dej. Cat. Oriente.

2 (Dubia. Olivier. Gall. orient.* Verticalis, Illiger. Austria.

Erythrocephala.Panz,id.

3 Erythrocephala. Fabr.

Oliv. id.

4 Flabellicornis. Germar. Dalmatia. Dubia. Sturm. Cat. id.

159

\section{马6. FAM. OEDEMERE.}

\section{OSPHIA. Illiger.}

Notнus. (Ziegler.) Olivier. Latr.

1

$\begin{cases}\text { Præusta. Olivier. } & \text { Alsatia. }^{*} \\ \text { Clavipes. (Megerle.) } & \text { Germania. } \\ \text { Bipunctata. Fabr. } & \text { id. }\end{cases}$

NACERDES. Stéven. Wilh. Schmidt.

Dej. Cat. Necydalis, Linné. Fabr.

1 Melanura. Linné.

Notatata. Fabr.

Analis, Olivier.

Erminea. Germar.

Germania.*

Gall. merid.

id.

2 Sardea. Schmidt.

Germania.

3 Caucasica. Dej. Cat.

4 Vestita, Dej. Cat.

Sardinia.

Caucasus.

Barbaria.

5 Viridana. Dej. Cat. Lucas, id.
6 Suturalis. Olivier. Barbaria.? \{eucogramma. Latr. id.

XANTHOCHROA. Wilh. Schmidt.

1 Carniolica. Gistl. Carniolia.

Lippichii. Kunze in litt. id.

2 Gracilis, von Heyden. Styria. Tilia. Spitz in litt. id.

ASCLERA, Dej. Cat. Wilh. Schmidt.

Necydalis, Linné. Fabr. Isch omera. Steph.

1 ( Sanguinicollis, Fabr, P.*

Flavicollis. Panzer. Germania.

2 Hæmorrhoidalis. Schm. Turcia.

3 Cœerulea. Linné. Gallia.*

Cyanea. Fabr. Germania.

Nigripes. Olivier. Gallia.

Corulescens, Fabr. id.

ANONCODES. Dej. Cat. Wilh. Schmidt.

(Anogcodes. Dej. Cat.) Cantharis. Linné. Necydalis. Fab. Ischromera. Steph.

1 Adusta. Panzer. Germania.* $\{$ ㅇ Collaris. Panzer. id. Ustulata. Scopoli. Gallia.

2 Geniculata. Schmidt. Turcia. Y Var. Basalis. (Friw.) id.

3 Rufiventris. Scopoli. Carniolia. Bipartita. Schrank. Austria.

Melanocephala. Fabr. Algiria.

Dispar. (Megerle.) Germania.

Dorsalis. Olivier. Gallia.

Affinis. Dej. Cat. Germania.

4 Ustulata, Fabr. id. ${ }^{*}$

Fasciata. Villers. Gall. orient.

Melanura. Fabr. Germania.

Scutellaris. Waltl. id.

5 ơ Fulvicollis. Scopoli.Carniolia.*

(Dissimilis. (Sturm.) Austria.

or Nigra. Dahl. Cat. id.

Atra. (Dahl.) id.

Var. Maura. (Parr.) id.

6 (Ruficollis. Oliv. Fabr. Gall. merid.

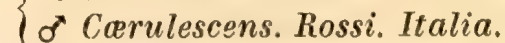

7 Turcica, Schmidt. Turcia. 
8 \{ Viridipes. (Meg.) Schm. Austria.* Dispar. Sturm. Cat. Russ. merid.

9 Amœna. Schmidt. Gall. merid.* Ruficollis. Dej. Cat. id.

10 Coarctata. Germar. Russ. merid. 11 Alpina. von Heyden. Styria. 12 Azurea. (Meg.) Schm. Tyrol.* 13 ? Difformis. Schmidt. Turcia.

\section{CHRYSANTHIA. Schmidt.}

Cantharis, Linné. Necydals. Fabr. Asclera, Dej. Cat.

1 (Viridissima. Linné. Austria.* $\{$ Viridis. de Geer. Suecia. Thalassina. Fabr. Germania.

2 Viridis. Illiger. Austria.* Viridissima Eabr. Tyrolis.

(V. Geniculata. (St. Ct.) Germania.

\section{OEDEMERA, olivier.}

Negydalis et Gantharis. Linné. NecyDALIS. Fabr.

1 (Podagrariæ. Linné.

Flavescens. Rossi.

우 Testacea. Fabr. Germania.

Melanocephala. Oliv. Gallia.

2 Penicillata. von Heyd. Turcia.

3 Ventralis. Dej. Cat.

Schmidt. Gall. merid.*

4 Brevicollis. Schmidt. Sicilia.

5 Flavimana. Hoffmsgg. Lusitania.

Marginata. Gyllenhal, id.

or Simplex. Linné. id.

6 Similis, von Heyden. Turcia.

7 Flavescens. Linné. Germania.* Femorata, Scopoli. Carniolia. 오 Simplex, Fabr. Volhynia.

Praterana. Schrank. Austria.

8 Flavipennis. vun Heyd. Caramania.

9 (Marginata. Fabr. Germania.*

Femorata. Panzer. id.

Subulata. Olivier. id.

10 Melanopyga. Kunze. Sicilia.

11 Lateralis. Eschscholtz. Hungaria. (Seladonia. (Megerle.) id.

12 Cœrulea. Linné. Germania.* Nobilis. Scopoli. Carniolia.

Ceramboides. Forster. Germania.

Var. Violacea. St. Cat. Sardinia.

Cyanescens. (Parr.) id.
13 Rufofemorata.Dej.Cat.

Germar. Schmidt. Dalmatia.

Cyanescens. Dej. Cat.

Schmidt. id.

Unicolor. Hoffmsgg.

Schmidt. Lusitania.

16 (Tristis. Ullrich. Schm. Germania.

Unicolor. Sturm. Cat. Carniolia.

Montana. (von Heyd.) id.

17 Atrata.Dej.Cat.Schm. Gall. merid..

18 Brevicornis. Schmidt, Austria.

19 (Croccicollis. (Sahlb.)

Gyll, jd.

Arundinis. Dahl. Cat. Findelia.

ơ Sanguinicollis.var.

Fabr. id.

20 Marmorata. Erichson. Algiria.*

21 Barbara. Fabr. Gall. merid.*

\{Terminata.(von Heyd.) id.

22 Flavipes. Fabr. id.*

Clavipes. Fabr. Germania.

(Enea. (Vill.) id.

23 Virescens. Linné. Gyll. id.*

Striata. Herbst. Austria.

24 Lurida. Marsham. Gallia.*

25 Tibialis. Lucas. Algiria.*

26 Linearis. Latreille. Gallia.*

\section{STENAXIS. Schmidt.}

OEdemera. Oliv.

1 Annulata. (Meg.) Germ. Tyrolis.

PROBOSCA. (Ziegler.) Schmidt.

1 Viridana. (Zieg.) Schm. Italia.

2 Incana.Friw. Schmidt. Hungaria.

CHITONA. Schmidt.

Stenostoma. de Castelnau, Sturm. Cat.

1 Variegata. Germar.

$\left\{\begin{array}{r}\text { Charp. Hispania. } \\ \text { Fasciata.Mus. Berol. id. }\end{array}\right.$

STENOSTOMA, Latreille.

Leptura, Fabr. Rhinomacer. Illig.

1 Rostrata. Fabr. Necydaloides. Latr. Lusitania. 
DRYOPS, Fabricius,

$1 \begin{cases}\text { Femorata. Fabr. } & \text { Gallia. } \\ \text { Simplex. Donovan. } & \text { Anglia. } \\ \text { Calapoides. Germar. } & \text { Helvetia. }\end{cases}$

CALOPUS. Fabricius.

Cerambex. Linné.

1 Serraticornis. Linné, Austria.*

SPAREDRUS. (Megerle.) Schmidt.

Calopus. Schönh.

1 Testaceus.And.Hoppe.Austria.*

DITYLUS. Fischer.

Helops. Fabr.

$1 \frac{\text { Lævis. Fabr. Austria.* }}{62}$

57. FAU. LAGRIE.

EUTRAPELA. Blanchard. (Dej. Cat.)

1 Suturalis. Lucas.

Algiria.

LAGRIA, Fabricius.

Ghrysomela. Linné. Cantharis, Geoff.

1 Viridipennis. Fabr.

2 Glabrata. Olivier.

3 Lata. Fabricius.

4 Hirta. Linné. Pubescens. Linné.

Algiria. $^{*}$ Gall. merid.* Hispania. ${ }^{*}$ Gallia.* id.
38. FAII, PYROCHROE.

PYROCHROA. Geoffroy.

Canthanis. Linné.

1 Coccinea. Linné. Gallia bor.*

2 Pectinicornis, Linné, Germania.*
3 Rubens. Fabr. \{ Satrapa. Schrank.

Gallia.*

Austria.
Dendroides. Latr,

1 Thoracicus. Fischer. Russ. merid.

\section{FAM, RHINOSIMI.}

MYCTERUS, Clairville.

Bruchus, Fabr. Rhin omacer, Latr.

1 f Curculionoides. Fabr. Gall. merid.* $\{$ Griseus. Clairville. Helvetia.

2 Umbellatorum. Fabr. Gall, merid.* Pulverulentus. Dahl.

Ruficornis. Dahl. Cat. id

3 Tibialis.(Parreyss.) St.

Cat. Corfou.

\section{SALPINGUS, Illiger.}

Sphariestes. Curtis.

1 Ater, Gyllenhal.

2 Foveolatus. Beck.

3 f Picex. Germar. \{Rufescens. Dej. Cat. id.

4 Quadriguttatus. Lepel. et Serv. $P{ }^{*}$

5 (Denticollis, Gyllenh. Austria. Humeralis, Dej. Cat, Lombardia.

6 Rufilabris, Dej. Cat. Suecia.

7 Cursor. Gyllenhal. id.

8 Nitidus. Sturm. Germania.

9 Limbatus, Dej, Cat. Gallia.

\section{RHINOSIMUS, Latreille.}

Anthribus, Fabr. Attelabus. Herbst. Salpingus. Gyllenh.

1 Eneus. Olivier. Gallia bor.*

2 Roboris. Fabr. P.* I Ruficollis. Hbst, Gyll. Germania.

3 Ruficollis. Panzer. Gallia.* Y Viridicollis. (Ziegler.) Germania. 
4 Planirostris. Fabr. Gallia. Fulvirostris. Paykull. Germania. 16

60. FAII, ANTHICI.

\section{STEROPES, Stéven.}

Blastanus. Illiger.

1 Caspius. Stév. \{ Colon. Illig.

Russ, merid. id.

\section{NOTOXUS. Geoffroy.}

Meloe, Attelabus. Linné. Notoxus. F. Anthicus. Paykull, et Fabr. Lvtra. Marsh. Monocerus. (Dej.) Falderm. Ceratoderus. Blanch.

1 (Brachycerus. Falderm. Caucasus. \{Major. Schm. (Dej.) Gall. merid.*

2 Mauritanicus. Laferté

et Lucas. Algiria.*

3 Monoceros. Linné. Gallia.*

Cucullatus. Fourcroy. $P$.

(Var. Integer. (Meg.) Germania.

4 Cavifrons, Laferté. Lusitania.

5 Platycerus. (Dej.) Laf. Hispania.

6 Siculus, Laferté. Sicilia.

7 Cornutus, Fabr. Gall. merid.*

Monoceros, v. $\beta$. Rossi. Italia.

Trifasciatus. Rossi. id.

Armatus, Schmidt, Tyrolis.

8 Numidicus. Lucas. Algiria.

9 Miles. Schmidt. Hungaria.

\section{AMBLYDERUS. Laferté.}

1 Scabricollis.Laf, et Luc, Algiria.

FORMICOMUS, Laferté.

Cantharis. Geoff. Charabus, Rossi. Notoxus et Anthicus. Fabr. AnthelePHILA. Saunders.

1 Pedestris. Rossi.

Italia.

Thoracicus, Panz.

Fuscus, Geoff.

Nobilis. Falderm. Cursor. Motschulsky. id.

2 Latro, Laferté.
Austria.

Gall. merid.

Russ, merid.

Sicilia.
TOMODERUS. Laferté.

Anthicus. Say, Motsch. Erich.

1 Compressicollis. Mot. Russ. merid. \{Melanophthalmus. Laf. Gall. merid.

ANTHICUS, Geoffroy.

Meloe, L. Lagria. F. Anthicus, Payk. LvtTA, Marsh.

1 Rodriguii, Latr.

Gall. merid.*

$\{$ Pulchellus. Schmidt. Hisp. merid.

2 Humilis, Germar. Bremei. Laferté.

Riparius. (Dej.)

3 Minutus. Laferté.

Sardeus. Schmidt.

Cursor. Géné.

Salinus. (Helfer.)

4 (Bimaculatus. Illig.

Sagitta. Krynicki.

Pictus. (Fisch. Dej.)

Bimaculatus. (Gyll.)

5 /Floralis, F. Payk.

Fuscus. Marsh.

Pedicularius. Schrk. Austria.

Formicoides, Fourc. P.

Myrmecocephalus, Ros.

Germania.*

Gall. merid.* id.

id.

Sardinia.

id.

id.

Germania.*

Russ, merid.

Sibiria.

Gall. bor.

Suecia.*

Anglia.

v. c. Italia.

Calycinus. Panz, v, b. Germania.

Formicarius, Oliv. Gallia.

Umbellatorum. (Dej.) Chili.

Basalis. Villa. v. b. Pedemont. ${ }^{*}$

6 Bifasciatus. Ross. Schm. Italia. ${ }^{*}$

4-Pustulatus, Dahl. Germania.

4-Guttatus. Latr. Gallia.

7 Sellatus. Panzer. Gall. or. ${ }^{*}$ $\{$ Arenarius. (Dahl.Dej.) Germania.

8 Instabilis. (Dej.) Schm, Gallia. *

Tibialis, Curtis. Anglia.

Mauritanicus, Lucas. Algiria.

Cursor. Stéven, v, c. Russ, merid.

9 Gracilis, Panzer. Germania.*

Lateripunctatus, St. Gall, or. mer.

(Stevenii. (Dej.) Russ, merid.

Cruciatus. (Stév.) v. b. id.

10 Subfasciatus. (Dej.) Laf. Gall. or.

Unipunctatus. (Dej.)

v. c. Hispania.

11 Longicollis. Schmidt. Gall, or.

12 Optabilis, Laferté. Alp. marit. 
13 (Tenellus, (Dej.) Laf. Gall. or. mer. 36 Sanguinicollis, Laf. Gall merid.

Transversalis. Villa. Pedemont.

Amanus. (Schm.) Italia.

14 Vittatus. Lucas.

$15\left\{\begin{array}{l}\text { Tristis. Schmidt. P.German } \\ \text { Sericeus.(Dej.) v. a. b.e.Hispania. }\end{array}\right.$

Algiria.

Fenestratus. (Dej.) v. $\gamma$. id. $^{*}$

16 Antherinus. Lin. (Dej.) Gallia.*

Cinctellus, Rossi. Italia.

17 4-Oculatus. Laferté. Gall. or.

4-Guttatus. Waltl. Hisp. merid.

(4-Maculatus. (Dej.) id.

18 (4-Maculatus. Lucas. Algiria.*

Var. b. Brunneus, Laf. Gall, merid.

19 4-Guttatus. Rossi. Italia.*

4-Notatus. Gyll. Suecia?

Bifasciatus, Casteln, Hispania.

Guttatus, Laferté. Gall.merid.

20 Hispidus. Rossi. Italia.*

Hirtellus. Fabr. Suecia.

Bicolor. Oliv.

21 Ater. Panzer, Payk.

Morio. (Dej.)

Fuscicornis, (Dej.)

Luteicornis. (Dej.)

Unicolor. (Dej.)

22 Morio. Laferté.

23 Fuscicornis. Laferté. Ater. (Dej.)

24 Luteicornis. Schm. Ater. (Dej.)

25 Biguttatus, Laferté.

26 Genei. Laferté.

27 Flavipes. Panzer.

Rufipes. Payk. (Dej.)

Gall. merid.

Germ. bor.

Suecia.

id.

id.

id.

Graecia.

Gall. merid.

Hispania.

Gall. or.

Germania.

Sardinia

id.

Gall. or. ${ }^{*}$

Brunnipennis.St. v.d. Germania.

Obscurus. St. v. d. id.

28 Nigriceps. Mannerh. Finlandia.

29 (Fenestratus. Schmidt. Gall, merid. Pecchioli. Melly. Sicilia.

Mitis. Mus, Berol. Lusitania.

30 Axillaris. Schmidt. Lombardia. Affinis. (Dej.)

31 fumosus. Lucas. Bicolor. Lucas. v. $b$.

32 Insignis. Lucas. $\{$ Venator. Duft. (Dej.) Argentatus. Klug.

33 Longiceps. Laferté.

34 Dichrous. Laferté.

35 Nectarinus. Panzer. Bicinctus, Hummel. (Sibiricus. (Dej.)
Austria.

Algiria.

id.

id.

Hisp. merid.

Algiria.

Sicilia.

id.

Germania.

Sibiria.

id.
Terminatus. Schmidt. Gracia.

Ruficollis. Schm, v. $\varepsilon$, Gall. merid.

Nectarinus. (Dej.) Lusitania.

Mylabrinus. Géné. Sardinia.

38 Terminatus. (Dej.) Laf. Gracia.

39 Dejeanii. Laferté. Sardinia.

Nectarinus. (Dej.) Dalmatia.

40 Corsicus. Laferté. Corsica.

41 Fasciatus. (Dahl.)Chev. Gall, merid. Affinis. Laferté. id.

오 Antonia. Laferté. id.

Monogrammus. Schm. Austria.

o Unifasciatus. (Dej.)

v. $\beta$. Gall. merid.

ㅇ Bicinctus. (Dej.) v. b. id.

Histrio. Géné. var. ßß. Sicil. Hisp.

42 | Venustus. Villa. Laf. Lombardia.

IUnifasciatus. Schm. id.

43 Ghilianii. Laferté. Hisp. merid.

44 Aubei. Laferté. Algiria.

45 Zonatus. (Géné.) Laf. Sardinia.

46 Ochreatus. Lucas. Laf. Sicil. Alg.

47 Olivaceus. Laferté. Hispania.

48 Depressus. Laferté. Caucasus.

49 Pauperculus. Lucas.

Laf. Algiria.

50 Posticus. Laferté. Hisp. merid.

51 (Plumbeus. (Dej.) Laf. Gall. or. mer.

o Callosus. Schmidt. id.

of Melanarius. Schm. id.

? Brevis. Schmidt. id.

52 Capito. Laferté. Hispania.

53 Velutinus. Laferté. Pyr. or.

54 Velox. Laferté. Sicilia.

55 Caliginosus. Laferté. Tyrolis.

Fuscus. (Dej.) Dalmatia.

56 Unicolor. Schmidt. Germania.

Fuscus. (Dej.) Dalmatia.

57 Validicornis. Laferté id.

Niger. Olivier. Italia.

५s Scrobicollis. Laferté. Hispania.

\{ Fuscus. (Dej.) Dalmatia.

59 Friwaldszkyi. Laferté. Hungaria.

60 ? Tennicollis. Rossi. Etruria.

61 ? Limbatus. Fabr. Dania.

62 Paykullii. Gyllenh, in

63 Niger. Olivier. Sch. Alyiria.

64 Tibialis. Waltl. Hisp. merid.

65 ? Nigrinus. Gyllenh. Lapponia.

Instabilis. (Dej.) id.

66 Palicari. Castelnau. Gracia. 


\section{OCHTHENOMUS. Schmidt.}

1 Punctatus, (Dej.) Laf. Lucas. Hispania
Gall. or.

Elongatus. (Dej.) Hisp. merid. Retrofasciatus. Mot. Russ. merid.

3 Angustatus, Laferté. Hispania. Elongatissimus. Cast. Sardinia. Tenuicollis. Schmidt. Algiria.

4 Similis. Motschulsky. Russia.

5 Maritimus. Motsch. id.

AGNATHUS, (Meg.) Germar.

1 Decoratus.(Dej.)Germ.Gall. or.

XYLOPHILUS, Latreille.

Anthicus, Fabr. Notoxus. Panzer. AdeRUS. Schuckard.

1 Populneus. Fabr.

Gallia.

2 Oculatus, Paykull.

3 Pygmæus. de Geer.

Germania.

4 Testaceus. Dej. Cat. Dalmatia.

$\dddot{3}$ Lividus. Dej. Cat. id.

6 Pumilus. Dej. Cat. Gallia.

7 Punctatus. Stm. Cat. Germania.

8 Albosignatus. Stm.Cat.Finlandia.

9 Dimidiatus. Kunze. Lombardia.* 95

\section{1, FAII, SCYDHENI.}

\section{SCYDMFNUS. Latreille.}

Pselaphus, Herbst. Anthicus, Fabr. NoToxus. Panzer. LvtTa. Marsham.

\section{Godartii. Latreille. Gallia.*}

2 Scutellaris. Müller. id.*

3 Helferi. (Märkel.)

Schaum. Sicilia.

4 (Collaris. Müller. Erich. Gallia.*

$\{$ Tuberculatus, Chaud, Podolia.

Propinquus. Chaud. id.

5 Chevrieri. Heer. Helvetia.

6 Pusillus. Müller. Gall. bor.*

Var. Minutus, Gyllenh.

$\left\{\begin{aligned} & \text { Schaum. Suecia. } \\ & \text { Panzeri. Dej, Cat. } \text { Germania. }\end{aligned}\right.$

7 Dalmanni. Gyllenhal. Suecia.

8 Angulatus. Müller. Gall, bor.

9 Impressus, Sahlberg. Helvetia.

10 Elongatulus. Müller. Gall. bor.*

11 Sparshali. Denny. Anglia.

12 Longicollis. Motsch. Russia.

13 Rubicundus. (Kunze.)

Schaum. Saxonia.

Wighami, Denny.

Anglia.

15 Kunzei. Géné. Schaum. Sardinia. \{ Schönherri. Dej. Cat. Austria.

16

17

Pubicollis. Müller. Oblongus. Sturm.

Hirtus. Sahlberg. Germania.* Austria.*

Motschulskyi. Schm. f Pumilio. Schaum. Minutus. Chaudoir.

Parallelus. Chaudoir. Podolia.

Denticornis. Müller. Helvetia. *

Styriacus. Schaum. Germania.* Ruficornis. Denny. Clavipes. Schaum. Brevicornis. Schaum. id. Rutilipennis. Müller. id.* (Hirticollis. Gyllenhal. Gallia.* Fimetarius. Chaud. Russia. Minutus. Fabr.

Schaumii. Lucas. Claviger. Müller. Maklinii. Mannerh.

(Nanus. Schaum. Luc. Exilis. Schaum. Minimus, Chaudoir. Podolia. Wetterhalii, Gyllenh. Gallia. Quadratus. Müller et Kunze. Helvetia.* Angustatus. Lucas. Algiria.

f Exilis. Erichson. Germaria.

\{ Vicinus. Chaudoir. Podolia. Antidotus. Germar. Sicilia.* \{ Hellwigii. Fabr. Erich. Gallia.* $\left\{\begin{array}{l}\text { Cornutus. Motsch. id.* } \\ \text { ( }\end{array}\right.$ Rufus. Muill, et Kunze. id.* Clavatus. Sahlberg. Finlandia. Geoffroyi. Dej. Cat. Germania.

40 Tarsatus. Muiller. (Eu$\{$ mierus. Lap.) Gallia.* Hellwigii. Latreille, id. Minutus, Panzer. Germania.

41 Tauricus, Motschulsky. Tauria.

12 Ilelvolus. Schaum. Germania.

43 Intrusus. Schaum. id.

14 Punctipennis. Steph. Anglia. 
45 Longulus. Kunze.

46 Grohmanni. Kunze.

47 Olivieri. Dej. Cat.

48 Sahlbergii. Mannerh. Finlandia.

49 Linnei. Dej. Cat. Styria.

50 Rossii. Dej. Cat. Dalmatia.

ऽ1 Reaumurii. Dej. Cat. Gall. merid.

EUTHEIA. Waterhouse.

SCYDMANUS. Erichson.

1 ('Truncatellus, Erich. Germania.

Plicatus, Gyllenhal. (Cryptocep.) Suecia.

2 Abbreviatellus, Erich. Germania. Scydmanoides, Wat. Helvetia.

CEPHENNIUM. Muiller et Kunze.

Megaladerus. Steph. Trttoma.Wesmael. Scydmenus. Schaum.

1 Thoracicum. Müller et

2 Laticolle. Aubé. $\boldsymbol{P}^{*}$ Kunze. Gall. bor.*

3 Minutissimum. Aubé. id. 56

\section{FAII, PSELAPHI.}

\section{BATRISUS. Aubé.}

Pselaphus, Reich. Bryaxis. Denny.

1 Formicarius. $A u b e ́$.

Gallia.*

2 Delaporti. Aubé.

3 Venustus. Aubé.

Nigriventris, Denny. Anglia.

Brullei. Aubé.

ơ Buqueti. Aubé.

4 Oculatus, Aubé.

id.

id.

5 Thoracicus. Motsch. Russia.

\section{CHENNIUM. Latreille.}

1 Bituberculatum. Latr. Gall. bor.*

TYRUS. Aubé.

Erich. Pselaphus, Panzer.

1 (Mucronatus. Panzer. Germania.* Insignis, Reichenbach, id.

Sanguineus, Paykull. Suecia.

\section{CTENISTES, Reichenbach.}

Latr. Dronyx. Aud. Serv, et Lepel. de Saint-Farg.

1 Palpalis. Reichenbach. Gall, merid.* Io Dejeanii. Aud.Serv. P.

2 Ghilianii. Aubé.

Hisp. merid.

\section{PHARONUS, Aubé.}

1 Lafertei. Aubé. Gallia.

\section{PSELAPHUS. Herbst.}

Reichenb. Anthicus, Paykull, Fabr.

1 Heisei. Herbst.

$P$.*

\{Herbstii. Erichson. Germania.

2 Caucasicus. Motsch. Caucasia.

3 f Dresdensis. Herbst. Gallia.*

\{ Longicollis, Reichenb. Germania.

BRYAXIS. Leach.

Denny. Aubé. Anthicus. Fabr. ReichenBACHIA. Leach.

1 (Sanguinea. Fabr. P. .

Longicornis, Leach. Anglia.

(o Laminata. Motsch. Russ. merid.

2 Albana. Motschulsky. Tauria.

3 Fossulata, Reichen $b . \boldsymbol{P}^{\star}$.

4 Tibialis. Aubé. Sardinia.

5 (Xanthoptera, Reichenb. Gallia.

ơ Rubripennis. Aubé. P.

오 Depressa. Aubé. id. Assimilis? Curtis. Anglia.

6 Hemoptera. Aubé. P.* Xanthoptera. Aubé. id.

Spinicoxis. Motsch. id.

7 Lefebvrii. Aubé. . id.*

8 Helferi. Schmidt. Sicilia.*

Pulchella. Schaum. Saxonia.

9 Schuppelii. Aubé. Térgeste.

" 10 Hæmatica, Reichenb. P.*

\{Nodosa.Motschulsky, Russ, merid.

11 Furcata. Motschulsky. Algiria.

12 Juncorum. Leach. $P$.*

13 Chevrieri. Aubé. Italia.

14 Opuntiæ. Schmidt. Hispania.

15 Impressa. Panzer. Gallia.*

16 Transversalis. Schaum.Dalmatia.*

17 Autennata. Aubé. $P_{\text {。 }}{ }^{\star}$ 
18 lleterocera. Aubé. Luc, Algiria.

19 Aubei, Schaum.

Dalmatia.*

20 Pulchella. Géné.

Germania.*

TYCHUS. Leach.

Denny. Aubé. Psecaphus, Payk, Gyll.

1 Niger. Paykull.

Gallia.*

2 Ibericus. Motschulsky. Italia. P.

Dichrous? Schmidt. Germania.

3 Castaneus. Aubé. Hispania.

4 Tuberculatus. Aubé. Gallia.

\{ Dichrous? Schmidt. Germania.

BYTHINUS, Leach. Denny.

Aubé. Psela phus. Panzer. Reichenb. Arcopagus, Leach. Denny.

1 Clavicornis. Panzer. Saxonia.*

Glabricollis? Erich.

Reich. $\sigma^{7}$. id.

2 Nigriceps. (Kunzea.)

Leach. Gallia.

3 (Puncticollis. Denny. id.

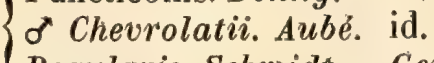

Regularis. Schmidt. Germania.

4 Validus. $A u b e ́$. id.*

5 Nigripennis. Aubé. Saxonia.*

6 (o Crassicornis. Motsch. Austria.

\& Longipalpis. Motsch. id.

Chaudoiri. Hochhuth. Russ. merid.

7 Femoratus. Aubé. Austria.

8 ( ${ }^{7}$ Bulbifer. Reichenb. Germania.*

$\{$ 을 Glabricollis, Gyll.

Aubé. Suecia.P.

9 Curtisii. Leach. P.*

10 Nodicornis. Aubé. Saxonia. *

$\{$ Steenbergii? Schmidt, Helvetia.

11 Securiger, Reichenb, $P^{*}$

Macropalpus. Aubé. id.

Globulipalpus, Aubé, id.

12 Burellii. Denny. Gallia.*

Luniger. Aubé. $P$.

13 Unicornis. Aubé. Gall, merid. \{ Burellii. Aubé.

id.

TRIMIUM. Aubé.

Lacord. Pselaphus. Reich. Gyll. Euplectus, Denny, Reich, Erich. Heer.

1 Brevicorne. Reichenb. Germania.*

2 Leiocephalum. Aubé. (Euplectus.) Gall, merid.
3 Brevipenne. Chaudoir. Russia.

4 (Schmidtii. (Euplectus.)

$\left\{\begin{aligned} \text { Märkel. Aubé. Pomerania. } & \text { Potiventris. Chaud. }\end{aligned}\right.$

EUPLEGTUS, Leach. Kirby.

Denny. Aubé. Lacord. Heer. Pselaphus. Illig. Anthicus, Fabr.

1 Märkelii. $A u b$ b́. $\quad \boldsymbol{P}^{*}$

Tuberculatus, Müller. Germánia.

Sulcicollis. Aubé. id.

2 Kunzei. Aubé. … Helvetia.

3 Erichsonii. Aubé. id.

4 Fischeri. Aubé. id.*

Tischeri. Heer. id.

5 Duponti. Aubé. P.

6 Signatus. Reichenbach. id.*

Kirbyi. Denny. Anglia.

7 Sanguineus. Denny. $P_{.}^{*}$

INigricans. Chaudoir. Russia.

8 Karstenii, Denny. Rei-

$\left\{\right.$ chenbach. $P .^{\star}$

Gracilis. Chaudoir. Russia.

9 Spinolæ. Aubé. Helvetia.

10 Nanus. Aubé. Gallia.

11 Piceus, Motschulsky. Russ, merid.

12 Ambiguus. Reichenb. Germ. bor.

$\{$ Pusillus.Denny, Aubé, P.

13 Minutissimus. Aubé. Saxonia.

14 Bicolor. Denny. Aubé. P.*

$\{$ Glabriusculus. Gyll. Suecia.

Fennicus, Maklin. Fennia.

15 Esterbroockianus.

16 Schmidtii. Märkel. Germania.

TRYCHONIX. Chaudoir.

Euplectus, Leach. Pselaphus, Reich.

1 (Sulcicollis. Reichenb. Gallia.

\{ Dresdensis, Illig, Fab. Germania.

71

\section{FAII. CLAVIGERI.}

\section{CLAVIGER, Preysster.}

Panz. Aubé. Gravifer. de Laporte.

1 Testaceus, Preyssler, Gallia.*

Foveolatus, Mïller, Germania. 
2 Coclchicus. Motsch. Russ. merid.

3 Longicornis. Panzer. Aubé. Gallia.

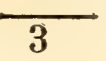

\section{FAII. STAPHYLINI.}

MYRMEDONIA, Erichson.

Paderus. Rossi. Drusilla. Mannerh. Boisduv, et Lacordaire. Aleochara. Gravenh, Latr. Gyll. Staphylivus. Fabr. Payk. Rossi, Oliv, Panz. Germar. Guérin.

1 Canaliculata. Fabr. Gallia.* Impressa, Rossi. Italia.

2 Plicata. Erichson. Gall. bor.

3 Limbata, Payk. Gallia.* Var, Foveicollis. Lac. P. Divisa. Marsh. Anglia. Lavis. Gravenhorst. id.

4 Humeralis. Gravenh. P.*

5 Funesta, Gravenhorst, id.*

6 Atrata. Heer. Helvetia.

7 Rigida. Erichson. Sardinia.

8 Lugens. Gravenhorst. Gallia.*

9 f Fulgida, Gravenhorst, id. Haworthi. Stephens. Anglia.

10 Collaris. Payk. Gall. bor.*

11 Memnonia. Märkel. Saxonia.

12 Tristis. Lucas. Algiria.

13 Laticollis. Märkel. Gallia.*

14 Cognata.Märkel. id.*

15 Similis. Märkel. id.

16 Nigricollis. Motsch. Petropolis.

AUTALIA. Leach.

Aleochara, Gravenh. Latreille. StaphyLINUS. Olivier.

1 Impressa, Olivier. Gallia."

2 Rivularis, Gravenh. id.*

FALAGRIA, Leach.

Aleochara. Gravenhorst.

1 S Sulcata. Paykull.

Gallia.* Casa, Erichson.
2 (Sulcatula, Gravenh. Gallia.*

Sulcata, var. B. Gyll, Suecia.

Polita. Curtis. Anglia.

3 (Thoracica. Curtis. Gallia.*

Lineolata. Lacord. $P$.

Ruficollis. Waltl. Germania.

4 Obscura. Gravenhorst. $P^{*}$

5 jigra. Gravenhorst. id.*

Fracticornis, Grav. id.

Picea. Gravenhorst. id.

6 Pusilla. Heer. Helvetia.

\section{BOLITOCHARA, Mannerheim.}

Aleochara. Gravenhorst.

1 Lucida, Gravenhorst, Germania.* $\{$ Var. Lunulata. Gyll. Suecia.

2 (Lunulata. Paykull. Gallia.*

Cincta. Gravenhorst. Germania.

Pulchra. Lacordaire. P.

3 Elongata, Heer. Helvetia.

4 Obliqua. Erichson. Germania.

Cincta. Lacordaire. $\boldsymbol{P}$.

5 Elegans. Heer. Helvetia.

6 Varia, Erichson. Sardinia.

7 Humeralis, Lucas. Algiria.

8 Bella. Märkel. Germania.

\section{OCALEA. Erichson.}

Aleochara. Gyllenhal. Sahlberg. BoliTOCHARA. Mannerheim. Gyll.

1 Castanea, Erichson. Germania.*

2 Alpina. Heer. Helvetia.

3 Badia. Erichson. Berolini.

4 Oblita. Chevrier. Heer. Helvetia.

5 Spadicea. Erichson. Berolini.

6 . Procera. Erichson. Germania.

7 Murina. Erichson Austria.

8 Decumana. Erichson. Gallia?

9 Prolixa. Gyllenhal. Suecia.

10 Rufilabris. Sahlberg. Finlandia.

\section{CALODERA. Mannerheim.}

Aleochara. Gravenh, Gyl. Bolitochara. Lacord. Staphylinus. Payk.

1 Nigricollis, Payle. Gallia.*

2 Forticornis. Lacord. P.

3 Nigrita. Mannerheim. Germania.

4 Humilis. Erichson. German, bor. 
5 Pusillima. Heer. Helvetia.

6 Longitarsis. Erichson, Gallia, ${ }^{*}$

7 Rubicunda. Erich. Austria.*

8 Rubens. Erich. Berolini.

9 Uliginosa. Erich.

10 Riparia, Erich.

11 Oculta. (Chevrier.) Germania.* id.

Heer. Helvetia.

12 Ethiops. Gravenh. Germania. * \{ Testacea. Mannerh. Suecia.

13 Linearis. Heer. Helvetia.

14 Umbrosa. Erich. Berolini.

15 Protensa, Gyllenhal.

Mannerh. Suecia.

\section{TAGHYUSA. Erichson.}

Drusilla. Mannerh. Aleochara. Gravenh. Bolitochara. Mannerh. Leucopus. Marsh.

\section{Constricta. Erichson. Gallia.* \\ 2 Nigrita.Chevrier.Heer.Helvetia. \\ 3 Coarctata, Erich. Gallia.* \\ 4 Concinna.Imhoff.Heer. Helvetia. \\ 5 (Balteata. Erichson. id.* \\ Flavocincta. Dahl. \\ Chevrier. id. \\ 6 Ferialis. Erich. \\ 7 Scitula. Erich. \\ Sardinia. \\ 8 Excavata. Mannerh. Austria. \\ 9 Læsa. Erich. \\ 10 Atra, Gravenh. Sardinia. \\ 11 Umbratica. Erich. \\ 12 Fugax. Erich. \\ Anglia. \\ 13 Lata. Kiesenwetter. \\ 14 Uvida. Erich. \\ 15 Chalybea. Erich. \\ 16 Immunita. Erich. \\ 17 Flavitarsis. Sahlberg. Finlandia. \\ 18 Coracina. Sahlberg. Lapponia. \\ 19 Carbonaria. Mannerh. Finlandia. \\ 20 Corulea. Sahlberg. id.}

\section{PHLOEOPORA, Erichson.}

Aleochara. Gravenhorst. Bolitochara. Mannerh.

1 Reptans. Gravenhorst.Suecia.*

2 Corticalis. Gravenh. Germania.* T Tenuis. Gravenhorst. id.
HYGRONOMA, Erichson.

Homalota, Curtis, Aleochara, Gravenh.

1 Dimidiata, Gravenh. P.*

2 Quercina. Chevrolat.

in lilt. id.

\section{HOMALOTA. Mannerheim.}

Bolitochara. Mannerheim. Lacordaire. Aleochara. Gyllenh. Gravenh. Latr. IIannerh. Zetterst. Sahlberg. Oxy poda. Mannerh. Paderus. Gravenh. StaphyLINUS. Marsham. Fabricius.

1 Graminicola. Gravenh. Gallia.*

Linearis. Gyllenh. Suecia.

Longiuscula. Gyllenh, id.

Longicornis, var. $b$.

Mosta. Zetterst. $\quad$ id.

Fracticornis, Lacord, $P$.

2 Umbonata. Erichson. Austria. ${ }^{*}$

3 f Ridigicornis. Erich. Geneva.

\{ Fusca. (Semiris.) Heer, Helvetia.

4 Occulta. Erichson. Germania.

5 Pagana. Aubé. Erich. $\boldsymbol{P}$.

6 Vestita. Gravenh. Suecia.

\{ Quisquiliara.Gyllenh.id.

7 (Callicera. Gravenh. Germania.* Obscura. Gravenh. id.

Spencei.Curtis. Anglia.

8 Paveus. Erichson. Germania.*

\{ Quisquiliarum. Erich. id.

9 Languida, Erich. id.

10 Gracilicornis, Erich, Austria.

11 Debilicornis. Erich. Sardinia.

12 Gregaria. Erich, Austria.

13 Ravilla. Erich. id.

14 Labilis. Erich. $P^{*}{ }^{*}$

15 Velata. Erich. - Gallia.*

16 Luteipes, Erich. Berolini.

17 Elongatula. Gravenh. Gallia*

Var, Terminalis, Grav. id.

Elongatula, Gravenh.

Latr, $P$.

Complana. Mannerh. Finlandia.

Oblonga. Lacordaire. P.

Exilis. Mannerh. Suecia.

Planiuscu?a, Mannh. id.

Depressiuscula. Mannerh, $P$. 
18 Rugulosá. Heer.

19 Linearis. Gravenh.

Helvetia.

Germania.

20

f Augustula. Gyllenh.

P.*

Linearis, var. Grav. Germania.

21 Tenuis. Heer.

22 Venustula. Heer.

23 Rufipes. Heer.

Helvetia.

24

25

26

27

28

29

30

31

32

33

34

35

36

Vaga. Chevrier. Heer.

Femoralis. Heer. id.

Fracticornis. Heer. id.

Equata. Erich.

Nigella. Erich.

\section{Germania.*}

Berolini.

id.

id.

d.

59

60

\section{1}

62

Niralis. Kiesenwelter. Germania."

Arcana. Erich.

Plana. Mannerh.

Thuringensi.

Germania.

Debilis. Erich. Berolini.*

Gracilenta. Aub. Erich, P.

Macella. Erich.

Bavaria mer. 66

Gallia. $^{*} \quad 67$

Cuspidata. Erich

Inconspicua. Gravenh. in litt. Heer.

Immersa. Erich.

37 Vilis. Erich.

38 Cæsula. Erich.

39 Circellaris. Gravenh.

40 Polita. Rosenhauer.

41 Procidua. Erich.

42 (Brunnea. Fabr.

Depressa. Gyllenh.

Nigriceps. Marsh.

43 Depressa. Gravenh.

44 Nigrifrons. Erich.

45 Atricila. Erich.

\{ Laticollis. Chevrier.

46 Oblonga. Erich.

Elongatula. v. Grav. id

47 Hepatica. Erich. id.

48 Socialis. Payk. Gallia.*

Boleti. Gravenh. id.

Sordida. Marsham. Anglia.

Sericans, Grav. Heer. Suecia.

Var. Castaneoptera.

Mannerh. id.

Pubescens. Heer. Helvetia.

Longicornis. var. $b$. Gyllenh. Suecia.

Boleti, Lacordaire, $P$.

Nigricula.v. c. Grav. Suecia.

49 Testaceipes. Imhoff.

$$
\text { Heer. Helvetia. }
$$

$\because 0$ Sodalis. Erich. Germania.

5) Erythrocera. Gravenh. in litt, Heer. Helvetia.
63

52

Heer.

Helvetia.

Ochracea. Erich. Gallia.*

34 Melanocephala. Heer. Helvetia

5 So Rubricollis. Chevrier.

56

57

58

Heer. id.

Marcida. Erich.

Alpestris. Heer.

Germania.

Planiuscula. Heer.

Helvetia.

Spelæa. Erich.

Incana. Erich.

Excavata. Gyllenh.

Deplanata. Gravenh.

Viduata. Erich.

id.

Carinthia.

Germania.

$P$.

Germania.

id.

(Atramentaria. Kirby.

Gyll. Erich. id.

Enescens. Zetterst. Lapponia.

Gemina. Erich. id.

Autumnalis. Erich. id.

Oblita. Aubé. Erich. $P$.

68 Talpa. Chevrier, Heer. Helvetia.

69 Glaucula. Erich. Berolini.

70 Contemta. Heer. Helvetia.

71 Analis. Gravenh. Gallia."

Evanescens. Mannerh. Suecia.

Var.Bifoveolata. Man-

(nerk. id.

72 Parallela. Mannerh. Finlandia.

73 Exilis. Erich. Germania.*

$\{$ Var. Analis. Gravenh. id.

74

Indigena. Heer.

Helvetia.

75

Palleola. Erich.

Germania.*

76 Indocilis, Chevrier.

77

Heer. Helvetia.

Nigriceps. Heer. id.

78 Inconspicua. Erich, $\boldsymbol{P}^{*}$

79 Longipennis. Heer. Helvetia.

80 Inquinula. Gravenh. Germania.

81 Minutissima. Heer. Helvetia.

82 Longula. Chevrier.

83 Canta. Erich. Germania.*

Pulicaria. Erich. id.

84 Egra. Heer.

Helvetia.

85 Cælata. Erich,

Germania.*

86 Morosa.Chevrier.Heer.Helvetia.

87 Sordidula. Erich. Berolini.*

88 Impressa. Heer. Helvetia.

89 Tibialis. Heer. id.

90 Luteicornis. Erich. Berolini.

91 Hæmorrhoidalis. Heer, Helvetia.

92 Flavipes. Gravenh. Gallia.

93 Subsinuata. Erich. Germ. merid.

94 Anceps, Erich.
Berolini. 
Morio. Heer.

96 Notha. Erich.

131 Macilenta. Sahlberg. Lapponia. Pygmaa: Zetterst. id.

132 Fusca. Sahlberg. id.
133 Scapularis, Sahlb. Finlandia.

134 Borealis. Sahlberg. Lapponia.

135 Marginalis. Gravenh. Gallia.

136 Mannerheimii. Sahlb. Finlandia.

137 Parva. Sahlberg. id.

P Parvula. Mannerh. id.

138 Pallidula. Mannerh. id.

139 Tenera. Sahlberg. id.

140 Pallipes. Lucas. Algiria.

141 Impressifrons. Manh. Finlandia.

142 Tarda. Motschulsky. Lithuania.

\section{OXYPODA. Mannerheim.}

Aleochara. Gyllenh. Sahlb. Gravenh. Latr. Zetterst. Bolitociana. Mannerh. SPhenoma, Mannerh.

1 Ruficornis. Gyllenhal. Gallia.

Luteipennis, var.

Erich. Germania.

2 Luteipennis. Erich. id.*

Ruficornis, vr. b. Gyl. Suecia.

3 Vittata. Märkel. Gallia. $\boldsymbol{P}^{*}$

4 Prospera. Erich. German.bor.

5 Opaca, Gravenh. Gallia.*

Pulla. Gravenh. Latr. id.

6 Nitidula. Heer. Helvetia.

7 Umbrata. Gyllenh. Germania.*

8 Longiuscula. Gravenh. id. Procelura. Mannerh. id.

9 Alternans, Gravenh, Gallia.*

10 Togata. Erich. Berolini.*

11 Abdominalis. Mannerh. Germania.

12 Testacea. Erich. Germ. sept.

13 Helvola. Erich. Germania.*

14 Ferruginea, Erich. id.*

15 Promiscua. Erich. id.*

16 Præcox, Erich. Austria.

17 Exoleta. Erich. Germania.

18 Cuniculina. Erich. Bavaria.*

19 Formiceticola, Mürk. Saxonia.*

20 Familiaris. Kiesenw. Germania.

21 Exigua, Erich, Berolini.

22 Litigiosa. Chevrier.

\section{Sericea. Heer. id."}

24 Mirmecophila, Märket. Saxonia.*

25 Myrmecobia. Märkel. id.*

26 Spectabilis. Märkel. id.

27 Cunctans. Erich. Berolini.

28 Lentula. Erich. id.

29 Curtula, Erich. id. 
Melanaria. Mannerh. Finlandia.

Pellucida. Mannerh. id.

Lateralis. Mannerh. id.

Sericata. Mannerh. Russia.

Cingulata. Mannerh, id.

Corticina. Erich, Germania.

Varia.(Chevrier.) Heer. Helvetia.

Amicta. Friwaldszky. Sardinia.

Analis, Gyllenh. Suecia.

Gracilis, Erich.

Prolixa, Erich.

Berolini.

Ruficollis. Erich. $\quad \boldsymbol{P}^{\star}{ }^{\star}$

Fumida. Erich. id. ${ }^{\star}$

Maura. Erich.

Infuscata. Kellner.

Similis. Kellner.

Leporina. Kiesenw.

Latiuscula. Mannerh. Gat

Gilvipes. Mannerh.

Suturalis. Sahlb.

Pulchella. Sahlb.

Elegantula. Sahlb.

Dimidiata, Motsch.

Inflexa. Motschulsky.

Diluta. Motschulsky.

Stabilis. Motschulsky. Russia.

Atramentaria, Motsch. id.
Tenuicornis. Motsch. Russ, orient.

\section{ALEOCHARA. Gravenhorst.}

Staphylinus, Fabr. Fourcroy. Geoffroy. Schrank.

$\cdot$ $\left\{\begin{array}{l}\text { Fuscipes. Fabr. } \\ \text { Brachyptera. Fourc. } \\ \text { Var, Lata. Gravenh. }\end{array}\right.$

2 Rufipennis. Erich. Lavigata. Lacordaire, $P$.

3 Tristis, Gravenhorst. Gallia.* Geometrica.Schrank. Germania. Var. Crassiuscula.

Sahlberg. Gallia.

4 (Bipunctata. Grav. Ol. id.*

Intricata. Mannerh. Finlandia.

5 Scutellaris, Lucas. Algiria.

6 Brevipennis, Gravenh. P.*

\{ Carnivora. Gyllenh. Suecia.

7 Fumata. Gravenh. Gallia.*

Curta. Sahlberg. id.

8 Bisignata. Erich. Germania.

9 Nitida. Gravenh. P.* I Var. Bilineata. Gyll. id.
10 Sparsa. Heer.

11 Morosa. Heer.

Helvetia.

12 Punctata. Motschulsky. Lithuania.

13 Lanuginosa. Gravenh. $P$.*

14 Monticola. Roserh. Tyrolis.

15 Brevis. Heer. Helvetia.

16 Mœrens, Gyllenh. Germania.

Fumata. var. Gravenhorst. id.

17 Møsta. Gravenh. Gallia*

Fumata. Gyllenh. Suecia.

Hamorrhoidalis.Man-

nerheim. Lapponia.

18 Rufitarsis. Heer. Helvetia.

19 Ruficornis. Gravenh. Germania.*

20 Erythroptera. Gravh. id.

Mosta. var. b. Erich. id.

21 Pulla. Gyllenh. P.*

22 Spissicornis. Erich. id. ${ }^{*}$

23 Lævigata. Gyllenh. Suecia.

24 Longula. Imhoff. Heer. Helvetia.

23 Morion. Gravenh. Germania.*

\{Exigua. Mannerh. Suecia.

26 Intractabilis. Chevrier.

27 Angulata, Erich. $\boldsymbol{P}^{*}$

28 Prætexta, Erich. Gallia.

29 Gentilis, Lunemann. Finlandia.

30 Obscurella. Gravenh. $\boldsymbol{P}$.

Sericea. Lacordaire. id.

31 Inquilina, Märkel. Saxonia.

32 Brevis, Heer. Helvetia.

33 Villosa. Mannerh. Finlandia.

34 Grassicornis. Lacord. $\boldsymbol{P}$.

PHYTOSUS. Rudd.

1 Spinifer. Curtis.

Anglia.

\section{OLIGOTA. Mannerheim.}

Aleochara. Gravenh. Hypocyptus. Lacord.

1 Pusillima. Gravenh. Gallia.*

2 Atomaria. Erich. Berolini.

3 Subtilis, Erich. Germania.*

4 Punctulata. Heer. Helvetia.

5 Granaria. Erich. Germania.

6 Flavicornis. Erich. 'P.

7 Apicata. Erich. Gallia.

8 Tantilla. Mannerh. Finlandia. ${ }^{*}$ 
GYROPHANA. Mannerheim.

Aleochara. Gyllenh. Saillb. Gravenh. Latr. Bolitochara. Mannerheim. Encepualus Westw. Steph. StaphyliNus. Payk. Marsh. Linne. Heer, Müll. Goze de Villers.

1 Complicans. Westw. Germania.

2 Nitidula, Gyllenh. Suecia.*

3 (Nana. Payk. $P_{\text {. }}^{*}$ Nitidula, Lacordaire, id. Fasciata. Marsham. Anglia.

4 Affinis. Sallberg. P.* Amabilis. Lacordaire. id. Nana. Lacordaire. id.

5 ) Pulchella. Heer. Gentilis. Erich.

6 Congrua. Erich.

7. Lucidula, Erich.

8 Manca. Erich.

9 Polita, Gravenh. Gallia.*

10 Exigua. Imhoff. Heer. Helvetia.

11 Strictula, Erich. Hungaria.

12 Boleti. Lin. (Homalot.)

Heer. Germania.

13 Minima, Erich. Berolini.

14 Pigmæa. Sturm. Germania.

15 Ceranota. Stephens, Anglia.

16 Daltoni. Kirby. id.

17 Lævigata. Märkel. Germania.*

\section{PLACUSA. Erichson.}

Aleochara, Gravenh, Latr, Gyll. Sahlb. Zett. Bolitochara. Mannerh. Lacord.

\section{Complanata, Erich.}

2 Pumilio. Gravenh.

3 Humilis. Erich.

4 Infirma. Erich.

Tachyporoides. Waltl. id.

5 Adscita, Erich. Sardinia. 6 Atrata. Mannerh. Finlandia.
Germania. Gallia. Germania. id.

\section{EURYUSA. Erichson.}

1 Sinuata, Erich.

2 Obtabilis. Heer.

3 Acuminata. Märkel.

4 Coarctata. Märkel.

5 Linearis. Märkel.

6 Formicaria, Motsch.
Germania. ${ }^{*}$ Helvetia. Gall. bor.* Saxonia.* id.* Lithuania.
DINARDA. Leach.

Lomechusa. Gravenh. Gryll. Zett. StaphyLinus. Payk.

1 Dentata. Gravenh, Gallia.* Strumosa. Payk. Suecia.

2 Märkelii. Kiesenwetter. Gall. bor.*

LOMECHUSA. Gravenh.

Staphylinus, Payk. Oliv, Fabr. AleoCHARA, Gravenh. Latr.

1 Strumosa. Fabr. Gallia.*

21 Paradoxa. Gravenh. Gallia.*

V. Emarginata. Payk. Suecia.

3 Emarginata. Payk. Gallia.*

4 Inflata. Zetterst. Lapponia.

SILUSA. Erichson.

1 Rubiginosa. Erich, $\quad \boldsymbol{P}_{\text {. }}^{*}$

2 Rubra. Erich. Germ. merid.

3 Rufa.Chevrier. Helvetia.

4 Alpicola. Heer. id.

\section{PRONOMEA. Erichson.}

1 Rostrata. Erich. Gallia.*

DIGLOSSA. Haliday.

1 Mersa. Haliday. Hibernia.

\section{MYLLANA. Erichson.}

Grunusa, Mannerh, Centroglossa. Mathews. Aleochara, Gravenh.

1 Dubia, Gravenh. Gallia." \{ Cornuroides. Mathews. Anglia.

2 Intermedia. Erich. Germania.*

3 Minuta. Erich. id.

4 Gracilis. Heer. Helvetia.* Grandicollis. Kiesenw. Germania.

GYMNUSA, Karsten.

AleocharA. Gyll. Sahlb, Zetterst, Gravenh. Staphylinus, Payk.

1 (Brevicollis. Payk, Germania.* Carnivora. Gravenh, id.

Excusa. Gravenh. Suecia. 
2 Laticollis, Erich.

3 Fuscata. Mathews.

Gallia.*

4 Variegata. Kiesenwett. id.

\section{HYPOCYPTUS. Schüppel.}

Scaphydum, Payk. Gyll, Sahlb. TachyPoRUS. Gravenh.

1 Longicornis. Payk. Gallia.* Acuminatus, Marsh. Anglia. Granulum. Gravenh. Suecia. Globulus, Lacordaire. P.

2 Ovulum. Heer.

Helvetia.

3 Discoideus. Erich. Berolini. *

Biguttalus. Mathews. id

4 Læviusculus. Mannh. Gallia.*

5 Nigripes. Heer.

Helvetia.

6 Seminulum. Erich.

7 Pulicarius. Erich.

8 Parvulus. Stephens, Anglia.

\section{CONURUS. Stephens.}

TAchyponus, Lacd. Gravenh, Latr, Erich. Gyll. Sahlb, Mannerh. Oxyponus, Fab. Staphylinus. Lin. Payk. Rossi. Fourc. Fabr. Geoff.

Littoreus. Linné. Cellaris. Fabr. Pubescens. Payk.

Gallia.*

id.

2-Maculatus. Fabr. id

Maculatus, Fourcroy. $P$.

2 Pubescens. Gravenh. Gallia.*

Cellaris.v. B.Payk. Suecia.

Tomentosus, $v$. B.Ross, Italia.

Sericeus, Lacordaire. $P$.

Testaceus. Fabr. id.

3 Bipustulatus. Gravenh. Hungaria.

Bimaculatus, Gravh. id.

4 Obscuripennis. Stephens. Anglia.

5 Binotatus. Gravenh. Germania.

6 Fusculus. Gravenh. id. ${ }^{*}$ Pedicularius. Lacordaire. $P$.

7 Lividus. Erich. id.*

8 (Pedicularius. Gravenh. Gallia.* Var. Truncatellus. Graven/. Suecia.

9 Bipunctatus. Gravenh. Germania.
TACHYPORUS. Gravenhorst.

Oxyponus. Panz, Fabr. Staphylinus. Fabr. Schr. Goze de Villers. Rossi. Oliv. Payk. Waltl. Müll, Geoff.

1 Obtusus. Linné. Analis. Fabr. Gallia.*

Vernalis. Müller. Germania.

Dispar. var. y. Pay-

kull. Suecia.
Chrysomelinus. var. 2. Walknoer. P.

Melanurus. Marsham. Anglia.

2 Abdominalis. Gyllenh. Gallia. *

3 Rufus. Erich. Germania.

4 (Formosus. Mathews. Anglia:

Abdominalis, Lacor-

5 Saginatus, Gravenh. Germania.*

6 Erythropterus. Panz. (Lamprinus.) Heer. Germ. merid.

7 Hypnorum. Fabr. Gallia.*

Nitidulus, Fabr. Oliv. id.

Minutus, Fabr. Suecia.

Marginatus, Panzer. Germania

Blattinus. Schrank. id.

Conicus. de Villers. $\boldsymbol{P}$.

Martialis. Schrank. id.

Dispar. var. . Payk. Suecia.

Chrysomelinus, var.

$$
\text { Walk. } P \text {. }
$$

8 Chrysomelinus. Linné. Gallia.

Melanocephalus. Fab. id.

Merdarius. Marsh. Anglia.

Chrysomelinus, var.a.

Payk. Suecia.

9 Solutus. Erich. Germania.*

Saginatus, Lacord. $P$.

10 Tersus. Erich. Germania.

11 Humerosus. Knoch. id.*

Lateralis. Gravenh. Gallia.

(Ruficollis. Runde. id.

12 Ruficollis. Gravenh. Germania.*

Chrysomelinus. var.

Rossi, id.

13 Pulchellus, Heer. Helvetia.*

14 Pusillus. Gravenh. $P_{\text {. }}^{*}$

15 (Scitılıs. Erich. Gallia. *

Prusillus, Gravenh. $\quad P$.

16 'Transversalis. Gravh. Gallia.* Ruficollis, Gyllenh. Suecia.

Dispar. var. Payk. id. 
17 Brunneus. Fabr. $\quad P^{*}$

Nitidulus. Olivier. Suecia.

Abdominalis. Gravh. id.

Scutellaris. Lacord. P.

18 Nigricornis, Gyllenh. Suecia.

19 Obscurellus. Zetterst. id.

20 Nigriceps. Mannerh. Finlandia.

21 Crassicornis. Mannh, id.

\section{HABROCERUS, Erich.}

1 Capillaricornis. Gravh.P.*

TACHINUS, Gravenhorst.

Oxyponus, Panzer, Fabr. Tachyporus. Gravenh. Staphylinus. Linné. Gœze. de Villers. Marsham. Payk. de Geer. Walk. Geoff. Panz.

1 Silphoides. Linné. Suturalis. Panzer.

Marginalis. Gravenh. id

Dispar. var. \&. Payk. Suecia.

2 (Rufipes. de Geer.

Pullus. Gravenh.

Signatus, Gravh. Latr. Gallia.

Var. Laticollis. Gyll. Suecia.

Pallens. Gyllenh.* id.

3 Flavipes. Fabr. Gallia.*

Rufipes. Marsham. Anglia.

Var.Castaneus. Grav,

Latr. Germania.

Dubius. Gyllenh. Suecia.

4 Humeralis. Gravenh. Gallia. ${ }^{*}$

\{ Cinclus, var. Marsh. Anglia.

5 Subterraneus. Linné. Gallia. *

Var, Marginatus. Fab. Germania.

Bicolor. Gravenh. Suecia.

Biplagiatus. Lacord. P.

Latus. Marsh. Anglia.

6 Bipustulatus. Fabr. Gallia.*

Subterraneus, var.

Humeralis. v. 1. Grav. Suecia.

8 Marginellus. Gravenh. Gallia.*

Laticollis. Gravenh. $P$.

Lavigatus. Marsh. Anglia.

Intermedius, Mannh. Suecia.

Rufipes. var. B. Payk. Suecia.

Marginalus. Fourc. $P$.

Immaturatus, Gravh. Germania.
9 Fimetarius. Gravenh. Gallia.* Sordidus. Gravenh. Suecia. Fuscipes. Panzer. Germania.

10 Collaris. Gravenh. Gallia.* $\{$ Var. Corticinus. Grav. Suecia. Flavellus. Zetterst. id.

11 Elongatus. Gyllenh. Helvetia.

12 Discoideus. Erich. Austria.

13 Rufipennis. Gyllenh. Suecia.

\section{TRICOPHYUS. Erich.}

Tricophia. Mannerh.

1 Pilicornis. Gyllenh. Germania.

\section{BOLETOBIUS, Leach.}

Tachinus, Gravenh. Gyll. Latr, Sahlb. Zett. Briocharis, Lacord. Oxyporus. Rossi. Fabr. Panz. Macronus. Steph. Staphylinus, Payk. Oliv. Fabr. Gaze. Panz. Schrank. Marsh. Lin. Müller. de Villers.

1 Analis. Payk. Gallia. ${ }^{*}$ Y Var. Merdarius, Gyll. Suecia.

2 Cingulatus. Mannerh. Gallia. Analis. var. 1 et 3. Gravenh. Suecia. Bicolor. Rossi. Italia.

3 Inclinans. Gravenh. Germania.

4 Punctulatus. Heer. Helvetia.

5 f Formosus. Gravenh. Germania. Merdarius. Gravenh. id.

6 Cernuus, Knoch. Gravenh. Gallia. Var. Merdarius. Oliv. $\boldsymbol{P}$.

7 Rufus. Fischer. Erich. Germania.

8 Bicolor. Gravenh. Hercynia.

9 iStriatus. Olivier. Gallia. ${ }^{*}$ \{Angularis. Payk. Suecia. Analis. var. y. Payk. id.

10 Atricapillus. Fabr. Gallia.* Lunulatus. Fabr. Germania.

11 Speciosus, Erich. Hungaria.

12 Lunulatus. Linné. Gall. bor.* Atricapillus. Zetterst, Suecia.

13 Trimaculatus. Fabr. Germ. bor.* \{Littoreus. Payk. Suecia.

14 Trinotatus. Erich. Germania.* \{ Bimaculatus. Schrank. id.

15 Exoletus, Erich. Gallia. 
16

$\left\{\begin{array}{cl}\text { Pygmæus. Fabr. } & \text { Gallia.* } \\ \text { Thoracicus. Fabr. } & \text { Germania. } \\ \text { Melanocephalus.Grav.id. } & \multicolumn{1}{c}{\text { Payk. }} \\ \text { 3-Maculatus,var. B.y. } \\ \text { Merdarius. Runde. } & \text { id. } \\ \text { Pallidus. Rossi. } & \text { Italia. }\end{array}\right.$

MYCETOPORUS. Mannerheim.

Ischnosoma, Steph. Tachyoros. Gyll. Zett. Sahlb. Tachy nus. Gravenh. Oxyporus. Fab. Staphylinus, Payk. Schr. Fabr. Gaze.

1 Splendens. Marsh. Germania.*

2 Punctus. Gyll. Erich. id.*

3 Longulus. Mannerh, $\boldsymbol{P}^{*}$

4 Semirufus. Heer. Helvetia.

5 Lepidus. Mannerh, Gallia.*

Punctatostriatus.var.

a. y. Payk. Suecia.

Merdarius. Schrank. Germania.

Bimaculatus, Lacord. P.

Tristis, Gravenh. Suecia.

6 Pronus. Erich. Germania.*

7 Nanus. Gyllenh. id.

8 Lucidus. Erich. id.*

9 Splendidus, Gravenh. $\boldsymbol{P}$.*

TANYGNATUS, Erichson.

1 Terminalis. Erich. Berolini.

\section{OTHIUS. Leach.}

Cafius. Lacord. Groohypus. Mannerh. Nordm. Penenus, Fabr. Staphy Linus. Fourcroy. Geoff. Gravenh. Latr. Gyll. Sahlb. Lacord. Payk. Schr. XanthoLINUS. Zetterst.

\footnotetext{
1 (Fulvipenuis. Erich. P.* Fulgidus. Payk. Suecia.

Fulminans. Gravenh, id.

Fulvopterus. Fourc. Gallia.

Ustulatus. Gravenh. Germania.

2 Sardeus. Sturm. Sardinia.

3 Melanocephalus. Gra-

$$
\text { venh. Gallia.* }
$$

4 Fuscicornis. Heer. Helvetia.

5 Punctipennis. Lacor-

daire. $P_{0}^{*}$
}

$6 \begin{aligned} & \text { Pilicornis. Payk, Suecia.* } \\ & \text { Var. Alternans. Grav. Gall. bor. } \\ & \text { Nigriceps, Mannerh. Germania. } \\ & \text { Affinis. Payk. Suecia. } \\ & \text { Myrmecophilus. Kie- }\end{aligned}$ senwetter. Germania.

\section{XANTHOLINUS, Dahl. Erichson.}

Staphylinus. Gravenh. Latr, Gyll. Sahlb Oliv, Marsh. Payk. Fabr, Panz. Fourc. Muill. Gæze. Paderus, Fabr. Panz. Gyrohyprus. Mannerh. Nordm. Runde

1 Fulgidus. Fabr. Pyropterus, Gravenh. $\boldsymbol{P}$.

2 Glabratus, Gravenh. Gall, orient.* Fulgidus, Gravenh. id.

Relucens. Nordmann. Suecia.

Nitidus. Panzer. Germania. $\{$ Cruentatus. Marsh. Anglia.

Merdarius. Nordm.

Var. Cadaverinus. Lacordaire. $\boldsymbol{P}$.

Ochropterus. Nordm.

3 Rufipennis, Erich.

4 Elegans. Olivier. $\{$ Meridionalis, Lacord, $P$.

5 Collaris. Erich. Germ. merid.

6 Decorus. Erich. Austria.

7 Glaber. Nordm. Germania. Lentus, var, b. Zett. id.

Diaphanus. Marsh. Anglia.

8 Ruficollis. Lucas. Algiria.

9 Rutipes. Lucas. id.

10 Lentus. Gravenh. Germania.* Glaber. var. 1. Grav. id. (Tricolor, var. U. Payk. Suecia.

11 Punctulatus. Payk. Gallia.*

Elongatus. Fourcroy. $P$.

Fracticornis. Müller. Germania.

Var, Ochraceus, Gyll. Suecia.

12 Atratus, Heer. Helvetia.

13 Hespericus. Erich. Lusitania.

14 Procerus. Schm. Erich, Germ, bor.

15 Tricolor. Fabr, Gallia.*

Elegans. Gravenh. Germania.

Affinis. Marsh. Anglia.

16 Linearis, Olivier. Gallia.*

Longiceps. Gravenh. Germania.

Punctulatus. Schrank. id.

Var. Ochraceus. Grav. P.

17 Longiventris. Heer. Helvetia 
LEPTAGINUS. Erichson.

Xantholinus. Lacordaire. Gyrohypnus. Mannerh.Runde. Nordm. StAPHylivus. Gyll. Sahlb. Gravenh. Latr.

1 Brevicornis, Erich. Austria.

2 Parumpunctatus. Gyll. Gallia.*

3 Batychrus. Knoch. id.*

Var. Linearis, Gravh. Germania.

Episcopalis. Lacord. $P$.

Minutus. Lacordaire. id.

4 Nothus. Erich. Germania.*

5 Formicetorum. Mannerh. Gallia."

6 Fuscus. Motschulsky, Russia.

7 Myrmecobius. Motsch. id.

\section{STAPHYLINUS. Linné.}

Fabr. Panz. Fourc. de Villers. Payk. Schr, Rossi. Walk. Oliv. Gravenh, Latr. Marsh. Gyll. Nordm. Erich. Mannerh. Curtis. Lacord. Runde. Enus. Mannerh. Lacord. Creophicus. Mannerh.

1 (Hirtus. Linné.

Gall. bor.*

Bombilius. de Geer.

Quintus. Schaffer.

Germania.

id.

2 Maxillosus. Linné. Gallia.*

Nebulosus. Fourcroy. $\boldsymbol{P}$.

Anonimus. Sulz. Germania.

Balteatus, de Geer. id.

Fasciatus. Fuesly. id.

Tertius. Schœff. id

3 Nebulosus. Fabr. Gallia.*

Tenellatus. Fourcroy. $\boldsymbol{P}$.

Hybridus, Marsh. Anglia.

Murinus. Panzer, Germania.

Villosus, var. de Geer. id.

Secundus. Scheff. id.

4 Marginalis.Géné.Erich.Sardinia.*

5 ) Murinus. Linné. Gallia.

\{Villosus, de Geer. Suecia.

6 Chrysocephalus. Fourc. Gallia.

$\{$ Pubescens. Rossi. Italia.

7 Pubescens, de Geer, Gallia.*

8 Chloropterus. Creutz.

$$
\text { Fabr. } P \text {. }
$$

9 Fossor. Scopol. Gallia.*

$$
\left\{\begin{aligned}
& \text { Erythropterus. Scop. } \\
& \text { var. } 2 . \text { id. } \\
& \text { Fodicus. Gravenh. } \text { Suecia. }
\end{aligned}\right.
$$

10 Erythropterus. Linné. Gall. merid.* Castanopterus. Gravenh. Germania.

Flavicornis, Dej. Cat. Gall. orient. ${ }^{*}$

11 Sculpticollis. (Gaubil.) Algiria.*

12 Cresareus. Cedh. Gallia.*

Erythropterus. Fabr. Germania.

Primus. Schoff. id.

13 Stercorarius. Olivier. Gallia.*

14 (Lutarius. Gravenh. Austria.*

Flavopunctatus, Latreille. Gallia.

15 Chalcocephalus. Fabr. id.*

Eneocephalus, Fabr. Germania.

Ochropterus. Germar. id.

Carinthiacus. Lacord. $P$.

16 Latebricola. Gravenh. Germania.

17 Meridionalis. Rosenh, Gall. merid.

18 Fulvipes. Scopol. Gallia.*

Bicinctus. Rossi. Italia.

Erythropus. Payk. Suecia.

Tricolor. Gravenh. id.

Azureus. Ledebour. Germania.

19 Dauricus. Mannerh. Sibiria.

20 Italicus, Géné. Italia.

21 Tricinctus, Géné. id.

22 Ventralis. Géné. … id.

\section{OCYPUS. Kirby.}

Emus. Lacord. Sta Phylinus. Fab. Payk. oliv. Geoff. de Villers. Rossi, Panz. Latr. Gravenh. Mannerh. Fourc. Scop. de Geer. Gœze. Schrank. Nordm. Marsh. Sahlb. Guérin. Müller. Heer. AstanPeUS, Latr. Anodus. Nordm.

1 Olens. Müller. Gallia. *

Unicolor. Herbst. id.

Maxillosus. Schrank. Germania.

Major. de Geer. Suecia.

2 Cyaneus. Payk. Gallia.*

Azurescens. Mannerh. Suecia.

Ophthalmicus. Scop. Italia.

Carulescens. Fourc. $\boldsymbol{P}$.

Atro-cœrulescens.

Gœze. Germania.

3 Nigrinus. Lucas. Alger.

4 Italicus, Géné. Erich. Italia.

5 Velutinus. Jan. Lombardia.

6 Macrocephalus, Grav. Carinthia.

7 Megacephalus. Nordm. id.

8 Alpestris, Erich. id. 
9

$\begin{cases}\text { Similis. Fabr. } & \text { Gallia.* } \\ \text { Lugens. Nordmann. } & \text { Suecia. } \\ \text { Nitens. Schrant. } & \text { Germania. } \\ \text { Niger. de Geer. } & \text { id. } \\ \text { Globulifer. Fourcroy. } & P .\end{cases}$

10 Masculus. Nordmann. Lusitania.

11 Ethiops. Waltl. Germania.

12 Picipes. Nordmann. Austria.

13 Brunnipes. Fabr. Gallia*

14 Rufipes. Latr. $P$.

I Brunnitarsis, Sturm. Illyria.

15 Alpicola. Erich. Carinthia.

16 Fuscatus. Gravenh. Gallia.*

Crassicollis. Graverh.

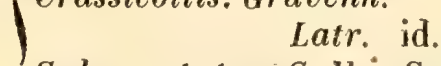

\{Subpunetatus, Gyll, Suecia.

Morio. Gyllenh. id.

Punctulatus. Marsh. Angtia.

Politus, de Geer. Suecia.

17 Picipennis. Fabr. Gallia. *

Eneocephalus, Payk. Suecia.

Penetrans. Müller. Germania.

Quartus, Schoffer. id.

Var. Tristis Fabr. id.

Sericeus, Marsh. Anglia.

Leucophthalmus. Marsh. id.

Chalcocephalus. Man-

18 Vagans. Heer. nerh. Suecia.

19 /Cupreus, Rossi.

Helvetia.

Eneocephalus de Geer Germania.

Aneicollis. Gyllenh. Suecia.

Sericeicollis, Ménétr. Russia.

Strigatus. Nordmann. id.

Puberulus. Runde. Anglia.

20 Fulvipennis. Erich. Gallia*

Chalcocephalus. Nord-

mann. Austria.

Picipennis. Lacord. $P$.

21 Tataricus. Pallas.

22 f Pedator. Gravenh.

Rufipes. Latr.

23 Siculus. Aubé.

24

25

26

Planipennis. Aube. id

Lusitanicus. Aube. Lusitania.

Ater. Gravenh.

Gallia.*

Fuscatus, Gylleni. Suecia.

obscurus. Nordmann. id.

Nigripes. Lacordaire. $P$.

Confinis. Curtis. Anglia.

Uralensis. Manner-

heim. Russia.
27 Morio. Gravenh.

Edentulus. Block.

Similis. Payk.

Angustatus. Lacord.

obscurus. Runde.

28 Luganensis. Heer.

29 Cerdo. Erich.

30 Melanarius, Heer.

31 ( Compressus. Marsh.

IRufipalpis. Lacord. $P$.

32 Falcifer. Nordmann. Italia. Messor. Nordmann. Suecia.

\section{PHILONTHUS, Leach.}

Erich. Heer, Nordmann, Staphylinus. Fabr. Payk. Latr. Gyll. Sahlb. Marsh. Mannerh.Lacord. Müll, Panz, Gravenh.

1. Splendèns. Fabr. Gallia.* Eneus. Müller. Germania. Sextus. Schaffer. id.

Niger. Müller. id.

2 Læevicollis. Sturm. Finlandia.

3 Punctipennis. Sturm. Germania.

4 Montivagus. Heer. Helvelia.

5 Intermedius. Dej. Cat.

Lacord. P.*

(Laminatus. Nordm. Germania.

6 Laminatus. Creutzer. Gallia.*

Viridanus. Nordm. Suecia.

Enews. Marsham. Anglia.

7 Cribratus. Erich. Gall. merid.

8 Lævicollis. Lacordaire. $P$.

9 Cyanipennis. Fabr. id.

Amcenus. Oliv. Latr. id.

Coruleipennis. Man-

nerh. Austria.

10 Nitidus. Fabr. Germania."

\{Conosus. Gravenh. Gallia.

11 Nitidipennis. Sturm. Finlandia.

12 Asphaltinus. Erich. Germ. merid.

13 Carbonarius. Gyllenh. Gallia.*

INigritus. Runde. Anglia.

14 Eneus. Rossi. Gallia. ${ }^{*}$

Laticeps. Zetterst. Germania.

Metallicus. Lacord. P.

Similis. Marsh. Anglia.

Var, Atratus, Lacord. $P$.

15 Scutatus. Erich. Germ.merid."

16 Atratus, Gravenh. Gallia.*

Metallicus. Grav. Latr.id.

Var. Corulescens. Lac.P. 
17 Lælus. Heer.

Helvetia.

Gallia.*

Germania.*

Gallia.*

Germania.

Gallia.*

$P .^{*}$

Suecia.

$P^{*}$

Subfuscus. Gyllenh.

23 Varius. Gyllenh.

Var. Carbonarius.

Gravenh. Gallia.

24 Gilvipes. Erichson. Germania.

25 (Bimaculatus. Gravenh. Gallia.*

Nitidicollis. Lacord. $\quad P$.

Dubius. Gravenh. Germania.

26 Albipes. Gravenh. Gallia.*

27 (Lepidus, Gravenh. id.*

Flavopterus. Gravenh. Suecia.

(Fulvipes, Runde. Germania.

28 Nitidulus. Gravenk, id.

\{ Denigrator. Gravenh. Suecia.

29 Xantholoma. Gravenh. Germania.*

30 Variegatus, Erich. Sicilia.

31 Fucicola. Curtis. Anglia.

32 Cicatricosus. Erich. Sicilia.

33 Cephalotes. Gravenh. Gallia.*

34 Fimetarius. Gravenh. id.*

\{Rigidicorris. Grav. Germania.

35 Sordidus, Gravenh, Gallia.*

36 Sparsus, Lucas. Algiria.

37 (Fuscus, Gravenh. Gallia.

Subiliformis. Gravh. Suecia.

Fragilis. Gravenh. id.

38 Placidus. Erich. Hungaria.

39 Suturalis. Nordm. Er. Russ, orient.

40 Microcephalus. Grav. Erich. $P$.

41 Ebeninus. Gravenh. Gallia.*

Var. Brevicornis. Gra-

venh. Suecia.

Varians. Gravenh. $P$.

Concinnus. Gravenh. Suecia.

42 Corvinus. Erich. Germania.*

43 (Fumigatus. Dhl. Erich. Gallia.*

Var. Intermedius. Gra-

I

Corruscus. Gravenh. Gallia.*

Nitidus, Marsh. Anglia.

Planus, Lacordaire. $P$.

45 Turbatus. Erich. Sardinia.

46 Sanguinolentus, Grav, Gallia.*

$\{$ Var, Contaminatus.

Gravenh. $P$.
47 Strangulatus. Erich. Gall. merid.*

48 Bipustulatus, Panzer. Gallia.*

49 Varians. Payk. . id.*

Opacus. Gravenh. $P$.

Var. Bipustulatus.

Gravenh, Suecia.

Bimaculatus, Marsh. Anglia.

Var. Opacus. Gravenh. $P$.

Aterrimus. Marsh. Anglia.

Scybalarius. Nordm. Suecia.

Fuscicornis. Nordm. id.

Simplex. Marsh. Anglia.

Agilis. Gravenh. Germania.

Discoideus, Lacord. $P$.

50 Debilis, Erich. id.*

Lucidus. Gravenh. id.

fimetarius, var. Grav, Suecia.

Agilis, Lacordaire. $P$.

51 Ventralis. Gravenh. Gallia.

Immundus, Gravenh. Suecia.

Anthrax, Gravenh. Germania.

52 Discoideus. Gravenh. Gallia.*

Conformis. Lacord. $P$.

Testaceus, Gravenh. Germania.

ऽ3 Melanocephalus, Heer. Helvetia.

54 Rubidus. Erich. Gallia.

Dimidiatus, Nordm.

Lacord. Germania.

5ัง Quisquiliarus. Gyllenh.

Lacord. Gallia.*

๖6 Alcyoneus, Erich. Sardinia.*

57 Rufimanus. Dej. Cat.

Erich. Carinthia.*

58 Vernalis. Gravenh. $\boldsymbol{P}$.*

59 Splendidulus. Gravh, Gallia.*

Nanus, Gravenh, P.

Parvulus. Gravenh. id.

60 Immundus. Gyllenh. Suecia.

61 Dimidiatus, Sahlberg. Finl, merid.

62 Celer. Gravenh. $P$.

63 Irregularis. Mannerh. Petropoli.

64 Nigrita. Gravenh. Germania.

65 Fumarius. Gravenh. id.*

66 Virgo. Gravenh. P.

67 Micans. Gravenh. Gallia.*

$\{$ Varians, var, Gyll. Suecia.

68 Fulvipes. Fabr. Gallia.*

69 Tenuis. Fabr. Germania.*

$\{$ Dimidiatus. Panzer, $\boldsymbol{P}$.

70 Pullus. Nordmann. Gallia.

71 Luxurians. Erich. Sardinia.

72 Exiguus. Nordm. Germania.

73 Pusillus, Heer. Helvetia. 
74 Astutus, Erich.

75 (Aterrimus. Gravenh. Gallia.*

Nigritulus. Gravenh. Suecia.

Pumilus. Mannerh. id.

76 Puella. Nordm.

77 Punctus. Gravenh

Punctatus. Latreille. Gall. merid.

Multipunctatus. Man-

nerheim. Suecia.

Politus. Panzer. Germania.

78 Parumpunctatus.Erich.Suecia.*

Punctus. Gyllenh. id.

79 Dimiatipennis. Erich. Gall. merid. ${ }^{*}$

80 Rufipennis, Gravenh. Gallia. ${ }^{*}$

81 Cinerascens. Gravenh. id. ${ }^{*}$

82 Sericeus. Holm. id.

83 Pruinosus. Erich. Austria.

84 Prolixus. Erich. id, ${ }^{*}$

85 Procerulus. Gravenh. Germania.*

Y Planatus. Gravenh. Suecia.

86 Elongatulus. Erich. Germania.*

87 Palmula. Gravenh. id.

Cinctus. Latreille. Gallia.

88 Rubripennis. Kiesen-

wetter. Germania.

89 Salinus. Kiesenwetter. id.

\section{HETEROTHOPS, Kirby.}

Emus. Lacord. TAchyporus, Gravenh. Tachinus. Gravenh. Trichopigus. Nordm. Staphylinus. Gyll. Mannerh. Zetterst.

1 ( Prævius. Erich. $\{$ Subuliformis, Lacord. $P$.

2 Limbatus. Knoch.

3 Binotalus, Erich. Heer. Helvetia.

4 Dissimilis, Gravenh. id. \{ Subuliformis. Gyll. Suecia.

5 \{uadripunctatus. Er. Germania.* \{ Subuliformis.Zett. Suecia.

6 Nitens. Nordmann. Germania.

\section{ACYI,OPHORUS. Nordmann.}

Staphylinus. Lacord. Zetterst.

1 (Glabricollis Lacord. P.* $\{$ Rufilabris. Zetterst. Suecia. Ahrensii. Nordmann. Germania.
QUEDIUS, Leach.

Microsaurus. Dej. Cat. Emus. Lacord. Staphylinus, Fabr. Panz. Payk. Latr. Gravenh. Gyllenh. Mannerh. Geoffroy. Sahlberg. Philonthus. Nordmann.

1 (Dilatatus, Fabr. (Vel$\{$ leius. Mannerh.) Gallia.* Serraticornis. Schrk. Germania. Concolor. Marsh. Anglia.

2 Lateralis. Gravenh. Gallia.*

3 Fulgidus. Fabr. Erich. id.* Variabilis. Gyllenh. Suecia. Nitidus. Gravenh. id. Rufitarsis. Marsham. Anglia. Fuscipennis, Block. Germania. Floralis. Lacordaire. $P$. Assimilis. Nordmann. Suecia. Mesomelinus. Marsh. Anglia. Ocultus, Lacordaire. $P$.

4 Cruentus. Olivier. Gallia.* Fulgidus. Marsham. Anglia.

5 Xanthopus. Erich. Germania. \{Variabilis. v. c. Gyll. Suecia.

6 Scitus. Gravenh. Gallia.* Var. Analis. Fabr. Germania. Atricilus. var.b.Grav. id. Pygmaus. Gravenh. Suecia.

7 Lævigatus. Gyllenh. Lapponia.*

8 Impressus. Panzer. Gallia.* Cinctus. Payk. Suecia. Marginellus. Marsh, Anglia.

Nitidus, var. Gravenh. Germania.

9 Punctatellus. Heer. Helvetia.

10 Montivagus. Heer. id.

Nigritus. Heer. id.

11 Curtus. Erich. Sardinia.

12 Brevis. Erich. $\boldsymbol{P}^{*}$

13 Unicolor. Kiesenwett. Germania.

14 (Molochinus. Gravenh. Gallia.*

Laticollis. Gravenh. Suecia.

Picipennis, Payk. id.

Lapponicus. Zetterst. Lapponia.

Lavicollis. Runde. Anglia.

15 Pallipes, Lucas. Algiria.

16 Frontalis, Nordmann, Gallia.*

17 Fuliginosus. Gravenh. id.*

Tristis. Lacordaire. $\boldsymbol{P}$.

Dilatatus, Marsh. Anglia.

18 Picipes. Mannerh. Germania.

Y Varicolor, Nordmann. Suecia.

19 Planeus. Erich. Sardinia. 
20 Ochropterus. Erich. Austria.*

21 Fimbriatus, Erich. id.

22 Peltatus. Erich.

\{ Pracox. Erich.

Præcox. Gravenh.

24 Umbrinus. Erich.

Maurorufus. Gyllenh. Suecia.

25 Montanus. Heer. Helvetia.

26 Paradisianus. Heer. id.

27 Maurorufus. Gravenh. Gallia.*

Attenuatus, Gravenh. Suecia.

Procox.Gyllenh. P.

28 Infuscatus. Erich. id.

29 (Rufipes. Gravenh. Gallia.*

Ruficornis. Gravenh. Germania.

Attenuatus. Lacord. P.

30 Monticola. Erich. Germania.*

31 Semi-obscurus. Marsh. Ang. Sardin. *

32 Collaris. Erich. Volhynia.

33 Attenuatus. Gyllenh. $\boldsymbol{P}^{*}$

Maurorufus. Runde. Anglia.

Scintillans, Lacord. Gallia.

34 Picipennis. Heer. Helvetia.

35. Virgnlatus. Erich. Sardinia.

36 Boops. Gravenh, Gallia.*

37 Satyrus, Kiesenwetter. Germania.

38 Obliteratus. Erich. Sardinia.

39 Scintillans. Gravenh. Germania.

40 Alpestris. Heer. Helvetia.

41 Lucidulus. Erich. Germania.

42 Tenellus. Gravenh. id.

43 Rufocinctus. Mannerh, Finland.mer.

44 Maurus. Sahlberg. id.

45 Vicinus, Lacordaire. $P$.

46 Suturalis. Kiesenwett. Germania.

47 Riparius. Kellner. id.

\section{ASTRAPAUS. Gravenh.}

Lacordaire. Staphylunus. Fabr, Panzer. Gravenh. Rossi. Oliv.

1 Ulmi. Rossi.

$\{$ Ulmineus. Fabr, Lacordaire. $P$.

Gall. merid.*

OXYPORUS, Fabricius.

Staphylinus, Lin. Payk. Marsh. Geoff.

1 Rufus, Linné. Gallia.*

2 Maxillosus, Fabr. Germania.*

Var. Angularis, Gebl. Russia.

Schonherri. Mannerh. Finlandia.

3 Mannerheimii. Gyll. Lapponia.

CRYPTOBIUM. Mannerh.

Pederus, Payk. Lathrobium. Gravenh. Latr. Gyllenh. Germar.

1 Fracticorne. Mannerh. Gallia.* \{ Glaberrimus. Payk. Lapponia.

DOLICAON, de Laporte.

Lathnовіом. Gravenh. Latr, Lacordaire.

1 Illyricus, Erich. Illyria.

2 Hæmorrhous. Erich. Sardinia.

3 Gracilis. Gravenh. "Lusitania.

4 Bigutlulus, Lacord. P. Gall.mer.*

SCIMBALIUM. Erichson.

1. Planicolle. Erich. Italia.

2 Testaceum. Erich. Sicilia.

\section{ACHENIUM. Leach.}

\section{LATHROBIUM. Gravenh, Latr.}

1 Depressum. Gravenh. Gall. merid.* Cordatum, Lacord. $\boldsymbol{P}$.

2 Ephippium. Erich. Hungaria.

3 Punctatum. Zetterst. Finlandia.

4 Basale. Erich. Sardinia.

5 Striatum. Latreille. Gallia.

6 Distinctum. Lucas. Algiria.

7 Humile. Nicolaï. German. P.*

Depressum. Curtis. Anglia.

8 Hæmorrhoidale. Lucas. Algiria.

9 Jejunum. Erich. Sardinia.

10 Tenellum. Erich. id.

\section{LATHROBIUM. Gravenhorst.}

EURYPORUS, Erichson.

Oxyporus, Payk, Gyll.

1 Picipes. Marsham.

2 Eneiventris, Lucas.
Gall, bor.* Algiria.
Paderus, Payle. Panz. Rossi. Oliv. StaPhylinus. Marsh. Geoff. Gravenh. Payk.

1 (Brunnipes, Fabr. Gallia.* Elongatus, v, c. Paylk. Suecia. Dertatus. Marsh. Anglia. 
2 Elongatum. Linné.

3 - Alpestre. Heer.

4 Basale. Kerl.

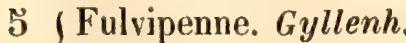
\{ Elongatum. Lacord.

6 Lævipenne. Heer.

7 Rufipenne. Gyllenh.

8 Bicolor. (Dahl.) Heer. Helvetia.

9 Multipunctatum. Grav, Gallia.* Lineare. Gravenh. Germania. Var. Testaceum. Oliv. Gallia.

10 Anale. Lucas. Algiria.

11 Spadiceum. Erich. Germania.

12 Brunneum. Stm. Cat. Germania.

13 Angusticolle. Lacord.

14 Bicolor. Erich. Erich. $P$.

15 Picipes, Erich.

16 Labile. Erich.

Quadratum. Gyllenh. Filiforme. Payk.

Var. Pilosum. Grav. Terminatum. Grav.

\section{Carniolia.} Germania. Sardinia. Gallia.* Suevia. $P$. id. Albipes. Lucas. Algiria.

19 Scutellare. Nordmann. Austria.

20 Angustatum. Lacord. $P$.

21 Lusitanicum. Grav.

22

23

Punctatum. Zetterst.

(Filiforme. Gravenh. id.* Lineare. Gyllenh.

Iusitania.

Germania.*

Suecia.

Megacephalum. Heer. Helvetia.

25 Pallidum. Nordmann. Germania.

26 Dividuum. Erich.

27 Dilitum. Erich.

28 Impressum. Heer.

Sardinia.

29 Longulum. Gravenh.

Minutum. Mannerh.

30 Agile, Heer.

Germ. sept.

Helvetia.

Gallia.*

Germania.

Ilelvetia.

31 Stilicinum. Erich. Germania.

32 Elegantulum. Sturm. Cat. id.

33 Scabricolle. Erich. Austria.

34. Brevicorne. Latreille, Gallia.

35 Dentatum. Kellner. Germania.

\section{SCOPAUS. Erichson.}

Paderus. Gyllenh. Rugicus. Mannerh. LA'ThRoBIUM. Erich. Heer.

1 Lævigatus. Gyllenh.

2 Didymus. Erich.

3 Minutus. Erich.
4 Minimus. Erich.

5 Punilus. Heer.

Germania. Helvetia.

\section{MICROPHIUS. Dejean.}

1 Colubrinus. Dejean. Sicilia.

\section{LITHOCHARIS. Dejean,}

Panerus. Lacord. Gravenh, Latr, Oliv. Panz, Fabr. Ruglius, Mannerh. StaPHYLINUS. Marsham.

1 Castanea. Gravenh. Gallia.

2 Fuscula. (Ziegl.) Lac. P.

3 Brunnea. Erich.

Germania.*

4 Diluta. Erich.

id.

5 Rufiventris. Nordm. id.

6 Ferruginea, Erich. Austria.

7 (Melanocephala. Fabr. Gallia.*

Tricolor. Marsh. Anglia.

Bicolor. Olivier. $\quad P$.

Ruficornis, Latreille. Gallia.

8 Ochracea. Gravenh. P.*

Rubricollis. Gravenh. Germania.

Testacea. Lacordaire. $P$.

9 Obsoleta. Nordmann. Germania.*

10 Minuta. Lucas. Algiria.

11 Obscurella. Erich. Sardinia.

12 Nigritula. Erich. Sicilia.

13 Exigua. Nees. Germania.

\section{STILICUS. Latreille.}

Pederus, Gravenh. Latr. Oliv. Fabr. Dahl. Rugilus. Germ. Lacord. Curtis.

1 (Dentipes. Tischer. Germania. Srabricollis.Dahl.Dej.

St. Cat. id.

2 Fragilis. Latreille. $\quad P^{*}$ \{ Sanguinicollis. Dahl. Germania.

3 Rulipes. Müller. Germ. id.* \{rbiculatus. Fab. Lac. P.

4 Subtilis, Erich. Austria.*

5 (Similis. Erich. Germania.* Orbiculatus. Gravenh. id.

6 Geniculatus. Ahrens. id.*

7 Affinis. Erich. Gallia. ${ }^{*}$

8 Ruficornis. Lucas. Algiria.

9 Fuscipes. Erich. Sardinia.

10 Orbiculatus. Payk. Succia.*

11 Exiguus. Gravenh. in litt. Heer. Irelvetia. 


\section{SUNIUS, Leach.}

Paderus, Latr, Oliv, Gravenh, Mannerh. Payk. Gyll. Astenus. Dej. Cat. Lacord. Staphylinus, Payk. Fabr. Panz.

1 Filiformis. Latreille. Gallia.* Procerus. Gravenh. $P$.

Extensus. Mannerh. Germania.

2 Intermedius. Dej. Cat. Erich. id.*

3 Linearis. Sturm. id

4 Lapidarius. Nees. id.

5 Angustatus. Payk. Gallia.*

Y Var. Gracilis. Payk. Germania.

6 Bimaculatus. Erich. Sardinia.*

7 Curtulus. Erich. id.

8 Tristis. Erich. id.

9 Exilis. Schüppel. Germania.

10 Fusculus, Dahl. Austria.

11 Kunzei. Heer. Helvetia.

12 Neglectus. Märck. Saxonia.

13 Pulchellus. Heer. Helvetia.

\section{PADERUS, Fabricius.}

Mannerh. Lacord. Latr. Oliv. Curtis. Gravenh. Erich. Payk. Panz. Gyllenh. StaPHYlinus. Schrank. Paykull. Marsh. Linné. Geoff.

1 Littoralis. Gravenh. Gallia.* $\{$ Riparius, var. Latr. id.

2 Lusitanicus. Aubé. Lusitania.

3 Brevipennis. Lacord. P.*

4 Longipennis. Erich. id." Riparius, Gravenh. Gallia. Fuscipes. Curtis. Anglia.

5 Caligatus. Erich. $P{ }^{*}$

6 Limnophilus. Märkel.

7 (Riparius. Linné. Gallia.* $\{$ Confinis. Zetterst. Germania.

8 Ruficollis. Fabr. Gallia.* $\{$ Thoracicus, Fourcroy. $P$.

9 Filiformis. Costa. Italia.

10 Melanurus, Géné. id.

OEDICHIRUS. Erichson,

1 Prederinus, Erich. Sicilia.

2 Unicolor. Aubé.
PROCIRRUS. Latreille.

1 Lefebvrei. Latreille. Sicilia.

DIANOUS. Leach.

Stenus. Gyll. Sahlb.

1 Cœerulescens. Gyllenh. Gall, bor.*

Coruleus. Curtis.Man-

nerheim. Finlandia.

STENUS. Latreille.

Paderus. Olivier. Staphylinus. Linné. Fabr. Panz, Rossi, Valk. Scopol. Gœze. Ljung. Weber.

1 (Biguttatus. Linné. Fab. Gallia.* $\{$ Bipustulatus. Mannh. Suecia.

2 Bipunctatus. Kirby. Gallia.*

3 Longipes. Heer. Helvetia.

4 Guttula. Müller. Gallia. Kirbyi. Gyllen'. Suecia.

(Biguttatus, var. Grav, Gallia.

5 Maculipes, Heer. Helvetia.

6 Geminus. Gravenh. in litt. Heer. id.

Bituberculatus. Aubé. id.

7 jimaculatus. Gyllenh. P.* Juno. Gravenh. id.

8 Stigmula. Erich. id.*

9 Juno. Fabr. Germania.*

Clavicornis. Fabr. id.

Buphthalmus. Latr. Gallia.

Boops. Gravenh. id.

10 Intricatus. Erich. Sardinia.

11 Asphaltinus. Erich. Tyrol.

12 Ater. Mannerh. Gallia.*

Maurus. Manneri. Germania.

13 Carbonarius. Gyllenh. Gallia. ${ }^{*}$

14 Labilis. Erici. Finlandia."

\{ Canaliculatus. Sahlb. id.

15 Ruralis. Erichson. Austria.*

16 Buphthalmus. Schrank.P.*

Boops. Ljung. Anglia.

Clavicornis. Panzer. Germania.

Canaliculatus, Lacd. $P$.

Palposus. Zettersted. Suecia.

17 Morio. Knoch. Germania.*

Buphthalmus. Zett. Suecia.

18 Incanus, Erich. Germania.

19 Cinerascens. Erich, Gallia. ${ }^{*}$

20 Atratulus. Erich. id.* 
Mendicus, Erich.

Lusitania. Germania.*

22 Incrassatus, Erich.

23 Foraminosus, Erich.

24 Nitidus. Lacordaire. id.

$P$.

23 Emulus. Erich.

Germania.

26 Canaliculatus. Knoch. Gyllenh. Gallia.* Buphthalmus. var. Gravenh. Suecia.

Niger. (Dahl.) Mannerh.

Helvetia.

28 Opacus. Erich. 29 Pusillus. Kirby. Erich. Germania.*

30 Exiguus. Erich. Anglia.

31 Speculator, Knoch.Lacordaire. $P^{*}$

Clavicornis. Scopol. Germania.

Buphthalmus. Schrk. id.

Boops. var. 1. Grav. Suecia.

Cicindeloides. Ljung. Anglia.

32 Obscurus. Lucas. Algiria.

33 Providus. Erich. Gallia.*

34 Eneus. Lucas. Algiria.

35 Scrutator. Erich. Germania.

\{Femoralis, Erich. Col. id.

36 Sylvester, Erich. id.

37 Fossulatus, Erich. Saxonia.

38 Lustrator. Erich. Germania.

39 Atlerrimus. Aubé. $\mathrm{Er} . \mathrm{P}^{*}$.

40 Alpestris, Heer. Helvetia.

41 Proditor. Erich. Germania.

42 Excubitor. Erich. Berolini.

43 Argus. Knoch. Grav. Gallia.*

Opticus. Gyllenh. Germania.

44 Cantus. Erich.

45 Vafellus. Erich.

Berolini.

46 Fuscipes. Gravenh. Gallia.* $\{$ Fulvipes. Lacordaire. P.

47

Humilis. Erich. Gallia.*

Fuscipes, Ljung. Anglia.

Argus. Gyllenh. Suecia.

Carbonarius. Lacord. $P$.

48 Circularis. Gravenh. Gallia.*

49 Declarntus. Tischer. id.*

\{ Circularis. Gravenh. $P$.

¿0 Pumilio. Erichson. Germania

51 Nigritulus. Gyllenh. Gallia.

52 Campestris. Erich. Germania.*

53 Unicolor. Erich. Gallia.*

54 Opticus. Knoch. id.*

F'emorellus. Zetterst, Suecia.

ii) Formicetorum. Mannerheim. Finlandia.
56

58

59

60

\section{1}

62

64

65

\section{6}

\section{7}

\section{8}

69

\section{0}

\section{1}

Binotatus. Ljung. Gallia.*

Subimpressus. Knoch. $\boldsymbol{P}$.*

(Plantaris. Erich. id.*

Binotatus. var. b. Gyl-

lenhal. Germania.

Rufimanus. Heer. Helvetia.

Plancus. Erich. Anglia.

Bifoveolatus. Gyllenh. P.*

Rusticus. Erich. id.*

Tempestivus. Erich. Gallia.*

Picipennis. Erich. Saxonia.

Languidus. Erich. Sicilia.

Cordatus. Gravenh. Sardinia.

Hospes. Erich. Coreyra.

(Subæneus. Erich. $\quad \boldsymbol{P}$.

\{Geniculatus. Marnerh. Gall. merid.

Erosus, Erich. Sardinia.

0 Glacialis. Chevrier. Helvetia.

(Impressus.Tischer.Ger-

mar. Gallia."

Proboscideus, Germar.Germania.

Aceris. Lacordaire. $P$.

Pallipes, var. Grav. Germania.

72 Obliquus. Heer. Helvetia.

73 Geniculatus. Knoch.

74 Flavipes, Erich. id.*

75 Palustris. Erich. Germania.*

\{Proboscideus. Gyllenh. Suecia.

76 Montivagus. Heer. Helvetia.

77 Fuscicornis, Erich. $\quad \boldsymbol{P}^{*}$

78 Pallipes. Gravenh. id. ${ }^{*}$

79 Annulipes, Heer. Helvetia.

80 Angustulus. Heer. id.

81 Filum. Tischer, Erich. Gallia.*

82 (Tarsalis. Ljung. id.*

Buphthalmus. Ljung. Germania.

Clavicornis. Rossi. $\quad P$.

Riparius. Runde. id.

83 Oculatus. Gravenh. Gallia.*

Similis. Herbst. Germania.

84 Solutus. Erich. $\quad \boldsymbol{P}^{*}$

83 (Cicindeloides. Schall. Gallia.*

Buphthalmus. Rossi. Italia.

Clavicornis. Rossi. Gall. merid.

Bigutlatus, var. Oliv. Germania.

Similis. Ljung. Anglia.

86 Paganus. Erich. Germania.

87 Latifrons. Knoch. Gallia.*

Morio, var. Gravenh. Germania.

88 Contractus. Dej. Cat,

Erich. Gallia.*

89 Rotundatus. Ljung. Germania. 


\section{EUASTETHUS, Knoch.}

Eristhetus. Mannerh. Sahlb. Lacord. Stenus. Ljung. Web.

1 S Scaber. Knoch.

$$
P_{.}^{*}
$$

I Bipunctatus. Ljung... id.

2 Læviusculus. Mannerh. Finlandia.

3 Ruficapillus. Lacord. P.*

\section{BLEDIUS. Leach.}

Oxytelus. Gravenh. Germ. Ahrens, Latr. oliv. Gyll. Guérin. Sahlb. StaphyLINUS. Payk. Block. Herbst.

1 (Taurus, Dej. Mannerh. Gall. merid.* Furcatus. Olivier. id.

Skrimshiri. Curtis. Anglia.

Ruddii. Stephens. id.

Armatus. Dej. Cat. Gall, merid.

2 Bicornis. Ahrens.

id. $^{*}$

Hinnulus. Erich. Russ. merid.

Juvencus, Erich.

Tricornis. Herbst.

id.

$P^{*}$

Littoralis. Heer.

Alpestris. Heer.

Helvetia.

id.

8 Monoceros. Stm. Cat. Sicilia.

9 Unicornis. Germar. Gall. merid.*

10 Aquarius. Erich. Geneva.

11 Fracticornis. Payk. Gallia.*

Pallipes. Grav. Lac. P.

Gallicus. Gravenh. Gallia.

Tricornis. var. Grav. Germania.

Femoralis. Gyllenh. Suecia.

Longulus, Erich.

Procelurus. Erich.

Nigricans. Erich.

Dissimilis, Erich.

Tristis. Aube.

Germania.*

Austria.

Russ, merid.

Gallia.*

Sicilia.

Rufipennis. Erich. : Austria.*

Crassicollis. Lacord. P.*

Cribricollis. Chevrier. Helvetia.

Filum. Chevrier, Heer, id.

Fossor. Chevr. Heer. id.

(Opacus. Block. Erich. Germania.*

Castaneipennis. Lacordaire. $P$.

Erraticus. Erich.

Germania.

Pallipes. Gravenh.

Gallia.

Atricapillus. Germar. Germania.

Nanus. Erich.

Austria.

Pusillus. Erich.

Gallia.

Pigmæus. Erich.
30 Verres. Erich.

Sardinia.

31 Talpa. Gyllenh. Gallia.*

Subterraneus. Märkel.

Erich. Germania.*

Morio. Heer. Helvetia.

34 Tibialis. Chevrier.

Heer. id

35 Agricultor. Chevrier.

Heer. id.

36 Arenarius. Payk. Suecia.

37 Debelis. Erich. Russ, merid.

38 Elongatus. Mannerh. Petropoli.

\section{PLATISTHETUS. Mannerh.}

\section{Oxytelus. Sahlb. Gyll. Staphylinus. Payk. Marsham.}

1 Morsitans. Payk. Gallia.* Trilobus. Olivier. id. Striolatus, Lacord. $P$. Mordax. Sahlberg. Germania.

2 Cornutus. Gravenh. : . $^{*}$ Scibalarius, Runde. Germania.

3 Longicornis. Lucas. Algiria.

4 Nodifrons. Sahlberg. Gallia.* Nitens. Sahlberg. Germania. Var. Pallidipennis.

Panzer, Helvetia.

Morsitans.var.c. Gyll. Suecia.

5 Splendens. Chevrier.

Heer. Helvetia.

6 Striatulus. Heer. id.

7 Spinosus. Erich. $P$.

8 Capito. Chevrier.Heer. Helvetia.

OXYTELUS. Gravenhorst.

Staphylinus, Fabr, Panz, Marsh, Oliv. Payk. Walk. Müller. Fourcroy. Geoff. Schr. Linné.

1 Rugosus. Fabr. P.* Carinatus, Panzer. Germania. Piceus, Olivier. Gallia.

Var. Striatus, Müller. Germania. Sulcatus. Fourcroy. P. Pulcher. Gravenh. .... id. Terrestris. Lacord. id.

Laqueatus, Marsham. Anglia.

2 Insectatus. Gravenh. Germania.* \{ Carinatus, var, Gyll, Gallia.

3 Fulvipes, Erich, Germania. 
4

Picens. Linné

Sulcatus. Muller.

Rugosus. Schrank

5 Humilis. Chevrier.

Heer. Helvetia.

6 Terrestris. Dahl. Heer. id.

7 Sculptus. Grav. Knoch. Gallia.* Longicornis. Mannerh. Germania.

8 Montivagus. Heer. Helvetia.

9 Sculptarus. Gravenh. Germania." $\{$ Flavipes. Lacordaire. $P$. Inustus. Gravenh.

Gallia. *

11 Luteipennis. Erich. Germania.*

12 Politus. Erich.

13 Intricatus. Erich.

14 (Nitidulus. Gravenh. Austria. id.

Piceus. Schrank.

Var. Pygmeus. Payk. Suecia. Rugulosus. Say.

Anglia.

15 Complanatus. Erich. Gallia.* $\{$ Depressus. Gyllenh. Suecia.

16 Depressus. Gravenh. Gallia.* Pusillus Mannerh. Suecia. Tetracarinatus. Block. Germania.

17 Pumilus, Erich. Berolini.

18 Fornicarius. Motsch. Russia.

\section{PHLOEONAUS. Erichson.}

Oxytelus. Gravenh. Latr. Oliv. Panz. Gyll. Sahlb. Zetterst. Mannerh. Lacord. StAPHYLINUS. Marsh. Block.

$1 \begin{cases}\text { Coelatus. Gravenh. } & \text { Gallia. } \\ \text { Brachypterus. Marsh. } & \text { Anglia. } \\ \text { Spinipes. Block. } & \text { Germania. }\end{cases}$

TROGOPHLEUS. Mannerh.

Oxytellus. Gyllenh. Sahlberg. Runde. Zetterst.

Dilatatus, Erich.

Gallia.* $^{*}$

2 Omalinus, Erich. Angustatus. Erich.

Saxonia.

Bavaria.

Scrobiculatus. Erich. Saxonia.

5 Bilineatus. Stephens.
Erich. Gallia.
Corticinus, Gyllenh. Suecia.
Var. Inquilinus, Erich. Germania.
6 Riparius. Lacordaire. $P$.
7 Elongatulus. Erich. Germania.*

8 Fuliginosus, Gravenh. Germania.

9 Corticinus. Gravenh. id.* Minimus, Runde. id.

10 Affinis. Heer. Helvetia.

11 Exiguus. Erich. Germania. Impressus. lacord. P.

12 Punctatellus. Erich. Carinthia.

13 Foveolatus. Erich.

Sahlb. Germania.

14 Troglodyies. Erich. Sardinia.

15 (Pusillus. Gravenh. Gallia.*

Fuliginosus. Gyllenh, Suecia.

Corticinus, Lacord. $P$.

16 Inquilinus. Erich. Germania.*

17 Obesus, Kiesenwetter, id.*

18 Tenellus, Erich. Germania.* Gracile. Mannerh. Gallia.

19 Subtilis. Erich. Berolini.

20 Affinis. Heer. Helvetia.

TRIGONURUS. Mulsant.

1 Mellyi. Mulsant. Alp. sup.

COPROPHILUS. Latreille.

Staphylinus. Fabr. Panz. Oliv. Grav. Anthoв. Lacord. Oxytel. Germar.

1 Striatulus. Fabr. P. ${ }^{*}$ Rugosus. Olivier. Gallia. Lessus. Germar. Germania.

\section{ACROGNATHUS. Erichson.}

Omalium. Gyllenh.

1 Mandibularis. Gyll. $\quad$ P. $^{*}$

2 Cephalotes. Erich. Corcyra.

3 Palpalis. Erich. Berolini.*

\section{DELEASTER. Erichson.}

Anthoph. Gravenh. Lesteua. Latr. Guérin. Mannerh. Lacord. Curtis. StAPHYLINUS. Schrank.

$1 \begin{cases}\text { Dichrous. Gravenh. } & \text { Gallia.* } \\ \text { Leachii. Curtis. } & \text { Anglia. } \\ \text { Brassica. Schrank. } & \text { Germania. }\end{cases}$ 
MICRALYMMA. Westwood.

Oмalium. Gyllenh. Zetterst.

1 Brevipenne. Gyllenh. Suecia. Johnstonis. Westw. Anglia.

SYNTOMIUM. Curtis.

Omalium. Müller. Gyllenh.

1 (Eneum. Müller. Germania.* \{ Nigro-aneum. Curtis. Anglia.

PROGNATHA. Latreille.

Siagona. Kirby.

1 Quadricornis. Kirby. Gallia.* $\{$ Rufpennis. Blondel. $P$.

OLISTHARUS. Dej. Cat. Erich.

Omalium, Zetterst. Gyll. Sahlb.

1 Megacephalus. Zett. Lapponia.

2 Substriatus. Paykull. id,*

\section{PHLOEOCHARIS. Mannerheim.}

1 Subtilissima. Mannerh. Finlandia.*

2 Minutissima. Heer. Helvetia.

CORYNOCERUS. Dejean. Cat.

1 Mandibularis, Dej. Cat. Russ, merid.

ANTHOPHAGUS. Gravenhorst.

LesteuA. Latr. Lepell. de Saint-Farg. Laoord. Mannerh. StaphyLINus. Block. de Geer. Fabr. Payk.

1 Armiger. Gravenhorst. Germania.*

2 Spectabilis. Heer. Helvetia.

3 Forticornis. Kiesenw. Germania.

4 Sudeticus. Kiesenw. id.

5 Alpinus. Fabr. Alp. Gall.*

Mandibularis, Gyll. Suecia.

Maxillosus. Zetterst. id.

Lapponicus. v. Sahlb. Lapponia.

6 Alpestris. Heer.

Helvetia.

7 Omalinus. Zetterst. Lapponia. Alpinus. Sahlberg. id.

8 Austriacus. Erichson. Austria.*
9 Caraboides. Linné. Gallia.*

Var. Fulvus. de Geer. Germania.

Testaceus. Lacord. $\quad P$.

10 Gracilis. Heer. Helvetia.

11 Melanocephalus. Heer. id.

12 Rotundicollis. Heer. id.

13 Abbreviatus. Fabr. Gallia." Angusticollis. Mannh. Suecia.

14 Scutellaris. Erichson. Helvetia.

15 Testaceus. Gravenh. Gallia.* I Caraboides. Lacord. $P$.

16 Patruelis. Chevrier.

$$
\text { Heer. Helvetia. }
$$

17 Præustus. Müller. Gallia.*

Bimaculatus. Lacord. $P$.

18 Plagiatus. Fabr. Gallia.*

Var. Suturalis. Lacord. $P$.

Nigritus. Müller. Germania.

Longipes. Mannerh. Suecia.

Globulicollis. Zetterst. Lapponia.

Var. Kunzei. Heer.

19

(Geobius.) Helvetia.

Emulus. Rosenhauer. Tyrolis.

\section{LESTEUA. Latreille.}

Anthophagus. Gravenh. Staphylinus. Gravenh. Geoffroy. Gyll. Zett. Bock. Carabus. Panz.

1 Monticola. Kiesenw. Germania.

2 Bicolor. Fabr. Gallia.*

Truncatellus, Fabr. id.

Obscurus. Gravenh. Germania.

Intermedius. Gravenh. id.

Multipunctatus. Block.id.

3 Pubescens. Mannerh. Germania.*

4 Maura. Erichson. Sardinia.

5 Riparia.Chevrier.Heer.Helvetia.

6 Sicula. Erichson. : Sardinia.

7 Punctata. Erichson. Germania.*

8 Longula. Mannerh. Finland.mer.

ARPEDIUM. Erichson.

Omalium. Gravenh. Oliv. Gyll. Zetterst. Mannerh, Lacord. Latr. ANтновіUм. Runde.

1 Quadrum. Gravenh. P.* $\{$ Castaneum. liunde. Suecia.

2 Brachypterum. Erich. Germanie" Impressum. Zetterst Lapponia.

3 Troglodytes. Kiesenw. Germania. 
4 Gyllenhalii, Sahlberg. Lapponia.

5 Latum. Sturm. Germania.

\section{ACIDOTA. Leach.}

Omalium. Gravenh. Latr. Oliv. StaphyLinus. Fabr. Payl. Panzer.

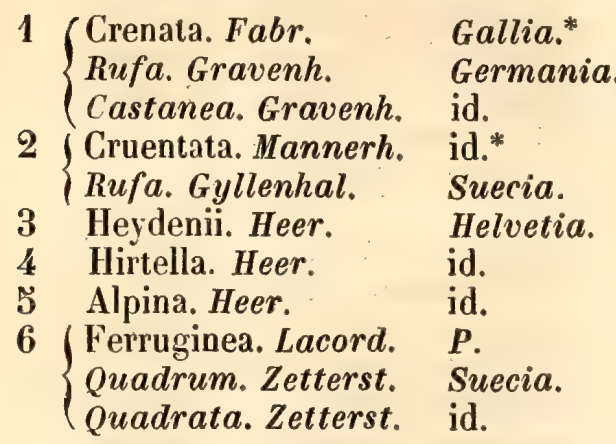

\section{OLOPHRƯ. Erichson.}

Omalium. Gyll, Zett. Mannerh. Lacord. Oliv. Gravenh. Sahlb. Silpha. Ahr. Staphylinus. Payk.

\section{Piceum. Gyllenhal. Gallia.*} $\{$ Blattoides. Ahrens. Germania.

2 Fuscum. Gravenhorst. id.*

3 Assimile. Paykull. id.

4 Boreale. Paykull. Lapponia.

5 Rotundicolle. Sahlb. Finlandia.

6 Alpestre. Heer. Helvetia.

7 f Consimile. Gyllenhal. Lapponia. \{ Boreale. Paykull. id.

\section{LATHRIMEUM. Erichson.}

Silpha. Illig. Schneid. Panz. Marsham. OmaLium. Gyllenh. Lacord.

1 Melanocephalum. Illig. Gallia.*

2 Luteum. Erichson. Lusitania.

3 (Atrocephalum. Gyll. Germania.* Melanocephalum.

Marsham. $\boldsymbol{P}$.

4 Fusculum. Erichson. Germania.

5 Canaliculatum. Erich. id.*

\section{DELIPHRUM. Erichson.}

Omalium. Grav. Oliv. Gyll. Sahlb, Zett. Lac. Mannh. Staphylinus, Payk. Oliv.
1 f Tectum. Paykull.
Germania.
\{ Love. Gravenhorst.

2 Crenatum. Gravenh.

3 Arcticum. Erichson. Lapponia.

4 Algidum. Erichson. Hercynia.

5 Angustatum. Erich. Thuringia.

Anthobium. Mannerh. Lacord. StaphyLINUs, Payk. Oliv. Panz. Marsh. Fabr. Linné. Xycodromus. Heer.

1 (Rivulare. Paykull. P.* Incisum. Gravenh. Germania. Cursor. Müller. id. Var. Oxyacantho. Lacordaire. $\boldsymbol{P}$.

2 Monilicorne. Gyllenh. Suecia.

3 Exiguum. Gyllenhal. Germania.*

4 Oxyacanthæ. Gravenh. Gallia. Casum. Lacordaire. $P$.

5 f Fossulatum. Erichson. Germania.* \{ Casum. Gyllenhal. id.

6 Impressum. Heer. Helvetia.

7 f Cæsum. Gravenhorst. Germania. * \{ Oxyacantho. Gyllenh. Suecia.

8 Minimum. Erichson. Berolini.

9 Pusillum. Gravenh. Gallia.*

10 Florale. Paykull. P.* Viburni. Gravenh. Germania.

11 Cribrosum. Gravenh. in litt. Heer. Helvetia.

12 Nigrum. Gravenhorst. Bavaria. Salicinum. Gyllenh. Suecia.

13 Salicis. Gyllenhal. Suecia.

14 Brunneum. Paykull. Gallia. Melanocephalum, Fab.Germania.

15 Lucidum. Erich. id.*

16 Vile. Erichson. Saxonia.

17 S Striatum. Gravenhorst.P. Minutum. Olivier. Gallia.

18 Amabile. Chevr. Heer. Helvetia.

19 Pygmæum. Gravenh. $P$.

20 Inflatum. Gyllenhal. Germania.

21 Rufulum. Erichson. Austria.

22 Brevicorne. Erichson. Sardinia.

23 Lineare. Zettersted. Germania.

24 (Testaceum. Gravenh. id.*

Pygmaum. Gravenh. id.

Brunneum. var. Grav. Suecia.

Deplanatum. var. Er. Germania.

25 (Deplanatum. Gyllenh. Gallia.* Depressum. Gravenh, $\boldsymbol{P}$. Oblongum. $v$. Lacord, id. 
26 Concinnum. Marsh. Germania.*

27 Planum. Paykull. P.* Flavipes. Fabr. Germania.

28 Læviusculum. Gyllenh. Suecia.

29 Fahræi. Zetterst. Lapponia.

30 Fenestrale. Zetterst. id.

31 Pubescens. Zetterst. id.

32 Alpinum. Zetterst. id.

33 Luridum. Gyllenhal. Finlandia.

34 Cursor. Gravenhorst. Suecia.

\section{ANTHOBIUM. Leach.}

Dermestes. Panz. Omalium. Gravenh. Oliv. Gyll. Mannerh. Latr. SiLPHA. Fabr. Panz. Herbst. Schönh, Germar. Marsh. Staphylinus. Panz.

1. Florale. Paykull. Gallia.*

2 Maculicorne. Heer. Helvetia.

3 Atrum. Chevrier.Heer. id.

4 Cribrosum. Heer. id.

5 Alpestre. Heer. id.

6 Robustum. Imh. Heer. id.

7 Paludosum. Chevrier.

8 Brevicolle. Heer. id.

9 Alpinum. Chevr. Heer. id.

10 Triviale. Erichson. P. ${ }^{*}$

Florale. Lacordaire. id.

11 Lævicolle. Heer. Helvetia.

12 Appendiculatum. Chevrier. Heer. id.

13 Dentipes, Heer. id.

14 Tenuipes. Heer. id.

15 Nigrum. Erichson. id.

16 Obsoletum. Erichson. Lusitania.

17 Excavatum. Erichson. Helvetia.

18 Semicoleoptratum.

$\left\{\begin{array}{l}\text { Panzer. Germania.* } \\ \text { Pravenh }\end{array}\right.$

Abdominale. Gravenh, id.

19 Adustum. Grav. Heer. Helvetia.

20 Fuscipenne. Heer. id.

21 Ophthalmicum. Payk. P.*

\{ Pallidum. Gravenh. Germania.

22 Montivagum. Chevrier.

23 Imhoffii, Heer.

Heer. Helvetia.

24 (Sorbi. Gyllenhal. Gallia.*

Ophthalmicum. Lac. P.

Luteum. Marsham. Anglia.

Var. Testaceum. Grav. Suecia.

Torquatum. Marsham. Anglia.
25 Tempestivum. Erich. Sardinia.

26 Scutellare, Erichson. Gallia.

T Testaceum. Lacord. . P.

27 Minutum. Fabr. Gallia." $\{$ Ranunculi. Gravenh. $P$.

28 Alpinum. Chevrier. Heer. Helvetia.

29 Flavipenne. Erichson. Lapponia.

30 Montanum. Erichson. Alp. Helvet. *

31 Luteiconne. Erichson, Sicilia. ${ }^{*}$

32 Longipenne. Erichson. Germania.

33 Longulum. Kiesenw. id.*

34 Anale. Erichson. Austria.

35 Luteipenne. Erichson. Germania.*

36 Lapponicum. Mannerh.Lapponia.

37 Palligerum. Kiesenw. Germania.

38 Signatum. Märkel. id.*

\section{BOREAPHILUS. Sahlberg.}

1 Henningianus. Sahlb, Lapponia.

CHEVRIERIA. Heer。

1 Velox. Heer.

Helvetia.

\section{CORYPHIUM. Kirby .}

1 Angusticolle. Kirby. Anglia.

PROTEINUS. Latreille.

Catenetes. Herb. Gyll. Sahlb. Omalium. Gyll. Gravenh, Oliv. Dermestes. Fab.

1 (Brachypterus. Fabr. Gallia.*

Ovatus. Gravenh. Germania.

2 Brevicollis. Erichson. $\boldsymbol{P}$.

3 Macropterus. Gyllenh, Germania.*

4 Atomarius. Erichson. id.*

5 Glaber. Newmann. Suecia.

\section{MEGARTHRUS, Kirby.}

Omalium. Gyll, Zett, Mannerh, Gravenh. oliv. Phloe obium. Lacord. StaphyexNus, Payk. Oliv, Silpha. Illig.

1 Depressus. Paykull. Gallia.* $\left\{\begin{array}{c}\text { Macropterus. Grav. Germania. } \\ \text { Var, Lineatocollis. La- } \\ \text { cordaire. } P^{*}\end{array}\right.$ 
2 Denticollis. Beck. Gallia.* Marginicollis. Lacord. $P$.

Hemiptera. Illig, var, id.

3 Hemipterus. Illiger. Gallia.* Depressus. var. Gyllenhal. Suecia. Melanocephalus. OliNitiduloides. Lacord. $\boldsymbol{P}$. Rufescens. Stephens. Anglia.

PHLOEOBIUM. Dej. Cat. Erichson.

Silpha. Müller. Germar.

1 Clypeatum. Müller. Germania.* Corticale. Lacordaire. $P$.

GLYPTOMA. Erichson. Thoraxaphorus. Motschulsky.

1 Corticinum. Motsch. $P$.
MICROPEPLUS. Latreille.

Nitidula. Marsh. Herbst. Panz. Schh. Omalium. Gyllenh.

1 Porcatus. Paykull. Gallia.* Sulcatus, Herbst. Germania.

2 Cælatus, Erichson. id.

3 Fulvus. Erichson. $\quad P$.

4 Staphylinoides. Marsh. Anglia.* \{Maillei. Guérin. Gallia.

5 Marietti. Kunze. Lombardia.

6 Tesserula. Curtis. Germania. \{ Staphylinoides. Gyll. Suecia.

PSEUDOPSIS. Newmann.

1 Obtusus. Newmann. Germania.* S Sulcatus. Newmann. Suecia.

HARPOGNATHUS. Westmaël.

1 Robynsii. Westmaël. Belgia. 


\section{ADDENDA.}

GICINDELA. $L$.

$8^{\text {bis }}$ Olivieri. Brullé.

Gracia. $12^{\text {bis }}$ Montana. Charpent. Russ. merid. $19^{\text {bis }}$ Hispanica. Gory. Hispania.

BLETHISA. Bonelli.

4 Zetterstedtii. Gyllenh.Lapponia.

NOTIOPHILUS. Duméril.

8 Marginatus. Géné. Pedemont?

9 Rufipes. Curtis. Anglia.

LEISTUS. Fröhlich.

$3^{\text {bis }}$ Montanus. Steph. Anglia.

GYCHRUS, $F$.

11 Prymnæus. Fisch. Russ. merid.

12 Torulosus. Fisch. id.

PROCRUSTES. Bonelli.

7 Excavatus, Charp. Russ, merid.

8 Caraboides. Waltl. $N$.

9 Vicinus. Waltl. id.

10 Kindermanni. Waltl. id.

\section{CARABUS. $L$.}

1 bis Planicollis. Fuessly. Transylvan. 19 is Paphius. Redtenbach. $N$.

29bis Carpathicus.Palliard.Grocia.

$55^{\text {is }}$ Luczotii. Laporte. id.

$63^{\text {bis }}$ Carinatus. Charpent. Turcia.

111bis Fossulatus. Dej.

139bis Bayardi. Solier.
Russ. merid.

Gracia?
CYMINDIS, Latr.

$6^{\text {bis }}$ Servillei. Sol. . N.

DROMIUS. Bonelli.

$10^{\text {bis }}$ Nigricornis, Brullé, Gracia. $28^{\text {bis }}$ Foveolatus. Dej. Barbaria. 28ter Cupreus. Waltl. N.

LEBIA. Latr.

7 bis Formosa. Comolli. Italia.

9 bis Nigricollis. Géné. Sardinia.

APTINUS, Bonelli.

$3^{\text {bis }}$ Cordicollis. Chaudoir. Turcia.

10bis Hispalensis. Ramb. Hisp. mer.

BRACHINUS. Weber.

20 bis Palicari. Laporte. Gracia.

22bis Longicollis. Waltl, id.

MASOREUS. $D e j$.

1 bis Affinis. Küster.

Sicilia?

NOMIUS 1. Laporte.

1 Græcus. Laporte. Græcia.

CARTERUS. $D e j$.

1 bis Megacephalus. Waltl. $N$.

$2^{\text {bis }}$ Microcephalus. Ramb. Hisp, merid.

1 Ce genre doit être placé après le genre Dyschirius. 
DITOMUS, Dej.

$\zeta^{\text {bis }}$ Lefeburei. Brullé.

Gracia.

$15^{\text {bis }}$ Latreillei. Solier.

id.

$15^{\text {ter }}$ Cœruleus. Brullé.

$18^{\text {bis }}$ Cephalotes. Dej.

$18^{\text {ter }}$ Boeticus. Ramb.

26bis Depressus. Brullé.

26ter Siagonoides. Brullé, id.

LICINUS. Latr.

$10^{\text {bis }}$ Angustus. Chevrolat. $N$.

POGONUS. Dej.

19 Smaragdinus. Waltl. N.

PATROBUS. Dej.

Ilpinus, Curtis. $\quad N$.

SPHODRUS, Bonelli.

5 Longicollis. Fisch. Russ. merid.

PRISTONICHUS. Dej.

$4^{\text {bis }}$ Boeticus. Ramb.

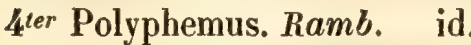

$28^{\text {bis }}$ Angusticollis. Fischer. Russ, merid.

\section{CAI_ATHUS. Bonelli.}

$2^{\text {bis }}$ Violatus. Germar. N.

$3^{\text {bis }}$ Boeticus. Ramb.

9 bis Brunneus. Brullé.

Hisp. merid.

Grocia.

23 $3^{\text {bis }}$ Angustatus. Ramb. Hisp. merid.

ANCHOMENUS. Erich.

10bis Distinctus. Chaud. N.

$48^{\text {bis }}$ Putridus. Zetterst. Lapponia.

48ter Fuliginosus. Dej.

Suecia.

$52^{\text {bis }}$ Consimilis. Gyllenh. id.

\section{OLISTHOPUS. Dej.}

5bis Græcus. Brullé.

Gracia.

POECILUS. Bonelli.

$8^{\text {bis }}$ Reichii. Waltl.

24 bis Decipiens. Waltl.
Hungaria.

id.?
ARGUTOR, Megerle.

1 bis Politus. Heer. Helvetia.

$18^{\text {bis }}$ Cincticollis. Chevrolat. $N$.

$20^{\text {bis }}$ Testaceus. Rambur. Hisp. merid.

$24^{\text {bis }}$ Velocissimus. Waltl. id.

$35^{\text {bis }}$ Acrogonus. Chaudoir. $N$.

OMASEUS. Ziegl.

$2^{b:}\{$ Furvus. Sahlb.

$\{$ Var. Ater. Sahlb.

Suecia.

3 bis Brevipennis. Chevrol. N.

$12^{b s}\{$ Pinctorum. Graëlls. Hispania.

Graëllii. L. Dufour. id.

12 ter Variolosus. Graëlls. . id.

CORAX ${ }^{1}$. Putzeys.

1 Ghiliani. Putzeys. Lusitania.

STEROPUS. Megerle.

2 bis Lacordairii. Putzeys. $N$. $4^{b i s}$ Convexus. Chaudoir. id.

PLATYSMA. Bonelli.

$14^{b}\{$ Latibula. Sturm. Hungaria.

\{Foveolata. (Megerle.\} id.

PTEROSTICHUS, Bonelli.

6 bis Pyrenæis. Chaudoir. Pyrenais.

11 bis Ecoffeti. Chevrolat. Gall. merid.

$20^{\text {bis }}$ Rugulosus. Heer. Helvetia.

20ter Heerii. Escher. Heer. id.

32 bis Auratus. Heer. id.

MYAS. $D e j$.

2 Rugicollis. Brullé. Gracia.

AGELAEA 2. Génẻ.

1 Fulva. Géné.

Sardinia.

ZABRUS. Clairville.

2 bis Rotundatus. Rambur. Hisp, merid. 2er Rotundicollis. Ramb. id. $3^{\text {bis }}$ Ambiguus. Ramb. id. $8^{\text {bis }}$ Angulatus. Ramb. id.

$1 \mathrm{Ce}$ genre doit être placé après le genre $O m a-$ seus.

2 Ce genre doit être placé après le genre Stomis. 
$11^{b / s}$ Intermedius. Zimm. N. 11 ter Convexus. Zimm. id. 24ter Silphoides. Zimm. id.

AMARA. Bonelli.

(Gela, Zimm.)

6is Rufoænea, Dej. Hisp.merid. 25 bis Planiuscula. Rosenh. Tyrolis.

(Bradytus. Zimm.)

75 $5^{\text {is }}$ Patri. Hummel. Germania.

(Leirus. Zimm.)

81 bis Borealis. Chaud. Lapponia. 95 bis Helopioides. Heer. Helvetia.

OPHONUS. Ziegl.

$5^{\text {bis }}$ Obscurus. Sturm. Germania. 18 bis Annulatus. Chaud. N. 19 bis Longicollis ${ }^{1}$. Ramb. Hisp. merid. 19ter Distinctus ${ }^{1}$. Ramb. id. $33^{b i s}$ Hispanus. Dej.: id. $34^{\text {bis }}$ Discicollis, Rosenh. $N$.

HARPALUS. Latr. $76^{\text {ss }} \begin{cases}\text { Flavicornis. Dej. } & \text { Hisp. merid. } \\ \text { Obscuricornis. St. } & \text { id. }\end{cases}$

91 bıs Caffer. Duftsch. Italia.

102 Lxvipes. Zetterst. Lapponia.

103 Ruficeps. Curtis. Anglia.

STENELOPHUS, Dej.

18bis Circumcinctus. Sahlb. Finlandia.

¿23bis Flavus. St. Germania.

23ter Humeralis, Redtenb. $N$.

\section{BRADYGELLUS. Erich.}

12 bis Rufulus. Dej.

12 er Verbasci. Sturm.

$13^{\text {bis }}$ Cordicollis. Westm

15 Testaceus. St.

16 Pallidus. St.

17 Platypterus. St.

HISPALIS, Rambur.

$2^{\text {bis }}$ Niger. Heer.

Helvetia.

\footnotetext{
1 Ces deux espèces doivent être supprimées au genre Harpalus.
} Germania.
ANOPHTHALMUS, $D e j . s t$.

2 Bilimekii. Sturm. Germania.

TRECHUS, Clairv.

38 bis Limacodes. Dej. Styria.

EPUS. Leach.

2 Robinii. Laboulbene. Gallia.

BEMBIDIUM. Latr.

$9^{\iota^{8}}$ (Bisulcatum, Nicolä. Germania. Latipenne. St. id.

Fockii. Hummel. id.

$23^{\text {bls }}$ Semistriatum. Duft. Austria.

24bis Bifoveolatum. Ramb. Hisp. merid.

(Notaphus. Meg.)

27 bis Semipunctatum. Donovan. Anglia.

42bis Kusteri. Schaum. Germania.

\section{(Peryphus, Meg.)}

104 ${ }^{\text {bis }}$ Planum. Letzn. Germania. 104ter Infuscatum. Letzn. id. $105^{b i s}$ Fornicatum. Beck. id.

$$
\text { (LEJA. Meg.) }
$$

105้ ter Dejeanii. Putzeys. Belgia.

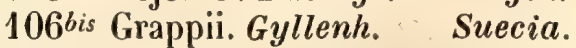
$110^{\text {bis }}$ Glaciale ${ }^{1}$. Heer. Helvetia. $111^{\text {bis }}$ Rhæticum ${ }^{1}$. Heer. id.

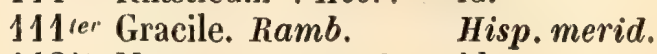
1126is Montanum. Ramb. id.

AGABUS. Leach.

$12^{\text {bis }}$ Nigroæneus. Erich. Germania. 24bis Silesiacus, Letzn. Silesia.

\section{HYDROPORUS, Clairville.}

89bis Thermalis. Germar. Germania?

HELOPHORUS, Fabr.

4 bis Villosus. Duftsch. Austria. $11^{\text {bis }}$ Borealis. Sahlb. Finlandia.

OCHTHEBIUS, Leach.

11bis Difficilis. Mulsant. Gallia.

1 Ces deux espèces doivent être supprimées à la sixième division dı même genre. 
$14^{b s}$ Hybernicus. Curtis, Hybernia. Punctatus, Stephens. id. Nobilis. Heer. Helvetia.

16 Quadricollis. Mulsant. Gallia.

\section{HYDR ANA, Kugelann.}

7 bis Polita. Kiesenwetter. Germania.

7ter Lapidicola. Kiesenw. id.

$8^{\text {bis }}$ Dentipes. Germar. id.

8ter Deplanata. Kiesenw. id.

9bis Lata. Märkel. id.

BEROSUS. Leach.

6 Hispanicus. Küster. Hispania.

HYDROBIUS, Leach.

7 Punctatostriatus, Letz, Germania.

GYGLONOTUM, Erich.

2 Hispanicum. Küster. Hispania.

3 Dalmatinum. Küster, Dalmatia.

PARNUS, Fabr.

6 ${ }^{\text {bis }}$ Lutulentus, Erich。 Sicilia.

\section{LIMNIUS, Müller.}

2 Variabilis, Steph.

3 Lacustris, Steph.

Anglia.

id.

STENELMIS. L. Dufour.

2 Consobrinus, L. Duf. Gall. merid.

HETEROCERUS, Fabr.

$6^{\text {bis }}$ Gravidus. Kiesenw. Germania.

7 bis Crinitus. Kiesenw. id.

7ter Minutus. (Dej.) Kiesw. Hispania.

\section{ADELOPS 1. Erich.}

1 Tellkampfii. Erich: N.

\section{TRICHOPTERYX. Kirby.}

8 bis Curta. Allibert.

$P$.

${ }^{1}$ Placer ce genre après le genre Leptinus.
NOSSIDIUM ${ }^{1}$, Erich.

Ptilium. Schüpp.

1 f Pilosellum. Marsh. Anglia. $\left\{\right.$ Ferrari ${ }^{2}$. Redtenb. Austria.

TOLYPHUS ${ }^{3}$. Erich

Phalacrus. (Lej.

1 Granulatus. (Dej.) Germar. Gall. merid.

OLIBRUS. Erich.

$3^{\text {bis }}$ Bimaculatus. Küster. Germania.

CRYPTARCHA. Schuck.

$2^{\text {bis }}$ 4-Signata. Küster. Germania.

PELTIS. Geoffroy.

3bis Pubescens. Erich. Germania.

TARPHIUS ${ }^{4}$. Germar.

1 Gibbulus, Germar. Sicilia.

ATOMARIA. Kirby.

39 Pallida. Wollaston. Anglia.

EPISTEMUS. Westwood.

6 Palustris. Wollaston. Anglia.

MYRMECHOXENUS, Chevrolat.

3 Opulo, Märkel.

Germania.

CORTICARIA, Mannerh.

1 bis Piligera. Mannerh. Austria.

ATTAGENUS, Latr.

4bis Maritimus. Géné. Italia.

7 bis Pocilus. Germar. N.

$12^{\text {bis }}$ Marginicollis. Küster. id.

1 Placer ce genre après I genre Ptenidium.

2 L'espèce sous le $n^{0}$ r 8 du genre Ptilium doit être supprimée.

3 Placer ce genre après le genre Phalacrus.

4 Placer ce genre après le genre Coxelus. 
ANTHIENUS, Geoffroy,

1 bis Gravidus. Küster. $\quad N$.

Iter Isabellinus. Küster. id.

$2^{\text {bis }}$ Nitidulus. Küster. Montenegro.

כ̧bis Apicalis. Küster. id.

GEORISSUS. Latr.

$5^{\text {bis }}$ Cælatus. Erich.

Germania.

PAROMALUS. Erich.

1 bis Pumilio, Erich.

Hispania.

\section{SAPRINUS, Erich.}

54 Godetii. Brullé.

Gracia.

53 Semi-æneus. Brullé. id.

56 Rugiceps. Duftsch. Austria.

\section{ABREUS, Leach.}

$8^{b i s}$ Minutus, Fabr.

Austria.

SCARABAUS. Linné.

1 bis Affinis. Brullé.

1 ter Retusus. Brullé.

Gracia.

id.

\section{ONITIS. Fabr.}

$3^{\text {bis }}$ Menalcas, Pallas.

8 bis Stevenii. Brullé.

\section{ONTHOPHAGUS, Illig.}

32bis Histeroides. Ménétriés. Europ. mer. 32 ıer Nitidicollis. Brullé.

33 bis Morio. Brullé.

Gracia.

id.

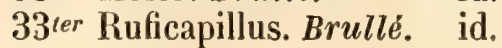

\section{APHODIUS. Illiger}

9 Alpinus, Scopoli. Austria. Rubens. (Dej.) Comoll. Tyrolis. Suturalis.(Voigt.Dhl.) Carinthia. $\{$ Var. Rubens. Mulsant, Alp. Gall.

- Constans.Schmidt.Germania.

- Carthusianus. Mul.Alp. Gall.

- Dilatatus. Schmd. Helvetia.

- Rupicola. Muls. Alp. Gall.

9 bis Corvinus. Erich.

13 (Piceus. Gyllenh.

Germania.

Alpicola. Mulsant.

Austria.

Alp. Gall.

Var, Orobius. Muls. id. $13^{\text {bis }}$ Nemoralis, Erich. Germania.

14 (Constans. (Meg.) Duft, Austria.

Vernus. Mulsant, Alp. Gall.

Var. A.Martialis. Muls. id.

Nomas, Kolenati. Russia.

Mastus. Ziegler. Austria.

24 is Convexus. Erich. Tyrolis.

29bis Sanguinolentus. Panz. Germania.

30bis Biguttatus. (Koy.) Germar. id.

31 bis Tersus. Erich. Hispania.

38 bis Tyrolensis. Rosenh. Tyrolis.

$65^{b i s}$ Conspurcatus, Linné. Germania.

65ter Zenkeri. Germar. : id.

69 Obscurus. Fabr. id.

Sericatus. Schm. Muls, Gall, merid.

Var. A. Immaturus.

70 Thermicola. Sturm. Germania.

Obscurus. Panz, Muls, Gall, merid. Var. A. Meridionalis.

Muls. id.

82 Macri. Costa. Sicilia.

83 Cribrarius. Brullé. Gracia.

84 Lateralis. Brullé. id.

85 4-Signatus. Brullé. id.

\section{ACROSSUS. Mulsant.}

$6^{\text {bis }}$ Montanus ${ }^{1}$. Rosenh. Tyrolis.

$6^{t^{r}}$ Atramentarius. Erich. Austria.

Nigripes, Kriechb. id.

9 Pollicatus. Erich. Illyria.

10 Præcox. Erich. Austria.

11 Montivagus, Erich, id.

12 f Picimanus. Erich. id.

Schmidtii. Rosenh. id.

13 Gagatinus. Ménétriẻs, Russ. merid.

\section{MELINOPTERUS. Mulsant.}

6 Rapax, Falderm. Russ, merid.

7 Costalis. Mannerh. id.

8 (Punctatosulcatus. St. Germania.

Marginalis. Steph. Anglia.

Prodromus, v. Gyll. Suecia.

Sphacelatus. Marsh. Anglia.

9 Tabidus. Erich. Dalmatia.

10 Limbatus. (Ziegl.) Er. Austria. ( Circumcinctus. Schm. id.

${ }^{1}$ L'espèce sous le no I doit être supprimée. 
11 Serotinus ${ }^{1}$. Creutzer.

Panz. Austria.

HEPTAULAUCUS. Muls.

2 (Carinatus. (Gebl.) Germ.Sibiria. Nivalis. Mulsant. Alp. Gall.

4 Esuricus, Helfer. Sicilia.

5 Villosus. Gyllenh. Saxonia.

6 Furvus. Erich. Constantinp.

AMMOEGIUS, Muls.

2 Brevis. Erich. Germania.

Elevatus, Panz. Gyll, id.

3 Gibbus. Germar. Austria. Anthracinus. Schm. Tyrolis.

RHYSSEMUS, Mulsant.

5 Plicatus. Germar.

Dalmatia.

6 Arenarius. Costa.

Sicilia.

7 Inæqualis. Erich.

id.

PSAMMODIUS, Gyllenh.

3 f Plicicollis. Erich. \{ Rugicollis. (Dahl.)

Sardinia. id.

4 Rugicollis. (Ziegl.) Er. Italia.

5 Lævipennis. Costa. Sicilia.

\section{AGIALIA. Latr.}

2 Sabuleti. Payk.

3 Rufa, Fabr.

Germania.

id.

GLARESIS 2. Friwaldszky.

1 Rufa. Friw. Erich. Hungaria.

\section{TROX.Fabr}

$1 \mathrm{et}$ ( Cadaverinus. Illig. Austria.

2 Morticini. Pallas. Russ. merid,

10 (Concinnus. (Dej.)Erich.Hungaria.

Setosus. (Ziegl.) St. Cat. id.

\section{PENTODON. Hope.}

\section{Puncticollis. Burm. N.}

Les espèces sous les $\mathrm{n}^{\mathrm{OS}} \mathrm{I9}, 4 \mathrm{I}, 42,73,79$ 56 et 58 du genre Aphodius doivent être supprimées.

"Placer ce genre après le genre Trox.
4 Elatus. Küster. Caucasus.

5 Emarginatus. Küster. $N$.

EUCHIRUS 1. Kirby.

1 Bimucronatus. Pallas. Russ. merid. RHIZOTROGUS. Latr.

$49^{b i s}$ Limbatipennis. Graëlls. Hispania.

AMPHIMALLUS. Latr.

10 Vernalis. Brullé. Gracia.

\section{ANOMALA. Koppe.}

12 Rugatipennis. Graëlls. Hispania.

PHYLLOPERTHA. Kirby.

3 Lineolata ${ }^{2}$. Fisch. Russ, merid.

4 Hirtella. Brullé. Grocia.

5 Rumeliaca. Waltl. Russ, merid.

TRIODONTA. Mulsant.

5 Puberula. Erich. Sicilia.

HYMENOPLIA. Eschsch. Muls.

12 Lineolata. Ramb. Hisp. merid.

13 Cortulata. Graëlls. id.

ANTHIPNA. Eschsch. Latr.

3 Carceli. Laporte. Grocia.

BUPRESTIS. Linné.

(Calcophora. Serv. Sol.)

1 bes Detrita. Klug. Grocia.

(Ancrlocheira. Dej. Cat.)

$23^{\text {ss}}$ (Splendida. Payk. Austria.

Pretiosa. Herbst. id.

Splendens. Fabr. (?) id.

AGRILUS, Curtis.

$4^{b i s}$ Coryli. Andersch. Germania. $36^{\text {bis }}$ Subuliformis. Mannh. Volhynia. 36ter Sahlbergi. Mannerh. Fennia.

1 Placer ce genre après le genre Calicnemis

2 Les espèces sous les nos 26 et 27 du genre Anisoplia doivent être supprimées. 
$38^{\text {bis }}$ Littlii. Curtis.

38ter Chryseis. Curtis.

Anglia.

id.

CORRABUS. Lap. et Gory.

13 Pruinosus, Küster. $N$.

14 Subfasciatus. Küster. Montenegro.

15 Laticollis, Olivier. Gall, merid.?

ANTHAXIA, Solier.

4 Præclara. Mannerh. Dalmatia.

46 Podolica. Mannerh. Podolia.

47 Praticola. Laferté. Gallia.

48 Nigritula, Erich. N.

49 Basalis. Küster. id.

EUCNEMIS. Ahrens.

1 bis Deflexicollis. (Meg.) Germania.

HYPOCALUS. Eschsch. Dej. Cat.

3 Spondyloides. Germar. $N$.

4 Unicolor. Latreille. id.

MELANOTUS. Eschsch, Redtenb.

18 Subvillosus. Brullé. Gracia.

LACON. Laporte.

2 Kokeilii. Küster. Carniolia.

ATHOUS, Eschsch, Redtenb.

56 Cavus. Germar. Dalmatia?

57 Sutura-nigra. Chevrol. Gallia?

CAMPYLUS. Fisch.

3 Borealis. Payk.

Lapponia.

LIMONIUS, Eschsch, Redtenb.

22 Longulus. Gyllenh. Suecia.

CARDIOPHORUS. Eschsch.

33bis Musculus. Erich.

Germania.

DIACANTHUS. Latreille.

1 bis Bifasciatus. Küster. Transylvan.

AGRIO'TES. Eschsch. Redtenb.

15 Rulipalpis. Brullé. Gracia.

16 Punctulatus. Brullé. id.
CEBRIO. Olivier.

18 Rufifrons, Graëlls. Hisp. merid.

DYCTIOPTERUS. Latreille.

9 Hybridus, Mannerh. Fennia.

LAMPYRIS. Linné.

7 Germari. Küster. Dalmatia.

TELEPHORUS. Geoffroy.

92 Annularis. Ménétr. Russ. merid.

93 Consobrinus, Märk. Germania.

94 Alpicola. Märk. id.

95 Morosa. Märk. id.

96 Distinguendus. Märk. id.

97 Sulcifrons. Märk. id.

98 Maculicollis. Märk. id.

99 Boreellus. Zetterst. Lapponia.

100 Figurata. Mannerh. Fennia.

MALACHIUS, $\boldsymbol{F}$.

1bis Labiatus, Brullé, Gracia.

ATTALUS. Erich.

$4^{\text {bis }}$ Genei. Küster. Sardinia.

$8^{b i s}$ Nigricollis. Küster. Dalmatia.

ANTHOCOMUS. Erich.

$\check{5}^{\text {bis }}$ Parallelus. Küster. $N$.

COLOTES. Erich.

3 Nigripennis, Küster, $N$.

DASYTES. Fabr.

25bis Virens. Suffrian. Germania.

53 $3^{\text {bis }}$ Tarsalis. Gyllenh. Austria.

82 Testaceus. Oliv. P.

CORYNETES. Payk.

2 Cueruleus, de Geer. Gall. merid.

3 Ruficornis. Sturm. Germania.

4 Pusillus. Klug. N.

5 Geniculatus. Klug. id.

PTINUS, Linné.

1 bis Hololeucus, Fald. Russ, merid. 
$5^{\text {bis }}$ Ornatus. Müller. Germania.

7bis Hirtellus. Sturm. id.

Tter Subpilosus. St. id.

22bis Dubius. St. id.

22ter Coarcticollis. St. id.

\section{MASTIGUS. Hoffmansegg.}

2 Prolongatus, Gory. N.

ANOBIUM. Fabr.

$2^{\text {bis }}$ Excisum. Mannerh, Fennia.

$14^{\text {bis }}$ Explanatum. Mannh. id.

15 bis Rufum. Illig. Austria.

37 his Immarginatum. Müll. Germania.

CIS. Latreille.

$13^{\text {his }}$ Fissicornis: (Motsch.) Mellié. Russ. merid.

DORCATOMA. Herbst.

$4^{b i s}$ Affinis. Sturm.

Germania.

XYLETINUS, Latreille.

1 bis Serratus. Fabr.

Germania.

8 bis Hæmorrhoidalis, Illig. Austria.

16 Ochraceus. Sturm. Hungaria. T Testaceus. St. Cat. id.

17 Bucephalus. Müller. Germania.

18 Pilosus. Müller. id.

LYMEXYLON. Latr.

2 Flabellicornis. Sahlb. Fennia.

TOMICUS, Latreille.

6 bis Euphorbiæ. Küster. Dalmatia. 26is Fuscus. Marsh: Anglia.

HYLESINUS. Fabr.

2 bis Suturalis. W. Redtenb. Austria.

HYLASTES. Erich.

10bis Corticiperda, Illig. Austria.

$13^{\text {bis }}$ Brunneus. Erich. id.

23 Spartii. Nordlinger. id.

NANOPHYES. Schönh'.

16bis Gi'acilis. Redtenb. Austria.

21 Marmoratus. Fourcroy. $P$.
ACALLES. Schönh.

19bis Rulirostris. Schönh. Germania.

CELIODES, Schönh.

20bis Affinis. Steph.

Anglia.

BARIDIUS, Schönh.

3S bis Angustus, Brullé. Gracia.

SIBYNES. Schönh.

20 Vittatus. Germar. Austria.

TYCHIUS. Germar.

$6^{\text {bis }}$ Squamulatus. Sch. Germania.

DORYTOMUS. Germar.

33 Atomarius. Géné. Sardinia.

LARINUS. Germar.

39bis Lynx. I üster. $\quad N$.

79 Subcostatus. Brullé. Gracia.

PURPURICENUS. Serville.

10 Boryi. Brullé. Gracia.

AROMIA. Serville.

5 Chlorophana, Fischer. Russ, merid.

CALLIDIUM. Fabr.

16 Latreillei. Brullé. Gracia.

17 Fasciolatum. Stév. Russ, merid.

CLYTUS. Fabr.

27bis Lugens. Küster. N $\quad$.

\section{STENOPTERUS. Illig.}

6 Femoratus, Stév.

7 Decorus, Géné.

8 Gracilis. Brullé.

Russ. merid. Sardinia.

Gracia.

EDILIS. Serville.

5 Modestus. Schönh. Suecia.

DORGADION. Dalman.

44 Perecii. Graëlls.

Hispania. 
64 Rasumofskii. Fisch. Russ, merid.

65 Handschuchii. K ïster. $N$.

66 4-Maculatum. Küster. Sicilia.

67 Stevenii. Waltl. Russ. merid.

68 Virletii. Brullé. Gracia.

69 Femoratum. Brullé. id.

70 Bizanthinum. Waltl. id.

71 Laqueatum. Küster. id.

\section{SAPERDA. Fabr.}

7 Duponcheli. Brullé.

8 Flavescens. Brullé.

9 Genei, Aragona.

Grocia.

id.

Italia.

\section{(IBEREA. Mulsant.}

9 Cœса. Küster.

$N$.

PHYT ECIA. (Dej.) Muls.

35 Cyclops. Krister. N.

36 Baccueti. Brullé. Gracia.

37 Icterica. Schaller. N.

38 Humeralis, Fisch. Constantinp.

39 Languida. Ménétr.

40 Fumigata. Küster.

41 Trilineata. Schönh.

42 Decora. Stév. Turcia.

N.

id.

Russ, merid.

AGAPANTHIA. Serville.

18 Umbellatorum. Waltl. $N$.

PACHYTA. (Dej.) Serville.

13 Picta. Märk.

Germania.

14 Erratica. Dalman.

$N$.

15 7-Signata. Kuister. id.

16 Erythrura. Ktister. id.

LEPTURA. Linné.

19bis Mieziana, Graëlls. Hispania.

CASSIDA. Linné.

$12^{\text {bis }}$ Lata. Suffrian.

Germania.

12 er Deflorata. Illig.

Austria.

$13^{\text {bis }}$ Hexatigma. Suffrian. Germania.

$13^{\text {er }}$ Depressa. Suffrian. id.

28bis Puncticollis. Heyden, id.

ADIMONIA. Laichart.

$13^{\text {bis }}$ Circumcincta.Mannh. Fennia. 17 bis OElandica. Gyllenh. OElandia.
GYRTONUS. Dalman.

7 Montanus. Graëlls. Hispania.

8 Ruficornis. Graëlls. id.

TIMARCHA. Redtenbach.

35 Erosa. Herr. Sch. N.

36 Immarginata, Herr.

Sch. id.

37 Stricticollis. Géné. Sardinia.

38 Scortea. Germar. N.

39 Splendens. Koch. id.

CHRYSOMELA. Fabr.

123 Sulcicollis. Graëlls. Hispania.

\section{LINA. Redtenb.}

$2^{\text {bis }}$ Salicetti, Suffrian. Germania.

PHEDON. Redtenb.

12 Salicinum. Heer. Helvetia.

13 Concinnum. Steph. Anglia.

14 Tumidulum. Kirby. id.

COLASPIDEA. de Castelnau.

5 Oblonga, Blanchard. Sicilia.

GONIOCTENA. Redtenb.

8 Nivosa. Heer.

9 Lasserrei. Heer. id.

10 Lineata. Géné. Italia.

PACHNEPHORUS, Redtenb.

8 Globosus. Küster. $N$.

9 Nitidulus, Dahl. Sicilia.

EXOCHOMUS. Redtenb.

3 Pubescens, Küster, N.

DAILOGNATA. Stéven.

10 Lævigata. Brullé. Gracia.

TENTYRIA. Latreille.

56 (Rugosostriata, Ramb. Hisp. merid.

(53) Dorsosulcata.Chevrl. Algiria.

71 Curculionides. Host. it. 
72 Angustata, Brullé.

73 Rotundata. Brullé. id

74 Wiedmanni. Ménét. Turcia.

\section{PIMELIA, Fabr.}

43 ( Payraudii. Latreille. Corsica. (52) Nitida. (Gaubil.) id.

73 Timarchoides. Ménét. Russ. merid.

74 Varicosa. Ménétr. id.

\section{SEPIDIUM. Fabr.}

12bis Castillanum, Graëlls, Hisp. merid.

\section{SCAURUS. Fabr.}

1 bis Giganteus.(Meg.) Kiist. Sardinia.

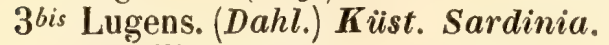
14 Gracilis. Küster. N.

\section{BLAPS. Fabr.}

4 bis Plana. Solier. Hispania.

4 ter Abbreviata. (Dej.) Sol. Hisp. merid. $4 q^{\text {ter }}$ Requieni. Solier. Algiria.

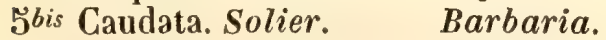

9 Hispanica. (Dej.) Sol. Hisp. merid. 12 bis Vicina. Solier.

12 er Barbara. Solier. Algiria.

$12 q^{2}$ Substriata. Solier. Barbaria.

Sulcata. Mus. Taur.

13 Prodigiosa. Erich. Multicostata. Sol.

$13^{\text {bis }}$ Nitidula. Solier. id.

id.

Algiria.

id.

$15^{\text {bis }}$ Brachyura. Kïter. N

$16^{\text {bis }}$ Mucronata. Cristofori. Gall. merid.

16 ter Græca. Solier.

$16 q^{\text {ter }}$ Proxima. Solier.

2| bis Rotundata. Solier.

Gracia.

Gall. merid.

27bis Rectangularis. Solier, Barbaria.

39 bis Striolata. Küster. Sardinia.

39ter Clypeata. Germar. - N.

42bis Gibbosa. Brullé. Gracia.

42ter Affinis. Brulle.

42 $q^{\text {ter }}$ Subrugosa. Duftsch. $\quad N$.

\section{MISOLAMPUS. Latr.}

$1 \begin{cases}\text { Gibbula. Herbst. } & \text { Lusitania. } \\ \text { Hoffmanseggii. Latr. Hisp. merid }\end{cases}$ 1 bis Lusitanicus. de Brême. Lusitania.

1 ter Scabricollis, Graëlls. Hisp, merid.
LENA. Latreille.

4 Ferruginea. Küster. Dalmatia.

PEDINUS. Latr.

2 is Politus. Sturm. N.

כois Sulcatus. Ménétr. Russ. merid.

$7^{\text {bis }}$ Byzantinicus. Waltl. Gracia.

PANDARUS, Latr.

11 bis Cælatus. Brullé.

Gracia.

PHILAX. Brullé.

$3^{\text {bis }}$ Gravidus. Brullé. Gracia.

3er Plicatulus. Brullé. id.

$3 q^{\text {ter }}$ Messenius. Brullé. $\quad$ id.

3quq.Obscuripennis. Brullé. Gracia.

3sext.Tentyrioides, Brullé. id.

\section{OPATROIIIES 1 , Brullé.}

1 Punctulatus. Brullé. Gracia.

\section{OPATRUM. Fabr.}

19bis Gemellatum. Brullé. Gracia.

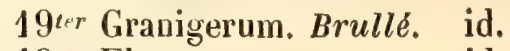

$19 q^{\text {ter }}$ Elevatum. Brullé. $\quad$ id.

26 $6^{\text {bis }}$ Hispidosum. Brullé. id.

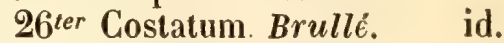

42 Obesum. Brullé. id.

43 Intermedium. Fisch. Russ. merid.

CHEIRODES. Dej. Cat.

2 Sardeus. Géné. Sardinia.

PLATYDEMA, Laporte.

5 Azurea. Waltl. N.

6 Europæa. Laporte. Europa.

ULOMA. Latreille.

2 Picea, Küster. Dalmatia.

TRIBOLIUM, Mac-Leay.

3 Bifoveolatum, Duft. Austria.

HELOPS. Fabr.

9bis Azureus, Brullé.

Gracia.

Ce genre doit être placé après le genre Philax. 
$62^{\text {bis }}$ Maurus. Waltl.

63bis Morio. Brullé.

$64^{b s}\{$ Tenebricosus. Brullé. id.

Obesus. Waltl. id

64ter Genei. Géné.

PRIONYGHUS. Solier.

3 Melanarius. Germar. N.

CISTELA. Geoffroy.

18 Sturmii. Küster. N.

19 Quadricollis. Brullé. Groecia.

20 Rugosicollis. Brullé. id.

CONOPALPUS. Gyllenh.

$1^{6}\{$ Testaceus. Olivier. Gallia.

I Nigricornis. Germar. Germania.

ABDERA 1. Stephens.

1 4-Fasciata. Steph. Anglia.

PELECOTOMA ${ }^{2}$. Fisch.

1 Fennica, Payk. Fennia.

EMENADIA. de Castelnau.

$3^{\text {bis }}$ Apicalis. Küster. Gall. meria.

MORDELLA, Fabr.

16 $6^{\text {bis }}$ Parvula. Gyllenh. Suecia.

ANASPIS, Geoffroy.

$5^{\text {bis }}$ Fusca. Schrank.

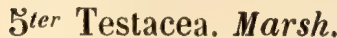

7 bis Bimaculata. Rossi.

16 bis Nigricollis, Marsh.

$25^{\text {bis }}$ Biguttata. Marsh.

25 ter Atra. Fabr.

Germania. Anglia.

Italia.

Anglia.

id.

Germania.

ZONITIS. Fabr.

13 Bimaculata. Tausch. Russ. merid.

MELOE. Linné.

17bis Erythrocnemus. Pall. Russ, merid.

1 Placer ce genre après le genre Hallomenus.

2Placer ce genre après le genre Ripidius.
34 Cyanellus. Brullé.: Gracia.

35 Rugulosus. Brulle. id.

MYLABRIS. Fabr.

$8^{b i s}$ Schreibersii. Heeger. Sicilia.

30bis Dufourii. Gruëlls. Hisp. merid.

ら1bis Caspica. Ménét. Russ. merid.

62 Bis-5-punctata. Friw. N.

63 Contigua, Schm. et

Helf. Smyrna.

64 Tenera. Germar. N.

65 Salonica. Pallas. Russ, merid

OENAS. Latreille.

3 Dispar. St. Cat. Hispania.

CEROCOMA. Geoffroy.

9 Micans. Ménétr. Russ merid.

PROBOSCA. Schmidt.

$1^{6 s}$ Unicolor. Küster. Hispania.

\{ Plumbea. Suffrian. id.

CHITONA. Schmidt.

1 Connexa. Fabr. Hispania.

Variegata. Germar. id.

Fasciata. Mus, Berol. id.

2 Ornata. Küster. id.

\{trigilata. Suffrian. id.

SALPINGUS. Illig.

$2^{\text {bis }}$ Bimaculatus. Gyllenh. Suecia.

ANTHICUS. Geoffroy.

7bis Schmidtii. Rosenh. Tyrolis.

$30^{\text {bis }}$ Genistæ. Rosenh. id.

\section{SCYDM ENUS, Latr.}

2 Scutellaris. Mill. et

$\left\{\begin{array}{cl}\text { Kunze. } & \text { Gallia. } \\ \text { Schönherri. (Dej.) } & \text { Germania. }\end{array}\right.$

4 Collaris. Müll. et Kze. Gallia.

Tuberculatus. Chaud. Podolia.

Propinguus. Chaud. id.

Rossii. (Dej.) Dalmatia.

8 Angulatus. Müll, et Kz.Alsatia.

Impressus, Sahlb. Fennia.

(14) Wighami, Denny. Anglia. 
19 Motschulskyi. Schaum. Germania.

(49) ơ Linnei. (Dej.) Styria.

(o Fabricii. (Dej.) id.

22 Denticornis. Mül. et Kz. Helvetia.

Latreillei. (Dej.) Germania.

Dennii. Steph.

28 Hirticollis. Gyllenh. Gallia.

Fimetarius. Chaud, Podolia.

Minutus. Fabr. Germania.

Illigeri. (Dej.) P.

33 Wetterhalii. Gyllenh, Gallia.

Hirtus. Sahlb. Fennia.

(34) Quadratus. Mül. et Kz. Germania. Megerlei. (Dej.) Austria.

36 Exilis. Erich. Germania.

Vicinus. Chaud. Podolia.

(48) Sahlbergi. (Dej.) Fennia.

BYTHINUS, Leach.

$12^{\text {bis }}$ Distinctus. Chaud. Pomerania.

\section{MYRMEDONIA. Erich.}

9 bis Haworthi ${ }^{1}$ Steph.

17 Gracilis. Hochhuth. Caucasus.

18 Subnitida. Hochh. id.

19 Confragosa. Hochh. id.

HOPLONOTUS². Schm.-Gœbel.

1 Laminatus, Sch.-Gæb. Germania.

FALAGRIA, Leach.

7 Elongata. Kolenati, Caucasus.

BOLITOCHARA. Mannerh.

9 Pubescens. Kolenati. Caucasus.

10 Venusta. Hochh. id.

11 Lrviuscula, Hochh. jd.

OCALEA. Erich.

$8^{\text {bis }}$ Concolor. Kiesenw. Germania.

TACHYUSA. Erich.

1 Coarctata. Erich. Gallia.

(2) Nigrita. Chevr. Heer. Helvetia.

1 Cette espéce a été reconnuc distincte de la Fulgida.

¿Placer ce genre après te genre Myrmedonia.
HOMALOTA. Mannerh.

17 bis Palustris. Kiesenw. Germania.

94 Anceps. Erich. Gallia.

(110) Angularis. Heer. Helvelia.

142 Lævigata. Hocht, Caucasus.

143 Forticornis. Hochh. il.

144 Fuvicollis. Hochh. id.

145 Granosa. Hochh. id.

146 Tæniata. Kolenati. id.

147 Biguttula. Kolenati. id.

OXYPODA. Mannerh.

$10^{\text {bis }}$ Hospita. Grimm. Germania.

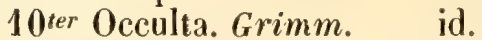

38 bis Rufipennis. Heer. Helvetia.

58 Stevenii. Mannerh. Caucasus.

59 Gotsehii. Hochh. id.

60 Angusticollis. Hochh. id.

ALEOCHARA. Gravenh.

$4^{\text {bis }}$ Biguttata. Heer. Helvetia.

4 ter Lateralis. Ull. L. Redt. Austria.

18 bis Alpicola. Heer. Helvetia.

31 bis Clavicornis. Redtenb. Austria.

35 Flavomaculata.Ménét. Caucasus.

36 Convexiuseula.Kolen. id.

37 Apicalis. Ménét. id.

38 Erythroptera. Grav. id.

39 Solida. Hochh. id.

\section{GYROPHENA. Mannerh.}

18 Glacialis. Kolenati. Caucasus.

CONURUS. Steph.

10 Erythrocephalus. Heh.Caucasus.

11 Rufulus. Hochh. id.

12 Dimidiatus. Hochh. id.

13 Caucasicus. Kolenat. id.

TACHYPORUS. Gravenh.

$12^{\text {bis }}$ Pisciformis. Heer. Helvetia.

LAMPRINUS 1 . Heer.

1 Lasserrei. Heer. Helvetia.

TACHINUS, Gravenh.

14 Caucasicus, Kolenati. Caucasus.

1 Placer ce genre après le genre Tachrporus. 
15 Pallipes. Gravenh.

16 Basalis. Erich.

17 Rufitarsis, Hochh.

\section{BOLETOBIUS. Leach.}

16 Pulchellus, Mannerh. Fennia.

17 Insignis. Hochh. Caucasus.

18 Flavicollis. Hoch. id.

19 Phædrus. Kolenati. id.

\section{MYCETOPORUS. Mannerh.}

$5^{\text {bis }}$ Piceus. Maklin.

$6^{\text {bis }}$ Ruficollis. Maklin.

Germania.

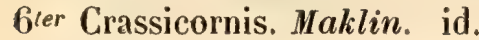

$6 q^{\text {tex }}$ Bicolor. Maklin. id.

$6 q^{n q}$ Longicornis. Maklin. id.

10 Debilis. Maklin. id.

OTHIUS, Leach.

1 bis Grandis. Hochh.

Caucasus.

XANTHOLINUS. Erich.

18 Sanguinipennis. Kolen. Caucasus.

19 Flavocinctus. Hochh. id.

20 Fasciatus. Chaud. id.

21 Scripticollis. Hochh. id.

22 Hæmatodes. Kolenati. id.

LEPTACINUS, Erich.

$3^{\text {bis }}$ Angustatus. Grimm. Germania.

\section{STAPHYLINUS, Linné.}

$3^{\text {bis }}$ Ciliaris. Steph.

23 Cingulus. Comolli. Italia.

OCYPUS. Kirby.

1 bis Brachypterus. Brullé. Gracia.

9 I Similis. Fabr.

Gallia.

Brevipennis. Heer. Helvetia.

PHILONTHUS. Leach.

33bis Megacephalus. Heer. Helvetia.

90 Binotatus. Gravenh. Germania.

9 Ephippium. Nordm. Fennia.

QUEDIUS. Leach.

$3^{\text {his }}$ Chrysurus, Kiesenw.

Küst. Germania.
30 ) Monticola. Erich. Germania.

(26) Paradisianus. Heer. Helvetia.

SCIMBALIUM. Erich.

3 Anale. Nordm.

Fennia.

ACHENIUM. Leach.

2 Planum. Erich. Germania.

LATHROBIUM. Gravenh.

$13^{b / s}$ Longicorne. Redtenb. Austria.

SCOPEUS. Erich.

6 Pusillus, Kiesenw. Germania.

SUNIUS, Leach.

14 Unicolor. Curtis. Anglia.

STENUS. Latr.

62 Rusticus. Erich. P.

(59) Rufimanus. Heer. Helvetia.

BLEDIUS. Leach.

38 Picipennis. Hochh. Caucasus.

PLATISTHETUS. Mannerh.

9 Lævis. Kiesenw. Germania.

OXYTELUS, Gravenh.

19 Stigifrons, Hochh. Caucasus.

TROGOPIIL EUS.

12 $2^{\text {his }}$ Halophilus. Kiesenw. Austria.

21 Caucasicus. Hoch'. Caucasus.

22 Tarsalis. Hochh. id.

23 Brevipennis. Hochh. id.

THINOBIUS 1, Kiesenwetter.

1 Longipennis. Heer. Helvetia. \{ Cilialus. Kiesenw. Austria.

DELEASTER, Erich.

2 Adustus. Küster. Austria.

1 Placer ce genre apjès le genre Trigonurus. 
PROGNATA. Latreille.

2 Humeralis. Germar. Germania.

ANTHOPHAGUS. Gravenh.

$5^{b i s}$ Fallax. Kiesenw. Germania.

8 Austriacus. Erich. Austria.

(6) Alpestris. Heer. Helvetia.

20 Arpedinus. Hochh. Caucasus,

GEODROMUS' ${ }^{1}$. Heer.

AnthopHagus. Gravenh.

1 Plagiatus. Fabr. Gallia.

ARPEDIUM. Erich.

2 Brachypterum. Erich. Germania.

(4) Gyllenhalii. Sahlb. id.

1 Genre créé aux dépens du genre Anthopha. gus, avec l'espèce de ce genre, $\mathrm{n}^{\circ}$ I8.
LATHRIM EUM. Erich.

6 Hirtellum. Heer. Helvetia.

17 Excavatum. Erich. Helvetia.

(6) Robustum. Heer. id.

$20^{\text {bis }}$ Limbatum. Erich. Austria.

24bis Pallens. Heer. Helvetia.

26 Scutellare. Erich. Gallia.

(22) Montivagum. Heer. Helvetia.

37 bis Puberulum. Kiesenw. Germania.

37ter Fimetarium. Mannerh. Fennia.

BOREAPHILUS, Sallberg.

2 Brevicollis. Halid. Germania. 


\section{TABLE ALPHABÉTIQUE}

\section{DES NOMS GÉNÉRIQUES CONTENUS DANS CE VOLUME.}

Nota. Les noms en italique ne sont pas adoptés et sont seulement cités comme synonymie.

Abax. 32.

Abdera. 273.

Abræus. 79. 267.

Acalles. 135. 270.

Acalyptus. 139.

Acanthocinus. 179.

Acanthoderes. 178.

Acanthopus. 218.

Acentrus. 131.

Achenium. 253. 275.

Acidota. 260.

Acilius. 44.

Acinopus. 36.

Acisba. 211.

Acmæodera. 101.

Acorius. 34.

Acrodon. 35.

Acrognathus. 258 .

Acrossus. 89. 267.

Actinophorus. 82.

Acupalpus. 39.

Acylophorus. 252.

Adelocera. 108.

Adelops. 266.

Adelostoma. 216.

Aderus. 237.

Adesmia. 213.

Adexius. 157.

Adimonia. 138. 271.

Adolus. 56.

Adonia. 206.

Idrastus. 112.

Edilis. 179. 270.

Egialia. 91. 268.

Egosoma. 174.
Folus. 110.

Epus. 40. 265.

Esalus. 80.

Ethiessa. 92.

Etophorus, 20.

Agabus. 45. 265.

Agapanthia. 182. 271.

Agaricophagus. 59.

Agathidium. 60.

Agelæa. 264.

Agelastica. 189.

Aglenus. 66.

Agnathus. 237.

Agonum. 28. 29.

Agrilus. 103. 268.

Agriotes. 112. 269.

Agrypnus. 107.

Agyrtes. 56.

Akis. 216.

Alaus. 108.

Albana. 179.

Aleochara. 244. 274.

Alexia. 70.

Allecula. 225.

Alophus. 160.

Alpous. 15.

Alphitobius. 222.

Nlica. 189.

Alticopus. 171.

Amalus. 141.

Amara. 34. 265.

Amarignus. 225.

Amauronia. 119.

Amaxobium. 114.

Amblis. 103.
Amblycerus. 172.

Amblychus. 25.

Amblyderus, 235 .

Amblystomus. 39.

Ammocius. 291. 268.

Amorphocephalus. 167.

Ampedus. 110.

Amphicoma. 100.

Amphicyliis. 60 .

Amphimallus. 94. 268.

Amphotis. 62.

Anærea. 181.

Anæsthetis. 181.

Anatia. 181.

Anaglyptus. 177.

Anaspis. 228. 273.

Anatis. 208.

Anatolica. 212.

Anchomenus. 27. 264.

Ancylocheira. 103. 268.

Anelastes. 112.

Anisarthria. 58.

Anisarthron. 177.

Anisodactylus. 36.

Anisonchus. 95.

Anisoplia. 95.

Anisorhynchus, 158.

Anisosticta. 202.

Anisotoma. 59.

Anobium. 122. 270.

Anogcodes. 232.

Anomaus. 20.

Inomala. 94. 268.

Inommatus. 66 .

Anoncodes. 232. 
Anophthalmus. 40. 265.

Anoplistes 175.

Anoplodera. 184.

Anoplus. 139.

Anoxia. 92.

Anthaxia. 104. 269.

Anthelephila. 235.

Antherophagus. 68.

Anthia. 22.

Anthicus. 23.5. 273.

Anthipna. 101. 268.

Anthobium. 260. 276.

Anthobium: 258.

Anthocomus 117. 269.

Anthonomus. 141.

Anthophagus. 2\%9. 276

Anthophagus. 258. 276.

Anthracias. 223.

Anthrenus. 74. 267.

Anthribus. 171.

Apales. 229.

Apalochrus. 117.

Apate. 125.

Apatura. 104.

Apeistus. 66.

Aphanisticus. 106.

Aphodius. 86. 267.

Aphthona. 190.

Apion. 167.

Aplidia, 94.

Aplolocnemis. 119.

Apoderus. 171.

Apotomus. 24.

Apteropeda. 192.

Aptinus. 21. 263.

Arcopagus. 239.

Argante. 102.

Argopus. 192.

Argutor. 29. 264.

Arhopalus. 141.

Aristus. 23.

Aromia. 175. 270.

Arpedium. 259. 276.

Artia. 93.

Asclera. 232.

Asemum. 176.

Asida. 213.

Aspidiphorus. 75.

Astenus. 255.

Astrapreus. 253.

Astrapaus 249.

Astynomus. 179.
Atelestus. 118.

Ateuchus. 85. 82, 83.

Athous. 108. 269.

Atomaria. 69. 266.

Atopa. 113.

Attagenus. 74. 266.

Attalus. 117. 269.

Attelabus. 171.

Auchenia. 186. 187.

Aulacocheilus. 70.

Aulacopus. 74.

Aulacus. 160.

Auletes. 170.

Aulonium. 65.

Aurigena. 102.

Autalia. 240.

259. Axinopalpis. 178.

Badister. 25.

Bagous, 137.

Balaninus. 141.

Balanomorpha. 191.

Barathrcea. 198.

Baridius. 136. 270.

Baris. 136.

Barynotus. 159.

Batrisus. 238.

Bembidium. 40. 265.

Berginus. 73.

Berosus. 54. 266.

Biphyllus. 68.

Bitoma. 69. 66. 68.

Bius. 223.

Blaps. 217. 272.

Blastanus. 235.

Bledius. 257. 275.

Blemus. 40.

Blethisa. 14. 263.

Bolboceras. 81 .

Boletaria. 73.

Boletobius. 247. 275.

Bolitochara. 240. 276.

Bolitophagus. 221.

Boreaphilus. 261. 276.

Boros. 223.

Bostrichus. 125.

Bothrideres. 66.

Bothynoderes. 161.

Brachinus. 21. 263.

Brachonyx. 142.

Brachycerus. 166.

Brachyderes. 164.

Brachypalpus. 52.

Brachyphylla. 96.
Brachypterus. 52.

Brachyrhinus. 147.

Brachytarsus. 172.

Bradybatus. 142.

Bradycellus. 39. 265.

Bradytus. 35. 265.

Brius. 147.

Bromius. 197.

Brontes. 67.

Broscosoma. 36 .

Broscus. 33.

Bruchella. 172.

Bruchus. 172.

Bryaxis. 238.

Bryocharis. 247.

Bubas. 83.

Bulbifer. 128.

Buprestis. 102. 268.

Byrrhus. 79.

Bythinus. 239. 274.

Byturus. 73.

Cacicula. 205.

Cafius. 248.

Calais. 108.

Calamobius. 182.

Calandra. 128.

Calathus. 27. 264.

Calcar. 223.

Calcophora. 102. 268.

Calicnemis. 92.

Calisthenes. 19.

Callichroma. 175.

Callidium. 175. 270.

Callimus. 178.

Callistus, 24.

Calodera. 240.

Caloderus. 109.

Calomicrus. 189.

Calopus. 224.

Calosoma. 19.

Calvia. 208.

Calyptobium. 71.

Calyptomerus. 60.

Calyptopsis. 211.

Calyptorhina. 198.

Camptolenes. 198.

Camptorhinus. 136.

Campylirhynchus. 139.

Campylus. 109. 269.

Cantharis. 231.

Capnodis. 102.

Carabus. 16. 263.

Carabus. 259. 
Cardiomera. 27.

Cardiophorus. 109. 269.

Carpophilus. 61.

Cartallum. 178.

Carterus, 23. 263.

Cassida. 187. 171.

Catalasis. 92.

Cataphronetis. 222.

Cateretes. 69. 61. 261.

Cathamistes. 75.

Cathops. 56.

Cathormiocerus. 154.

Cebrio. 113. 269.

Celia. 34. 265.

Celiodes. 135̌. 270.

Centroglossa. 245.

Cephalostenus. 217.

Cephalotes. 33.

Cephennium. 238.

Cerambyx. 174.

Cerandria. 222.

Ceratipes, 117.

Ceratoderus. 233.

Ceratophyus. 80.

Cercus. 61.

Cercyon. 53.

Cerocoma. 231. 273.

Cerophytum. 106.

Ceruchus. 80.

Cerylon, 66.

Cetonia. 98.

Ceutocerus. 77.

Ceutorhynchus. 132.

Chatocnema. 192.

Chatoporus. 75.

Charopus. 118.

Chasmatopterus. 97.

Checlotoma. 199.

Cheirodes. 221. 272.

Chennium. 238.

Cheporus. 33.

Chevrieria. 261.

Chilocorus. 203.

Chiloneus. 153.

Chitona. 233. 273.

Chlænius. 24.

Chlobius. 147.

Chlorophanus. 162.

Chœtarthria. 52.

Choleva. 56.

Choragus. 171.

Chrysanthia. 233.

Chrysobothris. 102.
Chrysochus. 197.

Chrysodera. 105.

Chrysomela. 171. 271.

Chypus. 171.

Cicindela. 13. 26.

Cicones. 65.

Cillenum. 40.

Cionus. 131.

Cis. 123. 269.

Cistela. 225. 273.

Clambus. 60.

Clavifer. 239.

Claviger. 239.

Cleonis. 160

Cleonus. 160.

Cleopus. 130.

Clerus. 120.

Clinidium. 66.

Clivina. 23.

Clunipes. 82.

Clypeaster. 202.

Clythra. 197.

Clytus. 176. 270.

Cneorhinus. 165.

Cnemidotus. 49.

Coccidula. 205.

Coccinella. 206.

Coculus. 102.

Colodera. 92.

Calostoma. 52.

Colaphus. 196.

Colaspidea. 196. 271.

Colaspidema. 196.

Colenis. 59.

Colobicus. 65 .

Colobopterus. 86.

Colon. 57.

Colophotia. 114.

Colorhinus. 92.

Colotes. 269.

Colovocera. 210.

Colydium. 65.

Colymbetes. 44.

Compsidia. 181.

Coniatus. 156.

Conopalpus. 226. 273.

Conurus. 246. 274.

Cophosus. 31.

Copris. 83.

Copromorphus. 86 .

Coprophilus. 258.

Coptocephala. 199.

Coptogaster. 127.
Corax. 264.

Corræbus. 104. 269.

Corsyra. 22.

Corticaria. 72, 266.

Corticus. 65.

Corylophus. 203.

Corymbites. 111.

Corynetes. 121. 269.

Corynocerus. 259.

Coryphium. 261.

Coryssomerus. 141.

Cossonus. 128.

Cossyphus. 222.

Coxelus. 65.

Cratomerus. 106.

Cratonychus. 107.

Cratoparis. 171.

Creophilus. 249.

Crepidodera. 190.

Criocephalum. 76.

Crioceris. 113. 187.

Criomorphus. 176.

Cryphalus. 126.

Cryptarcha. 64. 266.

Crypticus. 223.

Cryptobium. 253.

Cryptocephalus, 200.

Cryptohypnus. 110.

Cryptophagus. 68.

Cryptopleurum. 53.

Cryptorhynchus. 136.

Crypturgus. 126.

Cteniceras. 111.

Ctenicerus. 110.

Ctenidion. 114.

Cteniopus. 225.

Ctenistes. 238.

Ctenonychus. 107. 112.

Ctenopus. 227.

Cucujus. 67.

Curculio. 128.

Curimus. 76.

Cyaniris. 197.

Cybister. 44.

Cybocephalus. 64.

Cychrammus. 63.

Cychrus. 16. 263.

Cyclonotum. 52. 266.

Cylidrus. 120.

Cylindra. 125.

Cillidium. 52.

Cyllodes. 64.

Cymindis, 20, 263. 
Cynegetis. 209.

Cyngetis. 209.

Cyphogenia. 216.

Cyphon. 113.

Cyphonota. 102.

Cyphonotus. 92

Cyphosoma. 102.

Cyrtocephalus. 60.

Cyrtonus. 192. 27.

Cyrtusa. 59.

Cylillus. 76.

Dacne. 70.

Dailognata. 210. 271.

Dapsa. 209.

Daptus. 35.

Dascillus. 113. 162.

Dasycerus. 72.

Dasysterna. 93.

Dasytes. 118. 269.

Decamera. 97.

Deleaster. 258. 275.

Deliphrum. 260.

Demetrias. 20.

Demetrius. 20.

Dendarus. 219.

Dendroctonus. 127.

Dendroides. 234.

Dendrophagus. 67.

Dendrophilus. 78.

Denops. 120.

Dereloma. 137.

Dermestes. 73.

Dermestes. 261.

Deroplia. 179.

Deylus. 178.

Diacanthus. 111. 269.

Diachrommus. 36.

Dianous. 255.

Diaperis. 221.

Diastictus. 90.

Dibolia. 192.

Dicerca. 102.

Dices. 231.

Dichomma. 211.

Diglossa. 24.

Dima. 107.

Dinarda. 24.

Dinodes. 25.

Dinoderus. 125.

Diodesma. 6\%.

Diodyrhynchus. 170.

Dionyx. 238.

Diphyllus. 68.
Dircæa. 226.

Disopus. 202.

Ditoma. 65.

Ditomus. 23. 264.

Ditylus. 234.

Dolicaon. $2 \% 3$.

Dolichosoma. 119.

Dolichus. 26.

Dolopius. 112.

Donacia. 185.

Dorcadion. 180. 270.

Dorcatoma. 124. 270.

Dorcus. 80.

Dorylomus. 143. 270.

Drapetes. 106.

Drasterius. 111.

Drilus. 114.

Dromius. 20. 263.

Drusilla. 211.

Drymochares. 176.

Dryophthorus. 128.

Dryops. 234.

Dryops. 54 .

Drypta. 19.

Dyctialotus. 123.

Dyctiopterus. 114. 269.

Dyschirius. 23.

Dytiscus. 43.

Lbæus. 118.

Eccoptogaster. 129.

Ectinus. 112.

Etaphocera. 92.

Elaphrus. 14.

Elater. 110.

Eledona. 222.

Elenophorus. 216.

Ellescus. 142.

Elmis.

Elodes. 113.

Elophorus. 50. ริ1. 6 .

Elytrodon. 147.

Emenadia. 227. 273.

Emphylus. 68.

Emus. 249. 252.

Encephalus. 245.

Endecatomus. 123.

Endomychus. 209.

Endophlœus. 222.

Enedreytes. 171.

Engis. 70.

Enicocerus. 50.

Ennearthron. 124.

Enoplium. 121.
Entomoscelis. 196.

Entypus. 124.

Ephistemus. 69.

Epicauta. 232.

Epicometis. 99.

Epierus. 77.

Epilachna. 208.

Epimeces. 160.

Epistemus. 69. 266.

Epomis. 25.

Epuræa. 62.

Eremnus. 153.

Eretes. 44.

Ergates. 174.

Erirhinus. 142.

Eristhetus. 257.

Erodius. 210.

Erotylus. 70.

Eurstethus. 257.

Eubria. 113.

Euchirus. 268.

Euchlora. 94.

Eucinetus. 114.

Eucnemis. 106. 269.

Eulasia. 100.

Euinolpus. 197.

Eunectes. 44.

Euplectus. 239.

Eupleurus. 86.

Euryporus. 253.

Eurythyrea. 103.

Euryusa. 245.

Eusomatus. 164.

Eusomus. 164.

Eustrophus. 227.

Eutheia. 238.

Eutrapela. 234.

Eutroctes. 34.

Evaniocera. 227.

Exhophthalmus. 109.

Exocentrus. 179.

Exochommus. 203. 271.

Falagria. 240. 274.

Falciger. 152.

Faronus. 238.

Feronia. 29. 30. 31. 32.33.

Formicomus. 235.

Galerita. 19. 22.

Galeruca. 188.

Gasterocerus. 136.

Gastrodus. 159.

Gastrophysa. 196.

Geobius. 91. 
Geobius. $2: 39$

Geodromus. 276.

Geonemus. 159.

Geophilus. 159.

Geopyris. 114.

Georyssus. 75. 267.

Geotrupes. 80.

Gibbium. 122.

Glaphyrus. 101.

Glaresis. 268.

Globicornis. 74.

Glyptoma. 262.

Gnaptor. 217.

Gnorimus. 98.

Golgia. 209.

Gonioctena. 197. 271.

Gonocephalum, 220.

Gracilia. 178.

Grammoptera. 184.

Graphypterus. 22.

Graptodera. 189.

Gronops. 160.

Gryphinus. 203.

Grypidius. 142.

Grypus. 142.

Gymnætron. 130

Gymnodus. 98.

Gymnopleurus. 82.

Gymnusa. 245.

Gynandromorphus. 36 .

Gynandrophthalmus. 199.

Gyrohypnus. 249.

Gyrophæna. 245. 274.

Habrocerus, 247.

Hadrotoma. 74.

Hæmerophygus. 223.

Hæmonia. 186.

Haliplus. 48.

Hallomenus. 227

Halyzia. 208.

Hammaticherus. 174

Hammionus. 109.

Hammonia. 113.

Harmonia. 207.

Harpalus. 37. 265.

Harpognatus. 262.

Hedobia. 121.

Hegeter. 212.

Heliocantharus. 82.

Heliodromus. 211.

Heliopates. 219.

Hellodes. 196.

Helochares. 52.
Helophorus. 50. 265.

Helops. 223. 272.

Heptaphilus. 140

Heptaulaucus. 90. 268.

Hesperophanes, 176.

Hetarius. 78.

Heterocerus. $55,266$.

Heterophaga. 222.

Heterothops, 252.

Hexaphyllus. 80 .

Hippodamia. 205.

Hispa. 187.

Hispalis. 39. 265.

Hister. 77.

Halcorhinus. 153.

Holocnemis. 56 .

Hololepta. 77.

Holoparamecus. 71.

Homalopus, 200.

Homalorhinus, 166.

Homalota. 241, 276.

Hoplia. 97.

Hoplitus. 49. 48.

Hoplonotus. 174.

Hybosorus, 91.

Hycleus. 231.

Hydaticus. 44.

Hydnobius. 59 .

Hydrachna. 48.

Hydræena. 51. 266.

Hydrobius. ๑1. 266.

Hydrochus. 50 .

Hydronomus. 142.

Hydrophilus. 51 .

Hydroporus, 46. 265.

Hydrous. 51.

Hygrobia. 46.

Hygronoma. 21.

Hygrotophila. 70.

Hygrotus. 46 :

Hylastes. 127. 270.

Hylecotus, 125.

Hylesinus, 127. 270.

Hylobius. 158.

Hylochares. 107.

Hylotrupes. 176.

Hylurgus. 127.

Hymenontia. 97.

Hymenoplia. 97. 268

Hypera. 142.

Iyperaspis. 204.

1 yperops. 217.

Ilyphidrus. 48.
Hypoborus. 126.

Hypocœlus, 107. 269.

Hypocyptus. 246.

Hypophlœus. 222.

Hypothenemus. 127.

Hypulus. 226.

Idalia. 206.

Jlybius, 45.

Iphthinus. 223.

Ipidia. 62.

Ips. 64.

Isarthron. 176.

Ischnodes. 110.

Ischnomera. 232.

Ischnosoma. 248.

Isocerus. 218.

Isorhipis. 107.

Julodis. 101.

Labidostomis. 197.198.

Laccobius. 52.

Laccophilus. 46.

Lachnaia. 197. 198.

Lacon. 108. 269.

Læmophlœus. 67.

Lamostenus. 26.

Læna. 218. 272.

Lagria. 234.

Lamia. 179.

Lampra. 103.

Lamprias. 21

Lamprinus. 274.

Lamprosoma. 197.

I.ampyris. 114. 269.

Langelandia. $6 \breve{. ~}$

Laparocerus. 153.

Laria. 62.

Laricobius. 121.

Larinus. 140. 270.

Lasia. 209.

Lasiostola. 213.

Lathridius. 71.

Lathrimæum. 260, 276.

Lathrobium. 253. 275.

Lathrobium. 253. 254

Lathropus. 67.

Latipalpis. 102.

Lebia. 21. 263.

Leichenum. 221.

lejestes. 70 .

Leiochiton. 22.

Leiocnemis. 35.

Leioderes. 175.

Leiodes, 6? 
Leiopus. 178.

Leiosomus. 158.

Leirus. 35. $26 \%$.

Leistus. 15. 263.

Leja. 42. 265.

Lema. 187.

Liparus. 157.

Leptacinus. 249. 275.

Leptidea. 178.

Leptinus. 57.

Leptomorpha. 187.

Leptopalpus. 229.

Leptopus. 93.

Leptura. 181. 271.

Leptus. 67.

Lepyrus. 159.

Lestena. 299.

Lesteua. 258. 259.

Lethrus. 82.

Leucopus. 241.

Licinus. 24. 264.

Lignyodes. 142.

Limnebius. 51.

Limnesius. 68.

Limnichus. 75.

Limnius. 54. 266.

Limobius. 156.

Limonius. 109. 269。

Lina. 195. 271.

Liodes. 60.

Liophlous. 159.

Liparus. 158. 159.

Litargus. 73.

Lithonoma. 189.

Litocharis. 254.

Litodactylus. 139.

Litophilus. 70.

Lixus. 145.

Lomechusa. 245.

Longitarsus. 191.

Lopha. 43.

Lophoma. 211.

Loricera. 24.

Lucanus, 80.

Luciola. 114.

Ludius. 111.

Ludius. 111.

Luperus. 169.

Lycoperdina. 209

Lyctus. 64. 65.

Lycus, 114.

Lydus. 231.

Lygistopterus, 114.
Lymexylon. 125. 270.

Lymnäu. 40.

Lyprus. 138.

Lytta. 231.

Macipus. 130.

Macrocephalus. 171.

Macrocnema. 191.

Macrodes. 111.

Macrolenes. 198.

Macronus. 247.

Macronychus. รั5.

Macroplaa. 186.

Magdalinus. 143.

Malachius. 117. 269.

Malacogaster. 114.

Malacosoma. 188.

Malthinus. 116.

Manticora. 15.

Margus. 222.

Marmoropus. 135̆.

Masoreus. 22. 263.

Mastigus. 122. 270.

Mechoris. 170.

Mecinus. 130.

Megacephala. 13.

Megagenius. 213.

Megagnathus. 66.

Megaloderus, 238.

Megarthrus. 261.

Megasternum. 53.

Megatoma. 74.

Megischia. 225.

Meladema. 44.

Melandrya. 226.

Melanius. 30.

Melanophila. 104.

Melanostola. 214.

Melanotus. 107. 269.

Melasis. 106.

Melasoma. 195.

Meleus. 159

Meligethes. 63.

Melinopterus. 89. 267.

Meloë. 229. 273.

Melolontha. 92.

Melyris. 120.

Meriomus, 159.

Mesagroicus, 163.

Mesites. 129.

Mesosa. 179.

Metallites. 161.

Mezium. 122.

Miarus. 130.
Miccotrogus. 140.

Micralymma. 259.

Micraspis. 203.

Microdera. 211.

Micropeplus. 262.

Microphius. 254.

Micronyx. 140.

Microrhagus. 107.

Microsaurus. 252.

Microtelus. 217.

Microzoum. 221.

Milochus. 57.

Miniops. 159.

Miscodera. 22.

Miscrophara. 60.

Misolampus. 219, 272.

Mniophila. 192.

Matecus. 227.

Molops. 33.

Molorchus. 178.

Molytes. 158.

Monocerus, 235.

Monochamus. 179.

Monocrepidius. 107.

Monohammus. 179.

Monolepta. 189.

Mononychus. 135.

Monopis. 66.

Monotoma. 71.

Mordella. 227. 273.

Morica. 216.

Morimus. 179.

Morychus. 76.

Murenus. 162

Murhorhynchus. 136.

Myas. 33. 264.

Mycetæa. 70.

Mycetina. 209.

Mycetochares. 226.

Mycetoma. 226.

Mycetophagus. 73.275.

Mycetophila. 226.

Mycetoporus. 248.

Mycterus. 234.

Mylabris. 230. 273.

Myllæna. 245.

Myodes. 227.

Myorhinus. 138.

Myrmechixenus. 71 .

Myrmechoxenus. 71. 266.

Myrmecinomus. 68 .

Mvrmecobius. 77.

Myrmedonia. 210. 274. 
Mvrrha. 208.

Mysia. 208.

Nacerdes. 232.

Nanodes. 129.

Nanophyes. 129. 270.

Nastus. 147.

Nataris. 143.

Nebria. 15.

Necrobia. 121.

Necrodes. כ้อ.

Necrophilus. 56.

Necrophorus. 55.

Necydalis. 178.

Necydalis. 116, 233.

Nedyus. 132.

Nematodes. 107.

Nemognatha. 229.

Nemonyx. 170.

Nemosoma. 64.

Neomida. 221.

Nephodes. 225.

Neptaphilus, 129.

Nitidula. 62.

Nitidula. 262.

Nogrus. 44.

Nomius. 204. 263.

Nosodendron. 76.

Nossidium. 266.

Notaphus. 40, 265.

Noterus. 46.

Nothorhina. 176.

Nothus. 232.

Notiophilus. 14. 263.

Notoxus. 235.

Notoxus. 120.

Nundina. 205.

Nycteus. 114.

Oberea. 181. 271.

Obrium. 178.

Ocalea. 240. 274.

Oclina. 123.

Ochodæus. 91.

Ochthebius. 50. 265.

Ochthenomus. 237.

Octotemnus. 124.

Ocypus. 249. 275.

Odacantha. 19.

Odontceus. 81.

OEdemera. 233.

OEdichirus. $25 \%$.

OEnas. 231. 273.

Oiceoptoma. 5 ร็.

()librus. 61. 266.
Oligomerus. 123.

Oligota. 244.

Olisthærus. 259.

Olisthopus. 29. 264.

Olophrum. 260.

Omalisus. 114.

Omalium. 260.

Omalium.258.259.260.261. Pachybrachys. 200.

Omaloplia. 96.

Omaseus. 30. 264.

Omias. 153.

Omophlus. 225.

Omophron. 15.

Omosita. 62.

Omphreus. 26.

Oniticellus. 85.

Onitis. 83. 267.

Onthophagus. 83. 267.

Onthophilus. 79.

Oodes. 25.

Oomorphus. 197.

Oophorus. 111. 263.

Opatrinus. 220.

Opatroides. 272.

Opatrum. 220. 272.

Opetiopalpus. 121.

Ophonus. 36.

Opilus. 120.

Oplocephala. 221.

Orchesia. 227.

Orchestes. 138.

Orectochilus. 49.

Oreina. 195.

Orestia. 70.

Orobitis. 129.

Orophius. 124.

Orphilus. 74.

Orsodachna. 186.

Orsodacna. 186.

Orthocerus. 6 อ̆.

Ortochates. 138.

Orthoptevra. 121.

Oryctes. 91.

Osmoderma. 98.

Osphia. 232.

Othius. 248. 275.

Peryphus. 41. 265.

tiorhynchus. 147. 148.149. Perytclus. 153. 150.

Otophor'us. 86.

oxydromus. 40.

Oxylæmus. 66.

Oxyomus. 90.

Oxypleurus. 176.
Oxyporus. 253.

Oxytelus. 257. 275.

Oxytelus. 257. 258.

Oxythyrea. 99.

Oxystoma. 167.

Pachnephorus. 197. 271.

Pachycerus, 160.

Pachyehila. 211.

Pachygaster. 147.

Pachypterus. 216.

Pachypus. 92.

Pachyrinus. 139.

Pachyscelis. 213.

Pachystola. 179.

Pachyta. 183. 270.

Pæderus. 255.

Paderus. 253. 254.

Pales. 196.

Panagæus. 24.

Pandarus. 219. 276.

Paramecosoma, 69.

Parmena. 179.

Parnus. 54. 266.

Paromalus. 78, 267.

Paropsis. 197.

Patrobus. 26. 264.

Pediacus. 67.

Pedilophorus. 76.

Pedinus. 219. 272.

Pelamis. 167.

Pelecotoma. 273.

Pelecotoma. 227.

Pelmatopus. 226.

Pelophila. 14.

Pelor. 33.

Pelosoma. 53.

Peltarium. 218.

Peltis. 64. 266.

Pentaphyllus. 221.

Pentodon. 92. 268.

Percosia. 34 .

Percus. 32.

Perotis. 102.

Phrdon. 196. 271.

Phonops. 104.

Phalacrus. 60.

Phalacrus. 266.

Phaleria. 121.

Philax. 220.
Oxypoda. 243. 274. 
Philhydrus. 52.

Philonthus. 250. 275.

Philonthus. 252.

Philothermus. 66.

Phlexis. 93.

Phløobium. 262.

Phloobium. 261.

Phløeocharis. 259.

Phloeonæus. 258.

Phlœeophagus. 29.

Phlœopora. 241.

Phlœostichus. 68.

Phløotribus. 127.

Phloiophilus. 120.

Pholicodes. 153.

Phosphøenus. 114.

Phosphuga. 56.

Phratora. 196.

Phryganophilus. 226.

Phtora. 222.

Phylethus. 221.

Phyllobius. $150 \%$

Phyllobrotica. 189.

Phyllocerus. 106.

Phyllognathus. 91.

Phyllopertha. 95. 268.

Phyllotretra. 190.

Phymathodes. 175.

Phytobius. 139.

Phytæcia. 181. 271.

Phytonomus. 156.

Phytosus. 244.

Pimelia, 213. 272.

Pissodes. 144.

Pitophilus. 60.

Placusa. 245.

Plagiodera. 196.

Plagiogonus. 90.

Plagionotus. 176.

Platycerus. 79.

Platydema. 221. 272.

Platyderus. 77.

Platynaspis. 203.

Platynotus. 177:

Platynus. 27.

Platyope. 213.

Platypus. 125.

Platyrhinus. 171.

Platyscelis. 218.

Platysma. 31. 264.

Platysoma. 77.

Platysthethus. 257. 275.

Platytarsus. 164.
Platytomus. 90.

Plectes. 16.

Plectroscelis. 192.

Plegaderus. 79.

Pleurophorus.: 90.

Plinthus. 157.

Pocadius. 63.

Podabrus. 115.

Podagrica. 190.

Precilma. 141.

Pocilus. 29. 264.

Polobius. 46.

Pogonocerus. 234.

Pogonocherus. 179.

Pogonophorus. 15.

Pogonus. 26. 264.

Polistichus. 19.

Polydrosus. 162.

Polydrusus. 162.

Polygraphus. 127.

Polymus. 209.

Polyopsia. 181.

Polyphylla. 92.

Poophagus. 131.

Porthmidius. 107.

Pria. 62.

Prinobius. 174.

Prionus. 174.

Prionychus. 225. 273.

Pristilophus. 111.

Pristonychus. 26. 264.

Probosca. 233. 273.

Procas. 158.

Procerus. 16.

Procirrus. 255.

Procrustes. 16. 263.

Proctophysus. 200.

Prognatha. 259. 276.

Pronomæa. 245.

Propylea. 208.

Prostomis. 66.

Proteinus. 261.

Protophysus. 200.

Psallidium. 166.

Psammøecius. 68.

Psammacus. 48.

Psammodius. 91. 268.

Pselaphus. 238.

Pseudocolaspis. 197.

Pseudopsis. 262.

Psilodema. 100.

Psoa. 125.

Psychidium. 69.
Psylliodes. 191.

Ptenidium. 58.

Pterocoma. 213.

Pteroloma. 56.

Pterostichus. 31. 264.

Ptilinus. 124.

Ptilium. 58.

Ptilophorus, 227.

Ptinus. 122. 269.

Ptochus. 154.

Ptomophagus. 56.

Ptosima. 102.

Purpuricenus. 174. 270.

Pycnomerus. 66.

Pylidrus. 54 .

Pyrochroa. 234.

Pyropterus. 114.

Pytho. 226.

Quedius. 252. 275.

Ragonycha. 116.

Ramphus. 167.

Ramphus. 138.

Raphidopalpa. 189.

Raptor. 26.

Reichenbachia. 238.

Rhagium. 182.

Rhamnusium. 182.

Rhina. 143.

Rhinobatus. 144.

Rhinocyllus. 144.

Fhinodes. 142.143.

Rhinomacer, 270.

Rhinomacer, 270. 233.

Rhinoncus. 131.

Rhinosimus. 234.

Rhinusa. 130.

Rhipiphorus. 227.

Rhizobius. 205.

Rhizopertha. 125.

Rhizophagus. 64.

Rhizophagus. 66.

Rhizotrogus. 93. 268.

Rhopalocerus. 66.

Rhopalopus. 175.

Rhosthax. 212.

Rhynchites. 170.

Rhynchanus. 167.

Rhyncolus. 121.

Rhyncophorus. 128.

Rhysodes. 66.

Rhyssemus. 90. 268.

Rhytidosomus, 131.

Rhytirhinus. $15 \%$ 
Ripidius. 227.

Ropalodontus, 123.

Rosalia. 175.

Rugilus. 254.

Salpingus. 234. 273.

Saperda. 181. 271.

Saphanus. 175.

Saprinus. 78. 267.

Sarrotrium. 65.

Scaphidema, 221.

Scaphidium. 57.

Scaphisoma. 57.

Scaphium. 57.

Scarabæus. 82. 267.

Scarites. 22.

Scaurus. 217. 272.

Sciaphilus. 164.

Scimbalium. 253. 275.

Scirtes. 113.

Scleropterus. 135.

Sclerum. 220.

Scolitus. 15.

Scolytus. 127.

Scopæus. 254. 275.

Scotodes. 226.

Scraptia, 227.

Scutopterus, 44.

Scydmænus. 237. 273.

Scymnus. 204.

Scythropus. 163.

Selenophorus. 36.

Semanotus. 175.

Sepidium. 216. 272.

Serica. 96.

Sericosomus. 112.

Serrocerus. 124.

Serropalpus. 227.

Siagona. 22.

Siagona. 259.

Sibinia. 139. 140.

Sibines. 140. 270.

Silenus. 1 i2.

Silis. 116.

Silpha. 55.

Silpha. 260. 261. 262.

Silusa. 245.

Simplocaria. 75.

Singilis. 21.

Sinodendron. 80.

Sisyphus. 82.

Sitaris. 229.

Sitona. 163.

Sitones. 163.
Sitophilus. 129.

Smaragdina. 199.

Smicronyx, 140.

Solenophorus. 176.

Soronia. 62.

Sospita. 208.

Sparedrus. 234.

Spartophila. 197.

Spercheus. 50.

Spermophagus. 172.

Sphæridium. 53.

Sphariestes. 234.

Sphærites. 56.

Sphærius. 54.

Spheroderma. 192.

Spharulus. 129.

Sphenoma. 243.

Sphenophorus. 129.

Sphenoptera. 105.

Sphindus. 71.

Spharidium. 204.

Sphodrus. 26. 264.

Spondylis. 174.

Staphylinus. 249. 275.

Staphylinus. 249 à 261.

Steatoderus. 111.

Stenaxis. 223.

Stenchorus. 178.

Stenidea. 179.

Stene. 222.

Stenelmis. 55. 266.

Stenelophus. 39. 265.

Stenocorus. 182.

Stenopterus. 178. 270.

Stenorhynchus, 136.

Stenosis. 211.

Stenosis. 217.

Stenosoma. 179.

Stenostola. 181.

Stenostoma. 233.

Stenotrachelus. 225.

Stenus. 255. 275.

Stenus, 25๊. 257.

Steropes. 235.

Steropus. 30. 264.

Stilicus. 254.

Stomis. 33

Stomodes. 154.

Strangalia. 183.

Stromatium. 176.

Strongylus. 63.

Strophosomus, 165.

Stylosomus. 200.
Styphlus. 138.

Subcocinella. 209.

Sunius. 255. 275.

Sybines. $140^{\circ}$.

Sylvanus. 67.

Symbiotes. 70.

Synaptus. 107.

Synbius. 227.

Syncalypta. 75.

Synchita. 65.

Synoxylon. 125.

Syntomium. 259.

Synuchus. 27.

Tachinus. 247, 274.

Tachinus. 252.

Tachierges. 139.

Tachyporus. 246. 274.

Tachypus. 16.

Tachys. 40.

Tachyusa. 241. 274.

Tagenia. 217.

Tagona. 218.

Tamnophilus. 143.

Tanaos. 167.

Tanygnatus. 248.

Tanymecus. 164.

Tanysphyrus. 158.

Taphria. 27.

Tapinotus. 131.

Tarandus. 80.

Tarphius. 266.

Tarsostenus. 120.

Tarus. 20.

Teinodactyla. 191.

Telephorus. 115. 269.

Telephorus. 116.

Telmatophilus. 68.

Temnochila. 64.

Tenebrio. 223.

Tentyria. 211. 271.

Teredosoma. 66.

Teredus. 66.

Teretrius. 79 .

Tetracha. 13.

Tetratoma. 70.

Tetrops. 181.

Teuchestes. 86.

Thalicra. 63.

Thanasimus. 120.

Tharops. 107.

Thea. 208.

Thinobius. 275.

Thoraxophorus. $26^{\circ}$. 
Thorectes. 81.

Thorictus. 77.

Thriptera. 213.

Throscus. 77.

Thyamis. 191.

Thylacites. 165.

Thymalus. 65.

Tibesia. 113.

Tillus. 120.

Timarcha. 193. 271.

Tiresias. 75.

Tituraea. 198.

Tolyphus. 266.

Tomicus. 126. 270.

Tomoderus. 235.

Toxotus. 183.

Trachodes. 138.

Trachyderma. 213.

Trachyphlœus. 154.

Trachys. 106.

Trachyscelis. 221.

Tragosoma. 174.

Trechus. 40. 265.

Triarthron. 59.

Tribalus. 78.

Tribolium. 222. 272.

Trichius. 90.

Trichodes. 120.

Trichonotus. 90.

Trichopigus. 252.

Trichopteryx. 58. 266.
Tricophia. 247.

Tricophius. 247.

Trigonurus. 258.

Trimium. 239.

Trinodes. 74.

Triodonta. 96. 268.

Triplax. 70.

Tripocladus. 125.

Tritoma. 70.

Tritoma. 204.

Trixagus. 77.

Trochalus. 44.

Troglops. 118.

Trogoderma. 75.

Trogophlœus. 258. 275.

Trogosita. 64. 66 .

Trogus. 44.

Tropideres. 172.

Tropiphorus. 157.

Trox. 91. 268.

Trychonix. 239.

Tryphillus. 73.

Tripodendron. 127.

Trypopytis. 123.

Trysibius. 158.

Tychius. 140. 270.

Tychus. 239.

Tyloderes. 147.

Tylodes. 133.

Typhæa. 73.

Tyrus. 238.
Tyttoma. 238.

Ulciota. 67.

Uloma. 222. 272.

Upis. 223.

Urodon. 172.

Valgus. 98.

Velleius. 252.

Vesperus. 182.

Vibidia. 208.

Xantochroa. 232.

Xantholinus. 248. 275。

Xantholinus. 249.

Xyletinus. 124. 270.

Xylita. 226.

Xylobius. 107.

Xylodromus. 260.

Xylolæmus. 66.

Xylœcus. 107.

Xylographus, 123.

Xylopertha. 125.

Xylophilus. 237.

Xylophilus. 107.

Xylmurinus. 124.

Xylonotrogus. 77.

Xyloterus. 127.

Zabrus. 33. 264.

Zeugophora. 186.

Zonitis. 229. 273.

Zophosis. 210.

Zuphium. 19.

Zygia. 120. 


\section{DELENDA ET CORRIGENDA.}

\author{
P. 13 , c. $2,1.19$.
}

Soluta. (Meg.) Dej.

Ajoutez à la synonymie :

Var. Assimilis, Chaudsir. Russ. mer.

$$
\text { P. } 14 \text {, c. } 1,1.47 .
$$

Germanica, Fabr.

Gallia.

Ajoutez à la synonymie : \{ Subtruncata. Chaud. Angustata. Fisch.

Russ. mer. Sibiria.

$$
\text { P. 15, c. 1, 1. } 29 .
$$

Andalusia. Rambur.

$$
\text { Hisp. mer. }
$$

Lisez :

Andalusiaca. Ramb.

Et ajoutez :

Barbara. Chaud.

Hisp. mer.

Et supprimez le no 13.

$$
\text { P. } 16 \text {, c. 1, } 1.34 \text {. }
$$

Attenuatus. Fabr.

Gall. bor.

Réunissez dans la même accolade la ligne 36 .

$$
\text { P. } 17 \text {, c. } 1,1.46 .
$$

Cristoforii. Spence.

$$
\text { Lisez : }
$$

Cristofori. Spence.

$$
\text { P. } 20, \text { c. } 2,1.3 .
$$

Prosta (Odacanth,) Stéven. Russ. mer. Lisez :

Præusta (Odacanth.) Stév. Russ. mer.

$$
\text { P. } 22 \text {, c. } 2,1.14 .
$$

Gerardii. Buquet.

Algiria.

Ajoutez à la synonymie :

Rufa. Chaud.

Et supprimez la ligne 15.
P. 25, c. $2,1.3$.

Circunscriptus. Duft.

Gall. mer.

Lisez :

Circumscriptus. Duft. Gall. mer.

$$
\text { P. } 26, \text { c. 2, 1. } 22 .
$$

Cimmerius. Stéven. Dej. Gracia. Ajoutez à la synonymie :

Elegans. Brullé.

Grocia.

$$
\text { P. } 31 \text {, c. } 2,1.34 \text {. }
$$

Instusii. Lw. Redtenb. Austria. Lisez:

Justisii. Lw. Redtenb. Austria.

$$
\text { P. } 32 \text {, c. 2, 1. } 32 .
$$

Bilineatus.

Sicilia. Lisez :

Bilineatus. Dej.

Sicilia.

$$
\text { P. 33, c. } 2 \text {, 1. } 21 .
$$

Curtus. Latreille.

$$
P \text {. }
$$
Ajoutez à la synonymie:

Curtoides Chaud. Et supprimez la ligne 22.

$$
\text { P. } 34, \text { c. } 2,1.8 \text {. }
$$

Silvicola. Schmidt.

Suecia. Lisez :

Sylvicola. Schmidt. Austria. Ajoutez à la synonymie :

Maritima. Schiodte. Et supprimez la ligne 2\%.

$$
\text { P. } 34 \text {, c. 2, 1. } 39 .
$$

$$
\begin{array}{ll}
\text { Homatapa. (Parr.) } & \text { Corfou. } \\
\text { Lisez : } \\
\text { Hematopa. (Parr.) } & \text { Corfou. }
\end{array}
$$


DELENDA ET CORRIGENDA.

P. 38, c. $1,1.27$.

Qudripunctatus. Dej.

Lisez :

Quadripunctata. Dej.

$$
\text { P. 41, c. 2, 1. } 18 .
$$

Pallidipenne. Illiger. Dej. Gallia. Lisez :

Pallidipenne. 1lliger.

Gallia.

$$
\text { P. } 50 \text {, c. } 2,1.44 .
$$

Bicolor. (Kirby.) Germ. Gall. mer. Lisez :

Bicolon. (Kirby.) Germar. Gall. mer.

$$
\text { P. 5ร, c. 2, 1. } 40 .
$$

Tuberculata, Dej.

Hispania.

Lisez :

Tuberculata. (Dej.). Lucas. Hisp. mer. Et supprimez la ligne 43.

$$
\text { P. } 56, \text { c. } 1,1.40 .
$$

Orientalis. Dej. Cat. Küster. Gracia. Lisez :

Orientalis. (Dej.) Brul. Küst. Gracia.

$$
\text { P. } 60, \text { c. } 1,1.25 .
$$

Var. Staphylacum. Gyll. Suecia. Lisez:

Var. Staphylaum. Gyll. Suecia.

$$
\text { P. } 68, \text { c. 1, 1. } 29 .
$$

Bicolor. Perroud. Comalli, Gall, mer. Lisez :

Bicolor. Perroud. Comolli. Gall. mer.

$$
\text { P. } 71, \text { c. } 2,1.1 .
$$

MYRMECHIXENUS. Chevrol.

\section{Lisez :}

MYRMECOXENUS. Chevrol.

$$
\text { P. } 71, \text { c. } 2,1.25 .
$$

Chlatratus. (Dahl.)

Austria.

Lisez:

Clathratus. (Dahl.)

Austria.

$$
\text { P. } 78 \text {, c. } 1,1.10 .
$$

Mæreus. Erichson. Lisez:

Hœrens. Erichson.

Istria.

Austria.
P. 81, c. 1, 1. 49.

Germinatus, Dej. Cat.

Corsica. Lisez :

Geminatus. Dej. Cat. Corsica.

$$
\text { P. 84, c. 1, 1. } 49 .
$$

Fiscicornis. Stéven.

Gracia. Lisez:

Fissicornis. Stéven. Gracia.

P. 91 , c. 2, 1. 18. OCHODOEUS. (Meg.) Latreille. Lisez :

OCHODAUS. (Meg.) Latreille.

$$
\text { P. } 94 \text {, c. 1, 1. } 27 .
$$

Etuensis. Jan. St. Cat. Sicilia. Lisez:

Etnensis. Jan. St. Cat. Sicilia.

$$
\text { P. } 106 \text {, c. } 2 \text {, 1. } 31 .
$$

Feisthameli. Gräells. Lisez:

Feisthameli. Graëlls.

Hispania.

Hispania.

$$
\text { P. 107, c. 2, 1. } 6 .
$$

MONOCREPIDIUS. Esch. L. Redt. Lisez :

PORTHMIDIUS. Germar.

Et ajoutez :

Monocrepidus. Eschsch. L. Redtenb.

$$
\text { P. } 108, \text { c. } 1, \text { I. } 5 \text {. }
$$

Chrysoprasa. Herbst.

Austria.

Lisez :

Chrysoprara. Herbst. Austria.

$$
\text { P. } 112 \text {, c. } 1,1.9 \text {. }
$$

Inonatus. (Gaubil.)

Algiria.

Lisez :

Inornatus. (Gaubil.) Algiria.

$$
\text { P. 115, c. } 1,1.44 .
$$

Opacus. Germar.

Substituez à cette espèce:

Oralis. Germar.

Austria.

Germania.

$$
\text { P. } 117, \text { c. 1, 1. } 17 .
$$

APALOCRUS. Erich. Lisez: 
P. 118, c. 2, I. 4. ATTELESTUS. Erich.

Lisez :

ATELESTUS. Erich.

P. 124, c. $2,1.31$.

Cylindricub. Germar.

Lisez :

Cylindricus. Germar.

$$
\text { P. 124, c. 2, 1. } 46 .
$$

Pectinatus. Linné. $\quad \boldsymbol{P}$.

Lisez :

Pectinicornis. Linné.

P.

P. 127, c. 2, 1. 20.

DENTROCTONUS. Erichson.

\section{Lisez :}

DENDROCTONUS. Erichson.

$$
\text { P. } 128 \text {, c. } 2 \text {, 1. } 25 .
$$

Ovalis. (Marietté.)

Lisez :

Ovalis. (Marietti.)

Lombardia.

Lombardia.

$$
\text { P. } 130 \text {, c. 1, 1. } 42 .
$$

Labialis. Herbst.

Lisez :

Labilis. Herbst.

Gallia.

Gallia.

$$
\text { P. } 130 \text {, c. } 1,1.44 .
$$

obiquus. (St. Cat.)

Lisez :

Obliquus. (St. Cat.)

Germania.

Germania.
P. 137, c. 2, 1. 26.

Linnosus. Gyllenh. Lisez:

Limosus. Gyllenh.

Suecia.

Suecia.

$$
\text { P. 137, c. 2, 1. } 43 .
$$

Biglyptus. Germar.

Saxonia.

Lisez :

Diglyptus. Germar.

Saxonia.

$$
\text { P. } 138 \text {, c. } 1 \text {, 1. } 3 .
$$

Binotutus. Steph.

Anglia.

Lisez:

Binotatus. Steph.

Anglia.

P. 138 , c. $2,1.8$.

Lisez :

RaMpUUs. Thunberg.

RamphUs. Thunberg.

P. 139 , c. $1,1.6$.

Laniceræ. Fabr.

Germania.

Lisez :

Loniceræ. Fabr.

Germania.

P. 139 , c. 2 , 1. 7.

Fitidulus. Steph. var.

Anglia.

Lisez:

Nitidulus. Steph. var. Anglia.

P. 142, c. 1, 1. 5 .

Pomonæ. Linné.

Gallia.

Lisez :

Pomorum. Linné.

Gallia.

$$
\text { P. } 132 \text {, c. } 1,1.25 .
$$

P. 142, c. 1, 1. 10.

Albicinetus. Schh.

Gallia.

Lisez :

Albicinctus. Schh.

Gallia:

P. 132 , c. $2,1.7$.

Alauda. Fabr.

Gallia.

Lisez :

Alanda. Fabr.

Gallia.

P. 132 , c. 2 , 1. 10.

Alauda. Herbst.

Lisez :

Alanda. Herbst.

Germania.

Gallia.

P. 134, c. 1, 1. 31 .

Alauda. Fabr.

Gallia.

Lisez :

Alanda. Fabr.

Gallia.
Pomarum. var, b. Gyll. Suecia. Lisez :

Pomorum. var. b. Gyll. Suecia.

$$
\text { P. } 148, \text { c. } 2 \text {, 1. } 49 \text {. }
$$

Polycocos. Schh.

Constantinp. Lisez .

Polycocus. Schh.

Constantinp.

P. 149, c. $2,1.50$.

Geminatus. Fabr. Styria. Lisez :

Gemmatus. Fabr.

Germania.

$$
\text { P. } 150 \text {, c. } 1 \text {, } 1.19 \text {. }
$$

Transversempressus. (Chevr. Sch.) Algiria. Lisez :

Transverseimpressus. (Chevr. Sch.)Algiria. 


$$
\text { P. } 153 \text {, с. } 2,1.8 \text {. }
$$

Noscius. Chevrol. Sch.

Italia.

Lisez :

Noxius. Chevrol.Sch. Italia.

$$
\text { P. } 154, \text { c. } 2,1.43 .
$$

Fasciculatus. Gebler.

Russ. mer.

Lisez:

Fasciolatus. Gebler.

Russ. mer.

$$
\text { P. } 156 \text {, c. 1, 1. } 25 .
$$

Circumvagus. (Chevr.) Algiria.

Supprimez ici cette espèce et voyez le $\mathbf{n}^{\circ} \mathbf{2 5}$ du même genre.

$$
\text { P. } 156, \text { c. } 1,1.27 \text {. }
$$

Danci. Olivier.

Lisez :

Dauci, Olivier.

Algiria.

Algiria.

$$
\text { P. } 160 \text {, c. } 2, \text { 1. } 4 \text {. }
$$

Dalbatus, Linné.

Suecia.

Lisez :

Dealbatus, Linné.

$$
\text { P. } 160 \text {, c. } 2,1.5 .
$$

Rodirus, Voet.

Suecia.

Lisez :

Roridus. Voet.

Suecia.

$$
\text { P. } 162 \text {, c. } 2,1.39 .
$$

Voluptiferus, Schönh.

Austria. Lisez :

Voluptificus. Schönh.

Austria.

$$
\text { P. 163, c. 1, 1. } 26 .
$$

Tennis. Rosenhauer.

Tyrolis.

Lisez :

Tenuis. Rosenhauer. Tyrolis.

$$
\text { P. } 165, \text { c. } 1,1.4 \text {. }
$$

Carinatus, Oliv. Dej. Cat. Lusitania. Lisez :

Carinula. Oliv. (Dej.)

Lusitania.

$$
\text { P. } 166, \text { c. 1, I. } 26 .
$$

Rignus. Erichson. Lisez :

Riguus, Erichson.

Algiria.
P. 167, c. $1,1.1$. AMOPHOCEPHALUS, Schh. Lisez :

AMORPHOCEPHALUS. Schh.

$$
\text { P. } 167 \text {, c. } 2,1.14 \text {. }
$$

Tennæ. Kirby. Steph.

Gallia.

\section{Lisez:}

Tenuæ. Kirby. Steph. Gallia.

$$
\text { P. } 169 \text {, c. } 2, \text { ]. } 12 .
$$

Translaticeum. Schh.

German.bor. Lisez:

Translatitium. Schh. German, bor.

$$
\text { P. } 170 \text {, c. } 1,1.44 \text {. }
$$

Violacipenne. Dej. Cat. Dalmatia. Lisez :

Violaceipenne. Dej. Cat. Dalmatia.

$$
\text { P. 174, c. 1, 1. } 23 .
$$

MACROTOMA. Dej. Cat. Serville. Substituez à ce genre le genre : PRINOBIUUS. Mulsant.

$$
\text { P. } 174, \text { c. } 2,1.27 .
$$

Nodicornis. Küster. $\quad N$.

$$
\text { Lisez : }
$$

Nodicornis. Küster. Dalmatia.

$$
\text { P. } 174 \text {, c. } 2,1.28 .
$$

Carinatus. Küster. _ N N.

$$
\text { Lisez : }
$$

Carinatus. Küster. Dalmatia.

$$
\text { P. } 175, \text { c. } 2,1.37 .
$$

Similare. Küster.

\section{Germania.}

$$
\text { Lisez : }
$$

Similare, Küster.

Dalmatia.

$$
\text { P. } 180 \text {, c. } 1,1.10 .
$$

Hirsuta. Küster. N.

$$
\text { Lisez: }
$$

Hirsuta. Küster. Dalmatia.

$$
\text { P. } 180, \text { c. } 2,1.8 \text {. }
$$

Axillare. Küster.

Italia.

Lisez:

Axillare. Küster.

Turcia.

$$
\text { P. } 180, \text { c. 2, 1. } 12 .
$$

Sulcipenne. Küster.

$$
\text { Lisez : }
$$


P. 181, c. $1,1.40$.

Tiliæ. Küster.

Lisez :

Tiliæ. Küster.

P. 181 , c. $2,1.32$.

Cephalotes. Küster.

Lisez :

Cephalotes. Küister.

Gracia.

P. 182, c. $1,1.22$.

Saperda, Fabr. Augapanthia, Serville. Lisez :

Saperda. Fabr, Agapanthia. Serville P. 184 , c. 1 , I. 6.

Strangulata, Ill. Germ.

Lusitania.

Supprimez cette ligne et voyez le $n^{\circ} 23$ du genre Leptura.

$$
\text { P. } 184 \text {, c. } 2,1.25 .
$$

Strangulata. Germ. Charp. Pyr. or. Lisez :

Stragulata. Germ. Charp. Pyr, or. P. 184, c. $2,1.35$.

ANAPLODERA. Mulsant.

Lisez:

ANOPLODERA. Mulsant.

$$
\text { P. } 193, \text { c. } 1,1.12 .
$$

Confragoso. Buquet.

Lisez:

Confragosa. Buquet.

Algiria.

Algiria.

P. 193, c. 2, 1. 21.

Gennensis. Dej. Cat.

Italia.

Lisez:

Genuensis. Dej. Cat.

Italia.

P. 193, c. 2, 1. 42.

Globosa. Meg. St. Cat. Illyria.

Supprimez cette ligne et voyez le $n^{\circ} 34$ du genre $T i$ marcha.

$$
\text { P. } 198, \text { c. } 1,1.51 \text {. }
$$

Foncipifera, Lej.Cat.Lucas, Algiria. Lisez:

Forcipifera, Dej. Cat,Lucas. Algiria,
P. $211 ;$ c. $2,1.47$.

Livicollis. Solier.

Corsica.

Lisez :

Lævicollis. Solier. $\quad$ Corsica.

P. 216, c. 2, 1. 42.

Discoideus. Schönherr.

Hispania.

Supprimez cette ligne et voyez la ligne 8 , même page, mểme colonne.

P. 217, c. $1,1.37$.

HYPEROPS. Eschscholtz.

Ce genre faisant double emploi, supprimez-le ici et voyez la p. 211.

$$
\text { P. } 217, \text { c. } 2,1.36 \text {. }
$$

Eymondi. Solier.

Algiria.

Lisez :

Emondi. Solier.

Algiria.

P. 217 , c. $2,1.43$.

Producta. Dej. Fisch.

Gall. mer.

Lisez :

(Producta. (Dej.) Brul. Sol. Gall. mer.

Gigas. Olivier. id.

P. 218, c. 1, 1. 15.

Australis. Dej. Cat. Italia.

Lisez:

(Australis. (Dej.) Solier. Corsica.

Var. A. Planicollis, Sol. Sicilia.

(Var. B. Impressicollis.Sol.id.

P. 218, c. 2, 1. 12.

Ramburii. Dej. Cat.

Hisp. mer.

Lisez :

Ramburii. (Dej.) de Brême. Hisp. mer.

P. 220 , c. 1, 1. 25.

Striatus. Solier.

Supprimez cette ligne et voyez la ligne 12.

$$
\text { P. } 220 \text {, с. } 2,1.11 .
$$

Perlatum. Dej. Cat.

Hisp. mer.

Lisez:

Perlatum. (Dej.) Germar. Hisp. mer.

P. 223 , c. $2,1.35$.

Lacertosus. Dej. Cat. Smyrna. Lisez:

Lacertosus. (Dej.) Küster, Smyrna. 
P. 224, c. 1, 1. 20.

Celestinus. Klug.

Lisez :

Coelestinus. (Klug.) Waltl. Constantinp. Wagneri. Küster.

\section{P. 224, c. 1, 1. 36.}

Subrugosus. Creutzer.

Lisez :

\{ Schmidtii. Ahrens.

$\{$ Subrugosus. Duftsch.

$$
\text { P. } 225, \text { c. } 2,1.31 .
$$

Curvipes. Dej. Cat.

Gall. mer. Lisez :

Curvipes. (Dej.) Brullé. $\quad$ Gall. mer.

$$
\text { P. 226, c. 2, 1. } 19 .
$$

Quadriguttata. Fabr.

Supprimez cette espèce et voyez le no 6 du même genre.

Sturmii. Küster.

$$
\text { P. 227, c. } 2,1.28 .
$$

\section{Lisez :}

Sturmii. Küster.

P. 228, c. $1,1.51$ et 52 .

Decora. (Chevrolat.) Lucas. Algiria. Lisez :

Decora. Chevrolat. Lucas. Algiria.

$$
\text { P. } 229 \text {, c. 1, 1. } 35 .
$$

Necydalens. Pallas.

Lisez :

Necydaleus. Pallas.

$$
\text { P. } 230 \text {, c. 2, 1. } 42 .
$$

Affinis. Lucas.

Lisez :

Vicina, Lucas.

$$
\text { P. } 231, \text { c. } 1,1.42 .
$$

Similio. Sturm. Cat. Lisez:

Similis. Sturm. Cat.

Tyrolis.

Tyrolis.

$$
\text { P. } 231, \text { c. } 2,1.23 .
$$

Varians, Dej. Cat.

Lisez:

Varians. (Dej.) Schh.

Russia.

Russ. mer.

Algiria.

Algiria. 

P. 243, c. 2 , 1. 28.
P. 257, c. 1, 1. 32.

Procelura. Mannerh.

\section{Lisez:}

Procerula. Mannorh. P. 243 , c. $2,1.45$.

Mirmecophila. Märkel.

Lisez :

Myrmecophila. Märk.

$$
\text { P. } 245 \text {, c. } 1 \text {, 1. } 36 .
$$

Infirma. Erich.

$$
\text { Lisez: }
$$

Infima, Erich.

$$
\text { P. 245, c. 2, 1. } 28 .
$$

Cornuroides. Mathews. Anglia.

\section{Lisez:}

Conuroides, Mathews. Anglia.

$$
\text { P. } 251 \text {, c. } 1 \text {, l. } 3 .
$$

Luceus. Mannerh.

Germania.

- Lisez :

Lucens. Mannerh.

Germania.

$$
\text { P. } 251 \text {, c. } 2,1.29 .
$$

Quisquiliarus. Gyll. Lacord. Gallia.

$$
\text { Lisez : }
$$

Quisquiliarius. Gyll. Lacord. Gallia.

$$
\text { P. 253, c. 1, 1. } 27 .
$$

Obliteratus. Erich.

$$
\text { Sardinia. }
$$

Lisez:

Oblitteratus. Erich.

$$
\text { P. } 254 \text {, c. 1, } 1.34 \text {. }
$$

Dilitum, Erich.

Lisez :

Dilutum. Erich.

$$
\text { P. } 256 \text {, c. } 1,1.35 \text {. }
$$

\section{Cantus. Erioh.}

Lisez :

Cautus. Erich.

\section{Sardinia.}

German. sep. German. sep.

Berolini.

Berolini.
Procelurus. Erich.

Austria. Lisez :

Procerulus, Erich.

Austria.

$$
\text { P. 257, c. 1, 1. } 49 .
$$

Pigmæus, Erich.

\section{Gallia.} Lisez:

Pygmæus, Erich.

Gallia.

$$
\text { P. 257, c. } 2,1.11 .
$$

Debelis. Erich.

Russ. mer. Lisez :

Debilis. Erich.

Russ. mer.

P. 257 , c. 2 , 1. 13 , et p. 275 , c. $2,1.18$.

PLATISTHETUS. Mannerh. Lisez :

PLATYSTHETUS. Mannerh.

$$
\text { P. } 258 \text {, c. } 1,1.10 .
$$

Sculptarus. Gravenh.

Germania. Lisez :

Sculpturatus. Gravenh. Germania.

$$
\text { P. } 260 \text {, c. } 1,1.27 .
$$

Alpestre. Heer.

Helvetia. Lisez :

Alpestre. Erich.

Germania.

$$
\text { P. } 261 \text {, c. } 2,1.39 \text {. }
$$

Var. Lineatocollis. Lacord. $P$. Lisez :

Sinuatocollis, Lacord. $\quad-P$.

$$
\text { P. } 264 \text {, c. } 1,1.17 .
$$

PRISTONICHUS. Dej.

\section{Lisez :}

PRISTONYGHUS, Dej. 


\title{
RESULTAT
}

DES

\section{Observations sur les genres Trachyphlous, Omias, Otiorhyn- chus, Sitones, Cneorhinus et Strophosomus d'Angleterre, par M. John WaLton.}

\author{
(Annals of nat. hist., vol. XIX, p. 217, 314, 445.) \\ P. 147, c. 2, 1. 26. \\ P. 150, c. $2,1,42$.
}

\section{OTIORHYNCHUS :}

0. Caudatus. Rossi. Ajoutez à la synonymie de cette espèce :

\{ Lima. Marsh.

\{Bisulcalus. Steph.

Anglia.

id.

$$
\text { P. } 148 \text {, c. } 1,1.25 .
$$

0. Fuscipes. Olivier. Ajoutez :

(Fagi. (Chevrol.) Schh. Hypolaus. Kirby.

Sacer. Kirby.

Ilalia.

$$
\text { P. } 150, \text { c. } 2,1.9 .
$$

O. Atroapterus, de Geer. Ajoutez :

Ater. Steph.

$\{$ Niger. Steph.

Arenarius. Kirby.

$$
\text { P. } 150 \text {, c. } 2,1.13 .
$$

0. Nonticola. Germar.

$$
\text { Ajoutez: }
$$

Lavigatus. Gyll, Sehh.
Gall. mer.

Gallia.

Anglia.

id.

Pomerania.

Anglia.

id.

id.

Germania.

Suecia.
0. Picipes. Fabr. Ajoutez :

Vastator. Marsh. Anglia.

Notatus. Steph. id.

Septentrionis. Steph. id.

Marquardtii. Schönh. id.

Chevrolati. Schh. var. id.

$$
\text { P. 152, c. 1, 1. } 8 \text {. }
$$

o. Verticosus. Schönh. Gallia.

Lisez:

Ovatus. Linné. F. Gallia.

$\{$ Vorticosus. (Chev.) Schh. id.

$22^{\text {bıs }}$ (Pabulinus. Panzer. $\left\{\begin{array}{r}\text { Germar. Germania. } \\ \text { Confinis. Kirby. Anglia. }\end{array}\right.$

P. 154 , c. 2, I. 6.

Trachyphloeus :

T. Scabriculus. Schönh. $\quad \boldsymbol{P}$. Lisez:

(Scaber.L.(Mus. Lin.) Germ.P.

Tesselatus. Marsh. Steph. Anglia.

Confinis. Steph. $\quad$ id.

Scabriculus. Payk. Gyll.

Schh. non Linné. P.

Nigricans. Steph. Kirby.

(Stroph.) Anglia.

Grisescens. Kirb. (Thylacit.) id. 


$$
\text { P. } 154, \text { c. } 2,1.13 .
$$

T. Waltoni. Schh. Ajoutez :

Anglia.

Ventricosus. Stph, non Germ. Anglia. P. 154, c. 2, 1. 20.

T. Squamulatus. Oliv. Gallia. Ajoutez :

(Aristatus. Gyll. Schh.

Stipulatus. Germar.

(Setosus, Kirby.

Anglia.

Anglia.
1) 154 , c. $2,1.17$.

T. Setarius. Schönh.

Scabriculus. L: F. Mus.

Banks. Germania.

Germania. Lisez :

of Setarius. Sehh. id.

Scaber. Schh. Gallia.

Maculatus, var. $\beta$. (Schh.) id.

Germania.

Digitalis. Steph.

Occultus. (Chevrol.)
Anglia.

Gallia.

(Annals of nat. hist., vol. XVII, p. 227 and 304.)

P. 163 , c. $1,1.21$.

Sitones :

S. Griseus. Fabr.

Ajoutez :

\{ Fuscus. Marsh.

$\{$ Trisulcus. Kirby.

$$
\text { P. } 163 \text {, c. } 1,1.31 \text {. }
$$

S. Regensteinensis. Herbst. $\boldsymbol{P}$. Ajoutez :

Spartii, Femoralis et

$\left\{\begin{array}{r}\text { Pleuritica. Steph. } \\ \text { Spartii. Kirby. }\end{array}\right.$

Anglia.

id.

P. 163, c. 1, 1. 41.

S. Tibialis. Herbst.

Gallia.

Ajoutez:

(Chloropus. Marsh.

Anglia.

Lineellus. Gyll. Schh.

Ambiguus, var. Schh.

Albescens, Affinis et $\mathrm{Li}$ -

Germania. id. neatulus. Kirby. Anglia.

24 ${ }^{\text {bis }}$ Waterhousei. (Schh.)

Walton. id.

$$
\text { P. } 163 \text {, c. } 2,1.1 \text {. }
$$

S. Sulcifrons. Thunb.

\section{Suecia.}

Ajoutez :

fChloropus, Marsh.?

Subauratus. Kirby.

Plewriticus. Kirby.

Anglia.

id.

id.

$$
\text { P. } 163 \text {, c. } 2,1.6 \text {. }
$$

S. Crinitus, Oliv. Dej. Cat. Gallia. Ajoutez:

$\begin{cases}\text { Lineellus, var. Steph, } & \text { Anglia. } \\ \text { Albescens. var. Steph, } & \text { id. } \\ \text { Macularis, Marsh. } & \text { id. }\end{cases}$
P. 163, c. $2,1.14$.

S. Octopuntatus. Schh. Gallia.

Lisez :

Flavescens. Marsh. Anglia.

Ajoutez :

8.Punctatus. Schh. Gallia.

Nigriclavis Marsh. Anglia.

Longiclavis. Marsh. id.

Griseus. Kirby. id.

Lineatus. Fabr. (Mus.

Banks.)

P. 163 , c. $2,1.30$.

S. Lineatus. Linné.

Lisez :

(Lineatus. L. (Mus. Linn.)

F. Germ. Schh. Gallia. Ajoutez :

Ruficlavis. Marsh. Anglia.

Griseus. Marsh. id.

Griseus. v. $\beta$. Kirby. id.

$$
\text { P. } 163 \text {, c. } 2,1.48 .
$$

S. Hispidulus, Fabr.

P. Ajoutez :

Pallipes. Steph.

Anglia.

$$
\text { P. } 164 \text {, c. } 1,1.9 \text {. }
$$

S. Puncticollis. Kirby. Anglia. Ajoutez:

(8-Punctatus. Germar. Gallia. Insulsus..Schh.

Flavescens, $v$. Kirby. Anglia.

$$
\text { P. } 164, \text { c. } 1,1.11 .
$$

S. Suturalis. Steph.

Ajoutez :

Anglia.

Rufipes. Marsh, non Linné. Anglia. 


$$
\text { P. } 164, \text { c. } 1,1.13 .
$$

S. Pisi. Stephens. Lisez :

(Humeralis. Kirby.

Pisi. Steph.

Promptus. Schh.

$63^{\text {bis }}$ Meliloti. Walton.

$$
\text { P. } 164, \text { c. } 1,1.16 .
$$

S. Cambricus. Kirby. Anglia. Ajoutez:

\{ Cribricollis. Schh. Austria.

\{ Rugulosus. (Dillw.) Kirb. Anglia.

$$
\text { P. } 165, \text { c. } 1,1.19 .
$$

Strophosomus :

S. Coryli. Fabr. Mus. Banks. P. Ajoutez:

$\left\{\begin{array}{l}\text { Illibatus. Schh. } \\ \text { Coryli. Marsh. Gyll. } \\ \text { Obesus. Marsh. } \\ \text { Cognatus. Steph. } \\ \text { Cervinus. Fabr. }\end{array}\right.$

\section{Gallia.} Anglia.

id.

id.

id.

$$
\text { P. } 165 \text {, c. } 1,1.23 .
$$

\section{S. Alternans. Schh.}

\section{Lisez :}

Gall. mer.

(Retusus. Marsh. Steph. Anglia. Squamulatus. Steph.

Alternans. Schh.

id.

Gall. mer

Oxyops. (Chevrol.) Schh. Gallia.

Obesus. v. $\alpha$. $\beta . \gamma$. Kirby. Anglia.

$$
\text { P. 165, c. 1, 1. } 24 .
$$

S. Faber. Herbst.

$$
\text { Ajoutez : }
$$

(Chctoporus, Steph.

Limbatus. Marsh.

Septentricnis. Steph.

Sus. v. $\alpha . \beta$. Kirby.

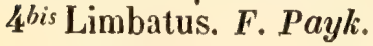

$P$.

Anglia.

id.

id.

id.

Gallia.
P. 165 , c. $1,1.43$.

S. Rufipes. Steph.

Anglia.

Lisez :

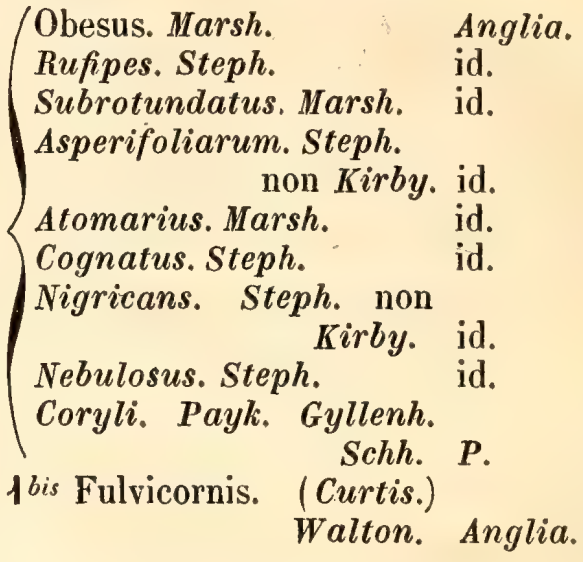

P. 165, c. 2 , I. 13 .

\section{Coneorhinus :}

C. Geminatus. Fabr.

Gallia.

Ajoutez :

$\begin{cases}\text { Albicans. Schh. } & \text { Gall. occid. } \\ \text { Parapleurus. Marsh. } & \text { Anglia. } \\ \text { Maritimus. Marsh. } & \text { id. } \\ \text { Scrobiculatus. Marsh. } & \text { id. }\end{cases}$

$$
\text { P. } 165 \text {, c. } 2,1.18 .
$$

C. Exaratus. Marsh.

Anglia.

Ajoutez :

\{ Plumbeus. Marsh.

6-Striatus, Marsh.
Anglia. id. 


\section{ESPÈGES NOUVELLES}

publiées par M. Léon Fairmaire, dans les Annales de la Sociélé entomologique de France.

P. 21, c. 2, avant le genre Aptinus, P. 160, c. 2, après la lig. 17, ajoutez: ajoutez:

9bis Helferi. L. Fairm. Sicilia. COPTODERA. Dejean.

1 Massiliensis, L. Fairm. Gall. mer.

P. 113 , c. 1, après la lig. 7 , ajoutez: $1^{\text {bis }}$ Benedicti, L. Fairm. Sicilia.

P. 175 , c. 1, après la lig. 36 , ajoutez : $2^{\text {bis }}$ Cylindraceus. L. Fairm. Hisp. sept.

P. 226 , c. 2, après la lig. 34 , ajoutez : $10^{\text {bis }}$ Griseoguttata. L. Fairm. $P$.

P. 160, c. $\mathbf{2}$, après la lig. 5 , ajoutez :

P. 234, c. 2, après la líg. 2, ajoutez: $2^{\text {bis }}$ Tesselatus. L, Fairm. Hisp. mer, 4 Kiesenwetteri. L. Fairm, Sicilia. 



20. $3 x$

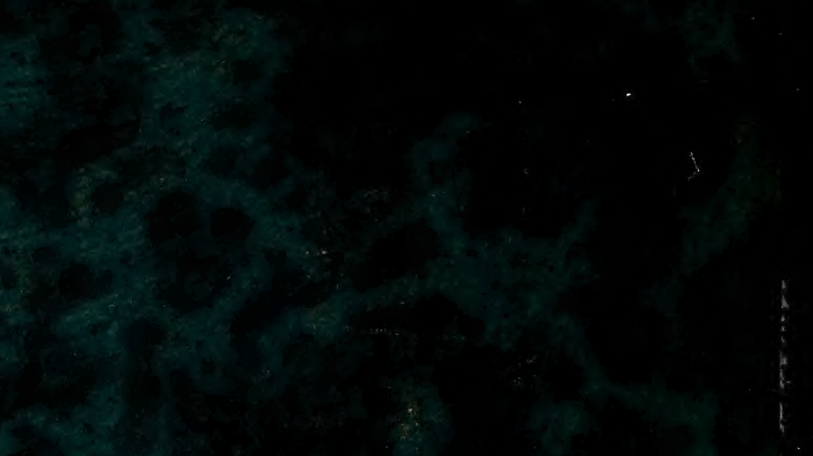

s.

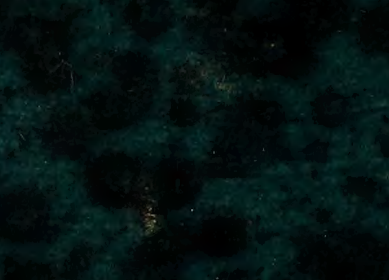

19.9

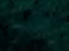

$2 \ln ^{2}+15$

13

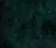

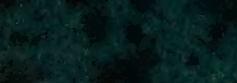

2.

$3 x+3$
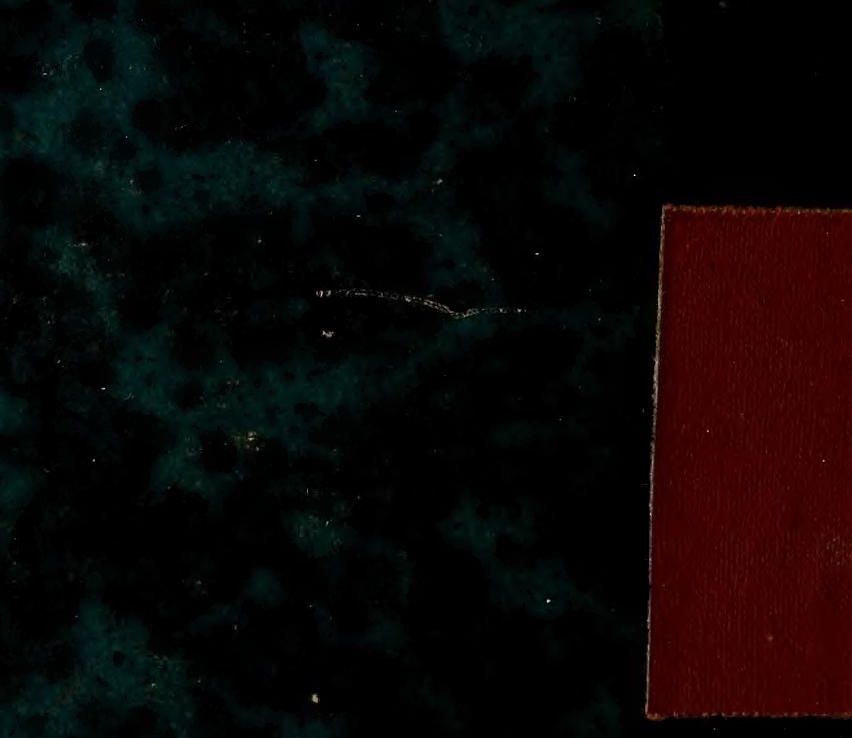